\title{
atmosphere
}

Atmospheric

Composition

Observations

Edited by

Armin Sorooshian

Printed Edition of the Special Issue Published in Atmosphere 
Armin Sorooshian (Ed.)

\section{Atmospheric Composition Observations}


This book is a reprint of the Special Issue that appeared in the online, open access journal, Atmosphere (ISSN 2073-4433) from 2015-2016, available at:

http://www.mdpi.com/journal/atmosphere/special_issues/Atmospheric_Composit ion_Observations

Guest Editor

Armin Sorooshian

Department of Chemical and Environmental Engineering

University of Arizona

USA

Editorial Office

MDPI AG

St. Alban-Anlage 66

Basel, Switzerland

Publisher

Shu-Kun Lin

Managing Editor

Lucy Lu

\section{Edition 2016}

MDPI • Basel • Beijing • Wuhan • Barcelona • Belgrade

ISBN 978-3-03842-284-6 (Hbk)

ISBN 978-3-03842-285-3 (PDF)

Articles in this volume are Open Access and distributed under the Creative Commons Attribution license (CC BY), which allows users to download, copy and build upon published articles even for commercial purposes, as long as the author and publisher are properly credited, which ensures maximum dissemination and a wider impact of our publications. The book taken as a whole is @ 2016 MDPI, Basel, Switzerland, distributed under the terms and conditions of the Creative Commons by Attribution (CC BY-NC-ND) license (http://creativecommons.org/licenses/by-nc-nd/4.0/). 


\section{Table of Contents}

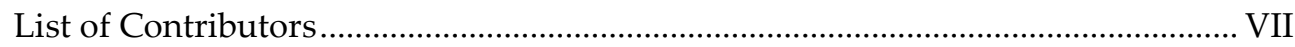

About the Guest Editor........................................................................................... XII

Stefano Falcinelli, Fernando Pirani and Franco Vecchiocattivi

The Possible Role of Penning Ionization Processes in Planetary Atmospheres

Reprinted from: Atmosphere 2015, 6(3), 299-317

http://www.mdpi.com/2073-4433/6/3/299. 1

Seung-Myung Park, Beom-Keun Seo, Gangwoong Lee, Sung-Hyun Kahng and Yu Woon Jang

Chemical Composition of Water Soluble Inorganic Species in Precipitation at Shihwa Basin, Korea

Reprinted from: Atmosphere 2015, 6(6), 732-750

http://www.mdpi.com/2073-4433/6/6/732

Evgueni Kassianov, Larry K. Berg, Mikhail Pekour, James Barnard, Duli Chand, Connor Flynn, Mikhail Ovchinnikov, Arthur Sedlacek, Beat Schmid, John Shilling, Jason Tomlinson and Jerome Fast

Airborne Aerosol in Situ Measurements during TCAP: A Closure Study of

Total Scattering

Reprinted from: Atmosphere 2015, 6(8), 1069-1101

http://www.mdpi.com/2073-4433/6/8/1069. .44

Grzegorz Majewski, Wioletta Rogula-Kozłowska, Piotr O. Czechowski, Artur Badyda and Andrzej Brandyk

The Impact of Selected Parameters on Visibility: First Results from a Long-Term Campaign in Warsaw, Poland

Reprinted from: Atmosphere 2015, 6(8), 1154-1174

http://www.mdpi.com/2073-4433/6/8/1154 82 
Haider Abbas Khwaja, Omar Siraj Aburizaiza, Daniel L. Hershey, Azhar Siddique, David A. Guerrieri P. E., Jahan Zeb, Mohammad Abbass, Donald R. Blake, Mirza Mozammel Hussain, Abdullah Jameel Aburiziza, Malissa A. Kramer and Isobel J. Simpson

Study of Black Sand Particles from Sand Dunes in Badr, Saudi Arabia Using Electron Microscopy

Reprinted from: Atmosphere 2015, 6(8), 1175-1194

http://www.mdpi.com/2073-4433/6/8/1175.....

\section{Zhihui Wu, Fenwu Liu and Wenhua Fan}

Characteristics of PM10 and PM2.5 at Mount Wutai Buddhism Scenic Spot, Shanxi, China

Reprinted from: Atmosphere 2015, 6(8), 1195-1210

http://www.mdpi.com/2073-4433/6/8/1195.

Hugo Saldarriaga-Noreña, Rebecca López-Márquez, Mario Murillo-Tovar, Leonel Hernández-Mena, Efrén Ospina-Noreña, Enrique Sánchez-Salinas, Stefan Waliszewski and Silvia Montiel-Palma

Analysis of PAHs Associated with Particulate Matter PM2.5 in Two Places at the City of Cuernavaca, Morelos, México

Reprinted from: Atmosphere 2015, 6(9), 1259-1270

http://www.mdpi.com/2073-4433/6/9/1259

Cameron B. Faxon, Jeffrey K. Bean and Lea Hildebrandt Ruiz

Inland Concentrations of $\mathrm{Cl}_{2}$ and $\mathrm{ClNO}_{2}$ in Southeast Texas Suggest Chlorine Chemistry Significantly Contributes to Atmospheric Reactivity

Reprinted from: Atmosphere 2015, 6(10), 1487-1506

http://www.mdpi.com/2073-4433/6/10/1487. 156

Akua Asa-Awuku, Armin Sorooshian, Richard C. Flagan, John H. Seinfeld and Athanasios Nenes

CCN Properties of Organic Aerosol Collected Below and within Marine Stratocumulus Clouds near Monterey, California

Reprinted from: Atmosphere 2015, 6(11), 1590-1607 http://www.mdpi.com/2073-4433/6/11/1590. 


\section{Minmin Yang, Yan Wang, Qiang Liu, Aijun Ding and Yuhua Li}

The Influence of Sandstorms and Long-Range Transport on Polycyclic Aromatic Hydrocarbons (PAHs) in PM2.5 in the High-Altitude Atmosphere of

Southern China

Reprinted from: Atmosphere 2015, 6(11), 1633-1651

http://www.mdpi.com/2073-4433/6/11/1633.

María A. Rubio, Eduardo Lissi, Ernesto Gramsch and René D. Garreaud

Effect of Nearby Forest Fires on Ground Level Ozone Concentrations in

Santiago, Chile

Reprinted from: Atmosphere 2015, 6(12), 1926-1938

http://www.mdpi.com/2073-4433/6/12/1838.

David H. Lopez, Michael R. Rabbani, Ewan Crosbie, Aishwarya Raman, Avelino F. Arellano and Armin Sorooshian

Frequency and Character of Extreme Aerosol Events in the Southwestern United

States: A Case Study Analysis in Arizona

Reprinted from: Atmosphere 2016, 7(1), 1

http://www.mdpi.com/2073-4433/7/1/1.

Michael Stovern, Héctor Guzmán, Kyle P. Rine, Omar Felix, Matthew King,

Wendell P. Ela, Eric A. Betterton and Avelino Eduardo Sáez

Windblown Dust Deposition Forecasting and Spread of Contamination around

Mine Tailings

Reprinted from: Atmosphere 2016, 7(2), 16

http://www.mdpi.com/2073-4433/7/2/16.....

Aishwarya Raman, Avelino F. Arellano and Armin Sorooshian

Decreasing Aerosol Loading in the North American Monsoon Region

Reprinted from: Atmosphere 2016, 7(2), 24

http://www.mdpi.com/2073-4433/7/2/24.

\section{Ivan Gregorio Hetem and Maria de Fatima Andrade}

Characterization of Fine Particulate Matter Emitted from the Resuspension of Road and Pavement Dust in the Metropolitan Area of São Paulo, Brazil

Reprinted from: Atmosphere 2016, 7(3), 31

http://www.mdpi.com/2073-4433/7/3/31 



\section{List of Contributors}

Mohammad Abbass Civil Engineering Department, King Abdulaziz University, Jeddah 21589, Saudi Arabia.

Omar Siraj Aburizaiza Unit for AinZubaida and Groundwater Research, King Abdulaziz University, Jeddah 21589, Saudi Arabia.

Abdullah Jameel Aburiziza School of Medicine, Umm Ul Qura University, Mecca 21955, Saudi Arabia.

Maria de Fatima Andrade Atmospheric Science Department, Institute of Astronomy, Geophysics and Atmospheric Sciences, University of São Paulo, São Paulo 05508-090, Brazil.

Avelino F. Arellano Department of Hydrology and Atmospheric Sciences, University of Arizona, Tucson, AZ 85721, USA.

Akua Asa-Awuku Bourns College of Engineering, Center for Environmental Research and Technology, and Department of Chemical and Environmental Engineering, University of California, Riverside, Riverside, CA 92521, USA.

Artur Badyda Warsaw University of Technology, Faculty of Environmental Engineering,20 Nowowiejska St., 00-653 Warszawa, Poland.

James Barnard Department of Physics, University of Nevada, Reno, NV 89557, USA.

Jeffrey K. Bean Center for Energy and Environmental Resources, The University of Texas at Austin, Austin, TX 78758, USA.

Larry K. Berg Pacific Northwest National Laboratory, Richland, WA 99352, USA.

Eric A. Betterton Department of Chemical and Environmental Engineering, and Department of Atmospheric Sciences, University of Arizona, Tucson, AZ 85721, USA.

Donald R. Blake Department of Chemistry, University of California-Irvine, Irvine, CA 92697, USA.

Andrzej Brandyk Water Centre Laboratory, Faculty of Civil and Environmental Engineering, Warsaw University of Life Sciences, 166 Nowoursynowska St., 02-776 Warszawa, Poland.

Duli Chand Pacific Northwest National Laboratory, Richland, WA 99352, USA. 
Ewan Crosbie National Aeronautics and Space Administration Langley Research Center, Chemistry and Dynamics Branch, Hampton, VA 23681, USA; Oak Ridge Associated Universities, Oak Ridge, TN 37831, USA.

Piotr O. Czechowski Gdynia Maritime University, Information Systems Department, 83, Morska St., 81-225 Gdynia, Poland.

Aijun Ding Institute for Climate and Global Change Research and School of Atmospheric Sciences, Nanjing University, 163 Xianlin Avenue, Nanjing 210023, China.

David A. Guerrieri P. E. New York State Department of Environmental Conservation, 625 Broadway, Albany, New York, NY 12233, USA.

Wendell P. Ela Department of Chemical and Environmental Engineering, University of Arizona, Tucson, AZ 85721, USA.

Stefano Falcinelli Department of Chemistry, Stanford University, Stanford, CA 94305, USA; Department of Civil and Environmental Engineering, University of Perugia, Via G. Duranti 93, Perugia 06125, Italy.

Wenhua Fan Environmental Engineering Laboratory, College of Resource and Environment, Shanxi Agricultural University, Jinzhong 030801, China.

Jerome Fast Pacific Northwest National Laboratory, Richland, WA 99352, USA.

Cameron B. Faxon Center for Energy and Environmental Resources, The University of Texas at Austin, Austin, TX 78758, USA.

Omar Felix Department of Chemical and Environmental Engineering, University of Arizona, Tucson, AZ 85721, USA.

Richard C. Flagan Department of Chemical Engineering, California Institute of Technology, Pasadena, CA 91125, USA.

Connor Flynn Pacific Northwest National Laboratory, Richland, WA 99352, USA.

René D. Garreaud Center for Climate and Resilience Research and Department of Geophysics, Universidad de Chile, Santiago 8320000, Chile.

Ernesto Gramsch Facultad de Ciencia, Depto Física, Universidad Santiago de Chile, USACH, Santiago 9160000, Chile.

Héctor Guzmán Department of Chemical and Environmental Engineering, University of Arizona, Tucson, AZ 85721, USA.

Leonel Hernández-Mena Centro de Investigación y Asistencia en Tecnología y Diseño del Estado de Jalisco, Guadalajara 44270, Mexico. 
Daniel L. Hershey New York State Department of Environmental Conservation, 625 Broadway, Albany, New York, NY 12233, USA.

Ivan Gregorio Hetem Atmospheric Science Department, Institute of Astronomy, Geophysics and Atmospheric Sciences, University of São Paulo, São Paulo 05508-090, Brazil.

Mirza Mozammel Hussain Wadsworth Center, New York State Department of Health, Albany, New York, NY 12201, USA; Department of Environmental Health Sciences, School of Public Health, University at Albany, Albany, New York, NY 12201, USA.

Yu Woon Jang Department of Environmental Science, Hankuk University of Foreign Studies, 449-791 Yongin, Korea.

Sung-Hyun Kahng Korea Institute of Ocean Science and Technology, 426-744 Ansan, Korea.

Evgueni Kassianov Pacific Northwest National Laboratory, Richland, WA 99352, USA.

Haider Abbas Khwaja Wadsworth Center, New York State Department of Health, Albany, New York, NY 12201, USA; Department of Environmental Health Sciences, School of Public Health, University at Albany, Albany, New York, NY 12201, USA.

Matthew King Department of Atmospheric Sciences, University of Arizona, Tucson, AZ 85721, USA.

Malissa A. Kramer New York State Department of Environmental Conservation, 625 Broadway, Albany, New York, NY 12233, USA.

Gangwoong Lee Department of Environmental Science, Hankuk University of Foreign Studies, 449-791 Yongin, Korea.

Yuhua Li Shandong Environmental Monitoring Center, 3377 Jingshi Road, Jinan 250101, China.

Eduardo Lissi Facultad de Quimica y Biologia, Universidad Santiago de Chile, USACH, Av. L. B. O’Higgins 3363, Santiago 9160000, Chile.

Fenwu Liu Environmental Engineering Laboratory, College of Resource and Environment, Shanxi Agricultural University, Jinzhong 030801, China.

Qiang Liu Institute for Climate and Global Change Research and School of Atmospheric Sciences, Nanjing University, 163 Xianlin Avenue,

Nanjing 210023, China. 
David H. Lopez Department of Chemical and Environmental Engineering, University of Arizona, Tucson, AZ 85721, USA.

Rebecca López-Márquez Centro de Investigaciones Químicas, IICBA, Universidad Autónoma del Estado de Morelos, Cuernavaca 62209, Mexico.

Grzegorz Majewski Division of Meteorology and Climatology, Faculty of Civil and Environmental Engineering, Warsaw University of Life Sciences, 166 Nowoursynowska St., 02-776 Warszawa, Poland.

Silvia Montiel-Palma Centro de Investigaciones Químicas, IICBA, Universidad Autónoma del Estado de Morelos, Cuernavaca 62209, Mexico.

Mario Murillo-Tovar Centro de Investigaciones Químicas, IICBA, Universidad Autónoma del Estado de Morelos, Consejo Nacional de Ciencia y Tecnología, Cuernavaca 62209, Mexico.

Athanasios Nenes Schools of Chemical and Biomolecular Engineering and Earth and Atmospheric Sciences, Georgia Institute of Technology, Atlanta, GA 30332, USA; Institute of Chemical Engineering Sciences, FORTH, Patras, GR-26504, Greece; Institute of Environmental Research and Sustainable Development, National Observatory of Athens, Palea Penteli, GR-15236, Greece.

Efrén Ospina-Noreña Facultad de Ciencias Agrarias, Departamento de Agronomía, Universidad Nacional de Colombia, Bogotá 111321, Colombia.

Mikhail Ovchinnikov Pacific Northwest National Laboratory, Richland, WA 99352, USA.

Seung-Myung Park National Institute of Environmental Research, 404-708 Inchon, Korea.

Mikhail Pekour Pacific Northwest National Laboratory, Richland, WA 99352, USA.

Fernando Pirani Department of Chemistry, Biology and Biotechnologies, University of Perugia, Via Elce di Sotto 8, Perugia 06123, Italy.

Michael R. Rabbani Department of Chemical and Environmental Engineering, University of Arizona, Tucson, AZ 85721, USA.

Aishwarya Raman Department of Hydrology and Atmospheric Sciences, University of Arizona, Tucson, AZ 85721.

Kyle P. Rine Department of Atmospheric Sciences, University of Arizona, Tucson, AZ 85721, USA.

Wioletta Rogula-Kozłowska Institute of Environmental Engineering, Polish Academy of Sciences, 34 M. Skłodowska-Curie St., 41-819 Zabrze, Poland. 
María A. Rubio Facultad de Quimica y Biologia, Universidad Santiago de Chile, USACH, Av. L. B. O’Higgins 3363, Santiago 9160000, Chile; Centro Para el Desarrollo de la Nanociencia y Nanotecnología (CEDENNA-USACH), Santiago 9160000, Chile.

Lea Hildebrandt Ruiz Center for Energy and Environmental Resources, The University of Texas at Austin, Austin, TX 78758, USA.

Avelino Eduardo Sáez Department of Chemical and Environmental Engineering, University of Arizona, Tucson, AZ 85721, USA.

Hugo Saldarriaga-Noreña Centro de Investigaciones Químicas, IICBA, Universidad Autónoma del Estado de Morelos, Cuernavaca 62209, Mexico.

Enrique Sánchez-Salinas Centro de Investigación en Biotecnología, Universidad Autónoma del Estado de Morelos, Cuernavaca 62209, Mexico.

Beat Schmid Pacific Northwest National Laboratory, Richland, WA 99352, USA. Arthur Sedlacek Brookhaven National Laboratory, Upton, NY 11973, USA.

John Seinfeld Schools of Chemical and Biomolecular Engineering and Earth and Atmospheric Sciences, Georgia Institute of Technology, Atlanta, GA 30332, USA.

Beom-Keun Seo National Institute of Meteorological Research, 697-845 Seogwipo, Korea.

John Shilling Pacific Northwest National Laboratory, Richland, WA 99352, USA.

Azhar Siddique Unit for AinZubaida and Groundwater Research, King Abdulaziz University, Jeddah 21589, Saudi Arabia; Chemistry Department, University of Karachi, Karachi 75270, Pakistan.

Isobel J. Simpson Department of Chemistry, University of California-Irvine, Irvine, CA 92697, USA.

Armin Sorooshian Department of Chemical and Environmental Engineering, and Atmospheric Sciences, and Department of Hydrology and Atmospheric Sciences, University of Arizona, Tucson, AZ 85721, USA.

Michael Stovern Department of Atmospheric Sciences, University of Arizona, Tucson, AZ 85721, USA.

Jason Tomlinson Pacific Northwest National Laboratory, Richland, WA 99352, USA.

Franco Vecchiocattivi Department of Civil and Environmental Engineering, University of Perugia, Via G. Duranti 93, Perugia 06125, Italy. 
Stefan Waliszewski Instituto de Medicina Forense, Universidad Veracruzana, Veracruz 91980, Mexico.

Yan Wang School of Environmental Science and Engineering, Shandong University, 27 Shanda Nanlu, Jinan 250100, China.

Zhihui Wu Environmental Engineering Laboratory, College of Resource and Environment, Shanxi Agricultural University, Jinzhong 030801, China.

Minmin Yang School of Environmental Science and Engineering, Shandong University, 27 Shanda Nanlu, Jinan 250100, China.

Jahan Zeb Unit for AinZubaida and Groundwater Research, King Abdulaziz University, Jeddah 21589, Saudi Arabia. 


\section{About the Guest Editor}

Armin Sorooshian is an associate professor in the Department of Chemical and Environmental Engineering at the University of Arizona (UA) with joint appointments in the Department of Hydrology and Atmospheric Sciences and also the College of Public Health. He received his BS degree in Chemical Engineering from UA (2008) and his PhD in Chemical Engineering from Caltech Institute of Technology, Pasadena, California (2008). After a one-year postdoctoral fellowship through the Cooperative Institute for Research in the Atmosphere, Fort Collins, Colorado (CIRA), he began his faculty career at UA in 2009. Armin specializes in understanding the sources, nature, and impacts of particulate matter in the atmosphere. He also studies the formation and evolution of clouds and rainfall using a blend of field data, satellite data, and models. 



\section{The Possible Role of Penning Ionization Processes in Planetary Atmospheres}

\section{Stefano Falcinelli, Fernando Pirani and Franco Vecchiocattivi}

Abstract: In this paper we suggest Penning ionization as an important route of formation for ionic species in upper planetary atmospheres. Our goal is to provide relevant tools to researchers working on kinetic models of atmospheric interest, in order to include Penning ionizations in their calculations as fast processes promoting reactions that cannot be neglected. Ions are extremely important for the transmission of radio and satellite signals, and they govern the chemistry of planetary ionospheres. Molecular ions have also been detected in comet tails. In this paper recent experimental results concerning production of simple ionic species of atmospheric interest are presented and discussed. Such results concern the formation of free ions in collisional ionization of $\mathrm{H}_{2} \mathrm{O}, \mathrm{H}_{2} \mathrm{~S}$, and $\mathrm{NH}_{3}$ induced by highly excited species (Penning ionization) as metastable noble gas atoms. The effect of Penning ionization still has not been considered in the modeling of terrestrial and extraterrestrial objects so far, even, though metastable helium is formed by radiative recombination of $\mathrm{He}^{+}$ions with electrons. Because helium is the second most abundant element of the universe, Penning ionization of atomic or molecular species by $\mathrm{He}^{*}\left(2^{3} \mathrm{~S}_{1}\right)$ is plausibly an active route of ionization in relatively dense environments exposed to cosmic rays.

Reprinted from Atmosphere. Cite as: Falcinelli, S.; Pirani, F.; Vecchiocattivi, F. The Possible Role of Penning Ionization Processes in Planetary Atmospheres. Atmosphere 2015, 6, 299-317.

\section{Introduction}

In general, atomic or molecular species can be ionized by using photons of sufficient energy: in this case we have photoionization processes. Alternately, it is possible to induce ionization via collision events involving energetic and excited particles, like energetic electrons and metastable atoms or molecules. In the latter case we have the so-called Penning ionization or chemi-ionization processes. As will be discussed in the next section, we are suggesting Penning ionization as an important route of formation for ionic species in the upper planetary atmospheres. The knowledge of cross sections, rate constants, and the stereodynamics driving such reactions should be useful to provide full basic physical chemical data needed as input for calculations that model the atmospheric chemistry of Earth and other planets of the solar system, like Mars and Mercury. 
Free ionic species are extremely important for the transmission of radio and satellite signals. In fact, due to the presence of high amounts of ions and free electrons, the ionosphere plays an important role in the transmission of electromagnetic waves (especially HF radio and satellite waves). Indeed, the incidence of a HF radio wave on an ionized layer can produce total reflection under appropriate conditions, contrary to what happens on atmosphere without ionization events (whose refractive index exhibits variations generally too small to produce the total reflection of a wave) [1-3]. In addition, experimental observations demonstrate a significant improvement in the propagation of these waves after thunderstorms, especially concerning high HF bands (21-24-28 MHz) [4]. This effect is not yet fully understood and is correlated to the presence of electrical discharges in the Earth's atmosphere (statistically, from thunderstorms about 100 electrical discharges occur every second) with a huge amount of energy able to ionize and excite atomic and molecular species. For more than 20 years, studies have clearly shown that, when atoms and simple molecules (for example, $\mathrm{H}, \mathrm{O}$, rare gas atoms, and $\mathrm{N}_{2}$ molecules) are subjected to electrical discharges, they produce a large amount of species in highly excited electronic metastable states (see Table 1). These metastable atoms or molecules can induce Penning ionization by collisions with neutral atomic or molecular targets with high efficiency [5-7]. In particular, the presence in our terrestrial atmosphere of argon (the third component of air at $0.93 \%$ ), and of a minor amount of neon and helium (with concentrations of about 18 and 5 ppm, respectively), makes highly probable, in these circumstances, the production of ionized species by Penning ionization induced by these rare gas metastable atoms. It has to be noted that these processes are still not fully considered in kinetic models used to describe the physical chemistry of such environments. We are confident that our research is able to clarify the possible role played by Penning ionization processes in Earth's atmosphere, contributing to the attempt to explain some unclear phenomena, for example the "sprites" formation, i.e., phenomena similar to lightning, showing a big influence on radio wave propagation. They are formed in the stratosphere (between 10 and $100 \mathrm{~km}$ ) through electrical discharges of the duration of a few tenths of a second, which are created by the electrical potential difference between the clouds and the upper atmosphere even in the absence of thunderstorms [8]. Because these phenomena happen in certain meteorological conditions and in the presence of electrical discharges, we suggest that they could be related to the formation in the atmosphere of a strong local increase in the ionic concentration due to Penning ionization processes induced by argon, neon, and helium metastable atoms that would facilitate the transmission of the radio waves by reflection. 
Table 1. Some characteristics of metastable atomic and molecular species [5].

\begin{tabular}{ccc}
\hline Excited Species & Excitation Energy (eV) & Lifetime (s) \\
\hline $\mathrm{H}^{*}\left(2 \mathrm{~s}^{2} \mathrm{~S}_{1 / 2}\right)$ & 10.1988 & 0.14 \\
$\mathrm{He}^{*}\left(2^{1} \mathrm{~S}_{0}\right)$ & 20.6158 & 0.0196 \\
$\mathrm{He}^{*}\left(2^{3} \mathrm{~S}_{1}\right)$ & 19.8196 & 9000 \\
$\mathrm{Ne}^{*}\left({ }^{3} \mathrm{P}_{0}\right)$ & 16.7154 & 430 \\
$\mathrm{Ne}^{*}\left({ }^{3} \mathrm{P}_{2}\right)$ & 16.6191 & 24.4 \\
$\mathrm{Ar}^{*}\left({ }^{3} \mathrm{P}_{0}\right)$ & 11.7232 & 44.9 \\
$\mathrm{Ar}^{*}\left({ }^{3} \mathrm{P}_{2}\right)$ & 11.5484 & 55.9 \\
$\mathrm{Kr}^{*}\left({ }^{3} \mathrm{P}_{0}\right)$ & 10.5624 & 0.49 \\
$\mathrm{Kr}^{*}\left({ }^{3} \mathrm{P}_{2}\right)$ & 9.9152 & 85.1 \\
$\mathrm{Xe}^{*}\left({ }^{3} \mathrm{P}_{0}\right)$ & 9.4472 & 0.078 \\
$\mathrm{Xe}^{*}\left({ }^{3} \mathrm{P}_{2}\right)$ & 8.3153 & 150 \\
$\mathrm{O}^{*}\left(2 \mathrm{p}^{3} 3 \mathrm{~s}^{5} \mathrm{~S}_{2}\right)$ & 9.146 & $1.85 \times 10^{-4}$ \\
$\mathrm{H}_{2}^{*}\left({ }^{3} \Pi_{\mathrm{u}}{ }^{-}\right)$ & 11.764 & $1.02 \times 10^{-3}$ \\
$\mathrm{~N}_{2}^{*}\left(\mathrm{~A}^{3} \Sigma_{\mathrm{u}}{ }^{+}\right)$ & 6.169 & 1.9 \\
$\mathrm{~N}_{2}^{*}\left(\mathrm{~A}^{1} \Pi_{\mathrm{g}}\right)$ & 8.549 & $1.20 \times 10^{-4}$ \\
$\mathrm{~N}_{2}^{*}\left(\mathrm{E}^{3} \Sigma_{\mathrm{g}}{ }^{+}\right)$ & 11.875 & $1.90 \times 10^{-4}$ \\
$\mathrm{CO}^{*}\left(\mathrm{a}^{3} \Pi_{1}\right)$ & 6.010 & $\geq 3 \times 10^{-3}$ \\
\hline
\end{tabular}

The presence of ions in the upper terrestrial atmosphere was soon recognized after the experiment of Marconi in 1901, who succeeded in transmitting radio waves from the United Kingdom to Canada. Later, the ion $\mathrm{CH}^{+}$was detected in space, practically at the same time as the detection of the first neutral molecules, $\mathrm{CH}$ and $\mathrm{CN}$ [9]. Ions are extremely important in the upper atmosphere of planets, where they govern the chemistry of ionospheres. Furthermore, molecular ions have also been detected in comet tails [9]. In planetary ionospheres and in space, ions are formed in various ways, the importance of which depends on the specific conditions of the considered environment. The interaction of neutral molecules with cosmic rays, UV photons, X-rays, and other phenomena such as shock waves are all relevant processes for their production [10]. In particular, the absorption of UV photons with an energy content higher than the ionization potential of the absorbing species can induce photoionization (for most species, extreme-UV or far-UV photons are necessary). Cosmic rays are also important since they are ubiquitous and carry a large energy content (up to $100 \mathrm{GeV}$ ).

The detailed laboratory characterization of relevant elementary processes and their relative role, leading to the formation of atomic and molecular ions, is therefore of crucial importance. In this paper recent experimental results concerning production and characterization via Penning ionization of simple ionic species of atmospheric interest are presented. The discussed data concern the formation of free ions in collisional ionization of $\mathrm{H}_{2} \mathrm{O}, \mathrm{H}_{2} \mathrm{~S}$, and $\mathrm{NH}_{3}$ induced by highly excited species. As mentioned above, the effect of Penning ionization has not yet been 
fully considered in the modeling of terrestrial and extraterrestrial objects, even though metastable helium is known to be formed by photoelectron impact and radiative recombination of $\mathrm{He}^{+}$ions with electrons [11,12]. In fact, it is well-known that the $1083 \mathrm{~nm}$ twilight airglow arises from resonant scattering of solar photons by metastable $\mathrm{He}^{*}\left(2^{3} \mathrm{~S}\right)$ atoms in the upper thermosphere and exosphere (see, for instance [13], and references therein). Having a radiative lifetime of $2.5 \mathrm{~h}$ and an energy content of $19.82 \mathrm{eV}$, Penning ionization of molecules by $\operatorname{He}^{*}\left(2^{3} \mathrm{~S}_{1}\right)$ (helium is the second most abundant element of the universe) is plausibly an active route of ionization in relatively dense environments exposed to cosmic rays for two main reasons: (i) recent studies have shown that the $\mathrm{He}^{*}\left(2^{3} \mathrm{~S}\right)$ density profiles in the Earth's atmosphere reach their maximum values at about $600 \mathrm{~km}$ altitude, changing with the investigated solar zenith angle, and ranging between 0.6 and 1.1 atoms $/ \mathrm{cm}^{3}$ [13]; and (ii) with respect to ionization by VUV photons and cosmic rays, Penning ionization processes are characterized by much higher cross-sections [14,15]. Moreover, concerning the role of $\mathrm{He}^{*}\left(2^{3} S\right)$ metastable atoms in the interstellar medium, in a recent paper Indriolo et al. derived new equations for the steady state abundance of $\mathrm{He}^{*}$ using its $1083 \mathrm{~nm}$ absorption line data observed through the diffuse clouds toward HD 183143 [11]. By their analysis the authors inferred an upper limit for the cosmic-ray ionization rate of helium of $1.2 \times 10^{-15} \mathrm{~s}^{-1}$, which is an important route of $\mathrm{He}^{*}\left(2^{3} \mathrm{~S}\right)$ formation via cosmic-ray ionization of helium atoms, followed by radiative recombination of the ions with electrons, as mentioned before. This ionization rate value is higher than those related to the ionization of interstellar hydrogen (various models have predicted ionization rates from $10^{-18} \mathrm{~s}^{-1}$ up to $10^{-15} \mathrm{~s}^{-1}$ ) in the diffuse interstellar medium, or in comparison with the ionization of other molecules such as $\mathrm{HD}$ and $\mathrm{OH}$ having estimated ionization rates of the order of $10^{-17} \mathrm{~s}^{-1}$ [11]. These data allow us to highlight the importance of the chemistry associated with interstellar metastable helium, and the possible use of $\operatorname{He}^{*}\left(2^{3} S\right)$ as a widely applicable probe of the cosmic-ray ionization rates, as suggested by Indriolo et al. [11].

Penning ionization is an autoionizing process involving the collision complex between an excited species and an atomic or molecular target. The collision will yield various ion products when the ionization potential of the target particle is lower than the excitation energy of the primary metastable species. Very common species able to induce Penning ionization are the rare gas atoms excited to their first electronic level, which is a metastable state (their lifetime is long enough to allow them to survive at least several seconds); this makes them able to ionize upon collision many atomic and molecular targets (they are characterized by a high excitation energy which ranges from $8.315 \mathrm{eV}$, in the case of $\mathrm{Xe}^{*}\left({ }^{3} \mathrm{P}_{2}\right)$, up to $20.616 \mathrm{eV}$, in the case of $\left.\mathrm{He}^{*}\left({ }^{1} \mathrm{~S}_{0}\right)\right)$. 
These ionization reactions were widely studied starting in 1927 by F.M. Penning [16]. They can be schematized as follows:

$$
\operatorname{Rg}^{*}+\mathrm{M} \rightarrow[\operatorname{Rg} \ldots \mathrm{M}]^{*} \rightarrow[\operatorname{Rg} \ldots \mathrm{M}]^{+}+\mathrm{e}^{-} \rightarrow \text { ion products }
$$

where $\mathrm{Rg}$ is a rare gas atom, $\mathrm{M}$ is an atomic or molecular target, $[\mathrm{Rg} . . \mathrm{M}]^{*}$ represents the intermediate autoionizing collisional complex, and $[\operatorname{Rg} \ldots \mathrm{M}]^{+}$the intermediate transient ionic species formed by the electron ejection. This is the most efficient way for the collisional system $[\mathrm{Rg} \ldots \mathrm{M}]^{*}$ to lose its excitation energy, since the autoionization time is usually shorter than the characteristic molecular collision time at thermal energies $\left(\sim 10^{-12} \mathrm{~s}\right)$. Then, various final product ions can be formed depending on the characteristics of the involved potential energy surfaces. Some specific features make these processes very interesting from a fundamental point of view. In fact, at high interatomic separation the metastable rare gas atoms behave as alkaline metals because of their weakly bound external electron, whereas at shorter distances the role of their "open shell" core becomes prominent, looking like halogen atoms. Therefore, their phenomenological behavior when interacting with hydrogenated molecules $\left(\mathrm{H}_{2} \mathrm{O}, \mathrm{H}_{2} \mathrm{~S}, \mathrm{NH}_{3}\right)$ is very interesting for fundamental and applied science, as shown by the many discussions in the literature about the role of hydrogen and halogen bonds [17].

However, many applications of collisional autoionization to important fields, like radiation chemistry, plasma physics, and chemistry, and the development of laser sources, are also possible. In some cases, this technique is also used to induce gentle ionization of complex molecules.

In general, the role of metastable rare gas atoms in Earth's atmospheric reactions is of interest for a number of reasons: (i) the quantity of rare gas atoms in the atmosphere is not negligible (argon is the third component of the air, after nitrogen and oxygen molecules: $0.934 \% \pm 0.001 \%$ by volume compared to $0.033 \% \pm 0.001 \%$ by volume of $\mathrm{CO}_{2}$ molecules); (ii) as mentioned in the previous section, recent studies have shown that the upper atmosphere of our planet is relatively rich in $\operatorname{He}^{*}\left(2^{2} \mathrm{~S}_{1}\right)$ metastable atoms [18,19]; and (iii) rate constants for ionization processes induced by metastable rare gas atoms are generally larger than those of common bimolecular chemical reactions of atmospheric interest. For example, the rate constants for Penning ionization processes are of the same order of magnitude as gas phase bimolecular reactions of $\mathrm{O}\left({ }^{1} \mathrm{D}\right), \mathrm{Cl}\left({ }^{2} \mathrm{P}\right)$, or $\mathrm{Br}\left({ }^{2} \mathrm{P}\right)$, which are known to be relevant in the modeling of atmospheric chemistry, whereas other important reactions (as for example those involving $\mathrm{Br}+\mathrm{O}_{3}, \mathrm{O}+\mathrm{HOCl}$, and $\mathrm{OH}+\mathrm{HOCl}$ ), exhibit rate constants that are at least one order of magnitude smaller [20].

Considering the planetary atmospheric compositions, we can suppose that rare gas atoms are involved in several atmospheric phenomena not only on Earth, 
but also on other planets of the solar system, like Mars and Mercury. In fact, argon is the third component of the Martian atmosphere at $1.6 \%$, while helium is a relatively abundant species (about $6 \%$ ) in the low density atmosphere of Mercury. As mentioned in the previous section, a basic step in the chemical evolution of planetary atmospheres and interstellar clouds (where $89 \%$ of atoms are hydrogen and $9 \%$ are helium) is the interaction of atoms and molecules with electromagnetic waves ( $\gamma$ and $X$ rays, $U V$ light) and cosmic rays. Therefore, $\mathrm{He}^{*}$ and $\mathrm{Ar}^{*}$ formation by collisional excitation with energetic target particles (like electrons, protons, or alpha particles) and the possible subsequent Penning ionization reactions could be of importance in these environments. In particular, considering the tenuous exosphere of Mercury, it is interesting to note that in 2008 the NASA spacecraft MESSENGER discovered surprisingly large amounts of water and several atomic and molecular ionic species including $\mathrm{O}^{+}, \mathrm{OH}^{-}, \mathrm{H}_{2} \mathrm{O}^{+}$, and $\mathrm{H}_{2} \mathrm{~S}^{+}$(NASA and the MESSENGER team will issue periodic news releases and status reports on mission activities and make them available online). Taking into account the suggested polar deposits of water ice in this planet, McClintock and Lankton in 2007 have suggested impact vaporization mechanisms [21]. We argue that in these parts of the planetary surface, the presence of $\mathrm{He}^{*}$ and its collisions can cause subsequent ion formation. In this respect, the study of the Penning ionization of water and hydrogen sulfide by metastable rare gas atoms $\left(\mathrm{He}^{*}\right.$ and $\mathrm{Ne}^{*}$, see Section 3$)$, producing $\mathrm{H}_{2} \mathrm{O}^{+}, \mathrm{OH}^{+}$, $\mathrm{O}^{+}$, and $\mathrm{H}_{2} \mathrm{~S}^{+}$, respectively, could help in explaining the possible routes of formation for these ionic species in Mercury's exosphere. The importance of these processes and, in particular, of Penning ionization phenomena of hydrogenated molecules by $\mathrm{He}^{*}$ and $\mathrm{Ne}^{*}$ metastable atoms has been widely discussed in [22-24], to which we refer for an overview of the experimental and theoretical works performed since the 1970s by several research groups, starting with the pioneering works by Čermák and Yencha [25] and by Brion and Yee [26].

We have recently studied the ionization of water molecules by Penning ionization coupled with both Mass Spectrometry (MS) and Electron Spectroscopy (PIES) techniques [22-24]. The analysis of the obtained results suggests the selective role of a quite pronounced anisotropic attraction that controls the stereodynamics in the entrance channels of the analyzed systems. In the case of $\mathrm{He}^{*}$ and $\mathrm{Ne}^{*}-\mathrm{H}_{2} \mathrm{O}$ autoionizing collisions, we were able to confirm the presence of a strong anisotropic interaction, after previous experimental and theoretical evidences by Ohno et al. [27], Haug et al. [28], Bentley [29], and Ishida [30], respectively. In particular, we rationalized our experimental findings, taking into account the critical balancing between molecular orientation effects in the intermolecular interaction field and the ionization probability. In analyzing our PIES spectra, a novel semiclassical method was proposed: (i) it assumes ionization events as mostly occurring in the vicinities of the collision turning points (where the main part of the collision time is accumulated 
because the relative velocity tends to zero); and (ii) the potential energy driving the system in the relevant configurations of the entrance and exit channels, employed in the spectra simulation, has been represented by the use of a semiempirical method and given a proper analytical form [24]. This procedure was able to clearly point out how different approaches between the metastable atom and water molecules promote the formation of ions in different electronic states. In particular, it provides the angular acceptance cones where the stereo-selectivity of the processes leading to the specific formation of each one of the two energetically possible electronic states of $\mathrm{H}_{2} \mathrm{O}^{+}$final product ions [24] (the $\mathrm{X}^{2} \mathrm{~B}_{1}$ ground state and the first $\mathrm{A}^{2} \mathrm{~A}_{1}$ excited ones) mainly manifests.

In the present work we analyze our recent PIES spectra, collected in $\mathrm{He}^{*}$, $\mathrm{Ne}^{*}-\mathrm{H}_{2} \mathrm{~S}$, and $\mathrm{NH}_{3}$ collisions, by using the same procedure already applied to water molecules, in order to compare the three sets of data, obtained under the same experimental conditions (see next Section), and to identify relevant differences in the stereodynamics of Penning ionization of the three hydrogenated molecules. In particular, our recent results recorded in $\mathrm{He}^{*}$ and $\mathrm{Ne}^{*}-\mathrm{H}_{2} \mathrm{~S}$ collisional experiments clearly suggest again the formation of molecular ions both in the ground and in the first excited electronic state. They are also indicating similar anisotropic behavior for the potential energy surface describing the $\mathrm{Rg}^{*}-\mathrm{H}_{2} \mathrm{~S}$ incoming channel with an attractive component less "effective" with respect to $\mathrm{Rg}^{*}-\mathrm{H}_{2} \mathrm{O}$ (see Section 3). In the case of ammonia, anisotropic behavior is still present, with a strong attractive interaction between $\mathrm{Rg}^{*}-\mathrm{NH}_{3}$ collisional partners (where $\mathrm{Rg}=\mathrm{He}, \mathrm{Ne}$ ), as in the $\mathrm{Rg}^{*}-\mathrm{H}_{2} \mathrm{O}$ case (see Section 3$)$.

\section{Experimental Techniques}

The experimental setup, consisting of a crossed molecular beam apparatus (three vacuum chambers which are differentially pumped at a pressure of about $10^{-7}$ mbar) is schematically sketched in Figure 1 and described in detail in some previous works [31-33].

In such an apparatus a molecular beam of excited atoms, mostly metastable rare gas atoms, is crossing at right angles a second beam of molecules (to be ionized). Electrons produced in photo- and/or chemi-ionization reactions are extracted and analyzed by an appropriate electron energy selector, while product ions are sent into a quadrupole mass spectrometer (located below the beam's crossing volume), where they are mass analyzed and detected. A powerful improvement of our apparatus could be realized by introducing the coincidence detection technique for the related electrons and ions coming out of the same autoionizing $[\mathrm{Rg} \ldots \mathrm{M}]^{*}$ collisional complex (see Equation (1)). 


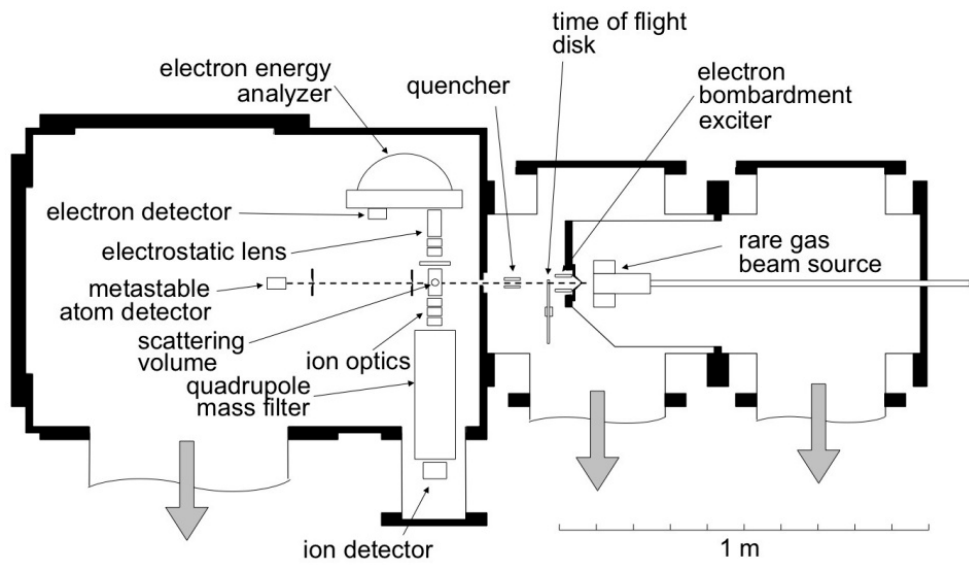

Figure 1. The crossed molecular beam apparatus used in Penning ionization studies.

The metastable rare gas beam, produced by electron bombardment or by a microwave discharge beam source, crosses at a right angle an effusive beam of $\mathrm{H}_{2} \mathrm{O}, \mathrm{H}_{2} \mathrm{~S}$, or $\mathrm{NH}_{3}$ target molecules. The kinetic energy of emitted electrons is recorded by the use of a hemispheric electrostatic analyzer, which is located above the crossing beam volume. In such a way we can perform, in the same experimental conditions, both photoionization (PES) and Penning Ionization Electron Spectroscopy (PIES) measurements. This can be easily achieved because the microwave discharge produces not only metastable atoms but also many $\mathrm{He}(\mathrm{I})$ and $\mathrm{Ne}(\mathrm{I})$ photons, having an energy comparable to that of metastable atoms. To calibrate the electron transmission efficiency and the electron energy scale, some $\mathrm{He}^{*}-\mathrm{H}_{2} \mathrm{O}, \mathrm{H}_{2} \mathrm{~S}, \mathrm{NH}_{3}$ PIES, and $\mathrm{Ne}(\mathrm{I})-\mathrm{H}_{2} \mathrm{O}, \mathrm{H}_{2} \mathrm{~S}, \mathrm{NH}_{3}$ PES spectra have been measured by exploiting the microwave discharge beam source: in the first case we use for the energy calibration the weak electron signal by $\mathrm{He}(\mathrm{I})$ photons of $21.22 \mathrm{eV}$, whereas in the latter case we record a PES spectrum, using the discharge source in pure neon producing a high intensity of $\mathrm{Ne}\left(\mathrm{I}_{\alpha, \beta}\right)$ photons [22,34], essentially of 16.84 and $16.66 \mathrm{eV}$, respectively, in a $\alpha: \beta$ ratio of about 5.3 , checked by photoelectron spectrometric measurements of $\mathrm{Kr}$ atoms [35,36]. By comparison of our measurements with the expected spectrum as obtained from the $\mathrm{He}(\mathrm{I})$ radiation by Kimura et al. [37], we calibrate the ionization energy scale. The resolution of our electron spectrometer is $\sim 45 \mathrm{meV}$ at a transmission energy of $3 \mathrm{eV}$, as determined by measuring the photoelectron spectra of $\mathrm{Ar}, \mathrm{O}_{2}$, and $\mathrm{N}_{2}$ by $\mathrm{He}(\mathrm{I})$ radiation with the procedure described in previous papers $[18,36]$. Spurious effects due to the geomagnetic field have been reduced to $\leq 20 \mathrm{mG}$ by a $\mu$-metal shielding. Finally, the metastable atom velocity can be analyzed by a time-of-flight technique: the beam is pulsed by a rotating slotted disk and the metastable atoms are counted, using a multiscaler, as a function of the delay time from the beam opening. The secondary molecular beam can be prepared in two 
ways: (i) by effusion of $\mathrm{H}_{2} \mathrm{O}, \mathrm{H}_{2} \mathrm{~S}$, or $\mathrm{NH}_{3}$ from a glass microcapillary array kept at room temperature, or (ii) by using a supersonic source whose nozzle temperature can be changed between 300 and $700 \mathrm{~K}$. The collision energy resolution is largely defined by the thermal spread of target molecules effusing from the microcapillary source (about 30\% full with a half-maximum (FWHM) velocity spread at the lowest relative velocity of $1100 \mathrm{~m} / \mathrm{s}$, and about $10 \%$ FWHM for the highest probed relative velocity of $1800 \mathrm{~m} / \mathrm{s}$ ), and in our experiment the average collision energy is about $55 \mathrm{meV}$. Absolute values of the total ionization cross-section for different species can be obtained by the measurement of relative ion intensities in the same conditions of metastable atom and target gas density in the crossing region. This allows the various systems to be put on a relative scale, which can be normalized by reference to a known cross-section such as, in the present case, the $\mathrm{Ne}^{*}-\mathrm{Ar}$ absolute total ionization cross-section by West et al. [38]. By analyzing the kinetic energy of the emitted electrons, we can perform a real spectroscopy of the collisional intermediate transient species for such a process, obtaining detailed information about the intermolecular potentials controlling the microscopic collision dynamics, the preferential geometries of approach between the collision partners, and the molecular orbitals involved in the autoionization event. On the other hand, looking at the product ions, we can determine the cross-sections for each open ionization channel, obtaining complementary information that enables us to achieve a complete understanding of the reaction dynamics for the system under study.

\section{Results and Discussion}

In this section recent experimental results of relevance for the characterization of simple ionic species of atmospheric interest, produced by Penning ionization of hydrogenated molecules $\left(\mathrm{H}_{2} \mathrm{O}, \mathrm{H}_{2} \mathrm{~S}\right.$, and $\left.\mathrm{NH}_{3}\right)$ with $\mathrm{He}^{*}$ and $\mathrm{Ne}^{*}$ metastable atoms, are presented and discussed in a comparative way. First of all, following the observations made on the importance of Penning ionization of water by $\operatorname{He}^{*}\left(2^{3} \mathrm{~S}_{1}\right.$, $\left.2^{1} \mathrm{~S}_{0}\right)$ and $\mathrm{Ne}^{*}\left({ }^{3} \mathrm{P}_{2,0}\right)$ in the understanding of the chemical composition of Mercury's atmosphere, we report here the mass spectrometric determination of the channel branching ratios for both systems as obtained at an averaged collision energy of $70 \mathrm{meV}$ in the case of $\mathrm{He}^{*}$ and of $55 \mathrm{meV}$ for $\mathrm{Ne}^{*}$ experiments:

$$
\begin{aligned}
\mathrm{He}^{*} & +\mathrm{H}_{2} \mathrm{O} \rightarrow \mathrm{He}+\mathrm{H}_{2} \mathrm{O}^{+}+\mathrm{e}^{-}(77.3 \%) \\
\rightarrow & \mathrm{He}+\mathrm{H}+\mathrm{OH}^{+}+\mathrm{e}^{-}(18.9 \%) \\
& \rightarrow \mathrm{He}+\mathrm{H}_{2}+\mathrm{O}^{+}+\mathrm{e}^{-}(3.8 \%) \\
\mathrm{Ne}^{*}+ & \mathrm{H}_{2} \mathrm{O} \rightarrow \mathrm{Ne}+\mathrm{H}_{2} \mathrm{O}^{+}+\mathrm{e}^{-}(96.2 \%) \\
\rightarrow & \mathrm{Ne}+\mathrm{H}+\mathrm{OH}^{+}+\mathrm{e}^{-}(3.2 \%)
\end{aligned}
$$




$$
\rightarrow \mathrm{Ne}+\mathrm{H}_{2}+\mathrm{O}^{+}+\mathrm{e}^{-}(0.6 \%)
$$

No evidence has been recorded for the $\mathrm{HeH}^{+}, \mathrm{NeH}^{+}$, and $\mathrm{HeH}_{2} \mathrm{O}^{+}, \mathrm{NeH}_{2} \mathrm{O}^{+}$ ion production, at least under our experimental conditions. In the case of Penning ionization of ammonia by collisions with neon metastable atoms, the measured yield of possible product ions at an averaged collision energy of $55 \mathrm{meV}$ is:

$$
\begin{gathered}
\mathrm{Ne}^{*}+\mathrm{NH}_{3} \rightarrow \mathrm{Ne}+\mathrm{NH}_{3}^{+}+\mathrm{e}^{-}(58.8 \%) \\
\rightarrow \mathrm{Ne}+\mathrm{NH}_{2}{ }^{+}+\mathrm{e}^{-}(41.2 \%),
\end{gathered}
$$

while, for $\mathrm{Ne}^{*}-\mathrm{H}_{2} \mathrm{~S}$ autoionizing collision in a thermal energy range $(0.02-0.25 \mathrm{eV})$, our spectrometric measurements showed the formation of only the parent $\mathrm{H}_{2} \mathrm{~S}^{+}$ product ion.

The ionization of hydrogenated molecules by metastable helium and neon atoms strongly depends on how the molecule is oriented with respect to the approaching excited rare gas atom, determining the electronic state of the intermediate ionic complex $[\mathrm{Rg} \ldots \mathrm{M}]^{+}$and therefore its following reactivity. In fact, for example, a direct probe of the anisotropy of the potential energy surface for $\mathrm{Ne}^{*}-\mathrm{H}_{2} \mathrm{O}$ system can be provided by a comparative analysis of the features of electron energy spectra measured under the same conditions both in photoionization (see Figure 2-upper panel) and in Penning ionization (see Figure 2-lower panel) experiments. All the electron spectra reported in Figure 2 appeared to be composed by two bands: one (at higher electron kinetic energies) for the formation of $\mathrm{H}_{2} \mathrm{O}^{+}$ions (in case of photoionization measurements) and of the $\left[\mathrm{Ne} \ldots \mathrm{H}_{2} \mathrm{O}\right]^{+}$intermediate ionic complex (in Penning ionization determinations) in its ground $X\left({ }^{2} B_{1}\right)$ electronic state, and another one (at lower electron energy) for the formation of $\mathrm{H}_{2} \mathrm{O}^{+}$and $\left[\mathrm{Ne} \ldots \mathrm{H}_{2} \mathrm{O}\right]^{+}$ in their first excited $\mathrm{A}\left({ }^{2} A_{1}\right)$ electronic state. The formation of these two electronic states corresponds to the removal of one electron from one of the two non-bonding orbitals of the neutral molecule. The ground state $\mathrm{X}\left({ }^{2} B_{1}\right) \mathrm{H}_{2} \mathrm{O}^{+}$ion is formed by the removal of one electron from the $2 \mathrm{p} \pi$ lone pair orbital, while for the formation of the excited $\mathrm{A}\left({ }^{2} A_{1}\right) \mathrm{H}_{2} \mathrm{O}^{+}$ion, the electron must be removed from the $3 \mathrm{a}_{1} \mathrm{sp}^{2}$ lone pair orbital, as can be seen in Figure 3. Moreover, the "exchange mechanism" for Penning ionization suggests that one outer shell electron of the molecule to be ionized is transferred into the inner shell hole of the excited atom, and then the excess of energy produces the ejection of one electron from the collision complex [14,15].

Therefore, we can assert that the two bands in the spectra of Figures 2 and 3 must be related to ionization events occurring with two different geometries of the system: the $\mathrm{X}\left({ }^{2} B_{1}\right)$ band originates from a collision complex with the metastable neon atom perpendicular to the plane of the molecule, while for the $\mathrm{A}\left({ }^{2} A_{1}\right)$ band, at the instant of the ionization, the metastable atom approaches water in the direction 
of the $C_{2 v}$ symmetry axis. In a recent paper [24], by analyzing the energy spectra of the emitted electrons reported in Figure 2 using a novel semiclassical method based on: (i) the assumption that the ionization events are mostly occurring in the vicinities of the collision turning points, and (ii) the formulation of the potential energy driving the system in the relevant configurations of the entrance and exit channels by the use of a semiempirical method developed in our laboratory, we were able to show clearly how different collisional approaches of the metastable atom to the water molecule lead to ions in different electronic states. In particular, we provided the angular acceptance cones where the selectivity of the process leading to the specific formation of each of the two energetically possible ionic product states of $\mathrm{H}_{2} \mathrm{O}^{+}$emerges, showing how the ground state ion is formed when neon metastable atoms approach water mainly perpendicularly to the molecular plane, while the first excited electronic state is formed when the approach occurs preferentially along the $\mathrm{C}_{2 \mathrm{v}}$ axis, on the oxygen side [24].
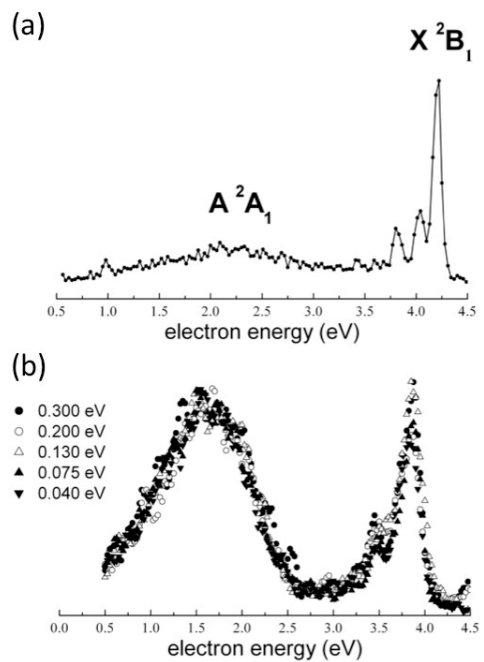

Figure 2. The obtained (a) PES (upper panel) and (b) PIES (lower panel) spectra in photoionization and Penning ionization studies of water molecules using $\mathrm{Ne}(\mathrm{I})$ photons and $\mathrm{Ne}^{*}\left({ }^{3} \mathrm{P}_{2,0}\right)$ metastable atoms, respectively. The PIES spectra reported in the lower panel are recorded at five different collision energies $(0.300,0.200$, $0.130,0.075$, and $0.040 \mathrm{eV}$ ), by varying the nozzle temperature (between 300 and $700 \mathrm{~K}$ ) of the supersonic source and by using, besides pure neon, two different $\mathrm{Ne}: \mathrm{H}_{2}$ mixtures (50:50 and 20:80 ratios). By looking at the relative position of the corresponding peak maxima in the PES and PIES spectra, it is possible to evaluate the evident negative energy shifts $\left(-140 \mathrm{meV}\right.$ and $-300 \mathrm{meV}$ for $\mathrm{X}^{2} \mathrm{~B}_{1}$ and $\mathrm{A}^{2} \mathrm{~A}_{1}$ electronic states of $\mathrm{H}_{2} \mathrm{O}^{+}$, respectively) that depend on the binding energy of the autoionizing collision complex (see the text). 

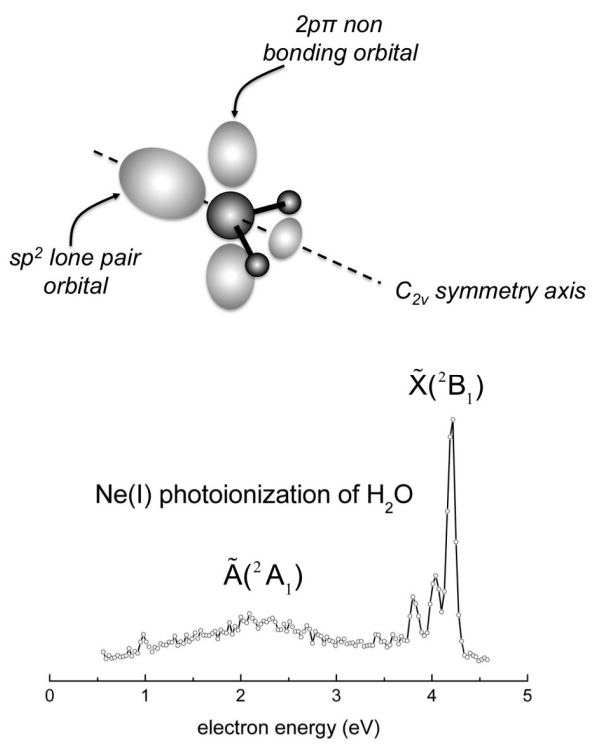

Figure 3. The structure of water molecules in the gas phase, with a $\mathrm{C}_{2 \mathrm{v}}$ molecular axis and a sp ${ }^{2}$ hybridization, is depicted. The ground state $X\left({ }^{2} B_{1}\right) \mathrm{H}_{2} \mathrm{O}^{+}$ion is formed by the removal of one electron from the $2 \mathrm{p} \pi$ lone pair orbital, while for the formation of the excited $\mathrm{A}\left({ }^{2} A_{1}\right) \mathrm{H}_{2} \mathrm{O}^{+}$ion, the electron must be removed from the $3 \mathrm{a}_{1} \mathrm{sp}^{2}$ lone pair orbital. The electron energy spectrum obtained in photoionization of water molecules by using $\mathrm{Ne}(\mathrm{I})$ photons is also shown. The two bands in the spectrum are related to the formation of $\mathrm{H}_{2} \mathrm{O}^{+}$in its ground $\mathrm{X}\left({ }^{2} B_{1}\right)$ electronic state (at higher electron energy), and in the first excited $\mathrm{A}\left({ }^{2} A_{1}\right)$ electronic state (at lower electron energy).

By analyzing the PES and PIES spectra of Figure 2, recorded in $\mathrm{Ne}(\mathrm{I})$ and $\mathrm{Ne}^{*}-\mathrm{H}_{2} \mathrm{O}$ autoionizing collisions, respectively, we are able to obtain important findings such as the so-called "energy shifts" for the analyzed systems (see Table 2). It is well known that these observables depend on the strength, range, and anisotropy of the effective intermolecular interaction controlling the stereodynamics in the entrance $\mathrm{Rg}^{*}-\mathrm{M}$ (neutral) and the exit $\mathrm{Rg}-\mathrm{M}^{+}$(ionic) channels of ionizing collisional events. In particular, when the position of the maximum of a certain peak in the PIES spectrum exhibits a sizeable negative shift, here referred to as $\varepsilon_{\max }$, with respect to the nominal energy, $\varepsilon_{0}$ (defined as the difference between the excitation energy of the metastable $\mathrm{He}^{*}$ or $\mathrm{Ne}^{*}$ atoms and the ionization potential of the relevant molecular state), this is an indication that a phenomenological global attractive interaction drives the collision dynamics of the partners on a multidimensional potential energy surface, including entrance and exit channels [23,36]. These energy shifts are also determined by looking at the relative position of the corresponding peak maxima in 
the PES and PIES spectra. For example, analyzing in a comparative way the electron spectra of Figure 2, it is possible to clearly note that both peak maxima for $X$ and A states of produced $\mathrm{H}_{2} \mathrm{O}^{+}$ions are shifted at lower electron kinetic energy values in the case of Penning ionization spectra with respect to the photoionization ones. The absolute values of these shifts are much higher than the difference between the energy content of the $\mathrm{Ne}^{*}$ metastable atoms and the energy of the $\mathrm{Ne}(\mathrm{I})$ photons, because of the strong attractive interaction potential between the incoming collisional partners $\mathrm{Ne}^{*}-\mathrm{H}_{2} \mathrm{O}$ (for a detailed description, see for instance [22]). In general, as the $\mathrm{Rg}^{*}-\mathrm{M}$ attractive interaction is stronger, a more negative energy shift is expected between PES- and PIES-recorded spectra. These considerations are confirmed by our mass spectrometric determinations, and, in particular, by the total ionization cross-section measurement as a function of the collision energy reported in Figure 4. It is interesting to note that the cross-section shows a decreasing trend as the collision energy increases and, further, its absolute value is quite big (of about $50 \AA^{2}$ at $70 \mathrm{meV}$ ). Accordingly to the well-known peculiarity of Penning ionization processes [14,15], both these characteristics are a clear confirmation of the strong binding behavior of the $\mathrm{Ne}^{*}-\mathrm{H}_{2} \mathrm{O}$ autoionizing collision complex, due to the strong attractive nature of the system because of the intense molecular dipole of the water molecule $(\mu=1.85 \mathrm{D})$.

Analyzing the PES and PIES spectra for $\mathrm{Ne}(\mathrm{I})$ and $\mathrm{Ne}^{*}-\mathrm{H}_{2} \mathrm{~S}, \mathrm{NH}_{3}$ systems, respectively, by using the same procedure already described for water molecules, we have been able to determine the related energy shifts, as shown in Figure 5 (see also Table 2). All the measured shifts have a negative value, indicating again an attractive behavior for the potential energy surface in the entrance $\mathrm{Ne}^{*}-\mathrm{M}$ channel (where $\mathrm{M}=\mathrm{H}_{2} \mathrm{~S}, \mathrm{NH}_{3}$ ) as in the case of $\mathrm{Ne}^{*}-\mathrm{H}_{2} \mathrm{O}$ autoionizing collisions. This attractive behavior is stronger in the formation of $\mathrm{Ne}^{*}-\mathrm{H}_{2} \mathrm{O}$, whose energy shifts are $-140 \pm 20$ and $-300 \pm 20 \mathrm{meV}$ for the $\mathrm{X}\left({ }^{2} B_{1}\right)$ and $\mathrm{A}\left({ }^{2} A_{1}\right)$ bands, respectively; it is less intense in the case of $\mathrm{Ne}^{*}-\mathrm{H}_{2} \mathrm{~S}$ collisions (energy shifts of $-80 \pm 20$ and $-120 \pm 20$ for the same two $X\left({ }^{2} B_{1}\right)$ and $\mathrm{A}\left({ }^{2} A_{1}\right)$ bands, respectively); while in the case of $\mathrm{Ne}^{*}-\mathrm{NH}_{3}$ (energy shifts of $-290 \pm 20$ and $-140 \pm 20$ for the two $\mathrm{X}\left({ }^{2} A_{2}{ }^{\prime \prime}\right)$ and $\mathrm{A}\left({ }^{2} E\right)$ bands, respectively) the attractive interaction component, affecting the entrance channel of the potential energy surface, favors those collisions producing the $\left[\mathrm{Ne} \ldots \mathrm{NH}_{3}\right]^{+}$intermediate ionic complex in its $\mathrm{X}\left({ }^{2} A_{2}{ }^{\prime \prime}\right)-3 \mathrm{a}_{1}$ ground electronic state. The formation of $\left[\mathrm{Ne} \ldots \mathrm{NH}_{3}\left(X^{2} A_{2}{ }^{\prime \prime}\right)\right]^{+}$occurs preferentially when the neon metastable atom is approaching ammonia molecules towards the direction of the $3 \mathrm{a}_{1}$ non-bonding lone pair located on the nitrogen atom and removes one electron from this orbital, producing Penning ionization. 

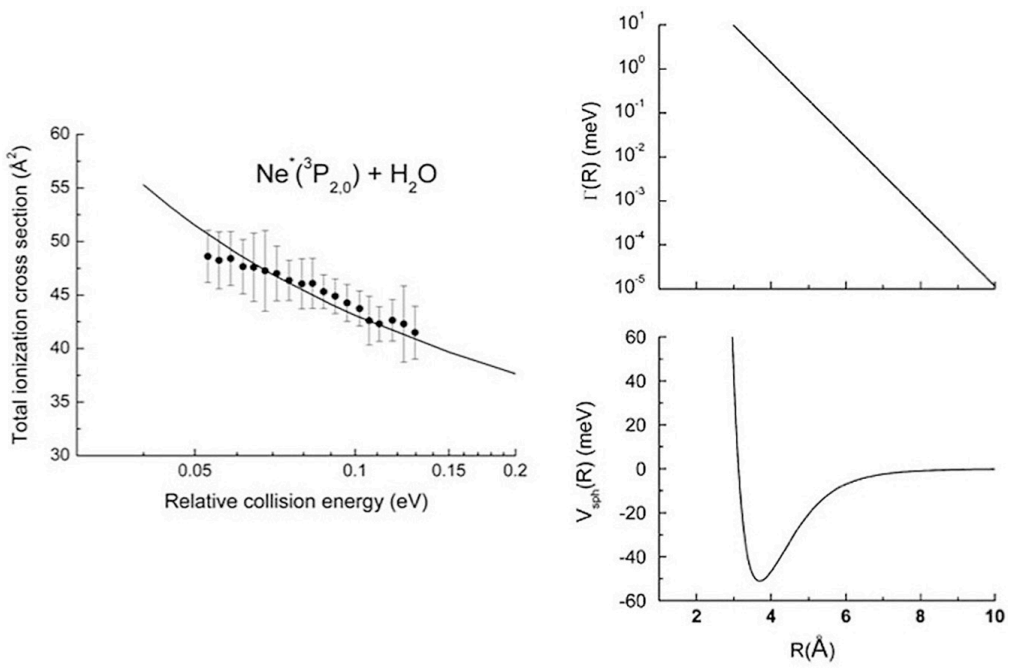

Figure 4. Left side: The total ionization cross-sections of the $\mathrm{Ne}^{*}-\mathrm{H}_{2} \mathrm{O}$ autoionizing collisions as a function of the relative collision energy. The curve represents the calculation of the cross-section by applying the theory defined in terms of the so-called optical potential model, which exploits the recorded energy shifts from the electron kinetic energy spectra to probe the main characteristics of the potential energy surface for the $\mathrm{Ne}^{*}-\mathrm{H}_{2} \mathrm{O}$ entrance channel (see the text and Figure 2) $[14,15,22]$. Right side: The spherical optical potential used for the cross-section calculation (see left side of this figure). Lower panel represents the real part of the optical potential, while the upper panel is the imaginary part (see the text and [23]).

The measured energy shifts for the three investigated systems are compared in Table 2, where are also reported those extracted from the measured PIES spectra for $\mathrm{He}^{*}-\mathrm{H}_{2} \mathrm{~S}$ and $\mathrm{NH}_{3}$ autoionizing collisions (see Figure 6). Also in these cases we recorded negative energy shifts having a bigger value, indicating a more attractive behavior in the case of $\mathrm{He}^{*}-\mathrm{M}$ collisions respect to $\mathrm{Ne}^{*}-\mathrm{M}$ collisions (with $\mathrm{M}=\mathrm{H}_{2} \mathrm{~S}$, $\mathrm{NH}_{3}$ ). This observation is in fairly good agreement with previous results obtained by Ohno et al. [27] and Ben Arfa et al. [39] for $\mathrm{He}^{*}-\mathrm{H}_{2} \mathrm{O}$ collisions, and can be justified in terms of the higher electronic polarizability of $\mathrm{He}^{*}$ atoms (118.9 and $46.9 \AA^{3}$ for $2^{1} \mathrm{~S}_{0}$ and $2^{3} S_{1}$ spin-orbit states, respectively) with respect to that of $\mathrm{Ne}^{*}\left(27.8 \AA^{3}\right.$ for the ${ }^{3} \mathrm{P}_{2}$ state), which is the fundamental physical property of the partners, enhancing the attractive interaction behavior of the potential energy surfaces [22]. 


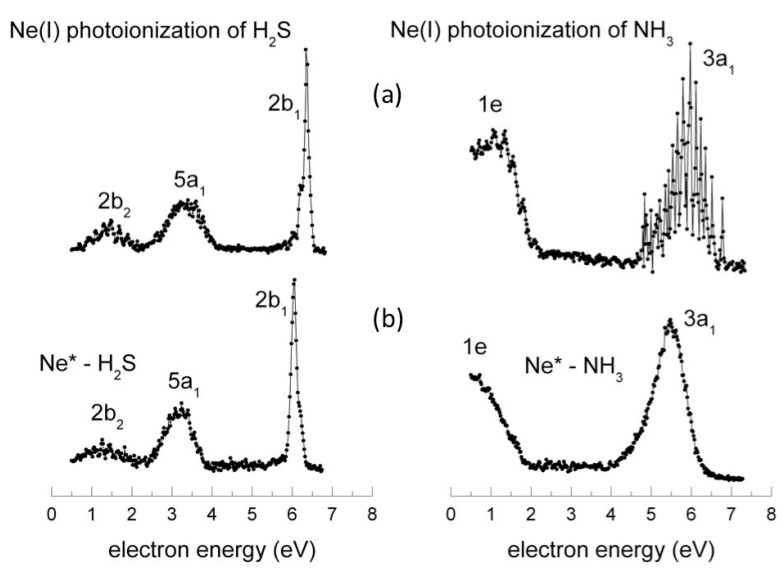

Figure 5. The obtained (a) PES (upper panel) and (b) PIES (lower panel) spectra in photoionization and Penning ionization studies of hydrogen sulfide (left side) and ammonia (right side) molecules by using $\mathrm{Ne}(\mathrm{I})$ photons and $\mathrm{Ne}^{*}\left({ }^{3} \mathrm{P}_{2,0}\right)$ metastable atoms, respectively. By looking at the relative position of the corresponding peak maxima in the PES and PIES spectra for both investigated systems, it is possible to measure the negative energy shifts $\left(-80 \mathrm{meV}\right.$ and $-120 \mathrm{meV}$ for $2 \mathrm{~b}_{1}$ and $5 \mathrm{a}_{1}$ electronic states of $\mathrm{H}_{2} \mathrm{~S}^{+}$, respectively; and $-290 \mathrm{meV}$ and $-140 \mathrm{meV}$ for $3 \mathrm{a}_{1}$ and 1e electronic states of $\mathrm{NH}_{3}{ }^{+}$), with the same procedure used in the $\mathrm{Ne}^{*}-\mathrm{H}_{2} \mathrm{O}$ case (see Figure 2 and the text).

As already discussed in previous papers [22,23], by a simple analysis of obtained PIES spectra, in the case of $\mathrm{H}_{2} \mathrm{O}^{+}-1 b_{1}, \mathrm{H}_{2} \mathrm{~S}^{+}-2 b_{1}$, and $\mathrm{NH}_{3}{ }^{+}-3 \mathrm{a}_{1}$ ground states it is possible to extract not only the negative shift, $\varepsilon_{\max }$, from the change in position of the maximum of peak but also the shift between the electron energy value where the peak intensity drops down to $44 \%$ of its maximum at the lower energy side, $\varepsilon_{\mathrm{A}}$, with respect to the nominal energy, $\varepsilon_{0}$ [40]. For a detailed description of the theory of Penning ionization with a full discussion of the relation between the measured energy shifts from recorded PIES spectra and the main characteristics of the related potential energy surfaces, we refer to the important and basic paper of H.W. Miller published in 1970 [40]. The obtained values amount to $-320 \pm 20 \mathrm{meV}$ for $\mathrm{Ne}^{*}-\mathrm{H}_{2} \mathrm{O}$ collisions, $-195 \pm 20 \mathrm{meV}$ for $\mathrm{Ne}^{*}-\mathrm{H}_{2} \mathrm{~S}$ collisions, and $-840 \pm 20 \mathrm{meV}$ for $\mathrm{Ne}^{*}-\mathrm{NH}_{3}$ collisions, and they are also reported in Table 2. These findings confirm the occurrence of a very strong attractive behavior of $\mathrm{Ne}^{*}-\mathrm{NH}_{3}$ interaction promoting the Penning process, which leads to the formation of the molecular ion in the ground electronic state. A still quite strong attractive behavior drives the $\mathrm{Ne}^{*}-\mathrm{H}_{2} \mathrm{O}$ collisions, while the corresponding attraction in the $\mathrm{Ne}^{*}-\mathrm{H}_{2} \mathrm{~S}$ collisions appears to be about $40 \%$ smaller than in the case of water. This consideration comes from the suggestion by Haug et al. [28], who noted that the energy shift $\varepsilon_{\mathrm{A}}$ can be used as a proper 
indication of the depth of the attractive potential well of the interaction between the colliding particles in the entrance channel. This suggestion is reasonable considering the weaker attraction in the exit channel due to the very low polarizability of neon atoms $\left(0.40 \AA^{3}\right)$ in the ground electronic state with respect to that in the excited ones $\left(27.8 \AA^{3}\right)$. In our case, the estimate of $\varepsilon_{\mathrm{A}}$ can be done only for the interaction of $\mathrm{Ne}^{*}$ approaching the $\mathrm{H}_{2} \mathrm{O}, \mathrm{H}_{2} \mathrm{~S}$, and $\mathrm{NH}_{3}$ molecules and producing $\left[\mathrm{Ne} \ldots \mathrm{H}_{2} \mathrm{O}^{+}\right.$, $\left[\mathrm{Ne} \ldots \mathrm{H}_{2} \mathrm{~S}\right]^{+}$and $\left[\mathrm{Ne} \ldots \mathrm{NH}_{3}\right]^{+}$intermediate ionic complexes in the $\left(\mathrm{X}^{2} B_{1}\right)-1 \mathrm{~b}_{1}$, $\left(\mathrm{X}^{2} B_{1}\right)-2 \mathrm{~b}_{1}$, and $\left(\mathrm{X}^{2} A_{2}{ }^{\prime \prime}\right)-2 \mathrm{~b}_{1}$ ground electronic states, respectively. An analogous evaluation of $\varepsilon_{\mathrm{A}}$ for the $\left(\mathrm{A}^{2} A_{1}\right)$ and $\left(\mathrm{A}^{2} E\right)$ excited states of $\left[\mathrm{Ne} \ldots \mathrm{H}_{2} \mathrm{O}\right]^{+},[\mathrm{Ne} \ldots$ $\left.\mathrm{H}_{2} \mathrm{~S}\right]^{+}$and $\left[\mathrm{Ne} \ldots \mathrm{NH}_{3}\right]^{+}$intermediate ionic complexes is not possible because in these cases the recorded broad bands in the PIES spectra do not satisfy the features required by the theory $[28,40-42]$.

Table 2. Negative energy shifts from the PIES $\mathrm{He}^{*}$ and $\mathrm{Ne}^{*}-\mathrm{H}_{2} \mathrm{O}, \mathrm{H}_{2} \mathrm{~S}$ and $\mathrm{NH}_{3}$ spectra measured at an average collision energy of 70 and $55 \mathrm{meV}$, respectively.

\begin{tabular}{|c|c|c|}
\hline & \multicolumn{2}{|c|}{ Shifts with Respect to Nominal Energy $\varepsilon_{0}(\mathrm{meV})$} \\
\hline Band in PIES & $\mathrm{H}_{2} \mathrm{O}^{+}\left(\mathrm{X}^{2} B_{1}\right)-1 \mathrm{~b}_{1}$ & $\mathrm{H}_{2} \mathrm{O}^{+}\left(\mathrm{A}^{2} A_{1}\right)-3 \mathrm{a}_{1}$ \\
\hline $\begin{array}{l}\text { maximum of peak }(\mathrm{v}=0) \varepsilon_{\max } \\
\text { for } \mathrm{He}^{*}\left({ }^{3} \mathrm{~S}_{1}\right) \text { collisions }\end{array}$ & $-440^{1}$ & $-485^{1, *}$ \\
\hline $\begin{array}{l}\text { maximum of peak }(\mathrm{v}=0) \varepsilon_{\max } \\
\text { for } \mathrm{He}^{*}\left({ }^{1} \mathrm{~S}_{0}\right) \text { collisions }\end{array}$ & $-720^{2}$ & $-700^{2, *}$ \\
\hline $\begin{array}{l}\text { maximum of peak }(\mathrm{v}=0) \varepsilon_{\max } \\
\text { for } \mathrm{Ne}^{*} \text { collisions }\end{array}$ & $-140 \pm 20$ & $-300 \pm 20 *$ \\
\hline $\begin{array}{l}44 \% \text { of maximum }(\mathrm{v}=0) \varepsilon_{\mathrm{A}} \\
\text { for } \mathrm{Ne}^{*} \text { collisions }\end{array}$ & $-320 \pm 20$ & Not evaluable \\
\hline Band in PIES & $\mathrm{H}_{2} \mathrm{~S}^{+}\left(\mathrm{X}^{2} \mathrm{~B}_{1}\right)-2 \mathrm{~b}_{1}$ & $\mathrm{H}_{2} \mathrm{~S}^{+}\left(\mathrm{A}^{2} A_{1}\right)-5 \mathrm{a}_{1}$ \\
\hline $\begin{array}{l}\text { maximum of peak }(\mathrm{v}=0) \varepsilon_{\max } \\
\text { for } \mathrm{He}^{*}\left({ }^{3} S_{1}\right) \text { collisions }\end{array}$ & $-150 \pm 20$ & $-190 \pm 20 *$ \\
\hline $\begin{array}{l}\text { maximum of peak }(\mathrm{v}=0) \varepsilon_{\max } \\
\text { for } \mathrm{He}^{*}\left({ }^{1} \mathrm{~S}_{0}\right) \text { collisions }\end{array}$ & $-220 \pm 20$ & $-230 \pm 20 *$ \\
\hline $\begin{array}{c}\text { maximum of peak }(\mathrm{v}=0) \varepsilon_{\max } \\
\text { for } \mathrm{Ne}^{*} \text { collisions }\end{array}$ & $-80 \pm 20$ & $-120 \pm 20 *$ \\
\hline $\begin{array}{l}44 \% \text { of maximum }(\mathrm{v}=0) \varepsilon_{\mathrm{A}} \\
\text { for } \mathrm{Ne}^{*} \text { collisions }\end{array}$ & $-195 \pm 20$ & Not evaluable \\
\hline Band in PIES & $\mathrm{NH}_{3}{ }^{+}\left(\mathrm{X}^{2} \mathrm{~A}_{2}^{\prime \prime}\right)-3 \mathrm{a}_{1}$ & $\mathrm{NH}_{3}{ }^{+}\left(\mathrm{A}^{2} \mathrm{E}\right)-1 \mathrm{e}$ \\
\hline $\begin{array}{l}\text { maximum of peak }(\mathrm{v}=0) \varepsilon_{\max } \\
\text { for } \mathrm{He}^{*}\left({ }^{3} S_{1}\right) \text { collisions }\end{array}$ & $-450 \pm 20$ & $-350 \pm 20 *$ \\
\hline $\begin{array}{l}\text { maximum of peak }(\mathrm{v}=0) \varepsilon_{\max } \\
\text { for } \mathrm{He}^{*}\left({ }^{1} \mathrm{~S}_{0}\right) \text { collisions }\end{array}$ & $-585 \pm 20$ & Not evaluable \\
\hline $\begin{array}{l}\text { maximum of peak }(\mathrm{v}=0) \varepsilon_{\max } \\
\text { for } \mathrm{Ne}^{*} \text { collisions }\end{array}$ & $-290 \pm 20$ & $-140 \pm 20 *$ \\
\hline $\begin{array}{l}44 \% \text { of maximum }(\mathrm{v}=0) \varepsilon_{\mathrm{A}} \\
\text { for } \mathrm{Ne}^{*} \text { collisions }\end{array}$ & $-840 \pm 20$ & Not evaluable \\
\hline
\end{tabular}

*: evaluated from the maximum of band; ${ }^{1}$ : from [19]; ${ }^{2}$ : from [32]. 


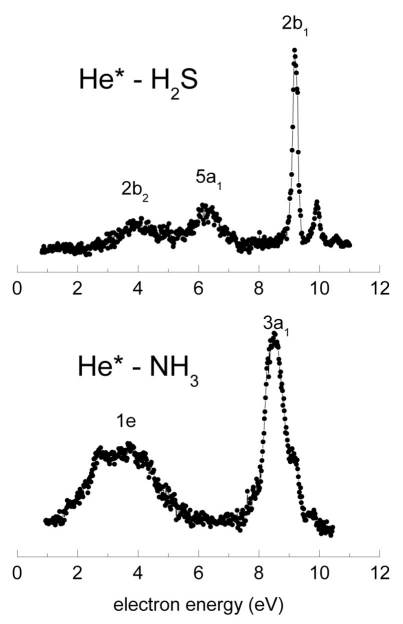

Figure 6. The obtained PIES spectra in Penning ionization studies of hydrogen sulfide (upper panel) and ammonia (lower panel) molecules by using $\mathrm{He}^{*}\left({ }^{3} \mathrm{~S}_{1}\right.$, ${ }^{1} \mathrm{~S}_{0}$,) metastable atoms. In the $2 \mathrm{~b}_{1}$ band of the $\mathrm{He}^{*}-\mathrm{H}_{2} \mathrm{~S}$ spectrum, the two peaks recorded at an electron energy value of 9.18 and $9.94 \mathrm{eV}$, and related to $\mathrm{He}^{*}\left({ }^{3} \mathrm{~S}_{1}\right)$ and $\mathrm{He}^{*}\left({ }^{1} \mathrm{~S}_{0}\right)$ collisional ionization events, respectively, appear well separated (see upper panel). The two $\mathrm{He}^{*}\left({ }^{3} \mathrm{~S}_{1},{ }^{1} \mathrm{~S}_{0},\right)$ metastable components are still present, even if less separated, in the wide $3 \mathrm{a}_{1}$ band of the $\mathrm{He}^{*}-\mathrm{NH}_{3}$ spectrum (see lower panel).

\section{Conclusions}

Considering that a basic step in the chemical evolution of planetary atmospheres and interstellar clouds (where $89 \%$ of atoms are hydrogen and $9 \%$ are helium) is the interaction of atoms and molecules with electromagnetic waves ( $\gamma$ and $X$ rays, UV light) and cosmic rays [43], the formation of excited metastable species like $\mathrm{He}^{*}$ and $\mathrm{Ar}^{*}$ (argon is the third component of Earth and Mars' atmosphere) by collisional excitation with energetic target particles (electrons, protons, or alpha particles, coming from the solar wind) and the possible subsequent Penning ionization reactions, could be of importance in these environments. It has to be stressed that the effect of Penning ionization has not yet been fully considered in the modeling of terrestrial and extraterrestrial objects, even though metastable excited species can be formed in such environments. In this paper recent experimental results concerning production and characterization of simple ionic species of atmospheric interest are presented and discussed. Such results concern the formation of free ions in collisional ionization of $\mathrm{H}_{2} \mathrm{O}, \mathrm{H}_{2} \mathrm{~S}$, and $\mathrm{NH}_{3}$ induced by highly excited species, like $\mathrm{He}^{*}$ and $\mathrm{Ne}^{*}$ metastable atoms.

The analysis of the recorded electron spectra for the three presented systems clearly shows: (i) a very strong and anisotropic attractive behavior of $\mathrm{Ne}^{*}-\mathrm{NH}_{3}$ 
interaction promoting the Penning process; (ii) a still quite strong attractive behavior for $\mathrm{Ne}^{*}-\mathrm{H}_{2} \mathrm{O}$ collisions; and (iii) a corresponding attractive interaction in the $\mathrm{Ne}^{*}-\mathrm{H}_{2} \mathrm{~S}$ collisions, which appear to be about $40 \%$ that of water. The analyzed experimental observables, in term of energy shifts, clearly indicate that $\mathrm{H}_{2} \mathrm{O}$ and $\mathrm{H}_{2} \mathrm{~S}$ show similar behavior when these hydrogenated molecules are involved in Penning ionization induced by $\mathrm{He}^{*}$ and $\mathrm{Ne}^{*}$ metastable atoms, and in particular: (i) the interaction potential driving the formation of $\mathrm{A}^{2} \mathrm{~A}_{1}$ excited state of $\mathrm{H}_{2} \mathrm{O}^{+}$and $\mathrm{H}_{2} \mathrm{~S}^{+}$product ions exhibits an effective interaction potential well depth in the entrance channel of the ionization reaction, quite deeper (about $50 \%$ more in the case of water and about $25 \%$ more in the case of hydrogen sulfide) than that involved in the production of the same final ions in their $\mathrm{X}^{2} \mathrm{~B}_{1}$ ground state (see the relative $\varepsilon_{\max }$ values reported in the Table 2); (ii) a quite pronounced anisotropic attraction controls the stereodynamics in the entrance channels of both $\left(\mathrm{Ne}^{*}-\mathrm{H}_{2} \mathrm{O}, \mathrm{H}_{2} \mathrm{~S}\right)$ analyzed systems: in the case of $\mathrm{Ne}^{*}-\mathrm{H}_{2} \mathrm{O}$ autoionizing collisions a strong anisotropic interaction is operative, while in the $\mathrm{Ne}^{*}-\mathrm{H}_{2} \mathrm{~S}$ system the attractive interaction appears to be weaker than the system involving water; and (iii) the entity of this smaller attraction, characterizing $\mathrm{Ne}^{*}-\mathrm{H}_{2} \mathrm{~S}$ collisions, is dependent on the approach orientation between $\mathrm{Ne}^{*}$ atoms and the hydrogenated target molecules: it is about $43 \%$ with respect to the $\mathrm{Ne}^{*}-\mathrm{H}_{2} \mathrm{O}$ case for those collisions producing $\mathrm{H}_{2} \mathrm{X}^{+}$parent ions (where $\mathrm{X}$ stands for $\mathrm{O}$ or $\mathrm{S}$ atom) in their $\mathrm{X}^{2} \mathrm{~B}_{1}$ ground electronic state (this is the case for the $\mathrm{Ne}^{*}-\mathrm{H}_{2} \mathrm{X}$ collisions towards the orthogonal direction respect to the molecular plane), while it reaches about $60 \%$ of the $\mathrm{Ne}^{*}-\mathrm{H}_{2} \mathrm{O}$ interaction when the exchange mechanism of Penning ionization can exploit $\mathrm{Ne}^{*}$ collisions along the $\mathrm{C}_{2 \mathrm{v}}$ molecular axis in the opposite side with respect to hydrogen atoms, with the formation of $\mathrm{H}_{2} \mathrm{X}^{+}$final ions in their first $\mathrm{A}^{2} \mathrm{~A}_{1}$ excited electronic state. In the case of $\mathrm{Ne}^{*}-\mathrm{NH}_{3}$ collisions, a very strong and anisotropic attractive interaction is observed, characterizing the potential energy surface of the entrance channel with a much deeper well depth in the case of those collisions producing the $\left[\mathrm{Ne} \ldots \mathrm{NH}_{3}\right]^{+}$intermediate ionic complex, leading product ion in its $\mathrm{X}\left({ }^{2} A_{2}{ }^{\prime \prime}\right)-3 \mathrm{a}_{1}$ ground electronic state (which is an opposite situation to that observed in $\mathrm{H}_{2} \mathrm{O}$ and $\mathrm{H}_{2} \mathrm{~S}$ systems). In this case the formation of $[\mathrm{Ne} \ldots$ $\left.\mathrm{NH}_{3}\left(X^{2} A_{2}{ }^{\prime \prime}\right)\right]^{+}$occurs preferentially when the neon metastable atom approaches ammonia molecules towards the direction of the $3 \mathrm{a}_{1}$ non-bonding lone pair located on the nitrogen atom, removes one electron from this orbital, and gives rise to Penning ionization. Finally, the analysis of PIES spectra recorded for $\mathrm{He}^{*}-\mathrm{H}_{2} \mathrm{~S}$ and $-\mathrm{NH}_{3}$ collisions, confirms the attractive and anisotropic behavior of the potential energy surfaces already discussed for $\mathrm{Ne}^{*}-\mathrm{M}$ interactions (with $\mathrm{M}=\mathrm{H}_{2} \mathrm{O}, \mathrm{H}_{2} \mathrm{~S}$, and $\mathrm{NH}_{3}$ ) governing the entrance channel for Penning ionization of such hydrogenated molecules. A similar situation for metastable helium and metastable neon atoms interacting with $\mathrm{N}_{2} \mathrm{O}$ molecules [19] has been explained by the use of a semiempirical method that accounts for the metastable atom orbital deformation because of the 
permanent dipole of the molecule. We have plans to extend such a model also to the systems presented here, and to test the potential energy surfaces so obtained in a comparative way. This work, is in progress in our laboratory, will allow us to achieve a deeper understanding of the stereodynamics of the collisional autoionization processes involving $\mathrm{He}^{*}$ and $\mathrm{Ne}^{*}$ with the hydrogenated molecules here discussed, and of the propensity to form ions.

Acknowledgments: Stefano Falcinelli is very grateful to Richard N. Zare of the Chemistry Department of Stanford University, CA, USA, for his constant encouragement and the useful suggestions during the period between June and September 2014 when Stefano Falcinelli was working in his laboratory as a visiting scholar and was conceiving the paper here presented.

Author Contributions: Stefano Falcinelli and Franco Vecchiocattivi conceived and designed the experiment; Stefano Falcinelli performed the experiment; Fernando Pirani developed the semiempirical method for potential energy surfaces calculations; all the authors analyzed the data, participated to the discussion on the obtained results, and contributed to write the paper.

Conflicts of Interest: The authors declare no conflict of interest.

\section{References}

1. Davies, K.; Rush, C.M. Reflection of high-frequency radio waves in inhomogeneous ionospheric layers. Radio Sci. 1985, 20, 303-309.

2. Hruska, F. Electromagnetic Interference and Environment. Int. J. Circuits Syst. Signal Process. 2014, 8, 22-29.

3. Qiu, H.; Chen, L.T.; Qiu, G.P.; Zhou, C. 3D visualization of radar coverage considering electromagnetic interference. WSEAS Trans. Signal Process. 2014, 10, 460-470.

4. Egano, F. Radio Propagation Observatory. Available online: http://www.qsl.net/ik3xtv (accessed on 14 August 2014).

5. Hotop, H. Detection of metastable atoms and molecules. In Atomic, Molecular, and Optical Physics: Atoms and Molecules; Dunning, F.B., Hulet, R.G., Eds.; Academic Press, Inc.: San Diego, CA, USA, 1996; pp. 191-216.

6. Brunetti, B.G.; Falcinelli, S.; Giaquinto, E.; Sassara, A.; Prieto-Manzanares, M.; Vecchiocattivi, F. Metastable-Idrogen-Atom scattering by crossed beams: Total cross sections for $\mathrm{H}^{*}(2 \mathrm{~s})-\mathrm{Ar}, \mathrm{Xe}$, and $\mathrm{CCl}_{4}$ at thermal energies. Phys. Rev. A 1995, 52, 855-858.

7. Falcinelli, S. Penning ionization of simple molecules and their possible role in planetary atmospheres. In Recent Advances in Energy, Environment and Financial Planning-Mathematics and Computers in Science and Engineering Series; Batzias, F., Mastorakis, N.E., Guarnaccia, C., Eds.; WSEAS Press: Athens, Greece, 2014; pp. 84-92.

8. Rodger, C.J.; Nunn, D. VLF scattering from red sprites: Application of numerical modeling. Radio Sci. 1999, 34, 923-932.

9. Larsson, M.; Geppert, W.D.; Nyman, G. Ion chemistry in space. Rep. Prog. Phys. 2012, $75,066901$.

10. Stauber, P.; Doty, S.D.; van Dishoeck, E.F.; Benz, A.O. X-ray chemistry in the envelopes around young stellar objects. Astron. Astrophys. 2005, 440, 949-966. 
11. Indriolo, N.; Hobbs, L.M.; Hinkle, K.H.; McCall, B.J. Interstellar metastable helium absorption as a probe of the cosmic-ray ionization rate. Astrophys. J. 2009, 703, 2131-2137.

12. Waldrop, L.S.; Kerr, R.B.; González, S.A.; Sulzer, M.P.; Noto, J.; Kamalabadi, F. Generation of metastable helium and $1083 \mathrm{~nm}$ emission in the upper thermosphere. J. Geophys. Res. 2005, 110, A08304.

13. Bishop, J.; Link, R. He $\left(2^{3} \mathrm{~S}\right)$ densities in the upper thermosphere: Updates in modeling capabilities and comparison with midlatitude observations. J. Geophys. Res. 2005, 104, 17157-17172.

14. Siska, P.E. Molecular-beam studies of Penning ionization. Rev. Mod. Phys. 1993, 65, 337-412.

15. Brunetti, B.G.; Vecchiocattivi, F. Autoionization dynamics of collisional complexes. In Ion Clusters; Ng, C., Baer, T., Powis, I., Eds.; Springer: New York, NY, USA, 1993; pp. 359-445.

16. Penning, F.M. Über Ionisation durch metastabile Atome. Naturwissenschaften. J. 1927, 15.

17. Legon, A.C. The halogen bond: An interim perspective. Phys. Chem. Chem. Phys. 2010, 12, 7736-7747.

18. Biondini, F.; Brunetti, B.G.; Candori, P.; de Angelis, F.; Falcinelli, S.; Tarantelli, F.; Teixidor, M.M.; Pirani, F.; Vecchiocattivi, F. Penning ionization of $\mathrm{N}_{2} \mathrm{O}$ molecules by $\mathrm{He}^{*}\left(2^{3,1} \mathrm{~S}\right)$ and $\mathrm{Ne}^{*}\left({ }^{3} \mathrm{P}_{2,0}\right)$ metastable atoms: A crossed beam study. J. Chem. Phys. 2005, 122, 164307:1-164307:10.

19. Biondini, F.; Brunetti, B.G.; Candori, P.; de Angelis, F.; Falcinelli, S.; Tarantelli, F.; Pirani, F.; Vecchiocattivi, F. Penning ionization of $\mathrm{N}_{2} \mathrm{O}$ molecules by $\mathrm{He}^{*}\left(2^{3,1} \mathrm{~S}\right)$ and $\mathrm{Ne}^{*}\left({ }^{3} \mathrm{P}_{2,0}\right)$ metastable atoms: Theoretical considerations about the intermolecular interactions. J. Chem. Phys. 2005, 122, 164308:1-164308:11.

20. McClintock, W.E.; Lankton, M.R. The mercury atmospheric and surface composition spectrometer for the MESSENGER mission. J. Phys. Chem. Ref. Data 1989, 21, 1125-1499.

21. Atkinson, R.; Baulch, D.L.; Cox, R.A.; Hampson, R.F.; Kerr, J.A., Jr.; Troe, J. Evaluated kinetic and photochemical data for atmospheric chemistry: Supplement IV. IUPAC Subcommittee on kinetic data evaluation for atmospheric chemistry. Space Sci. Rev. 2007, 131, 481-521.

22. Brunetti, B.G.; Candori, P.; Cappelletti, D.; Falcinelli, S.; Pirani, F.; Stranges, D.; Vecchiocattivi, F. Penning ionization electron spectroscopy of water molecules by metastable neon atoms. Chem. Phys. Lett. 2012, 539-540, 19-23.

23. Balucani, N.; Bartocci, A.; Brunetti, B.G.; Candori, P.; Falcinelli, S.; Pirani, F.; Palazzetti, F.; Vecchiocattivi, F. Collisional autoionization dynamics of $\mathrm{Ne}^{*}\left({ }^{3} \mathrm{P}_{2,0}\right)-\mathrm{H}_{2} \mathrm{O}$. Chem. Phys. Lett. 2012, 546, 34-39.

24. Brunetti, B.G.; Candori, P.; Falcinelli, S.; Pirani, F.; Vecchiocattivi, F. The stereodynamics of the Penning ionization of water by metastable neon atoms. J. Chem. Phys. 2013, 139, 164305:1-164305:8.

25. Čermák, V.; Yencha, A.J. Penning ionization electron spectroscopy of $\mathrm{H}_{2} \mathrm{O}, \mathrm{D}_{2} \mathrm{O}, \mathrm{H}_{2} \mathrm{~S}$ and $\mathrm{SO}_{2}$. J. Electron Spectr. Rel. Phenom. 1977, 11, 67-73. 
26. Brion, C.E.; Yee, D.S.C. Electron spectroscopy using excited atoms and photons IX. Penning ionization of $\mathrm{CO}_{2}, \mathrm{CS}_{2}, \mathrm{COS}, \mathrm{N}_{2} \mathrm{O}, \mathrm{H}_{2} \mathrm{~S}, \mathrm{SO}_{2}$, and $\mathrm{NO}_{2}$. J. Electron Spectr. Rel. Phenom. 1977, 12, 77-93.

27. Ohno, K.; Mutoh, H.; Harada, Y. Study of electron distributions of molecular orbitals by Penning ionization electron spectroscopy. J. Am. Chem. Soc. 1983, 105, 4555-4561.

28. Haug, B.; Morgner, H.; Staemmler, V. Experimental and theoretical study of Penning ionisation of $\mathrm{H}_{2} \mathrm{O}$ by metastable Helium He ( $2^{3}$ S). J. Phys. B At. Mol. Phys. 1985, 18, 259-274.

29. Bentley, J. Potential energy surfaces for excited neon atoms interacting with water molecules. J. Chem. Phys. 1980, 73, 1805-1813.

30. Ishida, T. A quasi-classical trajectory calculation for the Penning ionization $\mathrm{H}_{2} \mathrm{O}-\mathrm{He}^{*}\left(2^{3} \mathrm{~S}\right)$. J. Chem. Phys. 1996, 105, 1392-1401.

31. Falcinelli, S.; Candori, P.; Bettoni, M.; Pirani, F.; Vecchiocattivi, F. Penning ionization electron spectroscopy of hydrogen sulfide by metastable Helium and Neon atoms. J. Phys. Chem. A 2014, 118, 6501-6506.

32. Arfa, M.B.; Lescop, B.; Cherid, M.; Brunetti, B.; Candori, P.; Malfatti, D.; Falcinelli, S.; Vecchiocattivi, F. Ionization of ammonia molecules by collision with metastable neon atoms. Chem. Phys. Lett. 1999, 308, 71-77.

33. Brunetti, B.; Candori, P.; Falcinelli, S.; Vecchiocattivi, F.; Sassara, A.; Chergui, M. Dynamics of the Penning ionization of fullerene molecules by metastable Neon atoms. J. Phys. Chem. A 2000, 14, 5942-5945.

34. Brunetti, B.; Candori, P.; Falcinelli, S.; Kasai, T.; Ohoyama, H.; Vecchiocattivi, F. Velocity dependence of the ionization cross section of methyl chloride molecules ionized by metastable argon atoms. Phys. Chem. Chem. Phys. 2001, 3, 807-810.

35. Brunetti, B.; Candori, P.; Falcinelli, S.; Lescop, B.; Liuti, G.; Pirani, F.; Vecchiocattivi, F. Energy dependence of the Penning ionization electron spectrum of $\mathrm{Ne}^{*}\left({ }^{3} \mathrm{P}_{2,0}\right)+\mathrm{Kr}$. Eur. Phys. J. D 2006, 38, 21-27.

36. Kraft, T.; Bregel, T.; Ganz, J.; Harth, K.; Ruf, M.-W.; Hotop, H. Accurate comparison of Hel, NeI photoionization and $\mathrm{He}\left(2^{3,1} \mathrm{~S}\right), \mathrm{Ne}\left(3 s^{3} P_{2},{ }^{3} P_{0}\right)$ Penning ionization of argon atoms and dimers. Z. Phys. D 1988, 10, 473-481.

37. Kimura, K.; Katsumata, S.; Achiba, Y.; Yamazaky, T.; Iwata, S. Handbook of HeI Photoelectron Spectra of Fundamental Organic Molecules; Japan Scientific Societies Press: Tokyo, Japan, 1981.

38. West, W.P.; Cook, T.B.; Dunning, F.B.; Rundel, R.D.; Stebbings, R.F. Chemiionization involving rare gas metastable atoms. J. Chem. Phys. 1975, 63, 1237-1242.

39. Arfa, M.B.; le Coz, G.; Sinou, G.; le Nadan, A.; Tuffin, F.; Tannous, C. Experimental study of the Penning ionization of the $\mathrm{H}_{2} \mathrm{O}$ molecule by $\mathrm{He}^{*}\left(2^{3} \mathrm{~S}, 2^{1} \mathrm{~S}\right)$ metastable atoms. J. Phys. B At. Mol. Opt. Phys. 1994, 27, 2541-2550.

40. Miller, W.H. Theory of Penning ionization. I. Atoms. J. Chem. Phys. 1970, 52, 3563-3571.

41. Hotop, H.; Niehaus, A. Reactions of excited atoms molecules with atoms and molecules. II. Energy analysis of penning electrons. Z. Phys. D 1969, 228, 68-88. 
42. Falcinelli, S.; Rosi, M.; Candori, P.; Vecchiocattivi, F.; Bartocci, A.; Lombardi, A.; Lago, N.F.; Pirani, F. Modeling the intermolecular interactions and characterization of the dynamics of collisional autoionization processes. In ICCSA 2013, Part I, LNCS 7971 Computational Science and its Applications; Murgante, B., Misra, S., Carlini, M., Torre, C.M., Nguyen, H.Q., Taniar, D., Apduhan, B.O., Gervasi, O., Eds.; Springer-Verlag: Berlin Heidelberg, Germany, 2013; pp. 47-56.

43. Falcinelli, S.; Rosi, M.; Candori, P.; Vecchiocattivi, F.; Farrar, J.M.; Pirani, F.; Balucani, N.; Alagia, M.; Richter, R.; Stranges, S. Kinetic energy release in molecular dications fragmentation after VUV and EUV ionization and escape from planetary atmospheres. Planet. Space Sci. 2014, 99, 149-157. 


\title{
Chemical Composition of Water Soluble Inorganic Species in Precipitation at Shihwa Basin, Korea
}

\author{
Seung-Myung Park, Beom-Keun Seo, Gangwoong Lee, Sung-Hyun Kahng and \\ Yu Woon Jang
}

Abstract: Weekly rain samples were collected in coastal areas of the Shihwa Basin (Korea) from June 2000 to November 2007. The study region includes industrial, rural, and agricultural areas. Wet precipitation was analyzed for conductivity, $\mathrm{pH}$, $\mathrm{Cl}^{-}, \mathrm{NO}_{3}{ }^{-}, \mathrm{SO}_{4}{ }^{2-}, \mathrm{Na}^{+}, \mathrm{K}^{+}, \mathrm{Mg}^{2+}, \mathrm{NH}_{4}{ }^{+}$, and $\mathrm{Ca}^{2+}$. The major components of precipitation in the Shihwa Basin were $\mathrm{NH}_{4}{ }^{+}$, volume-weighted mean (VWM) of $44.6 \mu \mathrm{eq} \cdot \mathrm{L}^{-1}$, representing $43 \%$ of all cations, and $\mathrm{SO}_{4}{ }^{2-}$, with the highest concentration among the anions (55\%) at all stations. The $\mathrm{pH}$ ranged from 3.4 to 7.7 with a VMM of 4.84 . $\mathrm{H}^{+}$was weakly but positively correlated with $\mathrm{SO}_{4}{ }^{2-}$ $(r=0.39, p<0.001)$ and $\mathrm{NO}_{3}{ }^{-}(r=0.38, p<0.001)$. About $66 \%$ of the acidity was neutralized by $\mathrm{NH}_{4}{ }^{+}$and $\mathrm{Ca}^{2+}$. The $\mathrm{Cl}^{-} / \mathrm{Na}^{+}$ratio of the precipitation was $37 \%$ higher than seawater $\mathrm{Cl}^{-} / \mathrm{Na}^{+}$. The high $\mathrm{SO}_{4}{ }^{2-} / \mathrm{NO}_{3}{ }^{-}$ratio of 2.3 is attributed to the influence of the surrounding industrial sources. Results from positive matrix factorization showed that the precipitation chemistry in Shihwa Basin was influenced by secondary nitrate and sulfate $(41 \% \pm 1.1 \%)$, followed by sea salt and Asian dust, contributing $23 \% \pm 3.9 \%$ and $17 \% \pm 0.2 \%$, respectively. In this study, the annual trends of $\mathrm{SO}_{4}{ }^{2-}$ and $\mathrm{NO}_{3}{ }^{-}(p<0.05)$ increased, different from the trends in some locations, due to the influence of the expanding power generating facilities located in the upwind area. The increasing trends of $\mathrm{SO}_{4}{ }^{2-}$ and $\mathrm{NO}_{3}{ }^{-}$in the study region have important implications for reducing air pollution in accordance with national energy policy.

Reprinted from Atmosphere. Cite as: Park, S.-M.; Seo, B.-K.; Lee, G.; Kahng, S.-H.; Jang, Y.W. Chemical Composition of Water Soluble Inorganic Species in Precipitation at Shihwa Basin, Korea. Atmosphere 2015, 6, 1069-1101.

\section{Introduction}

Rain is the most effective process transporting soluble gases and particles from the atmosphere to the ground [1,2]. Precipitation chemistry plays an important role in understanding the air quality in a study area, because the concentrations and distribution of chemical components in rain depend on a variety of emission sources including sea spray, soil particles, and industrial pollutants [3-7]. 
In coastal areas, the $\mathrm{Na}^{+} / \mathrm{Cl}^{-}$ratio, $\mathrm{SO}_{4}{ }^{2-}, \mathrm{Mg}^{2+}$, and $\mathrm{Ca}^{2+}$ are useful for evaluating both anthropogenic and natural influences [8]. Soil particles in northern China, Africa, and the Middle East play an important role in neutralizing the acidic components of rain [9]. The concentrations of anthropogenic components are related to energy consumption and the use of fertilizers [10,11].

Some anthropogenic acidic ions may greatly contribute to acidification and eutrophication of aquatic ecosystems [12]. Numerous long-term observations of precipitation have been carried out in Europe, North America, and Asia [13-17]. These studies have reported that $\mathrm{SO}_{4}{ }^{2-}$ concentrations have decreased commensurate with reductions in $\mathrm{SO}_{2}$ emissions. $\mathrm{NO}_{3}{ }^{-}$concentrations have also shown decreasing trends in regions where $\mathrm{NO}_{2}$ emissions have decreased [18-20].

In many regions of Asia, rapid economic growth has led to an increase in pollutant emissions and seriously compromised air quality [21]. In addition, air quality has also been affected by wind-blown soil particles originating from the deserts of Mongolia and northern China [22-24]. Several studies conducted in this region have found that the rain is acidic, with $\mathrm{H}^{+}$concentration ranges similar to that reported for Europe [25-28].

In Korea, previous studies have shown that most precipitation events are acidic and even in background areas of Korea precipitation $\mathrm{pH}$ is $<4.9$ [29-31]. Some studies have suggested that air masses from the Asian continent increase the concentration of anthropogenic components of rainwater in Korea [31,32]. Choi et al. (2015) [33] studied precipitation chemistry in the area near Shihwa Lake (located in the mid-western area of the Korean peninsula), using both urban and background stations to compare precipitation chemistry. To date, precipitation chemistry in the industrial area has not been studied yet.

This study aimed to provide a detailed evaluation of the chemical composition of precipitation in the Shihwa Basin and to evaluate the relative contributions of various sources. Because the Shihwa Basin includes areas with industrial, agricultural, and coastal land use, the study are has experienced serious problems with air pollution [34-37].

\section{Material and Methods}

\subsection{Sampling Sites}

The Shihwa Basin is located $60 \mathrm{~km}$ southwest of Seoul, the capital of South Korea. The basin of Shihwa Lake has an area of about $475 \mathrm{~km}^{2}$ and a population of more than 0.8 million people. The Shihwa and Banwol industrial complexes are located in Shihwa Basin. Three sampling stations were selected for precipitation monitoring, covering the southern (Hwasung), northern (Banwol), and western (Daeboo) areas of the Shihwa Basin (Table 1). Hwasung station is located inland about $20 \mathrm{~km}$ east 
of Shihwa Lake, in a residential and rural area. Banwol station is located $5 \mathrm{~km}$ east of Shihwa Lake and is important because the Banwol industrial complex is located there. This complex is a site for intensive chemical, leather, and metal processing industries, which have led to air pollution episodes, including pollutants such as ammonia, amines, hydrogen sulfide $\left(\mathrm{H}_{2} \mathrm{~S}\right)$ and mercaptans. Daeboo station is located on an island, about $2 \mathrm{~km}$ west of Shihwa Lake. The locations of the Shihwa Basin and the three sampling stations are shown in Figure 1.

Table 1. Characteristics of sampling stations in the Shihwa Basin.

\begin{tabular}{ccccc}
\hline Shihwa Basin & Latitude & Longitude & Elevation $(\mathbf{m})$ & Land Type \\
\hline Hwasung & 37.236 & 126.918 & 31 & Rural and \\
Banwol & 37.315 & 126.750 & 56 & residential \\
Daeboo & 37.268 & 126.568 & 25 & Industrial \\
\hline
\end{tabular}

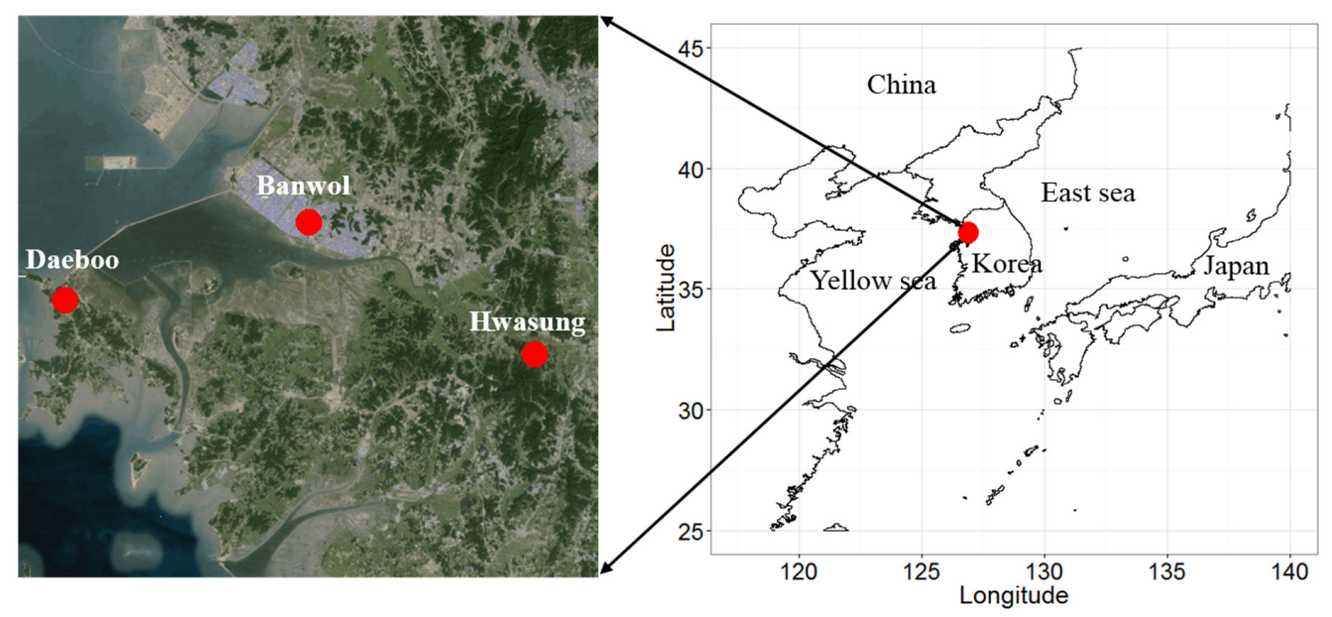

Figure 1. Locations of the study area and sampling stations.

The Shihwa Basin is strongly influenced by air masses driven by westerly winds. According to data from the Incheon meteorological administration (about $20 \mathrm{~km}$ north of Shihwa Lake), the average annual rainfall from June 2000 to November 2007 was $1187 \mathrm{~mm} \cdot$ year $^{-1}$. The average temperature and wind speed were $15^{\circ} \mathrm{C}$ and $2 \mathrm{~m} \cdot \mathrm{s}^{-1}$, respectively. In the summer (June to August), the mean temperature was $24.5^{\circ} \mathrm{C}$ and about $61 \%$ of the annual precipitation occurred during this season. In winter, the average temperature was $3.5^{\circ} \mathrm{C}$ with only $5 \%$ of the annual precipitation occurring during winter. The spring season is characterized by the influence of Asian dust, with an average wind speed of $3.67 \mathrm{~m} \cdot \mathrm{s}^{-1}, 58 \%$ higher than that in winter. According 
to AWS data from Korea Meteorological Administration near the three sites, the prevailing wind directions were north and south at Hwasung station, east and west at Banwol station and northwest at Daeboo station. At Hwasung, the wind during spring and summer was north, but south during fall and winter. At Banwol and Daeboo stations, the prevailing wind was constant throughout the year.

\subsection{Sample Collection}

Precipitation samples were collected weekly using wet and dry collectors from June 2000 to November 2007. Butler and Likens (1998) [38] found that daily samples may provide a better estimate of actual rain chemistry than weekly samples. Gilliland et al. (2002) [39] also suggested that considerable monthly variation existed between daily and weekly data for $\mathrm{H}^{+}, \mathrm{NH}_{4}{ }^{+}$and $\mathrm{SO}_{4}{ }^{2-}$. All of the samplers were placed $1 \mathrm{~m}$ from the ground, and the wet sample collector was covered with a lid to prevent any contamination from dry deposition. The wet collector uses a conductivity sensor, which automatically opens at the onset of each precipitation event and closes again when a rain event stops. The automated samplers consisted of two Teflon buckets $28 \mathrm{~cm}$ in diameter. The average precipitation intensity for onset was $40 \pm 59 \mathrm{~mm} /$ week during the study period. Before sampling, the buckets were carefully rinsed with deionized water several times until the conductivity of the water was $<1.5 \mu \mathrm{S} \cdot \mathrm{cm}^{-1}$. Precipitation samples were collected in a $30-\mathrm{mL}$ high-density polyethylene bottle. The samples were unrefrigerated during sampling but stored in a freezer at $-20^{\circ} \mathrm{C}$ until chemical analysis. Prior to analysis, the precipitation samples were filtered using $0.45-\mu \mathrm{m}$ membrane filters (Millipore).

\subsection{Sample Analysis}

After the precipitation samples were collected, the weight of the bucket was measured to calculate the volume-weighted mean (VWM) concentrations of the atmospheric components. The $\mathrm{pH}$ and conductivity of the unfiltered precipitation were also measured immediately with a $\mathrm{pH}$ and conductivity meters (Fisher Scientific) [40]. The $\mathrm{pH}$ meter was calibrated before each measurement using standard 4.00 and 7.01 buffer solutions. The conductivity meter was also calibrated with a standard solution. For this study, 528 rain samples were analyzed. Major anion and cation concentrations were determined using ion chromatography (Waters, USA). For anions $\left(\mathrm{Cl}^{-}, \mathrm{NO}_{3}{ }^{-}\right.$, and $\left.\mathrm{SO}_{4}{ }^{2-}\right)$, AS14 or AS11 columns were used; CS12A or CS14 columns were used to measure cations $\left(\mathrm{Na}^{+}, \mathrm{NH}_{4}{ }^{+}, \mathrm{K}^{+}, \mathrm{Mg}^{2+}\right.$, and $\left.\mathrm{Ca}^{2+}\right)$. The detection limits were $0.08 \mu \mathrm{eq} \cdot \mathrm{L}^{-1}$ for $\mathrm{Cl}^{-}, 0.12 \mu \mathrm{eq} \cdot \mathrm{L}^{-1}$ for $\mathrm{NO}_{3}{ }^{-}, 0.11 \mu \mathrm{eq} \cdot \mathrm{L}^{-1}$ for $\mathrm{SO}_{4}^{2-}, 0.54$ for $\mathrm{Na}^{+}, 2.32 \mu \mathrm{eq} \cdot \mathrm{L}^{-1}$ for $\mathrm{NH}_{4}{ }^{+}, 0.2 \mu \mathrm{eq} \cdot \mathrm{L}^{-1}$ for $\mathrm{K}^{+}, 0.44 \mu \mathrm{eq} \cdot \mathrm{L}^{-1}$ for $\mathrm{Mg}^{2+}$ and $2.87 \mu \mathrm{eq} \cdot \mathrm{L}^{-1}$ for $\mathrm{Ca}^{2+}$. Detection limits were calculated by dividing the standard deviation of the response by the slope of the calibration and then multiplying by 3.3 . 


\subsection{Quality Assurance}

Of the 678 precipitation samples collected, about $7 \%$ were discarded because the amount of precipitation was insufficient to perform the chemical analyses. An additional $12 \%$ of the rain samples were discarded due to noticeable contamination by dry deposition (soil, leafs and insects) or because of sampler malfunction [41]. The remaining samples were subjected to a quality check based on ionic balance and conductivity balance. When the $\mathrm{pH}$ was above 5.6, the concentration of $\mathrm{HCO}_{3}^{-}$(in $\mu \mathrm{eq} \cdot \mathrm{L}^{-1}$ ) was calculated using the formula $\left(\mathrm{HCO}_{3}{ }^{-}\right)=10^{(\mathrm{pH}-11.24)}$ [42-44]. According to Okuda et al. (2005) [15], the acceptable ion range ( $\sum$ cation $/ \sum$ anion) and conductivity ( $\sum$ measured conductivity $/ \sum$ anion conductivity) ratio for a rain sample is $0.67-1.5$. Data points outside this range were excluded. The percentage of samples excluded at each sampling station was 38\% at Hwasung, 21\% at Banwol, and 32\% at Daeboo, averaging 30\% overall. Linear regression of the relationship between cation sum vs. the anion sum showed an $r^{2}=0.95$ and slope of 0.95 (Figure 2a,b). Edmonds et al. (1991) [45] suggested that anion deficit means of the amount of unmeasured organic acids. Kim et al. (2013) [32] found that the organic acids $\left(\mathrm{CH}_{3} \mathrm{COO}^{-}, \mathrm{HCOO}^{-}\right)$contributed to $12.4 \%$ of the acidity in precipitation at Jeju Island in Korea. The relationship between the calculated and measured conductivity was also highly correlated with a $r^{2}=0.96$ and slope of 0.92 .

\subsection{Positive Matrix Factorization (PMF)}

Positive Matrix Factorization is a multivariable factor analysis tool to identify the contributions of various emission sources. PMF decomposes a speciated data matrix $X$ of $n$ by $m$ dimensions ( $n$ number of samples and $m$ chemical species) into factor profiles $(g)$ and factor contributions $(f)$ based on the correlation between the different components (Equation (1)). The objective of the PMF solution is to minimize the object function $Q$ based on the uncertainties $(u)$ as follows [46]:

$$
\mathrm{Q}=\sum_{i=1}^{n} \sum_{j=1}^{m}\left[\frac{X_{i j}-\sum_{k=1}^{p} g_{i k} f_{k j}}{u_{i j}}\right]^{2}
$$

where $x_{i j}$ are the measured concentrations (in $\mu \mathrm{eq} \cdot \mathrm{L}^{-1}$ ), $u_{i j}$ are the estimated uncertainty values (in $\mu \mathrm{eq} \cdot \mathrm{L}^{-1}$ ), $n$ is the number of samples, $m$ is the number of species and $p$ is the number of factors included in the analysis [47]. 

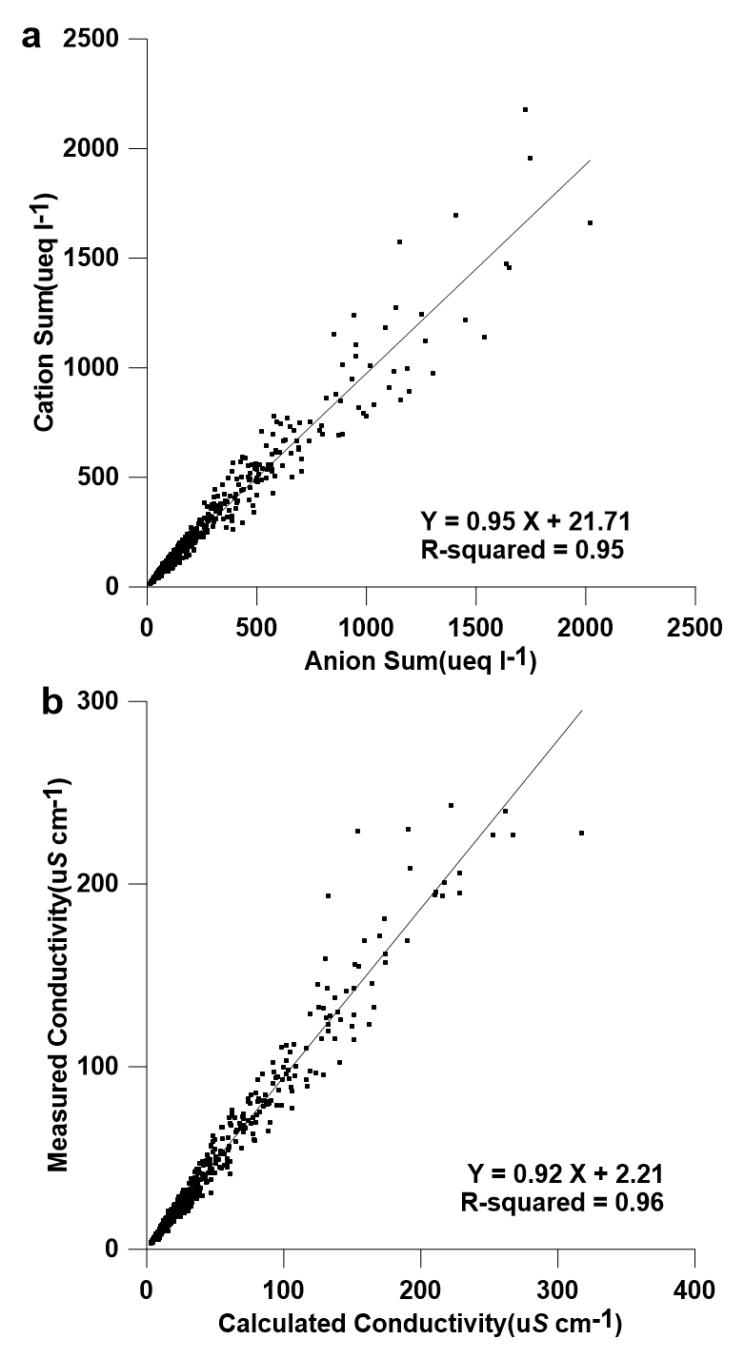

Figure 2. Linear regression (a) between the sum of cations and anions, and (b) between measured and calculated conductivity.

PMF needs two input files: one for the measured concentrations of the species and the other for the estimated uncertainties of the concentrations. The uncertainty greater than the detection limit was calculated using the concentration and detection limit (Equation (2)). If the concentration was less than or equal to the detection limit, the uncertainty was calculated with a fixed fraction (Equation (3)). Missing data and 
their uncertainty value were replaced with the value of the species-species median and four times the median value, respectively [48].

$$
\begin{gathered}
\text { uncertainty }=\sqrt{(\text { Error fraction } \times \text { Concentration })^{2}+(0.5 \times \text { Detection limit })^{2}} \\
\text { uncertainty }=\frac{5}{6} \times(\text { Detection limit })
\end{gathered}
$$

A variable was defined to be weak if its $\mathrm{S} / \mathrm{N}$ was between 0.2 and 2.0. Most of the scaled residuals were between -3.0 and 3.0. Fpeak values between -1 and 1 (in steps of 0.1 ) were examined to find out the most appropriate solution. Error estimate with bootstrap results showed $100 \%$ mapping for five factors at three sites.

\section{Results and Discussion}

\subsection{Acidity}

$\mathrm{SO}_{2}$ and $\mathrm{NO}_{\mathrm{x}}$ are the major precursors of acidity in precipitation. Wind-blown soil particles and sea spray species atmosphere play an important role in neutralizing acidic constituents. In uncontaminated precipitation, the equilibrium concentration of $\mathrm{CO}_{2(\mathrm{aq})}$ generates a $\mathrm{pH}$ of approximately 5.6, which serves as a reference value. Figure 3 shows the frequency distribution for $\mathrm{pH}$ in the Shihwa Basin. Among the samples, $84 \%$ of the $\mathrm{pH}$ values were $<5.6$. The $\mathrm{pH}$ of uncontaminated precipitation is considered to be 5.0-5.6; this acidity originates from natural levels of atmospheric $\mathrm{CO}_{2}, \mathrm{NOx}$, and $\mathrm{SO}_{x}[49]$. About $66 \%$ of the rain samples had a $\mathrm{pH}<5.0$, indicating that the rain samples were affected by additional acidic components. At Banwol station in the center of two industrial complexes, $69 \%$ of the samples had a $\mathrm{pH}$ of $<5.0$ and $14 \%$ had a $\mathrm{pH}<4.0$. At Hwasung station, $59 \%$ of the samples had a $\mathrm{pH}$ of $<5.0$ and $7 \%$ had a $\mathrm{pH}<4.0$. At Daeboo, $78 \%$ of the samples had a $\mathrm{pH}$ lower than 5.0 and $15 \%$ of the samples had a $\mathrm{pH}<4.0$.

Overall, the $\mathrm{pH}$ of the precipitation ranged from 3.4 to 7.7 (Table 2). The VWM $\mathrm{pH}$ was 4.84, indicating slight acidity in the study area. For Hwasung, located east of Banwol, the VWM pH was 4.96, higher than at the other sites $(p<0.001)$. At Banwol, the $\mathrm{pH}$ was 4.77 , lowest among all the stations $(p<0.001)$, reflecting its proximity in the industrial complex. Daeboo had the highest VWM pH (4.81), similar to the VWM $\mathrm{pH}$ at Banwol. 


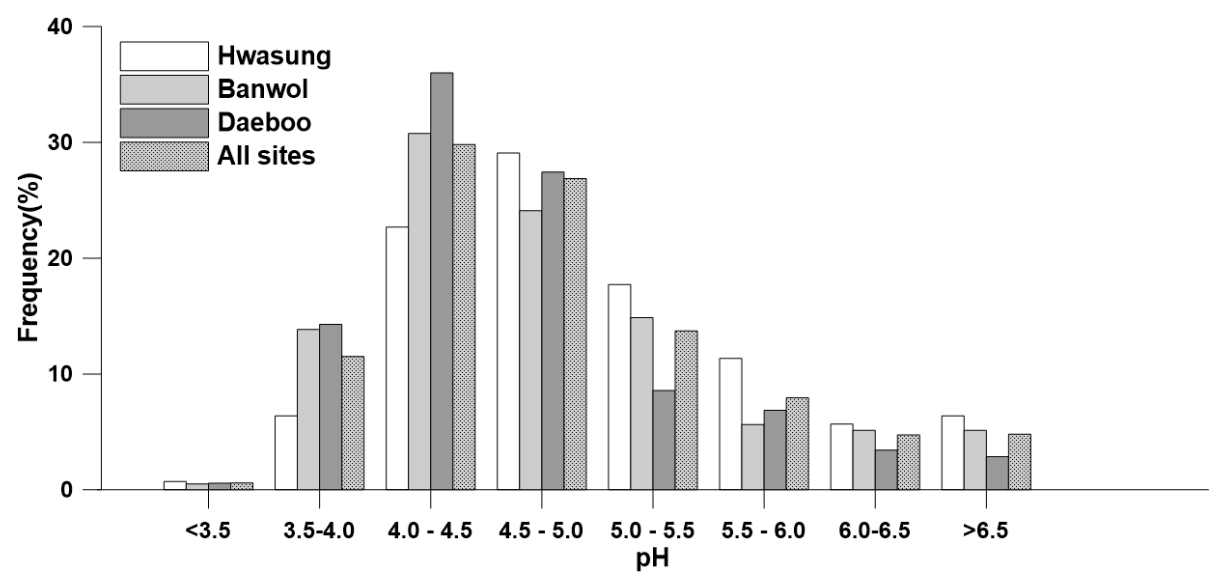

Figure 3. Frequency distribution for wet-only precipitation volume-weighted mean (VWM) $\mathrm{pH}$.

Table 2. Major ionic compositions of precipitation in the Shihwa Basin $\left(\mu \mathrm{eq} \cdot \mathrm{L}^{-1}\right.$ except $\mathrm{pH})$.

\begin{tabular}{|c|c|c|c|c|c|c|c|c|c|c|}
\hline Station & Parameter & $\mathrm{Cl}^{-}$ & $\mathrm{NO}_{3}{ }^{-}$ & $\mathrm{SO}_{4}{ }^{2-}$ & $\mathrm{Na}^{+}$ & $\mathrm{NH}_{4}{ }^{+}$ & $\mathrm{K}^{+}$ & $\mathrm{Mg}^{2+}$ & $\mathrm{Ca}^{2+}$ & $\mathrm{pH}$ \\
\hline \multirow{5}{*}{ Hwasung } & Min & 0.5 & 3.8 & 6.8 & 0.6 & 5.9 & 0.02 & 0.3 & 0.7 & 7.5 \\
\hline & Max & 472 & 482 & 1495 & 567 & 1758 & 163 & 297 & 781 & 3.4 \\
\hline & Mean & 45 & 68 & 127 & 33 & 117 & 9 & 15 & 57 & 4.5 \\
\hline & $\begin{array}{l}\text { Standard } \\
\text { deviation }\end{array}$ & 82 & 101 & 72 & 79 & 122 & 20 & 32 & 134 & 0.8 \\
\hline & VWM & 13.9 & 19.6 & 45.2 & 9.3 & 43.6 & 3.6 & 4.5 & 13.5 & 4.7 \\
\hline \multirow{5}{*}{ Banwol } & Min & 1.4 & 2.0 & 4.7 & 0.5 & 5.7 & 0.03 & 0.4 & 0.6 & 7.7 \\
\hline & Max & 683 & 598 & 839 & 506 & 539 & 308 & 144 & 639 & 3.5 \\
\hline & Mean & 58 & 71.3 & 145 & 34.7 & 116 & 9 & 14 & 65 & 4.3 \\
\hline & $\begin{array}{l}\text { Standard } \\
\text { deviation }\end{array}$ & 93 & 83 & 160 & 66 & 104 & 12 & 23 & 108 & 0.8 \\
\hline & VWM & 20 & 23 & 56 & 10 & 51 & 4.4 & 4.6 & 15 & 4.6 \\
\hline \multirow{5}{*}{ Daeboo } & Min & 1.2 & 1.9 & 3.5 & 0.4 & 1.6 & 0.11 & 0.3 & 0.6 & 6.9 \\
\hline & Max & 1505 & 594 & 753 & 1434 & 588 & 87 & 320 & 763 & 3.5 \\
\hline & Mean & 70 & 73 & 118 & 59 & 88 & 8.5 & 20 & 61 & 4.4 \\
\hline & $\begin{array}{l}\text { Standard } \\
\text { deviation }\end{array}$ & 119 & 89 & 134 & 88 & 89 & 20 & 33 & 115 & 0.7 \\
\hline & VWM & 25 & 22 & 40 & 17 & 40 & 5.1 & 6.6 & 15 & 4.6 \\
\hline
\end{tabular}

\subsection{Concentration of Inorganic Species}

The minimum and maximum concentrations of chemical components in the individual precipitation samples and their VWMs are shown in Table 2. $\mathrm{SO}_{4}{ }^{2-}$ and $\mathrm{NH}_{4}{ }^{+}$were the most abundant ions in precipitation at the three stations. At Hwasung, the concentrations of anthropogenic species were lower than those at the 
other stations. The VWM concentration of sea salts was also lowest in Hwasung, as it is located farthest from the seashore. The highest concentrations of $\mathrm{SO}_{4}{ }^{2-}, \mathrm{NH}_{4}{ }^{+}$ and $\mathrm{NO}_{3}{ }^{-}$were measured at Banwol, related to anthropogenic sources associated with two industrial complexes and motor vehicles. The VWM concentration of $\mathrm{H}^{+}$ was also highest in Banwol. In Daeboo, the precipitation acidity was similar to that in Banwol due to the low concentration of $\mathrm{NH}_{4}{ }^{+}$. The VWM conductivities in Hwasung, Banwol, and Daeboo were 18, 24, and $23 \mu \mathrm{S} \cdot \mathrm{cm}^{-1}$ with VWM total ions of 181, 253, and $235 \mu \mathrm{eq} \cdot \mathrm{L}^{-1}$, respectively.

For the combined data collected in the Shihwa Basin, the ion concentrations fell in the order $\mathrm{SO}_{4}{ }^{2-}>\mathrm{NH}_{4}{ }^{+}>\mathrm{H}^{+}>\mathrm{NO}_{3}{ }^{-}>\mathrm{Cl}^{-}>\mathrm{Ca}^{2+}>\mathrm{Na}^{+}>\mathrm{Mg}^{2+}>\mathrm{K}^{+}$. Among the anions, $\mathrm{SO}_{4}{ }^{2-}$ had the highest concentrations at all stations (54\%). The next most abundant anion was $\mathrm{NO}_{3}{ }^{-}$at nearly $24 \%$. The precipitation chemistry in the Shihwa Basin was mainly dominated by $\mathrm{NH}_{4}{ }^{+}$with an average VWM of $\mu \mathrm{eq} \cdot \mathrm{L}^{-1}$, representing $40 \%$ of all cations. Considering the sampling period, the concentration of $\mathrm{NH}_{4}{ }^{+}$can be as high as $49.5 \mu \mathrm{eq} \cdot \mathrm{L}^{-1}(10 \%)$. The next most abundant cations were $\mathrm{H}^{+}, \mathrm{Ca}^{2+}$, and $\mathrm{Na}^{+}$, representing $24 \%, 15 \%$, and $12 \%$ of the total, respectively. $\mathrm{NH}_{4}{ }^{+}$in the Shihwa Basin is associated with agricultural activities and an industrial wastewater treatment facility. $\mathrm{Ca}^{2+}$ is related to resuspension of dust from the soil and Asian dust originating from China mainly in the spring. [50]. These alkali components neutralize and decrease the acidity of the rainwater [51,52]. The ratios of individual ions to $\mathrm{Na}^{+}$are higher than seawater ratios, which indicates that contributions from anthropogenic and soil sources are important, while the marine contribution is negligible $[53,54]$. The $\mathrm{Cl}^{-} / \mathrm{Na}^{+}$ratio in the combined precipitation samples over six years was $45 \%$ higher than that of seawater $\mathrm{Cl}^{-} / \mathrm{Na}^{+}$(1.165). This suggests that the $\mathrm{Cl}^{-}$originated from industrial activities in the area such as coal combustion and incineration of polyvinyl chloride $[55,56]$. The $\mathrm{SO}_{4}{ }^{2-} / \mathrm{Na}^{+}, \mathrm{K}^{+} / \mathrm{Na}^{+}$, $\mathrm{Mg}^{2} / \mathrm{Na}^{+}$, and $\mathrm{Ca}^{2+} / \mathrm{Na}^{+}$ratios for precipitation in the Shihwa Basin were $4.02,0.32$, 0.45 , and 1.25 , respectively, higher than the seawater ratios $(0.12,0.23,0.04$, and 0.02 , respectively). This suggests that anthropogenic influences were present from local (two industrial complexes) or regional (Asian continent) sources.

The $\mathrm{SO}_{4}{ }^{2-} / \mathrm{NO}_{3}{ }^{-}$ratio has been used as an index to evaluate anthropogenic characteristic sources in rainfall samples [54,57]. $\mathrm{SO}_{4}{ }^{2-} / \mathrm{NO}_{3}{ }^{-}$ratios are diverse globally and temporally: 0.67 in the southwestern United States during 1995 2010 [58], 1.5 in Belgium in 2003 [41], 1.6 in Turkey in 2002 [51], 2.3 in central Pennsylvania during 1993 2001 [59], 2.4 in Mexico from 1994 to 2000 [60], 8.7 in Brazil in 2002 [55] and 18.6 in Costa Rica in 2009 [61]. Kulshrestha et al. (2003) [62] reported that the $\mathrm{SO}_{4}{ }^{2-} / \mathrm{NO}_{3}{ }^{-}$ratio in India increased as the degree of urbanization or industrialization increased. In Korea, $\mathrm{SO}_{4}{ }^{2-} / \mathrm{NO}_{3}{ }^{-}$ratios ranging from 0.6 to 3.6 have been reported (1990 to 2013) [30,33,63,64]. The mean $\mathrm{SO}_{4}{ }^{2-} / \mathrm{NO}_{3}{ }^{-}$at three EANET sites located in the western area of Korea averaged 0.97 during 2001 2007 
(EANET). The average $\mathrm{SO}_{4}{ }^{2-} / \mathrm{NO}_{3}{ }^{-}$ratio of 2.3 in this study was attributed to the close proximity to industrial areas in Korea [30]. The $\mathrm{H}^{+} /\left(\mathrm{NO}_{3}{ }^{-}+\mathrm{SO}_{4}{ }^{2-}\right)$ ratio can represent the relative contributions of $\mathrm{H}_{2} \mathrm{SO}_{4}$ and $\mathrm{HNO}_{3}$ to the acidity of rain, and deviations from unity indicate the degree of neutralization [65]. Earlier studies in Korea showed that acidity due to $\mathrm{H}^{+}$was less than $7 \%$ in rural areas, while that in urban areas ranged from $18 \%$ to $34 \%$. The combined contribution of $\mathrm{H}_{2} \mathrm{SO}_{4}$ and $\mathrm{HNO}_{3}$ to acidity in the Shihwa Basin was $0.36 \pm 0.26$, indicating that about $64 \%$ of the acidity was neutralized by cations [66]. The $\mathrm{H}^{+} /\left(\mathrm{NO}_{3}{ }^{-}+\mathrm{SO}_{4}{ }^{2-}\right)$ ratios for China, Southeast Asia, Korea, and Japan are about $20 \%, 60 \%, 30 \%$, and $37 \%$, respectively. The mean $\mathrm{pH}$ for wet-only precipitation (4.84) in the Shihwa Basin is higher than or similar to the results from other major cities in Asia.

\subsection{Temporal Variation}

During the study period, there were 26 episodic events in which the total ion concentration precipitation was $>2000 \mu \mathrm{eq} \cdot \mathrm{L}^{-1}$. There were 14 events in winter, 11 in spring, and one in autumn. In summer, there were no events when the total ion concentration was $>2000 \mu \mathrm{eq} \cdot \mathrm{L}^{-1}$. Rastogi and Sarin (2007) [67] reported that low-solute events are associated with heavy amounts of rain or successive events. The concentrations of most of the chemical components in the wet-only samples were inversely related to precipitation amount. About $74 \%$ of the rain in the Shihwa Basin fell from June to September (Figure 4). Concentrations during the rainy period (June to August) were significantly lower $\left(p<0.05\right.$ for $\mathrm{H}^{+}$and $p<0.01$ for other ions) than those in the dry period (September to May). The concentrations of the inorganic species decreased due to dilution during the rainy period, thus ion concentrations were lower during the rainy period than ion concentrations in the dry period. The concentrations of soil-derived species $\left(\mathrm{Ca}^{2+}\right.$ and $\left.\mathrm{Mg}^{2+}\right)$ in the dry season were about six times higher than those in the rainy season. In March, influenced by dust from Asian continental deserts such as the Taklamakan, Gobi, and Loess plateau, the concentrations of all chemical components were highest. The monthly VWM concentrations of $\mathrm{SO}_{4}{ }^{2-}$ and $\mathrm{NO}_{3}{ }^{-}$in the wet-only samples were correlated with concentrations of $\mathrm{SO}_{2}(r=0.466, p<0.001)$ and $\mathrm{NO}_{2}(r=0.559$, $p<0.001)$ in atmosphere (Figure 5). The $\mathrm{NO}_{2}$ and $\mathrm{SO}_{2}$ data are available from the National Institute of Environmental Research (NIER) in the same administrative district (Ansan city). Tu et al. (2005) [19] also reported that the $\mathrm{SO}_{4}{ }^{2-}$ concentration had a temporal trend corresponding to $\mathrm{SO}_{2}$ in atmosphere in China. Similar results have been found in a number of studies $[15,68]$. 


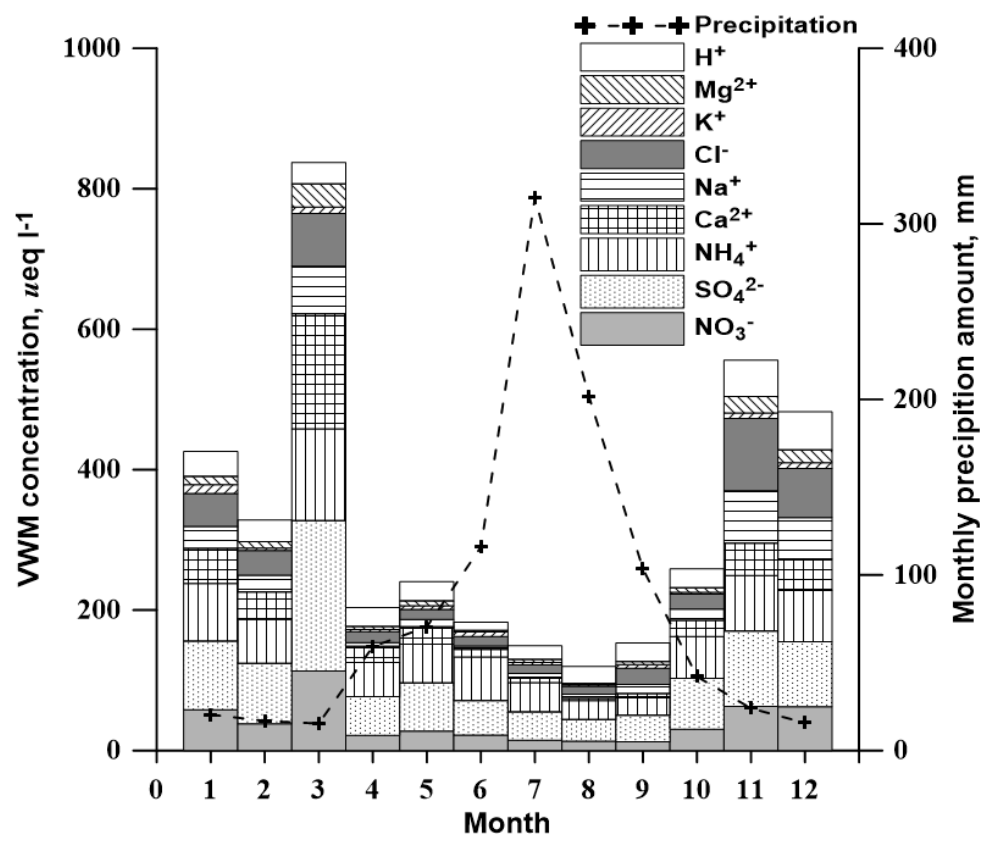

Figure 4. Monthly VWM concentrations of ions in rainfall and rainfall amount.

Figure 6 shows the annual VWM concentrations of the major components in precipitation. Anthropogenic $\mathrm{SO}_{2}$ and $\mathrm{NO}_{2}$ emitted from mobile and power plant sources contributed to the increasing of $\mathrm{SO}_{4}{ }^{2-}$ and $\mathrm{NO}_{3}{ }^{-}$in the wet-only samples [69]. In 2002, power plants (450 MW) near the study area expanded to Boryeong thermal power plant located about $100 \mathrm{~km}$ south of Daebudo station. Thermal power facilities (1600 MW) were also added to the Yeungheung thermal power plant located $13 \mathrm{~km}$ west from Daebudo station in 2004. In 2005, the Incheon thermal power plants located $20 \mathrm{~km}$ north of the Banwol industrial complex expanded to a $342 \mathrm{MW}$ power plant facility. Additional power generating capacity (5275 MW) has been added to these three power plants between 2008 and 2014. During the study period, the $\mathrm{NH}_{4}{ }^{+} / \mathrm{SO}_{4}{ }^{2-}$ ratio decreased from 1.01 to 0.68 . The $\mathrm{NH}_{4}{ }^{+} / \mathrm{NO}_{3}{ }^{-}$ratio also decreased from 2.39 to 1.20 . Results from studies of wet-only precipitation in Austria, Brazil, Canada, USA, Japan, and China have shown significant decreasing trends in the concentrations of $\mathrm{SO}_{4}{ }^{2-}$ and $\mathrm{H}^{+}$over long-term periods [15,16,70-72]. Table 3 shows VWM concentrations in recent precipitation studies in East Asia. The concentrations of water-soluble components in the Shihwa Basin were lower than those found in other Asian continental countries. The average concentrations of $\mathrm{NO}_{3}{ }^{-}$and $\mathrm{SO}_{4}{ }^{2-}$ ranged from 1.8 to 2.0 and were 2.0 to 2.4 times higher than average concentrations in Seoul [33]. 


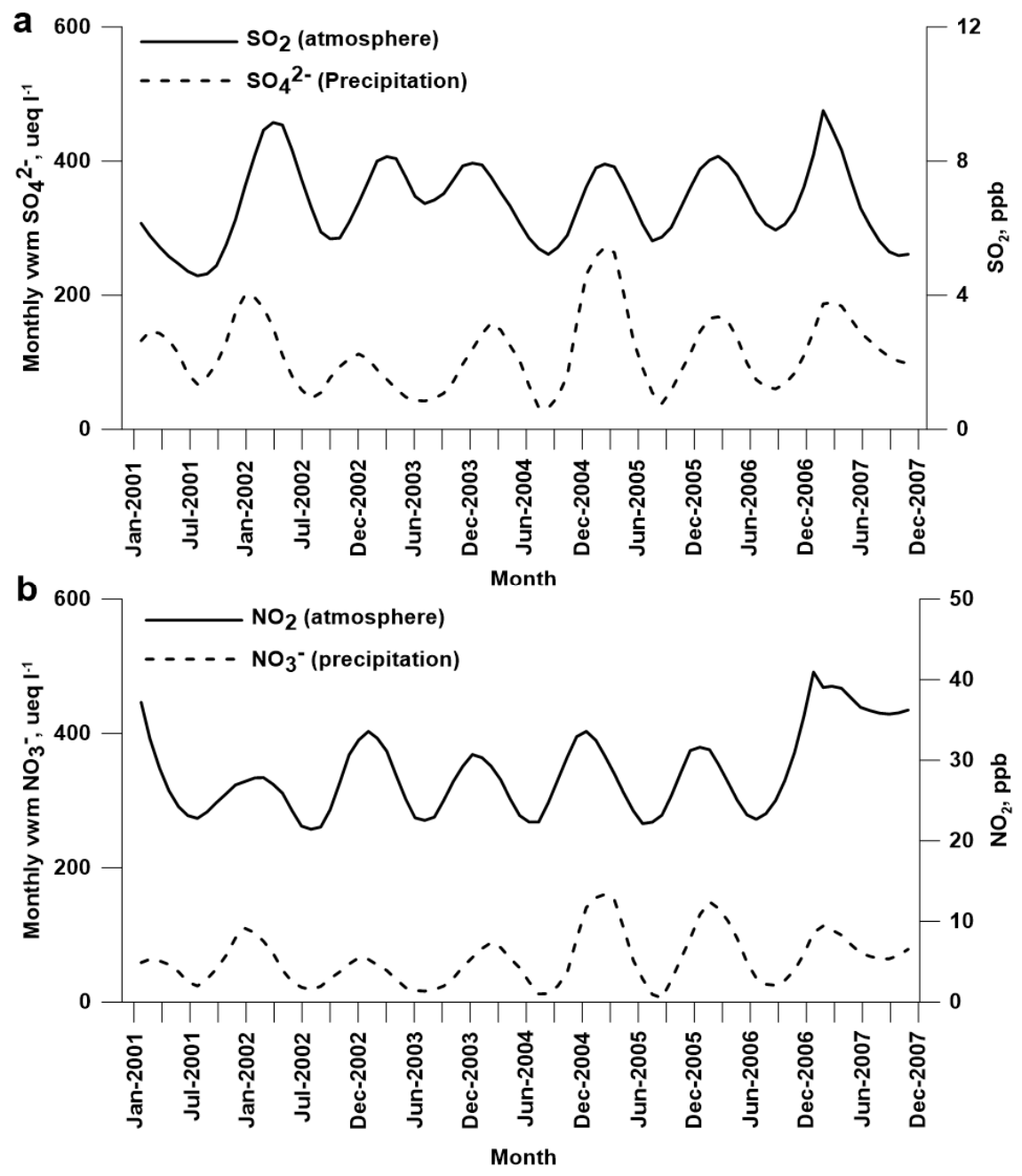

Figure 5. Trends with Lowess smoothing (span =0.2): (a) monthly $\mathrm{VWM} \mathrm{SO}_{4}{ }^{2-}$ concentration in wet-only precipitation and the monthly mean atmospheric $\mathrm{SO}_{2}$ concentration and (b) monthly $\mathrm{VWM} \mathrm{NO}_{3}{ }^{-}$concentration in wet-only precipitation and the monthly mean atmospheric $\mathrm{NO}_{2}$ concentration. 


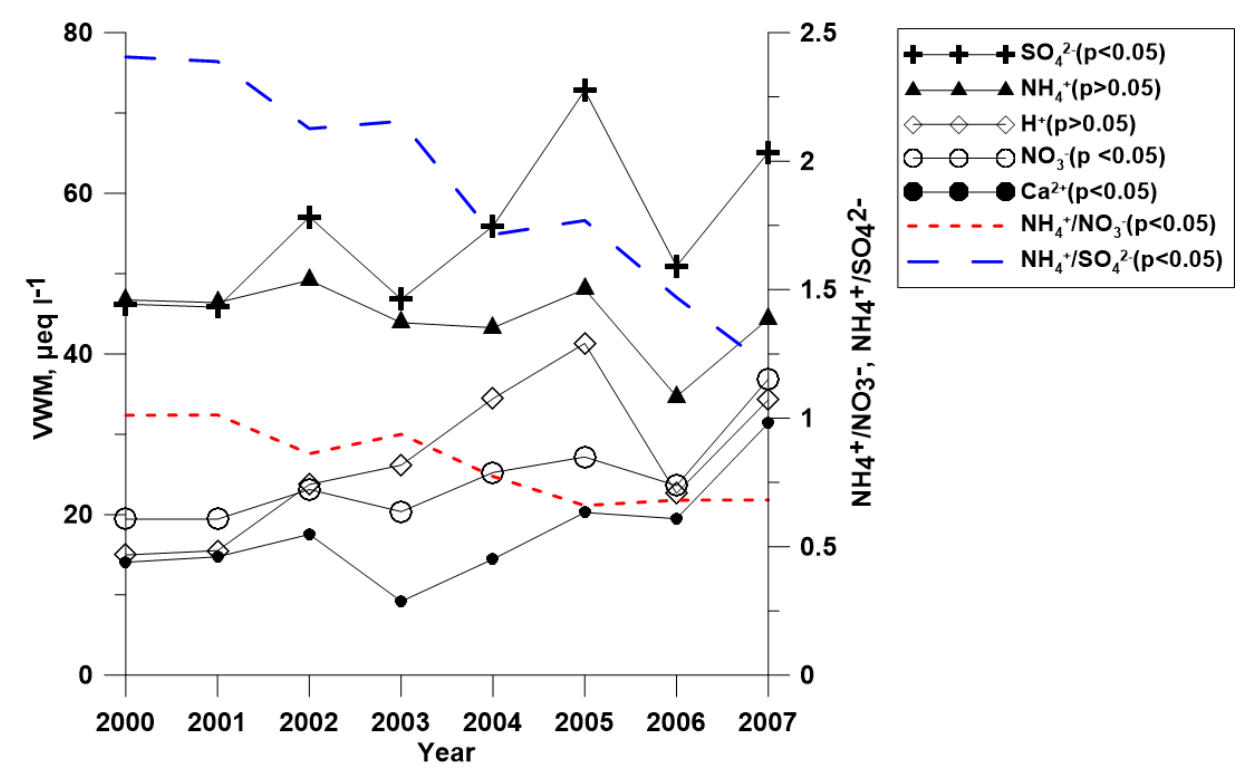

Figure 6. Annual variations of major inorganic VWM concentrations in the precipitation.

Table 3. Volume-weighted mean (VWM) concentrations of the chemical components in wet-only precipitation in East Asia ( $\mu$ eq. $\mathrm{L}^{-1}$ except $\mathrm{pH}$ ).

\begin{tabular}{|c|c|c|c|c|c|c|c|c|c|c|}
\hline East Asia & Year(s) & $\mathrm{Cl}^{-}$ & $\mathrm{NO}_{3}{ }^{-}$ & $\mathrm{SO}_{4}{ }^{2-}$ & $\mathrm{Na}^{+}$ & $\mathrm{NH}_{4}^{+}$ & $\mathrm{K}^{+}$ & $\mathrm{Mg}^{2+}$ & $\mathrm{Ca}^{2+}$ & $\mathrm{pH}$ \\
\hline Beijing a (N China) & 2003 & - & 118 & 380 & - & 211 & - & - & 159 & 6.48 \\
\hline China ${ }^{b}$ (SE China) & 2004 & 9 & 31 & 95 & 6 & 81 & 5 & 3 & 48 & 4.54 \\
\hline Cai Jia Tang ${ }^{c}$ (S China) & 2003 & 11 & 60 & 155 & 7 & 112 & 10 & 10 & 60 & 4.33 \\
\hline Hong Kong $\mathrm{d}$ & 1999-2000 & 43 & 27 & 74 & 37 & 22 & 4 & 7 & 16 & 4.20 \\
\hline Singapore $\mathrm{e}$ & $1999-2000$ & 34 & 22 & 84 & 33 & 19 & 7 & 7 & 16 & 4.20 \\
\hline Japan ${ }^{\mathrm{f}}$ (Tokyo) & $1990-2002$ & 55 & 31 & 50 & 37 & 40 & 3 & 12 & 25 & 4.52 \\
\hline Jejug (Korea) & $2000-2007$ & 40 & 23 & 22 & 24 & 40 & 21 & 3 & 9 & 5.37 \\
\hline This study & 2000-2007 & 23 & 23 & 54 & 13 & 44 & 4 & 6 & 17 & 4.84 \\
\hline
\end{tabular}

a Tang et al. (2005); ${ }^{\mathrm{b}}$ Zhang et al. (2007b); ${ }^{\mathrm{e}} \mathrm{Hu}$ et al. (2003); ${ }^{\mathrm{f}}$ Okuda et al. (2005);

g EANET(2013).

\subsection{Statistical Analysis}

Table 4 shows the correlation coefficients among chemical species for this study. Significant and strong correlations were found among $\mathrm{Na}^{+}, \mathrm{Cl}^{-}, \mathrm{Mg}^{2+}$ and $\mathrm{Ca}^{2+}$, indicating that this region is heavily affected by sea salts and soil. $\mathrm{Ca}^{2+}$ and $\mathrm{Mg}^{2+}$ correlations with $\mathrm{Cl}^{-}, \mathrm{NO}_{3}{ }^{-}$, and $\mathrm{SO}_{4}{ }^{2-}$ indicate that $\mathrm{CaCl}_{2}, \mathrm{MgCl}_{2}, \mathrm{Ca}\left(\mathrm{NO}_{3}\right)_{2}$, $\mathrm{Mg}\left(\mathrm{NO}_{3}\right)_{2}, \mathrm{CaSO}_{4}$, and $\mathrm{MgSO}_{4}$ were the primary soil-derived components in the wet-only precipitation [73]. The correlation among $\mathrm{NH}_{4}{ }^{+}, \mathrm{NO}_{3}{ }^{-}$, and $\mathrm{SO}_{4}{ }^{2-}$ indicate that they originated from similar anthropogenic sources. The significant correlation 
between $\mathrm{NH}_{4}{ }^{+}$and $\mathrm{NO}_{3}{ }^{-}(r=0.76, p<0.001)$ and between $\mathrm{NH}_{4}{ }^{+}$and $\mathrm{SO}_{4}{ }^{2-}(r=0.83$, $p<0.001)$ indicate that $\mathrm{NH}_{4} \mathrm{NO}_{3},\left(\mathrm{NH}_{4}\right) \mathrm{HSO}_{4}$, and $\left(\mathrm{NH}_{4}\right)_{2} \mathrm{SO}_{4}$ were the major forms of $\mathrm{NH}_{4}{ }^{+}$in precipitation. Although positive correlations between acidic anions and $\mathrm{H}^{+}$were observed, their correlations were weaker due to neutralization effect by basic soils components [74].

Table 4. Correlations between ionic components in wet-only precipitation over seven years in the Shihwa Basin ( $p<0.001$ for all correlation).

\begin{tabular}{cccccccc}
\hline & $\mathrm{Cl}^{-}$ & $\mathbf{N O}_{3}{ }^{-}$ & $\mathbf{S O}_{4}{ }^{2-}$ & $\mathbf{N a}^{+}$ & $\mathbf{N H}_{4}{ }^{+}$ & $\mathbf{K}^{+}$ & $\mathbf{M g}^{2+}$ \\
\hline $\mathrm{NO}_{3}{ }^{-}$ & 0.56 & & & & & & \\
$\mathrm{SO}_{4}{ }^{2-}$ & 0.62 & 0.82 & & & & & \\
$\mathrm{Na}^{+}$ & 0.89 & 0.58 & 0.64 & & & & \\
$\mathrm{NH}_{4}{ }^{+}$ & 0.52 & 0.76 & 0.83 & 0.48 & & & \\
$\mathrm{~K}^{+}$ & 0.66 & 0.43 & 0.54 & 0.61 & 0.55 & & \\
$\mathrm{Mg}^{2+}$ & 0.74 & 0.67 & 0.69 & 0.82 & 0.49 & 0.47 & \\
$\mathrm{Ca}^{2+}$ & 0.63 & 0.71 & 0.82 & 0.68 & 0.60 & 0.49 & 0.80 \\
\hline
\end{tabular}

Additional details on the patterns in the chemical constituents were analyzed using PMF. Similar studies have also used this technique to identify sources of air pollutants. Anttila et al. (1995) [75] determined the sources of bulk wet deposition in Finland using PMF analysis. Recently, Kitayama et al. (2010) [76] used PMF to identify the sources of $\mathrm{SO}_{2}$ in Japan. The number of factors was determined by the optimized values. Five factors were chosen (Figure 7). The factors were identified as sea salts $\left(\mathrm{Na}^{+}, \mathrm{ss}-\mathrm{Cl}^{-}\right)$, Asian dust $\left(\mathrm{Mg}^{2+}, \mathrm{Ca}^{2+}\right)$, sulfuric acid $\left(\mathrm{H}^{+}\right.$, $\left.\mathrm{SO}_{4}{ }^{2-}\right)$, ammonium salt with nitrate $\left(\mathrm{NH}_{4} \mathrm{NO}_{3}\right)$, and ammonium salt with sulfate $\left(\mathrm{NH}_{4} \mathrm{HSO}_{4}\right.$ and $\left.\left(\mathrm{NH}_{4}\right)_{2} \mathrm{SO}_{4}\right)$ components. The first factor was characterized by high concentrations of sea salts and its contribution to ions in precipitation was $18 \%-26 \%$. At the Banwol site, some anthropogenic components $\left(\mathrm{NO}_{3}{ }^{-}, \mathrm{NH}_{4}{ }^{+}\right.$, and $\left.\mathrm{SO}_{4}{ }^{2-}\right)$ were also included in this factor. The second factor, accounting for $17 \%$ of the total ions, was dominated by Asian dust at all three sites, indicated by high concentrations of $\mathrm{Mg}^{2+}$ and $\mathrm{Ca}^{2+}$. This factor explained $70 \%-79 \%$ of the variation in $\mathrm{Ca}^{2+}$ and $42 \%-74 \%$ of that in $\mathrm{Mg}^{2+}$. The average contribution of the Asian dust factor was especially high during the spring. The third factor represents $\mathrm{H}^{+}$and $\mathrm{SO}_{4}{ }^{2-}$, accounting for $16 \%-23 \%$ of the total. The acidity of the precipitation is largely affected by the dissolution of $\mathrm{SO}_{2}$ and $\mathrm{NO}_{2}$ as precursors of the acidic components. As noted above, the high $\mathrm{SO}_{4}{ }^{2-} / \mathrm{NO}_{3}{ }^{-}$ratio in the Shihwa basin indicates that emissions of $\mathrm{SO}_{2}$ from the industrial complex were dominant sources for acidity. $\mathrm{SO}_{4}{ }^{2-}$ accounted for $93 \%, 52 \%$, and $57 \%$ of this acidity factor at Hwasung, Banwol, and Daeboo, respectively. The fourth factor was characterized by ammonium nitrate, which explained $17 \%-21 \%$ of the total and originated from either local industrial or farmland sources. The presence 
of nitrate with ammonium ions indicates dissolutions of ammonium nitrate from aerosols. The fifth factor represents high loadings of $\mathrm{NH}_{4}{ }^{+}$and $\mathrm{SO}_{4}{ }^{2-}$. The highest $\mathrm{NH}_{4}{ }^{+}$concentrations were found in Hwasung where sizeable $\mathrm{NH}_{3}$ sources, such as farmland, exist. The relative contributions of five factors to measured components varied largely by the month of year (Figure 8). The acidity and secondary aerosol factors were mainly associated with the temperature and precipitation amount. Their contribution was highest in summer. However, Asian dust influences were dominant during the spring.

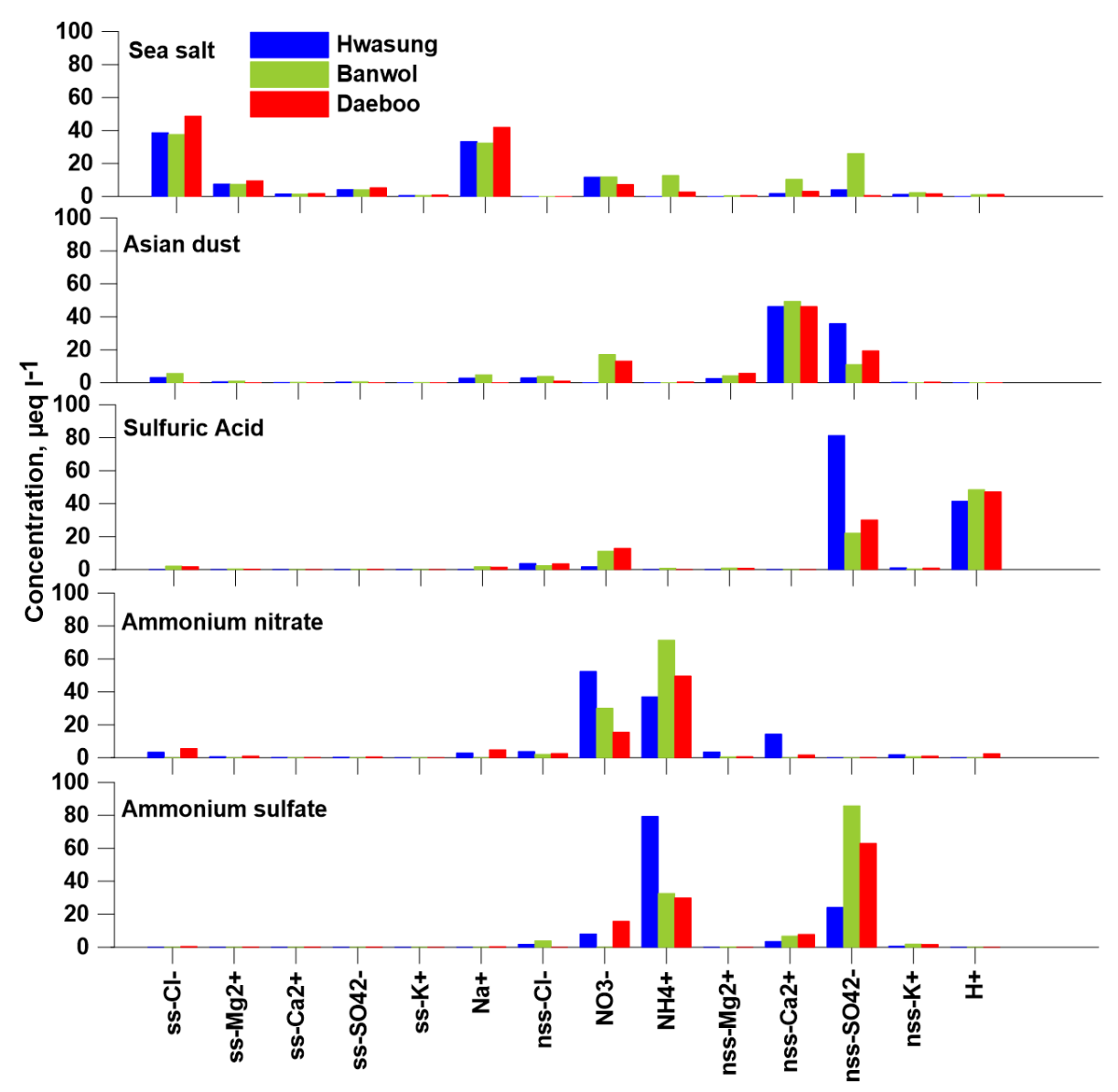

Figure 7. Source contributions of factors $1-5$ at the three stations. 


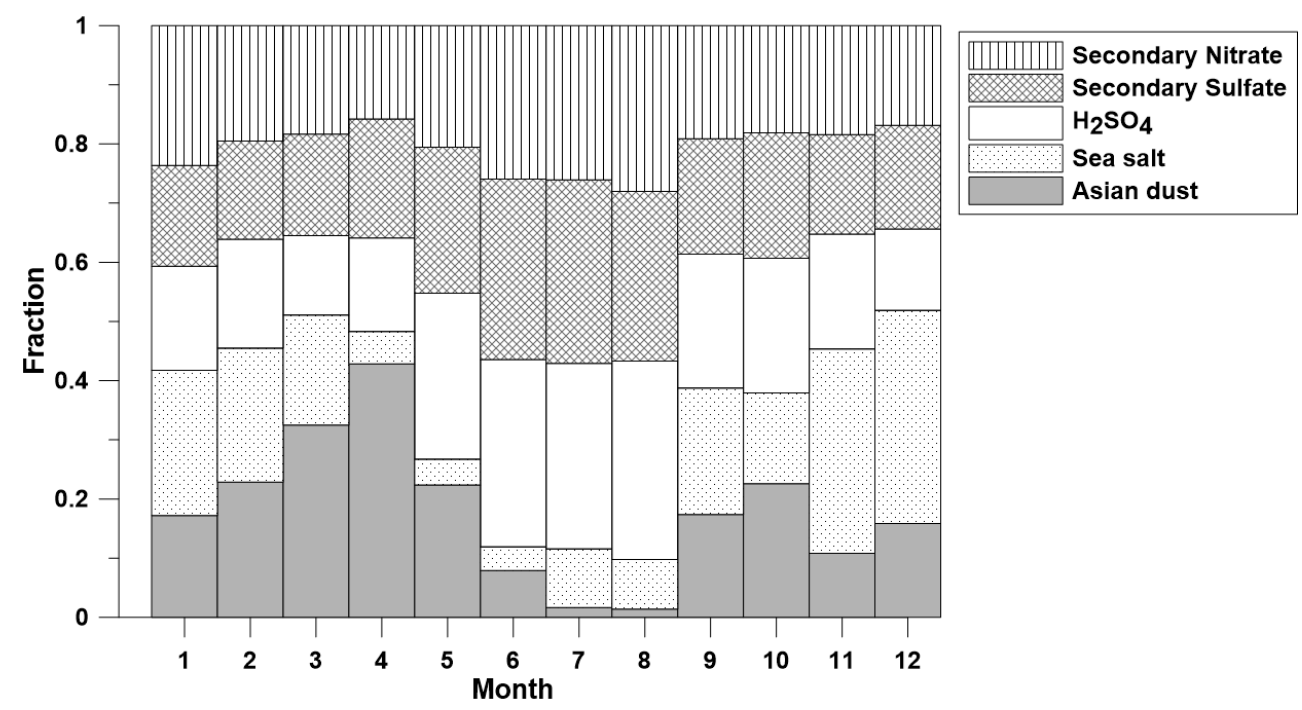

Figure 8. Temporal variations in the five source contributions.

\section{Conclusions}

The composition of precipitation at three sites in the Shihwa Basin was studied from June 2000 to November 2007. A total of 532 wet-only precipitation samples were used to characterize features of the precipitation and to evaluate the influence of anthropogenic sources.

The VWM for $\mathrm{pH}$ for all sites combined was 4.84, with $\mathrm{pH}<5.0$ in $66 \%$ of the precipitation samples, confirming that precipitation in the Shihwa Basin is acidic. The chemical composition of the precipitation was influenced by both natural (sea salt and soil components) and anthropogenic (acidic and alkali components) sources. $\mathrm{SO}_{4}{ }^{2-}$ and $\mathrm{NH}_{4}{ }^{+}$were the dominant ions, followed by $\mathrm{NO}_{3}{ }^{-}$and sea salts. Based on the $\mathrm{H}^{+} /\left(\mathrm{NO}_{3}{ }^{-}+\mathrm{SO}_{4}{ }^{2-}\right)$ ratio, $66 \%$ of the acidity in the Shihwa Basin was neutralized by alkaline particles. The $\mathrm{SO}_{4}{ }^{2-} / \mathrm{NO}_{3}{ }^{-}$ratio showed that the contribution of $\mathrm{SO}_{4}{ }^{2-}$ to acidity was twice as high as that of $\mathrm{NO}_{3}{ }^{-}$. Seventy-four percent of the rain fell during the summer, when the concentrations of all of the ions were significantly lower than those in other seasons.

Although the mean concentrations of the anthropogenic components $\left(\mathrm{NO}_{3}{ }^{-}+\right.$ $\mathrm{SO}_{4}{ }^{2-}+\mathrm{NH}_{4}{ }^{+}$) in the Shihwa basin were 1.8 2.2 times higher than those in Seoul, the VWM concentrations of most of the chemical components were lower than those in other areas of East Asia. To identify the sources of the inorganic species in wet-only precipitation, the components were evaluated using statistical analysis. The moderate correlations of $\mathrm{H}^{+}$with $\mathrm{NO}_{3}{ }^{-}$and $\mathrm{SO}_{4}{ }^{2-}$ and the strong correlations of $\mathrm{NO}_{3}{ }^{-}$and $\mathrm{SO}_{4}{ }^{2-}$ with $\mathrm{NH}_{4}{ }^{+}, \mathrm{Mg}^{2+}$, and $\mathrm{Ca}^{2+}$ suggest that neutralization reactions 
have a strong effect on acidity in the Shihwa Basin. Power generating facilities have expanded in the vicinity of the Shihwa basin from 2002 to 2013. This trend may have impacted the trend of increasing $\mathrm{SO}_{4}{ }^{2-}$ concentrations during the study period. The results from PMF indicated that the main contributors to the wet-only precipitation chemical components in this study were secondary aerosols (40\%-42\%) followed by acidity (16\%-23\%), while sea salts and Asian dust contributed $19 \%-26 \%$ and $17 \%-18 \%$, respectively.

Acknowledgments: This work was supported by the National Research Foundation of Korea (NRF-2009-413-B00004) and funded by the Korean Ministry of Education, Science and Technology.

Author Contributions: Seung-Myung Park: Sampling, Data analysis, Drafting of Manuscript Beom-Keun Seo: Sampling, Data analysis

Gangwoong Lee: Study conception and design, Research performance, Critical revision Sung-Hyun Kahang: Study conception and design

Yu Woon Jang: Sampling, Data analysis, Drafting of Manuscript

Conflicts of Interest: The authors declare no conflict of interest.

\section{References}

1. Draaijers, G.; Erisman, J.; Lövblad, G.; Spranger, T.; Vel, E. Quality and Uncertainty Aspects of Forest Deposition Estimation using Throughfall, Stemflow and Precipitation Measurements; TNO-MEP Report 98/093; TNO Institute of Environmental Sciences, Energy Research and Process Innovation: Apeldoorn, The Netherlands, 1998.

2. Duhanyan, N.; Roustan, Y. Below-cloud scavenging by rain of atmospheric gases particulates. Atmos. Environ. 2011, 39, 7201-7217.

3. Saxena, A.; Kulshrestha, U.C.; Kumar, N.; Kumari, K.M.; Srivastava, S.S. Characterization of precipitation at Arga. Atmos. Environ. 1996, 30, 3405-3412.

4. Hara, H. Acid deposition chemistry in Asia, Europe, and North America. Progress Nuclear Energy 1998, 32, 331-338.

5. Akkoyunlu, B.O.; Tayanç, M. Analyses of wet and bulk deposition in four different regions of Istanbul, Turkey. Atmos. Environ. 2003, 37, 3571-3579.

6. Vázquez, A.; Costoya, M.; Peña, R.M.; García, S.; Herrero, C. A rainwater quality monitoring network: A preliminary study of the composition of rainwater in Galicia (NW Spain). Chemosphere 2003, 51, 375-386.

7. Wai, K.M.; Wang, S.H.; Taner, P.A.; Lin, N.H. A dual site study of the rainwater chemistry within the western Pacific region. J. Atmos. Chem. 2007, 57, 85-103.

8. Santos, M.A.; Illanes, C.F.; Fornaro, A.; Pedrotti, J.J. Acid rain in downtown São Paulo City, Brazil. Water Air Soil Pollut. Focus 2007, 7, 85-92.

9. Avila, A.; Alarcó, M.; Queralt, I. The chemical composition of dust transported in red rains-its contribution to the biogeochemical cycle of a holm oak forest in Catalonia (Spain). Atmos. Environ. 1997, 32, 179-191. 
10. Galloway, J.N. Acidification of the world: Natural and anthropogenic. Water Air Soil Pollut. 2001, 130, 17-24.

11. Bashkin, V.N.; Radojevic, M. Acid rain and its mitigation in Asia. Int. J. Environ. Stud. 2003, 60, 205-214.

12. Luo, J.; Wang, X.; Yang, H.; Yu, J.Z.; Yang, L.; Qin, B. Atmospheric phosphorous in the northern part of Lake Taihu, China. Chemosphere 2011, 84, 785-791.

13. Munger, J.W.; Eisenreich, S. Continental-scale variations in precipitation chemistry. Environ. Sci. Technol. 1983, 17, 32A-42A.

14. Downing, C.E.H.; Vincent, K.J.; Campbell, G.W.; Fowler, D.; Smith, R.I. Trends in wet and dry deposition of sulphur in the United Kingdom. Water Air Soil Pollut. 1995, 85, 659-664.

15. Okuda, T.; Iwase, T.; Ueda, H.; Suda, Y.; Tanaka, S.; Dokiya, Y.; Fushimi, K.; Hosoe, M. Long-term trend of chemical constituents in precipitation in Tokyo metropolitan area, Japan, from 1990 to 2002. Sci. Total Environ. 2005, 339, 127-141.

16. Tu, J.; Wang, H.; Zhang, Z.; Jin, X.; Li, W. Trends in chemical composition of precipitation in Nanjing, China, during 1992-2003. Atmos. Res. 2005, 73, 283-298.

17. Noguchi, I.; Hara, H. Ionic imbalance due to hydrogen carbonate from Asian dust. Atmos. Environ. 2004, 38, 6969-6976.

18. Fowler, D.; Smith, R.I.; Muller, J.B.A.; Hayman, G.; Vincent, K.J. Changes in the atmospheric deposition of acidifying compounds in the UK between 1986 and 2001. Environ. Pollut. 2005, 137, 15-25.

19. Sicard, P.; Coddeville, P.; Sauvage, S.; Galloo, J.C. Trends in chemical composition of wet-only precipitation at rural French monitoring stations over the 1990-2003 period. Water Air Soil Pollut. Focus 2007, 7, 49-58.

20. Zbieranowski, A.; Aheme, J. Long-term trends in atmospheric reactive nitrogen across Canada: 1899-2007. Atmos. Environ. 2011, 45, 5853-5862.

21. Larssen, T.; Seip, H.M.; Semb, A.; Mulder, J.; Muniz, I.P.; Vogt, R.D.; Lydersen, E.; Angell, V.; Dagang, T.; Eilertsen, O. Acid deposition and its effects in China: An overview. Environ. Sci. Policy 1999, 2, 9-24.

22. Park, S.U.; Kim, J.W. Aerosol size distributions observed at the Seoul National University campus in Korea during the Asian dust and non-Asian dust periods. Atmos. Environ. 2006, 40, 1772-1730.

23. Fu, P.; Huang, J.; Li, C.; Zhong, S. The properties of dust aerosol and reducing tendency of the dust storms in northwest China. Atmos. Environ. 2008, 42, 5896-5904.

24. Kim, J. Transport routes and source regions of Asian dust observed in Korea during the past 40 years (1965-2004). Atmos. Environ. 2008, 42, 4778-4789.

25. Zhao, D.; Xiong, J.; Xu, Y.; Chan, W.H. Acid rain in southwestern China. Atmos. Environ. 1988, 22, 349-358.

26. Herut, B.; Starinsky, A.; Katz, A.; Rosenfeld, D. Relationship between the acidity and chemical composition of rainwater and climatological conditions along a transition zone between large deserts and Mediterranean climate, Israel. Atmos. Environ. 2000, 34, 1281-1292. 
27. Basak, B.; Alagha, O. The chemical composition of rainwater over Büyüqkçekmece Lake, Istanbul. Atmos. Res. 2004, 71, 275-288.

28. Wei, H.; Wang, J.L. Characteristics of acid rain in Jinyun Mountain, Chongqing, China. Appl. Ecol. Environ. Res. 2005, 3, 29-37.

29. Park, C.J.; Noh, H.R.; Kim, B.G.; Kim, S.Y.; Jung, I.U.; Cho, C.R.; Han, J.S. Evaluation of acid deposition in Korea. Water Air Soil Pollut. 2001, 130, 445-450.

30. Kang, G.; Collettjr, J.L.; Shin, D.Y.; Fujita, S.I.; Kim, H.K. Comparison of the chemical composition of precipitation on the western and eastern coasts of Korea. Water Air Soil Pollut. 2004, 151, 11-34.

31. Kim, S.B.; Choi, B.C.; Oh, S.Y.; Kim, S.; Kang, G.U. Acidity and chemical composition of precipitation at background area of the Korean Peninsula (Anmyeon, Uljin, Gosan). J. Korean Soc. Atmos. Environ. 2006, 22, 15-24.

32. Kim, K.J.; Bu, J.-O.; Kim, W.H.; Lee, Y.S.; Hyeon, D.R.; Kang, C.H. Pollution characteristics of rainwater at Jeju Island during 2009 2010. J. Korean Soc. Atmos. Environ. 2013, 29, 818-829.

33. Choi, J.S.; Park, J.S.; Ahn, J.Y.; Oh, J.; Son, J.S.; Kim, H.J.; Sung, M.Y.; Lee, Y.H.; Lee, S.D.; Hong, Y.D.; et al. The characteristics of long-term variation of acid deposition in Korea. J. Korean Soc. Environ. Anal. 2015, 1, 26-37.

34. Lee, S.I.; Kim, B.C.; Oh, H.J. Evaluation of lake modification alternatives for Lake Sihwa. Korea Environ. Manag. 2002, 29, 57-66.

35. Kim, K.T.; Kim, E.S.; Cho, S.R.; Chung, K.H.; Park, J.K. Distribution and pollution of heavy metals in the environmental samples of the Lake Sihwa. J. Korean Soc. Marine Environ. Eng. 2005, 8, 148-157.

36. Im, M.S.; Kim, K.H.; Choi, Y.J.; Jeon, E.C. Emission characteristics of VOC due to major industrial activities in the Banwol industrial complex. J. Korean Soc. Atmos. Environ. 2006, $22,325-336$.

37. Jung, J.; Jang, Y.W.; Arimoto, R.; Uematsu, M.; Lee, G. Atmospheric nitrogen deposition and its impact to Lake Sihwa in South Korea. Geochem. J. 2009, 43, 305-314.

38. Butler, T.J.; Likens, G.E. Weekly and daily precipitation chemistry network comparisons in the eastern U.S.: NADP/NTN vs. MAP3S/AIRMoN. Atmos. Environ. 1998, 32, 3749-3765.

39. Gilliland, A.B.; Butler, T.J.; Likens, G.E. Monthly and annual bias in weekly (NADP/NTN) vs. daily (AIRMoN) precipitation chemistry data in the Eastern USA. Atmos. Environ. 2002, 36, 5197-5206.

40. Sequeira, R.; Lung, F. A critical data analysis and interpretation of the $\mathrm{pH}$, ion loadings and electrical conductivity of rainwater from the territory of Hong Kong. Atmos. Environ. 1995, 29, 2439-2447.

41. Staelens, J.; Schrijver, A.D.; Avermaet, P.V.; Genouw, G.; Verhoest, N. A comparison of bulk and wet-only deposition at two adjacent sites in Melle (Belgium). Atmos. Environ. 2005, 39, 7-15.

42. Satsangi, G.S.; Khare, L.P.; Singh, S.P.; Kumari, K.M.; Srivastava, S.S. Composition of rain water at a semi-arid rural site in India. Atmos. Environ. 1998, 32, 3783-3793. 
43. Jain, M.; Kulshrestha, U.C.; Sarkar, A.K.; Parashar, D.C. Influence of crustal aerosols on wet deposition at urban and rural sites in India. Atmos. Environ. 2000, 34, 5129-5137.

44. Al-Khashman, O.A. Ionic composition of wet precipitation in the Petra Region, Jordan. Atmos. Environ. 2005, 78, 1-12.

45. Edmonds, R.L.; Thomas, T.B.; Rhodes, J.J. Canopy and Soil Modification of Precipitation Chemistry in a Temperate Rain Forest. Soil Sci. Soc. Am. J. 1991, 55, 1685-1693.

46. Environmental Protection Agency (EPA). EPA Positive Matrix Factorization (PMF) 3.0 Fundamentals and User Guide; USEPA Office of Research and Development: Research Triangle Park, NC, USA, 2008.

47. Gugamstty, B.; Wei, H.; Liu, C.N.; Awasthi, A.; Hsu, S.C.; Tsai, C.J.; Roam, G.D.; Wu, Y.C.; Chen, C.F. Source characterization and apportionment of $\mathrm{PM}_{10}, \mathrm{PM}_{2.5}$ and $\mathrm{PM}_{0.1}$ by using positive matrix factorization. Aerosol Air Qual. Res. 2012, 12, 476-491.

48. Environmental Protection Agency (EPA). EPA Positive Matrix Factorization (PMF) 5.0 Fundamentals and User Guide; Environmental Protection Agency: Washington, DC, USA, 2014.

49. García, R.; Ma, D.T.; Padilla, H.; Belmont, R.; Azpra, E.; Arcega-Cabrera, F.; Báez, A. Measurement of chemical elements in rain from Rancho Viejo, a rural wooded area in the State of Mexico, Mexico. Atmos. Environ. 2006, 40, 6088-6100.

50. Kai, Z.; Huiwang, G. The characteristics of Asian-dust storms during 2000-2002: From the source to the sea. Atmos. Environ. 2007, 41, 9136-9145.

51. Topcu, S.; Incecik, S.; Atimtay, A.T. Chemical composition of rainwater at EMEP station in Ankara, Turkey. Atmos. Res. 2002, 65, 77-92.

52. Hegde, P.; Sudheer, A.K.; Sarin, M.M.; Manjunatha, B.R. Chemical characteristics of atmospheric aerosols over southwest coast of India. Atmos. Environ. 2007, 41, 7751-7766.

53. Das, R.; Das, S.N.; Misra, V.N. Chemical composition of rainwater and dustfall at Bhubaneswar in the east coast of India. Atmos. Environ. 2005, 39, 5908-5916.

54. Tanner, P.A. Analysis of Hong Kong daily bulk and wet deposition data from 1994 to 1995. Atmos. Environ. 1999, 33, 1757-1766.

55. Migliavacca, D.M.; Teixeira, E.C.; Wiegand, F.; Machado, A.C.M.; Sanchez, J. Atmospheric precipitation and chemical composition of an urban site, Guaíba hydrographic basin, Brazil. Atmos. Environ. 2005, 39, 1829-1844.

56. Sanusi, A.; Wortham, H.; Millet, M.; Mirabel, P. Chemical composition of rainwater in eastern France. Atmos. Environ. 1996, 30, 59-71.

57. Al-Khashman, O.A. Study of chemical composition in wet atmospheric precipitation in Eshidiya area, Jordan. Atmos. Environ. 2005, 39, 6175-6183.

58. Sorooshian, A.; Shingler, T.; Harpold, C.; Feagles, C.W.; Meixner, T.; Brooks, P.D. Aerosol and precipitation chemistry in the southwestern United States: Spatiotemporal trends and interrelationship. Atmos. Chem. Phys. 2013, 13, 7361-7379.

59. Dayan, U.; Lamb, D. Meteorological indicators of summer precipitation chemistry in central Pennsylvania. Atmos. Environ. 2003, 37, 1045-1055.

60. Báez, A.P.; Belmont, R.D.; García, R.M.; Torres, M.C.B.; Padilla, H.G. Rainwater chemical composition at two sites in Central Mexico. Atmos. Res. 2006, 80, 67-85. 
61. Herrera, J.; Rodriguez, S.; Baez, A.P. Chemical composition of bulk precipitation in the metropolitan area of Costa Rica, Central America. Atmos. Res. 2009, 94, 151-160.

62. Kulshrestha, U.C.; Kulshrestha, M.J.; Sekar, R.; Sastry, G.S.R.; Vairamani, M. Chemical characteristics of rainwater at an urban site of south-central India. Atmos. Environ. 2003, 37, 3019-3026.

63. Acid Deposition Monitoring Network in East Asia (EANET). Data report on the acid deposition in the East Asian region 2001-2012; Network Center for EANET: Nigata-si, Japan, 2013.

64. Kang, G.U.; Lim, J.H.; Kim, H.K. An analysis of long-term trends in precipitation acidity of Seoul, Korea. J. Korea Air Pollut. Res. Assoc. 1997, 13, 9-18.

65. Safai, P.D.; Rao, P.S.P.; Momin, G.A.; Ali, K.; Chate, D.M.; Praveen, P.S. Chemical composition of precipitation during1984-2002 at Pune, India. Atmos. Environ. 2004, $38,1705-1714$.

66. Zhang, G.S.; Zhang, J.; Liu, S.M. Chemical composition of atmospheric wet depositions from the Yellow Sea and East China Sea. Atmos. Res. 2007, 85, 84-97.

67. Rastogi, N.; Sarin, M.M. Chemistry of precipitation events and inter-relationship with ambient aerosols over a semi-arid region. J. Atmos. Chem. 2007, 56, 149-163.

68. Aikawa, M.; Hiraki, T.; Tamaki, M. Comparative field study on precipitation, throughfall, stemflow, fog water, and atmospheric aerosol and gases at urban and rural sites in Japan. Sci. Total Environ. 2006, 366, 275-285.

69. Hand, J.L.; Gebhart, K.A.; Schichtel, B.A.; Malm, W.C. Increasing trends in wintertime particulate sulfate and nitrate ion concentrations in the great Plains of the United States (2000-2010). Atmos. Environ. 2012, 55, 107-110.

70. Aherne, J.; Farrell, E.P. Deposition of sulphur, nitrogen and acidity in precipitation over Ireland: Chemistry, spatial distribution and long-term trends. Atmos. Environ. 2002, 36, 1379-1389.

71. Fornaro, A.; Gutz, I.G.R. Wet deposition and related atmospheric chemistry in the São Paulo metropolis, Brazil, Part 3: Trends in precipitation chemistry during 1983-2003. Atmos. Environ. 2006, 40, 5893-5901.

72. Puxbaum, H.; Simeonov, V.; Kalina, M.; Tsakovski, S.; Löffler, H.; Heimburger, G.; Biebl, P.; Weber, A.; Damm, A. Long-term assessment of the wet precipitation chemistry in Austria (1984-1999). Chemosphere 2002, 48, 733-747.

73. Zhang, M.; Wang, S.; Wu, F.; Yuan, X.; Zhang, Y. Chemical compositions of wet precipitation and anthropogenic influences at a developing urban site in southeastern China. Atmos. Res. 2007, 84, 311-322.

74. Báez, A.; Belmont, R.; García, R.; Padilla, H.; Torres, M.C. Chemical composition of rainwater collected at a southwest site of Mexico City, Mexico. Atmos. Res. 2007, 86, 61-75.

75. Anttila, P.; Paatero, P.; Tapper, U.; Järvinen, O. Source identification of bulk wet deposition in Finland by positive matrix factorization. Atmos. Environ. 1995, 29, 1705-1718.

76. Kitayama, K.; Murao, N.; Hara, H. PMF analysis of impacts of SO2 from Miyakejima and Asian continent on precipitation sulfate in Japan. Atmos. Environ. 2010, 44, 95-105. 


\title{
Airborne Aerosol in Situ Measurements during TCAP: A Closure Study of Total Scattering
}

\author{
Evgueni Kassianov, Larry K. Berg, Mikhail Pekour, James Barnard, Duli Chand, \\ Connor Flynn, Mikhail Ovchinnikov, Arthur Sedlacek, Beat Schmid, \\ John Shilling, Jason Tomlinson and Jerome Fast
}

\begin{abstract}
We present a framework for calculating the total scattering of both non-absorbing and absorbing aerosol at ambient conditions from aircraft data. Our framework is developed emphasizing the explicit use of chemical composition data for estimating the complex refractive index (RI) of particles, and thus obtaining improved ambient size spectra derived from Optical Particle Counter (OPC) measurements. The feasibility of our framework for improved calculations of total scattering is demonstrated using three types of data collected by the U.S. Department of Energy's (DOE) aircraft during the Two-Column Aerosol Project (TCAP). Namely, these data types are: (1) size distributions measured by a suite of OPC's; (2) chemical composition data measured by an Aerosol Mass Spectrometer and a Single Particle Soot Photometer; and (3) the dry total scattering coefficient measured by a integrating nephelometer and scattering enhancement factor measured with a humidification system. We demonstrate that good agreement $(\sim 10 \%)$ between the observed and calculated scattering can be obtained under ambient conditions $(\mathrm{RH}<80 \%)$ by applying chemical composition data for the RI-based correction of the OPC-derived size spectra. We also demonstrate that ignoring the RI-based correction or using non-representative RI values can cause a substantial underestimation $(\sim 40 \%)$ or overestimation $(\sim 35 \%)$ of the calculated scattering, respectively.
\end{abstract}

Reprinted from Atmosphere. Cite as: Kassianov, E.; Berg, L.K.; Pekour, M.; Barnard, J.; Chand, D.; Flynn, C.; Ovchinnikov, M.; Sedlacek, A.; Schmid, B.; Shilling, J.; Tomlinson, J.; Fast, J. Airborne Aerosol in Situ Measurements during TCAP: A Closure Study of Total Scattering. Atmosphere 2015, 6, 1069-1101.

\section{Introduction}

Although the importance of atmospheric aerosol in modifying the Earth's radiation budget has been recognized by many studies [1,2], the extent to which aerosol shapes the regional and global climate is still ambiguous [3,4]. The magnitude and sign of the aerosol-induced changes of the radiation budget at the regional and global scales are highly uncertain, since these changes are influenced substantially by strong temporal and spatial variations of aerosol loading, chemical composition 
and mixing state [5-7]. Since the advent of observational techniques for monitoring these variations from surface, air and space, the diversity of sensors with improved precision and accuracy has increased and corresponding innovative methods have been developed [8-11]. Aircraft measurements are becoming increasingly important for model validation studies because they can document aerosol variations in remote regions where access to ground-based observations is difficult or unavailable, and offer observations with higher temporal resolution than can typically be attained with satellites [12-14].

Comprehensive and integrated measurements of aerosol properties provide an important observational basis for evaluations of climate model predictions and necessarily involve combining data collected by several instruments with different designs and uncertainties. To determine whether these data are consistent and reasonable, a special kind of quantitative comparison experiment is commonly performed. Such an experiment, traditionally referred to as a closure study, compares the measured values of a selected aerosol property with those calculated from independent measurements [15-18]. For example, an optical closure experiment compares the measured values of an aerosol optical property, such as total scattering coefficient, with those calculated from independently measured size distributions and chemical composition under a variety of conditions [19-21]. Good agreement between the measured and calculated aerosol properties (within error bars) indicates consistency of the observational dataset, and bolsters its relevance for further use in global and regional climate model evaluations.

Optical closure studies have become an essential part of testing integrated datasets where simultaneous measurements of the optical, microphysical and chemical properties of aerosol at dry and ambient conditions are available [21-23]. Compared to the ground-based instrumentation suites, instrumentation on board aircraft platforms requires particular attention to its design and operation [24] mainly due to payload restrictions (requiring instruments with smaller dimensions and less weight; $[25,26])$ and abrupt changes in atmospheric and aerosol characteristics during the aircraft's rapid (about $100 \mathrm{~m} / \mathrm{s}$ ) motion (requiring instruments with faster response time and data acquisition speeds; [27]), which directly impact spatial resolution. While airborne instrumentation and associated data synergy are continuing to evolve on many fronts, rigorous scrutiny of airborne integrated measurements has not always been achieved. Moreover, demands to assess the consistency and reasonableness of integrated airborne data sets have been growing, given the increasingly heavy reliance of process-oriented model evaluations on aircraft measurements [6].

Optical Particle Counters (OPCs) are a common type of airborne instrument for deriving size spectra $[24,28,29]$. The fundamental quantity measured by OPCs is the amount of light scattered by individual particles over a large solid angle. The 
amount of scattered light depends on aerosol characteristics, such as size, shape and complex refractive index (RI), which is a function of the particle's chemical composition. The measured scattered light is converted into particle size using an appropriate scattering theory (e.g., Mie theory for spherical particles) and an assumed or estimated refractive index. For example, several parameterizations have been developed for correcting OPC-derived size distributions for weakly absorbing aerosol using Mie calculations and assuming that the RI-based correction depends on the real part of the complex RI only [30,31]. It is important to note that the assumptions employed for the refractive index may or may not be representative of the observed ambient conditions.

Although Mie theory does allow for the RI-based corrections associated with both the real and imaginary parts of the complex RI [32-34], airborne measurements of aerosol chemical composition and absorbing components that are required for the RI estimation are demanding and not always available. The mass loading of black carbon (BC) is an example of one of these absorbing components with relatively sparse relevant measurements [4,35]. As a result, iterative schemes that use assumed values of complex RI in combination with other assumptions are commonly applied to minimize differences between the measured and calculated aerosol properties of interest, such as $\mathrm{PM}_{10}$ [34]. For humid conditions, the particle size distributions exhibit a sensitivity to water uptake by particles [36], and therefore the hygroscopic growth factor (HGF) and its dependence on particle chemical composition must be considered in closure-related studies.

While closure studies using the microphysical, optical, chemical components, and ambient relative humidity (RH) are a well-known framework that has been used intensively for decades [37-39], its successful applications are mainly limited to the ground-based observations [21,40,41]. Given the complexity of conducting airborne measurements and the growing demand to use these measurements for process-oriented model validation and climate model assessments, there is a strong need to extend this framework to comprehensive airborne datasets [22,42,43]. The primary purpose of our work is to attempt to formally extend the framework for ground-based optical closure studies to airborne data sets by answering the following three main questions:

(1) What level of agreement can be achieved between the in-flight measured and calculated values of total scattering coefficient at ambient $\mathrm{RH}$ ?

(2) What is the effect of ignoring the influence of chemical composition data on this agreement?

(3) How sensitive is this agreement to the assumed RI value, particularly if the assumed RI is non-representative of the ambient aerosol? 
The first question is associated with the consistency of the airborne measurements of the particle size distributions and optical properties when aerosol chemical composition data are available (a preferred "complete" dataset). The second and third questions can be considered as "practical-oriented" because they are focused mostly on practical situations when information on the chemical composition is not available (an "incomplete" dataset) and assumptions about aerosol composition are required. Given that the dimension/weight of several instruments commonly deployed to measure the aerosol chemical composition, such as the miniaturized version of the aircraft-compatible single particle mass spectrometer (miniSPLAT; [44]) and the Aerodyne Aerosol Mass Spectrometer (AMS), is substantial, they are deployed less frequently on mid-to-large size aerial platforms. Moreover, they cannot be deployed on small aerial platforms, such as small or unmanned aircraft. To assess the extended framework and evaluate the relevant assumptions through answering these three important questions, we use integrated airborne data collected during the recent Two-Column Aerosol Project (TCAP; http:/ / campaign.arm.gov/tcap/) over the North Atlantic Ocean and US coastal region (Cape Cod, MA, USA).

We outline in the next section our approach for extending the ground-based framework for conducting optical closure experiments to airborne data. In Section 3 we briefly describe the TCAP data, which represent mainly non-absorbing aerosol and include measured size spectra, chemical composition and total scattering [45]. The complementary model components of our approach and the corresponding assumptions are discussed in Section 4. These assumptions, such as the homogeneous internal mixture, are reasonable and permit us to calculate the HGF and complex RI at ambient $\mathrm{RH}$ when additional information is missing. In Section 5, the calculated and measured total scattering coefficients are compared for the wide range of atmospheric conditions observed during TCAP flights conducted in July of 2012, including conditions with low and high ambient RH. The sensitivity of the calculated scattering to the RI and related issues are further discussed in Section 6. In particular, this section emphasizes that the ability to make complementary measurements of chemical composition holds promise for properly specifying the RI, and thus for improving the accuracy of total scattering calculations. The last section presents a summary of key findings.

\section{Approach}

Figure 1 outlines the major components and main steps in conducting our optical closure experiment by obtaining total scattering coefficients at ambient $\mathrm{RH}$ from airborne measurements and Mie calculations. Although our approach relies heavily on several important components and assumptions, such as homogeneous internal mixture and spherical geometry of particles, introduced earlier by previous studies $[21,36,46]$, well-known challenges experienced in collection and interpretation 
of airborne data make evaluation of this unified approach mandatory. Our approach, as displayed in Figure 1, involves three major components: (1) integrated measurements of aerosol properties (Figure 1; top panel); (2) calculations of the ambient scattering coefficient using Mie theory and estimated hygroscopic growth factor (HGF) (Figure 1; middle panel), and (3) comparison of the scattering coefficients observed and calculated at ambient conditions (Figure 1; bottom panel). Note in Figure 1 how chemical composition information becomes encoded into the improved size spectra, making calculations of scattering more accurate. The left-hand part of diagram (Figure 1; top and bottom panels) illustrates the process of obtaining the observed total scattering at ambient conditions. For the TCAP data set used in this work, this step involves analyzing the total scattering measured by an airborne nephelometer at low $\mathrm{RH}$ and the light scattering hygroscopic growth $\mathrm{f}(\mathrm{RH})$ measured using a humidification system. The central part of diagram (Figure 1; top and middle panels) illustrates how the complex RI at dry conditions, derived from the chemical composition data, is used to adjust the particle size distributions. Note that the chemical composition data are also used to estimate the RH-dependent HGF. The latter is required for converting the dry complex RI and dry size spectra into their ambient counterparts. The right-hand part of diagram (Figure 1; top and middle panels) demonstrates how the ambient size spectra are obtained from the original (without RI-based correction) and adjusted (with RI-based correction) OPC-derived size distributions. The ambient complex RI and size distributions (both original and adjusted) are used as input for the Mie calculations. The output is the corresponding model total scattering coefficients calculated at ambient conditions. As we shall see, differences between model coefficients calculated using the original and the adjusted size distributions illustrate the importance of the RI-based correction for the total scattering calculation.

\section{Data}

This section is meant to survey the observational components of our framework (Figure 1; top panel) by reviewing the TCAP airborne data used in this investigation. The TCAP field campaign was designed to provide a comprehensive data set that can be used to investigate important climate science questions, including those related to aerosols. Conducted from June 2012 through June 2013, TCAP involved summer and winter periods of intensive aircraft observations that included the U.S. Department of Energy (DOE) Gulfstream-159 (G-1) aircraft. The G-1 typically sampled at multiple altitudes within two atmospheric columns, one located over Cape Cod, MA and a second over the Atlantic Ocean several hundred kilometers from the coast. Details of TCAP are given in Berg et al. [45]. To illustrate performance of our unified approach (Figure 1), we focus on the TCAP summertime data. 


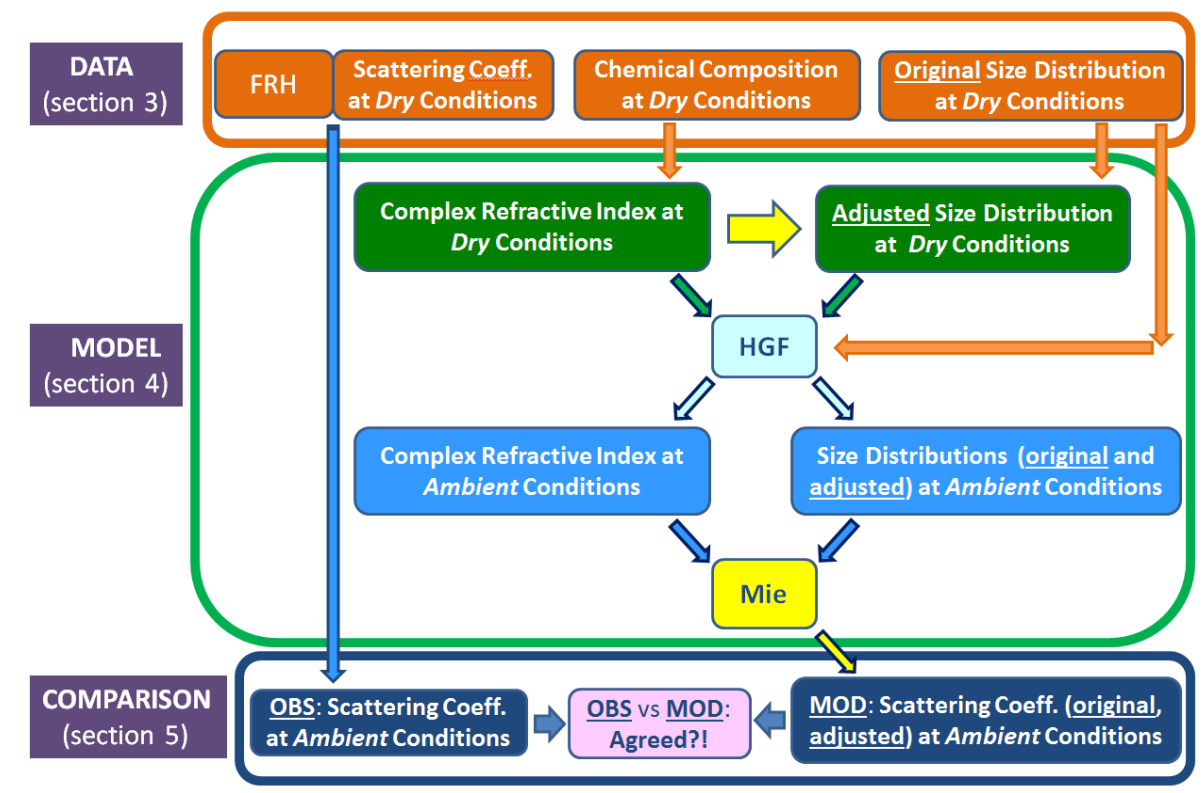

Figure 1. Schematic diagram summarizing the framework for an optical closure experiment using airborne data. The figure illustrates the link between the measured dry and ambient scattering coefficients (left part of diagram; top and bottom panels) and the connection between the measured dry chemical composition/size spectra and the calculated ambient scattering coefficient (center and right parts of diagram). Ambient size spectra (light blue, right) are obtained from the dry size distributions without (orange) and with (green) the RI-based correction, respectively. The estimated ambient size spectra (light blue, right) together with the ambient RI (light blue, left) are required as input for Mie calculations (yellow) of the corresponding total scattering coefficients (navy blue, right). See indicated text section for details of each component (Data, Model, Comparison).

An in situ instrumentation suite on board the DOE G-1 aircraft [24] together with airborne remote sensing sensors, such as the Spectrometer for Sky-Scanning, Sun-Tracking Atmospheric Research (4STAR; [26]) and the High Spectral Resolution Lidar (HSRL-2; [8]), were deployed during TCAP. Note that the HSRL-2 was operated aboard a NASA B-200 aircraft. Here, we focus on the instruments relevant to our study to improve calculations of the total scattering coefficient at ambient conditions. These calculations are based on Mie theory and require the ambient aerosol size distribution and complex RI. Aerosol size spectra, chemical composition and total scattering data were collected with high temporal resolution $(<1 \mathrm{~min})$ during the TCAP flights. We use these data to compute the corresponding averaged 
characteristics for each flight leg (FL), which is defined as a straight level run at different altitudes with variable duration of approximately 5-15 min. We employ these FL-averaged aerosol characteristics in our investigation, consistent with earlier studies $[28,29,46]$.

Particle size distributions were measured simultaneously by three airborne OPC instruments: an Ultra-High Sensitivity Aerosol Spectrometer (UHSAS, size range 0.06-1 $\mu \mathrm{m}$ ), a Passive Cavity Aerosol Spectrometer (PCASP; size range 0.13-3 $\mu \mathrm{m}$ ) and a Cloud and Aerosol Spectrometer (CAS; size range 0.6->10 $\mu \mathrm{m}$ ). These three instruments were mounted within PMS canisters on the same pylon underneath the right wing of the G-1 aircraft. The UHSAS and PCASP were operated with anti-ice heaters enabled and therefore the measured aerosol distributions are assumed to be dry. However, the CAS measured particle size distributions at ambient conditions. All three instruments were calibrated using polystyrene latex sphere (PSL) beads. The measured size distributions from the aforementioned probes are recovered from the raw counts (taking into account collection efficiencies and sampling volumes), merged, and smoothed (Appendix A) using a kernel based on Twomey's algorithm $[42,47,48]$. Figure 2 shows the resulting size distributions averaged over each FL on 21 July 2012. The altitude of each FL is shown in Figure 3.

Particle size spectra measured by these three OPC instruments (UHSAS, PCASP, and CAS) cover different particle size ranges. However, these instruments employ a similar underlying operating principle for determining particle size, namely light scattering by individual particles. The conversion of the scattered light into particle size requires the RI, which depends on the chemical composition of particles, thus demanding an accurate RI estimation. During TCAP, information on the organic and inorganic species mass loading and black carbon (BC) mass in individual aerosol particles came from complementary Aerodyne AMS and Droplet Measurement Technology Single Particle Soot Photometer (SP2; 0.06-0.6 $\mu \mathrm{m}$ range of mass-equivalent diameter; $[49,50]$ ) measurements, respectively (Figure 3 ). The AMS has a near unity transmission efficiency for particles with vacuum aerodynamic diameters between 0.06 and $0.6 \mu \mathrm{m}$, which falls to $50 \%$ at $1 \mu \mathrm{m}$, and is negligible above $1.5 \mu \mathrm{m}$ and below $0.06 \mu \mathrm{m}$. The RH inside the SP2 does not exceed $10 \%$ and the AMS can differentiate particle-phase water from other species; therefore, the acquired chemical composition data represent dry conditions. Figure 3 illustrates that the aerosol chemical composition on 21 July 2012 was dominated by organic matter (OM); dominance of OM (generally greater than $70 \%$ ) was observed for all TCAP flights [45]. 


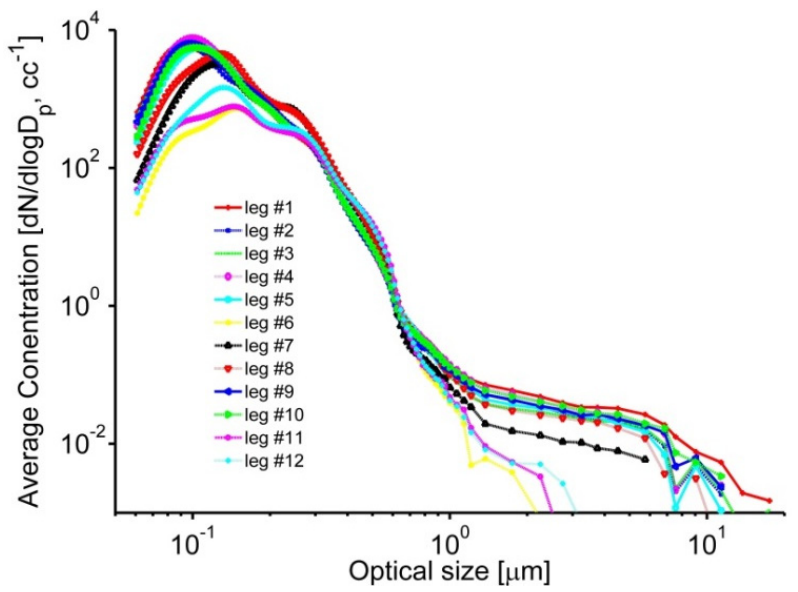

Figure 2. Example of combined size distributions generated for each FL during a given day (21 July 2012). Here and in the following plots, aerosol characteristics represent FL-averaged values. Elevation and time of each FL are shown in Figure 3.

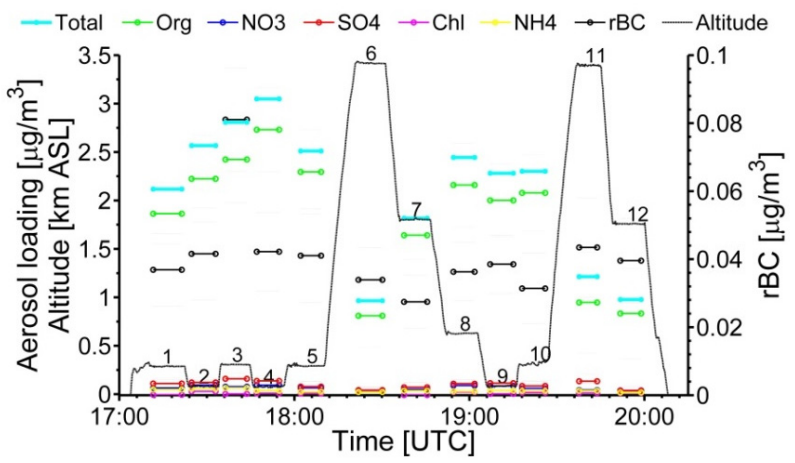

Figure 3. Example of FL-dependent chemical compositions (colored lines) and BC (thick black lines) mass measured by the AMS and SP2, respectively (21 July 2012). Additionally, altitude (thin black line) as a function of FL is included. FLs are labeled with numbers 1 through 12 on top of the thin black altitude line.

The total scattering coefficient at three wavelengths $(0.45,0.55,0.7 \mu \mathrm{m})$ was measured at dry $(\mathrm{RH}<20 \%)$ conditions using a TSI integrating nephelometer (Figure 4), while the light scattering hygroscopic growth, known as $\mathrm{f}(\mathrm{RH})$, was measured using a humidification system at three defined RHs (near $45 \%, 65 \%$ and $90 \%)$ at a single wavelength $(0.525 \mu \mathrm{m})$ [51]. Similar to Shinozuka et al. [52], we adjust the $\mathrm{f}(\mathrm{RH})$ obtained at the $0.525 \mu \mathrm{m}$ wavelength to the nephelometer wavelengths $(0.45,0.55,0.7 \mu \mathrm{m})$ by multiplying the obtained $\mathrm{f}(\mathrm{RH})$ by $0.98,1.01$ and 1.04, respectively. The conventional truncation error correction [53] has been 
applied to the total scattering measured by the integrating nephelometer. We obtain the total scattering at ambient conditions $\left(\sigma_{\text {obs }}\right)$ at three wavelengths $(0.45,0.55$, $0.7 \mu \mathrm{m})$ using both the adjusted $\mathrm{f}(\mathrm{RH})$ and spectrally-dependent measured dry total scattering. It is to be noted that measurement uncertainties in the reported total scattering by the integrating nephelometer are quite small $(\sim 10 \%)$ for sub- $\mu \mathrm{m}$, but can be considerable for super- $\mu$ m particles $(\sim 50 \%)[53,54]$. For a given $\mathrm{FL}$, we assume that the combined uncertainty of $\sigma_{\text {obs }}$ depends on its variability within FL (defined here as the standard deviation) and the measurement uncertainty [46]. In other words, the FL-dependent combined uncertainty in the ambient total scattering coefficient is comparable with the measurement uncertainty (10\%) for a homogeneous FL and can exceed it substantially for an inhomogeneous FL where the standard deviation is large.

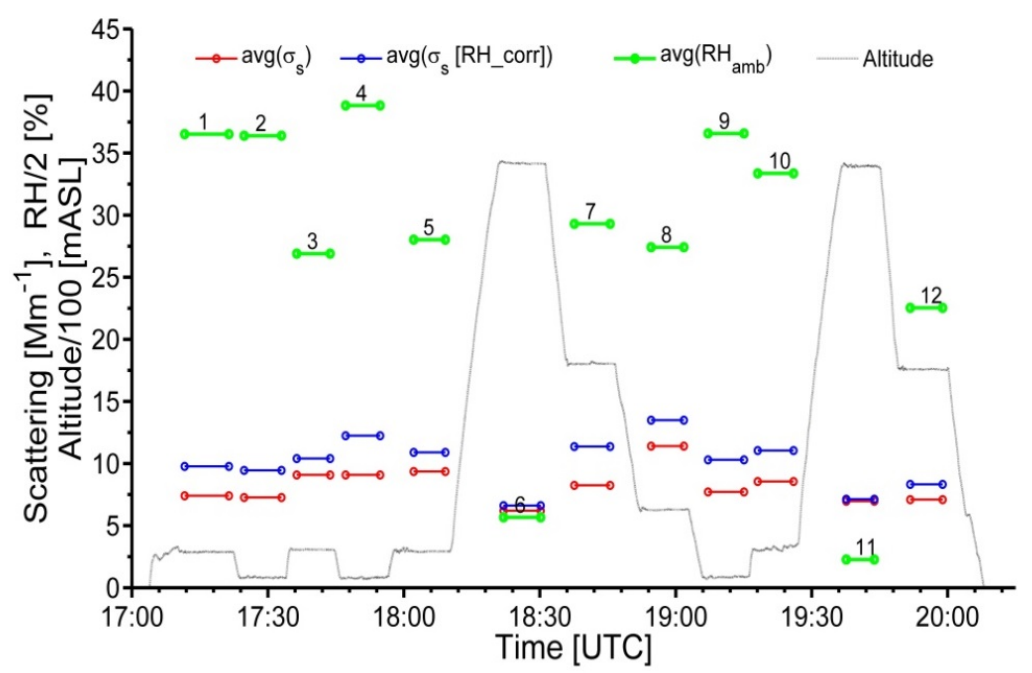

Figure 4. The same as Figure 3, except for the dry scattering measured by nephelometer (red lines) and ambient scattering obtained with measured $f(R H)$ (blue lines) at $0.55 \mu \mathrm{m}$ wavelength and ambient RH (green lines). FLs are labeled with numbers 1 through 12 on top of green lines.

TCAP aircraft data were screened prior to use in our study. To ensure that only high quality data are used, two quality assurance screening criteria are applied. First, we check the data streams for consistency between the size spectra, chemical composition and total scattering to prevent invalid data entry. Second, we consider only periods in which all data streams are available. For example, PCASP-measured size distributions were not available for several flights (e.g., 14 and 15 July) and the corresponding combined size distributions were generated from spectra measured by two instruments (UHSAS and CAS) only. These combined distributions obtained 
without PCASP data are excluded from our analysis. Similar to the size distributions, ambient scattering coefficients were not available for some episodes as well. Given the large uncertainties of the obtained $\mathrm{f}(\mathrm{RH})$ at very humid conditions $(\mathrm{RH}>80 \%)$, the corresponding cases are also excluded from our analysis. Using the quality assurance screening criteria reduces the size of the original dataset by about $30 \%$. A total of 45 TCAP FLs with the good quality data are included in our analysis. Further discussion of TCAP flight data quality can be found in Berg et al. [45].

\section{Model and Adjustments}

This section outlines the model components of our framework for an airborne optical closure experiment (Figure 1; middle panel) and describes the major assumptions required for estimating the hygroscopic growth factor (Section 4.1), ambient values of complex refractive index (Section 4.2) and correcting the OPC-derived size distributions (Section 4.3). The RI-based corrections of the size spectra together with estimates of HGF and complex RI form the basis for calculating the ambient total scattering (Section 4.4) with improved accuracy.

\subsection{Hygroscopic Growth Factor}

Water uptake by aerosol particles results in increased particle size and modifies the complex RI [36]. To account for changes associated with water absorption, information on aerosol chemical composition and hygroscopicity is needed. Typically, aerosol particles are a mixture of organic and inorganic substances. We estimate the hygroscopic growth factor of the mixture $\left(\mathrm{HGF}_{\text {mix }}\right)$ from the volume fractions of individual components $(\varepsilon)$ and their growth factors as the volume-weighted average [46]:

$$
\mathrm{HGF}_{\text {mix }}=\left(\sum_{\mathrm{i}} \varepsilon_{\mathrm{i}} \mathrm{HGF}_{\mathrm{i}}^{3}\right)^{1 / 3}
$$

We convert the mass fractions measured by the AMS and SP2 instruments (Section 2) into the required volume fractions using densities reported in literature and listed in Table 1. We emphasize that the AMS and SP2 data are capturing a limited size range (sub-micron particles; Section 2) only. However, we use the AMS/SP2 data to infer the chemical composition of the entire size range (both sub- and super-micron particles). The application of the AMS/SP2 data for the entire size range should be appropriate for cases where the relative contribution of sub-micron particles to the scattering coefficient is dominant. Given that the aircraft data collected during the TCAP data represent such a favorable case with large contributions of sub-micron particles (Appendix B), application of the AMS/SP2 data for the entire size range is appropriate. 
Table 1. Assumed size-independent density, real and imaginary parts of complex refractive index (RI) at $0.55 \mu \mathrm{m}$ wavelength, and hygroscopic growth factor (HGF) values used in this study. Values are taken from $[46,55,56]$.

\begin{tabular}{cccccccc}
\hline & $\mathrm{OM}$ & $\mathrm{SO}_{4}$ & $\mathrm{NO}_{3}$ & $\mathrm{Chl}$ & $\mathrm{NH}_{4}$ & $\mathrm{BC}$ & Water \\
\hline Density & 1.4 & 1.8 & 1.8 & 1.53 & 1.8 & 1.8 & 1.0 \\
$\left(\mathrm{~g} / \mathrm{cm}^{3}\right)$ & & & & & & & \\
RI (real) & 1.45 & 1.52 & 1.5 & 1.64 & 1.5 & 1.85 & 1.33 \\
RI (imag) & 0.0 & 0 & 0 & 0 & 0 & 0.71 & 0 \\
HGF (RH = 80\%) & 1.07 & 1.50 & 1.50 & 1.9 & 1.50 & 1.0 & - \\
\hline
\end{tabular}

Although the growth factor can be quite sensitive to the particle size [21,57], we assume that particles with different sizes have the same $\mathrm{HGF}_{\text {mix }}$. HGF mix from Equation (1) represents the hygroscopic growth factor at a specified relative humidity $\left(\mathrm{RH}_{\text {wet }}=80 \%\right)$. To obtain $\mathrm{HGF}_{\text {mix }}$ for a different humidity, we assume that $\mathrm{HGF}_{\text {mix }}$ follows the power law form $[19,21]$ :

$$
\mathrm{HGF}_{\text {mix }}(\mathrm{RH})=\left(\frac{100-\mathrm{RH}_{\mathrm{dry}}}{100-\mathrm{RH}}\right)^{\gamma}
$$

where $\mathrm{RH}_{\mathrm{dry}}$ is $30 \%$. The dimensionless exponent $\gamma$ is calculated as:

$$
\gamma=\frac{\log \left(\mathrm{HGF}_{\operatorname{mix}}\left(\mathrm{RH}_{\mathrm{wet}}\right)\right)}{\log \left[\left(100-\mathrm{RH}_{\mathrm{dry}}\right) /\left(100-\mathrm{RH}_{\mathrm{wet}}\right)\right]}
$$

Recall that OM is the dominant component of aerosol sampled during the TCAP flights (Figure 2 and [45]). Given that the hygroscopic growth factor of the OM is relatively small compared to other chemical components (Table 1), the calculated RH-dependent $\mathrm{HGF}_{\text {mix }}$ does not exceed 1.3 and approaches 1 as $\mathrm{RH}$ decreases (Figure 5).

\subsection{Dry and Wet Refractive Indices}

We apply a volume weighting approach to calculate the real $\left(\mathrm{n}_{\mathrm{dry}}\right)$ and imaginary $\left(k_{d r y}\right)$ parts of the complex RI $\left(m_{d r y}\right)$ of particles at dry conditions:

$$
\mathrm{m}_{\mathrm{dry}}=\sum_{\mathrm{i}} \varepsilon_{\mathrm{i}} \mathrm{m}_{\mathrm{dry}, \mathrm{i}}
$$

where $m_{d r y, i}$ represents the real $\left(n_{d r y, i}\right)$ or imaginary $\left(k_{d r y, i}\right)$ part for each measured chemical component (Table 1). The underlying assumption of this popular approach is that the contribution of each chemical component to the light scattering and absorption is proportional to its volume fraction $\left(\varepsilon_{i}\right)$. It should be emphasized that 
chemical composition, in general, depends on the particle size [57]. The same is true for the real $\left(\mathrm{n}_{\mathrm{dry}}\right)$ and imaginary $\left(\mathrm{k}_{\mathrm{dry}}\right)$ parts of the complex RI [40]. Here we assume that particles with different sizes have the same chemical composition, and therefore the same RI.
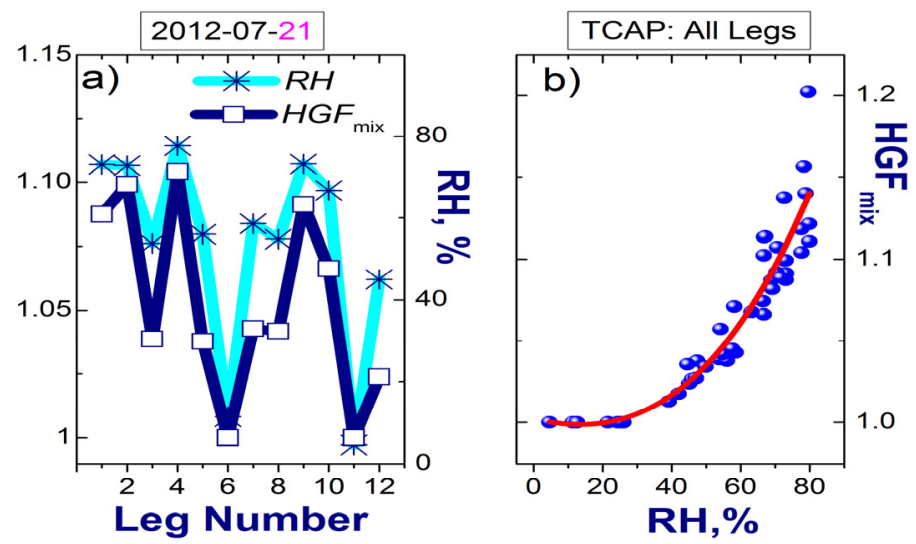

Figure 5. Example of ambient $\mathrm{RH}$ and $\mathrm{RH}$-dependent $\mathrm{HGF}_{\text {mix }}$ calculated for each FL during a given day (21 July 2012) (a); scatterplot of RH-dependent HGF $_{\text {mix }}$ (blue dots) for all TCAP FLs used in this study (Section 3) with polynomial fit (red line) (b).

The calculated dry RIs are applied to compute the corresponding ambient values [21,58]:

$$
\mathrm{m}_{\mathrm{wet}}=\frac{\mathrm{m}_{\mathrm{dry}}+\mathrm{m}_{\text {water }}\left(\mathrm{HGF}_{\text {mix }}^{3}-1\right)}{\mathrm{HGF}_{\text {mix }}^{3}}
$$

where $\mathrm{HGF}_{\text {mix }}$ is the $\mathrm{RH}$-dependent parameter defined in the previous section. As $\mathrm{HGF}_{\text {mix }}$ increases noticeably (near 1.1; Figure 5a), water becomes an influential component and the ambient RI decreases toward the water RI (Figure 6). In contrast, when $\mathrm{HGF}_{\text {mix }}$ is quite small (near 1; Figure 5a), the ambient RI increases toward the dry RI (Figure 6). Note that large values of the imaginary part of the RI (e.g., FL numbers 3, 6, 11 and 12) represent conditions where the relative contribution of $\mathrm{BC}$ to the total loading is substantial ( $>3 \%$; Figure 3 ) and exceeds those for other FLs roughly by a factor of two. 

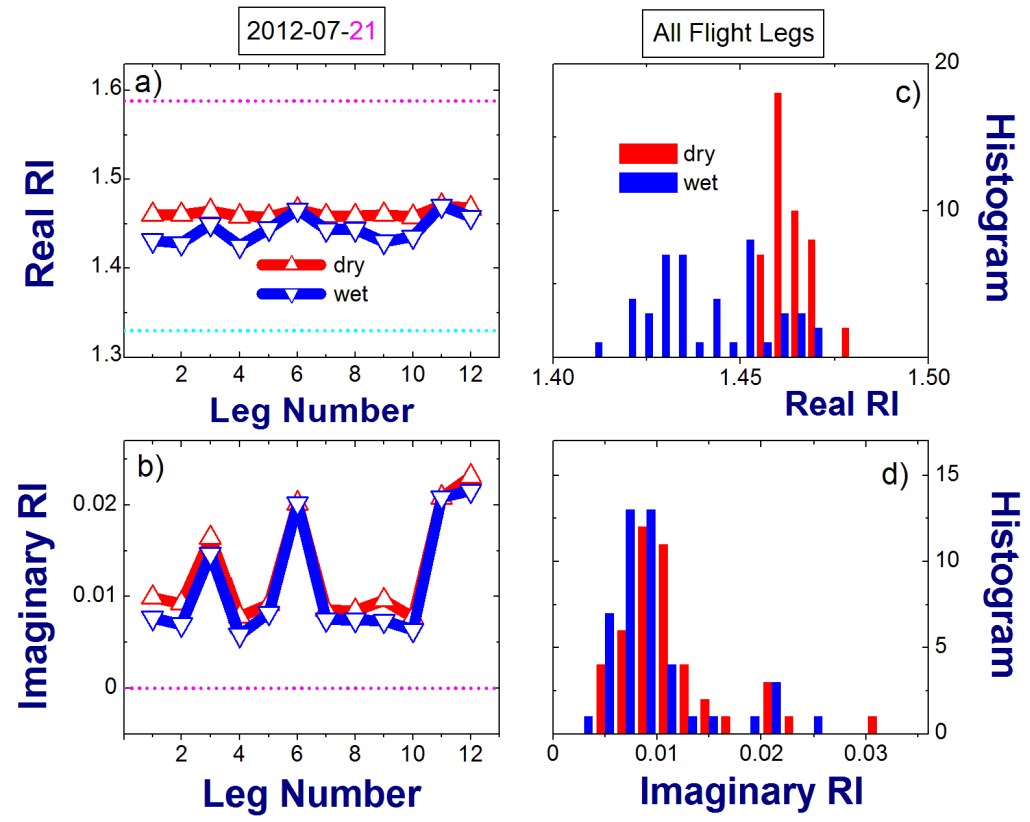

Figure 6. Example of RH-dependent dry and ambient values of the real and imaginary parts of the complex RI calculated for each FL during a given day (21 July 2012) (a,b); the corresponding histograms obtained for all TCAP FLs (c,d). The real $\left(\mathrm{n}_{\mathrm{OPC}}=1.588\right)$ and imaginary $\left(\mathrm{k}_{\mathrm{OPC}}=0\right)$ parts of the complex RI used for OPC calibration are shown in (a) (magenta) and (b) (magenta), respectively. The real RI of water $\left(\mathrm{n}_{\text {water }}=1.33\right.$ ) is also shown $(\mathrm{a}, \mathrm{cyan})$. The imaginary RI of water $\left(k_{\text {water }}=0\right)$ is equal to the imaginary RI used for OPC calibration $\left(k_{\text {OPC }}=0\right)$.

\subsection{Size Distribution}

There is a substantial difference between the RI used for OPC calibration $\left(\mathrm{n}_{\mathrm{OPC}}=1.588 ; \mathrm{k}_{\mathrm{OPC}}=0\right)$ and the FL-dependent dry RI calculated from chemical composition data (Figure 6). To take into account this difference, we apply the RI-based correction to adjust the OPC-derived size distributions (see Appendix C for further discussion). It should be emphasized that the PSL-based OPC calibration applies a RI that very likely overestimates those for the typical sub-micron aerosol. Since a higher RI produces a larger scattering signal, a particle with smaller size and higher RI scatters the same as a particle with larger size and smaller RI. As a result, all of the adjustments of the OPC calibration to the actual RI will increase the size of measured particles, and consequently will increase the total scattering for almost any reasonable size distribution. Moreover, adding an absorbing component further increases the size of the measured particles-after adjustment-due to the reduction 
of the particle's scattering and consequent decrease of its optical size. We will illustrate such increases of particle size and scattering in the following two sections.

The size spectra adjustment involves modification of the OPC-measured dry diameter of particles:

$$
\mathrm{D}_{\text {dry,adj }}=\mathrm{f}\left(\mathrm{D}_{\text {dry }}\right) \mathrm{D}_{\text {dry }}
$$

where $f\left(D_{\text {dry }}\right)$ is the size-dependent scaling factor obtained from theoretical response calculations by extending well-established approaches for correcting the OPC-derived size distributions [30,31,34]. In our work we take advantage of available AMS and SP2 measurements (Section 3) for estimating the complex RI (Section 4.2), and thus our approach can be applied to both non-absorbing and absorbing aerosol.

The original OPC-derived and corrected dry size distributions are related as:

$$
\frac{\mathrm{dN}}{\mathrm{d} \log \mathrm{D}_{\mathrm{dry}, \mathrm{adj}}}=\frac{\mathrm{dN}}{\mathrm{d} \log \mathrm{D}_{\mathrm{dry}}} \frac{\mathrm{d} \log \mathrm{D}_{\mathrm{dry}}}{\mathrm{d} \log \mathrm{D}_{\mathrm{dry}, \mathrm{adj}}}
$$

In other words, the size-dependent correction (Equation (6)) modifies the original OPC-derived size spectra in two ways by (1) changing bin boundaries (horizontal shifting; replacement of $D_{\text {dry }}$ with $D_{\text {dry,adj }}$ ) and (2) scaling of the normalized number concentration (vertical shifting; term $\mathrm{d} \log \mathrm{D}_{\text {dry }} / \mathrm{d} \log \mathrm{D}_{\mathrm{dry}, \mathrm{adj}}$ ).

The increase of particle diameter due to the water uptake is expressed as:

$$
\begin{aligned}
D_{\text {wet }} & =\operatorname{HGF}_{\text {mix }}(R H) D_{\text {dry }} \\
D_{\text {wet,adj }} & =H_{G G} \text { mix }(R H) D_{\text {dry,adj }}
\end{aligned}
$$

where the size-independent $\mathrm{HGF}_{\text {mix }}(\mathrm{RH})$ is calculated from the chemical composition measurements (Section 4.1).

The corresponding dry and wet size distributions are related by the following equations:

$$
\begin{gathered}
\frac{\mathrm{dN}}{\mathrm{d} \log \mathrm{D}_{\text {wet }}}=\frac{\mathrm{dN}}{\mathrm{d} \log \mathrm{D}_{\mathrm{dry}}} \frac{\mathrm{d} \log \mathrm{D}_{\mathrm{dry}}}{\mathrm{d} \log \mathrm{D}_{\text {wet }}}=\frac{\mathrm{dN}}{\mathrm{dlog} \mathrm{D}_{\mathrm{dry}}} \\
\frac{\mathrm{dN}}{\mathrm{d} \log \mathrm{D}_{\text {wet,adj }}}=\frac{\mathrm{dN}}{\mathrm{d} \log \mathrm{D}_{\text {dry,adj }}} \frac{\mathrm{d} \log \mathrm{D}_{\mathrm{dry}, \text { adj }}}{\mathrm{d} \log \mathrm{D}_{\text {wet,adj }}}=\frac{\mathrm{dN}}{\mathrm{dlog} \mathrm{D}_{\text {dry,adj }}}
\end{gathered}
$$

Note that functions in Equation (9) (left-hand versus right-hand side) have different arguments; for example, $\mathrm{dN}$ ( $\left.\mathrm{D}_{\text {wet }}\right) / \mathrm{d}$ log $\mathrm{D}_{\text {wet }}$ (Equation (9a); left-hand side) and $\mathrm{dN}\left(\mathrm{D}_{\mathrm{dry}}\right) / \mathrm{d} \log \mathrm{D}_{\mathrm{dry}}$ (Equation (9a); right-hand side). In comparison with the size-dependent scaling factor (Equation (6)), the size-independent adjustment (Equation (8)) associated with water uptake modifies the original OPC-derived size spectra by changing bin boundaries only (horizontal shifting; e.g., replacement of 
$D_{\text {dry }}$ with $D_{\text {wet }}$; Equation (9a)); this adjustment does not cause the vertical scaling of the normalized number concentration (Equation (7) versus Equation (9)).

To illustrate the conversion of the measured dry size distribution into the corresponding wet size spectra (Equations (8a) and (9a)), we select two flight legs with high ( $\mathrm{RH} \sim 78 \%)$ and low (RH 5\%) values of relative humidity (Figure 5a). The RH-related increase of particle size results in the horizontal shifting of the size distribution (wet size spectra versus dry size spectra) and this shifting is seen for the humid conditions (Figure 7a versus c). Since the theoretical response curves are multivalued for a resonance region (particle diameter $>0.5 \mu \mathrm{m}$ ) (Appendix C), the adjusted size distributions obtained at ambient $\mathrm{RH}$ with the size-dependent scaling factor (Equations (8b) and (9b)) exhibit a "bumpy" behavior for this region as well (Figure $7 b, d)$.
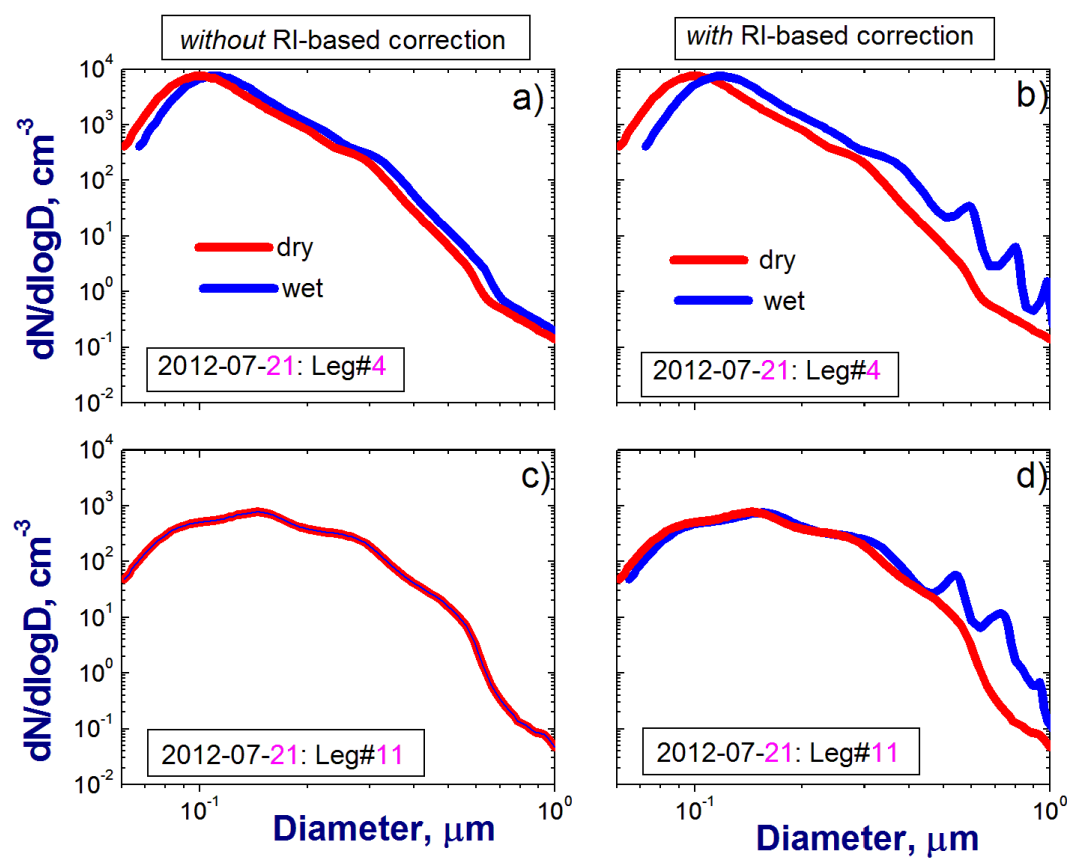

Figure 7. Example of size distributions obtained for two FLs (21 July 2012) with high $(\mathbf{a}, \mathbf{b})$ and low $(\mathbf{c}, \mathbf{d})$ values of ambient $\mathrm{RH}$, respectively. Measured dry size distributions (red) are converted into their wet counterparts (blue) without $(a, c)$ and with $(b, d)$ the size-dependent scaling factor.

\subsection{Scattering Coefficient Calculations}

We calculate the ambient total scattering coefficient using both the ambient complex RI with real $\left(\mathrm{n}_{\text {wet }}\right)$ and imaginary $\left(\mathrm{k}_{\mathrm{wet}}\right)$ parts (e.g., Figure $\left.6 \mathrm{a}, \mathrm{b}\right)$ and the 
ambient size distributions (e.g., Figure $7 \mathrm{~b}, \mathrm{~d}$ ). The calculations of total scattering coefficients $\sigma_{\text {mod,org }}$ and $\sigma_{\text {mod,adj }}$ are performed for ambient size distributions obtained without ( $\left.\mathrm{dN} / \mathrm{d} \log \mathrm{D}_{\text {wet }}\right)$ and with $\left(\mathrm{dN} / \mathrm{d} \log \mathrm{D}_{\text {wet,adj }}\right)$ the RI-based correction, respectively. Recall the RI is obtained at a single wavelength $(0.55 \mu \mathrm{m})$. Results from previous studies $[59,60]$ suggest that the spectral variability of the real part of RI is quite small within the visible spectral range considered here $(0.45-0.7 \mu \mathrm{m})$. Therefore, the obtained real RI $(0.55 \mu \mathrm{m})$ is likely representative for this spectral range and it can be applied to calculate the spectrally-resolved total scattering coefficient of weakly-absorbing aerosol. Note that the values of the imaginary part of RI at $0.55 \mu \mathrm{m}$ wavelength are quite small (Figure 6b) and thus they represent weakly-absorbing aerosol. For such aerosol, the total scattering is only slightly affected by changes of the imaginary part of RI $[59,60]$; therefore its spectral dependence can be ignored for the dataset considered here. We perform calculations of the total scattering at three wavelengths $(0.45,0.55,0.7 \mu \mathrm{m})$ using the same complex RI obtained at $0.55 \mu \mathrm{m}$ wavelength.

Our calculations are based on the Mie code developed by Barber and Hill [61] assuming that particles are homogeneous spheres and effective values of the complex $\mathrm{RI}$ are size-independent (particles are assumed to be a homogeneous internal mixture; Section 4.2). Note that other computational methods should be applied to calculate optical properties of particles with inhomogeneous internal mixing and aggregate morphology [62] although the influence of the internal mixing and particle geometry on the total scattering is quite small for submicron particles $[20,63,64]$. We calculate the total scattering coefficients using the original and corrected size distributions with different cut-offs (1- and 2- $\mu \mathrm{m}$ ). Large uncertainties (up to $50 \%$ ) of the measured total scattering associated with coarse mode particles (particle diameter $>1 \mu \mathrm{m}$ ) [54], the limited size range of chemical composition data (particle diameter $<1.0 \mu \mathrm{m}$ ) (Section 2), and small ( $<7 \%$ on average) relative contribution of coarse mode particles to the scattering coefficient (Appendix B) are the three main factors that led to the selected cut-offs. Note that there is a small difference ( $\sim 2 \%$ on average) between total scattering values calculated for the 1- and 2- $\mu \mathrm{m}$ cut-offs (Appendix B), mainly due to the small fraction of supermicron particles for FLs considered here. Below, we show the ambient scattering coefficients $\left(\sigma_{\text {mod,org }}\right.$ and $\left.\sigma_{\text {mod,adj }}\right)$ calculated with the $2-\mu \mathrm{m}$ cut-off only.

Uncertainties for the calculated scattering coefficient are associated mainly with ambiguities of the required inputs (ambient size distribution and complex RI) and model assumptions (homogeneous internal mixture). According to previous studies with similar model inputs [65], the uncertainties for the calculated dry total scattering are about $20 \%$. Although assumptions associated with the hygroscopic growth factor estimation (Section 4.1) are likely to introduce additional ambiguities associated with the required inputs, we assume that the uncertainties obtained earlier 
for the dry scattering (20\%) are also representative (at least as a lower limit) for the ambient total scattering considered in our study. The influence of the uncertainties mentioned above on agreement between the observed and calculated values at ambient conditions is considered in the next section.

\section{Results}

This Section outlines the comparison component of our framework for an airborne optical closure experiment (Figure 1; bottom panel) and includes time series and statistics of the observed and calculated total scattering coefficients at ambient conditions. This Section is designed to address questions 1 (level of agreement for "complete" dataset) and 2 (level of agreement for "incomplete" dataset given that the impact of chemical composition data on improved size spectra is ignored).

Let us start with the time series (Figure 8). The total modeled scattering $\sigma_{\text {mod,org }}$ calculated for the original size distribution substantially underestimates the observed scattering $\sigma_{\mathrm{obs}}$ for the majority of FLs considered on 21 July 2012. In contrast to $\sigma_{\text {mod,org }}$, the total scattering $\sigma_{\text {mod,adj }}$ calculated for the adjusted size distribution matches the observed scattering $\sigma_{\text {obs }}$ reasonably well.

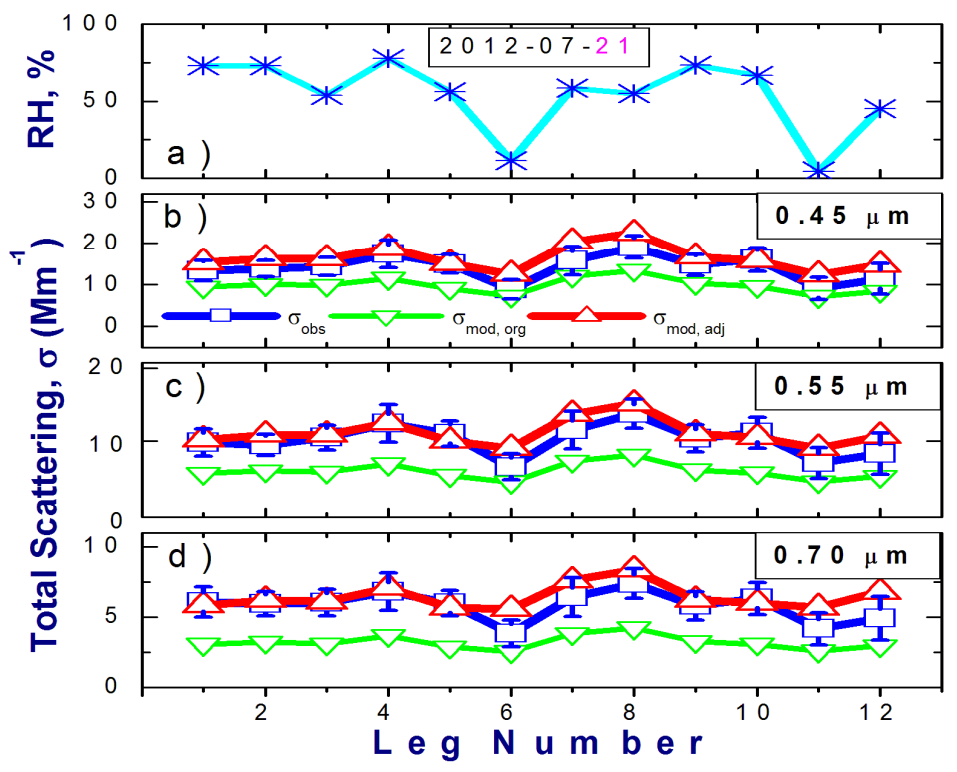

Figure 8. Ambient RH (a) and spectral values (b-d) of the total scattering coefficient measured (blue) and calculated for the original (green) and RI-based adjusted (red) size distributions for twelve FLs on 21 July 2012 at three wavelengths: (b) 0.45 ; (c) 0.55 and (d) $0.70 \mu \mathrm{m}$. Error bars represent uncertainties of measured scattering coefficient. 
The differences in Figure 8 between calculated $\left(\sigma_{\text {mod,org }}\right.$ and $\left.\sigma_{\text {mod,adj }}\right)$ and observed $\left(\sigma_{\text {obs }}\right)$ scattering illustrate three main points. First, the observed scattering $\sigma_{\text {obs }}$ tends to follow the ambient $\mathrm{RH}$ variations. For example, the lowest values of $\sigma_{\text {obs }}$ occurred for dry conditions (FL numbers 6 and 11, where $\mathrm{RH}<10 \%$ ). Second, inclusion of the hygroscopic growth of particles associated with water uptake alone (Section 4.3) is not sufficient for matching the observed ambient scattering ( $\sigma_{\text {mod,org }}$ versus $\left.\sigma_{\mathrm{obs}}\right)$. In addition to the hygroscopic growth, application of the RI-based correction to the dry size distributions measured by the OPCs (Section 4.3) is required $\left(\sigma_{\text {mod,adj }}\right.$ versus $\left.\sigma_{\text {obs }}\right)$. Finally, the calculated scattering $\sigma_{\text {mod,adj }}$ reproduces reasonably well the observed scattering $\sigma_{\text {obs }}$ at all three wavelengths considered here $(0.45,0.55$ and $0.70 \mu \mathrm{m})$, suggesting that our assumption of a spectrally-independent RI seems reasonable for the weakly-absorbing aerosol sampled during the TCAP flights.

The substantial underestimation of $\sigma_{\text {mod,org }}$ versus $\sigma_{\text {obs }}$ noticeable in Figure 8 is more obvious in the scatterplot of all considered TCAP FLs shown in Figure 9a. Since both the measured and calculated scattering can exhibit large uncertainties, the bivariate weighted method [66] is used to find the best linear fit. This method owes its popularity $[67,68]$ to its versatility, robustness and ability to use uncertainties of both $\mathrm{x}$ and $\mathrm{y}$ variables to find the slope and intercept of a best fit straight line to the data [69]; additionally, standard errors of the slope and intercept may be estimated.
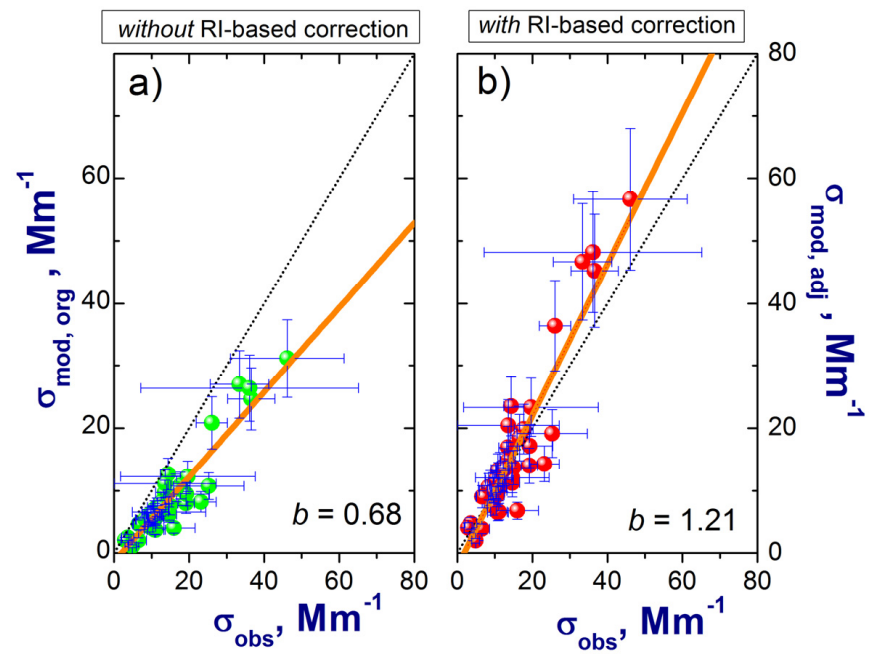

Figure 9. Comparison of the ambient total scattering observed $\left(\sigma_{\text {obs }}\right)$ with ambient total scattering calculated ( $\sigma_{\text {mod }}$ ) for the original (a) and adjusted (b) size distributions at $0.55 \mu \mathrm{m}$ wavelength for all TCAP FLs. Here $b$ is the slope of the linear regression fits to the data (straight orange lines). Error bars represent uncertainties of measured (Section 3) and calculated (Section 4.4) scattering coefficients. 
The slope of the corresponding linear regression fit is quite small (0.68; Figure 9a). The slope increases from 0.68 to 1.21 when the RI-based correction is applied to the OPC-derived size distributions (Figure 9a versus b). There are four points with large observed values of scattering coefficient $\left(\sigma_{\text {obs }}>30 \mathrm{Mm}^{-1}\right.$, Figure 9$)$. Close examination reveals that these points represent either large values of $\mathrm{RH}(>67 \%)$ or highly inhomogeneous FLs (standard deviation of $\sigma_{\mathrm{obs}} \sim 30 \mathrm{Mm}^{-1}$ ). Removal of these points reduces slightly the slope (from 0.68 to 0.65, Figure 9a; and from 1.21 to 1.17 , Figure $9 \mathrm{~b}$ ) and increases the intercept (from -1.30 to -1.11 , Figure $9 \mathrm{a}$; and from -2.25 to -1.91 , Figure $9 b$ ). Additionally, such removal reduces the difference between the mean values of $\sigma_{\text {obs }}$ and $\sigma_{\text {mod,adj }}$ considerably (roughly from $10 \%$ to $0.5 \%$ ), while having little effect on the discrepancy between the mean values of $\sigma_{\mathrm{obs}}$ and $\sigma_{\text {mod }}$ (about $40 \%$ ).

Similar changes of parameters (slope and intercept) obtained with the bivariate weighted method can be achieved by increasing the uncertainties of the calculated scattering coefficient but keeping uncertainties of the observed scattering the same (Figure 10). These changes are mostly caused by the influence of four points with large values of the observed/calculated scattering coefficient on the parameters of fitting line. The influence of these points decreases when assumed uncertainties of the calculated scattering are increased. Note that the assumed uncertainties $(20 \%)$ of the calculated scattering represent dry conditions (Section 4) and thus, they likely are underestimated for FLs with moderate and high RHs (e.g., due to inaccurate treatment of the $\mathrm{RH}$ dependence of the $\mathrm{HGF}_{\text {mix }}$ and its components).

Comparable slopes for linear regression fits $\left(\sigma_{\text {mod,adj }}\right.$ versus $\left.\sigma_{\text {obs }}\right)$ are obtained at the other two wavelengths $(0.45$ and $0.7 \mu \mathrm{m})$ (Table 2$)$. The weak spectral dependence of these slopes suggests that the complex RI obtained at $0.55 \mu \mathrm{m}$ wavelength could be applied to estimate the spectrally-resolved total scattering within the mid-visible range $(0.45-0.70 \mu \mathrm{m})$ reasonably well.

On average, the total scattering $\sigma_{\text {mod,org }}$ calculated for the original OPC-derived size distribution underestimates the observed scattering $\sigma_{\mathrm{obs}}$ substantially (Table 2 ). For example, underestimation of the mean value exceeds $40 \%$ at $0.55 \mu \mathrm{m}$ wavelength. However, the total scattering $\sigma_{\text {mod,adj }}$ calculated for the RI-adjusted size distributions and the observed scattering $\sigma_{\text {obs }}$ have comparable mean values (Table 2 ): the relative difference between them is quite small at three given wavelengths (about 13\% at $0.45 \mu \mathrm{m}, 7 \%$ at $0.55 \mu \mathrm{m}$ and $12 \%$ at $0.70 \mu \mathrm{m})$. In comparison with the mean values, the corresponding standard deviations are in moderate agreement $(\sim 40 \%)$. In addition to the basic statistics of mean and standard deviation, we calculate the corresponding Root Mean Squared Error (RMSE), which is defined as the root mean squared difference between the observed $\sigma_{\text {obs }}$ and the calculated scattering coefficients in question. The corresponding RMSEs are about 7 and $5 \mathrm{Mm}^{-1}$ for 
$\sigma_{\text {mod,org }}$ and $\sigma_{\text {mod,adj }}$ at $0.55 \mu \mathrm{m}$ wavelength, respectively. In other words, RMSE $\left(\sigma_{\text {mod,org }}\right)$ overestimates RMSE $\left(\sigma_{\text {mod,adj }}\right)$ noticeably $(\sim 30 \%)$.
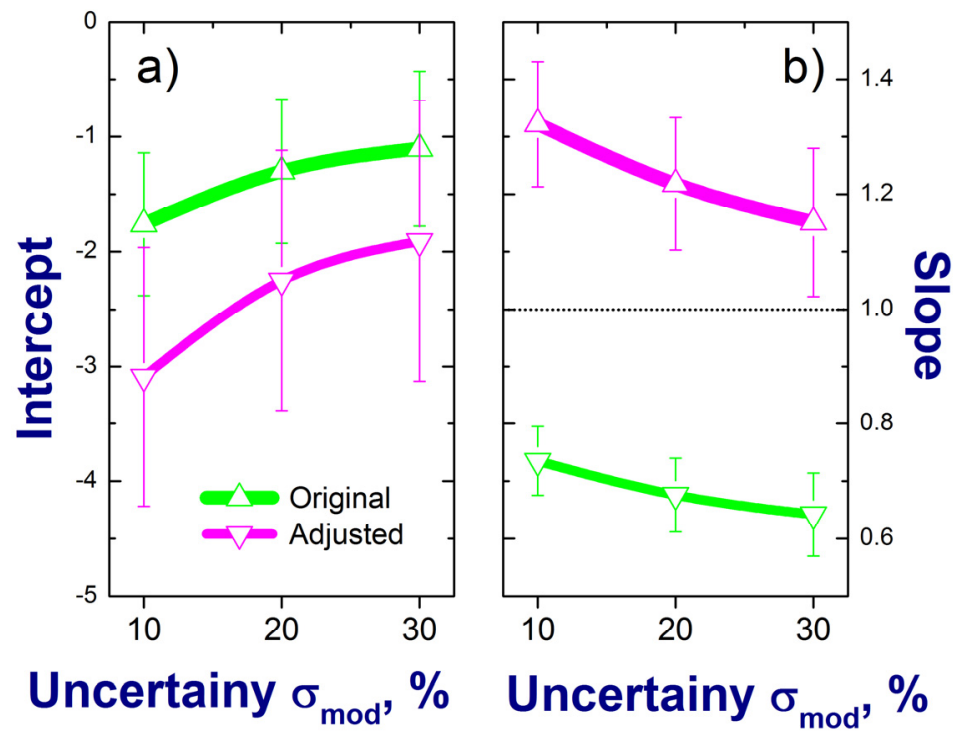

Figure 10. Intercept (a) and slope (b) of the linear regression fits (e.g., Figure 9) as a function of uncertainty for the ambient total scattering calculated for the original (without RI-based correction) and adjusted (with RI-based correction) size distributions. Error bars represent the standard errors of the displayed parameters (see text for details).

Overall, the quantitative comparisons (Figure 9 and Table 2) demonstrate clearly that application of the RI-based correction improves the agreement between observed and calculated scattering coefficients, most notably in terms of the mean values and RMSE. Thus, our results are in line with findings from previous studies [30,31], which highlighted the importance of such RI-based corrections and suggested corresponding parameterizations for non-absorbing aerosol. These valuable parameterizations were designed assuming that the RI-based correction was a function of the real part of complex RI only. In our approach, both the real and imaginary parts of the complex RI are used as input and thus it can be applied for both non-absorbing and absorbing aerosol. It should be noted that the single-scattering albedo is moderate $(0.93 \pm 0.03)$ for the TCAP dataset considered here, therefore this dataset represents slightly-absorbing aerosol. Further studies are needed to examine the feasibility of our approach for improved calculations of total scattering/absorption for strongly-absorbing aerosol. 
Table 2. Mean and Standard Deviation (StDv) of the observed ( $\left.\sigma_{\mathrm{obs}}\right)$ and calculated $\left(\sigma_{\text {mod,org }}\right.$ and $\left.\sigma_{\text {mod,adj }}\right)$ scattering coefficients obtained for all TCAP FLs at three wavelengths: $0.45 \mu \mathrm{m}$ (top part), $0.55 \mu \mathrm{m}$ (middle part), and $0.70 \mu \mathrm{m}$ (bottom part). The corresponding intercept $(a)$, slope $(b)$, their standard errors (in parenthesis) and Root Mean Squared Error (RMSE) also are included. The RMSE is defined as the root mean squared difference between the observed and the calculated scattering coefficients.

\begin{tabular}{|c|c|c|c|c|c|}
\hline & Mean & StDv & RMSE & $a$ & $b$ \\
\hline \multicolumn{6}{|c|}{$0.45 \mu \mathrm{m}$} \\
\hline$\sigma_{\text {obs }}$ & 20.05 & 12.26 & - & - & - \\
\hline$\sigma_{\text {mod,org }}$ & 13.70 & 10.42 & 7.49 & $\begin{array}{l}-1.58 \\
(0.80)\end{array}$ & $\begin{array}{c}\mathbf{0 . 7 5} \\
(0.06)\end{array}$ \\
\hline$\sigma_{\text {mod,adj }}$ & 22.75 & 17.09 & 7.10 & $\begin{array}{l}-2.54 \\
(1.33)\end{array}$ & $\begin{array}{c}1.24 \\
(0.10)\end{array}$ \\
\hline \multicolumn{6}{|c|}{$0.55 \mu \mathrm{m}$} \\
\hline$\sigma_{\text {obs }}$ & 14.85 & 8.98 & - & - & - \\
\hline$\sigma_{\text {mod,org }}$ & 8.80 & 6.78 & 6.99 & $\begin{array}{l}-1.30 \\
(0.63)\end{array}$ & $\begin{array}{c}\mathbf{0 . 6 8} \\
(0.06)\end{array}$ \\
\hline$\sigma_{\text {mod,adj }}$ & 15.89 & 12.17 & 5.01 & $\begin{array}{l}-2.25 \\
(1.13)\end{array}$ & $\begin{array}{c}1.21 \\
(0.11)\end{array}$ \\
\hline \multicolumn{6}{|c|}{$0.70 \mu \mathrm{m}$} \\
\hline$\sigma_{\mathrm{obs}}$ & 8.73 & 5.28 & - & - & - \\
\hline$\sigma_{\text {mod,org }}$ & 4.87 & 3.78 & 4.61 & $\begin{array}{l}-\mathbf{0 . 7 3} \\
(0.45)\end{array}$ & $\begin{array}{c}\mathbf{0 . 6 4} \\
(0.07)\end{array}$ \\
\hline$\sigma_{\text {mod,adj }}$ & 9.77 & 7.57 & 3.85 & $\begin{array}{l}-1.40 \\
(0.90)\end{array}$ & $\begin{array}{c}1.26 \\
(0.15)\end{array}$ \\
\hline
\end{tabular}

\section{Sensitivity Study}

This section is designed to supplement the previous one and to address question 3 (level of agreement for "incomplete" dataset given that a non-representative RI is used to correct size spectra). Note that we are able to use aerosol chemical composition data from the TCAP data set to obtain RI-based corrections for each FL. However, as discussed in Section 1, a more common scenario is one where chemical composition information is available only on a limited basis, or not at all. We wish to investigate the sensitivity of the agreement between calculated and observed scattering to the assumed RI value, particularly if the assumed RI is non-representative of the ambient aerosol. Further motivation for this sensitivity test is the reported lack of agreement (outside the $30 \%$ measurement uncertainty) between measured and calculated total scattering coefficients obtained recently for the VOCALS-Rex marine atmosphere campaign [46] where a universal refractive index $\left(\mathrm{n}^{*}=1.41\right.$ and $\left.\mathrm{k}^{*}=0\right)$ derived 
from the entire VOCALS-Rex dataset [29] was used in adjusting observed PCASP size distributions.

Given the strong sensitivity of VOCALS-Rex calculated scattering to size spectra uncertainties [46], it can be hypothesized that the lack of agreement can be associated (at least partially) with the RI specification and its strong impact on the adjusted size distributions. To confirm this hypothesis, we calculate the total scattering from the TCAP data (Section 2) using the procedure previously employed [46] for the VOCALS-Rex dataset. We emphasize that the main difference between their sensitivity-driven procedure and our framework approach (Figure 1) is specification of the RI required for adjusting the OPC-derived size spectra: an assumed universal real RI estimated implicitly from all available aerosol composition measurements (sensitivity-driven procedure) versus a variable complex RI estimated explicitly from the complementary chemical composition data (our framework approach, Sections 3-5).

We apply an assumed universal $\mathrm{RI}\left(\mathrm{n}^{*}=1.41\right.$ and $\left.\mathrm{k}^{*}=0\right)$, equivalent to that used in VOCALS-Rex analyses, to the entire TCAP dataset and compare the results with those from the variable RI estimated from the TCAP AMS and SP2 data. This assumed RI might reasonably be adopted if TCAP had an "incomplete" data set and we searched the literature for a reasonable universal RI applicable to coastal/marine aerosols sampled aloft. Note that there is a noticeable difference between the assumed RI and the estimated RI (Section 3) for many FLs. Compared with the variable RI, the use of the universal $\mathrm{RI}$ in the size spectra adjustment increases the relative contribution of particles with moderate diameter (within 0.4-0.8 $\mu \mathrm{m}$ range) (Figure 11), which scatter light in the visible spectral range most effectively. This relative increase in optically important particles, in turn, is responsible for a substantial rise of the calculated scattering coefficient (Figures 12 and 13a). As a result, the corresponding mean value and RMSE (calculations based on variable RI versus the universal RI) are enhanced by about $25 \%$ and $80 \%$, respectively (Tables 2 and 3). The mean value of the calculated scattering coefficient (based on universal RI) overestimates the mean value of observed scattering by about $35 \%$ (Table 3 ). This substantial overestimation $(\sim 35 \%)$ of the calculated scattering coefficient: (1) confirms the hypothesis made above regarding the potential strong impact of the RI specification on the calculated scattering and (2) suggests that use of a universal RI specification could be one possible explanation for the lack of agreement noted in the VOCALS-Rex closure study [46]. 


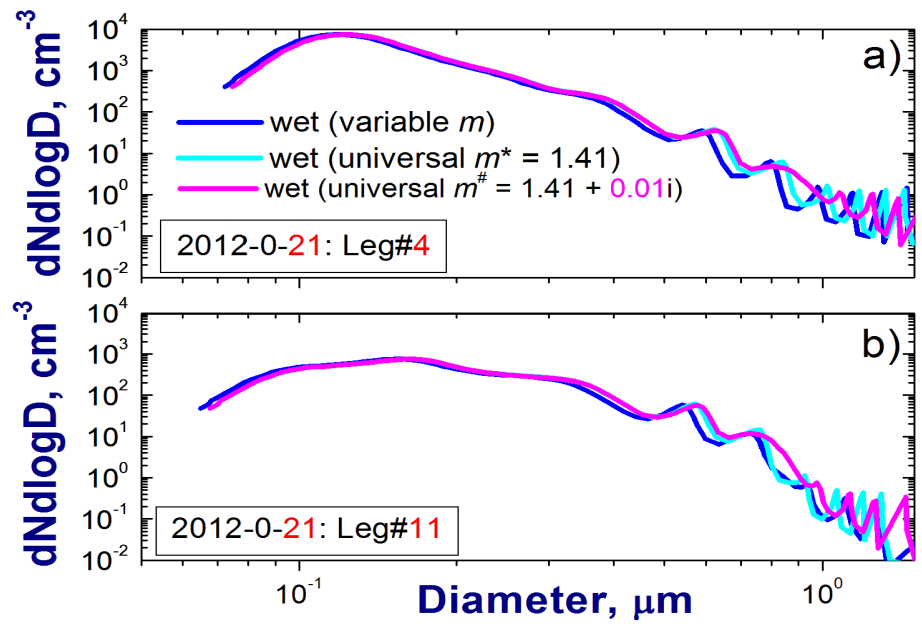

Figure 11. Example of size distributions obtained for two FLs (21 July 2012) and different values of RI. Measured dry size distributions are converted into their wet counterparts with the size-dependent scaling factor calculated for universal (cyan, magenta) and variable (blue) RI. Two values of universal RI are assumed: $\mathrm{m}^{*}=1.41$ (cyan) for a non-absorbing aerosol and $\mathrm{m}^{\#}=1.41+0.01 \mathrm{i}$ (magenta) for an absorbing aerosol.

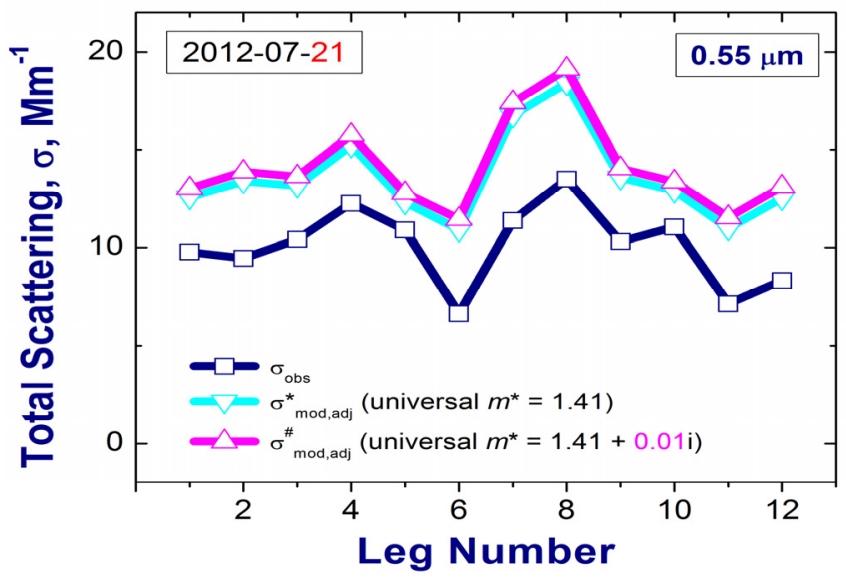

Figure 12. Total scattering coefficient at $0.55 \mu \mathrm{m}$ wavelength measured (navy blue) and calculated (cyan, magenta) for size distributions measured on 21 July, 2012 and corrected using two assumed universal RIs: $\mathrm{m}^{*}=1.41$ (cyan) and $\mathrm{m}^{\#}=1.41+0.01 \mathrm{i}$ (magenta). 

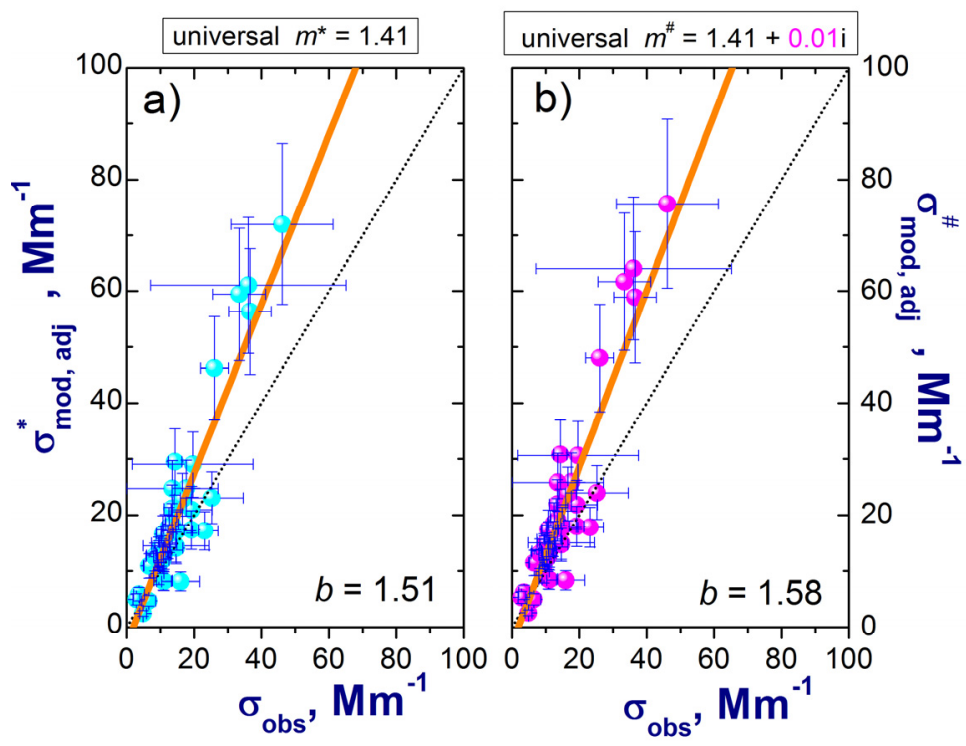

Figure 13. Comparison of the ambient total scattering observed $\left(\sigma_{\mathrm{obs}}\right)$ with ambient total scattering calculated $\left(\sigma_{\bmod }\right)$ for the size distributions adjusted with the universal RI. There are two values of assumed universal RI: $\mathrm{m}^{*}=1.41$ (a) and $\mathrm{m}^{\#}=1.41+0.01 \mathrm{i}(\mathbf{b})$. The observed and calculated values of total scattering are obtained at $0.55 \mu \mathrm{m}$ wavelength for all TCAP FLs. Here, $b$ is the slope of the linear regression fits to the data (straight orange lines). Error bars represent uncertainties of measured (Section 3) and calculated (Section 4.4) scattering coefficients.

To examine the importance of the imaginary part of the complex RI in changing the adjusted size distribution and thereby in modifying the calculated scattering, we focus on the complex RI for an absorbing aerosol $\left(\mathrm{m}^{\#}\right)$. We assume that the corresponding universal complex RI has the real $\left(\mathrm{n}^{\#}=1.41\right)$ and imaginary $\left(\mathrm{k}^{\#}=0.01\right)$ parts. This selected value of the imaginary part $\left(\mathrm{k}^{\#}=0.01\right)$ represents roughly the most frequent value observed under dry conditions during the TCAP campaign (Figure 6). Therefore, the imaginary part is the only difference between the complex RIs assumed for non-absorbing $\left(\mathrm{m}^{*}=1.41\right)$ and absorbing $\left(\mathrm{m}^{\#}=1.41+0.01 \mathrm{i}\right)$ aerosol.

Replacement of the imaginary part of the complex RI $\left(k^{*}=0\right.$ versus $\left.k^{\#}=0.01\right)$ increases the difference between the corresponding size distributions (Figure 11; cyan curve versus magenta curve). Note that this replacement $\left(\mathrm{k}^{*}=0\right.$ versus $\mathrm{k}^{\#}=0.01$ ) makes the adjusted size distributions "look smoother" within the resonance region (Figure 11; cyan curve versus magenta curve). The difference between the adjusted size distributions (Figure 11) is responsible for the difference between the corresponding total scattering coefficients (Figure 12, Figure 13 and Table 3). In particular, the replacement of the imaginary part of the complex RI $\left(\mathrm{k}^{*}=0\right.$ 
versus $\mathrm{k}^{\#}=0.01$ ) increases the RMSE by about $10 \%$ (Table 3 ). Therefore, selection of an inappropriate imaginary part of the assumed complex RI can also increase the discrepancy between the calculated total scattering from the observed total scattering.

Table 3. The same as Table 2 (middle part) except for the scattering coefficients $\sigma_{\text {mod,adj }}^{*}$ (middle row) and $\sigma_{\text {mod,adj }}^{\#}$ (bottom row) calculated for size distributions adjusted with two universal RIs $\mathrm{m}^{*}=1.41$ and $\mathrm{m}^{\#}=1.41+0.01 \mathrm{i}$, respectively.

\begin{tabular}{cccccc}
\hline & Mean & StDv & RMSE & $\boldsymbol{a}$ & $\boldsymbol{b}$ \\
\hline$\sigma_{\text {obs }}$ & 14.85 & 8.98 & - & - & - \\
$\sigma_{\text {mod,adj }}^{*}$ & 19.82 & 15.46 & 9.17 & -2.85 & 1.51 \\
$\sigma_{\text {mod,adj }}^{\#}$ & 20.67 & 16.19 & 10.20 & -2.96 & $(0.14)$ \\
\hline
\end{tabular}

The results presented in this Section illustrate that selecting inappropriate values of the real part of RI when adjusting OPC-derived size distributions in an optical closure study can cause substantial overestimation of the calculated scattering coefficient. Moreover, selecting inappropriate values of the imaginary part of RI can increase this overestimation noticeably, even for weakly absorbing aerosol. Therefore, one should not expect closure studies based on such "incomplete" data sets to be as exact as closure studies using "complete" data sets because of all the assumptions that must be made and the high probability that the RI estimates based on these assumptions potentially will be inappropriate. The error in RI estimates likely will be larger for aircraft sampling regimes because of the strong temporal and spatial variability of aerosol sampled by research aircraft. When faced with an "incomplete" data set, another possible approach is to use conventional iterative or optimization schemes, which apply a set of assumed representative RI values for minimizing differences between the measured and calculated aerosol properties of interest $[34,40,70]$. Such iterative or optimization schemes may possibly improve the RI estimation relative to the approach of assuming a universal RI.

\section{Summary}

We extend methods for calculating total aerosol scattering at ambient RH, originally developed for ground-based measurements $[17,21,41]$ to the challenging situation of airborne measurements. The importance of such extension is now widely recognized [6,45]. Our extended framework is suitable for conducting optical closure studies using "complete" aircraft data sets, where "complete" means that collocated and concurrent information on particle chemical composition is available. Our approach takes advantage of the existing information on aerosol chemical constituents and explicitly uses it to obtain improved ambient size spectra 
derived from complementary Optical Particle Counter (OPC) data, and therefore to obtain improved estimates of the total scattering under ambient conditions with low-to-moderate values of relative humidity $(\mathrm{RH}<80 \%)$.

To illustrate the performance of our approach, we use "complete" aerosol data collected by the DOE G-1 aircraft during the recent Two-Column Aerosol Project (TCAP; http:/ / campaign.arm.gov/tcap/) over the North Atlantic Ocean and US coastal region (Cape Cod, Massachusetts). The integrated data set collected by the G-1 aircraft includes: (1) size distributions measured by three OPCs: an Ultra-High Sensitivity Aerosol Spectrometer (UHSAS; 0.06-1 $\mu \mathrm{m}$ ), a Passive Cavity Aerosol Spectrometer (PCASP; 0.1-3 $\mu \mathrm{m}$ ) and a Cloud and Aerosol Spectrometer (CAS; 0.6-> $10 \mu \mathrm{m}$ ), (2) chemical composition data measured by an Aerosol Mass Spectrometer (AMS; 0.06-0.6 $\mu \mathrm{m}$ ) and a Single Particle Soot Photometer (SP2; 0.06-0.6 $\mu \mathrm{m}$ ) and (3) the dry total scattering coefficient measured by TSI integrating nephelometer at three wavelengths $(0.45,0.55,0.7 \mu \mathrm{m})$ and $\mathrm{f}(\mathrm{RH})$ measured with a humidification system at three RHs (near 45\%,65\% and 90\%) at a single wavelength $(0.525 \mu \mathrm{m})$. To illustrate the importance of the chemical composition data in the scattering closure study, we also utilize "incomplete" aerosol data, where "incomplete" means that information on particle chemical composition is not used to obtain the corrected ambient size spectra. The main conclusions are organized along the three main questions we posed at the start of our study:

- Analysis based on using the "complete" data set addresses our first question, namely: What level of agreement between the in-flight measured and calculated values of total scattering coefficient can be achieved at ambient $R H$ ? We demonstrate that despite the well-known limitations of airborne measurements and the assumptions required by our approach, we can obtain good agreement between the observed and calculated scattering at three wavelengths (about 13\% at $0.45 \mu \mathrm{m}, 7 \%$ at $0.55 \mu \mathrm{m}$, and $12 \%$ at $0.7 \mu \mathrm{m}$ on average) using the RI-based correction for OPC-derived size spectra and the best available chemical composition data for the RI estimation. We calculate the total scattering coefficient from the combined size spectra (UHSAS, PCASP and CAS data) and aerosol composition (AMS and SP2 data) at ambient conditions with a wide range of relative humidity values (from 5\% to $80 \%$ ). These calculations involve several assumptions, such as the homogeneous internal mixture assumption for estimating the hygroscopic growth factor and complex refractive index (RI) at ambient conditions, and simplified specification of particle geometry (homogeneous spheres) for Mie calculations.

- Analysis based on using an "incomplete" dataset addresses our second question, namely: What is the effect of ignoring the influence of chemical composition data on this agreement? We illustrate that ignoring the RI-based correction in the TCAP data can cause a substantial underestimation (about $40 \%$ on average) of the ambient 
calculated scattering when noticeable discrepancies between the actual RIs and those used for the OPC calibration have occurred. Our findings are in harmony with previous studies, which have highlighted the importance of the RI-based correction and have suggested its parameterization for non-absorbing aerosol assuming that the RI-based correction is a function of real RI only [30,31]. In comparison with these important parameterizations, our approach is more flexible in terms of available inputs (complex RI is estimated explicitly from the complementary chemical composition data), and therefore in terms of the expected applications (both non-absorbing and absorbing aerosol sampled by ground-based and airborne instruments).

- Analysis based on using an "incomplete" dataset also addresses our third question, namely: How sensitive is this agreement to the assumed RI value, particularly if the assumed RI is non-representative of the ambient aerosol? We illustrate in a sensitivity study that using a non-representative universal RI instead of the actual RI can result in a large overestimation (about 35\% on average) of the calculated total scattering at ambient $\mathrm{RH}$, and this overestimation is sensitive to specification of the imaginary part of the complex RI, even for weakly-absorbing aerosol. This sensitivity study suggests that the usefulness of assumptions required for universal RI estimation could be marginal, particularly when applied to the strong temporal and spatial variability of aerosol sampled by research aircraft. As a result, calculations of aerosol optical properties based on these assumptions should be used with caution and other possible approaches should be considered to improve the RI estimation. These possibilities include application of conventional iterative or optimization schemes where a set of assumed representative RI values is used to minimize differences between the measured and calculated aerosol properties of interest $[34,40,70]$.

To our knowledge, this work represents the first optical closure study that uses explicitly airborne chemical composition measurements of both non-absorbing and absorbing aerosol components in improving the OPC-derived size spectra. These measurements are employed to extend the capability of well-established methods originally developed for use with comprehensive ground-based measurements. Given the extended flexibility of these methods and the increasing availability of aerosol composition data collected from aircraft platforms, we expect that our approach can be successfully applied for improved understanding of a wide range of sophisticated processes and phenomena related to aerosols, including the time evolution of aerosol properties and dynamical aerosol-cloud interactions [71,72]. We further expect that closer agreement between measured and calculated aerosol properties, indicating the consistency of the observational data set, will improve confidence in, and use of, such observational data sets in global and regional climate 
model evaluations. For example, appropriate adjustments to optical particle counter data are needed to better understand and evaluate predictions of cloud-aerosol interactions, since cloud condensation nuclei (CCN) calculations are dependent on the aerosol size distribution.

Acknowledgments: The ARM Aerial Facility team is gratefully acknowledged for collecting the aircraft data during TCAP which was supported by the Department of Energy (DOE) Office of Science Atmospheric Radiation Measurement (ARM) and Atmospheric System Research (ASR) Programs. The ARM Aerial Facility is an integral part of the DOE ARM Program. This research was supported by the ARM and ASR Programs. The Pacific Northwest National Laboratory is operated by Battelle Memorial Institute under contract DE-AC06-76RLO 1830. We appreciate valuable discussions with Elaine Chapman (PNNL) and thoughtful comments from two anonymous reviewers that helped improve our paper.

Author Contributions: Abstract (E.K., L.B.), Introduction (E.K., L.B.), Approach (E.K., L.B., M.P., C.F., J.B.), Data (M.P., D.C., J.T., J.S., A.S., B.S.), Model and adjustments (E.K., L.B., M.P., M.O., J.S.), Results (all), Sensitivity study (E.K., L.B., M.P., M.O.), Summary (all). E.K. led the research and manuscript preparation. All authors contributed to data analysis. J.F. supervised research and manuscript writing.

Conflicts of Interest: The authors declare no conflict of interest.

\section{Appendix A. Merging of Size Distributions}

This appendix explains how data from overlapping measurements from three instruments (UHSAS, PCASP, and CAS) are merged into the combined size distributions (Figure 2). The best estimate aerosol size distribution $(\vec{N})$ can be recovered from the merged raw counts $(\vec{C})$ if a Kernel function $(\mathbf{R})$ can first be quantified:

$$
\overrightarrow{\mathrm{N}} \mathbf{R}=\overrightarrow{\mathrm{C}}
$$

or

$$
\left[\begin{array}{c}
\mathrm{n}_{1} \\
\mathrm{n}_{2} \\
\vdots \\
\mathrm{n}_{\mathrm{j}-1} \\
\mathrm{n}_{\mathrm{j}}
\end{array}\right]\left[\begin{array}{ccccc}
\mathrm{R}_{\text {UHSAS11 }} & \mathrm{R}_{\text {UHSAS12 }} & & & \\
\mathrm{R}_{\text {UHSAS21 }} & \mathrm{R}_{\text {UHSAS22 }} & & & \\
& & \ddots & & \\
& & & \mathrm{R}_{\mathrm{CASx}-1 \mathrm{j}-1} & \mathrm{R}_{\mathrm{CASx}-1 \mathrm{j}} \\
& & & \mathrm{R}_{\mathrm{CASx} \mathrm{j}-1} & \mathrm{R}_{\mathrm{CASx} \mathrm{j}}
\end{array}\right]=\left[\begin{array}{c}
\mathrm{C}_{\mathrm{UHSAS}_{1}} \\
\mathrm{C}_{\mathrm{UHSAS}_{2}} \\
\vdots \\
\mathrm{C}_{\mathrm{CAS}_{\mathrm{x}-1}} \\
\mathrm{C}_{\mathrm{CAS}_{\mathrm{x}}}
\end{array}\right]
$$

The raw counts are measured by the UHSAS, PCASP, and CAS. Furthermore, $\mathbf{R}$ can be defined as:

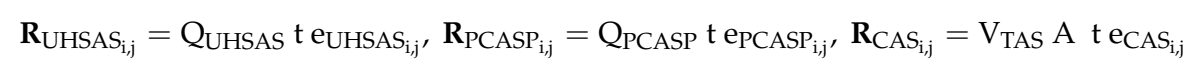

where $\mathrm{Q}, \mathrm{V}_{\mathrm{TAS}}, \mathrm{e}_{\mathrm{i}, \mathrm{j}}$, A and $\mathrm{t}$ are the flow, airspeed, collection efficiency for each probe, CAS laser sample area and the data collection integration time, respectively. From calibrations we know the collection efficiency $\left(e_{i, j}\right)$ of each probe within a specified 
bin size, and the CAS laser sample area (A). The other variables $\mathrm{Q}$ (flow) and $\mathrm{V}_{\mathrm{TAS}}$ (airspeed) are measured by the probes themselves during operation.

To start the recovery process, a rough estimate of $\vec{N}$ has to be provided. The size distribution values $\mathrm{dN} / \mathrm{d} \log \mathrm{D}_{\mathrm{p}}$ for UHSAS, CAS, PCASP are first interpolated to the same bin space. The concentration values from the UHSAS, CAS, and or PCASP that overlap in the same bin are averaged together then converted back to $\mathrm{dN}$ by multiplying by the $d \log D_{p}$ value for that bin. Using the estimation for $\vec{N}$, Twomey smoothing is started. The initial number distribution is smoothed until the roughness of the solution has decreased to a set value, normally around 0.96 . Roughness of the solution is measured by the average value of the second derivative. This initial trial solution is then ingested into a loop which continues until either the roughness of the solution has decreased below a set limit (typically 0.98), the goodness of the fit decreases, or the maximum number of iterations has been reached. After the loop successfully exits the resulting number distribution is considered the best estimate aerosol size distribution.

\section{Appendix B. Contributions from Particles of Different Sizes to Scattering}

To quantify contributions from particles of different sizes to the total scattering, we calculate the ambient scattering coefficient $\sigma_{\text {mod,adj }}=$ $\int_{D_{\min }}^{D^{*}} C_{\text {sca }}(\mathrm{m}, \mathrm{D}) \mathrm{dN} / \mathrm{d} \log \mathrm{D}$ dlog $\mathrm{D}$ at single wavelength $(0.55 \mu \mathrm{m})$, where $\mathrm{C}_{\mathrm{sca}}$ is the scattering cross section, which is a function of particle diameter $\mathrm{D}$ and size-independent complex refractive index (m; Equation (5)), $\mathrm{dN} / \mathrm{d} \log \mathrm{D}$ is the ambient and corrected number size distribution (Equation (9b)), $\mathrm{D}_{\min } \sim 0.07 \mu \mathrm{m}$ and $\mathrm{D}^{*}$ is an assumed cut-off (between 0.1 to $5 \mu \mathrm{m}$ ). Then we calculate normalized scattering coefficient $\rho\left(D^{*}\right)=\sigma_{\text {mod,adj }}\left(D^{*}\right) / \sigma_{\text {mod,adj }}\left(D^{*}=5 \mu \mathrm{m}\right)$, which represents the relative contribution of particles with $\mathrm{D}<\mathrm{D}^{*}$ to the total scattering from all particles smaller than $5 \mu \mathrm{m}$. Figure B1a shows the mean and standard deviation for $\rho\left(D^{*}\right)$ computed for all 45 TCAP flight legs considered in this study (Section 3). Sub-micron particles clearly dominate the total scattering in this case with $\rho\left(D^{*}\right)$ reaching about 0.93 and 0.96 for $D^{*}=1 \mu \mathrm{m}$ and $D^{*}=2 \mu \mathrm{m}$, respectively (Figure B1a). The obtained small ( $7 \%$, on average) contribution by the super-micron particles to the ambient scattering coefficient confirms the applicability of the simplified approach (Appendix C) to the TCAP airborne data. For events where super-micron particles dominate, for example, aerosol plumes resulting from dust storms [73] or volcanic eruptions [34] application of the strict approach (Appendix C) would be more relevant. 

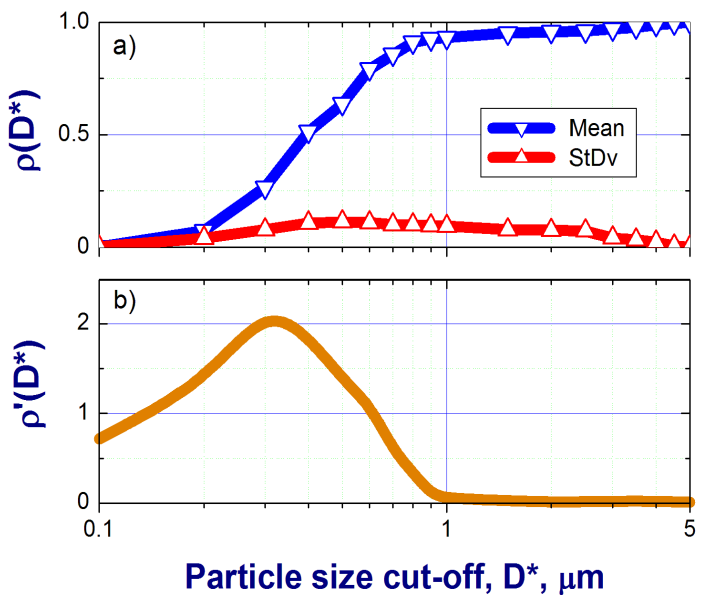

Figure B1. The normalized scattering coefficient (a) and its derivative (b) as a function of particle size cut-off. The blue line shows the mean for all TCAP flight legs and the red line represents the corresponding standard deviations.

To further illustrate the relative contribution to $\sigma_{\text {mod,adj }}$ from different particle size ranges (for a given complex refractive index), we note that the above relation for the ambient scattering coefficient can be rearranged as $\sigma_{\text {mod,adj }}=\int_{D_{\min }}^{D^{*}}\left(\mathrm{~d} \sigma_{\text {mod,adj }}(\mathrm{D}) / \mathrm{dD}\right) \mathrm{dD}$. Using this rearranged relation, we calculate the derivative of the normalized scattering coefficient as $\rho^{\prime}\left(\mathrm{D}^{*}\right)=$ $\sigma_{\text {mod,adj }}^{\prime}\left(D^{*}\right) / \sigma_{\text {mod,adj }}\left(D^{*}=5 \mu \mathrm{m}\right)$, where $\sigma_{\text {mod,adj }}^{\prime}(\mathrm{D})=\mathrm{d} \sigma_{\text {mod,adj }}(\mathrm{D}) / \mathrm{dD}$. Figure $\mathrm{B} 1 \mathrm{~b}$ shows that on average particles in the size range between 0.3 and $0.4 \mu \mathrm{m}$ contribute most strongly to the ambient scattering coefficient, while the corresponding contribution of particles in the "bumpy" region (particle sizes larger than $0.5 \mu \mathrm{m}$ ) is smaller.

\section{Appendix C. Correction of OPC-derived Size Spectra}

This appendix includes details related to the calculations of the size-dependent theoretical response curves. The latter are required to obtain an adjusted dry diameter of particles (Equation (6)) and the corresponding dry (Equation (7)) and ambient (Equation (9b)) size distributions using the OPC-derived size spectra. Recall that during the OPC measurements individual particles are illuminated by a laser beam and then the light scattered by the particles is collected over a large solid angle [32-34,74]. The wavelength of the illuminating light and solid angle limits are known properties of a given detector [12]. For example, the UHSAS and PCASP collect side-scattered light ( 35-135 degrees) at $0.6328 \mu \mathrm{m}$ and $1.054 \mu \mathrm{m}$ wavelength, respectively; while the CAS collects forward-scattered ( 4-13 degrees) 
and back-scattered ( 5-14 degrees) light at $0.685 \mu \mathrm{m}$ wavelength. Therefore, strictly speaking, the scaling factors should be calculated for each instrument independently using the specified properties, and then the individual corrected size spectra (UHSAS, PCASP and CAS) should be used to obtain the corresponding merged size distributions. We shall call this the "strict approach." Another potential approach could include the development of empirical relationships between the scattering measured over the limited range of angles and the total scattering for given compositions and ambient conditions. Such an "empirical approach" would not involve Mie calculations and therefore would relax assumptions and data requirements for the RI-based corrections.

For our study, the individual uncorrected size spectra (UHSAS, PCASP and CAS) are combined to obtain the corresponding merged size distributions (Section 3) and then these merged distributions are corrected. We shall call this the "simplified approach." The basis of this simplified approach is the assumption that the properties of the PCASP (both the wavelength of the illuminating light and the solid angle limits) are representative of all merged size distributions. Such a simplification is motivated by the fact that the PCASP-derived size distributions represent particles in the $0.13-3 \mu \mathrm{m}$ size range (Section 2), which scatter light most effectively in the visible spectral range considered here (about $0.2-0.7 \mu \mathrm{m}$ ). It can therefore be expected that the PCASP-derived size distributions contribute most of the total scattering measured by this airborne nephelometer.

We apply the simplified approach to calculate the theoretical response curves (Figure C1) and adjust particle diameters (Figure C2). Let us start with Figure C1. The first curve represents an assumed "experimental" one, which would be obtained from laboratory calibrations for particles with a known RI. Given that uncertainties of the experimental calibration are unknown, the "experimental" curve is the smoothed version of the corresponding theoretical response curve. A polynomial (curvilinear) regression model ([75]; pages 342-347) is applied to generate this smoothed version. Similar smoothed versions have been used previously for different OPCs [34]. The second and third curves represent low and high values of the imaginary part of RI obtained for two FLs (Figure 6b) and illustrate two important points. First, the corresponding curves are comparable and "smooth" for particles with diameter smaller than $0.75 \mu \mathrm{m}$; (Figure C1; upper zoom-in panel). In contrast, the curves are quite different and "bumpy" for large particles with diameter between $1.5 \mu \mathrm{m}$ and $3.0 \mu \mathrm{m}$ (Figure C1; lower zoom-in panel). Second, the observed "bumpy" behavior is responsible for the well-known multivalued issue, when particles with different diameter can produce the same response (Figure C1; lower zoom-in panel, green curve). In the presented calculations, we assume that the corresponding response is due to a particle with minimal diameter (Figure C1; lower zoom-in panel, red open circle on green curve). The observed differences between the second and third curves 
(Figure C1) are responsible for the corresponding differences between the adjusted diameters (Figure C2).

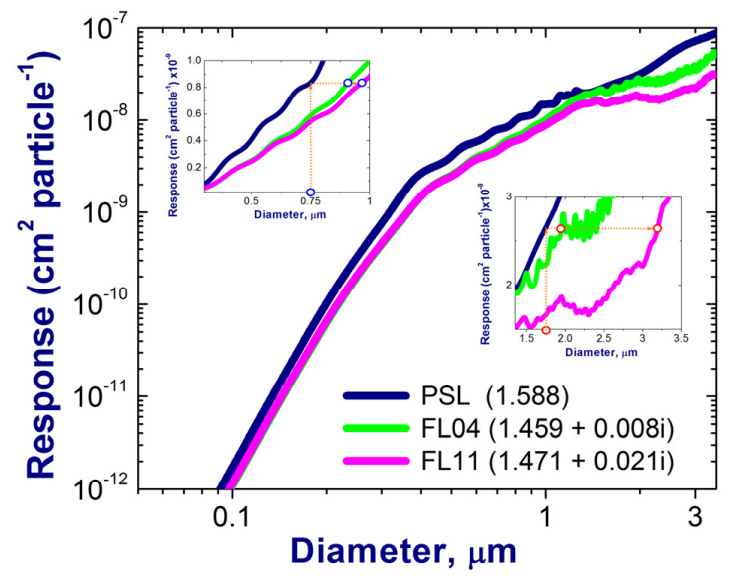

Figure C1. Example of theoretical response curves as a function of dry diameter $\left(D_{\text {dry }}\right)$ calculated for spherical particles and different values of complex RI. These values represent polystyrene latex spheres (PSL; navy blue) and those calculated for two FLs (FL = 4, green; FL = 11, magenta; 21 July 2012) from chemical composition measurements under dry conditions. Two complementary zoom-in versions of the calculated response curves are also included to illustrate that these curves may exhibit "smooth" and "bumpy" behavior for different ranges of diameter (see text for details).

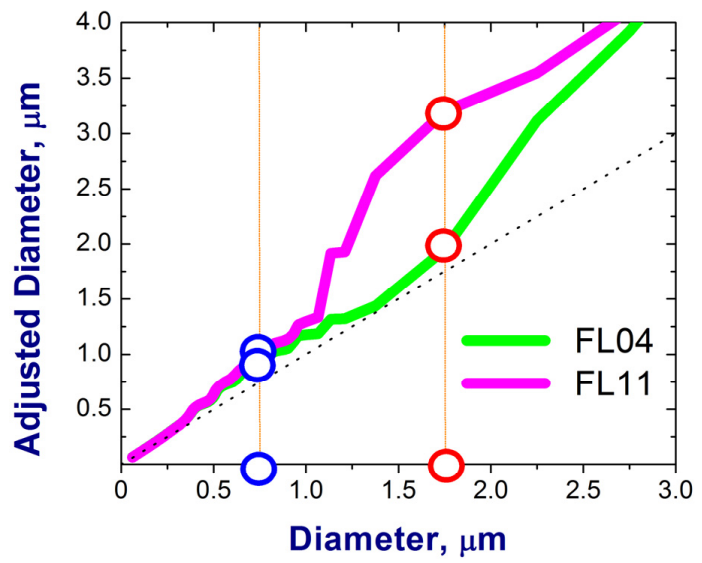

Figure C2. Adjusted dry diameter $\left(\mathrm{D}_{\mathrm{dry}, \text { adj }}\right)$ as a function of original dry diameter $\left(D_{\text {dry }}\right)$ obtained for two FLs (FL = 4, green; $F L=11$, magenta; 21 July 2012) from the calculated response curves (Figure C1). 


\section{References}

1. Wang, X.; Heald, C.L.; Ridley, D.A.; Schwarz, J.P.; Spackman, J.R.; Perring, A.E.; Coe, H.; Liu, D.; Clarke, A.D. Exploiting simultaneous observational constraints on mass and absorption to estimate the global direct radiative forcing of black carbon and brown carbon. Atmos. Chem. Phys. 2014, 14, 10989-11010.

2. Sundström, A.-M.; Arola, A.; Kolmonen, P.; Xue, Y.; de Leeuw, G.; Kulmala, M. On the use of a satellite remote-sensing-based approach for determining aerosol direct radiative effect over land: A case study over China. Atmos. Chem. Phys. 2015, 15, 505-518.

3. Adachi, K.; Chung, S.H.; Buseck, P.R. Shapes of soot aerosol particles and implications for their effects on climate. J. Geophys. Res. 2010, 115, D15206.

4. Bond, T.C.; Doherty, S.J.; Fahey, D.W.; Forster, P.M.; Berntsen, T.; DeAngelo, B.J.; Flanner, M.G.; Ghan, S.; Kärcher, B.; Koch, D.; et al. Bounding the role of black carbon in the climate system: A scientific assessment. J. Geophys. Res. Atmos. 2013, 118, 5380-5552.

5. China, S.; Mazzoleni, C.; Gorkowski, K.; Aiken, A.C.; Dubey, M.K. Morphology and mixing state of individual freshly emitted wildfire carbonaceous particles. Nat. Comm. 2013, 4, 2122.

6. Fast, J.D.; Allan, J.; Bahreini, R.; Craven, J.; Emmons, L.; Ferrare, R.; Hayes, P.L.; Hodzic, A.; Holloway, J.; Hostetler, C.; et al. Modeling regional aerosol and aerosol precursor variability over California and its sensitivity to emissions and long-range transport during the 2010 CalNex and CARES campaigns. Atmos. Chem. Phys. 2014, 14, 10013-10060.

7. Washenfelder, R.A.; Attwood, A.R.; Brock, C.A.; Guo, H.; Xu, L.; Weber, R.J.; Ng, N.L.; Allen, H.M.; Ayres, B.R.; Baumann, K.; et al. Biomass burning dominates brown carbon absorption in the rural southeastern United States. Geophys. Res. Lett. 2015, 42, 653-664.

8. Müller, D.; Hostetler, C.A.; Ferrare, R.A.; Burton, S.P.; Chemyakin, E.; Kolgotin, A.; Hair, J.W.; Cook, A.L.; Harper, D.B.; Rogers, R.R.; et al. Airborne Multiwavelength High Spectral Resolution Lidar (HSRL-2) observations during TCAP 2012: Vertical profiles of optical and microphysical properties of a smoke/urban haze plume over the northeastern coast of the US. Atmos. Meas. Tech. 2014, 7, 3487-3496.

9. Russell, P.B.; Kacenelenbogen, M.; Livingston, J.M.; Hasekamp, O.P.; Burton, S.P.; Schuster, G.L.; Johnson, M.S.; Knobelspiesse, K.D.; Redemann, J.; Ramachandran, S.; Holben, B. A multiparameter aerosol classification method and its application to retrievals from spaceborne polarimetry. J. Geophys. Res. Atmos. 2014, 119, 9838-9863.

10. Chaikovskaya, L.; Dubovik, O.; Litvinov, P.; Grudo, J.; Lopatsin, A.; Chaikovsky, A.; Denisov, S. Analytical algorithm for modeling polarized solar radiation transfer through the atmosphere for application in processing complex lidar and radiometer measurements. J. Quant. Spectrosc. Radiat. Trans. 2015, 151, 275-286.

11. Kokhanovsky, A.A.; Davis, A.B.; Cairns, B.; Dubovik, O.; Hasekamp, O.P.; Sano, I.; Mukai, S.; Rozanov, V.V.; Litvinov, P.; Lapyonok, T.; et al. Space-based remote sensing of atmospheric aerosols: The multi-angle spectro-polarimetric frontier. Earth-Sci. Rev. 2015. 
12. Baumgardner, D.; Brenguier, J.L.; Bucholtz, A.; Coe, H.; DeMott, P.; Garrett, T.J.; Gayet, J.F.; Hermann, M.; Heymsfield, A.; Korolev, A.; et al. Airborne instruments to measure atmospheric aerosol particles, clouds and radiation: A cook's tour of mature and emerging technology. Atmos. Res. 2011, 102, 10-29.

13. McFarquhar, G.; Schmid, B.; Korolev, A.; Ogren, J.A.; Russell, P.B.; Tomlinson, J.; Turner, D.D.; Wiscombe, W. Airborne instrumentation needs for climate and atmospheric research. Bull. Amer. Meteor. Soc. 2011, 92, 1193-1196.

14. Konwar, M.; Panicker, A.S.; Axisa, D.; Prabha, T.V. Near-cloud aerosols in monsoon environment and its impact on radiative forcing. J. Geophys. Res. Atmos. 2015, 120.

15. Russell, P.B.; Kinne, S.A.; Bergstrom, R.W. Aerosol climate effects: Local radiative forcing and column closure experiments. J. Geophys. Res. 1997, 102, 9397-9407.

16. Schmid, B.; Livingston, J.M.; Russell, P.B.; Durkee, P.A.; Jonsson, H.H.; Collins, D.R.; Flagan, R.C.; Seinfeld, J.H.; Gassó, S.; Hegg, D.A.; et al. Clear-sky closure studies of lower tropospheric aerosol and water vapor during ACE-2 using airborne sunphotometer, airborne in-situ, space-borne, and ground-based measurements. Tellus B 2000, 52, 568-593.

17. Malm, W.C.; Day, D.E.; Carrico, C.; Kreidenweis, S.M.; Collett, J.L., Jr.; McMeeking, G.; Lee, T.; Carrillo, J.; Schichtel, B. Intercomparison and closure calculations using measurements of aerosol species and optical properties during the Yosemite Aerosol Characterization Study. J. Geophys. Res. 2005, 110, D14302.

18. Mack, L.A.; Levin, E.J.T.; Kreidenweis, S.M.; Obrist, D.; Moosmüller, H.; Lewis, K.A.; Arnott, W.P.; McMeeking, G.R.; Sullivan, A.P.; Wold, C.E.; et al. Optical closure experiments for biomass smoke aerosols. Atmos. Chem. Phys. 2010, 10, 9017-9026.

19. Marshall, J.; Lohmann, U.; Leaitch, W.R.; Lehr, P.; Hayden, K. Aerosol scattering as a function of altitude in a coastal environment. J. Geophys. Res. 2007, 112, D14203.

20. Ma, N.; Birmili, W.; Müller, T.; Tuch, T.; Cheng, Y.F.; Xu, W.Y.; Zhao, C.S.; Wiedensohler, A. Tropospheric aerosol scattering and absorption over central Europe: A closure study for the dry particle state. Atmos. Chem. Phys. 2014, 14, 6241-6259.

21. Zieger, P.; Fierz-Schmidhauser, R.; Poulain, L.; Müller, T.; Birmili, W.; Spindler, G.; Wiedensohler, A.; Baltensperger, U.; Weingartner, E. Influence of water uptake on the aerosol particle light scattering coefficients of the Central European aerosol. Tellus B 2014, $66,22716$.

22. Wang, J.; Flagan, R.C.; Seinfeld, J.H.; Jonsson, H.H.; Collins, D.R.; Russell, P.B.; Schmid, B.; Redemann, J.; Livingston, J.M.; Gao, S.; et al. Clear-column radiative closure during ACE-Asia: Comparison of multiwavelength extinction derived from particle size and composition with results from sunphotometry. J. Geophys. Res. 2002, 107, 4688.

23. Parworth, C.; Fast, J.; Mei, F.; Shippert, T.; Sivaraman, C.; Tilp, A.; Watson, T.; Zhang, Q. Long-term measurements of submicrometer aerosol chemistry at the Southern Great Plains (SGP) using an Aerosol Chemical Speciation Monitor (ACSM). Atmos. Environ. 2015, 106, 43-55. 
24. Schmid, B.; Tomlinson, J.M.; Hubbe, J.M.; Comstock, J.M.; Mei, F.; Chand, D.; Pekour, M.S.; Kluzek, C.D.; Andrews, E.; Biraud, S.C.; et al. The DOE ARM Aerial Facility. Bull. Am. Meteor. Soc. 2014, 95, 723-742.

25. Kassianov, E.; Flynn, C.; Redemann, J.; Schmid, B.; Russell, P.B.; Sinyuk, A. Initial assessment of the Spectrometer for Sky-Scanning, Sun-Tracking Atmospheric Research (4STAR)-based aerosol retrieval: Sensitivity study. Atmosphere 2012, 3, 495-521.

26. Dunagan, S.; Johnson, R.; Zavaleta, J.; Russell, P.; Schmid, B.; Flynn, C.; Redemann, J.; Shinozuka, Y.; Livingston, J.; Segal-Rosenhaimer, M. Spectrometer for Sky-Scanning Sun-Tracking Atmospheric Research (4STAR): Instrument technology. Remote Sens. 2013, 5, 3872-3895.

27. Segal-Rosenheimer, M.; Russell, P.B.; Schmid, B.; Redemann, J.; Livingston, J.M.; Flynn, C.J.; Johnson, R.R.; Dunagan, S.E.; Shinozuka, Y.; Herman, J.; et al. Tracking elevated pollution layers with a newly developed hyperspectral Sun/Sky spectrometer (4STAR): Results from the TCAP 2012 and 2013 campaigns. J. Geophys. Res. Atmos. 2014, $119,2611-2628$.

28. Allen, G.; Coe, H.; Clarke, A.; Bretherton, C.; Wood, R.; Abel, S.J.; Barrett, P.; Brown, P.; George, R.; Freitag, S.; et al. South East Pacific atmospheric composition and variability sampled along $20^{\circ} \mathrm{S}$ during VOCALS-Rex. Atmos. Chem. Phys. 2011, 11, 5237-5262.

29. Kleinman, L.I.; Daum, P.H.; Lee, Y.-N.; Lewis, E.R.; Sedlacek, A.J., III; Senum, G.I.; Springston, S.R.; Wang, J.; Hubbe, J.; Jayne, J.; et al. Aerosol concentration and size distribution measured below, in, and above cloud from the DOE G-1 during VOCALS-REx. Atmos. Chem. Phys. 2012, 11, 207-223.

30. Liu, Y.; Daum, P.H. The effect of refractive index on size distributions and light scattering coefficients derived from optical particle counters. J. Aerosol. Sci. 2000, 31, 945-957.

31. Ames, R.B.; Hand, J.L.; Kreidenweis, S.M.; Day, D.E.; Malm, W.C. Optical measurements of aerosol size distributions in Great Smokey Mountains National Park: Dry aerosol characterization. J. Air Waste Manag. Assoc. 2000, 50, 665-676.

32. Garvey, D.M.; Pinnick, R.G. Response characteristics of the particle measuring systems active scattering aerosol spectrometer probe (ASASP-X). Aerosol Sci. Technol. 1983, 2, 477-488.

33. Kim, Y.J.; Boatman, J.F. Size calibration corrections for the Forward Scattering Spectrometer Probe (FSSP) for measurement of atmospheric aerosols of different refractive indices. J. Atmos. Oceanic Technol. 1990, 7, 681-688.

34. Bukowiecki, N.; Zieger, P.; Weingartner, E.; Juranyi, Z.; Gysel, M.; Neininger, B.; Schneider, B.; Hueglin, C.; Ulrich, A.; Wichser, A.; et al. Ground-based and airborne in-situ measurements of the Eyjafjallajökull volcanic aerosol plume in Switzerland in spring 2010. Atmos. Chem. Phys. 2011, 11, 10011-10030.

35. Kondo, Y. Effects of black carbon on climate: Advances in measurement and modeling. Monogr. Environ. Earth Planets 2015, 3, 1-85.

36. Kreidenweis, S.M.; Asa-Awuku, A. Aerosol Hygroscopicity: Particle water content and its role in atmospheric processes. Treat. Geochem.: Second Ed. 2013, 5, 331-361. 
37. Jensen, T.L.; Kreidenweis, S.M.; Kim, Y.; Sievering, H.; Pszenny, A. Aerosol distributions measured in the North Atlantic marine boundary layer during ASTEX/MAGE. J. Geophys. Res. 1996, 101, 4455-4467.

38. Swietlicki, E.; Zhou, J.; Berg, O.H.; Martinsson, B.G.; Frank, G.; Cederfelt, S.-I.; Dusek, U.; Berner, A.; Birmili, W.; Wiedensohler, A.; et al. A closure study of sub-micrometer aerosol particle hygroscopic behavior. Atmos. Res. 1999, 50, 205-240.

39. Dusek, U.; Frank, G.P.; Massling, A.; Zeromskiene, K.; Iinuma, Y.; Schmid, O.; Helas, G.; Hennig, T.; Wiedensohler, A.; Andreae, M.O. Water uptake by biomass burning aerosol at sub- and supersaturated conditions: Closure studies and implications for the role of organics. Atmos. Chem. Phys. 2011, 11, 9519-9532.

40. Kassianov, E.; Barnard, J.; Pekour, M.; Berg, L.K.; Shilling, J.; Flynn, C.; Mei, F.; Jefferson, A. Simultaneous retrieval of effective refractive index and density from size distribution and light-scattering data: Weakly absorbing aerosol. Atmos. Meas. Tech. 2014, 7, 3247-3261.

41. Titos, G.; Jefferson, A.; Sheridan, P.J.; Andrews, E.; Lyamani, H.; Alados-Arboledas, L.; Ogren, J.A. Aerosol light-scattering enhancement due to water uptake during the TCAP campaign. Atmos. Chem. Phys. 2014, 14, 7031-7043.

42. Collins, D.R.; Flagan, R.C.; Seinfeld, J.H. Improved inversion of scanning DMA data. Aerosol Sci. Technol. 2002, 36, 1-9.

43. Schmid, B.; Ferrare, R.; Flynn, C.; Elleman, R.; Covert, D.; Strawa, A.; Welton, E.; Turner, D.; Jonsson, H.; Redemann, J.; et al. How well do state-of-the-art techniques measuring the vertical profile of tropospheric aerosol extinction compare? J. Geophys. Res. 2006, 111, D05S07.

44. Zelenyuk, A.; Imre, D.; Wilson, J.; Zhang, Z.; Wang, J.; Mueller, K. Airborne Single Particle Mass Spectrometers (SPLAT II\& miniSPLAT) and new software for data visualization and analysis in a geo-spatial context. J. Am. Soc. Mass Spectrom. 2015, 26, 257-270.

45. Berg, L.; Fast, J.D.; Barnard, J.C.; Burton, S.P.; Cairns, B.; Chand, D.; Comstock, J.M.; Dunagan, S.; Ferrare, R.A.; Flynn, C.J.; et al. The Two-Column Aerosol Project: Phase I overview and impact of elevated aerosol layers on aerosol optical depth. J. Geophys. Res. Atmos. 2015. under review.

46. Esteve, A.R.; Highwood, E.J.; Morgan, W.T.; Allen, G.; Coe, H.; Grainger, R.G.; Brown, P.; Szpek, K. A study on the sensitivities of simulated aerosol optical properties to composition and size distribution using airborne measurements. Atmos. Environ. 2014, $89,517-524$.

47. Twomey, S. Comparison of constrained linear inversion and an iterative nonlinear algorithm applied to indirect estimation of particle-size distributions. J. Comput. Phys. 1975, 18, 188-200.

48. Markowski, G.R. Improving Twomey's Algorithm for Inversion of Aerosol Measurement Data. Aerosol Sci. Technol. 1987, 7, 127-141.

49. Moteki, N.; Kondo, Y. Effects of mixing state on black carbon measurements by laser-induced incandescence. Aerosol Sci. Technol. 2007, 41, 398-417. 
50. Sedlacek, A.J., III; Lewis, E.R.; Kleinman, L.; Xu, J.; Zhang, Q. Determination of and evidence for non-core-shell structure of particles containing black carbon using the Single-Particle Soot Photometer (SP2). Geophys. Res. Lett. 2012, 39.

51. Pekour, M.S.; Schmid, B.; Chand, D.; Hubbe, J.M.; Kluzek, C.D.; Nelson, D.A.; Tomlinson, J.M.; Cziczo, D.J. Development of a new airborne humidigraph system. Aerosol Sci. Technol. 2013, 47, 201-207.

52. Shinozuka, Y.; Johnson, R.R.; Flynn, C.J.; Russell, P.B.; Schmid, B.; Redemann, J.; Dunagan, S.E.; Kluzek, C.D.; Hubbe, J.M.; Segal-Rosenheimer, M.; et al. Hyperspectral aerosol optical depths from TCAP flights. J. Geophys. Res. Atmos. 2013, 118, 12180-12194.

53. Anderson, T.L.; Ogren, J.A. Determining aerosol radiative properties using the TSI 3563 Integrating Nephelometer. Aerosol Sci. Technol. 1998, 29, 57-69.

54. Hallar, A.G.; Strawa, A.W.; Schmid, B.; Andrews, E.; Ogren, J.; Sheridan, P.; Ferrare, R.; Covert, D.; Elleman, R.; Jonsson, H.; et al. Atmospheric Radiation Measurements Aerosol Intensive Operating Period: Comparison of aerosol scattering during coordinated flights. J. Geophys. Res. 2006, 111, D05S09.

55. Barnard, J.C.; Fast, J.D.; Paredes-Miranda, G.; Arnott, W.P.; Laskin, A. Technical note: Evaluation of the WRF-Chem "Aerosol chemical to aerosol optical properties" module using data from the MILAGRO campaign. Atmos. Chem. Phys. 2010, 10, 7325-7340.

56. Hu, D.; Chen, J.; Ye, X.; Li, L.; Yang, X. Hygroscopicity and evaporation of ammonium chloride and ammonium nitrate: Relative humidity and size effects on the growth factor. Atmos. Environ. 2011, 45, 2349-2355.

57. Healy, R.M.; Evans, G.J.; Murphy, M.; Jurányi, Z.; Tritscher, T.; Laborde, M.; Weingartner, E.; Gysel, M.; Poulain, L.; Kamilli, K.A.; et al. Predicting hygroscopic growth using single particle chemical composition estimates. J. Geophys. Res. Atmos. 2014, 119, 9567-9577.

58. Pilinis, C.; Charalampidis, P.E.; Mihalopoulos, N.; Pandis, S.N. Contribution of particulate water to the measured aerosol optical properties of aged aerosol. Atmos. Environ. 2014, $82,144-153$.

59. Dubovik, O.; Holben, B.; Eck, T.F.; Smirnov, A.; Kaufman, Y.J.; King, M.D.; Tanré, D.; Slutsker, I. Variability of absorption and optical properties of key aerosol types observed in worldwide locations. J. Atmos. Sci. 2002, 59, 590-608.

60. Kokhanovsky, A.A. Aerosol Optics: Light Absorption and Scattering by Particles in the Atmosphere; Springer-Berlin: Heidelberg, Germany, 2008; p. 148.

61. Barber, P.W.; Hill, S.C. Light Scattering by Particles: Computational Methods; World Scientific Publishing: Singapore, 1990.

62. Scarnato, B.V.; Vahidinia, S.; Richard, D.T.; Kirchstetter, T.W. Effects of internal mixing and aggregate morphology on optical properties of black carbon using a discrete dipole approximation model. Atmos. Chem. Phys. 2013, 13, 5089-5101.

63. Lesins, G.; Chylek, P.; Lohmann, U. A study of internal and external mixing scenarios and its effect on aerosol optical properties and direct radiative forcing. J. Geophys. Res. Atmos. 2002, 107, 4094-4106. 
64. Freney, E.J.; Adachi, K.; Buseck, P.R. Internally mixed atmospheric aerosol particles: Hygroscopic growth and light scattering. J. Geophys. Res. Atmos. 2010, 115, D19210.

65. Wex, H.; Neususs, C.; Wendisch, M.; Stratmann, F.; Koziar, C.; Keil, A.; Wiedensohler, A.; Ebert, M. Particle scattering, backscattering, and absorption coefficients: An in situ closure and sensitivity study. J. Geophys. Res. Atmos. 2012, 107, 8122.

66. York, D.; Evensen, N.M.; Lopez Martinez, M.; de Basabe Delgado, J. Unified equations for the slope, intercept, and standard errors of the best straight line. Am. J. Phys. 2004, 72, 367-375.

67. Petäjä, T.; Mauldin, R.L., III; Kosciuch, E.; McGrath, J.; Nieminen, T.; Paasonen, P.; Boy, M.; Adamov, A.; Kotiaho, T.; Kulmala, M. Sulfuric acid and $\mathrm{OH}$ concentrations in a boreal forest site. Atmos. Chem. Phys. 2009, 9, 7435-7448.

68. Zieger, P.; Weingartner, E.; Henzing, J.; Moerman, M.; de Leeuw, G.; Mikkilä, J.; Ehn, M.; Petäjä, T.; Clémer, K.; van Roozendael, M.; et al. Comparison of ambient aerosol extinction coefficients obtained from in-situ, MAX-DOAS and LIDAR measurements at Cabauw. Atmos. Chem. Phys. 2011, 11, 2603-2624.

69. Cantrell, C.A. Technical Note: Review of methods for linear least-squares fitting of data and application to atmospheric chemistry problems. Atmos. Chem. Phys. 2008, 8, 5477-5487.

70. Hand, J.L.; Kreidenweis, S.M. A new method for retrieving particle refractive index and effective density from aerosol size distribution data. Aerosol Sci. Tech. 2002, 36, 1012-1026.

71. McComiskey, A.; Feingold, G.; Frisch, A.S.; Turner, D.D.; Miller, M.A.; Chiu, J.C.; Min, Q.; Ogren, J.A. An assessment of aerosol-cloud interactions in marine stratus clouds based on surface remote sensing. J. Geophys. Res. 2009, 114, D09203.

72. Modini, R.L.; Frossard, A.A.; Ahlm, L.; Russell, L.M.; Corrigan, C.E.; Roberts, G.C.; Hawkins, L.N.; Schroder, J.C.; Bertram, A.K.; Zhao, R.; et al. Primary marine aerosol-cloud interactions off the coast of California. J. Geophys. Res. Atmos. 2015, 120.

73. Yu, H.; Chin, M.; Bian, H.; Yuan, T.; Prospero, J.M.; Omar, A.H.; Remer, L.A.; Winker, D.M.; Yang, Y.; Zhang, Y.; Zhang, Z.; et al. Quantification of trans-Atlantic dust transport from seven-year (2007-2013) record of CALIPSO lidar measurements. Remote Sens. Environ. 2015, 159, 232-249.

74. Barnard, J.C.; Harrison, L.C. Monotonic responses from monochromatic optical particle counters. Appl. Opt. 1988, 3, 584-592.

75. Kennedy, W.J.; Gentle, J.E. Statistical Computing; Marcel Dekker: New York, NY, USA, 1980. 


\title{
The Impact of Selected Parameters on Visibility: First Results from a Long-Term Campaign in Warsaw, Poland
}

\author{
Grzegorz Majewski, Wioletta Rogula-Kozłowska, Piotr O. Czechowski, \\ Artur Badyda and Andrzej Brandyk
}

\begin{abstract}
The aim of this study was to investigate how atmospheric air pollutants and meteorological conditions affected atmospheric visibility in the largest Polish agglomeration. The correlation analysis, principal component analysis (PCA) and generalized regression models (GRMs) were used to accomplish this objective. The meteorological parameters (temperature, relative humidity, precipitation, wind speed and insolation) and concentrations of the air pollutants $\left(\mathrm{PM}_{10}, \mathrm{SO}_{2}, \mathrm{NO}_{2}\right.$, $\mathrm{CO}$ and $\mathrm{O}_{3}$ ) were recorded in 2004-2013. The data came from the Ursynów-SGGW, MzWarszUrsynów and Okęcie monitoring stations, located in the south of Warsaw (Poland). It was shown that the $\mathrm{PM}_{10}$ concentration was the most important parameter affecting the visibility in Warsaw. The concentration, and indirectly the visibility, was mainly affected by the pollutant emission from the flat/building heating (combustion of various fuels). It changed intensively during the research period. There were also periods in which this emission type did not have a great influence on the pollutant concentrations (mainly $\mathrm{PM}_{10}$ ) and visibility. In such seasons, the research revealed the influence of the traffic emission and secondary aerosol formation processes on the visibility.
\end{abstract}

Reprinted from Atmosphere. Cite as: Majewski, G.; Rogula-Kozłowska, W.; Czechowski, P.O.; Badyda, A.; Brandyk, A. The Impact of Selected Parameters on Visibility: First Results from a Long-Term Campaign in Warsaw, Poland. Atmosphere 2015, 6, 1154-1174.

\section{Introduction}

The visibility deterioration caused by atmospheric pollution is a global problem. It occurs in many densely populated areas that have experienced population growth and industrialization. However, visibility is a complex issue. On one hand, it is directly affected by the anthropogenic air pollution. On the other hand, it is influenced by the meteorological conditions [1].

The anthropogenic air pollution effect on human health and visibility has been examined for decades. Many studies were conducted not only to assess the benefits for human health resulting from air pollutant emission reduction but also to understand how air pollutants negatively affect visibility. Generally, visibility 
makes a good index for the air pollution extent. It can also be used as a surrogate for assessing the human health effects $[2,3]$.

The visibility impairment is mainly attributed to the scattering and absorption of the visible light caused by suspended particles and gaseous pollutants in the atmosphere [4,5]. The visibility impairment in the urban atmosphere is closely related to the air pollution from anthropogenic sources, such as car exhaust fumes, fuel combustion, solid waste incineration, and industrial emissions [6-10].

The visibility impairment is mainly influenced by the airborne particulate matter (PM), particularly its fine particles with aerodynamic diameters smaller than $2.5 \mu \mathrm{m}$ $\left(\mathrm{PM}_{2.5}\right)$. In urban areas, the major $\mathrm{PM}_{2.5}$ components, such as ammonium, sulphates, nitrates, organic matter and elemental carbon [11], are the main factors contributing to the light absorption and scattering. Therefore, their presence effectively reduces visibility $[12,13]$. The specific content of $\mathrm{PM}_{2.5}$ is the most important aspect when analysing the $\mathrm{PM}_{2.5}$ effect on visibility. The size and chemical composition of each component particle affects its ability to refract, scatter, and absorb light [14]. There is a strong correlation between the presence of $\mathrm{PM}_{2.5}$ and $\mathrm{PM}_{10}$ (particles with aerodynamic diameters smaller than $10 \mu \mathrm{m}$ ), to the extent that a targeted reduction in $\mathrm{PM}_{10}$ is likely to lead to an increase in the atmospheric visibility $[15,16]$. In addition to the air pollutants, the meteorological parameters (i.e., wind speed and direction, relative air humidity, air temperature, atmospheric pressure and precipitation) can also directly or indirectly affect atmospheric visibility as they influence the local and regional air quality in urban areas [17-22].

Air quality monitoring has already been performed in Warsaw for about 20 years. Nevertheless, the measurement standards were adjusted to comply with the European Union (EU) regulations and requirements in 2004. Since then, the air quality has been successfully recorded in order to warn the community of high pollutant levels. The system also contributes to the research on air pollutant influence on human health $[23,24]$. The collected data also enables investigations into the air pollution impact on visibility. Taking into consideration the necessity to improve the visibility in urban areas, the underlying mechanisms must be well understood, particularly when it comes to aspects such as the main contributing air pollutants and their origin. This research field has a worldwide significance. Nonetheless, it needs further development in Poland. Among the key statistical methods applied in this study, the researchers found the correlation analysis and the related Principal Component Analysis (PCA) and Generalized Regression Models (GRMs) particularly useful. All the models served to identify the air pollutants and meteorological parameters influencing visibility in an urban area. 


\section{Materials and Methods}

\subsection{Research Area}

Warsaw is the biggest city in Poland and the ninth biggest city in Europe $\left(517.24 \mathrm{~km}^{2}\right)$. It is under the influence of the warm transitional temperate climate. The mean yearly air temperature is $8.6^{\circ} \mathrm{C}$. The mean precipitation sum is $550-650 \mathrm{~mm}$. In Warsaw, the visible influence of a large urban agglomeration on the climate is seen as the so-called urban heat island [25]. The mean air temperatures and precipitation sums are higher whereas the wind speed is lower in the city center. As the air in the city is highly polluted, the cloud cover is bigger and the air transparency deteriorates. Consequently, the direct solar radiation decreases, whereas the diffuse sky radiation increases.

Even though the air quality has improved tremendously in Poland over the last 30 years [26-28], it is still unsatisfactory in Warsaw. What is definitely positive is the fact that the air pollutant levels (especially $\mathrm{CO}$ and $\mathrm{SO}_{2}$ ) have been gradually lowered over the last few years. Nonetheless, the permissible levels of $\mathrm{PM}_{10}$ are still exceeded in the capital of Poland [29,30]. PM comes from many sources there, including, among others, the energy production sector and vehicular transport. The area of Warsaw is additionally threatened by the air inflowing from heavily polluted southern Poland [31]. PM composition in large urban areas of Poland differs significantly from the compositions observed in other urban areas in Europe [32-34]. There are higher contents of elemental (soot) and organic carbon and lower percentage of the secondary inorganic matter in PM. It seems that the differences must have a considerable impact on the visibility in the research area.

\subsection{Air Quality Data and Visibility Observation}

The study was based on the measurement results obtained from the MzWarszUrsynów monitoring station of the atmospheric air quality $\left(\lambda \mathrm{E}=21^{\circ} 02^{\prime}\right.$; $\left.\varphi \mathrm{N}=52^{\circ} 09^{\prime}\right)$, located in the south of Warsaw (Figure 1). The researchers used the data on the mean hourly concentrations of the following air pollutants, measured by proper type of analyzers: sulphur dioxide $\left(\mathrm{SO}_{2}\right)-\mathrm{MLU} 100 \mathrm{~A}$, carbon monoxide (CO)-MLU 300, ozone $\left(\mathrm{O}_{3}\right)$-MLU 400, nitrogen oxides $\left(\mathrm{NO}_{2}\right)$ - $\mathrm{MLU}$ 200A, and PM 10 -TEOM1400a. They were monitored with pulsed fluorescence, infrared absorption, ultraviolet light absorption, chemiluminescence, and a $\beta$-gauge automated particle sampler, respectively. The meteorological data came from the Ursynów-SGGW meteorological station $\left(\lambda_{\mathrm{E}} 21^{\circ} 02^{\prime} ; \varphi_{\mathrm{N}} 52^{\circ} 09^{\prime}\right)$. The following information was investigated: mean hourly air temperature values $(\mathrm{T})$, insolation intensity (Rad), relative air humidity $(\mathrm{RH})$, precipitation intensity $(\mathrm{P})$ and wind speed (Ws). The measurements taken at the station were performed according to the instruction for network of stations belonging to the Institute of Meteorology 
and Water Management (IMGW). The data on the visibility were obtained from the only station taking such measurements, i.e., the Okęcie station $\left(\lambda_{\mathrm{E}} 20^{\circ} 59^{\prime}\right.$; $\left.\varphi_{\mathrm{N}} 52^{\circ} 09^{\prime}\right)$. The distance between the stations was approximately $6 \mathrm{~km}$. The visibility measurements were carried out with a visibility meter equipped with an atmospheric phenomenon detector-Vaisala FS11 (wavelength $875 \mathrm{~nm}$ ). It performed the functions of a visibility meter using light dispersion measurements and an atmospheric phenomenon detector. The horizontal visibility measurements were performed in the range of $10 \mathrm{~m}-50 \mathrm{~km}$. The data (1-h values) were shared by the IMGW. The information used in the study came from 2004-2013. For the whole research period, the following numbers of data $(n)$ were obtained: visibility $n=87,634$; $\mathrm{SO}_{2}, n=85,416 ; \mathrm{PM}_{10} n=83,016 ; \mathrm{NO}_{2} n=85,985 ; \mathrm{O}_{3} n=85,148 ; \mathrm{T} n=85,936$; RH $n=86,205 ; \operatorname{Rad} n=85,384$.

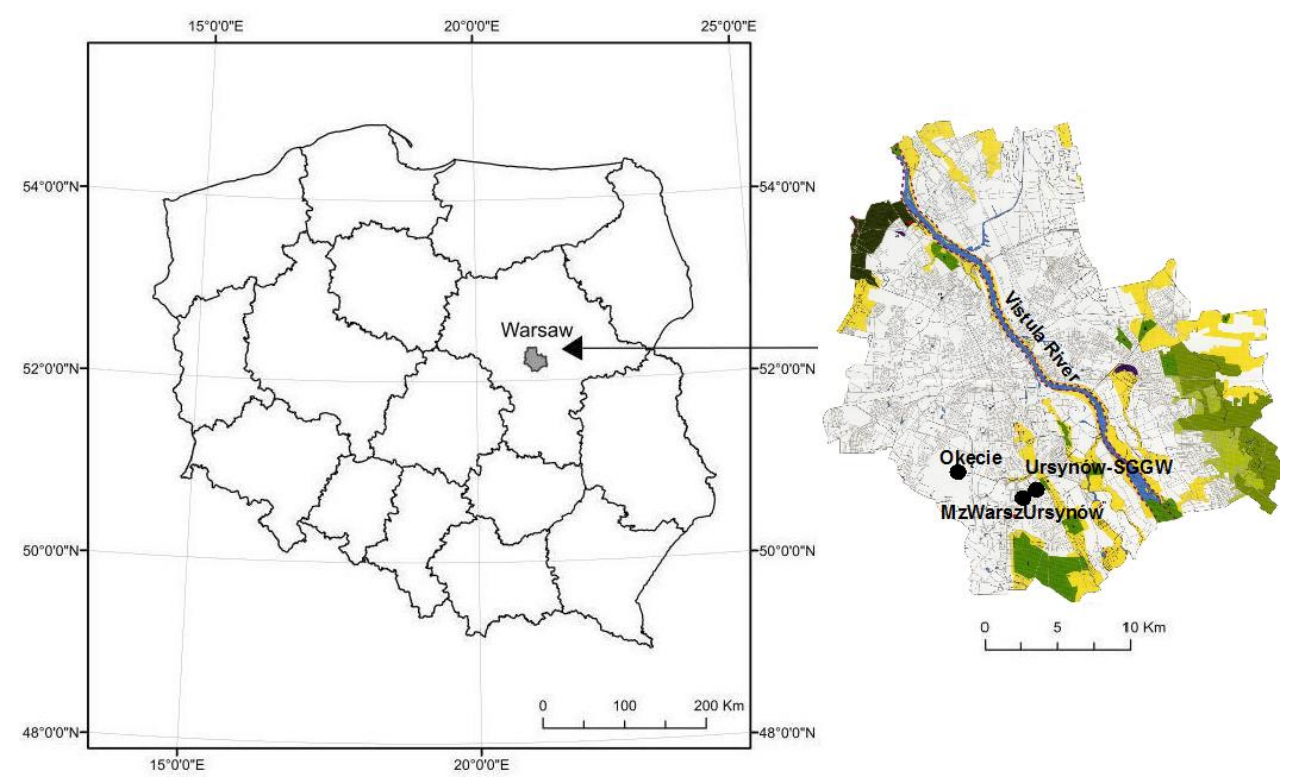

Figure 1. Location of the measurement stations in Warsaw (Poland).

\subsection{Statistical Method}

The correlation analysis, principal component analysis (PCA) and generalized regression models (GRMs) were applied in this research. The analyses helped to identify factors affecting visibility.

Generally, the PCA is a data reduction exercise. It is achieved through finding linear combinations (principal components) of the original variables, which account for as much of the original total variance as possible $[16,17,19,35,36]$. PCA is used to identify factors and their synergies in strong measurements scales: interval and 
ratio. Here, a scale or a level of measurement is the classification that describes the nature of information within the numbers assigned to variables. The interval type allows for the degree of difference between items, but not the ratio between them. The ratio type takes its name from the fact that measurement is the estimation of the ratio between a magnitude of a continuous quantity and a unit magnitude of the same kind.

GRM's models were used to identify factors in weak measurements scales: nominal and ordinal (ex. seasonality), and additionally, jointly with variables in strong scales. Nominal scales refer to the construction of classification of items, while ordinal ones allow for rank order by which data can be sorted. GRM is considered to be a path, embracing more than a model. It enabled to find the "best" model, describing the analysed phenomenon, out of an available range of models, and to replace many classical ones (e.g., ANOVA, MANOVA). Such an approach is more efficient for replication and cross-validation studies, less costly to put into practice in predicting and controlling the outcome in the future. Finally, it allows use of a wider range of fit statistics and diagnostic calculations, than using single models separately $[21,37,38]$.

\section{Results and Discussion}

\subsection{General Description of Visibility, Meteorology and Air Pollution}

The yearly course of the air temperature (average in the period 2004-2013) was typical for the temperate and transitional climate in Poland. July was the warmest month (mean air temperature $=20.5^{\circ} \mathrm{C}$ ). January was the coldest month (mean air temperature $=-2.1^{\circ} \mathrm{C}$ ). The lowest wind speed values were measured in August and September (approx. $2.1 \mathrm{~m} / \mathrm{s}$ ), whereas the highest ones were observed in March $(3.1 \mathrm{~m} / \mathrm{s})$-Figure 2.

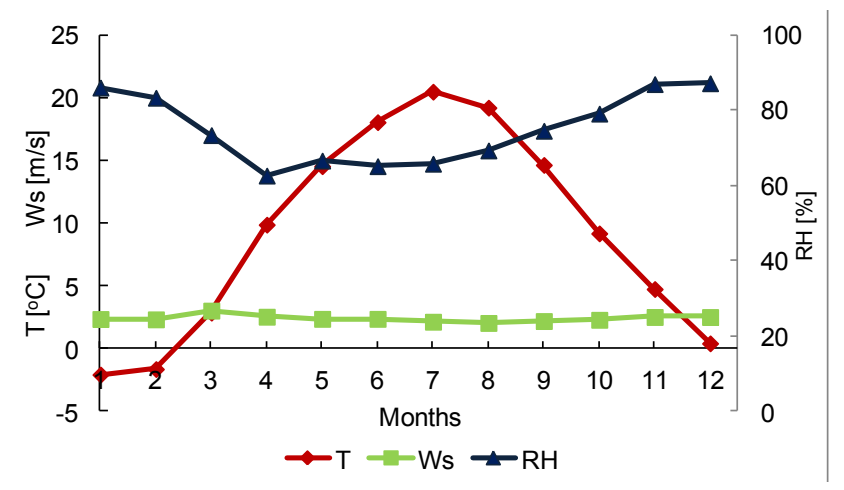

Figure 2. Monthly variations of meteorological parameters over Warsaw in 2004-2013. 
Figure 3 presents the diurnal patterns of visibility for the period 2004-2013. Visibility shows an obvious diurnal variation in each season of the year. In spring (March, April, May) and summer (June, July, August), a valley appears in early morning, from 04:00 to 06:00, while in autumn (September, October, November) and spring it is observed slightly later, between 06:00 and 07:00. The peak appears generally in the afternoon, from 13:00 to 15:00, except for winter (December, January, February), for which the peak is slightly later, at about 18:00. Visibility shows stronger diurnal cycles in summer and autumn, while it exhibits a much weaker variation in winter. Apart from this, the diurnal patterns during different seasons are desynchronized, which is attributed to the difference in weather patterns and stability of atmospheric boundary layer. The diurnal variation of visibility, characteristic for the city of Warsaw, is similar to that over several cities in China, however, hourly visibility values are found to be three times higher than those recorded in the Chinese cities [19].

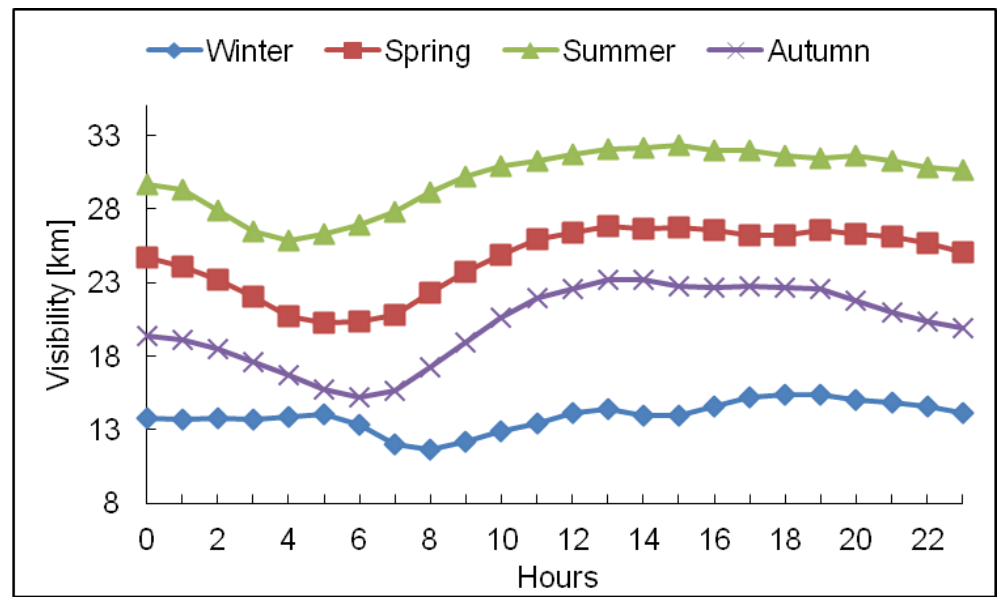

Figure 3. Diurnal variations of visibility over Warsaw for winter (December, January, February), spring (March, April, May), summer (June, July, August) and autumn (September, October, November) in 2004-2013.

For the whole research period (2004-2013) monthly visibility was in a wide range of 6.7-34.1 km (Figure 4). Within the researched period, noticeable seasonal changes in visibility were found. Visibility was generally higher in summer and lower in late autumn and winter. The average seasonal visibilities were 24.5, 30.1, 20.1 , and $13.9 \mathrm{~km}$ in spring, summer, autumn, and winter, respectively (Figure 4). 


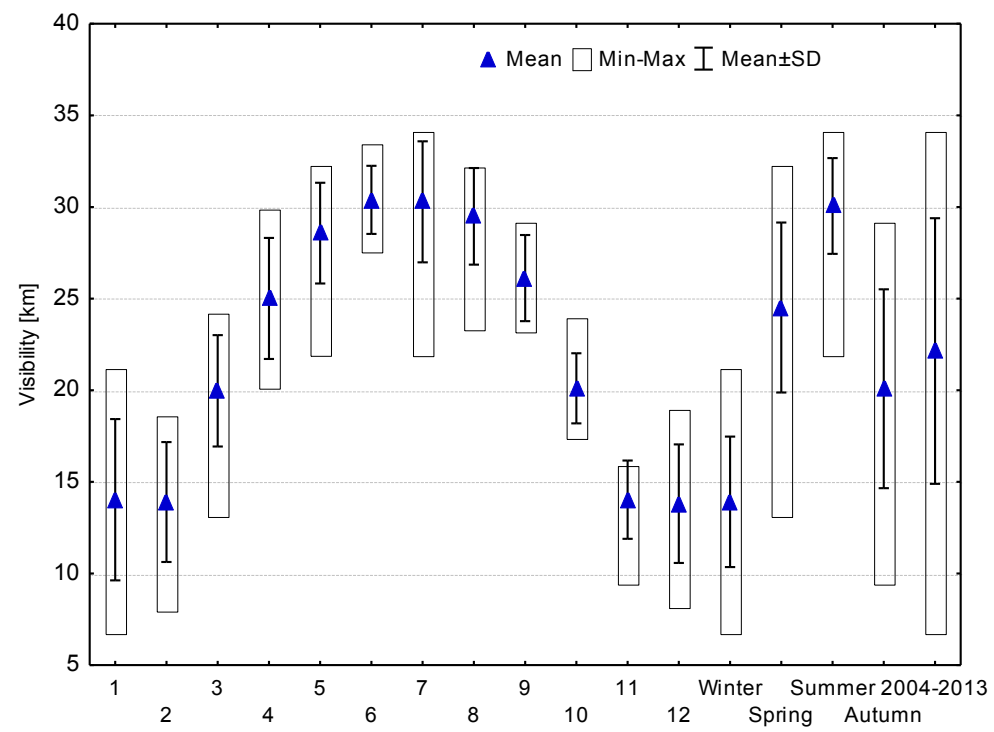

Figure 4. Descriptive statistics of monthly and seasonal visibility over Warsaw in 2004-2013.

Monthly visibility in Warsaw exhibited, in general, a considerable winter period variation (January-March, October-November) from 2004-2013. The summer period was characterized by much lower variation.

The mean yearly visibilities were in the range of $19.8-23.7 \mathrm{~km}$ (Figure 5). This proves that it did not show significant variability year by year. However, monthly values exhibit an increasing trend throughout the research period, but are statistically insignificant; $p<0.05$ (Figure 6). Average visibility for the whole period 2004-2013 was equal to $22.1 \mathrm{~km}$ and is from $5.3 \mathrm{~km}$ to over $13.1 \mathrm{~km}$ higher than the one over large, highly urbanized cities of China [1,19].

From 2004-2013 yearly air pollutants concentration didn't show much variation, alike visibility. In the analysed years, the mean yearly concentrations of $\mathrm{NO}_{2}$ were $21.4-28.0 \mu \mathrm{g} \cdot \mathrm{m}^{-3}$, which made $53.6 \%-70 \%$ of the permissible value $\left(40 \mu \mathrm{g} \cdot \mathrm{m}^{-3}\right)$-Figure 5 . The mean yearly concentrations of $\mathrm{SO}_{2}$ were $5.5-11.5 \mu \mathrm{g} \cdot \mathrm{m}^{-3}$ (the permissible value; $20 \mu \mathrm{g} \cdot \mathrm{m}^{-3}$ ), and the mean yearly concentrations of $\mathrm{CO}$ were $365.7-549.5 \mu \mathrm{g} \cdot \mathrm{m}^{-3}$. The mean yearly concentrations of $\mathrm{PM}_{10}$ did not exceed the permissible value as well $\left(40 \mu \mathrm{g} \cdot \mathrm{m}^{-3}\right)$ and were in the range of $28.0-37.2 \mu \mathrm{g} \cdot \mathrm{m}^{-3}$. The only pollutant, that, according to the Polish applicable laws, exceeded the permissible limit, was the ozone $\mathrm{O}_{3}$. The mean yearly concentrations of $\mathrm{O}_{3}$ were $43.5-50.4 \mu \mathrm{g} \cdot \mathrm{m}^{-3}$. The permissible level of the $8-\mathrm{h} \mathrm{O}_{3}$ concentration is $120 \mu \mathrm{g} \cdot \mathrm{m}^{-3}$ and can be exceeded about 25 days in each year. The biggest number of days with the exceeded value was observed in 2005. In the research area, there was no steady 
trend in the changes for $\mathrm{O}_{3}$, which is a secondary pollutant. The changes in its concentration mainly resulted from the changes in the weather conditions (insolation intensity, air temperature) and the participation of the $\mathrm{O}_{3}$ precursors (e.g., nitrogen oxides, hydrocarbons and other pollutants participation in the $\mathrm{O}_{3}$ formation) in the atmospheric air [30,39].

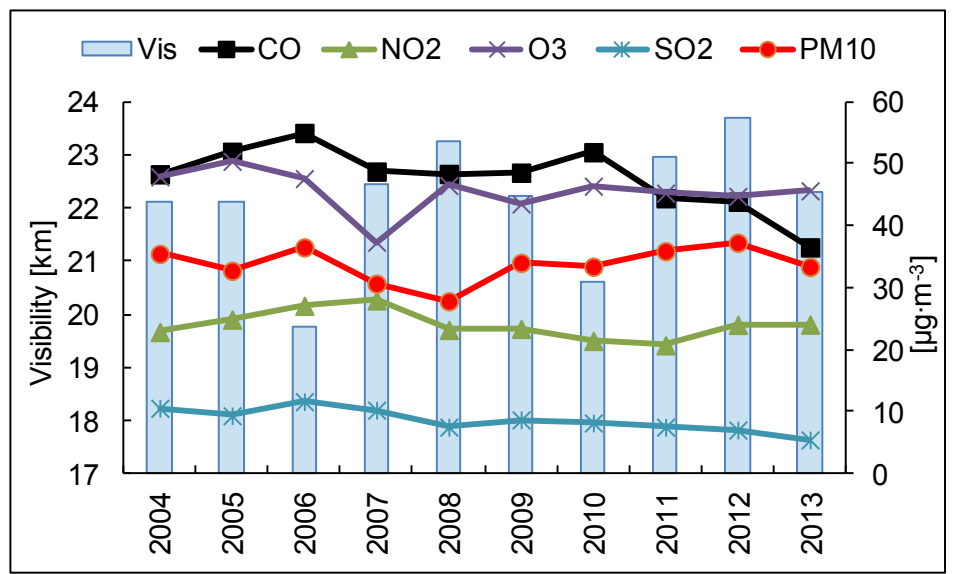

Figure 5. Yearly variations of visibility and ambient concentrations of $\mathrm{NO}_{2}, \mathrm{O}_{3}$, $\mathrm{SO}_{2}, \mathrm{PM}_{10}$ and $\mathrm{CO}$ (divided by 10 in the figure) over Warsaw in 2004-2013.

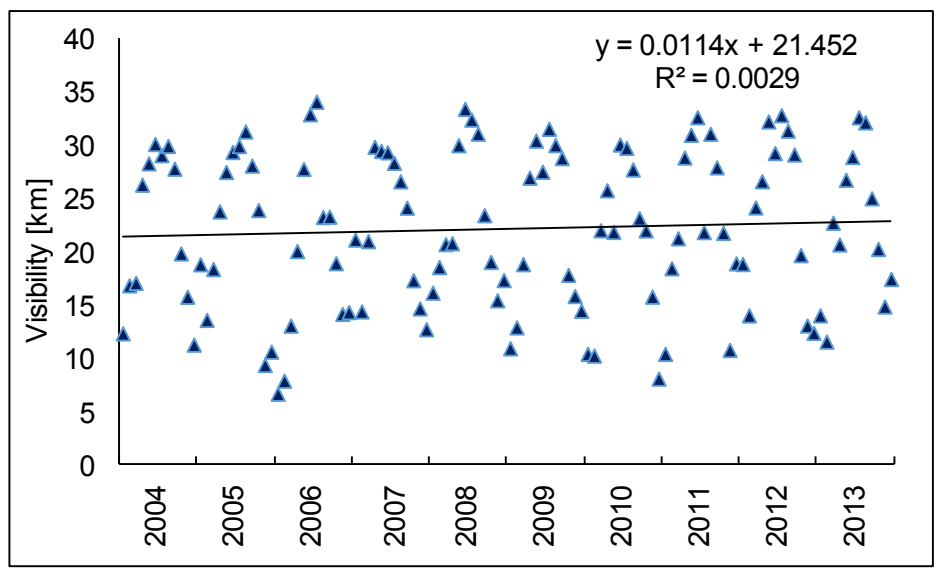

Figure 6. Visibility time series of monthly mean and its' linear regression trend over Warsaw in 2004-2013.

Since the visibility is strongly affected by air pollutants $[1,40,41]$, the presence of its' weaker variation over Warsaw from 2004-2013 is found. In Poland, considerable changes in air pollution were observed from 1980-2000 [42]. In that period, 
political and economical transformation was related with a sudden decrease of industrial emission due to large factories closure and limited production in remaining ones $[43,44]$. On the other hand, such transformation contributed to the knowledge on negative consequences of air pollution, and for this reason in 1980s industrial emissions were largely restricted in Poland. Unfortunately, no reliable air pollution measurements were then performed within the research area.
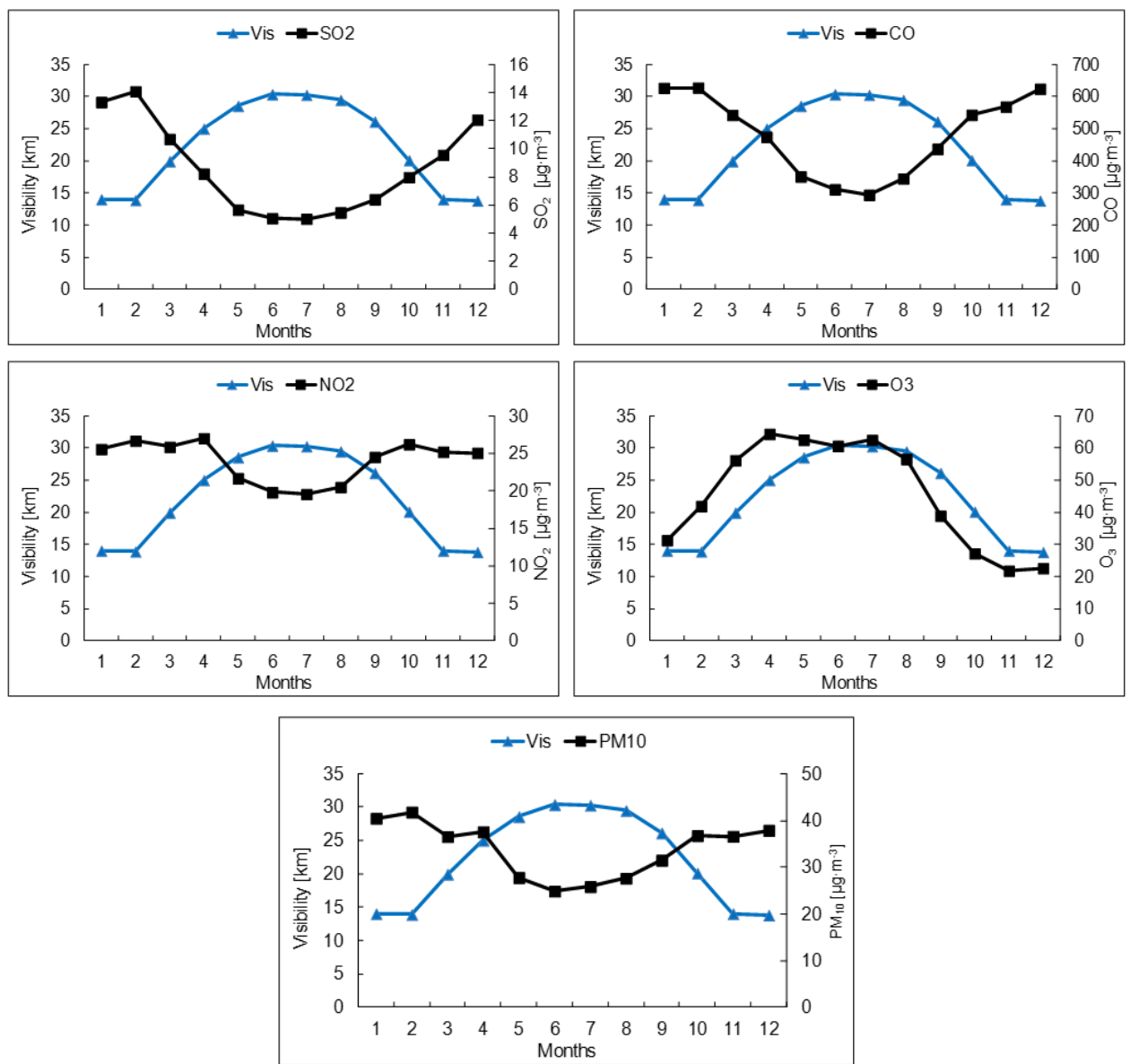

Figure 7. Monthly variations of visibility and ambient concentrations of air pollutants over Warsaw in 2004-2013.

Monthly visibilities and monthly ambient concentrations of air pollutants (averages for the whole period 2004-2013) are shown in Figure 7. Comparing the maximum and minimum values for the monthly concentrations shows that $\mathrm{O}_{3}, \mathrm{CO}$ and $\mathrm{SO}_{2}$ have the largest variability; while much lower variability was 
shown by $\mathrm{PM}_{10}$ and $\mathrm{NO}_{2}$. Visibility values were inversely proportional to all analysed pollutants, except for $\mathrm{O}_{3}$. Ambient concentrations of $\mathrm{PM}_{10}, \mathrm{SO}_{2}, \mathrm{NO}_{2}$ and $\mathrm{CO}$ tend to be strongly affected by the emission from fuel combustion for heating purposes [31,45]. It is obvious that their concentration is higher in winter months, while the visibility becomes lower (Figure 7). Except for the higher winter emission in Poland, the increase of air pollution is also attributed to meteorological conditions (lowering of air mixing layer, stagnant air) that deteriorate ventilation and ability of self-cleaning [46]. The fact that concentrations of $\mathrm{CO}, \mathrm{NO}_{2}$ and $\mathrm{SO}_{2}$ are higher in the cold season (Figure 7) is attributed to increased emissions-however, the atmospheric lifetime of these compounds is also generally longer in the winter and this is likely to explain some of the increase as well.

The mean $\mathrm{NO}_{2}$ concentration was slightly higher in the cold season than in the warm one. Such a situation was related to the low variation of the yearly $\mathrm{NO}_{2}$ emission. Typically, the data indicated two daily peaks in the ambient concentrations of both $\mathrm{NO}_{\mathrm{x}}$ forms ( $\mathrm{NO}$ and $\mathrm{NO}_{2}$ ), which pointed to the traffic-related pollution [30,39]. Emission from the traffic contributes also to ambient concentrations of volatile organic compounds (VOCs). VOCs and $\mathrm{NO}$ react with $\mathrm{O}_{3}$ giving a loss of $\mathrm{O}_{3}$ near to pollution sources. On the other hand, higher $\mathrm{O}_{3}$ in summer is probably because of higher solar insolation, and visibility is lower because of the lack of cold season emissions and boundary layer effects. For those reasons, the course of monthly ozone concentrations is different from $\mathrm{NO}_{2}$ and other pollutants, and resembles the course of visibility.

\subsection{Visibility during Periods Differing in the Air Pollution with PM}

Table 1 presents the mean values of visibility, $\mathrm{PM}_{10}$, gaseous pollutants and meteorological parameters (calculated on the basis of 1-h values from the entire research period) grouped within three categories: clear days $\left(\mathrm{PM}_{10}\right.$ concentration did not exceed $\left.50 \mu \mathrm{g} \cdot \mathrm{m}^{-3}\right)$, moderate days $\left(\mathrm{PM}_{10}\right.$ concentration was $50-200 \mu \mathrm{g} \cdot \mathrm{m}^{-3}$ ) and episode days $\left(\mathrm{PM}_{10}\right.$ concentration exceeded $\left.200 \mu \mathrm{g} \cdot \mathrm{m}^{-3}\right)$.

The $\mathrm{NO}_{2}$ concentration was 3.7 times higher during the episodes than on the clear days $\left(20.4 \mu \mathrm{g} \cdot \mathrm{m}^{-3}\right)$, while the $\mathrm{CO}$ concentration was approximately six times higher. A similar situation was observed for $\mathrm{SO}_{2}$. For $\mathrm{O}_{3}$, an inverse correlation was observed. Its lowest concentrations were found for the episode days. The episodes were characterized by low mean visibility $(6.0 \mathrm{~km})$, low air temperature, low wind speed, and higher relative air humidity. On the other hand, the clear days were characterized by the highest mean visibilities $(24.3 \mathrm{~km})$, higher wind speeds and lower relative air humidity. Episode days occurred mostly in winter, while clear days were found in warm periods, mostly in summer. Unfortunately, the researchers did not have data on the mixed layer height and atmospheric pressure. As different studies show, high atmospheric pressure leads to the lower mixed layer height and low wind speed, which causes increased pollutant concentrations close 
to the pollutant sources and visibility deterioration. Low atmospheric pressure results in the higher mixed layer height and high wind speed, which provides effective ventilation for cities and good dispersion of pollutants [6]. The research conducted by Majewski et al. [29] into the atmospheric pressure influence on the $\mathrm{PM}_{10}$ concentration in Warsaw showed that the increase in the PM concentration was significantly statistically related to the increase in the atmospheric pressure.

Table 1. Values of visibility and other parameters calculated as arithmetic means on the basis of the hourly values from 2004-2013 for three periods differing in the air pollution with PM.

\begin{tabular}{|c|c|c|c|}
\hline \multirow{2}{*}{ Parameter } & \multicolumn{3}{|c|}{ Air Quality } \\
\hline & $\begin{array}{c}\mathrm{PM}_{10}<50^{\mathrm{a}} \\
\mu \mathrm{g} \cdot \mathrm{m}^{-3} \text { Clear Days }\end{array}$ & $\begin{array}{c}\mathrm{PM}_{10}>50^{\mathrm{a}} \mu \mathrm{g} \cdot \mathrm{m}^{-3} \\
\text { Moderate Days }\end{array}$ & $\begin{array}{c}\mathrm{PM}_{10}>200^{\mathrm{b}} \\
\mu \mathrm{g} \cdot \mathrm{m}^{-3} \text { Episode }\end{array}$ \\
\hline $\begin{array}{c}\text { Occurrence } \\
\text { number (hours) }\end{array}$ & 68,652 & 14,285 & 146 \\
\hline $\mathrm{PM}_{10}\left(\mu \mathrm{g} \cdot \mathrm{m}^{-3}\right)$ & 25.7 & 75.1 & 236.1 \\
\hline $\mathrm{SO}_{2}\left(\mu \mathrm{g} \cdot \mathrm{m}^{-3}\right)$ & 6.8 & 12.6 & 28.1 \\
\hline $\mathrm{CO}\left(\mu \mathrm{g} \cdot \mathrm{m}^{-3}\right)$ & 378.8 & 822.1 & 2342.4 \\
\hline $\mathrm{NO}_{2}\left(\mu \mathrm{g} \cdot \mathrm{m}^{-3}\right)$ & 20.4 & 40.5 & 76.0 \\
\hline $\mathrm{O}_{3}\left(\mu \mathrm{g} \cdot \mathrm{m}^{-3}\right)$ & 48.5 & 28.9 & 14.0 \\
\hline Visibility $(\mathrm{km})$ & 24.3 & 13.6 & 6.0 \\
\hline Temperature $\left({ }^{\circ} \mathrm{C}\right)$ & 10.5 & 4.7 & -6.9 \\
\hline Wind speed $\left(\mathrm{m} \cdot \mathrm{s}^{-1}\right)$ & 2.7 & 1.7 & 1.1 \\
\hline Relative humidity (\%) & 73.4 & 77.7 & 78.9 \\
\hline
\end{tabular}

a Permissible level for the 24-h $\mathrm{PM}_{10}$ concentration due to the human health protection in Poland. ${ }^{b}$ Threshold value for informing the inhabitants about the risk of exceeding the alert level for $\mathrm{PM}_{10}$ in Poland.

\subsection{Weekend/Weekday Differences in Visibility and Air Pollution}

When referring to the visibility and air quality studies, there is a phenomenon known as the weekend effect, Studies on this phenomenon can help to better understand the emission characteristics of air pollutants in urban areas and the weekend effect has been reported in America since the 1970s [47,48]. Contemporary research is especially focusing on visibility variations and the effect of air quality on visibility on weekdays compared with weekend days. In order to investigate potential weekend effect over Warsaw, mean weekend and weekday levels of visibility as well as air pollutants were calculated and subjected to Fisher-Snedecore test. Visibilities on weekends were slightly better than on weekdays, and the differences were statistically significant. Mean hourly visibility was equal to 22.61 $\pm 12.57 \mathrm{~km}$ at weekends and slightly over $22.02 \pm 12.71$ on weekdays (Table 2 ). For $\mathrm{PM}_{10}$, slightly higher and statistically significant weekday concentrations were observed, as well as for $\mathrm{SO}_{2}$, which was slightly lower during weekends. We also 
found $\mathrm{CO}$ concentrations to become lower on weekends (statistically significant differences) and, moreover, mean weekend and weekday $\mathrm{NO}_{2}$ concentrations over Warsaw showed statistically significant differences, with $\mathrm{NO}_{2}$ concentration higher on weekdays. This is probably due to less vehicular emission on weekends.

All concentrations of airborne pollutants were higher during weekdays, (statistically significant differences), except ozone. $\mathrm{O}_{3}$ concentration was higher at weekends even though the $\mathrm{O}_{3}$ pollutant precursor concentrations (such as $\mathrm{NO}_{x}$ and volatile organic compounds) are lower on weekends $[47,48]$. The weekend effect in the $\mathrm{O}_{3}$ concentrations is the most likely to be attributable to decreased $\mathrm{O}_{3}$ destruction by $\mathrm{NO}_{x}$, as there are lower emissions from its' main source-communication and vehicular transportation $[48,49]$. The result of this study, concerning lower concentrations of airborne pollutants on weekends, excluding ozone, is similar to that obtained by Tsai [17].

Table 2. Visibility and air pollutant concentrations calculated as arithmetic means on the basis of the hourly values from 2004-2013 for two different periods-weekends and week days.

\begin{tabular}{|c|c|c|c|c|c|c|}
\hline & Vis & $\begin{array}{c}\mathrm{PM}_{10} \\
\left(\mu \mathrm{g} \cdot \mathrm{m}^{-3}\right)\end{array}$ & $\begin{array}{c}\mathrm{SO}_{2} \\
\left(\mu \mathrm{g} \cdot \mathrm{m}^{-3}\right)\end{array}$ & $\begin{array}{c}\mathrm{CO} \\
\left(\mu \mathrm{g} \cdot \mathrm{m}^{-3}\right)\end{array}$ & $\begin{array}{c}\mathrm{NO}_{2} \\
\left(\mu \mathrm{g} \cdot \mathrm{m}^{-3}\right)\end{array}$ & $\mathrm{O}_{3}\left(\mu \mathrm{g} \cdot \mathrm{m}^{-3}\right)$ \\
\hline Weekend & $\begin{array}{c}22.61 \pm 12.57 \\
(n=25,044)\end{array}$ & $\begin{array}{c}32.03 \pm 24.20 \\
(n=23,652)\end{array}$ & $\begin{array}{l}8.08 \pm 6.95 \\
(n=24,485)\end{array}$ & $\begin{array}{c}456.45 \pm \\
327.25 \\
(n=23,782)\end{array}$ & $\begin{array}{c}20.20 \pm 15.74 \\
(n=24,585)\end{array}$ & $\begin{array}{c}48.94 \pm 29.46 \\
(n=24,444)\end{array}$ \\
\hline Weekday & $\begin{array}{c}22.02 \pm 12.71 \\
(n=62,590)\end{array}$ & $\begin{array}{c}34.66 \pm 25.38 \\
(n=59,364)\end{array}$ & $\begin{array}{l}8.75 \pm 8.15 \\
(n=60,931)\end{array}$ & $\begin{array}{c}485.49 \pm \\
340.51 \\
(n=59,495)\end{array}$ & $\begin{array}{c}25.50 \pm 17.60 \\
(n=61,400)\end{array}$ & $\begin{array}{c}44.51 \pm 29.94 \\
(n=60,704)\end{array}$ \\
\hline $\begin{array}{c}\text { Fisher test } \\
p \text {-value }\end{array}$ & $\begin{array}{l}62,590 \\
0.0000\end{array}$ & $\begin{array}{l}59,364 \\
0.0000\end{array}$ & $\begin{array}{l}60,931 \\
0.0000\end{array}$ & $\begin{array}{l}59,495 \\
0.0000\end{array}$ & $\begin{array}{l}61,400 \\
0.0000\end{array}$ & $\begin{array}{l}60,704 \\
0.0000\end{array}$ \\
\hline
\end{tabular}

\subsection{Correlations between Pollutants, Meteorological Variables and Visibility}

Total correlations found between the results of the visibility measurements and other measurements (mean hourly values) in the whole research period are shown in Table 3. The visibility measurement results were negatively correlated with $\mathrm{CO}, \mathrm{PM}_{10}, \mathrm{SO}_{2}$ and $\mathrm{NO}_{2}$. Therefore, the increase in $\mathrm{CO}$ and $\mathrm{SO}_{2}$ concentrations corresponds with visibility decrease, and $\mathrm{PM}_{10}$ and $\mathrm{NO}_{2}$ are those species, that can directly contribute to visual range limitations.

Visibility was positively correlated with the $\mathrm{O}_{3}$ concentrations. The atmospheric $\mathrm{O}_{3}$ is a secondary air pollutant formed in photochemical reactions. Hence, summer is the period of the most intense $\mathrm{O}_{3}$ formation. It resulted from the high insolation intensity during this season. The observed correlation was caused by the fact that both parameters (visibility and $\mathrm{O}_{3}$ concentrations) increased and decreased in the same periods (Figure 7). 
Table 3. Correlation matrix for total parameters measured for the entire period of 2004-2013 (correlations calculated for hourly values) and arithmetic means and standard deviations of the hourly value sets for the measured parameters.

\begin{tabular}{ccccccccccccc}
\hline & $\mathbf{A}$ & $\mathbf{S D}$ & $\mathbf{P M}_{\mathbf{1 0}}$ & $\mathbf{S O}_{2}$ & $\mathbf{C O}$ & $\mathbf{N O}_{2}$ & $\mathbf{O}_{\mathbf{3}}$ & $\mathbf{T}$ & Ws & $\mathbf{R H}$ & $\mathbf{R a d}$ & $\mathbf{P}$ \\
\hline Vis $(\mathrm{km})$ & 22.19 & 12.67 & -0.33 & -0.27 & -0.37 & -0.21 & 0.47 & 0.52 & 0.14 & -0.60 & 0.37 & -0.10 \\
$\mathrm{PM}_{10}\left(\mu \mathrm{g} \cdot \mathrm{m}^{-3}\right)$ & 33.91 & 25.07 & 1.00 & 0.37 & 0.66 & 0.56 & -0.26 & -0.23 & -0.25 & 0.05 & -0.13 & -0.04 \\
$\mathrm{SO}_{2}\left(\mu \mathrm{g} \cdot \mathrm{m}^{-3}\right)$ & 8.56 & 7.83 & & 1.00 & 0.34 & 0.25 & -0.15 & -0.38 & -0.10 & 0.10 & -0.07 & -0.03 \\
$\mathrm{CO}\left(\mu \mathrm{g} \cdot \mathrm{m}^{-3}\right)$ & 477.20 & 337.03 & & & 1.00 & 0.62 & -0.41 & -0.38 & -0.28 & 0.21 & -0.25 & -0.03 \\
$\mathrm{NO}_{2}\left(\mu \mathrm{g} \cdot \mathrm{m}^{-3}\right)$ & 23.98 & 17.26 & & & & 1.00 & -0.52 & -0.19 & -0.36 & 0.15 & -0.31 & -0.03 \\
$\mathrm{O}_{3}\left(\mu \mathrm{g} \cdot \mathrm{m}^{-3}\right)$ & 45.78 & 29.87 & & & & & 1.00 & 0.47 & 0.22 & -0.71 & 0.56 & 0.02 \\
$\mathrm{~T}\left({ }^{\circ} \mathrm{C}\right)$ & 9.33 & 9.33 & & & & & & 1.00 & 0.03 & -0.52 & 0.48 & 0.05 \\
$\mathrm{Ws}(\mathrm{m} / \mathrm{s})$ & 2.42 & 1.68 & & & & & & & 1.00 & -0.08 & 0.16 & 0.04 \\
$\mathrm{RH}(\%)$ & 74.97 & 18.82 & & & & & & & & 1.00 & -0.58 & 0.06 \\
$\mathrm{Rad}\left(\mathrm{W} / \mathrm{m}^{2}\right)$ & 117.77 & 200.98 & & & & & & & & & 1.00 & -0.03 \\
$\mathrm{P}(\mathrm{mm})$ & 0.07 & 0.52 & & & & & & & & & & 1.00 \\
\hline
\end{tabular}

Notes: A: Mean value; SD: Standard deviation; Vis: Visibility; T: Temperature; RH: Relative air humidity; Ws: Wind speed; Rad: Insolation intensity; P: Precipitation.

In addition to their impact on visibility via changing the concentration of pollutants, meteorological variables can affect the visibility more directly - as humidity increases, hygroscopic aerosols increase in size and thus the scattering of light by them increases. At some point, aerosols activate and become fog [50,51]. This transition is very complex and has a very large impact on visibility [50,51].

Hourly visibility exhibited low, negative correlation with precipitation (Table 3). Generally, the precipitation lowered the air pollutant concentrations through the precipitation scavenging. Thereby, visibility increased [52]. However, the air purification effect and related visibility improvement appears with a delay after rainy days. During heavy precipitation visibility will be reduced. However, after the precipitation has stopped, the aerosols concentration are likely to be lower, thus giving two opposite impacts with a visibility increase following rain. In fact, the visibility reduction is more likely caused by the scattering of light by the hydrometeors. There is a negative correlation between precipitation and the primary pollutants, but this is weak and may relate to improved boundary layer ventilation when there is precipitation rather than scavenging. This is suggested by the fact that the relatively insoluble $\mathrm{CO}$ and $\mathrm{NO}_{2}$ have a negative correlation with precipitation, which is the same size as that of the more soluble $\mathrm{SO}_{2}$.

Visibility was positively correlated with the three remaining meteorological parameters, air temperature, insolation intensity, and wind speed. Most likely under clear sky conditions, the temperatures increase, the relative humidity falls and so the aerosols shrink, thus increasing the visibility. 


\subsection{Principal Component Analysis (PCA)}

PCA served to extract four principal components (new variables: PC1, PC2, PC3 and PC4) with eigenvalues $>1.0$, which accounted for $73 \%$ of the total variance (Table 4). PC1, PC2, PC3 and PC4 can be interpreted as major factors that control visibility $[1,6,17,40]$. Visibility (Vis), $\mathrm{O}_{3}$ concentration $\left(\mathrm{O}_{3}\right), \mathrm{NO}_{2}$ concentration $\left(\mathrm{NO}_{2}\right), \mathrm{CO}$ concentration $(\mathrm{CO})$, air temperature $(\mathrm{T})$, relative air humidity $(\mathrm{RH})$, $\mathrm{PM}_{10}$ concentration $\left(\mathrm{PM}_{10}\right)$ and insolation intensity (Rad) were most strongly correlated with PC1. Knowing the research area and the influence of various emission sources in this region, it can be assumed that PC1 could be related to fuel combustion for heating (mainly hard coal, wood/biomass and heating/crude oil) $[31,44]$. The emission increased over the year along with the $\mathrm{T}$ drop and Rad decrease. At the same time, the pollutant concentrations (i.e., $\mathrm{PM}_{10}, \mathrm{NO}_{2}, \mathrm{CO}$, or $\mathrm{SO}_{2}$ ) in the air increased. Simultaneously, the photochemical pollutant concentrations (represented by $\mathrm{O}_{3}$ ) decreased. Visibility was reduced in periods of high air pollution, related with heating (opposite signs for Vis/PC1 correlation and $\mathrm{PM}_{10}, \mathrm{NO}_{2}, \mathrm{CO}$, $\mathrm{SO}_{2} /$ PC1 correlation).

The PCA performed only for the cold season data (Table 5) confirmed the same correlations between PC1 and the air pollution with $\mathrm{NO}_{2}, \mathrm{SO}_{2}$ and $\mathrm{CO}$, and the inverse (opposite signs) correlation between $\mathrm{PC} 1$ and visibility, $\mathrm{O}_{3}$ concentration, air temperature and insolation. For the warm season, the PCA revealed a very strong correlation between $\mathrm{PC} 1$ and $\mathrm{NO}_{2}$ and an inverse strong correlation (opposite signs) between $\mathrm{PC} 1$ and visibility, $\mathrm{O}_{3}$ concentration and insolation. Most likely, in those periods when there is less pollution from heating, and temperature rises, air pollution is mainly shaped by traffic emissions [31]. Then, the increase of $\mathrm{NO}_{2}$ is observed, which reacts with $\mathrm{O}_{3}$ and in consequence the concentration of $\mathrm{O}_{3}$ in the air decreases. The reaction intensity becomes higher when the insolation intensity is stronger $\left(\mathrm{O}_{3}\right.$ concentrations and insolation were correlated with $\mathrm{PC} 1$ in the same way). Under such conditions, visibility may be improved (contrary signs of Vis/PC1 and $\mathrm{NO}_{2} / \mathrm{PC} 1$ ). Nonetheless, it is not possible to discuss the cause-and-effect correlation between the traffic emission (and the related photochemical reactions) and the visibility increase or decrease. Most probably, visibility increased because the air pollution with PM was lower and the temperature and insolation were higher in summer.

PC2 was most strongly but differently (opposite signs) correlated with the $\mathrm{PM}_{10}$ concentrations and relative air humidity (Table 4). Visibility, pollutant concentrations, temperature and insolation were correlated with PC2 in the same way as $\mathrm{PM}_{10}$ but to a lesser extent. Thus, PC2 reflected the situation when the concentrations of all the observed pollutants and visibility decreased whereas the relative air humidity and precipitation increased. While humidity increases, hygroscopic aerosols increase in size and thus the scattering of light by them increases, so visibility drops. Air 
humidity concentration in the air was high due to precipitation at high temperature. During precipitation, concentrations of all the pollutants decreased due to leaching. The situation concerned $\mathrm{PM}_{10}$ to the largest extent.

Table 4. Correlations between the principal components (PC1, PC2, PC3, PC4) and measured parameter values. The PCA was performed for the hourly data collected in 2004-2013.

\begin{tabular}{ccccc}
\hline Component & PC1 & PC2 & PC3 & PC4 \\
\hline Vis & $\mathbf{0 . 7}$ & 0.3 & 0.2 & 0.2 \\
$\mathrm{PM}_{10}$ & $-\mathbf{0 . 6}$ & $\mathbf{0 . 6}$ & -0.1 & -0.1 \\
$\mathrm{SO}_{2}$ & -0.4 & 0.3 & $-\mathbf{0 . 6}$ & 0.0 \\
$\mathrm{CO}$ & $\mathbf{- 0 . 7}$ & 0.5 & 0.0 & -0.1 \\
$\mathrm{NO}_{2}$ & $-\mathbf{0 . 7}$ & 0.5 & 0.3 & 0.0 \\
$\mathrm{O}_{3}$ & $\mathbf{0 . 8}$ & 0.3 & -0.3 & -0.1 \\
$\mathrm{~T}$ & $\mathbf{0 . 7}$ & 0.3 & 0.4 & -0.2 \\
$\mathrm{Ws}$ & 0.4 & -0.3 & -0.5 & 0.0 \\
$\mathrm{RH}$ & $-\mathbf{0 . 7}$ & $\mathbf{- 0 . 6}$ & 0.1 & 0.0 \\
$\mathrm{Rad}$ & $\mathbf{0 . 6}$ & 0.4 & -0.3 & -0.1 \\
$\mathrm{P}$ & 0.0 & -0.2 & 0.0 & $-\mathbf{0 . 9}$ \\
eigenvalue & 4.06 & 1.79 & 1.12 & 1.02 \\
\% total variance & 37 & 16 & 10 & 9 \\
Cumul. \% variance & 37 & 53 & 63 & 73 \\
\hline
\end{tabular}

Table 5. Correlations between the principal components (PC1, PC2, PC3) and measured parameter values. The PCA was performed for the hourly data collected in 2004-2013, separately for the cold (January-March and October-December) and warm (April-September) seasons.

\begin{tabular}{ccccccccc}
\hline Component & Season & PC1 & PC2 & PC3 & Season & PC1 & PC2 & PC3 \\
\hline Vis. & & $-\mathbf{0 . 7}$ & 0.3 & -0.1 & & -0.5 & -0.3 & -0.5 \\
$\mathrm{PM}_{10}$ & & $\mathbf{0 . 7}$ & 0.5 & -0.1 & & 0.4 & $-\mathbf{0 . 7}$ & 0.2 \\
$\mathrm{SO}_{2}$ & & 0.4 & 0.4 & 0.4 & & 0.1 & -0.4 & 0.5 \\
$\mathrm{CO}$ & & $\mathbf{0 . 8}$ & 0.4 & -0.1 & & $\mathbf{0 . 6}$ & -0.5 & 0.1 \\
$\mathrm{NO}_{2}$ & & $\mathbf{0 . 7}$ & 0.3 & -0.3 & & $\mathbf{0 . 7}$ & -0.5 & -0.1 \\
$\mathrm{O}_{3}$ & & $-\mathbf{0 . 7}$ & 0.4 & 0.4 & & $-\mathbf{0 . 8}$ & -0.2 & 0.2 \\
$\mathrm{~T}$ & cold & -0.4 & -0.1 & $-\mathbf{0 . 8}$ & warm & $-\mathbf{0 . 6}$ & -0.4 & 0.0 \\
$\mathrm{Ws}$ & & $-\mathbf{0 . 6}$ & 0.0 & 0.0 & & -0.4 & 0.3 & 0.4 \\
$\mathrm{RH}$ & & 0.5 & $-\mathbf{0 . 8}$ & 0.1 & & $\mathbf{0 . 7}$ & $\mathbf{0 . 6}$ & 0.0 \\
$\mathrm{Rad}$ & & -0.4 & $\mathbf{0 . 6}$ & -0.1 & & $-\mathbf{0 . 7}$ & -0.2 & 0.2 \\
$\mathrm{P}$ & & 0.0 & -0.3 & 0.0 & & 0.0 & 0.2 & $\mathbf{0 . 6}$ \\
Eigen & & 3.59 & 1.84 & 1.12 & & 3.46 & 1.99 & 1.14 \\
value & & & & & & & \\
\% total & & & 17 & 10 & & $31 \%$ & $18 \%$ & $10 \%$ \\
variance & & & & & & & & \\
\hline
\end{tabular}




\subsection{Generalized Regression Model (GRM)}

The GRM identification was performed to finally confirm the influence of the analysed factors on visibility. It concerned the observations of the measured parameters and other defined factors, such as the influence of a season or specific year or combination of these factors. It was assumed that a given factor would be introduced into the model, if the value $F$ ( $F$-Fischer-Snecedor distribution) characterizing the significance of the factor contribution into the dependable variable forecasting (visibility) was higher than $F 1$. The factor was removed if its $F$ was lower than F2 (Table 6).

Table 6. Generalized Regression Model (GRM) summary: Variables introduced into the model due to estimation.

\begin{tabular}{|c|c|c|c|c|c|c|c|}
\hline Variable & $\begin{array}{l}\text { Model } \\
\text { Steps }\end{array}$ & $\begin{array}{l}\text { Degrees of } \\
\text { Freedom }\end{array}$ & $F 2$ for out & $p 2$ for out & $F 1$ for in & P1 for in & Effect \\
\hline $\mathrm{RH}$ & 16 & 1 & 1594.25 & 0.00000 & & & In model \\
\hline $\ln \mathrm{PM}_{10}$ & & 1 & 389.95 & 0.00000 & & & In model \\
\hline Season & & 1 & 64.60 & 0.00000 & & & In model \\
\hline $\begin{array}{c}\text { Precipitation } \\
Y \mid N\end{array}$ & & 1 & 233.19 & 0.00000 & & & In model \\
\hline $\mathrm{O}_{3}$ & & 1 & 66.46 & 0.00000 & & & In model \\
\hline $\operatorname{lnCO}$ & & 1 & 92.79 & 0.00000 & & & In model \\
\hline $\mathrm{T}$ & & 1 & 24.39 & 0.00000 & & & In model \\
\hline Rad & & 1 & 41.06 & 0.00000 & & & In model \\
\hline Ws & & 1 & 36.40 & 0.00000 & & & In model \\
\hline YEAR & & 9 & 6.42 & 0.00000 & & & In model \\
\hline $\begin{array}{l}\text { Year * } \\
\text { Season }\end{array}$ & & 9 & 5.78 & 0.00000 & & & In model \\
\hline $\operatorname{lnSO} 2$ & & 1 & 21.37 & 0.00000 & & & In model \\
\hline $\begin{array}{c}\text { Year }^{*} \\
\text { Prec.YIN }\end{array}$ & & 9 & 3.68 & 0.00013 & & & In model \\
\hline $\begin{array}{l}\text { Season * } \\
\text { Prec.YIN }\end{array}$ & & 1 & 13.53 & 0.00023 & & & In model \\
\hline $\begin{array}{l}\ln \mathrm{NO}_{2} \\
\text { Year * }\end{array}$ & & 1 & 10.67 & 0.00109 & & & In model \\
\hline $\begin{array}{c}\text { Year* } \\
\text { Season * } \\
\text { Prec.YIN }\end{array}$ & & 9 & & & 0.629 & 0.773 & $\begin{array}{l}\text { Out of } \\
\text { model }\end{array}$ \\
\hline
\end{tabular}

Before the identification, the variables underwent necessary analyses and transformations. The variables that were at least in the interval scales were submitted to the quality assessment and logarithming (Box-Cox transformation with the Lambda parameter $=0.5$ ). The precipitation variable was taken to the nominal scale, where 0 and 1 meant the hours without and with precipitation, respectively (variable: Prec.Y $\mid \mathrm{N}$ ).

Table 6 presents the results of the stepwise estimation for the GRM. The variables, seasonal factors and their interactions marked "in model" turned out to be significantly affecting visibility. Hence, they were introduced into the model. Table 7 presents the adjustment of the model that was finally selected as the best one 
with consideration for the maximum adjustment criterion and minimum number of the independent variables.

Table 7. Assessment of the GRM adjustment.

\begin{tabular}{|c|c|c|c|c|c|c|c|c|c|c|c|}
\hline & Multipl.-R & Multipl.- $\mathbf{R}^{2}$ & Correct.- $\mathrm{R}^{2}$ & SS-Model & df-Model & MS-Model & SS-Rest & Df-Rest & MS-Rest & $F$ & $p$ \\
\hline Vis & 0.75 & 0.56 & 0.56 & 6499948 & 39 & 166665.3 & 5033049 & 71764 & 70.133 & 2376.4 & 0.00 \\
\hline
\end{tabular}

To picture the significance of the variables, they were ordered following the values of the Student's $t$-distribution for the assessment of the model parameter significance (Figure 8-Pareto chart). The higher the $t$-value was, the more important the significance of the factor was for explaining its influence on visibility. Relative air humidity and $\mathrm{PM}_{10}$ were two most important factors affecting visibility on the basis of the identified model. The influence exerted by $\mathrm{SO}_{2}$ and $\mathrm{NO}_{2}$ on visibility was much lower from the impact that other analysed pollutants had. The results also show the influence of the seasonality, atmospheric precipitation (its presence or lack; variable: Prec.Y IN) and interactions between the periods (e.g., season * month) on visibility.

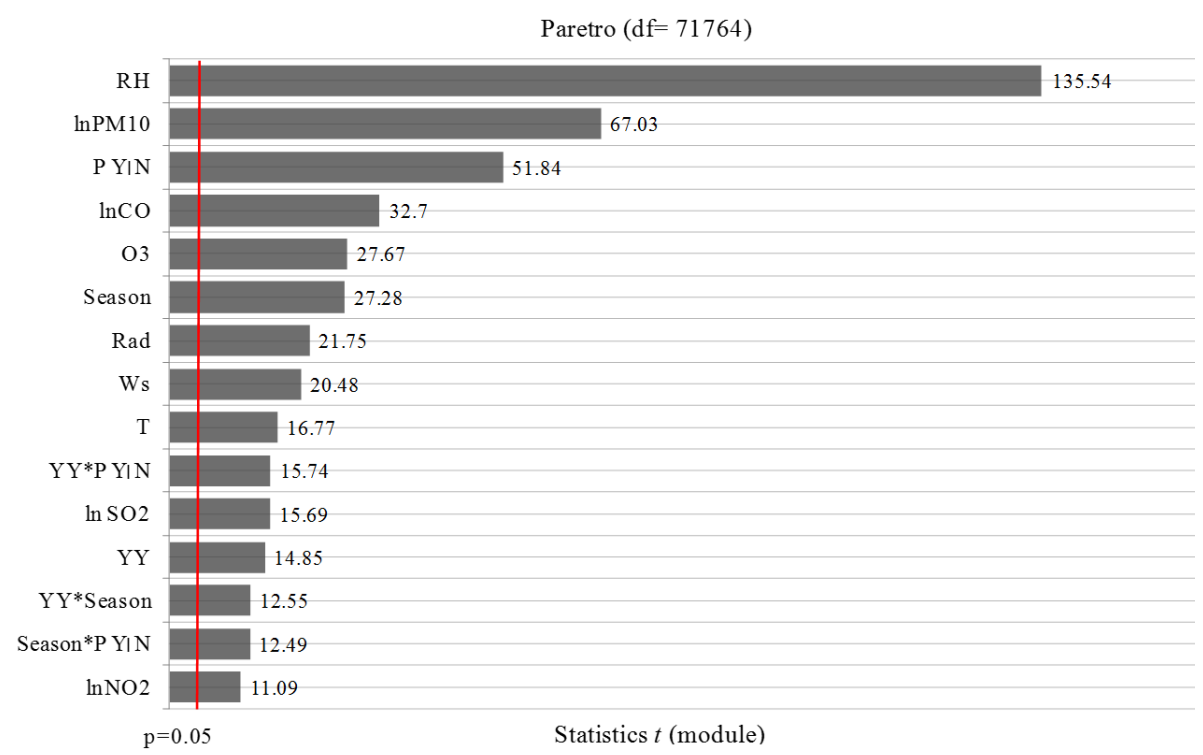

Figure 8. Pareto chart for the significance of factors affecting visibility in the GRM.

Table 8 presents estimated parameter values for the selected GRM (Tables 6 and 7), together with estimations for the parameter errors, $t$-distribution and $p$-value indicating the variable significance and coincidence with visibility. In a statistical sense, it was shown that the relative air humidity and wind speed variations 
corresponded with visibility more than the temperature. It was also revealed that visibility was most likely sensitive to the changes in the $\mathrm{PM}_{10}$ and $\mathrm{CO}$ concentrations. Specifically, a 50\% drop in the $\mathrm{PM}_{10}$ concentration could be associated with visibility improvement by $2.9 \mathrm{~km}$. On the other hand, a drop in the CO concentration by $50 \%$ corresponded with the visibility being increased by $2.2 \mathrm{~km}$, however, there was no evidence for a direct cause-and-effect relationship. The $50 \%$ decrease in the $\mathrm{O}_{3}$ concentration might only slightly affect the visibility increase, as suggested by the performed regression analyses.

Table 8. Estimated parameter values for the selected GRM with estimations of parameter errors, $t$-distribution and $p$-value indicating the variable significance.

\begin{tabular}{|c|c|c|c|c|c|}
\hline $\begin{array}{c}\text { Regression } \\
\text { Coefficien }\end{array}$ & Level/Effect & $\begin{array}{c}\text { Estimated } \\
\text { Regression } \\
\text { Coefficient }\end{array}$ & $\begin{array}{l}\text { Estimated } \\
\text { Standard } \\
\text { Deviation }\end{array}$ & $t$ & $p$ Value \\
\hline Intercept & & 82.615 & 0.633 & 130.468 & 0.000 \\
\hline $\operatorname{lnSO} \mathrm{O}_{2}$ & & -0.902 & 0.057 & -15.692 & 0.000 \\
\hline $\ln \mathrm{NO}_{2}$ & & 0.827 & 0.075 & 11.089 & 0.000 \\
\hline $\ln \mathrm{CO}^{2}$ & & -3.122 & 0.095 & -32.699 & 0.000 \\
\hline $\mathrm{O}_{3}$ & & -0.054 & 0.002 & -27.673 & 0.000 \\
\hline $\mathrm{T}$ & & 0.097 & 0.006 & 16.766 & 0.000 \\
\hline Ws & & 0.450 & 0.022 & 20.479 & 0.000 \\
\hline $\mathrm{RH}$ & & -0.395 & 0.003 & -135.537 & 0.000 \\
\hline $\mathrm{Rad}$ & & -0.005 & 0.000 & -21.751 & 0.000 \\
\hline $\ln \mathrm{PM}_{10}$ & & -4.220 & 0.063 & -67.032 & 0.000 \\
\hline$Y Y^{10}$ & 2004 & 0.727 & 0.163 & 4.467 & 0.000 \\
\hline$Y Y$ & 2005 & -0.736 & 0.164 & -4.495 & 0.000 \\
\hline$Y Y$ & 2006 & -1.829 & 0.168 & -10.867 & 0.000 \\
\hline$Y Y$ & 2007 & 0.880 & 0.175 & 5.024 & 0.000 \\
\hline$Y Y$ & 2008 & 0.913 & 0.165 & 5.540 & 0.000 \\
\hline$Y Y$ & 2009 & 2.028 & 0.137 & 14.847 & 0.000 \\
\hline$Y Y$ & 2010 & -1.619 & 0.162 & -9.997 & 0.000 \\
\hline YY & 2011 & 1.174 & 0.115 & 10.182 & 0.000 \\
\hline$Y Y$ & 2012 & -0.287 & 0.142 & -2.016 & 0.044 \\
\hline Season & warm & 1.695 & 0.062 & 27.284 & 0.000 \\
\hline Prec.Y|N & Non-raining & 2.698 & 0.052 & 51.837 & 0.000 \\
\hline Year * Season & 1 & 0.197 & 0.096 & 2.048 & 0.041 \\
\hline Year* Season & 2 & -0.857 & 0.095 & -9.028 & 0.000 \\
\hline Year* Season & 3 & -1.132 & 0.093 & -12.119 & 0.000 \\
\hline Year* Season & 4 & -0.189 & 0.104 & -1.815 & 0.070 \\
\hline Year* Season & 5 & -1.052 & 0.105 & -10.023 & 0.000 \\
\hline Year* Season & 6 & -0.276 & 0.092 & -3.010 & 0.003 \\
\hline Year * Season & 7 & 0.814 & 0.101 & 8.036 & 0.000 \\
\hline Year* Season & 8 & 0.531 & 0.093 & 5.730 & 0.000 \\
\hline Year* Season & 9 & 1.121 & 0.089 & 12.555 & 0.000 \\
\hline Year* Prec.YIN & 1 & 0.433 & 0.162 & 2.675 & 0.007 \\
\hline Year * Prec.YIN & 2 & 0.714 & 0.163 & 4.386 & 0.000 \\
\hline Year* Prec.YIN & 3 & -0.081 & 0.167 & -0.487 & 0.626 \\
\hline Year * Prec.YIN & 4 & 0.368 & 0.173 & 2.127 & 0.033 \\
\hline
\end{tabular}


Table 8. Cont.

\begin{tabular}{cccccc}
\hline $\begin{array}{c}\text { Regression } \\
\text { Coefficien }\end{array}$ & Level/Effect & $\begin{array}{c}\text { Estimated } \\
\text { Regression } \\
\text { Coefficient }\end{array}$ & $\begin{array}{c}\text { Estimated } \\
\text { Standard } \\
\text { Deviation }\end{array}$ & $t$ & $p$ Value \\
Year * Prec.Y|N & 5 & -0.136 & 0.161 & -0.843 & 0.399 \\
Year * Prec.Y|N & 6 & 0.132 & 0.137 & 0.964 & 0.335 \\
Year * Prec.Y|N & 7 & 1.426 & 0.161 & 8.880 & 0.000 \\
Year* Prec.Y|N & 8 & -1.756 & 0.112 & -15.736 & 0.000 \\
Year * Prec.Y|N & 9 & -0.045 & 0.140 & -0.319 & 0.750 \\
Season * & 1 & 0.624 & 0.050 & 12.487 & 0.000 \\
Prec.Y|N & & & & & \\
\hline
\end{tabular}

The results of the GRM analysis, performed herein, correspond with previous, abundant research, pointing that visibility degradation is due to particulate matter, as well as relative humidity, that can greatly enhance degradation in the presence of hygroscopic aerosols $[6,17,53]$.

\section{Conclusions}

In Warsaw, the changes in the visibility exhibited a seasonal character. The visibility increased in summer and decreased in late autumn and winter. Mean seasonal visibilities were $24.5,30.1,20.1$, and $13.9 \mathrm{~km}$ in spring, summer, autumn, and winter, respectively. The mean yearly visibility values were in the range of $19.8-23.7 \mathrm{~km}$.

When the meteorological conditions were unfavourable for dispersion and transportation, the visibility was adversely affected by high pollutant concentrations $\left(\mathrm{PM}_{10}-236.1 \mu \mathrm{g} \cdot \mathrm{m}^{-3} ; \mathrm{SO}_{2}-28.1 \mu \mathrm{g} \cdot \mathrm{m}^{-3} ; \mathrm{CO}-2342.4 \mu \mathrm{g} \cdot \mathrm{m}^{-3}\right.$; and $\left.\mathrm{NO}_{2}-76 \mu \mathrm{g} \cdot \mathrm{m}^{-3}\right)$. Consequently, the visibility was low $(6.0 \mathrm{~km})$. The unfavourable meteorological conditions involved low wind speed (approx. $1.1 \mathrm{~m} / \mathrm{s}$ ) and low air temperature.

On clear days, air quality was found to be good. Mean pollutant concentrations were: $\mathrm{PM}_{10}-25.7 \mu \mathrm{g} \cdot \mathrm{m}^{-3} ; \mathrm{SO}_{2}-6.8 \mu \mathrm{g} \cdot \mathrm{m}^{-3} ; \mathrm{CO}-378.8 \mu \mathrm{g} \cdot \mathrm{m}^{-3}$; and $\mathrm{NO}_{2}-20.4 \mu \mathrm{g} \cdot \mathrm{m}^{-3}$. Mean wind speed was $2.7 \mathrm{~m} / \mathrm{s}$. Generally, the mean visibility value was $24.3 \mathrm{~km}$ for the good air quality days in Warsaw.

PCA helped to find that the biggest changes in the visibility in Warsaw were observed with changes in air temperature, concentrations of $\mathrm{PM}_{10}, \mathrm{CO}, \mathrm{NO}_{2}$ and $\mathrm{O}_{3}$, and insolation. Generally, in the cold season, a fall in temperature corresponded to air pollution increase and visibility deterioration. An underlying cause for such a situation is the increase of emissions, related with fuel combustion for heating purposes.

There might also be an indirect correlation between the visibility and traffic emission in the warm (non-heating) season in Warsaw. Traffic emission influence on air quality in the warm season manifested itself with the increasing $\mathrm{NO}_{2}$ concentrations in the air without simultaneous increase of other pollutants' 
concentration. At the same time, there existed a decrease in concentrations of ozone, reacting photochemically under strong insolation. In such conditions the visibility increased.

Those conclusions correspond well with the GRM analyses, which demonstrated that the visibility in Warsaw was clearly affected by the measurement season and the factors-variables containing combinations of variables constructed from different measurement periods (e.g., season * month).

It was unequivocally proven that the $\mathrm{PM}_{10}$ concentration was the most important parameter affecting the visibility in Warsaw. The GRM results demonstrated that the reduction in the $\mathrm{PM}_{10}$ concentrations by $50 \%$ (with all the remaining parameters unchanged) contributed to the increase in the visibility by $2.9 \mathrm{~km}$.

Acknowledgments: The work was carried out within the project No 2012/07/D/ST10/02895 (ID 202319) financed by the National Science Centre Poland (NCN). This research was supported by The Faculty of Civil and Environmental Engineering basic (statutory) research projects.

Author Contributions: Conceived and designed the experiments: (GM). All authors (GM, WR-K, PC, AB, AB) contributed to the data analysis and manuscript writing.

Conflicts of Interest: The authors declare that there is no conflict of interests regarding the publication of this paper.

\section{References}

1. Deng, J.; Xing, Z.; Zhuang, B.; Du, K. Comparative study on long-term visibility trend and its affecting factors on both sides of the Taiwan Strait. Atmos. Res. 2014, 143, 266-278.

2. Huang, W.; Tan, J.; Kan, H.; Zhao, N.; Song, W.; Song, G.; Chen, G.; Jiang, L.; Jiang, C.; Chen, R.; et al. Visibility, air quality and daily mortality in Shanghai, China. Sci. Total Environ. 2009, 407, 3295-3300.

3. Thach, T.Q.; Wong, C.M.; Chan, K.P.; Chau, Y.K.; Chung, Y.N.; Ou, C.Q.; Yang, L.; Hedley, A.J. Daily visibility and mortality: Assessment of health benefits from improved visibility in Hong Kong. Environ. Res. 2010, 110, 617-623.

4. Latha, K.M.; Badarinath, K.V.S. Black carbon aerosols over tropical urban environment-A case study. Atmos. Res. 2003, 69, 125-133.

5. Lee, J.Y.; Jo, W.K.; Chun, H.H. Long-Term Trends in Visibility and Its Relationship with Mortality, Air-Quality Index, and Meteorological Factors in Selected Areas of Korea. Aerosol Air Qual. Res. 2015.

6. Tsai, Y.I.; Kuo, S.C.; Lee, W.; Chen, C.L.; Chen, P.T. Long-term visibility trends in one highly urbanized, one highly industrialized, and two rural areas of Taiwan. Sci. Total Environ. 2007, 382, 324-341.

7. Deng, X.; Tie, X.; Wu, D.; Zhou, X.; Bi, X.; Tan, H.; Li, F.; Jiang, C. Long-term trend of visibility and its characterizations in the Pearl River Delta (PRD) Region, China. Atmos. Environ. 2008, 42, 1424-1435. 
8. Pusheng, Z.; Zhang, X.; Xu, X.; Zhao, X. Long-Term Visibility trends and characteristics in the region of Beijing, Tianjin, and Hebei, China. Atmos. Res. 2011, 101, 711-718.

9. Fajardo, O.A.; Jiang, J.; Hao, J. Assessing young people's preferences in urban visibility in Beijing. Aerosol Air Qual. Res. 2013, 13, 1536-1543.

10. Zhuang, X.; Wang, Y.; Zhu, Z.; Querol, X.; Alastuey, A.; Rodríguez, S.; Wei, H.; Xu, S.; Lu, W.; Viana, M.; et al. Origin of PM10 pollution episodes in an industrialized mega-city in central China. Aerosol Air Qual. Res. 2014, 14, 338-346.

11. Rogula-Kozłowska, W.; Klejnowski, K.; Rogula-Kopiec, P.; Ośródka, L.; Krajny, E.; Błaszczak, B.; Mathews, B. Spatial and seasonal variability of the mass concentration and chemical composition of PM2.5 in Poland. Air Qual. Atmos. Health 2014, 7, 41-58.

12. Lee, C.G.; Yuan, C.S.; Chang, J.C.; Yuan, C. Effects of aerosol species on atmospheric visibility in Kaohsiung city, Taiwan. J. Air Waste Manag. Assoc. 2005, 55, 1031-1041.

13. Jung, J.; Lee, H.; Kim, Y.J.; Liu, X.; Zhang, Y.; Gu, J.; Fan, S. Aerosol chemistry and the effect of aerosol water content on visibility impairment and radiative forcing in Guangzhou during the 2006 Pearl River Delta campaign. J. Environ. Manag. 2009, 90, 3231-3244.

14. Appel, B.R.; Tokiwa, Y.; Hsu, J.; Kothny, E.L; Hahn, E. Visibility as related to atmospheric aerosol constituents. Atmos. Environ. (1967) 1985, 19, 1525-1534.

15. Malm, W.C.; Day, D.E. Estimates of aerosol species scattering characteristics as a function of relative humidity. Atmos. Environ. 2001, 35, 2845-2860.

16. Tsai, Y.I.; Lin, Y.H.; Lee, S.Z. Visibility variation with air qualities in the metropolitan area in Southern Taiwan. Water Air Soil Pollut. 2003, 144, 19-40.

17. Tsai, Y.I. Atmospheric visibility trends in an urban area in Taiwan 1961-2003. Atmos. Environ. 2005, 39, 5555-5567.

18. Wen, C.C.; Yeh, H.H. Comparative influences of airborne pollutants and meteorological parameters on atmospheric visibility and turbidity. Atmos. Res. 2010, 96, 496-509.

19. Deng, J.; Wang, T.; Jiang, Z.; Xie, M.; Zhang, R.; Huang, X.; Zhu, J. Characterization of visibility and its affecting factors over Nanjing, China. Atmos. Res. 2011, 101, 681-691.

20. Du, K.; Mu, C.; Deng, J.; Yuan, F. Study on atmospheric visibility variations and the impacts of meteorological parameters using high temporal resolution data: An application of Environmental Internet of Things in China. Int. J. Sustain. Dev. World Ecol. 2013, 20, 238-247.

21. Majewski, G.; Czechowski, P.O.; Badyda, A.; Brandyk, A. Effect of air pollution on visibility in urban conditions. Warsaw Case Study. Environ. Prot. Eng. 2014, 40, 47-64.

22. Chen, J.; Qiu, S.; Shang, J.; Wilfrid, O.M.F.; Liu, X.; Tian, H.; Boman, J. Impact of relative humidity and water soluble constituents of PM2.5 on visibility impairment in Beijing, China. Aerosol Air Qual. Res. 2014, 14, 260-268.

23. Badyda, A.; Dabrowiecki, P.; Lubiński, W.; Czechowski, P.O.; Majewski, G.; Chciałowski, A.; Kraszewski, A. Influence of traffic-related air pollutants on lung function. Adv. Exp. Med. Biol. 2013, 788, 229-235.

24. Badyda, J.; Dąbrowiecki, P.; Czechowski, P.O.; Majewski, G.; Doboszyńska, A. Traffic-Related Air Pollution and Respiratory Tract Efficiency. Adv. Exp. Med. Biol. 2015. 
25. Majewski, G.; Kleniewska, M.; Przewoźniczuk, W. The effect of urban conurbation on the modification of human thermal perception, as illustrated by the example of Warsaw (Poland). Theor. Appl. Climatol. 2014, 116, 147-154.

26. Pastuszka, J.S.; Wawroś, A.; Talik, E.; Paw U, K.T. Optical and chemical characteristics of the atmospheric aerosol in four towns in southern Poland. Sci. Total Environ. 2003, 309, 237-251.

27. Czarnecka, M.; Nidzgorska-Lencewicz, L. Impact of weather conditions on winter and summer air quality. Int. Agrophysics 2011, 25, 7-12.

28. Rogula-Kozłowska, W.; Kozielska, B.; Błaszczak, B.; Klejnowski, K. The mass distribution of particle-bound PAH among aerosol fractions: A case-study of an urban area in Poland. In Organic Pollutants Ten Years after the Stockholm Convention-Environmental and Analytical Update; Puzyn, T., Mostrag-Szlichtyng, A., Eds.; InTech: Rijeka, Croatia, 2012; pp. 163-190.

29. Majewski, G.; Kleniewska, M.; Brandyk, A. Seasonal variation of particulate matter mass concentration and content of metals. Pol. J. Environ. Stud. 2011, 20, 417-427.

30. Rozbicka, K.; Majewski, G.; Rozbicki, T. Seasonal variation of air pollution in Warsaw conurbation. Meteorol. Z. 2014, 23, 175-179.

31. Majewski, G.; Rogula-Kozłowska, W. The elemental composition and origin of fine ambient particles in the largest Polish conurbation: First results from the short-term winter campaign. Theor. Appl. Climatol. 2015.

32. Rogula-Kozłowska, W.; Klejnowski, K.; Rogula-Kopiec, P.; Mathews, B.; Szopa, S. A study on the seasonal mass closure of ambient fine and coarse dusts in Zabrze, Poland. Bull. Environ. Contam. Toxicol. 2012, 88, 722-729.

33. Rogula-Kozłowska, W.; Rogula-Kupiec, P.; Mathews, B.; Klejnowski, K. Effects of road traffic on the ambient concentrations of three PM fractions and their main components in a large Upper Silesian city. Ann. Wars. Univ. Life Sci. 2013, 45, 243-253.

34. Rogula-Kozłowska, W. Traffic-Generated Changes in the Chemical Characteristics of Size-Segregated Urban Aerosols. Bull. Environ. Contam. Toxicol. 2014, 93, 493-502.

35. Harrison, R.M.; Deacon, A.R.; Jones, M.R. Sources and processes affecting concentrations of $\mathrm{PM}_{10}$ and $\mathrm{PM}_{2.5}$ particulate matter in Birmingham (UK). Atmos. Environ. 1997, 31, 4103-4117.

36. Statheropoulos, M.; Vassiliadis, N.; Pappa, A. Principal component and canonical correlation analysis for examining air pollution and meteorological data. Atmos. Environ. 1998, 32, 1087-1095.

37. Kleinbaum, D.G.; Kupper, L.L.; Muller, K.E.; Nizam, A. Applied Regression Analysis and Other Multivariable Methods; Duxbury Press: Pacific Grove, CA, USA, 1998; p. 798.

38. Izenman, A.J. Modern Multivariate Statistical Techniques: Regression. Classification, and Manifold Learning; Springer Texts in Statistics: New York, NY, USA, 2008.

39. Rozbicka, K.; Rozbicki, T. Spatiotemporal variations of tropospheric ozone concentrations in the Warsaw Agglomeration (Poland). Ann. Wars. Univ. Life Sci. 2014, 46, 247-261.

40. Xue, D.; Li, C.; Liu, Q. Visibility characteristics and the impacts of air pollutants and meteorological conditions over Shanghai, China. Environ. Monit. Assess. 2015, 187, 363. 
41. Cao, J.J.; Wang, Q.Y.; Chow, J.C.; Watson, J.G.; Tie, X.X.; Shen, Z.X.; Wang, P.; An, Z.S. Impacts of aerosol compositions on visibility impairment in Xi'an, China. Atmos. Environ. 2012, 59, 559-566.

42. Rogula-Kozłowska, W.; Błaszczak, B.; Kozielska, B.; Klejnowski, K. The Mass Distribution of Particle-Bound PAH among Aerosol Fractions: A Case-Study of an Urban Area in Poland; InTECH Open Access Publisher: Rijeka, Croatia, 2012.

43. Houthuijs, D.; Breugelmans, O.; Hoek, G.; Vaskövi, É.; Miháliková, E.; Pastuszka, J.S.; Jirik, V.; Sachelarescu, S.; Lolova, D.; Meliefste, K.; et al. $\mathrm{PM}_{10}$ and $\mathrm{PM}_{2.5}$ concentrations in Central and Eastern Europe:: Results from the Cesar study. Atmos. Environ. 2001, 35, 2757-2771.

44. Pastuszka, J.S.; Okada, K. Features of atmospheric aerosol particles in Katowice, Poland. Sci. Total Environ. 1995, 175, 179-188.

45. Reizer, M.; Juda-Rezler, K. Explaining the high $\mathrm{PM}_{10}$ concentrations observed in Polish urban areas. Air Qual. Atmos. Health 2015.

46. Pastuszka, J.S.; Rogula-Kozłowska, W.; Zajusz-Zubek, E. Characterization of $\mathrm{PM}_{10}$ and $\mathrm{PM}_{2.5}$ and associated heavy metals at the crossroads and urban background site in Zabrze, Upper Silesia, Poland, during the smog episodes. Environ. Monit. Assess. 2010, 168, 613-627.

47. Cleveland, W.S.; Graedel, T.E.; Kleiner, B.; Warner, J.L. Sunday and workday variations in photochemical air pollutants in New Jersey and New York. Science 1974, 186, 1037-1038.

48. Qin, Y.; Tonnesen, G.S.; Wang, Z. Weekend/weekday differences of ozone, NOx, Co, VOCs, $\mathrm{PM}_{10}$ and the light scatter during ozone season in southern California. Atmos. Environ. 2004, 38, 3069-3087.

49. Marr, L.C.; Harley, R.A. Spectral analysis of weekday-weekend differences in ambient ozone, nitrogen oxide, and non-methane hydrocarbon time series in California. Atmos. Environ. 2002, 36, 2327-2335.

50. Stull, R.B. Meteorology for Scientists and Engineers; Brooks/Cole: Pacific Grove, CA, USA, 2000.

51. Elias, T.; Haeffelin, M.; Drobinski, P.; Gomes, L.; Rangognio, J.; Bergot, T.; Chazette, P.; Raut, J.C.; Colomb, M. Particulate contribution to extinction of visible radiation: Pollution, haze, and fog. Atmos. Res. 2009, 92, 443-454.

52. Geertsema, G.T.; Schreur, B.G.J. The effect of improved nowcasting of precipitation on air quality modeling. Atmos. Environ. 2009, 43, 4924-4934.

53. Pitchford, M.; Malm, W.; Schichtel, B.; Kumar, N.; Lowenthal, D.; Hand, J. Revised algorithm for estimating light extinction from IMPROVE particle speciation data. J. Air Waste Manag. 2007, 57, 1326-1336. 


\title{
Study of Black Sand Particles from Sand Dunes in Badr, Saudi Arabia Using Electron Microscopy
}

\author{
Haider Abbas Khwaja, Omar Siraj Aburizaiza, Daniel L. Hershey, \\ Azhar Siddique, David A. Guerrieri P. E., Jahan Zeb, Mohammad Abbass, \\ Donald R. Blake, Mirza Mozammel Hussain, Abdullah Jameel Aburiziza, \\ Malissa A. Kramer and Isobel J. Simpson
}

\begin{abstract}
Particulate air pollution is a health concern. This study determines the microscopic make-up of different varieties of sand particles collected at a sand dune site in Badr, Saudi Arabia in 2012. Three categories of sand were studied: black sand, white sand, and volcanic sand. The study used multiple high resolution electron microscopies to study the morphologies, emission source types, size, and elemental composition of the particles, and to evaluate the presence of surface "coatings or contaminants" deposited or transported by the black sand particles. White sand was comprised of natural coarse particles linked to wind-blown releases from crustal surfaces, weathering of igneous/metamorphic rock sources, and volcanic activities. Black sand particles exhibited different morphologies and microstructures (surface roughness) compared with the white sand and volcanic sand. Morphological Scanning Electron Microscopy (SEM) and Laser Scanning Microscopy (LSM) analyses revealed that the black sand contained fine and ultrafine particles (50 to $500 \mathrm{~nm}$ ranges) and was strongly magnetic, indicating the mineral magnetite or elemental iron. Aqueous extracts of black sands were acidic $(\mathrm{pH}=5.0)$. Fe, C, O, Ti, Si, V, and $\mathrm{S}$ dominated the composition of black sand. Results suggest that carbon and other contaminant fine particles were produced by fossil-fuel combustion and industrial emissions in heavily industrialized areas of Haifa and Yanbu, and transported as cloud condensation nuclei to Douf Mountain. The suite of techniques used in this study has yielded an in-depth characterization of sand particles. Such information will be needed in future environmental, toxicological, epidemiological, and source apportionment studies.
\end{abstract}

Reprinted from Atmosphere. Cite as: Khwaja, H.A.; Aburizaiza, O.S.; Hershey, D.L.; Siddique, A.; Guerrieri P. E., D.A.; Zeb, J.; Abbass, M.; Blake, D.R.; Hussain, M.M.; Aburiziza, A.J.; Kramer, M.A.; Simpson, I.J. Study of Black Sand Particles from Sand Dunes in Badr, Saudi Arabia Using Electron Microscopy. Atmosphere 2015, 6, 1175-1194. 


\section{Introduction}

Particulate air pollution significantly impacts public health in both developed and developing countries. Major sources of particulate matter (PM) include large industries, such as power plants, petrochemical plants, cement plants, vehicular traffic, windblown dust, crustal erosion, volcanic eruptions, wildfires, sea spray, and combustion processes [1]. Amongst all of these, combustion is the main source for pollutant emissions. PM varies in composition, origin, and size, ranging from submicron (sub- $\mu \mathrm{m}), 1-30 \mu \mathrm{m}$, and up to $50 \mu \mathrm{m}$ or more. Large particles $(>10 \mu \mathrm{m})$ have a relatively low residence time in the air and tend to settle quickly through gravitational subsidence. Fine particles with aerodynamic diameters less than $2.5 \mu \mathrm{m}$ $\left(\mathrm{PM}_{2.5}\right)$ and "coarse" particles with aerodynamic diameters less than $10 \mu \mathrm{m}\left(\mathrm{PM}_{10}\right)$ remain airborne for a longer period of time. Ultrafine particles are particulate matter of nanoscale size (less than $100 \mathrm{~nm}$ in diameter). Fine particles in the $0.05-2 \mu \mathrm{m}$ range can travel distances up to approximately $1000 \mathrm{~km} \mathrm{[2].}$

Exposure to PM has been associated with numerous effects on human health, including increased morbidity and mortality, respiratory problems, cardiovascular diseases, lung cancer, renal and brain damage, and human metal poisonings [3-6]. Both fine and coarse particles are readily inhaled into the human respiratory tract. It is suggested that fine particles are more strongly implicated in cardiovascular effects than coarse particles, while both impact respiratory end points [7]. In epidemiological studies, positive correlations have been established between elevated levels of inhaled airborne PM, especially $\mathrm{PM}_{2.5}$, and acute adverse health effects [8-10]. Aside from the amount of PM inhaled, particle-related parameters, such as size, composition, and solubility have also been linked to health effects in toxicology studies. Size affects the site deposition in the human respiratory tract and the consequent degree of toxicity that may result. Therefore, ultrafine particles are more toxic than coarse particles and are linked to pulmonary diseases in human and animals [11-14]. Particle sizes also reveal the origin and the formation of airborne particles: larger sized particles are of crustal origin whereas fine particles originate from combustion processes or gas-to-particle conversion in the atmosphere [15].

Particulate air pollution is a health concern among the residents of Badr, Saudi Arabia. Deposition of black sand particles has recently become common on Douf Mountain (Figure 1), located to the west of Badr. The soil surrounding Medina consists mostly of basalt, while the hills to the southeast and northeast are volcanic sand that dates to the Paleozoic era. The geology of Badr is represented by granophyre and alkali-feldspar granite. Dominant lithologies are andesitic and dacitic to rhyolitic tuffs, lavas, and volcanic rocks [16]. The town of Badr, in Medina province $\left(23^{\circ} 47^{\prime} \mathrm{N} 38^{\circ} 47^{\prime} \mathrm{E} ; 123 \mathrm{~m}\right.$ altitude), is located about $150 \mathrm{~km}$ southwest of the holy city of Medina, towards the western outlet of Wadi-e-Safra (Figure 2a). The total area of Badr is $8226 \mathrm{~km}^{2}$ with a population of 61,600 . Average annual rainfall is 
$80 \mathrm{~mm}$. June, July, August, and September are the hottest months in Badr with an average temperature of $40^{\circ} \mathrm{C}$, while the coldest are January and February at $14{ }^{\circ} \mathrm{C}$. Historically, Badr was a market, situated on the road which connects Sham (Syria, Lebanon and Palestine now) with Mecca (Figure 2b).

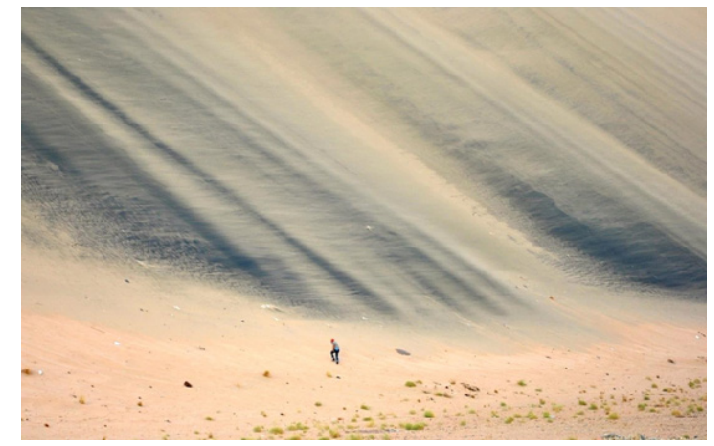

(a)

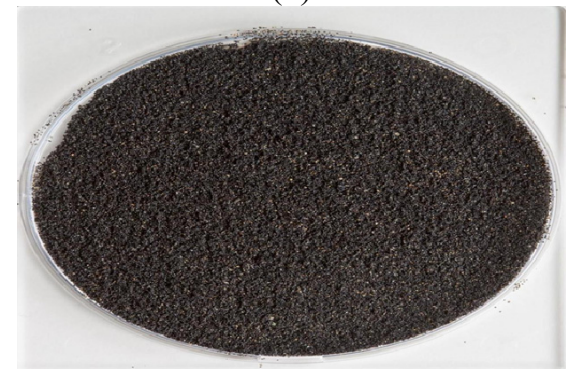

(b)

Figure 1. (a) Photograph of black sand on Douf Mountain, Badr, Saudi Arabia; (b) Photograph of black sand collected from Douf Mountain on February 2012.

Despite concerns about the health and environmental significance of the black sand particles, not much is known about the size, morphology, and specific chemical constituents of these sand particles. It is generally known that certain chemical forms are more toxic than others: in general, soluble forms are potentially more toxic than those tied up as insoluble oxides or forming glassy structures [17]. Knowledge of the morphology and chemical characterization of sand particles can aid in control strategies and help to interpret and predict chemical interactions in the atmosphere, downwind fallout rates, potential damage to vegetation, deterioration of materials and structures, and, in particular, impact on human health. Among microanalytical techniques for characterizing black sand particles, Scanning Electron Microscopy with an attached Energy Dispersive X-ray Spectrometer (SEM/EDS) is the most versatile technique, capable of simultaneously obtaining information on particle size, morphology, elemental composition, and microstructure [18,19]. Here the 
microscopic make-up of different varieties of sand particles collected from a dune near Badr is presented.

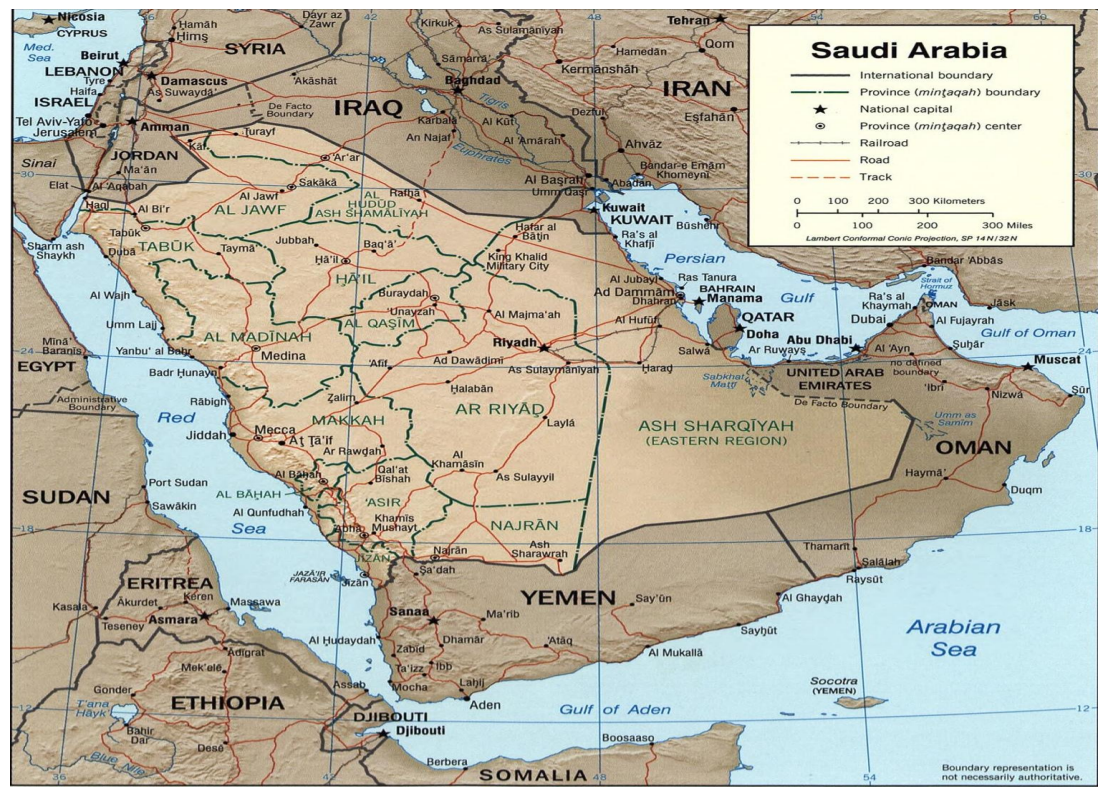

(a)

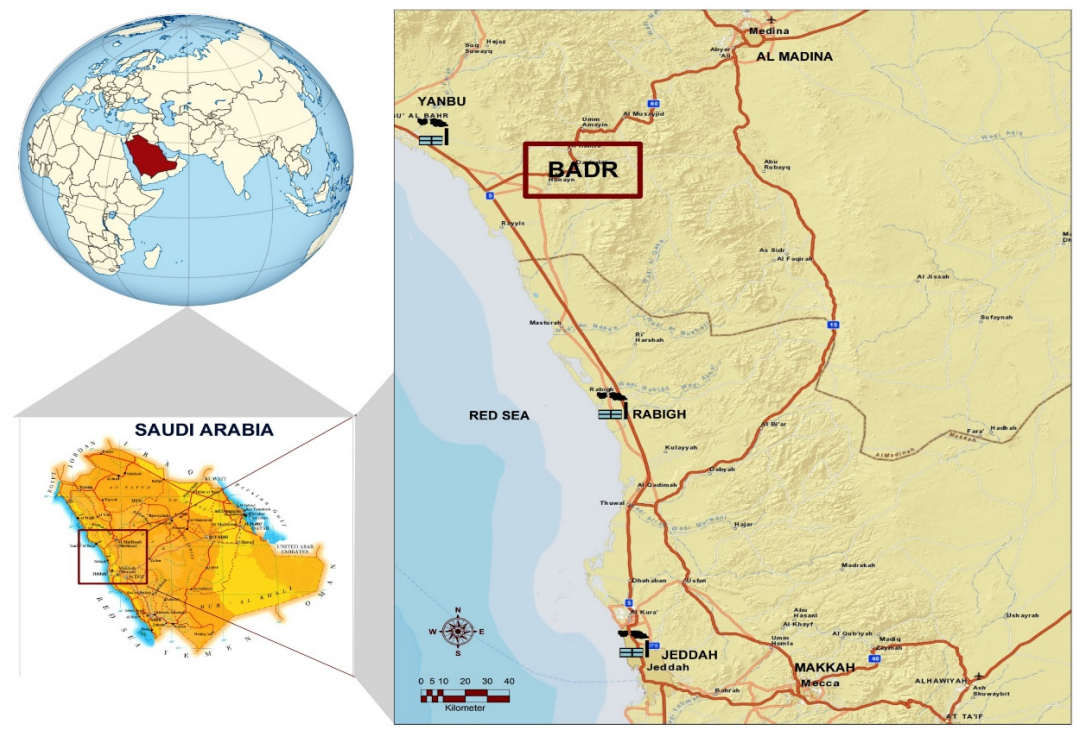

(b)

Figure 2. (a) Map of Saudi Arabia; (b) Map of western Saudi Arabia showing location of sampling site, Badr. 


\section{Experimental}

\subsection{Sample Collection}

Representative random composite sand samples about 4-6 inches thick were collected in 2012 from various locations in the sand dunes (Figure 1b) using hand towels and gloves. The randomly collected samples were composited and thoroughly mixed, and a representative sub-sample was taken for analysis. The sample collected was very black in color, and it was then separated into three groups in the laboratory: magnetic black sand, non-magnetic black sand, and white sand. Of the black sand sampled, there were two distinct types: magnetic and non-magnetic. Solutions of the black sands, immersed in deionized water gave a low $\mathrm{pH}$ of 5.0, indicating acidity. A volcanic sand sample was also collected from the area. For this study, the two types of black sand were compared to "normal" white sand and to volcanic sand. Four samples were selected for microscopic examination and morphology, size, and elemental composition were determined. Several hundred individual particles of each type (magnetic black sand, non-magnetic black sand, white sand, and volcanic sand) were viewed for comparison purposes.

\subsection{Equipment and Analytical Methodology}

Sand particles were characterized at the New York State Department of Environmental Conservation (NYS DEC) microscopy laboratory in Rensselaer NY, using an Olympus SZX12 optical stereo microscope (OSM), SEM/EDS (model JEOL 6490LV SEM equipped with a Sahara EDS), and an Olympus LEXT laser scanning microscope (LSM, model OLS 3100 LSM). This suite of techniques provided images showing morphology of particles, size, elemental composition, and specific source types. The stereo microscope provided a colored representation of the differences in the particles. The SEM provided much more detail at higher magnifications: high resolution of up to $300,000 \times$ magnification can be achieved. For this study, $12,000 \times$ microscope magnification was used. In addition, elemental composition was determined using an energy dispersive X-ray spectrometer (EDS) attachment to the SEM. An Olympus-LEXT instrument was used to locate, determine, and characterize fine particles adhered to the sand particles. An LSM or confocal scanning microscope (CLSM) was used to create high resolution optical images that show depth, creating a 3-dimensional image of the object being scanned, which can show how the particles are adhered to the particle surface. This technique allows the surface contours to be imaged for profile viewing. The LSM offers better detail on surface morphology and layering of the surface and produces a surface structure in submicron units (minimum resolution of $0.1 \mu \mathrm{m}$ ). 
The analysis of the samples was performed by a SEM/EDS to determine particle morphology and elemental composition. Electron microscopic particle identifications were based on visual comparisons to in-house reference standards and to data in McCrone's Atlas [20]. The EDS system can collect data from X-rays equivalent to the depth of the secondary electron formation. This is dependent on the sample density and $\mathrm{keV}$. The approximate secondary electron collection depth is between 0.5 and 1 micron. For this study, both secondary electron imaging and backscattered electron imaging were used. Operating conditions for the SEM analysis were: accelerating voltage $=15 \mathrm{keV}$; filament current $=112 \mu \mathrm{A}$; working distance $=19 \mathrm{~mm}$; analytical time $=200 \mathrm{~s}$; dead time $=$ varied from sample to sample. The X-ray spectra were generated from specified areas on sand grains placed on aluminum SEM stubs and collected for $90 \mathrm{~s}$ with probe current of $50 \mathrm{nA}$. The weight-percent of the elemental analysis was determined by using ZAF correction factors for each element.

Attached to the JEOL SEM was a Bruker-energy dispersive X-ray spectrometer (EDS; "Spirit" system), that was able to quantify elements with atomic number $Z \geq 6$. The EDS detector was controlled by Bruker "Spirit" system. In combination, the two provided detailed particle analysis information. A second EDS system, equipped with TEAM software, owned and operated by EDAX Inc., was used for back up and confirmation purposes. Intensities of the EDS spectrum lines were converted to corresponding weight-percent concentrations based on their excitation energy $(\mathrm{Kv})$. Calibration was referenced to the excitation energy of $\mathrm{Al}$ and $\mathrm{Cu}$. Weight-percent concentrations of elements were determined semi-quantitatively using the TEAM software. Forty-one $\mathrm{X}$-ray regions of interest were used to detect the presence of C, O, Na, Mg, Al, Si, P, S, Cl, K, Ca, Ti, V, Cr, Mn, Fe, Co, Ni, Cu, Zn, Ga, As, Se, $\mathrm{Br}, \mathrm{Sr}, \mathrm{Y}, \mathrm{Zr}, \mathrm{Mo}, \mathrm{Pd}, \mathrm{Ag}, \mathrm{Cd}, \mathrm{In}, \mathrm{Sn}, \mathrm{Sb}, \mathrm{Ba}, \mathrm{La}, \mathrm{Au}, \mathrm{Hg}, \mathrm{Tl}, \mathrm{Pb}$, and $\mathrm{U}$. An element was considered to be present if its peak counts were at least three times the square root of the background counts in the region of interest. Uncertainty in the reported percentages is within $\pm 3 \%-5 \%$ for each element studied.

Source contributions for individual samples were determined by comparing elemental ratios, morphological identifiers [20] and verified using a variety of microscopy techniques. Most of these techniques include visual observations, comparison to reference standards, comparisons to the source samples, and digital mapping. When setting up the individual source classes, miscellaneous and unidentified particles were placed in the biological/miscellaneous category. Particle size and elemental ratios were determined with the aid of multiple digital maps. The particle size was determined using the Feret diameter. In addition to the above, the identification of questionable particles was compared to reference standards and to McCrone's Atlas [20]. SEM/EDS elements were calibrated at different energies using computer generated standards, in-house standards, and a copper grid (ASTM) prior to sample analysis. 
The Dionex ICS 2500 ion chromatography system used for the determination of anions and cations was configured with an auto sampler, a pump, and a conductivity detector. Anions were eluted from an analytical column (AS14 $4 \mathrm{~mm} \times 250 \mathrm{~mm}$ ) and a guard column (AG14 $4 \mathrm{~mm}$ ) using $3.5 \mathrm{mM} \mathrm{Na}_{2} \mathrm{CO}_{3} / 1.0 \mathrm{mM} \mathrm{NaHCO}_{3}$ eluent. Cations were eluted from an analytical column (CS14 $4 \mathrm{~mm} \times 250 \mathrm{~mm}$ ) and a guard column (CG14 $4 \mathrm{~mm}$ ) using $10 \mathrm{mM}$ methylsulfonic acid eluent. For quantification, a linear calibration curve $(r>0.995)$ was established for each analyte and an independently made quality control sample was analyzed to validate the calibration curve. Sand samples were extracted in water ( $1 \mathrm{~g}$ dry weight $/ 5 \mathrm{~mL}$ of water), filtered through a pre-cleaned $0.2 \mu \mathrm{M}$ PTFE filter, and collected in a scintillation vial.

\subsection{Sample Preparation}

The sample was placed on a $12.5 \mathrm{~mm}$ diameter aluminum stub in order to directly observe the particles in an undisturbed condition. Each sample was affixed to the stub with double-sided carbon tape. To obtain representative elemental composition, and to avoid artificial damage and distortion of particles, the samples were not coated with carbon or gold.

\section{Results and Discussion}

\subsection{Morphology of Sand Samples}

OSM, SEM, LSM Analysis

Figure 3 shows four stereo microscope images $(63 \times)$ of non-magnetic black sand, magnetic black sand, white sand, and volcanic sand. White sand appears as a clean crystalline structure (quartz- $\mathrm{SiO}_{2}$ ), whereas the volcanic sand and the magnetic black sand both have reddish tints indicative of iron oxide. Even at relatively low SEM magnification $(220 \times)$, significant structural and elemental features were evident. The volcanic sand illustrates a very angular structure while ovoid structures were evident in the remaining three sands. The iron oxide particles were either irregular or spherical.

The surface differences became more pronounced at higher SEM magnification (450×; Figure 4a). Black sand particles, both magnetic and non-magnetic were more spherical than the oval white sand particles and angular volcanic sand. 

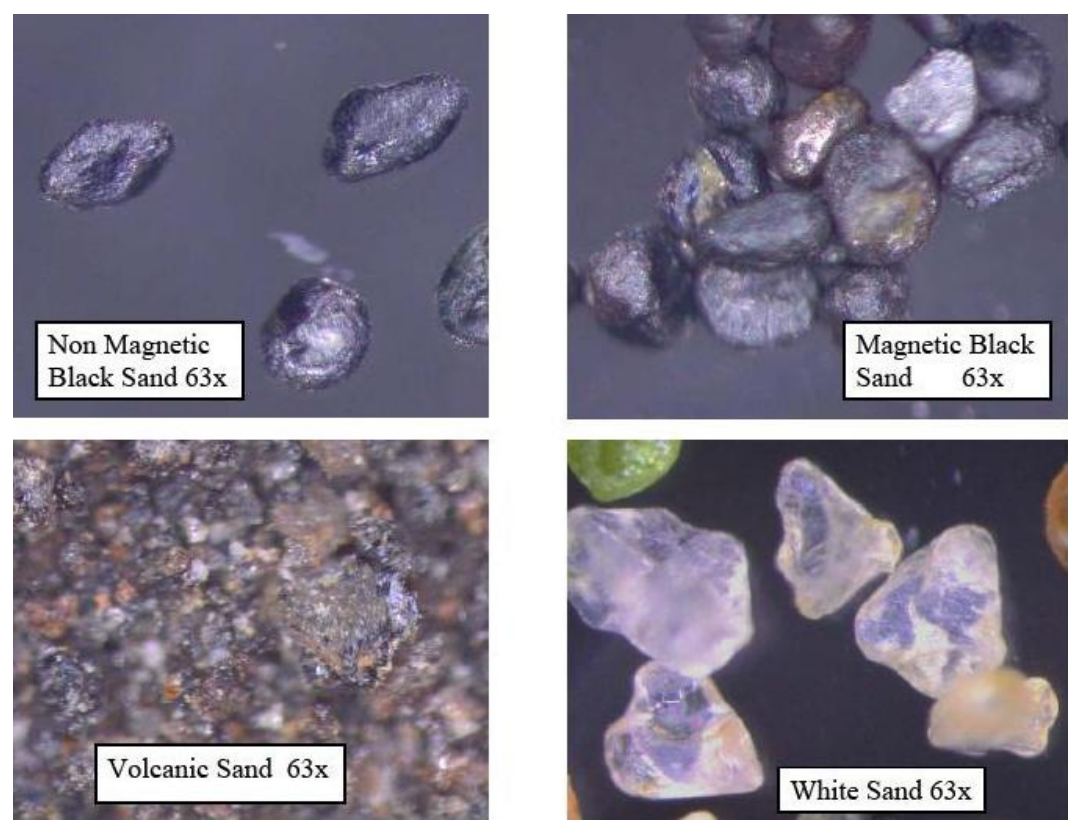

Figure 3. Stereo micrograph of non-magnetic black, magnetic black, white, and volcanic sand $(63 \times$ magnification).

SEM images at a magnification of $2000 \times$ (Figure $4 b$ ) revealed different surface structures in all four samples. At this magnification the fine particles began to appear, attached to the surfaces of the sand grains, especially the black sand particles. There are distinct differences between the images of the black sand particles and the images of the white sand and volcanic sand particles. While white sand and the volcanic sand are crustal in nature (Figure $4 \mathrm{~b}$ ), the white sand particles have been polished over time due to weathering; however, they otherwise appear similar to the volcanic sand. At $7000 \times$ magnification, fine particles attached to the surface of the white and black sand grains were round in shape, whereas particles on the volcanic sand were jagged and irregular. Examination at $7000 \times$ clearly indicated the predominance of fine and ultrafine particles (50 to $500 \mathrm{~nm}$ range) in the black sand sample. Morphology of these small round particles suggests industrial contributions and/or byproducts of fossil-fuel combustion. At higher magnification $(12,000 \times)$, differences in the fine particles embedded on the grains became more evident (Figure 4c). Since they are complex aggregates, their total surface area is enormous in contrast to coarser PM. Due to the longer residence times and better mobility of fine particles, their dispersion in the atmosphere is more efficient than that of coarse particles, and they are very readily transported long distances by air streams. 

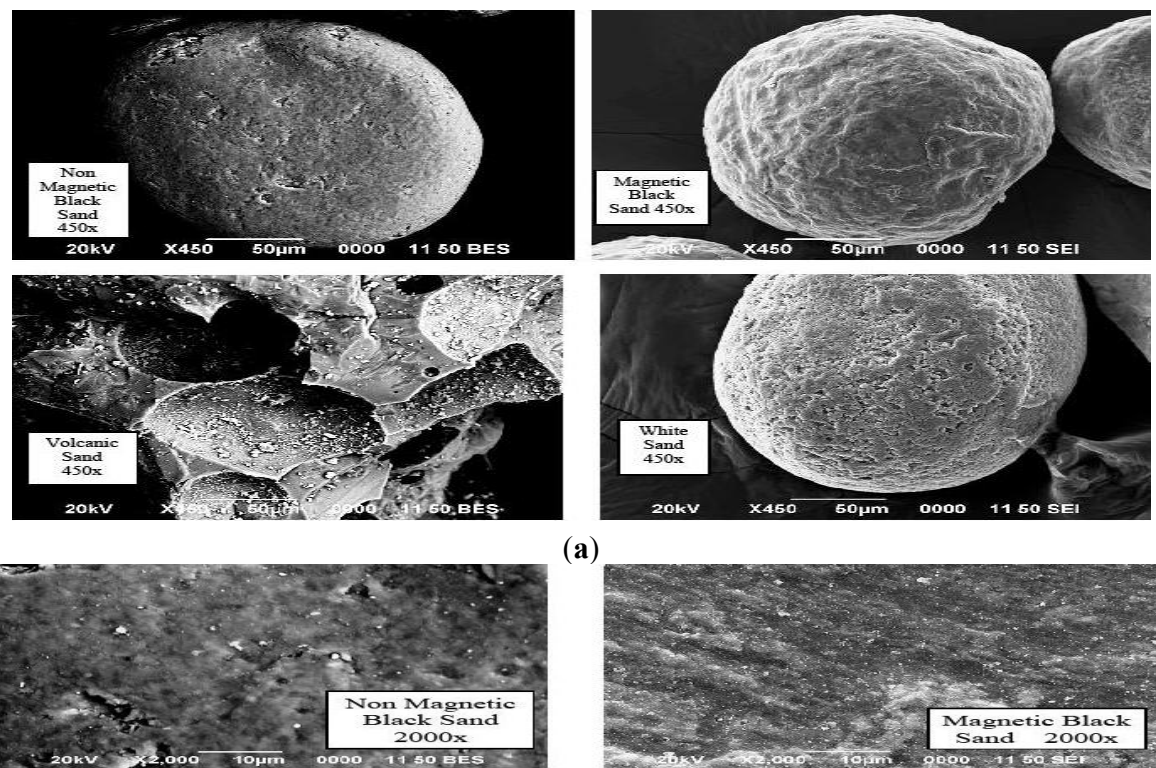

(a)
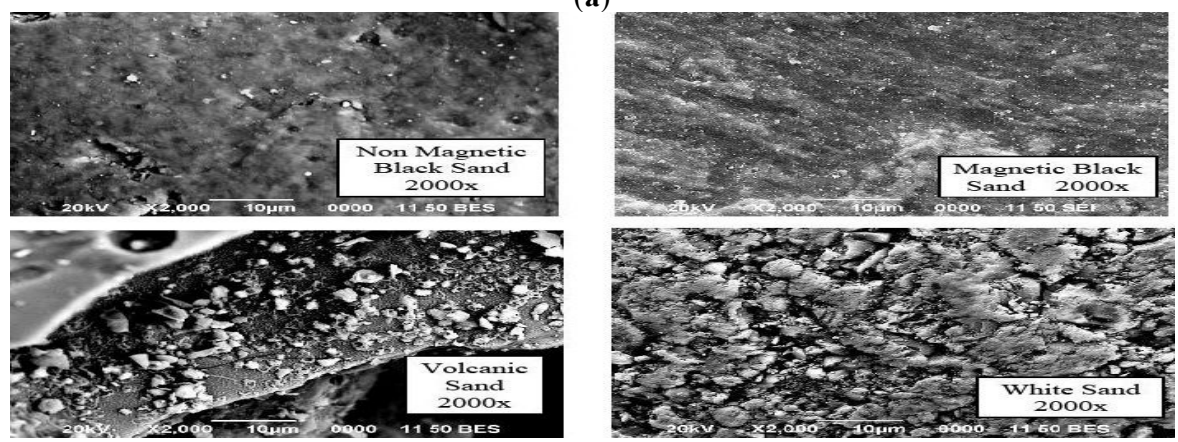

(b)
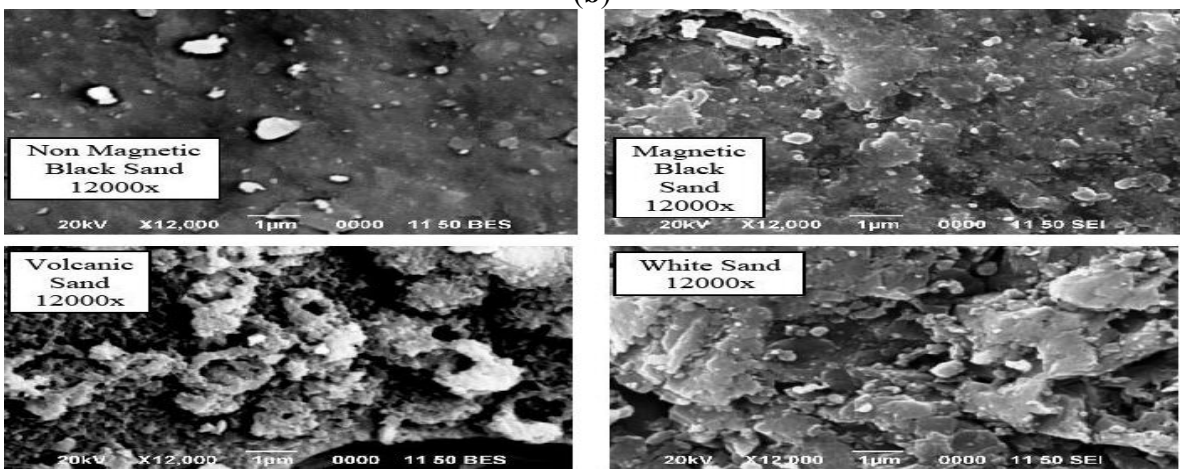

(c)

Figure 4. Scanning electron micrographs of sand at (a) $450 \times$ magnification; (b) $(2000 \times$ magnification showing ultrafine particles; and (c) $12,000 \times$ magnification.

The first series of LSM images, taken at $10 \times$ microscope magnification $(\mathrm{mm}$ optical, Figure 5a), showed characteristics of an oil-like product on the surface of the black sand samples with additional fine particles attached to the surface. The other three sand samples appeared similar in size, shape, and surface textures. The 
coloration of the three sand samples is somewhat similar; however, the magnetic black sand was less reflective because of the oil contribution. The LSM showed that the volcanic sand sample had a different appearance. It was less smooth and appeared more crystalline, and the surface appeared to be comprised of many smaller particles stuck together with many different color variations. At higher magnification $(20 \times)$, all four sand samples did show differences in shape, as well as color variations. Black sand samples appeared more spherical whereas the white sand sample appeared more oval, as seen previously by SEM. The surface of the magnetic black sand had some roughness, similar to the white sand, and there were variations in coloration that were not as apparent at lower magnification. The volcanic sand looked to be very rough and composed of material of many colors. The black sand appeared to be mostly gold-colored at this magnification, with similar surface roughness to that of the magnetic black sand.

The first four LSM images (Figure 5a) were created using the optical settings of the microscope. The next two images (Figure 5b,c) were created using the confocal imaging capabilities of the LSM. In Figure 5b, a $100 \times$ color image of a magnetic black sand particle, individual fine particles are seen coating the surface. Figure $5 c$ depicts a $100 \times$ color LSM image of a white sand particle, where fine black particles are seen coating the surface. They may be the beginning of a black coating, but most likely are anthropogenic. A cross section of a black sand particle $(90 \times$ magnification; Supplementary Figure S1), shows a white interior with a black coating on the outside. In this image, taken with the stereo microscope, the particle on the left shows where the surface coating was removed and the white core exists. The magnetic black sand clearly depicts the reddish iron oxide particles.

One of the black sand grains was fractured and divided into quarters, similar to an interior slice of an orange. In Figure 6a, an SEM image $(370 \times)$, the left side shows the interior of the sand grain with no deposition or evidence of fine and ultrafine particles. The roundish outside clearly shows a "fingernail" effect. There is a thin surface layer $(1 \mu \mathrm{m})$ embedding a large number of particles from the atmospheric deposition, and the coating can be seen as having a rough texture. A similar observation was seen using the LSM in the confocal mode $(100 \times)$, in which the rough textured outside coating is apparent with individual submicron particles attached to the surface, and the inside is smooth with no coating (Figure 6b). The $1 \mu \mathrm{m}$ deep surface coating is also apparent. It is known that emissions from combustion of fossil-fuel contain carbon soot and sulfur that is easily absorbed by soil particles during long-range transportation [21,22]. 


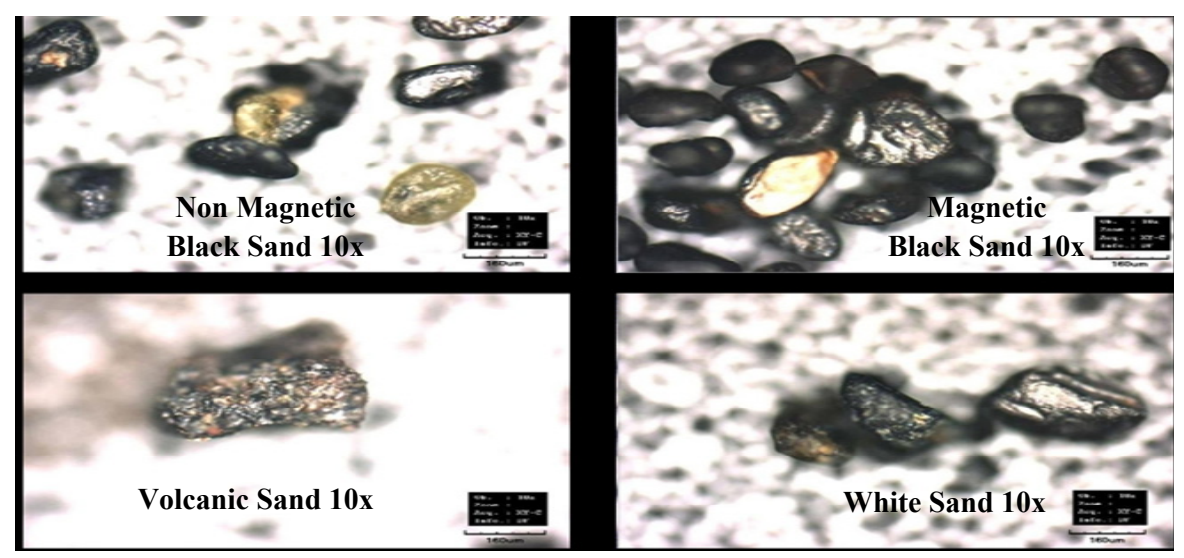

(a)

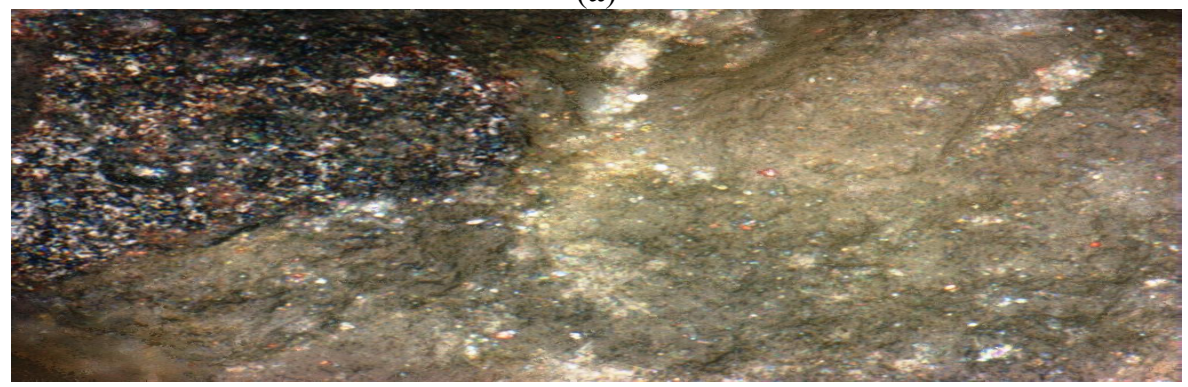

(b)

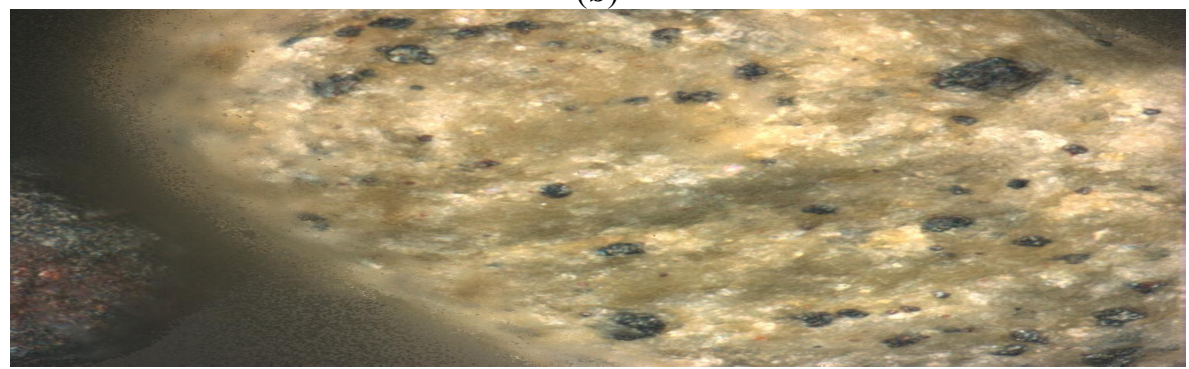

(c)

Figure 5. LSM images (a) sand (10× magnification); (b) magnetic black sand (100× magnification); and (c) white sand $(100 \times$ magnification).

The coating of the sand grains with the oil-like material and the fine and ultrafine particles were identified independently and are believed to be correct. It does not appear likely that the black sands originated from a natural oil seep (leaking oil field) as opposed to having anthropogenic origin. Oil fields are located in eastern Saudi Arabia, and not in western Saudi Arabia where the study took place. In addition, the predominant wind direction is from the northwest, in the direction of a 
highly industrialized area (Yanbu, Figure 2). Indeed, three of the country's largest oil refineries operate $83 \mathrm{~km}$ upwind of the sampling site. Together these results point to anthropogenic origin of the black sands, rather than impact from natural oil seeps.

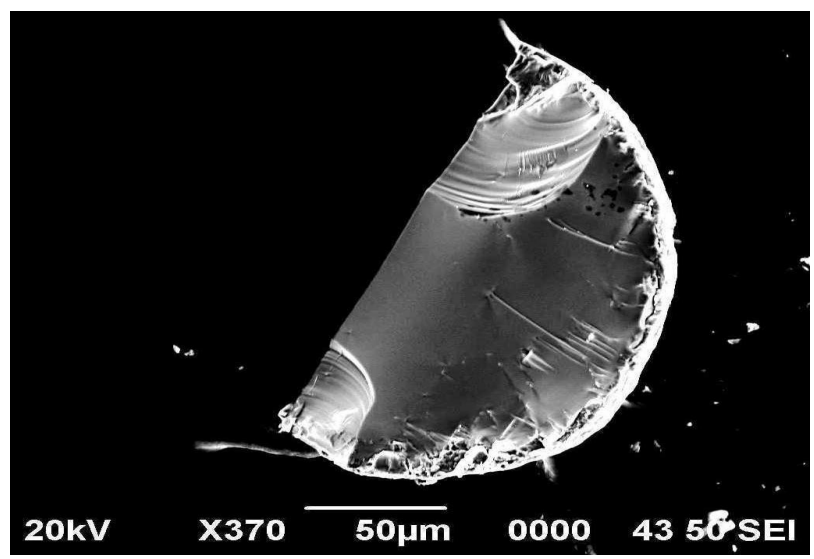

(a)

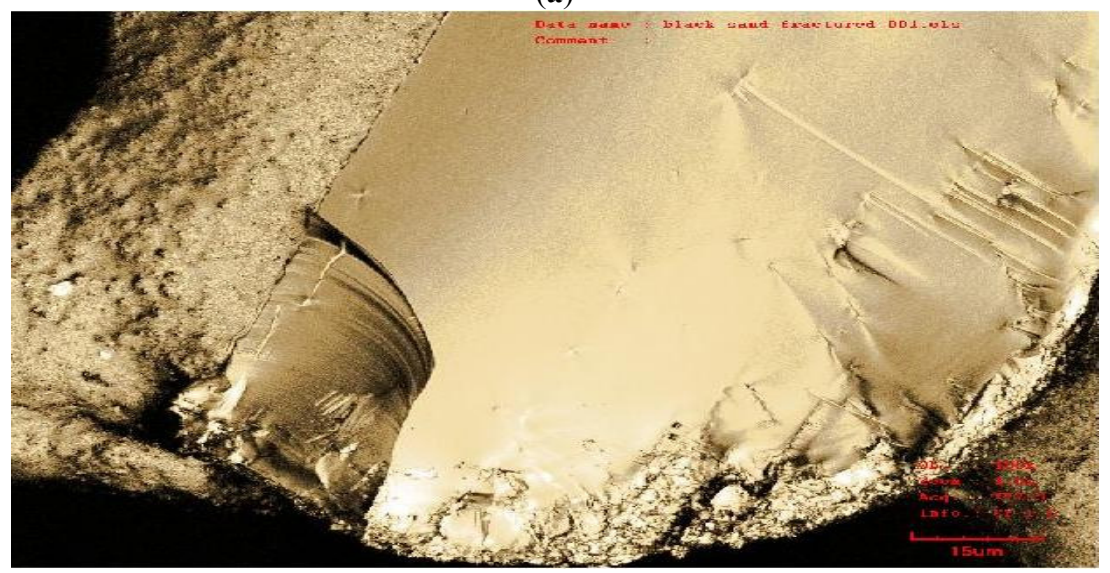

(b)

Figure 6. (a) Scanning electron micrograph of magnetic black sand (370 $\times$ magnification); (b) LSM image of magnetic black sand (100× magnification).

\subsection{Elemental Composition (SEM/EDS)}

Elemental determinations were performed to indicate the possible chemical species and quantities present. In an energy range of up to $7.5 \mathrm{keV}$, the following elements were detected: C, O, Na, Mg, Al, Si, P, S, K, Ca, Ti, V, Cr, Mn, and Fe. Carbon, $\mathrm{O}, \mathrm{Na}, \mathrm{Mg}, \mathrm{Al}, \mathrm{Si}, \mathrm{K}, \mathrm{Ca}, \mathrm{Ti}$, and Fe peaks were observed at 0.28, 0.53, 1.04, $1.25,1.50,1.74,3.31,3.69,4.51$, and $6.40 \mathrm{keV}$, respectively. These elements were the most common, found in varying intensity in all samples (Table 1). A clear P peak 
at $2.01 \mathrm{keV}$ appeared in the magnetic black sand and volcanic sand samples. Only the magnetic black sand sample exhibited a peak at $2.31 \mathrm{keV}$, which clearly indicates S. Well-defined V and Mn peaks at energies of 4.95 and $5.89 \mathrm{keV}$, respectively, were detected in the non-magnetic black and volcanic sand sample. A Cr peak at $5.41 \mathrm{keV}$ in the volcanic sand was clearly pronounced. The striking difference in elemental analysis between the black and white sand confirms that the two distinct entities were being separately resolved. The analysis of black sand and the comparison with white and volcanic sand samples that were performed in this study enables one to attribute the observed elements to anthropogenic emissions.

Table 1. Elemental Comparison of Four Sand Grains (Weight \%).

\begin{tabular}{ccccc}
\hline \multirow{2}{*}{ Element } & Magnetic & Non-Magnetic & White Sand & Volcanic Sand \\
\cline { 2 - 3 } & Black Sand & Black Sand & & \\
\hline $\mathrm{C}$ & 19.0 & 8.8 & 5.4 & 6.4 \\
$\mathrm{O}$ & 13.2 & 37.6 & 51.9 & 42.3 \\
$\mathrm{Na}$ & 0.3 & 0.5 & 3.1 & 2.5 \\
$\mathrm{Mg}$ & 0.3 & 0.7 & 3.5 & 3.1 \\
$\mathrm{Al}$ & 1.1 & 1.8 & 8.3 & 6.4 \\
$\mathrm{Si}$ & 2.0 & 3.4 & 20.0 & 16.8 \\
$\mathrm{P}$ & 0.1 & 0.0 & 0.0 & 0.4 \\
$\mathrm{~S}$ & 0.3 & 0.0 & 0.0 & 0.0 \\
$\mathrm{~K}$ & 1.0 & 0.1 & 1.9 & 0.7 \\
$\mathrm{Ca}$ & 1.0 & 0.4 & 1.6 & 6.2 \\
$\mathrm{Ti}$ & 6.7 & 18.0 & 0.8 & 1.5 \\
$\mathrm{~V}$ & 0.0 & 0.7 & 0.0 & 0.3 \\
$\mathrm{Cr}$ & 0.0 & 0.0 & 0.0 & 0.3 \\
$\mathrm{Mn}$ & 0.0 & 0.4 & 0.0 & 0.5 \\
$\mathrm{Fe}$ & 55.0 & 27.6 & 3.5 & 12.6 \\
\hline
\end{tabular}

The high sample to sample variability of the Fe concentration represents the most striking result shown on Table 1: the Fe weight contribution represents $55.0 \%$ of the magnetic black sand sample, compared to $27.6 \%, 12.6 \%$, and $3.5 \%$ in the non-magnetic black sand, volcanic sand, and white sand samples, respectively. The higher O peak in the energy dispersive X-ray spectrum of the magnetic black and non-magnetic black sand indicates that the Fe may be present as iron oxides specifically hematite $\left(\mathrm{Fe}_{2} \mathrm{O}_{3}\right)$, and magnetite $\left(\mathrm{Fe}_{3} \mathrm{O}_{4}\right)$. The black color of the sand is a result of the large quantity of $\mathrm{C}$-bearing particles with a nm size. The magnetic black sand sample had the highest $C$ content (19.0 wt \%), in contrast with the volcanic sand (6.4 wt \%) and white sand $(5.4 \mathrm{wt} \%)$. Elemental carbon is predominantly a product of the combustion process and is a good tracer for combustion-generated particles. Of the heavy metals, only Ti was present in levels approaching $6.7 \mathrm{wt} \%$. Potassium, $\mathrm{Ca}$, $\mathrm{Al}$, and $\mathrm{Si}$ were present at $1-2 \mathrm{wt} \%$. The presence of a low intensity but discernible 
$S$ peak in addition to $C$ and $O$ peaks in magnetic black sand suggests soot particles derived from fossil-fuel combustion. Similar results were observed in other electron microscopy studies of ambient particles [23-25]. A study by Chen et al. [25] reported that soot particles exhibiting a sulfur EDS peak, in addition to carbon and oxygen peaks, are prominently derived from combustion of coal, residual oil, gasoline, and diesel oil. Generally, the S content of soot particles is much less than that of char particles [23]. Since soot particles have smaller density compared to soil particles, their lifetime in the atmosphere is longer and they are more easily transported far away from their source areas [26]. A study carried out by Ali-Mohamed and Matter [27] revealed that soot from black smoke of the Kuwaiti oil-well fires settled $400 \mathrm{~km}$ downwind in Bahrain. Particles of combustion source origin included C, Al, S, $\mathrm{Ti}$, and Fe-rich particles from metal industries and iron deposits. Many studies have reported the prevalence of $\mathrm{C}$-rich particles in the fine fraction of $\mathrm{PM}_{10}$ in several urban environments [28-31]. By analyzing precipitation samples collected at Kanazawa, Japan, Tazaki et al. [32] reported that the high concentration of nm sized C-bearing particles and powdery dusts were produced by oil field combustion and a sandstorm in Iraq, and the particles were transported as cloud nuclei from Iraq to Japan by westerly winds.

Non-magnetic black sand is characterized by $\mathrm{C}, \mathrm{O}, \mathrm{Na}, \mathrm{Mg}, \mathrm{Al}, \mathrm{Si}, \mathrm{P}, \mathrm{K}, \mathrm{Ca}, \mathrm{Ti}, \mathrm{V}$, $\mathrm{Mn}$ and $\mathrm{Fe}$ (Table 1$)$ and is highly enriched in Ti (18.0 wt \%). Titanium oxide particles are common in ambient PM samples [33,34]. Carbon and Fe were observed in lesser amounts (8.8 and $27.6 \mathrm{wt} \%$ ) as compared to the magnetic black sand sample (19.0 and $55.0 \mathrm{wt} \%)$. Levels of $\mathrm{Mn}(0.40 \mathrm{wt} \%)$ in non-magnetic black sand were comparable to the volcanic sand sample $(0.50 \mathrm{wt} \%)$. The presence of transition metals on sand particles is consistent with the anthropogenic process where volatilized metal, $\mathrm{H}_{2} \mathrm{SO}_{4}$ vapor, and $\mathrm{SO}_{2}$ condense on submicron ash particles, forming a complex aerosol mixture that is eventually emitted into the ambient air [35]. Vanadium products can indicate a portion of fly ash from the combustion of heavy fuel oil [17]. The principal metals generally contained in fuel oils include C (1.5\%-69\%), $\mathrm{Na}(0.2 \%-3.91 \%), \mathrm{Mg}$ (2.26\%-18.4\%), Al (0.01\%-1.42\%), Si (0.05\%-0.31\%), K (0.1\%-0.13\%), Ca $(0.1 \%-1.0 \%)$, $\mathrm{V}(1.1 \%-12.85 \%)$, Fe $(0.40 \%-0.61 \%)$, and $\mathrm{Ni}(0.35 \%-2.28 \%)$. Possible compound compositions of oil-fired fly ash are: $\mathrm{C}$ as $\mathrm{C} ; \mathrm{Na}$ as $\mathrm{Na}_{2} \mathrm{SO}_{4} ; \mathrm{Mg}$ as $\mathrm{MgO}, \mathrm{Mg}$ as $\mathrm{MgSO}_{4} \cdot \mathrm{H}_{2} \mathrm{O} ; \mathrm{Al}$ as $\mathrm{Al}_{2} \mathrm{O}_{3}, \mathrm{Al}$ as $\mathrm{Al}_{2}\left(\mathrm{SO}_{4}\right)_{3} ; \mathrm{Si}$ as $\mathrm{SiO}_{2} ; \mathrm{K}_{2}$ as $\mathrm{K}_{2} \mathrm{SO}_{4} ; \mathrm{Ca}$ as $\mathrm{CaO}$, and $\mathrm{Ca}$ as $\mathrm{CaSO}_{4} ; \mathrm{V}$ as $\mathrm{V}_{2} \mathrm{O}_{5}, \mathrm{~V}$ as $\mathrm{VOSO}_{4} \cdot 3 \mathrm{H}_{2} \mathrm{O}$; and $\mathrm{Fe}$ as $\mathrm{Fe}_{2} \mathrm{O}_{3}, \mathrm{Fe}$ as $\mathrm{FeSO}_{4}$ [17].

White sand is rich in $\mathrm{C}, \mathrm{O}, \mathrm{Na}, \mathrm{Mg}, \mathrm{Al}, \mathrm{Si}, \mathrm{K}, \mathrm{Ca}, \mathrm{Ti}$, and $\mathrm{Fe}$ (Table 1). Oxygen (51.9 wt \%), Si (20 wt \%), $\mathrm{Al}(8.3 \mathrm{wt} \%), \mathrm{Mg}(3.5 \mathrm{wt} \%), \mathrm{Na}(3.1 \mathrm{wt} \%)$, and $\mathrm{K}(1.9 \mathrm{wt} \%)$ had the highest values of the four sand grain samples. The white sand had the lowest carbon content $(5.4 \mathrm{wt} \%)$, which is likely associated with $\mathrm{Ca}\left(\mathrm{as} \mathrm{CaCO}_{3}-\right.$ calcite, the main component of limestone) and other alkaline earth metals e.g., $\mathrm{Mg}$ (as $\mathrm{CaMg}$ $\left(\mathrm{CO}_{3}\right)$-dolomite), due to the corresponding presence of $\mathrm{O}$ [18]. It should be noted 
that the sample contained 2.4 times more Al than Fe. Both the white sand and the volcanic sand exhibit similar ratios of $\mathrm{O}$ to $\mathrm{Si}$ (2.59 and 2.52, respectively) and Si to $\mathrm{Al}$ (2.41 and 2.63, respectively). The silicates present in the white sand and volcanic sand are mainly silicon oxides and alumino-silicate particles originating from both natural (e.g., earth's crustal matter) and anthropogenic (e.g., combustion of fossil-fuels) sources [25]. Silica-rich particles have been reported as significant constituents of the fine fractions of aerosols collected in arid desert areas of southern Utah [36], the northern Sahara [37], Cairo, Egypt [38], and Phoenix, Arizona [30].

Table 1 reveals that predominant elements in volcanic sand are $\mathrm{Ca}, \mathrm{Al}, \mathrm{C}, \mathrm{Fe}$, $\mathrm{Si}$, and $\mathrm{O}$ : the weight concentration of these elements varies from $6.2 \%$ to $42.3 \%$. Particles are rich in $\mathrm{Ca}$, likely as $\mathrm{CaCO}_{3}$, and contain minor amounts of $\mathrm{V}, \mathrm{Cr}, \mathrm{P}, \mathrm{Mn}, \mathrm{K}$, $\mathrm{Ti}, \mathrm{Na}$, and $\mathrm{Mg}(0.30 \%$ to $3.1 \%)$. Phosphorous had the highest value $(0.40 \mathrm{wt} \%)$ of the four sand grain samples. Phosphorous-bearing particles normally occur as calcium phosphates [25]. Silicates present in the sample, within which Ca-rich silicates are prevalent, come from the soil [39].

\subsection{Enrichment Factors (EFs)}

Enrichment factors (EFs) were calculated to identify the role of potential sources of crustal or anthropogenic pollution:

$$
\mathrm{EF}=\left(\mathrm{C}_{\mathrm{x}} / \mathrm{C}_{\mathrm{Al}}\right)_{\text {sand }} /\left(\mathrm{C}_{\mathrm{x}} / \mathrm{C}_{\mathrm{Al}}\right)_{\text {crust }}
$$

where $C_{x}$ and $C_{A l}$ represent the concentrations of an element and the abundance of aluminum, respectively. The main advantage of the EF is that the enrichment of all elements can be promptly compared. Aluminum was selected as the reference element [40]. Usually, an EF value of $<10$ indicates an element of crustal origin, while an $\mathrm{EF} \geq 10$ is ascribed to an element of anthropogenic source [41]. The EFs of $\mathrm{Na}, \mathrm{Mg}$, $\mathrm{Si}, \mathrm{K}$, and $\mathrm{Ca}$ in magnetic black sand ranged from 0.5 to 3, demonstrating that these elements have a significant crustal origin. EFs of Fe, S, and $\mathrm{Ti}$ were 74, 84, and 88, respectively, revealing a mainly anthropogenic origin. For the non-magnetic black sand, a strong enrichment was observed for $\mathrm{Mn}(\mathrm{EF}=20), \mathrm{Fe}(\mathrm{EF}=23)$, Ti $(\mathrm{EF}=147)$, and $V(E F=237)$, while for all other elements EF values were less than 1 . The EFs of $\mathrm{V}$ and $\mathrm{Cr}$ in volcanic sand were 26 and 37, respectively, which suggests an anthropogenic component.

\subsection{Extractable Ion Analysis by IC}

In order to quantify anthropogenic contributions and further assess SEM/EDS results, ion chromatography analysis of magnetic black sand was also performed, and it confirmed the presences of the following water-soluble anions and cations: sulfate $\left(\mathrm{SO}_{4}{ }^{2-}\right)$, nitrate $\left(\mathrm{NO}_{3}{ }^{-}\right)$, chloride $\left(\mathrm{Cl}^{-}\right)$, sodium $\left(\mathrm{Na}^{+}\right)$, potassium $\left(\mathrm{K}^{+}\right)$, 
magnesium $\left(\mathrm{Mg}^{2+}\right)$, and calcium $\left(\mathrm{Ca}^{2+}\right)$. Among the anions, $\mathrm{SO}_{4}{ }^{2-}$ had the highest concentration (59 ppm), followed by $\mathrm{Cl}^{-}(17 \mathrm{ppm})$ and $\mathrm{NO}_{3}{ }^{-}$(5.2 ppm). Sulfate and $\mathrm{NO}_{3}{ }^{-}$are related to anthropogenic emissions. Generally, S-bearing particles exist in the forms of $\mathrm{SO}_{4}{ }^{2-}, \mathrm{HSO}_{4}{ }^{-}$, and/or $\mathrm{H}_{2} \mathrm{SO}_{4}$ with or without $\mathrm{NH}_{4}{ }^{+}$[26]. In fact, studies have concluded that atmospheric $\mathrm{SO}_{4}{ }^{2-}$ can either be emitted directly as primary particles or result from gas-to-particle conversion reactions in the atmosphere [15,42]. Sulfur dioxide from anthropogenic sources is oxidized into $\mathrm{H}_{2} \mathrm{SO}_{4}$, which in turn accumulates to form condensation nuclei [43]. Sulfur-bearing particles are often observed in airborne PM samples [25,44-46]. Sulfate has been found as the dominant chemical species in aerosol particles in urban atmospheres [47-49]. For example, a study by Zhang et al. [47] indicates the dominance of $\mathrm{SO}_{4}{ }^{2-}(>70 \%)$ in fine particles from the urban area of Beijing during non-dust-storm periods. Bassett and Seinfeld [50] reported that $\mathrm{NO}_{3}{ }^{-}$is hardly formed on fine particles owing to its volatility. Therefore, $\mathrm{NO}_{3}{ }^{-}$may be formed on soot particles through heterogeneous conversions of nitrogen oxides. Our observation is supported by an aerosol particle study by Ganor et al. [51] at Haifa Bay, which has revealed that $\mathrm{SO}_{4}{ }^{2-}$ and $\mathrm{NO}_{3}{ }^{-}$ concentrations were 5-10 times higher in the land breeze than in sea breeze, as a consequence of the emissions from local industries or the long-range transportation of pollutants. The high abundance of $\mathrm{Cl}^{-}$is likely to be linked to $\mathrm{Fe}, \mathrm{Na}, \mathrm{Ca}, \mathrm{K}$, and $\mathrm{Mg}$, and may have been transported long-range by way of sea [52].

As evidenced earlier, the black sand particles have very different morphologies and microstructures compared to the white sand and volcanic sand. An important finding in our study is the predominance of fine and ultrafine particles (50 to $500 \mathrm{~nm}$ ranges). Anthropogenic and natural sources have been implicated for the observed particles. It should be pointed out that Badr is situated $83 \mathrm{~km}$ southeast of Yanbu (Figure 2), which was established in 1975 as one of the country's two industrial centers, and has several oil-fueled power plants, three oil refineries, heavy petrochemical industries, a large cement factory, desalination plants, mineral and metal industries, and several other small industries and workshops. There are naturally occurring lava fields and iron deposits located upwind, near and around Badr. Also, Badr is about $800 \mathrm{~km}$ southeast of Haifa's (Figure 2) extensive industrial zone [52]. In fact, Badr is at a crossroads where PM from different sources may converge: long-range transported polluted air masses from Yanbu and Bay of Haifa, sea spray from the Red Sea itself, and mineral components from the deserts of North Africa (Sahara) and the Arabian Peninsula. Occasionally, Saudi Arabia and nearby countries are hit by sandstorms. The opaque mass of dust, debris, and fine particles in suspension is usually transported by strong winds to high mountains [32]. Backward-in-time trajectories using NOAA HYSPLIT model [53] suggest that the dominant air mass movement is northwesterly from the highly industrialized areas (Yanbu and Haifa) towards the area of concern (Badr). Thus, the morphological 
analysis, composition, proximity to the site, and air mass movements all suggest that emissions by these industries have contributed to particulate air pollution. They were formed as cloud condensation nuclei were readily long-range transported by northwesterly air currents, and finally settled on Douf Mountain.

\section{Conclusions}

High resolution electron microscopy, viz. optical stereo microscope (OSM), scanning electron microscopy with energy dispersive $X$-ray spectrometry (SEM/EDS), and laser scanning microscopy (LSM), was a powerful tool for in-depth analysis and identification of particles by revealing details of the microstructure, morphology, emission source types, size, and elemental composition of each type of sand particles. The physical and chemical characterization of sand samples from Badr, Saudi Arabia by different complimentary techniques has contributed to our knowledge and understanding of the sand particles. These particles could have long-term health consequences to the residents of Badr, but a dedicated health analysis would be needed to investigate this possibility.

White sand contained silicates (alumino-silicates), quartz $\left(\mathrm{SiO}_{2}\right.$, clean crystalline structure, milky, rose), calcite, olivine, feldspar, and magnetite. Compared to white sand and volcanic sand, the black sand particles exhibited very different morphologies and microstructures (surface roughness). Morphological SEM and LSM analyses showed that the black sand contained fine and ultrafine particles (50 to $500 \mathrm{~nm}$ ranges) and there was a thin surface layer $(1 \mu \mathrm{m})$ embedding a large number of particles from the atmospheric deposition. Ion chromatography analyses confirmed the presence of the following water-soluble anions and cations in variable concentrations in magnetic black sand: $\mathrm{SO}_{4}{ }^{2-}, \mathrm{NO}_{3}{ }^{-}, \mathrm{Cl}^{-}, \mathrm{Na}^{+}, \mathrm{K}^{+}, \mathrm{Mg}^{2+}, \mathrm{Ca}^{2+}$.

$\mathrm{X}$-ray energy dispersive microanalyses of sand samples revealed varying weight concentrations of $\mathrm{C}, \mathrm{O}, \mathrm{Na}, \mathrm{Mg}, \mathrm{Al}, \mathrm{Si}, \mathrm{S}, \mathrm{Ca}, \mathrm{Ti}, \mathrm{V}$, and $\mathrm{Fe}$, ranging from $0.1 \%$ to $55 \%$ in all four samples. The predominant elements in black sand were $\mathrm{Fe}, \mathrm{C}, \mathrm{O}, \mathrm{Ti}, \mathrm{Si}, \mathrm{Al}$, $\mathrm{V}$, and $\mathrm{S}$. Enrichment factor (EF) analysis results demonstrated that $\mathrm{Na}, \mathrm{Mg}, \mathrm{Al}, \mathrm{Si}, \mathrm{K}$, and $\mathrm{Ca}$ in black sand originated from natural sources, while $\mathrm{Fe}, \mathrm{C}, \mathrm{Ti}, \mathrm{V}$, and $\mathrm{S}$ were linked to anthropogenic sources. Fossil-fuel combustion and industrial emissions in Haifa and Yanbu can be significant sources of carbon and other contaminant particles, which can be easily adhered to soil particles during long-range transport.

This study presents the first in-depth characterization of black sand particles from sand dunes of Saudi Arabia. Reliable baseline data on particulate air pollution are needed for setting standards and objectives of air pollution controls, and to investigate the role of local and long-range transported anthropogenic emissions. Examining the types, dimensions, and the amount of carbon and other contaminant particles deposited in the coming years will indicate effectiveness of the pollution control devices which have been installed on industries producing energy from 
fossil-fuels. A comprehensive study should be considered to cover the whole area, trace wind pathways from source regions, and outreach to local people to locate other areas where black sand has been deposited. Future research into the issues such as the real size of the ultrafine particles, mechanisms of long-range transport in the region and mechanisms of adhesion of the ultrafine particles to the larger sand grains would help explain these issues more fully. Further research into health outcomes (respiratory diseases, cardiovascular) in the general population and susceptible groups in Badr and other major cities of Saudi Arabia should also be performed.

Acknowledgments: The authors express their appreciation to Abdul Rahman Al Youbi, Vice President for Academic Affairs, King Abdulaziz University and Abdullah Ahmad A. Ghamdi, Dean Research and Consulting Institute, King Abdulaziz University for their support. We gratefully acknowledge the support by the Wadsworth Center, New York State Department of Health. We also thank Curtis Boynton, a summer intern with the NYS DEC, for his help. The authors wish to thank to Tara Nylese of EDAX Inc. for providing the results for confirmation purposes. The authors also wish to thank Brian Frank, Katherine Alben, Simeen Tabatabai, and Jessica Stark for valuable comments and editing the manuscript.

Author Contributions: The study was completed with the cooperation between all the authors. Haider A. Khwaja, Omar S. Aburizaiza, Azhar Siddique, Abdullah J. Aburiziza, and Donald Blake conceived and designed the experiment. The corresponding authors Jahan Zeb, Mohammad Abbass, and Azhar Siddique were in-charge of sampling and collected the relevant data. Daniel Hershey was instrumental in the design of the study, sample analyses by SEM and LSM, and interpretation of the images and X-ray diffraction spectra. David Guerrieri performed all the LSM analyses and data interpretation. Malissa Kramer analyzed the sample by SEM, checked and reported the results. Mirza M. Hussain was in-charge of inorganic anions and cations analyses by Ion Chromatography. Isobel Simpson interpreted the results and critical revision. Paper was written by Haider A. Khwaja with a significant contribution by Omar Aburizaiza, Daniel Hershey and David Guerrieri.

Conflicts of Interest: The authors declare no conflict of interest.

\section{References}

1. D'Almeida, G.A.; Koepke, P.; Shettle, E.P. Atmospheric Aerosols: Global Climatology and Radiative Characteristics; A Deepak Publishing: Hampton, VA, USA, 1991.

2. Jaenicke, R. Physical properties of atmospheric particulate sulfur compounds. Atmos. Environ. 1978, 12, 161-169.

3. Goyer, R. Issue Paper on the Human Health Effects of Metals; U.S. Environmental Protection Agency: Lexington, MA, USA, 2004.

4. Jarup, L.; Akesson, A. Current status of cadmium as an environmental health problem. Toxicol. Appl. Pharmacol. 2009, 238, 201-208.

5. Mamtani, R.; Stren, P.; Dawood, I.; Cheema, S. Metals and Disease: A Global Primary Health Care Perspective. J. Toxicol. 2011, 2011.

6. Yaman, M. Comprehensive comparison of trace metal concentrations in cancerous and non-cancerous human tissues. Curr. Med. Chem. 2006, 13, 2513-2525. 
7. U.S. Environmental Protection Agency (US EPA). Third External Review Draft of Air Quality Criteria for Particulate Matter (April 2002); U.S. Environmental Protection Agency: Research Triangle Park, NC, USA, 2002; Volume 1.

8. Dockery, D.W.; Pope, C.A. Acute respiratory effects of particulate air pollution. Ann. Rev. Public Health 1994, 15, 107-132.

9. Donaldson, K.; MacNee, W. The mechanism of lung injury caused by $\mathrm{PM}_{10}$. In Air Pollution and Health, Issues in Environmental Science and Technology; Hester, R.E., Harrison, R.M., Eds.; Royal Society of Chemistry: Cambridge, UK, 1998; Volume 10, pp. 21-32.

10. Lipmann, M.; Ito, K.; Nadas, A.; Burnett, R.T. Association of Particulate Matter Components with Daily Mortality and Morbidity in Urban Populations; Research Report 95; Health Effects Institute: Cambridge, MA, USA, 2000.

11. Environmental Protection Agency (EPA). Air Quality Criteria for Particulate Matter; EPA 600/P-651001CF; National Center for Environmental Assessment: Research Triangle Park, NC, USA, 1996; Volume III.

12. HEI Perspectives. In Understanding the Health Effects of Components of the Particulate Matter Mix: Progress and Next Steps; Health Effects Institute: Cambridge, MA, USA, 2002.

13. Aust, A.; Smith, K.R.; Veranth, J.M.; Hu, A.; Lightly, J.S.; Ball, J.C.; Stracci, A.M.; Young, W.C. Particle Characteristics Responsible for Effects on Human Lung Epithelial Cells; Health Effects Institute: Cambridge, MA, USA, 2002.

14. Oberdorster, G.; Finkelstein, J.N.; Johnston, C.; Gelein, R.; Cox, C.; Baggs, R.; Elder, A.C.P. Acute Pulmonary Effects of Ultrafine Particles in Rats and Mice; Research Report 96; Health Effects Institute: Cambridge, MA, USA, 2000.

15. Post, J.E.; Buseck, P.R. Characterization of individual particles in the Phoenix urban aerosol using electron-beam instruments. Environ. Sci. Technol. 1984, 18, 35-42.

16. Clark, M.D. Geological Map of the Al Hamra Quadrangle, Sheet 23 C, Kingdom of Saudi Arabia; Directorate General of Mineral Resources: Jeddah, Saudi Arabia, 1981.

17. Henry, W.M.; Knapp, K.T. Compound forms of fossil fuel fly ash emissions. Environ. Sci. Technol. 1980, 14, 450-456.

18. Conny, J.M.; Norris, G.A. Scanning electron microanalysis and analytical challenges of mapping elements in urban atmospheric particles. Environ. Sci. Technol. 2011, 45, 7380-7386.

19. Perrone, M.R.; Turnone, A.; Buccolieri, A.; Buccolieri, G. Particulate matter characterization at a coastal site in south-eastern Italy. J. Environ. Monit. 2006, 8, 183-190.

20. McCrone, W.C. The Particle Atlas. Edition Two; Ann Arbor Science Publishers Inc.: Ann Arbor, MI, USA, 1980.

21. Friedlander, S.K. A review of the dynamics of sulfate containing aerosols. Atmos. Environ. 1978, 12, 187-195.

22. Kleefeld, S.; Hoffer, A.; Krivacsy, Z.; Jennings, S.G. Importance of organic and black carbon in atmospheric aerosols at Mace Head, on the West Coast of Ireland $\left(53^{\circ} 19^{\prime} \mathrm{N}\right.$, $9^{\circ} 54^{\prime}$ W). Atmos. Environ. 2002, 36, 4479-4490. 
23. Chen, Y.; Shah, N.; Huggins, F.E.; Huffman, G.P. Investigation of the microcharacteristics of $\mathrm{PM}_{2.5}$ in residual oil fly ash (ROFA) by analytical transmission electron microscopy. Environ. Sci. Technol. 2004, 38, 6553-6560.

24. Chen, Y.; Shah, N.; Huggins, F.E.; Huffman, G.P. Transmission electron microscopy investigation of ultrafine coal fly ash particles. Environ. Sci. Technol. 2005, 39, 1144-1151.

25. Chen, Y.; Shah, N.; Huggins, F.E.; Huffman, G.P. Microanalysis of ambient particles from Lexington, KY by electron microscopy. Atmos. Environ. 2006, 40, 651-663.

26. Zhang, D.; Iwasaka, Y.; Shi, G. Soot particles and their impacts on the mass cycle in the Tibetan atmosphere. Atmos. Environ. 2001, 35, 5883-5894.

27. Ali-Mohamed, A.Y.; Matter, H.A. Determination of inorganic particulates: (Cationic, anionic and heavy metals) in the atmosphere of some areas in Bahrain during the Gulf crisis in 1991. Atmos. Environ. 1996, 30, 3497-3503.

28. He, K.; Yang, F.; Ma, Y.; Zhang, Q.; Yao, X.; Chan, C.K.; Cadle, S.; Chan, T.; Mulawa, P. The characteristics of $\mathrm{PM}_{2.5}$ in Beijing, China. Atmos. Environ. 2001, 35, 4959-4970.

29. Hughes, L.S.; Cass, G.R.; Gone, J.; Ames, M.; Olmez, I. Physical and chemical characterization of atmospheric ultrafine particles in the Los Angeles area. Environ. Sci. Technol. 1998, 32, 1153-1161.

30. Katrinak, K.A.; Anderson, J.R.; Buseck, P.R. Individual particles types in the aerosol of Phoenix Arizona. Environ. Sci. Technol. 1995, 29, 321-329.

31. Chow, J.C.; Watson, J.G.; Lowenthal, D.H.; Solomon, P.A.; Magliano, K.L.; Ziman, S.D.; Richards, L.W. $\mathrm{PM}_{10}$ and $\mathrm{PM}_{2.5}$ composition in California's San Joaquin Valley. Aerosol Sci. Technol. 1993, 18, 105-128.

32. Tazaki, K.; Wakimoto, R.; Minami, Y.; Yamamoto, M.; Miyata, K.; Sato, K.; Saji, I.; Chaerun, S.K.; Zhou, G.; Morishita, T.; et al. Transport of carbon-bearing dusts from Iraq to Japan during Iraq's war. Atmos. Environ. 2004, 38, 2091-2109.

33. Posfai, M.; Anderson, J.R.; Buseck, P.R.; Shattuck, T.W.; Tindale, N.W. Constituents of a remote Pacific marine aerosol: A TEM study. Atmos. Environ. 1994, 28, 1747-1756.

34. Murr, L.E.; Bang, J.J. Electron microscope comparisons of fine and ultra-fine carbonaceous and non-carbonaceous, airborne particulates. Atmos. Environ. 2003, 37, 4795-4806.

35. Linak, W.P.; Wendt, J.O.L. Trace metal transformation mechanisms during coal combustion. Fuel Process. Technol. 1994, 39, 173-198.

36. Pitchford, M.; Flocchini, R.G.; Draftz, R.G.; Cahill, T.A.; Ashbaugh, L.L.; Eldred, R.A. Silicon in submicron particles in the Southwest. Atmos. Environ. 1981, 15, 321-333.

37. Gomes, L.; Bergametti, G.; Coude-Gaussen, G.; Rognon, P. Submicron desert dusts: A sandblasting process. J. Geophys. Res. 1990, 95, 13927-13935.

38. Hindy, K.T. Silicon, aluminum, iron, copper, and zinc levels in desert soil-related dust in Cairo. Atmos. Environ. 1991, 25, 213-217.

39. Paoletti, L.; de Berardis, B.; Diociaiuti, M. Physico-chemical characterization of the inhalable particulate matter $\left(\mathrm{PM}_{10}\right)$ in an urban area: An analysis of the seasonal trend. Sci. Total Environ. 2002, 292, 265-275.

40. Taylor, S.R. Abundance of chemical elements in the continental crust: A new Table. Geochim. Cosmochim. Acta 1964, 28, 1273-1285. 
41. Tang, X. Atmospheric Chemistry; Higher Education Publisher: Beijing, China, 1990. (In Chinese)

42. Kulmala, M.; Vehkamaki, H.; Petaya, T.; dal Maso, M.; Lauri, A.; Kerminem, V.M.; Birmili, W.; McMurry, P.H. Formation growth rates of ultrafine atmospheric particles: A review of observations. Aerosol Sci. 2004, 35, 143-176.

43. Seinfeld, J.H.; Pandis, S.N. Atmospheric Chemistry and Physics; Wiley: New York, NY, USA, 1998.

44. Buseck, P.R.; Posfai, M. Airborne minerals and related aerosol particles: Effects on climate and the environment. Proc. Natl. Acad. Sci. USA 1999, 96, 3372-3379.

45. Zhang, D.; Shi, G.-Y.; Iwasaka, Y.; Hu, M. Mixture of sulfate and nitrate in coastal atmospheric aerosols: Individual particle studies in Qingdao. Atmos. Environ. 2000, 34, 2669-2679.

46. Posfai, M.; Simonics, R.; Li, J.; Hobbs, P.V.; Buseck, P.R. Individual aerosol particles from biomass burning in southern Africa: 1. Compositions and size distributions of carbonaceous particles. J. Geophys. Res. Atmos. 2003, 104, 15941-15954.

47. Zhang, D.; Tang, X.; Qin, Y.; Iwasaka, Y.; Gai, X. Tests for individual sulfate containing particles in urban atmosphere in Beijing. Adv. Atmos. Sci. 1995, 12, 343-350.

48. Tsitouridou, R.; Voutsa, D.; Kouimtzis, T. Ionic composition of $\mathrm{PM}_{10}$ in the area of Thessaloniki, Greece. Chemosphere 2003, 52, 883-891.

49. Horng, C.L.; Cheng, M.T. Distribution of $\mathrm{PM}_{2.5}$, acidic and basic gases near highway in Central Taiwan. Atmos. Res. 2008, 88, 1-12.

50. Bassett, M.E.; Seinfeld, J.H. Atmospheric equilibrium model of sulfate and nitrate aerosols-II: Particle size analysis. Atmos. Environ. 1984, 18, 1163-1170.

51. Ganor, E.; Levin, Z.; Grieken, R.V. Composition of individual aerosol particles above the Israelian Mediterranean coast during the summer time. Atmos. Environ. 1998, 32, $1631-1642$.

52. Gilmour, P.S.; Brown, D.M.; Lindsay, T.G.; Beswick, P.H.; MacNee, W.; Donaldson, K. Adverse health effects of $\mathrm{PM}_{10}$ particles: Involvement of iron in generation of hydroxyl radical. Occup. Environ. Med. 1996, 53, 817-822.

53. Draxler, R.R.; Rolph, G.D. HYSPLIT (HYbrid Single-Particle Lagrangian Integrated Trajectory); NOAA Air Resources Laboratory: College Park, MD, USA, 2013. 


\title{
Characteristics of $\mathbf{P M}_{10}$ and $\mathbf{P M}_{2.5}$ at Mount Wutai Buddhism Scenic Spot, Shanxi, China
}

\author{
Zhihui Wu, Fenwu Liu and Wenhua Fan
}

Abstract: A survey was conducted to effectively investigate the characteristics of airborne particulate pollutants $\mathrm{PM}_{10}$ and $\mathrm{PM}_{2.5}$ during the peak tourist season at Mount Wutai Buddhism scenic spot, Shanxi, China. Characteristics of the $\mathrm{PM}_{10}$ and $\mathrm{PM}_{2.5}$ in Wu Ye Temple (core incense burners), Manjusri Temple (a traffic hub), Yang Bai Lin Village (a residential district), and Nan Shan Temple (located in a primitive forest district), were determined. The results showed that the $\mathrm{PM}_{10}$ concentration was more than 1.01-1.14 times higher than the threshold $\left(50 \mu \mathrm{g} / \mathrm{m}^{3}\right)$ of World Health Organization Air Quality Guidelines (2005), and the $\mathrm{PM}_{2.5}$ concentration was 1.75-2.70 times higher than the above standard $\left(25 \mu \mathrm{g} / \mathrm{m}^{3}\right)$. Particle size analysis indicated that the distribution of fine particulate matter in $\mathrm{Wu}$ Ye Temple ranged from 0 to $3.30 \mu \mathrm{m}$. In other sampling points, the fine particulate was mainly distributed in the range of 0-5.90 $\mu \mathrm{m}$. The particulates in $\mathrm{Wu}$ Ye Temple were mainly characterized by spherical, rod-like, and irregular soot aggregates $\left(\mathrm{PM}_{10}\right)$ and spherical particles of dust $\left(\mathrm{PM}_{2.5}\right)$. Manjusri Temple and Yang Bai Lin Village predominantly exhibited irregular soil mineral particulate matter $\left(\mathrm{PM}_{10}\right)$, and amorphous ultrafine soot particulate matter $\left(\mathrm{PM}_{2.5}\right)$.

Reprinted from Atmosphere. Cite as: Wu, Z.; Liu, F.; Fan, W. Characteristics of PM 10 and $\mathrm{PM}_{2.5}$ at Mount Wutai Buddhism Scenic Spot, Shanxi, China. Atmosphere 2015, 6, 1195-1210.

\section{Introduction}

Particulate matter impacts the environment, climate, and human health and has become one of the most important pollutants affecting air quality. However, particulate matter, including $\mathrm{PM}_{10}$ with an aerodynamic equivalent diameter less than $10 \mu \mathrm{m}$ and $\mathrm{PM}_{2.5}$ with an aerodynamic equivalent diameter less than $2.5 \mu \mathrm{m}$, has significant effect on human health in the last decades [1]. $\mathrm{PM}_{10}$ can be absorbed by the human body, thereby causing diseases following deposition in the respiratory tract and alveoli, and a number of studies have been conducted on this issue. For example, Wu et al. and Amador-Muñoz et al. studied the concentration and potential threats of $\mathrm{PM}_{10}$ in coal-fired industrial cities in China [2] and in Southwestern Mexico [3]. Makra et al. and Malandrino et al. studied the distribution of $\mathrm{PM}_{10}$ under different traffic patterns in Szeged and Bucharest [4], and the yearly and quarterly changes of the trace elements in $\mathrm{PM}_{10}$ in Turin, Italy [5], respectively. However, few studies 
have been conducted on $\mathrm{PM}_{10}$ distribution in Buddhist scenic spots, where a large number of incenses and candles are burned during the peak tourist season.

Studies on human health, particularly respiratory diseases, have increased in recent years. $\mathrm{PM}_{2.5}$ is likely to adsorb toxic substances and to be inhaled into the alveoli and blood [6], which has increased people's awareness regarding this matter. Compared with $\mathrm{PM}_{10}, \mathrm{PM}_{2.5}$ is characterized by small particle size, large area, and high activity. It is also likely to absorb toxic and harmful substances, have a long residence time in the air, and travel long distances. The majority of recent reports on $\mathrm{PM}_{2.5}$ have focused on the analysis of chemical components in organic pollutants, inorganic chemical components, and microbial components in particulate matter. For instance, Mikuška et al. analyzed all organic pollutants contained in the $\mathrm{PM}_{2.5}$ of Ostrava, an industrial city in the Czech Republic during winter [7]. Salameh et al. studied the seasonal and spatial distribution characteristics of the chemical components of $\mathrm{PM}_{2.5}$ in five cities around the Mediterranean Sea in Europe [8]. Cao et al. extracted DNA from some microorganisms in $\mathrm{PM}_{2.5}$ in Beijing for metagenomic analysis [9].

Work-related stress, fast-paced lifestyles, and severe atmospheric and environmental pollution have caused the degradation of living standards in urban areas. This has led to the popularity of tourism as it allows urban dwellers to get "close to nature". However, increasing vehicle flow and emissions from fossil fuel combustion have resulted in anthropogenic pollution in scenic spots and these effects cannot be underestimated any longer. In scenic spots, some studies on $\mathrm{PM}_{10}$ and $\mathrm{PM}_{2.5}$ focused on particulate matter concentration and analysis of chemical components. For instance, Zhang, et al. [10] studied the samples of aerosol from $\mathrm{PM}_{2.5}$ in the Yulong Scenic Spot southeast of the Qinghai Tibet Plateau, China, during the winter to determine the chemical composition and source. Lee, et al. [11] analyzed the impact of soot caused by biomass burning, the dispersion of $\mathrm{PM}_{2.5}$ concentrations in Southeast Asia, and the presence of water-soluble ions in Lulin Mountain Region, Taiwan.

Few studies have been carried out on single-particle morphology of $\mathrm{PM}_{2.5}$ in a natural scenic spot. Single-particulate matter research techniques can provide single-particle morphology, chemical composition, particle size distribution, and other pertinent information. Analytical data related to single-particulate matter can be used as "fingerprint information", to some extent, to show the corresponding natural or anthropogenic source. Unlike urban areas and other scenic spots, Mount Wutai Scenic Spot, located in Shanxi, China, a sacred place for Buddhists, is visited by many pilgrims who burn candles while praying. During tourists praying at Buddhist tourist spots at Mount Wutai, they burning a lot of incense and candle, which consisted with some combustible biomass materials, such as natural sticky powder, elm wood powder, and fragrant material. Strong pungent odor can be produced 
when incense, candles, and its plastic package materials burning accompanied with intense black smoke. Sometimes, the pungent odor can be easy-nosed by tourists in 30-50 m away. In addition, transportation and catering business are also expansion with the increasing of tourists. Furthermore, some tourists carry out their tourism activities in primitive forest district. During these activities, large amounts of particulate matter may be produced from incense and candles burning in praying, automobile exhaust, coal burning in catering business, and fugitive dust come from bared land caused by the tourists trample in primitive forest district. Unfortunately, to the best of our knowledge, none studies have investigated the $\mathrm{PM}_{10}$ or $\mathrm{PM}_{2.5}$ pollution at Buddhist scenic areas, such as Mount Wutai Scenic Spot.

The initial objective of the present work was to investigate the concentrations of $\mathrm{PM}_{10}$ and $\mathrm{PM}_{2.5}$ in the four different areas, including $\mathrm{Wu}$ Ye Temple (core incense burners), Manjusri Temple (a traffic hub), Yang Bai Lin Village (a residential district), and Nan Shan Temple (located in a primitive forest district), at Mount Wutai scenic spot during the peak season. In order to explore: (1) the typical pattern of particulate matter produced in Mount Wutai Scenic Spot and (2) the main elements, which may enter the human body by breathing these particulate matter. The morphology and elemental composition of $\mathrm{PM}_{10}$ and $\mathrm{PM}_{2.5}$ in the different areas were also investigated. The outcomes will provide scientific data for atmospheric pollution control at Mount Wutai scenic spot.

\section{Results and Discussion}

\subsection{Sampling Points}

The locations of the different sampling areas are shown in Table 1. Three sampling points were included in each area using a triangle model. The arrangement of sampling points in each area is shown in Figure 1.

Table 1. Information on the sampling areas in Mount Wutai, Shanxi, China.

\begin{tabular}{lllc}
\hline Sampling Point & Area & Geographical Position & Altitude (m) \\
\hline \multirow{2}{*}{ Wu Ye Temple } & a candle burning gathering & $113^{\circ} 35^{\prime} 22.9^{\prime \prime} \mathrm{E}$ & $39^{\circ} 03^{\prime} 23.3^{\prime \prime} \mathrm{N}$ \\
\multirow{2}{*}{ Manjusri Temple } & a ate in the scenic spot & $113^{\circ} 35^{\prime} 42.5^{\prime \prime} \mathrm{E}$ & 1672 \\
\multirow{2}{*}{ Yang Bai Lin Village } & a residential district in the & $39^{\circ} 02^{\prime} 31.2^{\prime \prime} \mathrm{N}$ & 1664 \\
& scenic spot & $33^{\circ} 35^{\prime} 21.5^{\prime \prime} \mathrm{E}$ & $16.4^{\prime \prime} \mathrm{N}$ \\
Nan Shan Temple & a primitive forest district & $113^{\circ} 34^{\prime} 18.4^{\prime \prime} \mathrm{E}$ & 1680 \\
& & $38^{\circ} 58^{\prime} 54.8^{\prime \prime} \mathrm{N}$ & 1678 \\
\hline
\end{tabular}



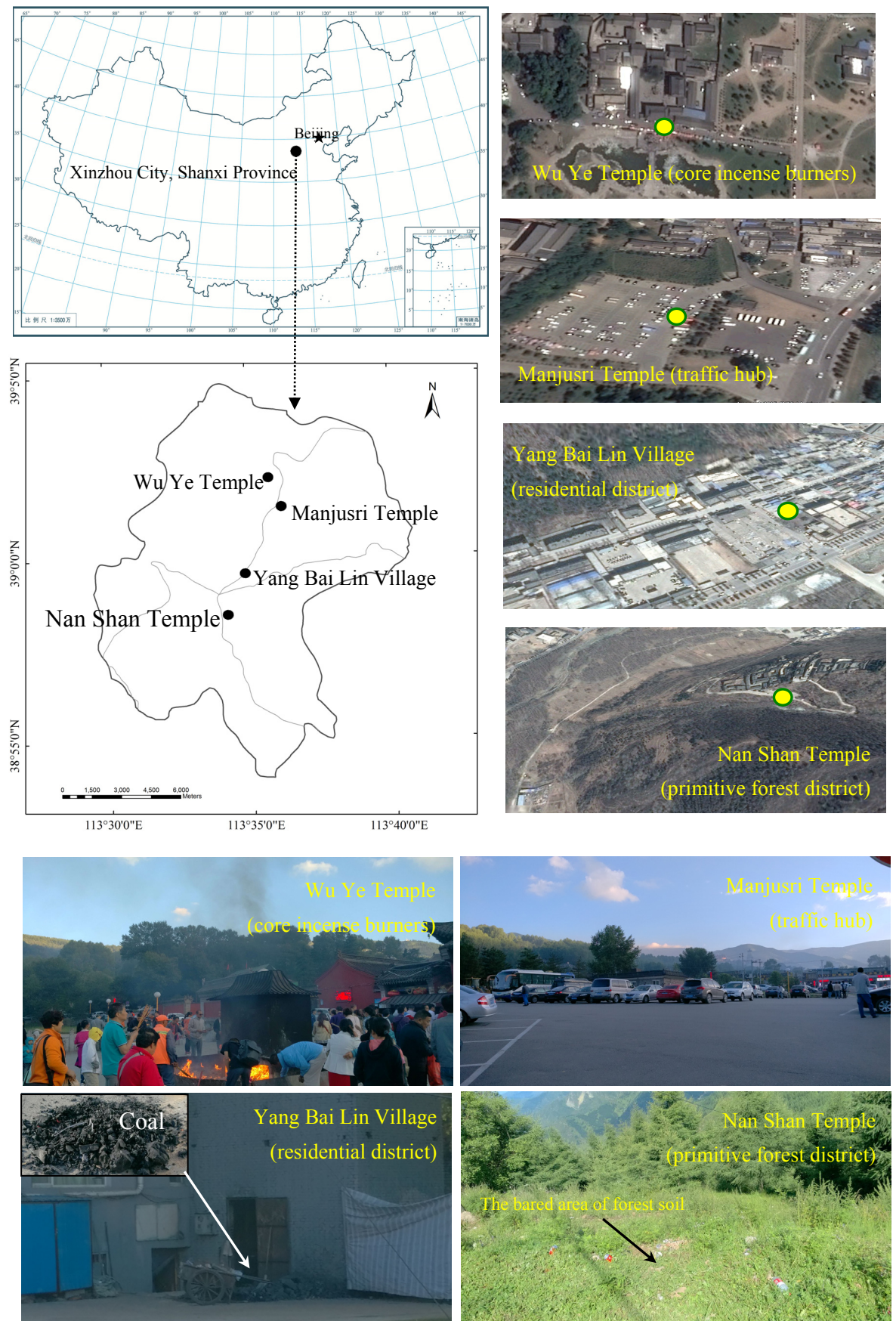

Figure 1. Distribution of sampling sites and its real scene pictures in the four different areas at Mount Wutai, Shanxi, China. 


\subsection{Sampling Methods}

August is the peak tourist time at Mount Wutai. Atmospheric pollution caused by tourism activities during this period is more than that observed in the other seasons. Samples of particulate matter were collected from the four different areas at Mount Wutai scenic spot (Manjusri Temple, Wu Ye Temple, Yang Bai Lin Village, and Nan Shan Temple Forest District) from 20-27 August. These four different areas represent the main area of Mount Wutai. A KB-6120 four-channel moderate-flow integrated atmospheric sampler, provided by Qingdao Xuyu Environmental Co., Ltd. (Qingdao, China), was used with $\mathrm{PM}_{10}$ and $\mathrm{PM}_{2.5}$ cutters to sample the airborne particulates, with a sampling flow of $100 \mathrm{~L} / \mathrm{min}$. A filter membrane of glass fiber (diameter: $47 \mathrm{~mm}$; bore diameter: $0.2 \mu \mathrm{m}$ ) was also used. During sampling, the daily mean temperature was $13.31^{\circ} \mathrm{C}$ and the daily mean wind speed was $2.6 \mathrm{~m} / \mathrm{s}$. Blank samples of collection and transportation were included during sampling. Samples were collected at each sampling point from 3:00 to 23:00 for seven consecutive days with measurements taken every $2 \mathrm{~h}$. To eliminate heterogeneity in the particulate matter settling rate at different altitudes, four different areas were selected at a similar altitude. In this study, eighty particulate matter samples for $\mathrm{PM}_{10}$ monitoring and eighty particulate matter samples for $\mathrm{PM}_{2.5}$ monitoring have been collected at each sampling point.

\subsection{Sample Preparation and Analytical Methods}

The weight of $\mathrm{PM}_{10}$ or $\mathrm{PM}_{2.5}$ was measured using a precision electronic balance. The concentration of $\mathrm{PM}_{10}$ or $\mathrm{PM}_{2.5}$ was calculated by the weight of $\mathrm{PM}_{10}$ or $\mathrm{PM}_{2.5}$ and the sampling volume. The micrograph or morphology of $\mathrm{PM}_{10}$ or $\mathrm{PM}_{2.5}$ were analyzed at the Shanxi Institute of Coal Chemistry of the Chinese Academy of Sciences. The detailed steps are as follows: (1) choose and cut a $5 \mathrm{~mm} \times 5 \mathrm{~mm}$ filter membrane for random sampling; (2) paste the membrane to a circular sample stage of aluminum with appropriate amounts of conductive adhesive; (3) under certain high-vacuum conditions, coat the filter membrane surface with a very thin Platinum (Pt) membrane by ion sputtering; (4) place the membrane in a JEOL JSM-7001F field-emission scanning electron microscope (SEM) for morphology analysis and to identify the pattern of particulate matter. A Bruker QX200 energy dispersive X-ray spectroscope (EDS, Bruker, Germany) was used for element analysis of the particulate matter. Each sample had five randomly selected micrographs that were obtained using the same optical zoom distance. Image-Pro Plus 6.0, micrograph analysis software, was used to process the particulate matter micrographs, obtain the number and equivalent spherical diameter of the particulate matter of different particle sizes, and calculate the average frequency of occurrence of each type of particulate matter of different particle sizes in every micrograph. 


\subsection{Statistical Methods}

Statistical software (SAS 9.2) was used to analyze the experimental data. All results shown in figures are mean values with their standard deviations to show reproducibility and reliability. In other words, the data are represented by means and error bars where the error bars are the standard deviation in all figures. All figures in this paper were drawn using Origin 7.5 software. Two numbers were connected by "-" in this article means the data ranges from minimum value (before "-") to maximum value (after "-"). Two numbers were connected by " \pm " means the mean value (before " \pm ") plus or minus standard deviation (after " \pm "). Significant differences among treatment means were determined using the Student-Newman-Keuls test $(p<0.05)$.

\section{Results and Discussions}

\subsection{Concentration Distribution of $P M_{10}$ and $P M_{2.5}$ at Mount Wutai Scenic Spot}

The mean daily concentrations of $\mathrm{PM}_{10}$ and $\mathrm{PM}_{2.5}$ are shown in Figure 2. The results showed that the $\mathrm{PM}_{10}$ concentration at the four different areas were within the range of $17.40 \mu \mathrm{g} / \mathrm{m}^{3}$ to $161.45 \mu \mathrm{g} / \mathrm{m}^{3}$, whereas the mean daily value at Wu Ye Temple was 1.01-1.14 times higher than the threshold $\left(50 \mu \mathrm{g} / \mathrm{m}^{3}\right)$ of the World Health Organization (WHO) Air Quality Guidelines (2005). The daily value for Nan Shan Temple Forest District was 0.99-1.46 times higher than the threshold of the above standard, respectively. However, at Manjusri Temple and Yang Bai Lin Village sampling points, the $\mathrm{PM}_{10}$ concentrations did not exceed the threshold of the WHO standard. The $\mathrm{PM}_{2.5}$ concentration at the four different areas was $1.43 \mu \mathrm{g} / \mathrm{m}^{3}$ to $59.20 \mu \mathrm{g} / \mathrm{m}^{3}$; the mean daily values of $\mathrm{PM}_{2.5}$ at sampling points $\mathrm{Wu}$ Ye Temple and Nan Shan Temple Forest District were 1.75-2.70 times and 0.99-1.87 times higher than the WHO standard $\left(25 \mu \mathrm{g} / \mathrm{m}^{3}\right)$, respectively. However, the $\mathrm{PM}_{10}$ and $\mathrm{PM}_{2.5}$ concentrations at the Manjusri Temple and Yang Bai Lin Village sampling points were lower than the WHO standard.

In addition, the results of this study show that the mean concentration ratios of $\mathrm{PM}_{2.5}$ to $\mathrm{PM}_{10}$ at $\mathrm{Wu}$ Ye Temple, Manjusri Temple, Yang Bai Lin Village, and Nan Shan Temple Forest District were 0.367, 0.435, 0.083 and 0.760, respectively. Thus, the mean concentration ratio of $\mathrm{PM}_{2.5}$ to $\mathrm{PM}_{10}$ in the four areas was 0.387 . It is concluded that the particulate matter around Manjusri Temple was mostly sub-fine particles with an equivalent spherical diameter range of 2.5-10 $\mu \mathrm{m}$. The sub-fine particle concentrations at Wu Ye Temple and Yang Bai Lin Village were lower that the level at Manjusri Temple. 


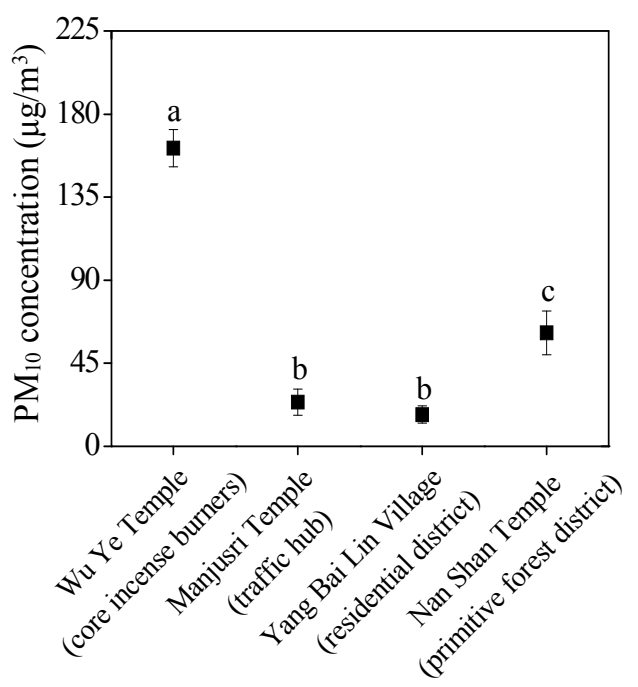

Different functional areas

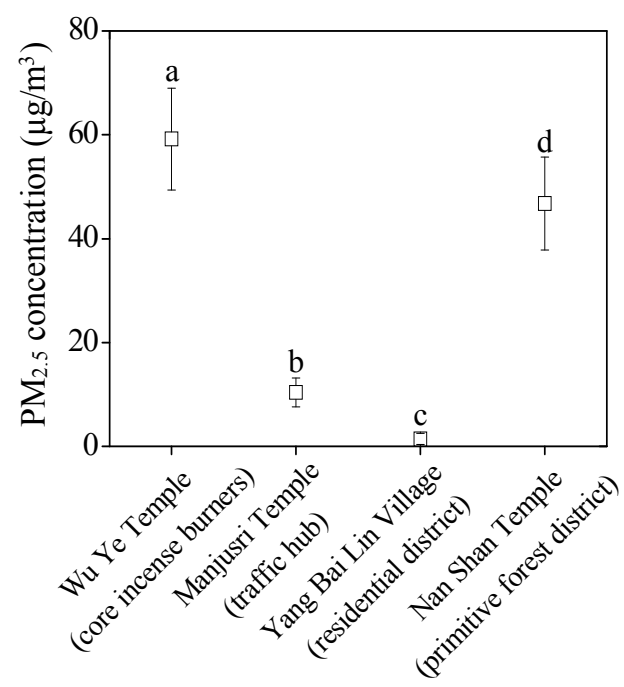

Different functional areas

Figure 2. $\mathrm{PM}_{10}$ and $\mathrm{PM}_{2.5}$ concentration distribution in the four different areas at Mount Wutai, Shanxi, China. Data labeled in the point with the same letter are not significantly different at $p<0.05$. The vertical T-bars indicated standard deviation about the means.

\subsection{Particle Size Distribution of Airborne Particulate Matter at Mount Wutai Scenic Spot}

It is now generally accepted that fine particles are more harmful to health effect than larger particles because fine particles offer a larger surface area and hence potentially larger concentrations of adsorbed or condensed toxic air pollutants per unit mass [12] or fine particles are more efficiently retained in the peripheral lung [13]. Based on equivalent spherical diameter, the particle size distributions of particulate matter in the four different areas are shown in Figure 3.

The main particulate matter at the Wu Ye Temple sampling point had an equivalent spherical diameter of $0-3.30 \mu \mathrm{m}$. The particulate numbers in the range of $0-3.30 \mu \mathrm{m}$ accounted for $80.0 \%$ of the particulate numbers of $\mathrm{PM}_{10}$ at this point, with the peak occurring at 1.10-2.20 $\mu \mathrm{m}$ (Figure 3a). However, no particulate matter in the range between 7.70 and $9.90 \mu \mathrm{m}$ was observed. Furthermore, the particulate numbers of $\mathrm{PM}_{2.5}$ accounted for $65.0 \%$ of the $\mathrm{PM}_{10}$ particulate numbers at this point. The particle size distribution of particulate matter in Manjusri Temple assumed an unimodal pattern (Figure 3b), with particulate matter mainly within the 0-4.60 $\mu \mathrm{m}$ range and accounted for $85.6 \%$ of the total particulate numbers in $\mathrm{PM}_{10}$ at this point. The peak interval was within 1.08-2.16 $\mu \mathrm{m}$. Unlike Wu Ye Temple, the number ratio of $\mathrm{PM}_{2.5}$ to $\mathrm{PM}_{10}$ was $37.1 \%$. Therefore, the proportion of $\mathrm{PM}_{2.5}$ that can penetrate into the lungs was smaller than the particulate matter at $\mathrm{Wu}$ Ye Temple (65.0\%). 
The particle size distribution of particulate matter at Yang Bai Lin Village assumed a bimodal pattern (Figure 3c), which was different to the other sampling points, and the individual peak-to-peak interval at this point was within 1.7-2.4 $\mu \mathrm{m}$ and 4.5-5.2 $\mu \mathrm{m}$ Furthermore, the particulate matter was mainly distributed within the range of $0-5.90 \mu \mathrm{m}$. The particulate number in the range of $0-5.90 \mu \mathrm{m}$ accounted for $86.9 \%$ of the total particulate numbers of $\mathrm{PM}_{10}$ at this point. In addition, the number ratio of $\mathrm{PM}_{2.5}$ to $\mathrm{PM}_{10}$ was $32.6 \%$. The particle size distribution of particulate matter in Nan Shan Temple Forest District assumed a unimodal pattern, with the particulate matter mainly distributed within the range of $0-4.32 \mu \mathrm{m}$, the particulate numbers in this scale accounted for $77.9 \%$ of the total $\mathrm{PM}_{10}$ particulate numbers. The peak occurred within the range of 1.08-2.16 $\mu \mathrm{m}$ (Figure 3d). The number ratio of $\mathrm{PM}_{2.5}$ to $\mathrm{PM}_{10}$ was $42.5 \%$ at this sampling point. Nan Shan Temple Forest District is located in a primitive forest district, and previous studies have shown that the plant leaf surfaces have a relatively strong absorptive capacity for fine particles, thus effectively reducing the airborne particulate matter concentration [14]. However, compared with Manjusri Temple and Yang Bai Lin Village sampling points, the particulate matter concentrations of $\mathrm{PM}_{10}$ and $\mathrm{PM}_{2.5}$ in this area were relatively high (Figure 2). The forest coverage is relatively small or a portion of the primitive forest has been damaged by human activities and may be the main reason for these results.

In conclusion, the number ratios of $\mathrm{PM}_{2.5}$ to $\mathrm{PM}_{10}$ were $65.0 \%, 37.1 \%, 32.6 \%$ and $42.5 \%$ at Wu Ye Temple, Manjusri Temple, Yang Bai Lin Village, and Nan Shan Temple Forest District sampling points. $\mathrm{PM}_{2.5}$ was the main air pollutant in $\mathrm{Wu} \mathrm{Ye}$ Temple (core incense and candle burners). The size of particulate matter in Yang Bai Lin Village (a residential district), Manjusri Temple (a traffic hub), and Nan Shan Temple Forest District (located in a primitive forest district) were mainly distributed between 2.5 and $10 \mu \mathrm{m}$.

\subsection{Single-Particle Morphology and Elemental Analysis of $P M_{10}$ in the Four Different Areas at Mount Wutai, Shanxi, China}

On the basis of field-emission scanning electron microscopy and energy dispersive X-ray spectroscopy (EDS) results of the collected samples (Figures 4 and 5), the single-particle morphology of $\mathrm{PM}_{10}$ was divided into two categories: soot particles and soil dust particles.

On the basis of the distribution of the major elements in particulate matter, the atomic percentages of $\mathrm{O}, \mathrm{Si}, \mathrm{C}, \mathrm{Na}, \mathrm{Al}, \mathrm{Ca}$, and $\mathrm{K}$ were $54.78 \%, 21.40 \%, 9.81 \%, 4.63 \%$, $3.54 \%, 1.96 \%$, and $1.19 \%$, respectively; among these elements, $\mathrm{O}$ and $\mathrm{Si}$ were the major components (Figure 5d). It is concluded that soot aggregates are found in $\mathrm{Wu} Y \mathrm{Ye}$ Temple and Yang Bai Lin Village, and soil particles are found in Manjusri Temple, Yang Bai Lin Village, and Nan Shan Temple Forest District. 

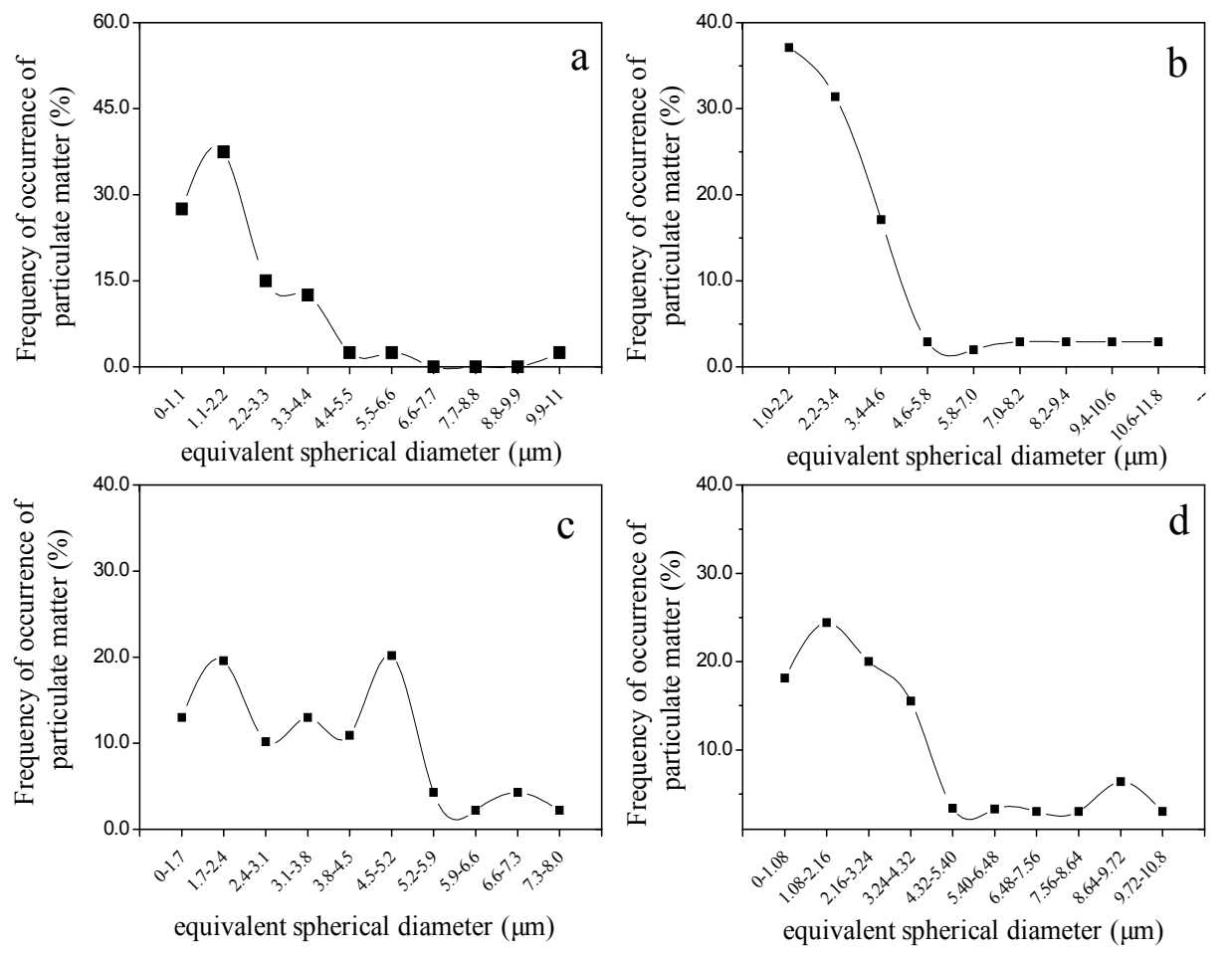

Figure 3. Equivalent spherical diameter distribution of atmospheric fine particles in the four different areas at Mount Wutai, Shanxi, China. ((a) Sampling points in the Wu Ye Temple; (b) sampling points in the Manjusri Temple; (c) sampling points in the Yang Bai Lin Village; (d) sampling points in the Nan Shan Temple Forest District).

Soot particles are formed by scaly fine particle aggregates, with a bitty appearance, loose structure, and particle size of 5.35-8.36 $\mu \mathrm{m}$ Most of the soot particles appeared near the primary tourist areas of $\mathrm{Wu}$ Ye Temple and Yang Bai Lin Village. Soot particles can be roughly divided into three categories on the basis of morphology: near-spherical (Figure 4a), rod-like (Figure 4b), and flocculent amorphous bodies (Figure 4c). The SEM-EDS results of particulate matter collected in the area of incense burners in $\mathrm{Wu}$ Ye Temple (Figure 5a) indicated that the distribution of major elements in such aggregates included $\mathrm{C}, \mathrm{O}, \mathrm{N}, \mathrm{Cl}, \mathrm{Na}$, and $\mathrm{K}$ of which the atomic percentages were $60.98 \%, 17.67 \%, 8.93 \%, 6.46 \%, 4.88 \%$, and $1.08 \%$, respectively, with high contents of $\mathrm{C}, \mathrm{O}$, and $\mathrm{N}$. The main sources of particulate matter were anthropogenic combustion. This phenomenon was described by Carrico et al. [15], who found that an increase in the particle size of soot aerosol particulate matter from biomass burning was mostly dependent upon its hygroscopic 
growth. The large number of soot particle aggregates distributed at the Mount Wutai scenic spot was closely associated with high rainfall and humidity during the summer. The surfaces of most soil dust particles have impurities. Thus, dust particles are not single-mineral particulate matter and had a relatively high frequency of occurrence in the samples collected in Manjusri Temple and from Yang Bai Lin Village. The SEM-EDS results in Figure 5b,c show that the distribution of the major elements in ellipsoidal particles collected from Manjusri Temple (Figure 4d) and Yang Bai Lin Village (Figure 4e) sampling points, which included $\mathrm{O}, \mathrm{Si}, \mathrm{Ca}, \mathrm{Al}$, and $\mathrm{Na}$ with atomic percentages of $57.87 \%, 16.60 \%, 16.32 \%, 4.71 \%$, and $4.49 \%$ (Figure $5 \mathrm{~b}$ ), and of $50.43 \%, 14.58 \%, 12.10 \%, 22.42 \%$, and $0.47 \%$ (Figure $5 c$ ), respectively. Particulate matter containing aluminosilicate minerals was likely formed during secondary atmospheric reactions. This result was verified previously by Dada et al. [16]. Another type of ellipsoidal particle (Figure 4f) was found in the Nan Shan Temple Forest District sampling point, and its surface had a slight depression and greater clastic particle adhesion.
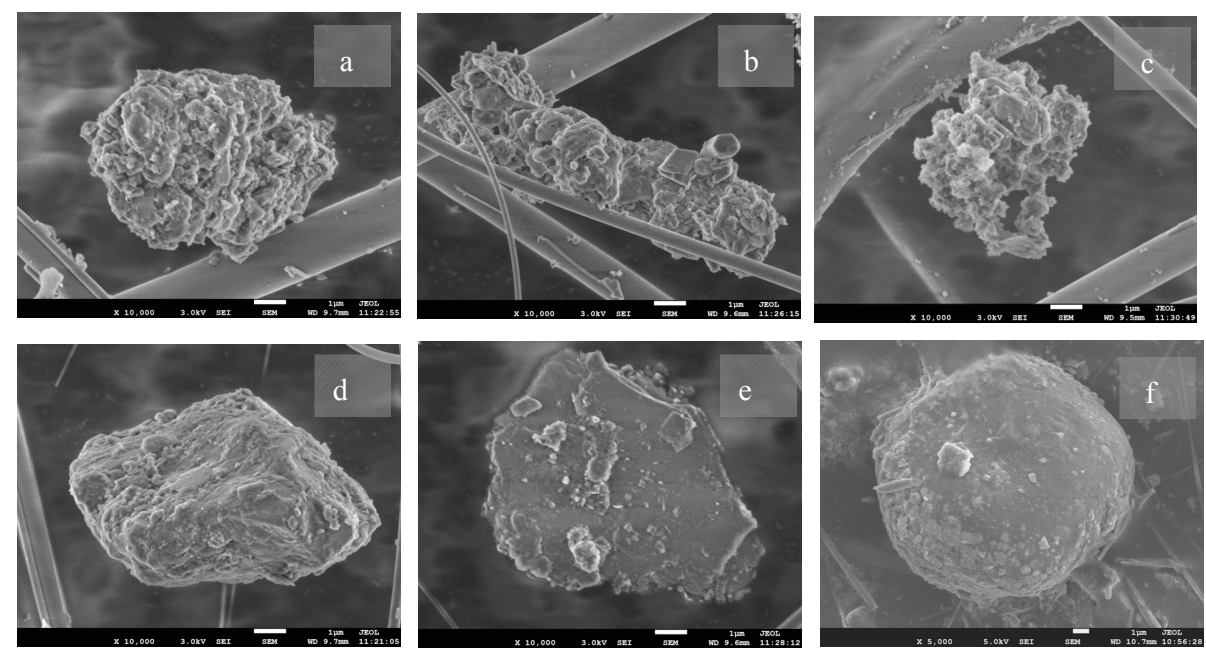

Figure 4. Scanning electron microscopy (SEM) images of atmospheric particles in $\mathrm{PM}_{10}$ at Wutai Mount scenic spot ((a) in the Wu Ye Temple sampling point; (b) in the $\mathrm{Wu}$ Ye Temple sampling point; (c) in the Yang Bai Lin Village sampling point; (d) in the Manjusri Temple sampling point; (e) in the Yang Bai Lin Village sampling point; (f) in the Nan Shan Temple Forest District sampling point). 

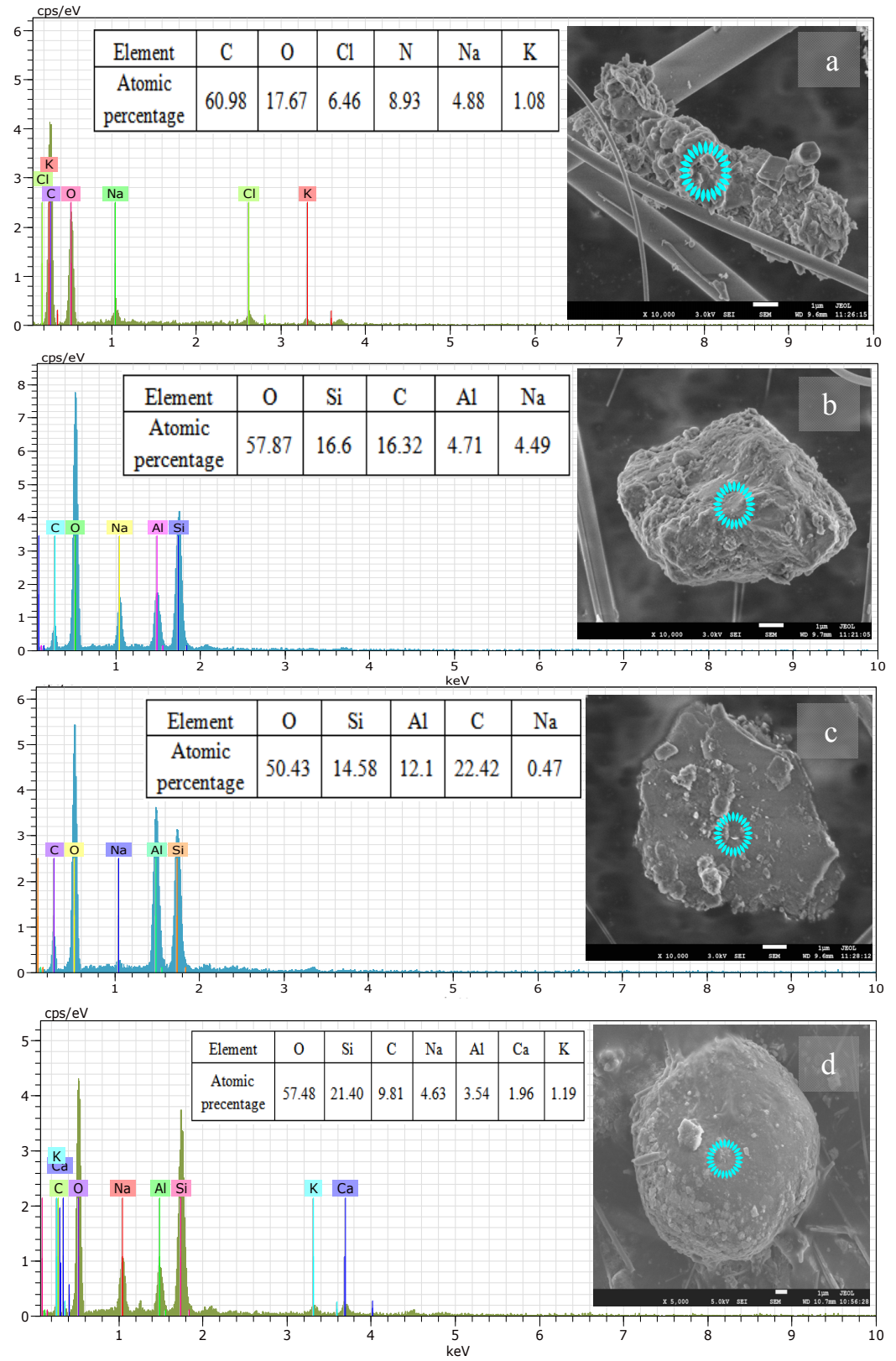

Figure 5. Element analysis of atmospheric particles in $\mathrm{PM}_{10}$ at Wutai Mount scenic spot ((a) in the Wu Ye Temple sampling point; (b) in the Manjusri Temple sampling point; (c) in the Yang Bai Lin Village sampling point; (d) in the Nan Shan Temple Forest District sampling point). 


\subsection{Single-Particle Morphology and Elemental Analysis of PM 2.5 in the Four Different}

Areas at Mount Wutai, Shanxi, China

Similar to $\mathrm{PM}_{10}$, the single-particle morphologies of $\mathrm{PM}_{2.5}$ were also divided into two categories: soot particles and soil dust particles. Figures 6 and 7 show the single-particle morphology and elemental analysis of $\mathrm{PM}_{2.5}$ in the four different areas at Mount Wutai. The shape of $\mathrm{PM}_{2.5}$ was roughly divided into the following three types: ultrafine soot particles, soil dust particles, and spherical soot particles. Soot particles of $\mathrm{PM}_{2.5}$ collected at Wu Ye Temple and Yang Bai Lin Village were characterized by scaly aggregates with a particle size distribution of $0.06-2.37 \mu \mathrm{m}$ (Figure 6a,b). The elemental composition of these particles included $\mathrm{C}, \mathrm{O}, \mathrm{N}, \mathrm{Cl}, \mathrm{Na}$, and $\mathrm{K}$ with atomic percentages of $60.98 \%, 17.67 \%, 8.93 \%, 6.46 \%, 4.88 \%$, and $1.08 \%$, respectively (Figure 7a). The elemental composition of $\mathrm{PM}_{2.5}$ was approximately consistent with that of $\mathrm{PM}_{10}$ (Figure 5a-c); therefore, $\mathrm{PM}_{2.5}$ and $\mathrm{PM}_{10}$ had similar emission sources.
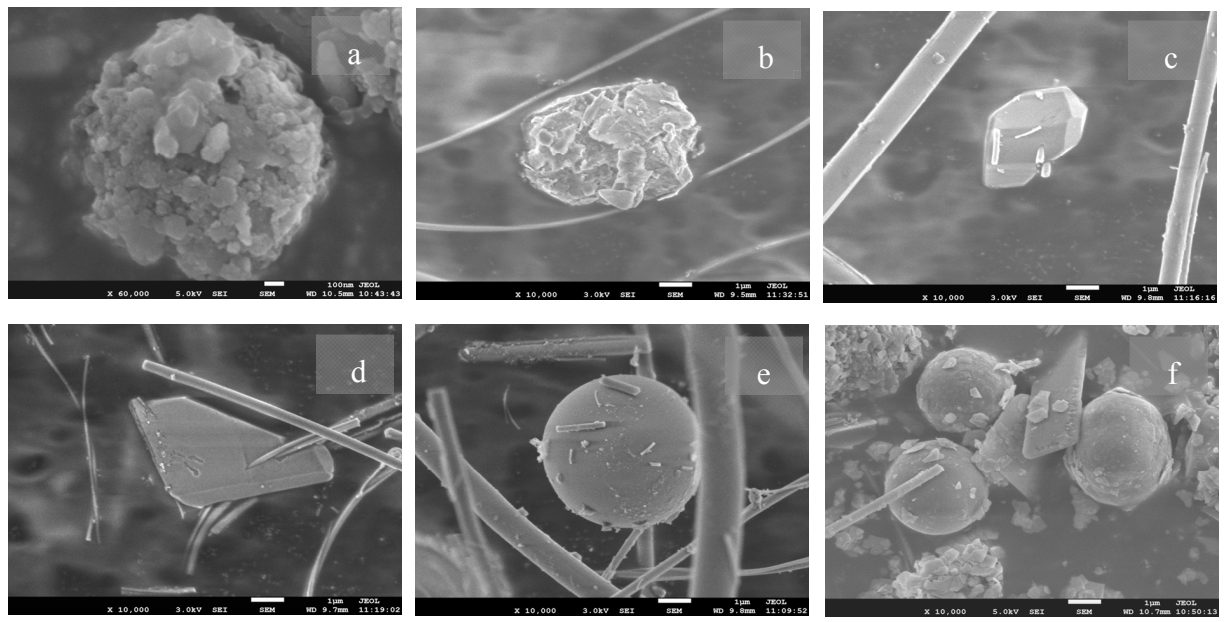

Figure 6. Scanning electron microscopy (SEM) images of atmospheric particles in $\mathrm{PM}_{2.5}$ at Wutai Mount scenic spot ((a) in the Wu Ye Temple sampling point; (b) in the Yang Bai Lin Village sampling point; (c) in the Manjusri Temple sampling point; (d) in the Nan Shan Temple Forest District sampling point; (e) in the Wu Ye Temple sampling point; (f) in the Yang Bai Lin Village sampling point).

Consistent with the findings of Baumgartner et al. [17], black carbon emissions from the area with the incense burners at the Mount Wutai scenic spot, as well as the emissions from six villages in Yunnan and Tibet, are primarily produced by biomass burning.

Soil dust particles of $\mathrm{PM}_{2.5}$ collected at Manjusri Temple and Nan Shan Temple Forest District were mostly characterized by an irregular morphology with distinct 
edges and corners and a particle size distribution of 0.03-1.49 $\mu \mathrm{m}$ (Figure $6 \mathrm{c}, \mathrm{d}$ ). Most fine dust debris were from crust-derived minerals and had elemental compositions of $\mathrm{O}, \mathrm{Ca}, \mathrm{Mg}$, and $\mathrm{C}$ with atomic percentages of $58.47 \%, 17.71 \%, 12.33 \%$, and $11.49 \%$, respectively (Figure $7 \mathrm{~b}$ ). The debris is presumed to be the mineral particulate matter of dolomite $\left(\mathrm{CaMg}\left(\mathrm{CO}_{3}\right)_{2}\right)$ [18] or magnesite $\left(\mathrm{MgCO}_{3}\right)$. [19] reporting on in-site observations in Hengshan, China, confirmed that gritty dust with a particle size of $15-50 \mu \mathrm{m}$ easily becomes the core of dust under light conditions, thus allowing photochemical isomerization with moisture and dust in the atmosphere, promoting the formation of new composite particles, and providing the carrier for the long-distance transport of pollutants. Reducing soil dust particles is important for the reduction of atmospheric particulate matter.
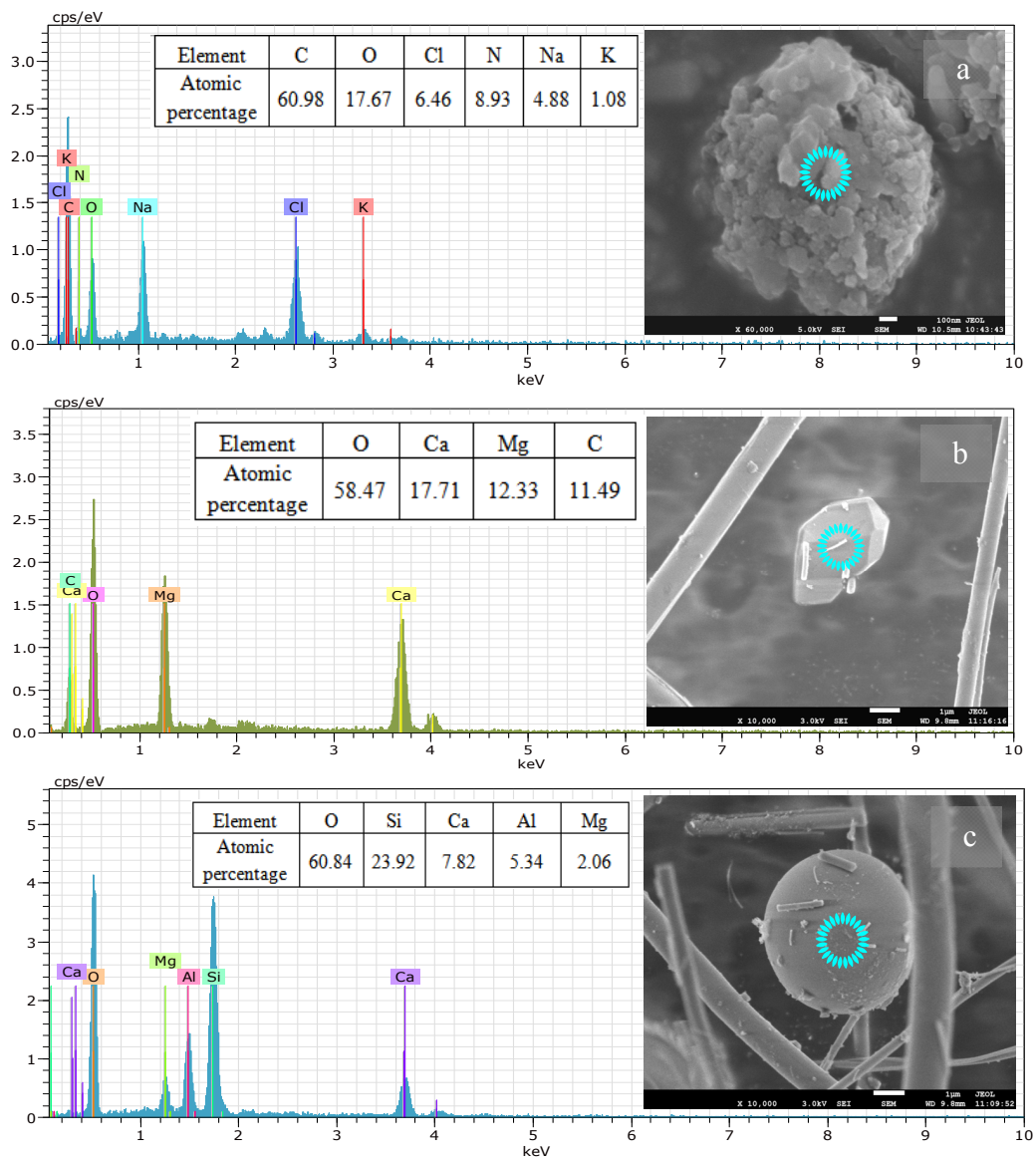

Figure 7. Element analysis of atmospheric particles in $\mathrm{PM}_{2.5}$ at Wutai Mount scenic spot ((a) in the Wu Ye Temple sampling point; (b) in the Manjusri Temple sampling point; (c) in the Wu Ye Temple sampling point). 
However, with the exception of scaly aggregates mentioned above, spherical particles with a smooth surface collected at Wu Ye Temple and Yang Bai Lin Village were another type of soot particle at these sampling points. SEM images combined with micrograph analysis method (Image-Pro Plus 6.0) showed that the average roundness of such particulate matter was $0.37 \pm 0.30 \mu \mathrm{m}$, and that the particle size distribution was 2.99-5.04 $\mu \mathrm{m}$. EDS revealed that the distribution of major elements in the particulate matter included $\mathrm{O}, \mathrm{Si}, \mathrm{Ca}, \mathrm{Al}$, and $\mathrm{Mg}$, with atomic percentages of $60.84 \%, 23.92 \%, 7.82 \%, 5.34 \%$, and 2.06\%, respectively (Figure 7c). Shi et al. [20] found that the particulate matter in the emissions from coal and other high-temperature combustion sources included spherical particulate matter with a smooth surface and a spherical particulate matter with the surface covered with particles. The particulate matter had the same morphology as that of fly ash particles determined by Geng et al. [21] in Tokchok Island, South Korea, during the summer; an 80\% similarity in the distribution of the major elements was found in this study and the current research. Considering the actual situation at the scenic spot, it is concluded that the particulate matter in the scenic spot was mainly sourced from the secondary atmospheric reactions of the emission from biomass burning, such as the fire coal or candles used in catering the primary tourist area.

In conclusion, the main single-particulate morphology of airborne particulate matter at Mount Wutai scenic spot included soot particles and soil dust mineral particles. Huang et al. [22] found that the main emission sources of soot particles are fossil fuel and biomass burning. Reducing the emission of such particulate matter can effectively reduce the concentration of secondary organic aerosol precursors and help avoid the occurrence of haze.

This research, which is consistent with other published literature, suggest implementing pollution control measures in the different functional areas of the Mount Wutai Buddhism Scenic Spot. For Wu Ye temple area, the incense and candles burning furnace should be covered by a smoke collector, in which the strong black smoke can be treated efficiency through absorption and precipitation devices. For Yang Bai Lin Village residential area, the natural gas, electromagnetic or solar energy types should be advocate use to replace coal as the main energy resource for heating and catering. For Manjusri Temple transport hub area, the green belt should be set at the both sides of the road. In addition, the water sprinkler working times and road cleaning should be increased. The vehicles numbers should be restricted in this area. The electric energy or other clean fuel should be employed for vehicles running. For Nanshan Temple next to the primeval forest area, it is should facilitate afforestation as more as possible. Some broad-leaved plants should be cultivated because the broad-leaved plants can intercept the dust produced from bare land and in further reduce the concentration of particulate matter in the atmosphere in this area. 
It is noted that in the process of this research, we focused on the elements kinds and contents in the typical particulate matters, but not the total particulate matters, in the different sampling points. In order to identify potential local origin or long-range transport of the elements, the enrichment factor (EF) of the elements in total particulate matter in different survey areas will be the focus of our further research.

\section{Conclusions}

Characteristics of $\mathrm{PM}_{10}$ with an aerodynamic equivalent diameter less than $10 \mu \mathrm{m}$ and $\mathrm{PM}_{2.5}$ with an aerodynamic equivalent diameter less than $2.5 \mu \mathrm{m}$ in four different areas, including Wu Ye Temple (core incense burners), Manjusri Temple (a traffic hub), Yang Bai Lin Village (a residential district), and Nan Shan Temple (located in a primitive forest district), were determined at Mount Wutai, Shanxi, China, a Buddhist scenic spot. To the best of our knowledge, this study is the first to present the characteristics of $\mathrm{PM}_{10}$ and $\mathrm{PM}_{2.5}$ in a Buddhist scenic spot. It was found that the daily average concentrations of $\mathrm{PM}_{10}$ and $\mathrm{PM}_{2.5}$ were $17.40-161.45 \mu \mathrm{g} / \mathrm{m}^{3}$ and $1.43-59.20 \mu \mathrm{g} / \mathrm{m}^{3}$, respectively. The number ratio of $\mathrm{PM}_{2.5}$ to $\mathrm{PM}_{10}$ was $65.0 \%$ at the $\mathrm{Wu}$ Ye Temple sampling point, and was 37.1\%-42.5\% at the other three sampling points. The particulates in $\mathrm{Wu}$ Ye Temple were mainly characterized by spherical, rod-like, and irregular soot aggregates $\left(\mathrm{PM}_{10}\right)$ and spherical particles of dust $\left(\mathrm{PM}_{2.5}\right)$. Manjusri Temple and Yang Bai Lin Village predominantly exhibited irregular soil mineral particulate matter $\left(\mathrm{PM}_{10}\right)$, and amorphous ultrafine soot particulate matter $\left(\mathrm{PM}_{2.5}\right)$. It is concluded that soot particles with high content of $\mathrm{C}, \mathrm{O}$, and $\mathrm{N}$ in $\mathrm{PM}_{10}$ or with high content of $\mathrm{O}, \mathrm{Si}$, and $\mathrm{Ca}$ in $\mathrm{PM}_{2.5}$, and soil dust particles with high content of $\mathrm{O}, \mathrm{Si}, \mathrm{Ca}$, and $\mathrm{Mg}$ elements in $\mathrm{PM}_{10}$ and $\mathrm{PM}_{2.5}$ are present at Mount Wutai. The findings in this study have great significance in developing measures to control atmospheric pollution at Mount Wutai scenic spot.

Acknowledgments: This work was supported by the Science and Technology Key Project of Shanxi Province (funded social development) (No.20120313011-1) and Shanxi Agricultural University Talent recruitment project (No. 2011017).

Author Contributions: Wenhua Fan conceived and designed the experiments. Zhihui Wu and Fenwu Liu collected and analyzed the data. Zhihui Wu and Fenwu Liu wrote the paper.

Conflicts of Interest: The authors declare no conflict of interest.

\section{References}

1. Pui, D.Y.H.; Chen, S.C.; Zuo, Z. PM 2.5 in China: Measurements, sources, visibility and health effects, and mitigation. Particuology 2014, 13, 1-26. 
2. Wu, D.; Wang, Z.S.; Chen, J.H.; Kong, S.F.; Fu, X.; Deng, H.B.; Shao, G.F.; Wu, G. Polycyclic aromatic hydrocarbons (PAHs) in atmospheric $\mathrm{PM}_{2.5}$ and $\mathrm{PM}_{10}$ at a coal-based industrial city: Implication for PAH control at industrial agglomeration regions, China. Atmos. Res. 2014, 149, 217-229.

3. Amador-Muñoz, O.; Bazán-Torija, S.; Villa-Ferreira, S.A.; Villalobos-Pietrini, R.V.; Bravo-Cabrera, J.L.; Munive-Colín, Z.; Hernández-Mena, L.; Saldarriaga-Noreña, H.; Murillo-Tovar, M.A. Opposing seasonal trends for polycyclic aromatic hydrocarbons and $\mathrm{PM}_{10}$ : Health risk and sources in southwest Mexico City. Atmos Res. 2013, 122, 199-212.

4. Makra, L.; Ionel, I.; Csépe, Z.; Matyasovszky, I.; Lontis, N.; Popescu, F.; Sümeghy, Z. The effect of different transport modes on urban $\mathrm{PM}_{10}$ levels in two European cities. Sci. Total Environ. 2013, 458, 36-46.

5. Malandrino, M.; Martino, M.D.; Ghiotti, G.; Geobaldo, F.; Grosa, M.M.; Giacomino, A.; Abollino, $\mathrm{O}$. Inter-annual and seasonal variability in $\mathrm{PM}_{10}$ samples monitored in the city of Turin (Italy) from 2002 to 2005. Microchem. J. 2013, 107, 76-85.

6. Maté, T.; Guaita, R.; Pichiule, M.; Linares, C.; Díaz, J. Shortterm effect of fine particulate matter $\mathrm{PM}_{2.5}$ on daily mortality due to diseases of the circulatory system in Madrid (Spain). Sci. Total Environ. 2010, 408, 570-575.

7. Mikuška, P.; Křůmal, K.; Večeřa, Z. Characterization of organic compounds in the $\mathrm{PM}_{2.5}$ aerosols in winter in an industrial urban area. Atmos. Environ. 2015, 105, 97-108.

8. Salameh, D.; Detournay, A.; Pey, J.; Pérez, N.; Liguori, F.; Saraga, D.; Bove, M.C.; Brotto, P.; Cassola, F.; Massabò, D.; et al. $\mathrm{PM}_{2.5}$ chemical composition in five European Mediterranean cities: A 1-year study. Atmos. Res. 2015, 155, 102-117.

9. Cao, C.; Jiang, W.; Wang, B.; Fang, J.; Lang, J.; Tian, G.; Jiang, J.; Zhu, T.F. Inhalable microorganisms in Beijing's $\mathrm{PM}_{2.5}$ and $\mathrm{PM}_{10}$ pollutants during a severe smog event. Environ. Sci. Technol. 2014, 48, 1499-1507.

10. Zhang, N.N.; Cao, J.J.; Liu, S.X.; Zhao, Z.Z.; Xu, H.M.; Xiao, S. Chemical composition and sources of $\mathrm{PM}_{2.5}$ and TSP collected at Qinghai Lake during summertime. Atmos. Res. 2014, 138, 213-222.

11. Lee, C.T.; Chuang, M.T.; Lin, N.H.; Wang, J.L.; Sheu, G.R.; Chang, S.C.; Wang, S.H.; Huang, H.; Chen, H.W.; Liu, Y.L.; et al. The enhancement of $\mathrm{PM}_{2.5}$ mass and water-soluble ions of biosmoke transported from Southeast Asia over the Mountain Lulin site in Taiwan. Atmos. Environ. 2011, 45, 5784-5794.

12. Chiu, H.F.; Peng, C.Y.; Wu, T.N.; Yang, C.Y. Short-term effects of fine particulate air pollution on ischemic heart disease hospitalizations in Taipei: A case-crossover study. Aerosol Air Qual. Res. 2013, 13, 1563-1569.

13. Calcabrini, A.; Meschini, S.; Marra, M.; Falzano, L.; Colone, M.; De, B.B.; Paoletti, L.; Arancia, G.; Fiorentini, C. Fine environmental particulate engenders alterations in human lung epithelial A549 cells. Environ. Res. 2004, 95, 82-91.

14. Song, Y.S.; Maher, B.A.; Li, F.; Wang, X.K.; Sun, X.; Zhang, H.X. Particulate matter deposited on leaf of five evergreen species in Beijing, China: Source identification and size distribution. Atmos. Environ. 2015, 105, 53-60. 
15. Carrico, C.M.; Kreidenweis, S.M.; Malm, W.C.; Day, D.E.; Lee, T.; Carrillo, J.; McMeeking, G.R.; Collett, J.L., Jr. Hygroscopic growth behavior of a carbon-dominated aerosol in Yosemite National Park. Atmos. Environ. 2005, 39, 1393-1404.

16. Dada, L.; Mrad, R.; Siffert, S.; Salib, N.A. Atmospheric markers of African and Arabian dust in an urban eastern Mediterranean environment, Beirut, Lebanon. J. Aerosol Sci. 2013, 66, 187-192.

17. Baumgartner, J.; Zhang, Y.; Schauer, J.J.; Huang, W.; Wang, Y.; Ezzati, M. Highway proximity and black carbon from cookstoves as a risk factor for higher blood pressure in rural China. P. Natl. Acad. Sci. USA. 2014, 111, 13229-13234.

18. Krueger, B.J.; Grassian, V.H.; Cowin, J.P.; Laskin, A. Heterogeneous chemistry of individual mineral dust particles from different dust source regions: The importance of particle mineralogy. Atmos. Environ. 2004, 38, 6253-6261.

19. Nie, W.; Ding, A.J.; Wang, T.; Kerminen, V.M.; George, C.; Xue, L.K.; Wang, W.X.; Zhang, Q.Z.; Petaja, T.; Qi, X.M.; et al. Polluted dust promotes new particle formation and growth. Sci. Rep. 2014.

20. Shi, Z.B.; Shao, L.Y.; Jones, T.P.; Whittaker, A.G.; Richards, R.J.; Zhang, P.F. Oxidative stress on plasmid DNA induced by inhalable particles in the urban atmosphere. Sci. Bull. 2004, 49, 692-697.

21. Geng, H.; Jung, H.J.; Park, Y.M.; Hwang, H.J.; Kim, H.K.; Kim, Y.J.; Sunwoo, Y.; Ro, C. Morphological and chemical composition characteristics of summertime atmospheric particles collected at Tokchok Island, Korea. Atmos. Environ. 2009, 43, 3364-3373.

22. Huang, R.J.; Zhang, Y.; Bozzrtti, C.; Ho, K.F.; Cao, J.J.; Han, Y.; Daellenbach, K.R.; Slowik, J.G.; Platt, S.M.; Canonaco, F.; et al. High secondary aerosol contribution to particulate pollution during haze events in China. Nature. 2014, 514, 218-222. 


\title{
Analysis of PAHs Associated with Particulate Matter $\mathbf{P M}_{2.5}$ in Two Places at the City of Cuernavaca, Morelos, México
}

\author{
Hugo Saldarriaga-Noreña, Rebecca López-Márquez, Mario Murillo-Tovar, \\ Leonel Hernández-Mena, Efrén Ospina-Noreña, Enrique Sánchez-Salinas, \\ Stefan Waliszewski and Silvia Montiel-Palma
}

\begin{abstract}
This study was carried out between January and February 2013, at two sites in the city of Cuernavaca, México, using low-volume equipment. Fifteen Polycyclic aromatic hydrocarbons (PAHs), were identified by gas chromatography coupled with mass spectrometry. The total average concentration observed for PAHs was $24.0 \mathrm{ng} \cdot \mathrm{m}^{-3}$, with the high molecular weight compounds being the most abundant. The estimated equivalent concentration for Benzo (a) P (BaPE) was $4.05 \mathrm{ng} \cdot \mathrm{m}^{-3}$. Diagnostic ratios together with the principal components analysis (PCA) allowed for establishing coal burning and vehicle emissions as being the main sources of these compounds in the area. The PAHs used to calculate this index account for $51 \%$ of the 15 PAHs identified, which probably involves a risk to the exposed population.
\end{abstract}

Reprinted from Atmosphere. Cite as: Saldarriaga-Noreña, H.; López-Márquez, R.; Murillo-Tovar, M.; Hernández-Mena, L.; Ospina-Noreña, E.; Sánchez-Salinas, E.; Waliszewski, S.; Montiel-Palma, S. Analysis of PAHs Associated with Particulate Matter $\mathrm{PM}_{2.5}$ in Two Places at the City of Cuernavaca, Morelos, México. Atmosphere 2015, 6, 1259-1270.

\section{Introduction}

Polycyclic aromatic hydrocarbons (PAHs) are one of the most studied families of organic compounds present in the atmosphere, given their proven negative effects on health and persistence in the environment. Different PAHs, specifically those with high molecular weight, which predominate in the particulate phase of atmospheric aerosols, have shown to be carcinogenic [1].

It is also known that PAHs accumulated in particles are mainly associated with fine particulate matter $\left(\mathrm{PM}_{2.5}\right)$. These fine particles are better able to penetrate the respiratory system, which increases their potential health effects [2,3].

PAHs are produced by incomplete combustion and pyrolysis of fossil fuels such as oil and coal, and from other organic materials from natural and anthropogenic sources [4-6], including vehicle emissions, wood burning, waste incineration, coke production, and metal production [7-9].

Their concentrations in ambient air are lower in rural than in urban and suburban areas. Moreover, they can have different profiles depending on the geographical 
location, the type of sources and the dominant atmospheric characteristics [10]. To identify possible sources of these compounds, diagnostic ratios were used. However, these should be used with caution, because sometimes the discrimination between these compounds is difficult, taking into account the reactivity of some of the PAHs with species such as $\mathrm{NO}_{x}, \mathrm{O}_{3}$, etc. [11].

For the city of Cuernavaca, there is little information on the chemical characterization of $\mathrm{PM}_{2.5}$, specifically in determining PAHs. Therefore, the present study has as its main objective to determine ambient levels of these compounds and their possible sources. The results will serve as a basis for mitigation programs of air pollution and/or generation of regulatory standards for the environmental authorities responsible for monitoring of air quality.

\section{Results and Discussion}

\subsection{Wind Trajectories}

During the study period, the winds came mainly from the southeast of the state $(66 \%)$, which probably suggests that part of air pollutants identified in the Autonomous University of State of Morelos (in the north) had its origin in the industrial zone of the city (located to the south) and the downtown area of the city. However, other possible sources could be the emissions from the Popocatepetl volcano, located to the south east of the State (Figure 1).

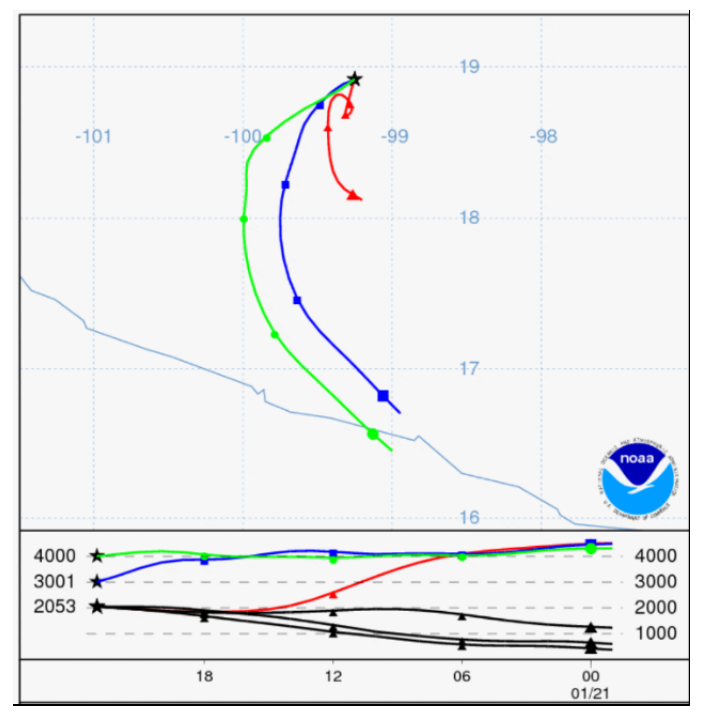

Figure 1. Behavior of wind trajectories in Cuernavaca during January and February 2013. Source: $18^{\circ} 55^{\prime} \mathrm{N}, 99^{\circ} 13^{\prime} \mathrm{W}$, heights: 1000,2000 and $3000 \mathrm{~m}$ above mean sea level (a.m.s.l.). 


\subsection{Concentration of PAHs}

PAHs with 2 and 3 aromatic rings (Acen, Ace, Flu, Phe and Ant) showed the lowest levels of concentration at both sites, whereas those with 4-6 rings (Flt, Pyr, BaA, $\mathrm{Chr}, \mathrm{BbF}, \mathrm{BkF}, \mathrm{BaP}, \mathrm{IP}, \mathrm{DBahA}, \mathrm{BghiP})$ were the most abundant. This suggests that the combustion of gasoline constitutes one of the main sources of these compounds in the study area, which is consistent with that observed by Zielinska et al. 2004 [12].

At the downtown site the average total PAHs concentration was $22.57 \mathrm{ng} \cdot \mathrm{m}^{-3}$ ( $\Sigma 15$ PAHs), while that at the CIQ site was $25.43 \mathrm{ng} \cdot \mathrm{m}^{-3}$ ( $\Sigma 15 \mathrm{PAHs}$ ), indicating that there were no statistically significant differences in the concentration of this family of compounds between sites $(p<0.05)$. Such behavior suggests relatively stable atmospheric conditions or similar emission sources in the study area (Table 1). Also the proximity of the University Campus to the México-Cuernavaca highway, probably is affecting the concentration of PAHs in this area, as well as transportation from the southern part of the city, such as was seen in the wind field.

\subsection{Comparison with Other Studies}

Comparisons with other studies, were carried out using the average between the two study sites. The average total concentration in Cuernavaca $\left(24.0 \mathrm{ng} \cdot \mathrm{m}^{-3}, \Sigma 15\right.$ PAHs) was similar to that reported in Taichung, China (22.29 ng $\cdot \mathrm{m}^{-3}, \Sigma 15$ PAHs) [13] and in Seoul, Korea (26.14 $\left.\mathrm{ng} \cdot \mathrm{m}^{-3}, \Sigma 14 \mathrm{PAHs}\right)$ [14]. It was lower than that reported for Nanjing (62.58 $\mathrm{ng} \cdot \mathrm{m}^{-3}, \Sigma 15$ PAHs) [6] and Jinzhou, China (190.86 ng. ${ }^{-3}, \Sigma 13$ PAHs) [15]. All these places have the characteristic of being large urban areas with high vehicular activity. However, the average total concentration was higher than that reported in Atlanta, USA (1.52 ng. $\mathrm{m}^{-3}, \Sigma 15$ PAHs) [16], Wanqingsha, Hong Kong (19.3 ng $\cdot \mathrm{m}^{-3}, \Sigma 17$ PAHs) [17] Porto, Portugal (13.3 ng $\cdot \mathrm{m}^{-3}, \Sigma 15$ PAHs) [18] and Mount Taishan, China (7.05 ng. $\mathrm{m}^{-3} \Sigma 15$ PAHs) [19]. These results are consistent with the type of industrial and/or vehicular activities, which occur in this urban area.

\subsection{Possible Sources of PAHs in Cuernavaca}

\subsubsection{Diagnostic Ratios}

Given the variability in the concentration of PAHs emitted into the atmosphere, often diagnostic ratios between them are used in order to estimate the possible sources [20]. However, they should be used with caution, if one considers, for example, reactivity with some of the $\mathrm{PAH}$ species such as $\mathrm{NO}_{\mathrm{x}}, \mathrm{O}_{3}$, etc. [9].

Diagnostic ratios used in this study, were calculated and reported by various authors, which established ranges or values indicating possible sources of origin of polycyclic aromatic hydrocarbons (Table 2). 
Table 1. Average concentration of PAHs associated with particulate matter $\mathrm{PM}_{2.5}$ in two places of the city of Cuernavaca, Morelos during the winter season of $2013\left(\mathrm{ng} \cdot \mathrm{m}^{-3}\right)$.

\begin{tabular}{ccccccccccc}
\hline & & \multicolumn{3}{c}{ CIQ } & \multicolumn{6}{c}{ GB (Downtown) } \\
- & Media & Min & Max & SD & Media & Media & Min & Max & SD & Median \\
\hline Acen & 0.81 & 0.72 & 0.90 & 0.06 & 0.80 & 1.29 & 0.67 & 6.17 & 1.62 & 0.82 \\
Ace & 0.96 & 0.79 & 1.25 & 0.16 & 0.94 & 0.93 & 0.63 & 1.46 & 0.23 & 0.89 \\
Flu & 0.95 & 0.77 & 1.09 & 0.12 & 0.98 & 0.94 & 0.72 & 1.15 & 0.14 & 0.99 \\
Phe & 1.27 & 1.00 & 1.45 & 0.14 & 1.34 & 1.18 & 0.79 & 1.46 & 0.25 & 1.23 \\
Ant & 1.07 & 0.87 & 1.24 & 0.10 & 1.10 & 1.14 & 0.77 & 1.64 & 0.24 & 1.15 \\
Flt & 1.73 & 1.07 & 2.76 & 0.51 & 1.61 & 1.24 & 0.63 & 1.69 & 0.34 & 1.32 \\
Pyr & 1.39 & 0.99 & 1.82 & 0.24 & 1.45 & 1.22 & 0.63 & 1.56 & 0.32 & 1.29 \\
BaA & 1.57 & 1.17 & 2.11 & 0.26 & 1.57 & 1.33 & 0.77 & 1.79 & 0.30 & 1.32 \\
Chr & 1.54 & 0.99 & 2.04 & 0.27 & 1.55 & 1.39 & 0.69 & 1.78 & 0.36 & 1.44 \\
BbF & 2.68 & 1.83 & 3.49 & 0.49 & 2.73 & 2.36 & 1.04 & 3.34 & 0.77 & 2.53 \\
BkF & 2.75 & 1.82 & 3.58 & 0.46 & 2.78 & 2.45 & 1.17 & 3.32 & 0.74 & 2.52 \\
BaP & 2.63 & 1.53 & 3.54 & 0.50 & 2.55 & 2.27 & 0.93 & 3.13 & 0.73 & 2.47 \\
IP & 2.14 & 1.56 & 2.81 & 0.35 & 2.14 & 1.70 & 0.89 & 2.56 & 0.56 & 1.64 \\
DBahA & 1.68 & 1.11 & 2.16 & 0.26 & 1.72 & 1.46 & 0.92 & 2.00 & 0.32 & 1.56 \\
BghiP & 2.25 & 1.57 & 3.36 & 0.50 & 2.16 & 1.68 & 0.53 & 3.13 & 0.83 & 1.72 \\
Total & 25.43 & - & - & - & - & 22.57 & - & - & - & - \\
$($ (EHAP) & & & & & & & & & & \\
\hline
\end{tabular}

$\mathrm{n}=11$; Min: minimum value; Max: maximal value; SD: standard deviation.

Table 2. Diagnostic ratios.

\begin{tabular}{cccccc}
\hline Diagnostic Ratios & $\begin{array}{c}\text { Gasoline } \\
\text { Engines }\end{array}$ & $\begin{array}{c}\text { Diesel } \\
\text { Engines }\end{array}$ & Coal & $\begin{array}{c}\text { Wood } \\
\text { Combustion }\end{array}$ & This Study \\
\hline Flt / (Flt + Pyr) [21,22] & $0.40-0.50$ & $0.40-0.50$ & $>0.50$ & $>0.50$ & $\mathbf{0 . 4 2}$ \\
IP/( IP + BghiP) [21,23-25] & 0.18 & $0.37-0.70$ & 0.56 & 0.62 & $\mathbf{0 . 5 0}$ \\
Phe/ ( Phe + Ant) [23,26] & 0.50 & 0.65 & 0.76 & - & $\mathbf{0 . 5 2}$ \\
IP/ BghiP [27,28] & 0.22 & 0.50 & 1.30 & - & $\mathbf{1 . 0 5}$ \\
\hline
\end{tabular}

The ratio Flt/(Flt + Pyr) was between 0.40 and 0.50, suggesting that the study area is influenced by emissions from gasoline and diesel [21,22]. Meanwhile the $\mathrm{IP} /(\mathrm{IP}+\mathrm{BghiP})$ ratio obtained in this study was 0.5 . Some previous studies that were done in other places have reported values between 0.18 and 0.40 corresponding to vehicle emissions, 0.56 corresponding to coal burning and 0.62 to wood combustion [21-25], indicating that in the study area coal burning is an important source. The Phe/(Ant + Phe) ratio was also estimated, giving a value of 0.52 . This value is associated with vehicle emissions, specifically gasoline [23,26]. Meanwhile the IP/BghiP (1.05) ratio indicated coal burning as a major source in this zone $[27,28]$. These results are congruent with the dimensions, anthropogenic activities and population density of the Cuernavaca city. Recently, Ortíz-Hernández et al. [29] performed an emissions inventory, this study suggests 
vehicle emissions, coal burning and forest activities as the principal sources of greenhouse gases in this region.

\subsection{Principal Component Analysis}

A principal component analysis (PCA) with varimax rotation was performed to determine the possible sources of these compounds in the city of Cuernavaca. The principal components (PC) were selected with eigenvalues greater than 1.0. In total 3 PC were extracted. The PC1 explained $75.6 \%$ of the total variance. In this component the compounds loads were similar. Although the BaA, Chr and BaP stand out slightly, these compounds are considered as markers of pyrogenic sources (combustion of oil and coal) [30]. While the PC2 represented $8.5 \%$ of the remaining variance, the compounds with the greatest burden in this component were BkF, IP and BghiP, which are associated with vehicle emissions [30,31]. Meanwhile, the PC3 explained $7.0 \%$, and the greatest burden was provided by Ace and Flu, considered as indicators of coke production (Table 3).

The results show that the study area is being specifically affected by emissions from gasoline and diesel.

The combined results of the PCA and diagnostic ratios suggests that vehicle emissions and coal combustion are probably the main sources of PAHs associated with the $\mathrm{PM}_{2.5}$ in the study area.

Table 3. Principal Component Analysis for Cuernavaca.

\begin{tabular}{cccc}
\hline- & PC1 & PC2 & PC3 \\
\hline Eigenvalues & 11.30 & 1.30 & 1.10 \\
\% Variance accumulated & 75.60 & 84.10 & 91.10 \\
Acen & 0.23 & 0.15 & -0.05 \\
Ace & 0.17 & -0.24 & $\mathbf{0 . 6 6}$ \\
Flu & 0.25 & 0.17 & -0.14 \\
Phe & 0.26 & 0.10 & -0.31 \\
Ant & 0.26 & -0.26 & -0.15 \\
Flt & 0.24 & 0.04 & $\mathbf{0 . 4 1}$ \\
Pyr & 0.27 & 0.14 & 0.27 \\
BaA & $\mathbf{0 . 2 8}$ & 0.06 & 0.20 \\
Chr & $\mathbf{0 . 2 9}$ & 0.12 & 0.02 \\
BbF & 0.27 & 0.16 & -0.02 \\
BkF & 0.26 & $\mathbf{0 . 3 7}$ & -0.19 \\
BaP & $\mathbf{0 . 2 9}$ & 0.12 & -0.13 \\
IP & 0.27 & $\mathbf{0 . 2 9}$ & 0.05 \\
DBahA & 0.27 & -0.06 & -0.27 \\
BghiP & 0.26 & $\mathbf{0 . 2 8}$ & -0.11 \\
\hline
\end{tabular}




\subsection{Evaluation of Health Risk}

The value obtained for the BaPE in this study was $4.05 \mathrm{ng} \cdot \mathrm{m}^{-3}$. This value is lower than that reported for Nanging, China, $\left(7.1 \mathrm{ng} \cdot \mathrm{m}^{-3}\right)$ [6] and Zonguldak, Turkey $\left(14.1 \mathrm{ng} \cdot \mathrm{m}^{-3}\right)$ [32]. However, it was higher than that found in Hamilton, Canada $\left(0.84 \mathrm{ng} \cdot \mathrm{m}^{-3}\right)$ [33] and in Florence, Italy $\left(0.92 \mathrm{ng} \cdot \mathrm{m}^{-3}\right)$ [34]. The compounds used to calculate $\mathrm{BaPE}(\mathrm{BaA}, \mathrm{BaP}, \mathrm{BbF}, \mathrm{BkF}, \mathrm{IP}, \mathrm{DBah}$ ) represent $51 \%$ of the PAHs identified in Cuernavaca, which constitutes a risk factor for the exposed population, taking into account the high degree of penetration in the respiratory system that have the $\mathrm{PM}_{2.5}$ particles.

\section{Experimental Section}

\subsection{Sampling Sites}

The study was conducted at two sites in the city of Cuernavaca. The first one is located in the downtown of the city (Government Building, GB), which is characterized by high commercial and vehicular activity. The second is located north of the city, at the Autonomous University of State of Morelos Campus ((Center of Chemical Research, CIQ), for its acronym in Spanish). This location is characterized as being surrounded by mountains, having a great variety of vegetation, and being influenced by the Mexico-Cuernavaca highway. The city has a total area of $207.5 \mathrm{~km}^{2}$ and a population of 338,620 inhabitants. During the study period, the average temperature was $23.1^{\circ} \mathrm{C}$ (minimum $9.6^{\circ} \mathrm{C}$, maximum $26.7^{\circ} \mathrm{C}$ ), the relative humidity was $36.2 \%$ and the average wind speed was $5.3 \mathrm{~m} \cdot \mathrm{s}^{-1}$ (Figure 2).

The particles were collected on quartz filters of $47 \mathrm{~mm}$ diameter and in a pore size of $2.0 \mu \mathrm{m}$, baked to $180{ }^{\circ} \mathrm{C}$ for at least $24 \mathrm{~h}$ to remove adsorbed organics, after which they were transferred to a chamber with relative humidity of $<40 \%$ at $20-23{ }^{\circ} \mathrm{C}$ for another $24 \mathrm{~h}$ for conditioning. Afterward, the filters with particles were equilibrated in the chamber for an additional $24 \mathrm{~h}$. Low volume equipment (Mini-Vol) were used at a constant flow of $5.0 \mathrm{~L} \cdot \mathrm{min}^{-1}$, equipped with impactors for $\mathrm{PM}_{10}$ and $\mathrm{PM}_{2.5}$. Sampling was carried out in periods of $24 \mathrm{~h}$ (12:00-12:00), from January 25 to 28 February 2013, every two days. In total, 11 samples were collected for each site. After sampling, the filters were refrigerated $\left(4^{\circ} \mathrm{C}\right)$ until extraction.

\subsection{Wind Trajectories}

To determine the behavior of winds in the study area, HYSPLIT4 model trajectories obtained from the NOAA (National Oceanic and Atmospheric Administration) were used. The trajectories were made for the entire study period [35]. 


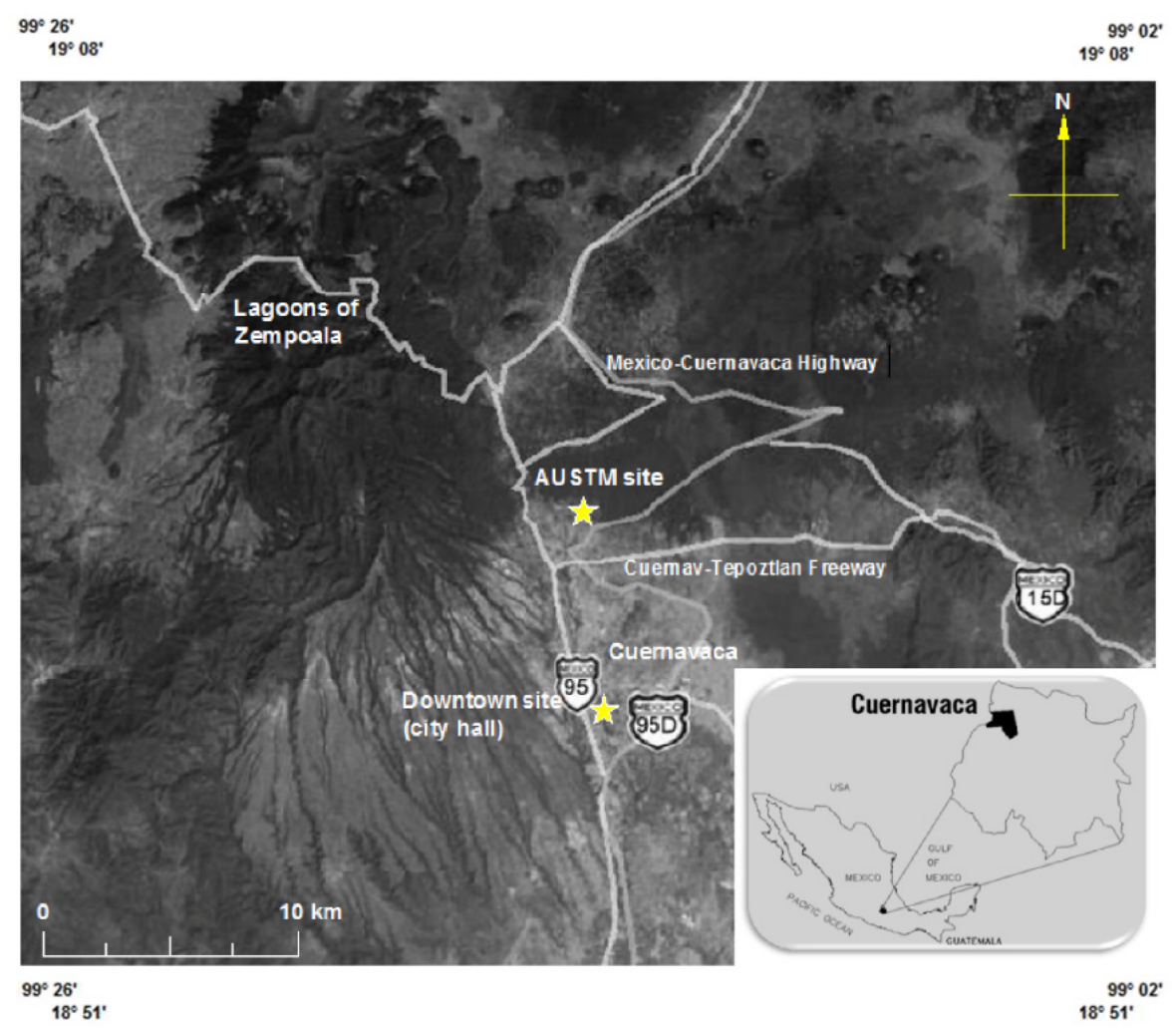

Figure 2. Sampling sites at the city of Cuernavaca, Morelos.

\subsection{Extraction of Organic Matter}

Sampled filters were extracted using $10 \mathrm{~mL}$ of dichloromethane (Burdick \& Jackson, HPLC grade) in an ultrasound bath (Branson 3210) for $30 \mathrm{~min}$. This procedure was performed twice. The Erlenmeyer flasks in which the filters were introduced, were fitted with cooled condensers with water at $10^{\circ} \mathrm{C}$. The extracts were filtered through a polytetrafluoroethylene (PTFE) membrane $(0.22 \mu \mathrm{m}$, Millipore), and then concentrated on a rotary evaporator (Buchi R-3) to about $1.0 \mathrm{~mL}$ and stored under refrigeration until analysis.

\subsection{Quality Control of the Analytical Method}

Field blanks (glass fiber filters) were performed once a week. Field blanks were stored in Petri dishes and returned to the laboratory until analysis. Further, blanks of laboratory material were used to discard the presence of analytes of interest during each one of the stages. The efficiency of the extraction methodology was evaluated by 
the enrichment of sampled filters with airborne particles with a solution containing 16 PAHs:

Acenaphthylene (Acen), Acenaphthene (Ace), Fluorene (Flu), Phenanthrene (Phe), Anthracene (Ant), Fluoranthene (Flt), Pyrene (Pyr), Benzo[a]Anthracene (BaA), Chrysene (Chr), Benzo[b]Fluoranthene (BbF), Benzo[k]Fluoranthene (BkF), Benzo[a]Pyrene (BaP), Indeno[1,2,3-cd]Pyrene (IP), Dibenzo[a,h]Anthracene (DBahA) and Benzo[g,h,i]Perylene (BghiP), at a concentration of 250 parts per billion (ppb). The amount recovered for each compound was compared to the amount added to calculate the recoveries. Once the percent recovery was determined on the enriched filters, the repeatability was calculated by estimating the coefficient of variation (\% CV). The recoveries ranged from $60 \%$ to $90 \%$, for Fluorene and IP, respectively. The repeatability in terms of \% CV was less than $10 \%$, which is consistent with the variation (30\%) set by the United States Environmental Protection Agency [36], for these samples.

Instrumental detection limits were performed based on a weight regression, proposed by Miller and Miller 2002 [37]; these ranged between 16.71 and $61.70 \mathrm{ng} \cdot \mathrm{mL}^{-1}$ for BghiP and BaP, respectively (Table 4). The concentrations of the compounds in ambient air were corrected using the recovery percentages.

Table 4. Detection limits ng $\mathrm{mL}^{-1}$.

\begin{tabular}{cc}
\hline Compound & LOD \\
\hline Acen & 33.62 \\
Ace & 32.20 \\
Flu & 31.19 \\
Phe & 35.17 \\
Ant & 37.30 \\
Flt & 46.85 \\
Pyr & 50.00 \\
BaA & 26.00 \\
Chr & 44.00 \\
BbF & 26.04 \\
BkF & 22.50 \\
BaP & 61.70 \\
IP & 59.22 \\
DahA & 44.30 \\
BghiP & 16.71 \\
\hline
\end{tabular}

LOD: limit of detection.

\subsection{Instrumental Analysis}

The extracts obtained were concentrated under a gentle stream of nitrogen to near dryness and subsequently resuspended with $150 \mu \mathrm{L}$ of dichloromethane 
in an insert injection. To this volume, $50 \mu \mathrm{L}$ of a solution containing 6 PAH-d (internal standard) were added to a final volume of $200 \mu \mathrm{L}$ (naphthalene-d8, acenaphthene-d10, phenanthrene-d10, pyrene-d10, chrysene-d12 and perylene-d12) (Chemservice West Chester, PA), at a final concentration of $2500 \mathrm{ppb}$ of internal standard.

The chromatographic analysis was performed on an Agilent Technologies, Model 6890 gas chromatograph (GC) coupled to a mass spectrometer (MS) 5973N with quadrupole mass filter. The separation of the compounds was carried out on a capillary column (J \& W Scientific, USA) with an internal diameter of $0.25 \mathrm{~mm}$ with $5 \%$ phenyl stationary phase and 95\% dimethyl polysiloxane and a film thickness of $0.25 \mu \mathrm{m}$. The oven temperature program was as follows: $60{ }^{\circ} \mathrm{C}$ for $10 \mathrm{~min}$ at a gradient of $5{ }^{\circ} \mathrm{C}$ per minute to $300{ }^{\circ} \mathrm{C}$ for $10 \mathrm{~min}$. The injector temperature was $300{ }^{\circ} \mathrm{C}$ in the splitless mode (purge time $30 \mathrm{sec}$ ) and the injection volume of $2.0 \mu \mathrm{L}$. Helium was used as carrier gas at a flow of $1.0 \mathrm{~mL} \cdot \mathrm{min}^{-1}$. The MS was operated in the electron impact $(70 \mathrm{eV})$ mode and the temperature of the ion source and the quadrupole filter were $230^{\circ} \mathrm{C}$ and $150^{\circ} \mathrm{C}$, respectively.

The mass-charge ratios $(\mathrm{m} / \mathrm{z})$ monitored were: Acenaphthylene (Acen, 152), Acenaphthene (Ace, 153), Fluoranthene (Flt, 202), Pyrene (Pyr, 202), Benzo[a]Anthracene (BaA, 228), Chrysene (Chr, 228), Benzo[a]Pyrene (BaP, 252), Benzo[b]Fluoranthene (BbF, 252), Benzo[k]Fluoranthene (BkF, 252), Indeno[1,2,3-cd]Pyrene (IP, 276), Dibenzo[a,h]Anthracene (DahA, 278), Benzo[g,h,i]Perylene (BghiP, 276). Multicomponent calibration curves were made for all PAHs in a concentration range of 12.5 to $1600 \mathrm{ng} \cdot \mathrm{mL}^{-1}$ ( $\left.\mathrm{r}>0.99, \mathrm{p}<0.001\right)$.

\subsection{Evaluation of Health Risk}

$\mathrm{BaP}$ is considered one of the most powerful mutagens, in many cases is used as a general indicator of PAHs and regarded by the World Health Organization (WHO) as a good index for whole PAH carcinogenicity. However, $\mathrm{BaP}$ it degrades easily in the presence of sunlight and some oxidants [38,39]. For this reason, $\mathrm{BaP}$ concentration alone does not give a good indication of the hazard presented by all the PAHs, and the PAHs' carcinogenic character, could be underestimated under determined conditions if only this compound is taken as the representative of carcinogenicity.

Therefore, an equivalent index for $\mathrm{BaP}(\mathrm{BaPE})$, was created with the objective of allowing a better estimation of the carcinogenic potential of PAHs associated with atmospheric particles. The concentration of BaPE for each PAH, was calculated by multiplying their concentration by its corresponding toxic equivalent factor (TEF), which represents the relative carcinogenic potency of the corresponding $\mathrm{PAH}$ [40]:

$$
B a P E=B a A \times 0.06+B F \times 0.07+B a P+D B a h A \times 0.6+I P \times 0.08
$$


where BF includes all the isomers of benzofluoranthene. The BaPE index tries to parameterize the health risk for human health related to ambient PAH exposure, and was calculated by multiplying the concentrations of each carcinogenic congener with its carcinogenic factor obtained by laboratory studies.

\subsection{Statistical Analysis}

The statistical analysis, including Kruskal-Wallis test, correlation analysis and principal component analysis (PCA), were realized using STATGRAPHICS program (Statistical Graphics Corp.).

\section{Conclusions}

The most abundant PAHs were those of high molecular weight, suggesting that in this area the combustion processes significantly contribute to atmospheric emissions. The diagnostic ratios and PCA revealed that coal burning and vehicle emissions are the main source of PAHs in the studied sites. The value obtained for the BaPE suggests a risk to the population, taking into account the degree of penetration of the $\mathrm{PM}_{2.5}$. It is important to mention that this result must be taken with caution, because the study was performed just for one month in the winter season, what suggests longer sampling periods in this urban zone for a better estimation of the risk.

The results obtained reflect the deterioration in air quality that is happening in the urban area of Cuernavaca as a result of high population growth, vehicle fleet growth and development in the industrial park that this region has experienced in recent years. This suggests that establishing strategies oriented to the reduction of atmospheric emissions is required, including the modernization of road transport and implementation of mass transportation systems, among other strategies.

Acknowledgments: The authors would like to express their appreciation to Winston Smith of the Peace Corps for the revision of this paper, to María Gregoria Medina Pintor for her support in the chromatographic analysis, to the Air Quality Monitoring Network of the State of Morelos (RAMAMOR, for its acronym in Spanish) for allowing the installment of the equipment in their locations. Special thanks also Program for the Professional Development of Teachers (PRODEP, for its acronym in English), for the financial support in this project. The authors gratefully acknowledge the NOAA Air Resources Laboratory (ARL) for the provision of the HYSPLIT transport and dispersion model and/or READY website (http:/ /www.ready.noaa.gov) used in this publication. Also to National Laboratory of Macromolecular Structures (LANEM, for its acronym in Spanish, project 251613).

Author Contributions: Saldarriaga-Noreña and López-Márquez wrote the article, Murillo-Tovar, Montiel-Palma and Hernández-Mena designed the study, Waliszewski realized the PAHs analysis, Ospina-Noreña and Sánchez-Salinas performed the statistical analysis.

Conflicts of Interest: The authors declare no conflict of interest. 


\section{References}

1. Monographs on the Evaluation of Carcinogenic Risks to Humans. Available online: http://monographs.iarc.fr/ENG/Monographs/vol32/volume32.pdf (accessed on 11 July 2015).

2. Aceves, M.; Grimalt, J.O. Seasonally dependent size distributions of aliphatic and polycyclic aromatic hydrocarbons in urban aerosols from densely populated areas. Environ. Sci. Technol. 1993, 27, 2896-2908.

3. Kiss, G.; Varga-Puchony, Z.; Rohrbacher, G.; Hlavay, J. Distribution of polycyclic aromatic hydrocarbons on atmospheric aerosol particles of different sizes. Atmos. Res. 1998, 46, 253-261.

4. Mazquiaran, M.A.B.; de Pinedo, L.C.O. Organic composition of atmospheric urban aerosol: Variations and sources of aliphatic and polycyclic aromatic hydrocarbons. Atmos. Res. 2007, 85, 288-299.

5. Ravindra, K.; Bencs, L.; Wauters, E.; de Hoog, J.; Deutsch, F.; Roekens, E.; Bleux, N.; Berghmans, P.; Grieken, R. Seasonal and site-specific variation in vapour and aerosol phase PAHs over Flanders Belgium and their relation with anthropogenic activities. Atmos. Environ. 2006, 40, 771-785.

6. Wang, G.; Huang, L.; Zhao, X.; Niu, H.; Dai, Z. Aliphatic and polycyclic aromatic hydrocarbons of atmospheric aerosols in five locations of Nanjing urban area, China. Atmos. Res. 2006, 81, 54-66.

7. Galarneau, E. Source specificity and atmospheric processing of airborne PAHs: Implications for source apportionment. Atmos. Environ. 2008, 42, 8139-8149.

8. Sklorz, M.; Kreis, J.S.; Liu, Y.B.; Orasche, J.; Zimmermann, R. Daytime resolved analysis of polycyclic aromatic hydrocarbons in urban aerosol samples-Impact of sources and meteorological conditions. Chemosphere 2007, 67, 934-943.

9. Liu, Y.; Liu, L.B.; Lin, J.M.; Tang, N.; Hayakawa, K. Distribution and characterization of polycyclic aromatic hydrocarbon compounds in airborne particulates of East Asia. China Partic. 2006, 6, 283-292.

10. Cotham, W.E.; Bidleman, T.F. Polycyclic aromatic hydrocarbons and polychlorinated biphenyls in air at an urban and a rural site near Lake Michigan. Environ. Sci. Technol. 1995, 29, 2782-2789.

11. Mantis, J.; Chaloulakou, A.; Samara, C. $\mathrm{PM}_{10}$-bound polycyclic aromatic hydrocarbons (PAHs) in the Greater Area of Athens, Greece. Chemosphere 2005, 59, 593-604.

12. Zielinska, B.; Sagebiel, J.; McDonald, J.; Whitney, K.; Lawson, D. Emission rates and comparative chemical composition from selected in-use diesel and gasoline-fueled vehicles. J. Air Waste Manag. Assoc. 2004, 54, 1138-1150.

13. Fang, G.C.; Wu, Y.S.; Chen, J.C.; Fu, P.P.C.; Chang, C.N.; Ho, T.T.; Chen, M.H. Characteristic study of polycyclic aromatic hydrocarbons for fine and coarse particulates at Pastureland near Industrial Park sampling site of central Taiwan. Chemosphere 2005, 3, 427-433.

14. Park, S.S.; Kim, Y.J.; Kang, C.H. Atmospheric polycyclic aromatic hydrocarbons in Seoul, Korea. Atmos. Environ. 2002, 36, 2917-2924. 
15. Kong, S.; Ding, X.; Bai, Z.; Han, B.; Chen, L.; Shi, J.; Li, Z.Y. A seasonal study of polycyclic aromatic hydrocarbons in $\mathrm{PM}_{2.5}$ and $\mathrm{PM}_{2.5-10}$ in five typical cities of Liaoning Province, China. J. Hazard Mater. 2010, 183, 70-80.

16. Li, Z.; Porter, E.N.; Sjödin, A.; Needham, L.L.; Lee, S.; Russell, A.G. Characterization of $\mathrm{PM}_{2.5}$-bound polycyclic aromatic hydrocarbons in Atlanta Seasonal variations at urban, suburban, and rural ambient air monitoring sites. Atmos. Environ. 2009, 43, 4187-4193.

17. Huang, B.; Liu, M.; Bi, X.; Chaemfa, C.; Ren, Z.; Wang, X.; Sheng, G.; Fu, J. Phase distribution, sources and risk assessment of PAHs, NPAHs and OPAHs in a rural site of Pearl River Delta region, China. Atmos. Pollut. Res. 2014, 5, 210-218.

18. Slezakova, K.; Castro, D.; Pereira, M.C.; Morais, S.; Delerue-Matos, C.; Alvim-Ferraz, M.C. Influence of Traffic Emissions on the Carcinogenic Polycyclic Aromatic Hydrocarbons in Outdoor Breathable Particles. J. Air Waste Manage. Assoc. 2010, 60, 393-401.

19. Li, P.H.; Wang, Y.; Li, Y.H.; Wang, Z.F.; Zhang, H.Y.; Xu, P.J. Characterization of polycyclic aromatic hydrocarbons deposition in $\mathrm{PM}_{2.5}$ and cloud/fog water at Mount Taishan (China). Atmos. Environ. 2010, 44, 1996-2003.

20. Kendall, M.; Hamilton, R.; Watt, J.; William, I.D. Characterization of selected speciated organic compounds associated with particulate matter in London. Atmos. Environ. 2001, 35, 2483-2495.

21. Kavouras, I.; Koutrakis, P.; Tsapakis, M.; Lagoudaki, E.; Stephanou, E.; Stephanou, E.G.; von Baer, D.; Oyola, P. Source apportionment of urban particulate aliphatic and polynuclear aromatic hydrocarbons (PAHs) using multivariate methods. Environ. Sci. Technol. 2001, 35, 2288-2294.

22. Yunker, M.; Macdonald, R.; Vingarzan, R.; Mitchell, R.; Goyette, D.; Sylvestre, S. PAHs in the Fraser River basin: A critical appraisal of PAH ratios as indicators of PAH source and composition. Org. Geochem. 2002, 33, 489-515.

23. Khalili, N.; Scheff, P.; Holsen, T. PAH source fingerprints for coke ovens, diesel and gasoline engines, highway tunnels, and wood combustion emissions. Atmos. Environ. 1995, 29, 533-542.

24. Sicre, M.A.; Saliot, A.; Aparicio, X.; Grimalt, J.; Albaiges, J. Aliphatic and aromatic hydrocarbons in different sized aerosols over the Mediterranean Sea: occurrence and origin. Atmos. Environ. 1987, 21, 2247-2259.

25. Rogge, W.F.; Hildemann, L.; Mazurek, M.A.; Cass, G.R.; Simoneit, B.R.T. Sources of fine organic aerosol: Non-catalyst and catalyst equipped automobiles and heavy duty diesel trucks. Environ. Sci. Technol. 1993, 27, 636-651.

26. Alves, C.; Pio, C.; Durate, A. Composition of extractable organic matter of air particles from rural and urban Portugese areas. Atmos. Environ. 2001, 35, 5485-5496.

27. Grimmer, G.; Jacob, J.; Naujack, K. Profile of the polycyclic aromatic compounds from crude oils-inventory by GC GC-MS. PAH in environmental materials: Part 3. Fresen. J. Anal. Chem. 1983, 316, 29-36.

28. Caricchia, A.M.; Chiavarini, S.; Pezza, M. Polycyclic Aromatic Hydrocarbons in the urban atmospheric particulate matter in the city of Naples (Italy). Atmos. Environ. 1999, 33, 3731-3738. 
29. Ortíz-Hernández, M.L.; Quiroz-Castañeda, R.E.; Sánchez-Salinas, E.; Castrejón-Godínez, M.L.; Macedo-Abarca, B. Emissions of Greenhouse Gases in the State of Morelos, 1st. ed.; Environmental Management Degree Program: Morelos, Mexico, 2013. (In Spanish)

30. Simcik, M.F.; Eisenreich, S.J.; Lioy, P.J. Source apportionment and source/sink relationships of PAHs in the coastal atmosphere of Chicago and Lake Michigan. Atmos. Environ. 1999, 33, 5071-5079.

31. Kulkarni, P.; Venkataraman, C. Atmospheric polycyclic aromatic hydrocarbons in Mumbai, India. Atmos. Environ. 2000, 34, 2785-2790.

32. Akyüz, M.; Cabuk, H. Meteorological variations of $\mathrm{PM}_{2.5} / \mathrm{PM}_{10}$ concentrations and particle-associated polycyclic aromatic hydrocarbons in the atmospheric environment of Zonguldak, Turkey. J. Hazard Mater. 2009, 170, 13-21.

33. Anastasopoulos, A.T.; Wheeler, A.J.; Karman, D.; Kulka, R.H. Intraurban concentrations, spatial variability and correlation of ambient polycyclic aromatic hydrocarbons (PAH) and $\mathrm{PM}_{2.5}$. Atmos. Environ. 2012, 59, 272-283.

34. Lodovici, M.; Venturini, M.; Marini, E.; Grechib, D.; Dolara, P. Polycyclic aromatic hydrocarbons air levels in Florence, Italy, and their correlation with other air pollutants. Chemosphere 2003, 50, 377-382.

35. Draxler, R.R.; Rolph, G.D. HYSPLIT-Hybrid Single-Particle Lagrangian Integrated Trajectory Model. Available online: http://www.arl.noaa.gov/HYSPLIT.php (accessed on 11 July 2015).

36. Environmental Protection Agency (EPA) of the United States. Compendium Method TO-13A, Determination of Polycyclic Aromatic Hydrocarbons (PAHs) in Ambient Air Using Gas Chromatography/Mass Spectrometry (GC/MS); EPA: Cincinnati, OH, USA, 1999.

37. Miller, J.N.; Miller, J.C. Estadística y Quimiometría para Química Analítica, 6th ed.; Prentice Hall: Harlow, UK, 2002.

38. Daisey, J.M.; Cheney, J.L.; Lioy, P.J. Profiles of organic particulate emissions from air pollution sources: status and needs for receptor source apportionment modelling. J. Air. Pollut. Control. Assoc. 1986, 36, 17-33.

39. Muller, J.F.; Hawker, D.F.; Connel, D.F. Polycyclic aromatic hydrocarbons in the atmospheric environment of Brisbane, Australia. Chemosphere 1998, 37, 1369-1383.

40. Nisbet, C.; LaGoy, P. Toxic equivalency factors (TEFs) for polycyclic aromatic hydrocarbons (PAHs). Regul. Toxicol. Pharmacol. 1992, 16, 290-300. 


\title{
Inland Concentrations of $\mathrm{Cl}_{2}$ and $\mathrm{ClNO}_{2}$ in Southeast Texas Suggest Chlorine Chemistry Significantly Contributes to Atmospheric Reactivity
}

\author{
Cameron B. Faxon, Jeffrey K. Bean and Lea Hildebrandt Ruiz
}

Abstract: Measurements of molecular chlorine $\left(\mathrm{Cl}_{2}\right)$, nitryl chloride $\left(\mathrm{ClNO}_{2}\right)$, and dinitrogen pentoxide $\left(\mathrm{N}_{2} \mathrm{O}_{5}\right)$ were taken as part of the DISCOVER-AQ Texas 2013 campaign with a High Resolution Time-of-Flight Chemical Ionization Mass Spectrometer (HR-ToF-CIMS) using iodide (I-) as a reagent ion. $\mathrm{ClNO}_{2}$ concentrations exceeding $50 \mathrm{ppt}$ were regularly detected with peak concentrations typically occurring between 7:00 a.m. and 10:00 am. Hourly averaged $\mathrm{Cl}_{2}$ concentrations peaked daily between 3:00 p.m. and 4:00 p.m., with a 29-day average of $0.9 \pm 0.3$ (1б) ppt. A day-time $\mathrm{Cl}_{2}$ source of up to $35 \mathrm{ppt} \cdot \mathrm{h}^{-1}$ is required to explain these observations, corresponding to a maximum chlorine radical $\left(\mathrm{Cl}^{\bullet}\right)$ production rate of $70 \mathrm{ppt} \cdot \mathrm{h}^{-1}$. Modeling of the $\mathrm{Cl}_{2}$ source suggests that it can enhance daily maximum $\mathrm{O}_{3}$ and $\mathrm{RO}_{2} \bullet$ concentrations by $8 \%-10 \%$ and $28 \%-50 \%$, respectively. Modeling of observed $\mathrm{ClNO}_{2}$ assuming a well-mixed nocturnal boundary layer indicates $\mathrm{O}_{3}$ and $\mathrm{RO}_{2}{ }^{\bullet}$ enhancements of up to $2.1 \%$ and $38 \%$, respectively, with a maximum impact in the early morning. These enhancements affect the formation of secondary organic aerosol and compliance with air quality standards for ozone and particulate matter.

Reprinted from Atmosphere. Cite as: Faxon, C.B.; Bean, J.K.; Ruiz, L.H. Inland Concentrations of $\mathrm{Cl}_{2}$ and $\mathrm{ClNO}_{2}$ in Southeast Texas Suggest Chlorine Chemistry Significantly Contributes to Atmospheric Reactivity. Atmosphere 2015, 6, 1487-1506.

\section{Introduction}

Research over the past several decades has shown that the presence of reactive gas phase chlorine species, particularly chlorine radicals $\left(\mathrm{Cl}^{\bullet}\right)$, can contribute significantly to atmospheric reactivity [1-7]. High concentrations of $\mathrm{Cl}^{\bullet}$ can lead to increased rates of Volatile Organic Compound (VOC) oxidation [8-13] and enhanced rates of $\mathrm{O}_{3}$ production in the troposphere $[1,3,7,14-16]$. Photochemical sources of $\mathrm{Cl}^{\bullet}$ include $\mathrm{Cl}_{2}$ and $\mathrm{HOCl}$ [17], which are the primary forms of anthropogenic chlorine emissions $[7,18,19]$. Chlorine radicals, once produced, can participate in reactions with VOCs to produce alkyl radicals. These reactions typically proceed via hydrogen 
abstraction as shown in Reaction (R1), where $\mathrm{R}$ represents a generic VOC, and $\mathrm{R}^{\bullet}$ represents the resulting alkyl radical $[17,20]$.

$$
\mathrm{Cl}^{\bullet}+\mathrm{RH} \rightarrow \mathrm{R}^{\bullet}+\mathrm{HCl} .
$$

Previous studies have reported field observations of elevated tropospheric $\mathrm{Cl}_{2}$ at various locations across North America. Many of these observations were made near coastal areas, and maximum observed concentrations range from $20 \mathrm{ppt}$ to $250 \mathrm{ppt}$ [21-24]. Additionally, concentrations of up to $400 \mathrm{ppt} \mathrm{Cl}_{2}$ were observed in the Arctic marine boundary layer in Barrow, Alaska [25]. $\mathrm{Cl}_{2}$ source strengths on the order of $10-100 \mathrm{ppt} \mathrm{Cl}_{2} \cdot \mathrm{h}^{-1}$ are required to explain such concentrations due to the rapid photolysis of $\mathrm{Cl}_{2}[22,23,25]$.

Anthropogenic sources [1,26], aqueous reaction pathways [27,28] and naturally occurring heterogeneous or surface reaction routes of $\mathrm{Cl}_{2}$ production $[25,29,30]$ have been proposed to explain these observations. Previous experiments have observed that $\mathrm{Cl}_{2}$ production from irradiated mixtures of $\mathrm{O}_{3}$ and particulate chloride proceeds at a rate that is too rapid to be explained unless heterogeneous chemistry is active [30]. Numerous mechanisms have been proposed to explain the exact pathway for the heterogeneous formation of $\mathrm{Cl}_{2}$. For example, the reaction of gas phase $\mathrm{O}_{3}$ at the particle surface has been suggested $[31,32]$.

$$
2 \mathrm{Cl}^{-}(\mathrm{aq})+\mathrm{O}_{3(\mathrm{~g})} \quad \stackrel{\mathrm{H}_{2} \mathrm{O}}{\longrightarrow} \mathrm{Cl}_{2(\mathrm{~g})}+2 \mathrm{OH}_{(\mathrm{aq})}^{-}+\mathrm{O}_{2(\mathrm{~g})}
$$

Other work suggests that the reaction of hydroxyl radicals $\left(\mathrm{OH}^{\bullet}\right)$ at the particle surface more accurately depicts the chemistry active during heterogeneous $\mathrm{Cl}_{2}$ production $[30,33,34]$, where the formation of a surface complex between particulate $\mathrm{Cl}^{-}$and gas phase $\mathrm{OH}^{\bullet}$ was suggested as the rate limiting step, as is shown in Reactions (R3) and (R4).

$$
\begin{gathered}
\mathrm{OH}_{(\mathrm{g})}+\mathrm{Cl}^{-}(\mathrm{aq}) \\
2 \mathrm{OH}^{\bullet \bullet} \mathrm{Cl}^{-} \rightarrow \mathrm{Cl}_{2(\mathrm{~g})}+2 \mathrm{OH}^{-} \mathrm{Cl}^{-}
\end{gathered}
$$

Regardless of the exact mechanism, the presence of a heterogeneous route for significant $\mathrm{Cl}_{2}$ production from particulate chloride has implications for air quality through the production of $\mathrm{O}_{3}[16]$ and particulate matter.

Heterogeneous production of gas phase $\mathrm{ClNO}_{2}$ from particulate chloride has also been shown to occur and can lead to the generation of $\mathrm{Cl}^{\bullet}$ in the presence of sunlight [35-38]. $\mathrm{ClNO}_{2}$ chemistry also decreases the loss of reactive nitrogen via $\mathrm{N}_{2} \mathrm{O}_{5}$ deposition by producing a photolytic form of reactive nitrogen that is 
reintroduced into the gas phase [39-42]. Heterogeneous routes for the production of $\mathrm{Cl}_{2}$ from particulate chloride have also been shown to exist [30,42-44].

Observations [24,37,45-47], modeling work [39-42] and laboratory studies [36-38,48-52] in the recent past have identified heterogeneous $\mathrm{ClNO}_{2}$ formation as a major route for the production of reactive gas phase chlorine. The mechanism of $\mathrm{ClNO}_{2}$ production is initiated by the reactive uptake of $\mathrm{N}_{2} \mathrm{O}_{5}$ on chloride-containing aerosol particles, as seen in Reaction (R5). This reaction competes with the heterogeneous hydrolysis of $\mathrm{N}_{2} \mathrm{O}_{5}$ (Reaction (R6)), which produces $\mathrm{HNO}_{3}$.

$$
\begin{gathered}
\mathrm{N}_{2} \mathrm{O}_{5(\mathrm{~g})}+\mathrm{HCl}_{(\mathrm{aq})} \rightarrow \mathrm{HNO}_{3(\mathrm{~g})}+\mathrm{ClNO}_{2} \\
\mathrm{~N}_{2} \mathrm{O}_{5(\mathrm{~g})}+\mathrm{H}_{2} \mathrm{O}_{(\mathrm{l})} \rightarrow 2 \mathrm{HNO}_{3}(\mathrm{aq})
\end{gathered}
$$

This results in relatively unreactive particulate chloride being transformed into reactive gas phase chlorine. The $\mathrm{ClNO}_{2}$ produced by the mechanism in Reaction (5) is photolytic and will decompose to produce gas phase $\mathrm{NO}_{2}$ and $\mathrm{Cl} l^{\bullet}$ [17].

Aside from coastal $[24,37,53,54]$ and oceanic [35] observations, several studies have recently reported significant $\mathrm{ClNO}_{2}$ concentration in inland and mid-continental regions [45-47,55]. Previous measurements and modeling work have indicated that ppb-level concentrations of $\mathrm{ClNO}_{2}$ are present in Houston, TX, USA and along the coast near the Houston Ship Channel [37,40,41]. The measurements reported here provide further insight into the formation and concentrations of $\mathrm{Cl}_{2}$ and $\mathrm{ClNO}_{2}$ at an inland location in southeast Texas and their implications to atmospheric reactivity.

\section{Results and Discussion}

The data presented in this work were collected during the DISCOVER-AQ 2013 campaign [56] in Houston, TX, USA for the period of 1 September 2013-1 October 2013. The data were obtained at an air quality monitoring ground site in Conroe, TX, USA $\left(30.350278^{\circ} \mathrm{N}, 95.425000^{\circ} \mathrm{W}\right)$ situated next to the Lone Star Executive Airport in Montgomery county. The site is located approximately $60 \mathrm{~km}$ north northwest from the Houston urban center and approximately $125 \mathrm{~km}$ northwest of the nearest coastline. The gas phase species $\mathrm{Cl}_{2}, \mathrm{~N}_{2} \mathrm{O}_{5}$ and $\mathrm{ClNO}_{2}$ were measured using an iodide High Resolution Time-of-Flight Chemical Ionization mass Spectrometer (HR-ToF-CIMS) and were identified by adduct ions $\mathrm{Cl}_{2} \mathrm{I}^{-}, \mathrm{N}_{2} \mathrm{O}_{5} \mathrm{I}^{-}$, and $\mathrm{ClNO}_{2} \mathrm{I}^{-}$, respectively. The concentration and bulk composition of particulate matter smaller than $1 \mu \mathrm{m}$ in diameter $\left(\mathrm{PM}_{1}\right)$ was measured using an Aerosol Chemical Speciation Monitor (ACSM, Aerodyne Research) [57]. Particle size distributions were measured using a Scanning Electrical Mobility System (SEMS, Brechtel Manufacturing).

Observations over the entire month of September 2013 revealed average peak concentrations of $\mathrm{Cl}_{2}, \mathrm{ClNO}_{2}$, and $\mathrm{N}_{2} \mathrm{O}_{5}$ of $1.6,25$, and $1.5 \mathrm{ppt}$, respectively. The highest concentrations of chlorine species were detected during the period 
of 11-20 September, and this time period is the focus of the following analysis. During this period, daily peak concentrations of $\mathrm{Cl}_{2}, \mathrm{ClNO}_{2}$, and $\mathrm{N}_{2} \mathrm{O}_{5}$ frequently exceeded 2 ppt, 60 ppt, and 20 ppt, respectively. Average nocturnal (8 p.m.-2 a.m.) concentrations of $\mathrm{N}_{2} \mathrm{O}_{5}$ were approximately 5 ppt. $\mathrm{ClNO}_{2}$ morning ( 5 a.m.- -11 a.m.) and nocturnal concentrations averaged 24 and $21 \mathrm{ppt}$, respectively. $\mathrm{Cl}_{2}$ typically peaked in the afternoon, and Figure 1 shows the time series for 11-20 September.

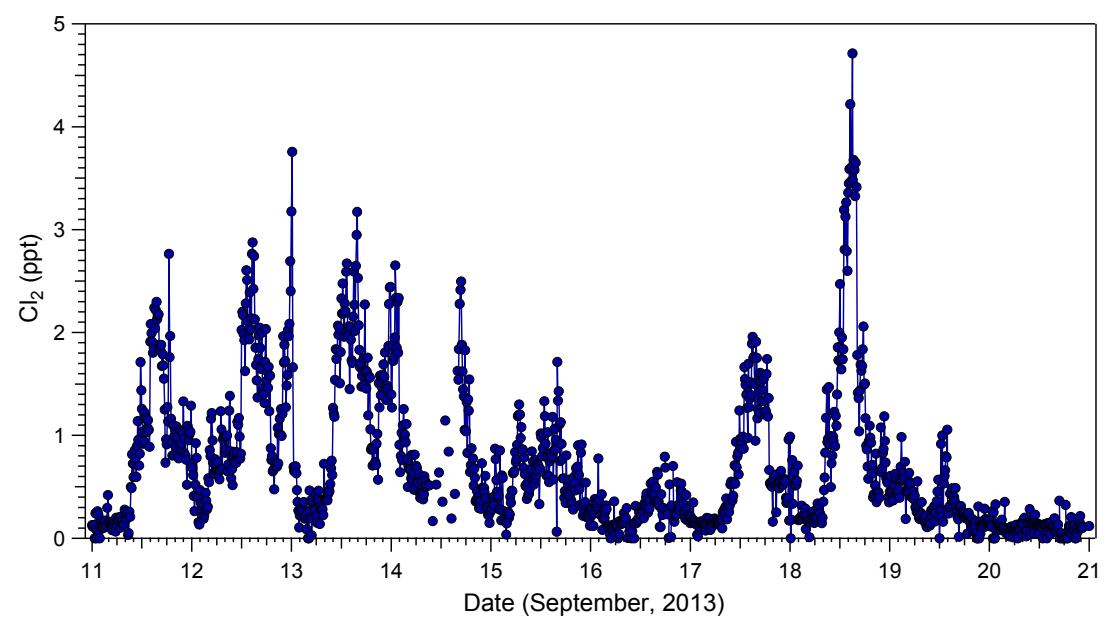

Figure 1. Time series of $\mathrm{Cl}_{2}$ (10-min averages) at the Conroe, Texas measurement site from 11-21 September.

$\mathrm{N}_{2} \mathrm{O}_{5}$ exhibited peak nocturnal concentrations exceeding $20 \mathrm{ppt}$ on the nights between 11-13 September. However, concentrations on most other nights rarely exceeded 2 ppt, even when increases in $\mathrm{ClNO}_{2}$ concentrations were observed during the same time periods. Concentration time series of $\mathrm{N}_{2} \mathrm{O}_{5}$ and $\mathrm{ClNO}_{2}$ from 11-21 September are shown in Figure 2. The implications of these observations are discussed further in the following sections.

\section{1. $\mathrm{Cl}_{2}$ Measurements}

Concentrations of $\mathrm{Cl}_{2}$ typically peaked in the afternoon at concentrations ranging 1-10 ppt. During the afternoon hours (12:00-6:00 p.m.) on many days, $\mathrm{Cl}_{2}$ concentrations remained above 1-2 ppt. On most days, $\mathrm{Cl}_{2}$ peaked between 3:00 p.m. and 4:00 p.m. (Figure 1). Figure 3 shows the diurnal pattern of $\mathrm{Cl}_{2}$ concentrations between 11-21 September. 


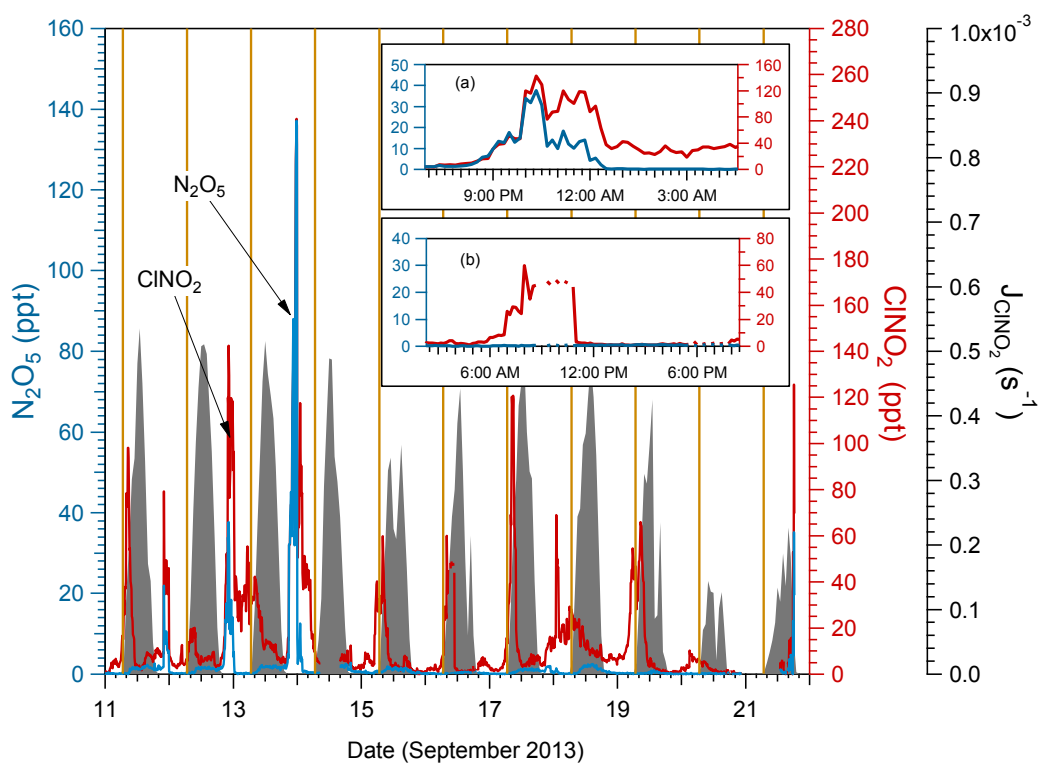

Figure 2. Time series of 10 -min average $\mathrm{ClNO}_{2}$ and $\mathrm{N}_{2} \mathrm{O}_{5}$ concentrations at the Conroe, Texas site from 11-21 September. Two distinct patterns were observed with respect to $\mathrm{N}_{2} \mathrm{O}_{5}$ and $\mathrm{ClNO}_{2}$. Inset (a) shows a time period $(9 / 13)$ when $\mathrm{ClNO}_{2}$ and $\mathrm{N}_{2} \mathrm{O}_{5}$ concentrations correlated. Inset (b) shows a time period (9/16) when little $\mathrm{N}_{2} \mathrm{O}_{5}$ concentrations remained below the detection limit despite elevated $\mathrm{ClNO}_{2}$ concentrations. A time series of the $\mathrm{ClNO}_{2}$ photolysis rate is shown in grey, and vertical lines represent sunrise.

These observed concentrations are slightly lower than concentrations observed in previous studies [22-24,58], and significantly lower than concentrations that have been detected in the arctic marine boundary layer [24,25]. However, the higher concentrations of $\mathrm{Cl}_{2}$ in these previous studies would be expected since they were taken in coastal areas, in close proximity to a large sea salt source. Although the $\mathrm{Cl}_{2}$ concentrations reported here are relatively low compared to other studies, the observation of a sustained concentration in spite of the rapid day-time photolysis of $\mathrm{Cl}_{2}$ suggests a significant source of $\mathrm{Cl}_{2}$ is present. For example, the average $\mathrm{Cl}_{2}$ concentration between 2:00 p.m. and 2:30 p.m. on 18 September was 3.8 ppt. The calculated photolysis rate constant for this same period, using the coordinates of the measurement site, was $2.5 \times 10^{-3} \mathrm{~s}^{-1}$. This implies an approximate $\mathrm{Cl}_{2}$ photolysis rate of $35 \mathrm{ppt} \mathrm{Cl}_{2} \cdot \mathrm{h}^{-1}$ for a mid-day $\mathrm{Cl}_{2}$ concentration of 4 ppt $\mathrm{Cl}_{2}$; calculated 30-min average photolysis rates between 10:00 a.m. and 4:00 p.m. ranged from $7-35 \mathrm{ppt} \mathrm{Cl}_{2} \cdot \mathrm{h}^{-1}$. Considering that concentrations on 18 September steadily increased from 10:00 a.m. and peaked at 2:00 p.m., this suggests that a minimum 
source strength of approximately $10-30 \mathrm{ppt} \cdot \mathrm{h}^{-1}$ during the mid-day is necessary to explain the observations. This is similar in strength to sources suggested by previous studies to explain $\mathrm{Cl}_{2}$ observations elsewhere [22,23].

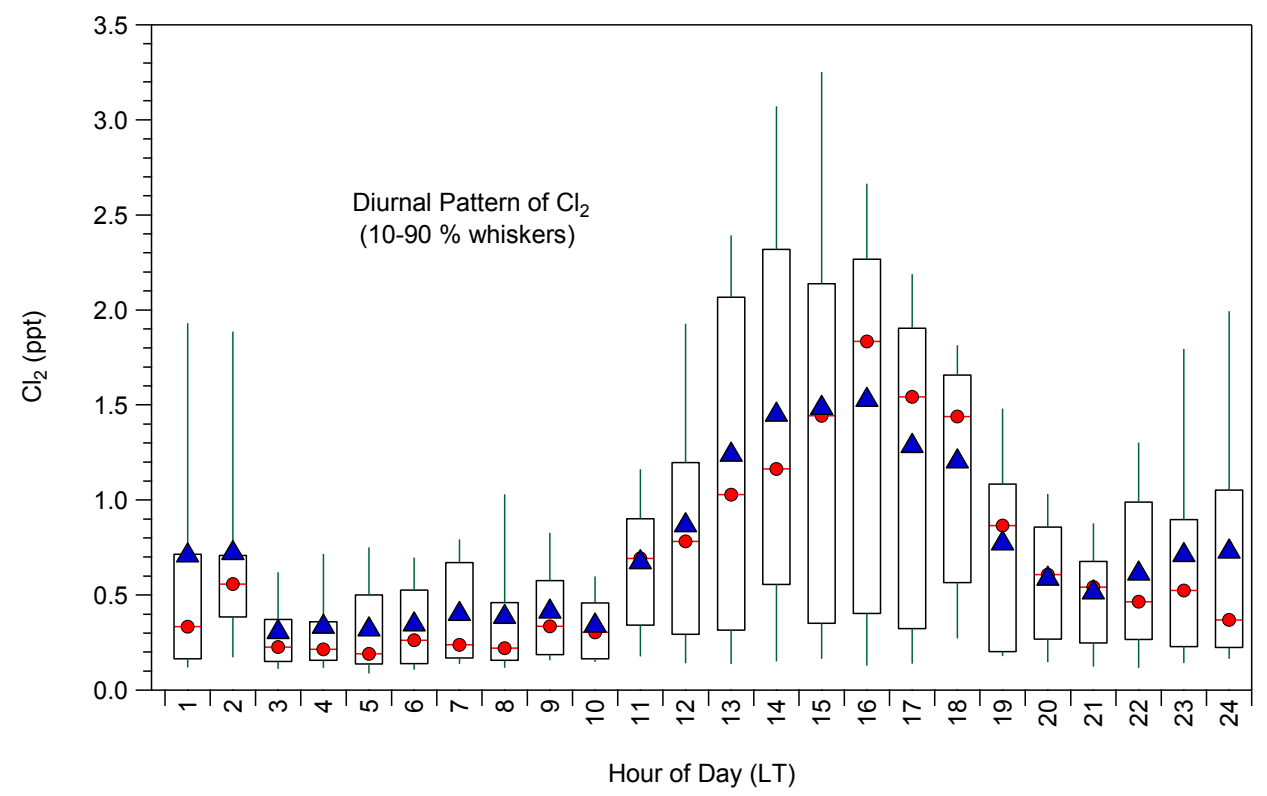

Figure 3. Diurnal pattern of $\mathrm{Cl}_{2}$ concentrations observed from 11-21 September. Concentrations were typically elevated in the mid to late afternoon, with a lower enhancement late at night due to late night peaks such as the night of 12 and 13 September. Boxes indicate 25th and 75th percentiles, and whiskers indicate 10th and 90 th percentiles.

Similar daytime $\mathrm{Cl}_{2}$ concentrations were observed for several other days during the campaign (Figure 1), suggesting that a similar source was present during those times as well. Such a $\mathrm{Cl}_{2}$ source could potentially lead to the enhanced oxidation of $\mathrm{CH}_{4}$ and other VOCs $[23,25,58]$, and therefore enhanced formation of secondary organic aerosol. Additionally, enhanced $\mathrm{O}_{3}$ production can result from the presence of a $\mathrm{Cl}^{\bullet}$ source $[1,33]$. The implications of this are discussed further in Section 2.4.

On several occasions (13-15 September, for example), increases in $\mathrm{Cl}_{2}$ were observed late at night or in the early morning, coinciding with increases in $\mathrm{ClNO}_{2}$. No significant anthropogenic emissions of gas phase $\mathrm{Cl}_{2}$ were known to be present in the area at the time. It is possible that the source of $\mathrm{Cl}_{2}$ in these situations was the same as $\mathrm{ClNO}_{2}$ since $\mathrm{N}_{2} \mathrm{O}_{5}$ has been shown to oxidize directly to $\mathrm{Cl}_{2}$ when particles are acidic [43]. Recent measurements in inland regions suggest that soil deflation could possibly play a larger role as a particulate chloride source than previously 
assumed [59-61]. Considering the diurnal pattern of $\mathrm{Cl}_{2}$, with peak concentrations occurring in the mid-afternoon, it is more likely that $\mathrm{Cl}_{2}$ is produced inland. Gas phase $\mathrm{Cl}_{2}$ transported from the coast would be degraded by photolysis by the time it reaches the measurement site, which could take several hours. Additionally, the heterogeneous production of reactive chlorine is thought to be driven by $\mathrm{O}_{3}$ or $\mathrm{OH}^{\bullet}$, species which peak during the mid-day. The timing of peak $\mathrm{Cl}_{2}$ concentrations thus suggest a heterogeneous mechanism as a possible source of observed $\mathrm{Cl}_{2}$.

\section{2. $\mathrm{ClNO}_{2}$ and $\mathrm{N}_{2} \mathrm{O}_{5}$ Measurements}

For the month of September, 2013, $\mathrm{ClNO}_{2}$ was frequently detected at concentrations exceeding 50 ppt (approximately $40 \%$ of the days in the month). Observations for the period spanning 11-21 September are shown in Figure 2. Although the timing of peak $\mathrm{ClNO}_{2}$ concentration varied between days (e.g., Figure 2a compared to Figure 2b), the typical pattern was a peak $\mathrm{ClNO}_{2}$ concentration between 25-60 ppt occurring between 7:00-10:00 a.m. Daily 1-hour maximum concentrations averaged $24 \mathrm{ppt}$ over the course of the month. On several days, however, $\mathrm{ClNO}_{2}$ concentrations peaked at night between 23:00-24:00, reaching concentrations over $100 \mathrm{ppt}$ by midnight. Examples of such episodes can be seen in Figure 2 on the nights of 13 and 14 September. Observations of $\mathrm{N}_{2} \mathrm{O}_{5}$ were also made, and during periods when the concentration was high (11-13 September), $\mathrm{N}_{2} \mathrm{O}_{5}$ correlated well with $\mathrm{ClNO}_{2}$. However, there were many days (e.g., 14-21 September) during which elevated concentrations of $\mathrm{ClNO}_{2}$ were observed while $\mathrm{N}_{2} \mathrm{O}_{5}$ remained low or was below the detection limit, perhaps suggesting that $\mathrm{ClNO}_{2}$ production was limited by $\mathrm{N}_{2} \mathrm{O}_{5}$ on these days.

Observed concentrations of $\mathrm{ClNO}_{2}$ were lower than previous measurements made in coastal areas. During the Texas Air Quality Study II (TexAQS II), $\mathrm{ClNO}_{2}$ concentrations reaching $1200 \mathrm{ppt}$ were detected in the Houston Ship Channel [37]. At another coastal location, in Los Angeles, CA, concentrations up to $1500 \mathrm{ppt}$ were detected off the California coast in the Los Angeles region [24]. The lower magnitude of $\mathrm{ClNO}_{2}$ concentrations reported here is likely due to the fact that measurements were taken further inland $(\sim 125 \mathrm{~km}$ from the coast) and therefore further from a sea salt chloride source. However, the $\mathrm{ClNO}_{2}$ concentrations observed in Conroe, TX, USA, are also lower than recent observations much further from the coast. For example, $\mathrm{ClNO}_{2}$ concentrations of up to $800 \mathrm{ppt}$ and $450 \mathrm{ppt}$ were detected at Kohler Mesa, CO ( 1400 km from coast) [46] and Hessen, Germany ( $\sim 380 \mathrm{~km}$ from coast) [45], respectively. The most likely explanation for these observations is that an inland source of chloride was present at these locations, and more recent work [59] suggest the presence of a significant soil source for chloride in Colorado. In this study, the measurements are consistent with the influence of a moderate particulate chloride source, and the observation that $\mathrm{N}_{2} \mathrm{O}_{5}$ was 
never present in the absence of $\mathrm{ClNO}_{2}$ suggests that heterogeneous production was limited by $\mathrm{N}_{2} \mathrm{O}_{5}$ availability. The concentrations reported here are within the range predicted by previous modeling studies of $\mathrm{ClNO}_{2}$ production in the region $[39,40,42]$. Concentrations of this magnitude were predicted to result in an enhancement of $\mathrm{O}_{3}$ production up to several $\mathrm{ppb}$ [42].

On nights when $\mathrm{ClNO}_{2}$ and $\mathrm{N}_{2} \mathrm{O}_{5}$ correlated well, the concentrations typically peaked around midnight (Figure 2a). However, when elevated $\mathrm{ClNO}_{2}$ was present in the absence of $\mathrm{N}_{2} \mathrm{O}_{5}$, the concentrations typically peaked in the early to mid-morning (Figure $2 \mathrm{~b}$ ). Figure 4 shows a diurnal pattern of $\mathrm{ClNO}_{2}$ and $\mathrm{PM}_{2.5}$ surface area (as measured by the SEMS). Within the diurnal cycle of $\mathrm{ClNO}_{2}$ at the measurement site, two distinct patterns of $\mathrm{ClNO}_{2}$ concentrations are clearly visible. One pattern peaks early in the morning between 8 and 9 a.m., shortly after sunrise. In the other, late night $\mathrm{ClNO}_{2}$ peaks are observed around midnight, coinciding with peak $\mathrm{N}_{2} \mathrm{O}_{5}$ concentrations (11-13 September). The peak 1-h average particulate surface area also occurs around the time of peak $\mathrm{ClNO}_{2}$ concentrations in the early mornings, suggesting that conditions are favorable for the heterogeneous production of $\mathrm{ClNO}_{2}$ from $\mathrm{N}_{2} \mathrm{O}_{5}$ in air masses that are advected to the site in the early mornings.

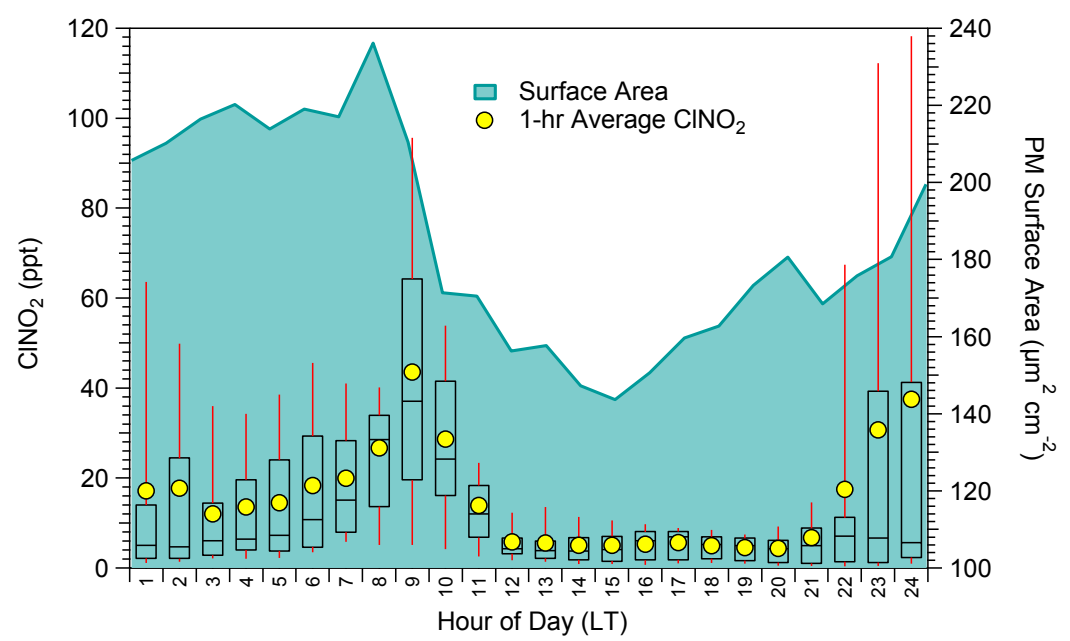

Figure 4. Diurnal cycle of $\mathrm{ClNO}_{2}$ concentrations, showing two distinct patterns that are present during the campaign: (1) an early morning peak concentrations where $\mathrm{ClNO}_{2}$ is advected to the site, and (2) late night peak concentrations, when $\mathrm{ClNO}_{2}$ and $\mathrm{N}_{2} \mathrm{O}_{5}$ concentrations correlate significantly, suggesting more local inland production of $\mathrm{ClNO}_{2}$.

Analysis of ACSM data collected during DISCOVER-AQ suggests high concentrations of particulate organic nitrates [62], which are formed either from photo-oxidation of hydrocarbons in the presence of $\mathrm{NO}_{x}$ or from the reactions of 
hydrocarbons with $\mathrm{NO}_{3}$ radicals. Nitrate radical chemistry acts as a $\mathrm{NO}_{\mathrm{y}}$ sink, reducing the formation of $\mathrm{N}_{2} \mathrm{O}_{5}$ and therefore $\mathrm{ClNO}_{2}$ and could be responsible for relatively low $\mathrm{N}_{2} \mathrm{O}_{5}$ concentrations observed throughout most of the campaign.

\subsection{Air Source Regions and Back Trajectories}

On several days during the campaign, differences in the correlation of $\mathrm{ClNO}_{2}$ and $\mathrm{N}_{2} \mathrm{O}_{5}$ concentrations and the timing of $\mathrm{ClNO}_{2}$ peak concentrations suggest possible differences in the production and transport of $\mathrm{ClNO}_{2}$. To investigate this difference further, HYSPLIT back trajectories were calculated for each case [63]. Starting height was set to $70 \mathrm{~m}$, the end point of the back trajectories was set to the coordinates of the Conroe field site, and the end time was set to the time of maximum observed $\mathrm{ClNO}_{2}$ concentration on each night. The model was used to generate a new back trajectory every two hours from the start time for a total of six 24-h back trajectories for each time period. Figure 5 shows a comparison of calculated back trajectories for the 13th and 16th of September.
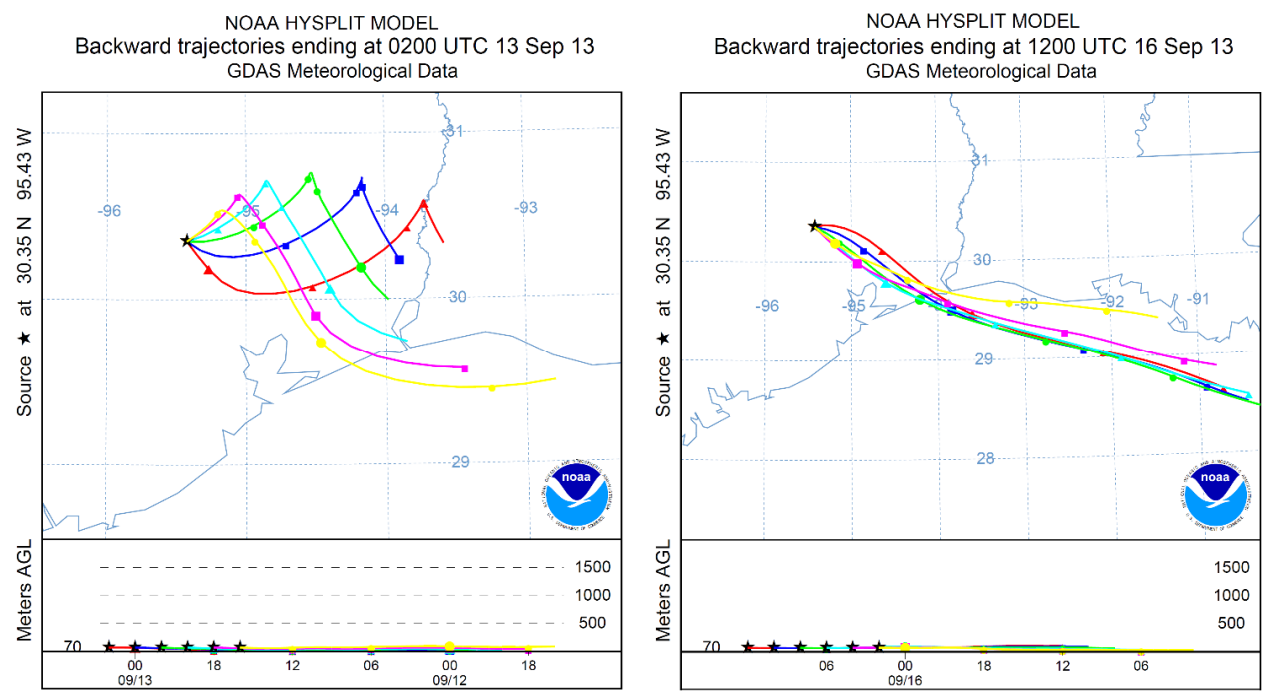

Figure 5. A comparison of HYSPLIT back trajectories for the days shown in the insets of Figure 2. Left: Back trajectories for the morning of 13 September 2013. The back trajectories ending at the time of peak $\mathrm{ClNO}_{2}$ concentrations (10-11 p.m.) show air that originated inland near the TX-LA border 24 hours prior. Right: Back trajectories for the morning of 16 September 2013. The back trajectory at the time of peak $\mathrm{ClNO}_{2}$ concentration (7-8 a.m.) indicates that the air mass originated in the Gulf of Mexico 24 hours prior, passing between the Houston, TX region and the LA-TX border. 
One major difference between the two back trajectories is the amount of time that the air mass spent over land or over sea prior to arriving at the site. For example, air that was sampled at the site on 15-16 September originated in the Gulf of Mexico, resulting in a lower residence time over industrialized regions relative to the air sampled on the night of 12-13 September. Observations on the night of the 15th of September indicated very low $\mathrm{N}_{2} \mathrm{O}_{5}$. Elevated morning-time concentrations of $\mathrm{ClNO}_{2}$ in these scenarios (e.g., 15-17 September) most likely resulted from transport of $\mathrm{ClNO}_{2}$ to the measurement site from the Gulf Coast. $\mathrm{ClNO}_{2}$ concentrations of up to $1.2 \mathrm{ppb}$ were previously detected along the coast [37] where HYSPLIT back trajectories indicate the air originated on 16 September (Figure 5). The lack of a corresponding increase in $\mathrm{N}_{2} \mathrm{O}_{5}$ concentrations during these early morning episodes also suggests that the $\mathrm{ClNO}_{2}$ observed at these times is produced elsewhere. The total amount of $\mathrm{ClNO}_{2}$ transported to the site on these mornings was probably limited by $\mathrm{N}_{2} \mathrm{O}_{5}$ availability since SEMS data indicate that the highest measured particulate surface area concentrations were observed during these times (Figure 4).

In contrast to the early morning peak concentrations, the air that was sampled on the night of 12 September originated inland, and the majority of its path was over land. On the night of 12 September, it might be expected that the observed air mass had been exposed to additional inland $\mathrm{NO}_{\mathrm{x}}$ sources that would explain the higher $\mathrm{N}_{2} \mathrm{O}_{5}$ concentration relative to the night of 15-16 September.

On several occasions, $\mathrm{ClNO}_{2}$ was found to be elevated in the afternoon during daylight hours. Examples of this include the afternoons of 12, 13 and 17 September. Back trajectories for the afternoon hours on these days indicate that air transported to the site originated along the Gulf coast. On the afternoon of 12 September between the hours of 1 and 5 p.m., incoming air originated along the gulf coast 18 hours prior to arriving at the site. The back trajectories for 13 and 17 September indicate that the air masses originated from the same region, frequently crossing the region between Lake Charles, LA and Houston, TX. The amount of $\mathrm{ClNO}_{2}$ that would need to be produced in the transported air can be estimated. For example, $\mathrm{ClNO}_{2}$ concentrations on the afternoons of 12 and 13 September were approximately 10-15 ppt. An air mass arriving at 2 p.m. would have been exposed to daylight for approximately 7 hours. Taking the average observed photolysis rate of $\mathrm{ClNO}_{2}$ between 7 a.m. and 2 p.m. for these dates, this corresponds to an average $\mathrm{ClNO}_{2}$ photolysis rate of $2.8 \times 10^{-4} \mathrm{~s}^{-1}$. This suggests that approximately 1-2 ppb of $\mathrm{ClNO}_{2}$ would need to be produced in the incoming air masses during transport to the site in order for concentrations on the order of $10 \mathrm{ppt}$ to be observed. Previous measurements and modeling predictions in this region indicate that elevated concentrations of $\mathrm{ClNO}_{2}$ can reach concentrations of this magnitude [37,39,40,42].

Back trajectories in the afternoons when $\mathrm{Cl}_{2}$ concentrations were typically elevated indicate that air is transported directly from the Gulf of Mexico, often 
passing over the metropolitan area of Houston, TX, USA. For example, air sampled at the site between 12-5 p.m. on the afternoon of 12, 13, 16 and 18 September consistently originated from the Gulf of Mexico 12 to 18 hours before being sampled. The transport of these air masses from the coast could suggest that long range sea salt transport contributed as a source of heterogeneously produced $\mathrm{Cl}_{2}$ at the site.

\subsection{Box Modeling Results}

The contribution of observed $\mathrm{Cl} 2$ and $\mathrm{ClNO} 2$ to $\mathrm{Cl}^{\bullet}$ production and resulting atmospheric reactivity were analyzed using ambient box modeling. The Statewide Air Pollution Research Center (SAPRC) software was used in combination with an updated version of the Carbon Bond 6 (CB6r2) chemical kinetics mechanism [64,65]. The CB6r2 mechanism was modified to include basic gas phase chlorine chemistry $[65,66]$ in addition to $\mathrm{Cl} 2$ and $\mathrm{ClNO} 2$ photolysis in a manner similar to Sarwar et al. [39]. A list of reactions that were added is provided in the supplementary material. The photolysis rate of $\mathrm{Cl} 2$ was calculated internally to the mechanism for a latitude and longitude matching the location of the measurement site. For the dates used in the simulations, this resulted in a peak $j_{C_{2}}$ of $2.8 \times 10^{-2} \mathrm{~s}^{-1}$, occurring at 12:30 p.m. Deposition was not included and boundary layer height was fixed for the duration of the model run.

Conditions on 18 September were modeled in order to assess the effects of $\mathrm{Cl}_{2}$ on atmospheric reactivity. Although this day did not have the highest concentration of $\mathrm{Cl}_{2}$ observed throughout the campaign (Figure 1), sustained concentrations over 2 ppt were observed for the entire period between 12:00-6 p.m., suggesting a continuous source of $\mathrm{Cl}_{2}$ was present. Emissions of $\mathrm{Cl}_{2}$ were added to the model so that the observed $\mathrm{Cl}_{2}$ concentration profile was replicated in the simulations. On this day, relative humidity was $98 \%$ at $6: 00$ a.m., dropping to a minimum of $43 \%$ by 2:00 p.m., and temperature rose from $294 \mathrm{~K}$ at 6:00 am to $308 \mathrm{~K}$ at 2:00 p.m. Initial concentrations included 0.4 ppt $\mathrm{Cl}_{2}, 8 \mathrm{ppb} \mathrm{NO}_{2}$, and $10 \mathrm{ppb} \mathrm{O}_{3}$, consistent with early morning observations at the site on 18 September. Using these initial conditions and meteorological inputs, the production of $\mathrm{OH}$ radicals was calculated Equation (1), and day-time $\mathrm{OH}$ production ranged $10^{6}-10^{7}$ molecules $\cdot \mathrm{cm}^{-3} \mathrm{~s}^{-1}$. A typical urban VOC mixture [67] was included in simulations at a concentration of 20 ppbC. Four scenarios were simulated: (1) no $\mathrm{Cl}_{2}$ and no VOCs, (2) including $\mathrm{Cl}_{2}$ emissions, no VOCs, (3) no $\mathrm{Cl}_{2}$ emissions with 20 ppbC VOCs and (4) including $\mathrm{Cl}_{2}$ emissions and 20ppbC VOCs. $\mathrm{Cl}^{\bullet}$ production was calculated from the photolysis of measured $\mathrm{Cl}_{2}$ concentrations Equation (2); an average emissions rate of $15 \mathrm{ppt} \cdot \mathrm{h}^{-1}$ between 8:00 a.m.-6:00 p.m. was found to be necessary to replicate the observed concentrations. A comparison of modeled $\mathrm{OH}^{\bullet}$ and $\mathrm{Cl}^{\bullet}$ production rates and concentrations are shown in Figure 6. 


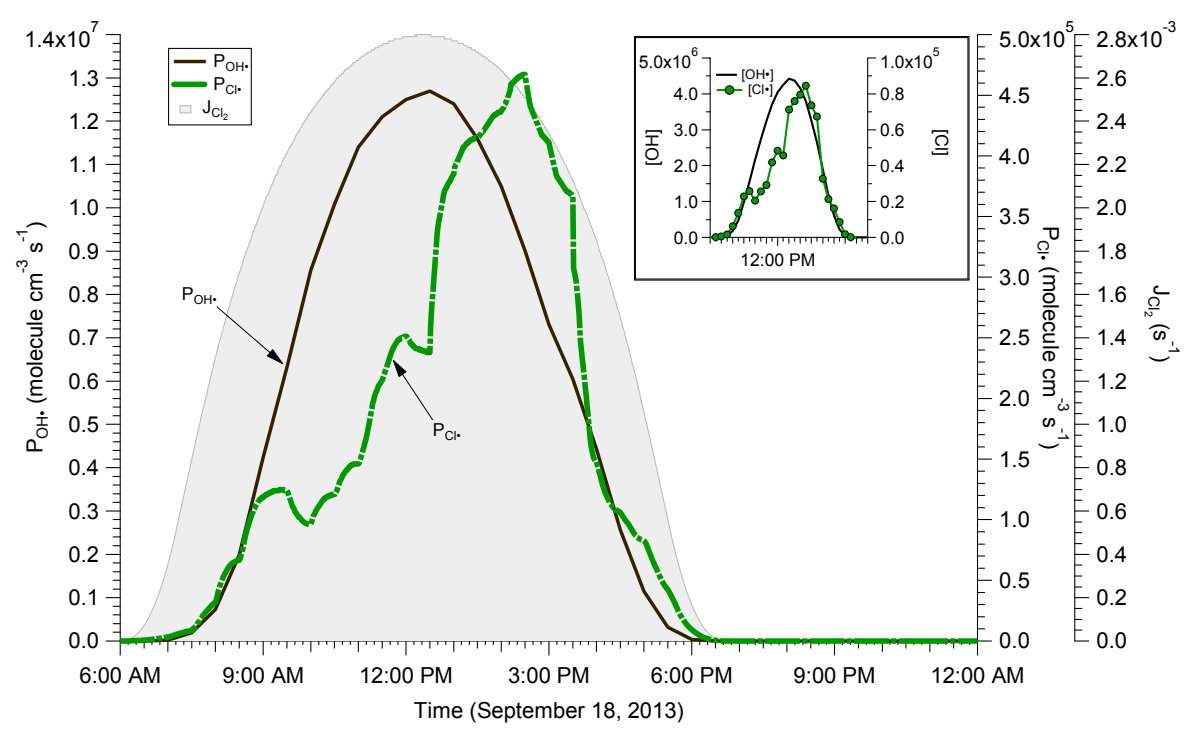

Figure 6. Production of $\mathrm{OH}$ and $\mathrm{Cl}$ radicals in a box modeling simulations for the conditions on 18 September 2013. The inset shows calculated concentrations of $\mathrm{OH}$ and $\mathrm{Cl}$ radicals in molecules $\cdot \mathrm{cm}^{-3} \cdot \mathrm{s}^{-1}$.

Although $\mathrm{Cl}^{\bullet}$ production rates and concentrations are approximately 1-2 orders of magnitude lower than those of $\mathrm{OH}^{\bullet}$, the calculated concentrations are significant when the relative reactivity of the two radicals is taken into account. The rate constants for the reactions of $\mathrm{Cl}^{\bullet}$ with many VOCs is significantly (up to 100 times) faster than the corresponding reactions of $\mathrm{OH}^{\bullet}[9-11,68,69]$. Thus, $\mathrm{Cl}^{\bullet}$ concentrations on the order of $10^{5}$ molecules $\cdot \mathrm{cm}^{-3} \mathrm{~s}^{-1}$ represent a significant radical source with respect to the oxidation of VOCs. For example, taking the maximum $\mathrm{Cl}^{\bullet}$ concentration $\left(8.5 \times 10^{4}\right.$ molecules $\left.\cdot \mathrm{cm}^{-3}\right)$ and the corresponding $\mathrm{OH}^{\bullet}$ concentration at the same time $\left(3.6 \times 10^{6}\right.$ molecules $\left.\cdot \mathrm{cm}^{-3}\right)$, the rate constant for the reaction of $\mathrm{Cl}^{\bullet}$ and $\mathrm{OH}^{\bullet}$ with $\mathrm{CH}_{4}$ at $298 \mathrm{~K}$ are approximately $1.0 \times 10^{-13}$ and $6.4 \times 10^{-15}$ $\mathrm{cm}^{3}$ molecules ${ }^{-1} \mathrm{~s}^{-1}$, respectively. Assuming a $\mathrm{CH}_{4}$ concentration of $1700 \mathrm{ppb}$, this corresponds to rates of reaction with $\mathrm{CH}_{4}$ for $\mathrm{Cl}^{\bullet}$ and $\mathrm{OH}^{\bullet}$ of $3 \times 10^{5}$ and $8 \times 10^{5}$ molecules $\cdot \mathrm{cm}^{-3} \mathrm{~s}^{-1}$, respectively. These rates of reaction indicate that the $\mathrm{Cl}^{\bullet}$ from observed concentrations of $\mathrm{Cl}_{2}$ could significantly contribute to atmospheric reactivity with respect to VOC oxidation. It is also important that $\mathrm{Cl}_{2}$ and $\mathrm{Cl}^{\bullet}$ peak in the mid to late afternoon when $\mathrm{OH}^{\bullet}$ production begins to wane.

Measured $\mathrm{O}_{3}$ concentrations on the morning of 18 September were low with concentrations reaching around $5 \mathrm{ppb}$ at 6:00 a.m. Simulations under-predicted day-time $\mathrm{O}_{3}$ concentrations by over $25 \%$ compared to measurements. Transport of $\mathrm{O}_{3}$ to the site in the afternoon (which is not included in this box modeling simulation) 
likely leads to higher observed $\mathrm{O}_{3}$ concentrations. $\mathrm{O}_{3}$ production was found to be enhanced by $\mathrm{Cl}_{2}$. The addition of $\mathrm{Cl}_{2}$ without VOC (model scenario 1 vs. 2) increased maximum modeled $\mathrm{O}_{3}$ concentration between 8:00 a.m.-6:00 p.m. by 10\% (1 ppb) and $\mathrm{RO}_{2} \bullet$ concentration by up to $50 \%$ (1.1 ppt). The addition of $\mathrm{Cl}_{2}$ in the presence of VOC (model scenario 3 vs. 4) increased the maximum day-time $\mathrm{O}_{3}$ concentration by $8 \%$ ( $2 \mathrm{ppb})$ and $\mathrm{RO}_{2} \bullet$ concentration by $28 \%$ (10 ppt). These results are summarized in Table 1.

Conditions on the morning of 13 September (scenario 5 and 6) and 16 September (scenarios 7 and 8) were modeled in order to assess the effects of $\mathrm{ClNO}_{2}$ on atmospheric reactivity. Relative humidity and temperature in the model were set to approximate conditions present at the Conroe site. The simulation start time was set to midnight in order to capture the changes in radical production between nocturnal and early morning conditions. Model inputs for 13 September also included initial concentrations of $35 \mathrm{ppb} \mathrm{O}_{3}$ and $20 \mathrm{ppb} \mathrm{NO}_{\mathrm{x}}$ (consistent with measurements), as well as $20 \mathrm{ppbC}$ VOC. A base case model run was performed without $\mathrm{ClNO}_{2}$ (scenario 5) and compared to the model run with $40 \mathrm{ppt}$ initial $\mathrm{ClNO}_{2}$ (scenario 6). Model inputs for 16 September included initial concentrations of $15 \mathrm{ppb} \mathrm{O}_{3}$ and $10 \mathrm{ppb} \mathrm{NO}_{\mathrm{x}}$ (consistent with measurements), as well as 20 ppbC VOC. A base case with no $\mathrm{ClNO}_{2}$ (scenario 7) was compared to a separate model run (scenario 8), where initial $\mathrm{ClNO}_{2}$ concentrations were set to 0 , and $\mathrm{ClNO}_{2}$ emissions were added to gradually increase the total amount of $\mathrm{ClNO}_{2}$ from 0 to 50 ppt between 6-8 a.m. (Figure $2 \mathrm{~b}$ ).

A comparison of the radical production rates of $\mathrm{OH}^{\bullet}$ and $\mathrm{Cl}^{\bullet}$ resulting from measured $\mathrm{ClNO}_{2}, \mathrm{O}_{3}$ and $\mathrm{H}_{2} \mathrm{O}$ concentrations is shown in Figure 7. Although $\mathrm{Cl}^{\bullet}$ production reaches a peak rate that is approximately 2 orders of magnitude lower than peak $\mathrm{OH}^{\bullet}$ production, its production in the early morning increases atmospheric reactivity during a time when $\mathrm{OH}^{\bullet}$ concentrations are low. Concentrations of $\mathrm{Cl}^{\bullet}$ peak in the very early morning (Figure 7 inset), approximately two hours before $\mathrm{OH}^{\bullet}$ concentrations begin to increase. Using the peak modeled $\mathrm{Cl}^{\bullet}$ concentration $\left(2.5 \times 10^{4}\right.$ molecules $\cdot \mathrm{cm}^{-3}$ and an approximate $\mathrm{OH}^{\bullet}$ concentration from the same time period $\left(1.5 \times 10^{6}\right.$ molecules $\left.\cdot \mathrm{cm}^{-3}\right)$, the reaction rates with $1700 \mathrm{ppb} \mathrm{CH}_{4}$ are similar at $1.1 \times 10^{5}$ and $4.1 \times 10^{5}$ molecules $\cdot \mathrm{cm}^{-3} \mathrm{~s}^{-1}$ for $\mathrm{Cl}^{\bullet}$ and $\mathrm{OH}^{\bullet}$, respectively.

The inclusion of 40 ppt $\mathrm{ClNO}_{2}$ enhanced $\mathrm{O}_{3}$ and $\mathrm{RO}_{2}{ }^{\bullet}$ concentrations between 7:00 a.m. $-3: 00$ p.m. by a maximum of $6.5 \%$ and $0.4 \%$, respectively, when compared to a simulation with no $\mathrm{ClNO}_{2}$ (scenarios 5 and 6 in Table 1). For simulations on the morning of 16 September, which included emissions of 50 ppt $\mathrm{ClNO}_{2}$ in the early morning, similar enhancements in $\mathrm{O}_{3}$ and $\mathrm{RO}_{2}{ }^{\bullet}$ concentrations were observed: in the presence of $20 \mathrm{ppbC}$ VOC, $\mathrm{O}_{3}$ and $\mathrm{RO}_{2}$ concentrations were enhanced by $11.5 \%$ and $1.0 \%$, respectively, when compared to a base case simulation without $\mathrm{ClNO}_{2}$ (scenarios 7 and 8 in Table 1). In the absence of VOCs the addition of $\mathrm{ClNO}_{2}$ did not significantly increase $\mathrm{O}_{3}$ or $\mathrm{RO}_{2}{ }^{\bullet}$ concentrations (not listed in 
Table 1). The increases in $\mathrm{RO}_{2} \bullet$ exemplify how $\mathrm{ClNO}_{2}$ at the observed concentrations can contribute significantly to atmospheric reactivity, particularly in the morning. A summary of these modeling results and the results of $\mathrm{Cl}_{2}$ simulations are listed in Table 1.

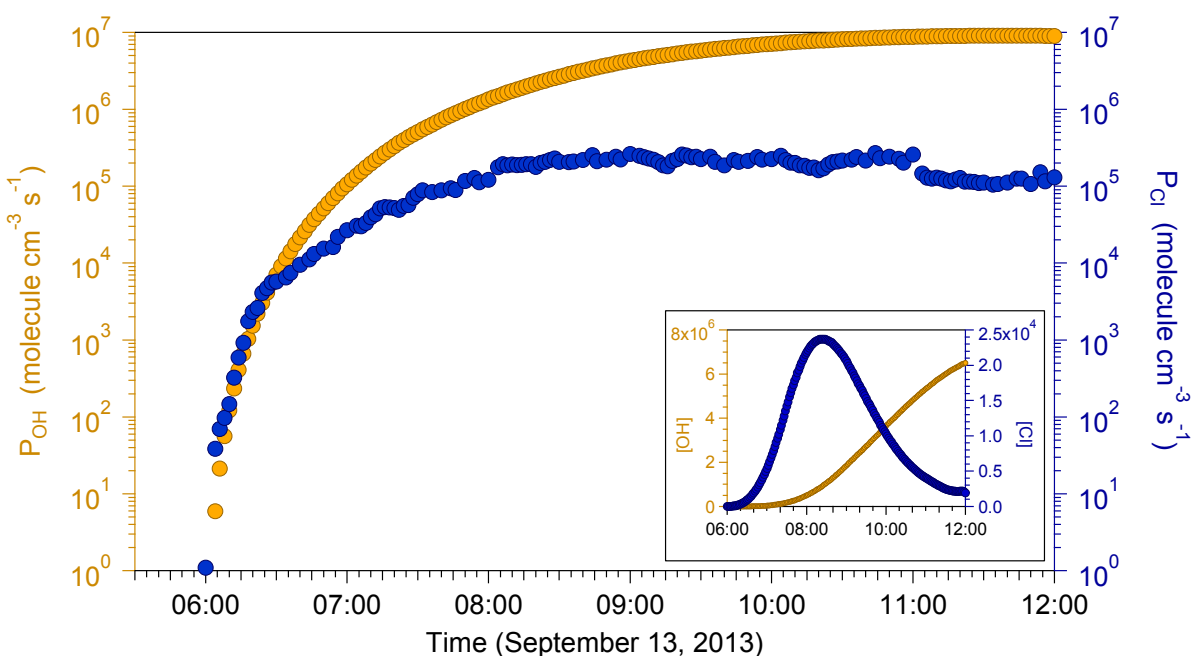

Figure 7. $\mathrm{OH}^{\bullet}$ production resulting from the photolysis of $\mathrm{O}_{3}$ compared to $\mathrm{Cl}^{\bullet}$ production from observed concentrations of $\mathrm{ClNO}_{2}$ on the morning of 13 September 2013. The inset shows a comparison of modeled $\mathrm{Cl}^{\bullet}$ and $\mathrm{OH}^{\bullet}$ radical concentrations (molecules $\cdot \mathrm{cm}^{-3}$ ) during the same time period, assuming an initial $\mathrm{ClNO}_{2}$ concentration of $40 \mathrm{ppt}$ (model scenario 6).

Table 1. Summary of Modeling Results.

\begin{tabular}{|c|c|c|c|c|c|}
\hline Scenario & $\begin{array}{l}\text { Chlorine } \\
\text { Species }\end{array}$ & Comparison & $\begin{array}{l}{[\mathrm{VOC}]} \\
(\mathrm{ppbC})\end{array}$ & $\Delta\left[\mathrm{O}_{3}\right]_{\max }(\%)$ & $\Delta\left[\mathrm{RO}_{2}{ }^{\bullet}\right]_{\max }(\%)$ \\
\hline $\begin{array}{l}1 \\
2\end{array}$ & - & 1 & $\begin{array}{l}0 \\
0\end{array}$ & 10 & 50 \\
\hline $\begin{array}{l}3 \\
4 \\
\end{array}$ & - & 2 & $\begin{array}{l}20 \\
20\end{array}$ & 8 & 28 \\
\hline $\begin{array}{l}5 \\
6\end{array}$ & $\stackrel{-}{\mathrm{ClNO}_{2}}$ & 3 & $\begin{array}{l}20 \\
20\end{array}$ & 6 & 0.4 \\
\hline $\begin{array}{l}7 \\
8\end{array}$ & $\stackrel{-}{\mathrm{ClNO}_{2}}$ & 4 & $\begin{array}{l}20 \\
20\end{array}$ & 12 & 1 \\
\hline
\end{tabular}




\section{Experimental Section}

The area surrounding the air-quality monitoring station at Conroe, TX is primarily affected by pollution in the outflow of air from Houston, which hosts significant energy and petrochemical industries in addition to a large urban population. The regional atmospheric chemistry is also influenced by marine air from the Gulf of Mexico. The site itself is located in the middle of a field adjacent to the airport, with a gravel parking lot nearby and bordered by trees approximately $200 \mathrm{~m}$ to the North.

A permanent Texas Commission on Environmental Quality (TCEQ) ambient measurement station exists at this site and provided continuous meteorological data (wind speed, wind direction, temperature and relative humidity) for the duration of the campaign. $\mathrm{NO}_{x}$ and $\mathrm{O}_{3}$ monitors were also present at the TCEQ site. During DISCOVER-AQ a temporary ground site was set up adjacent to the permanent station. Chlorine species were detected using a High Resolution Time-of-Flight Chemical Ionization Mass Spectrometer (HR-ToF-CIMS, henceforth referred to as CIMS) [70-73], which was operated in negative ionization mode utilizing the iodide reagent ion. Similar CIMS techniques have been used in previous studies for the detection of $\mathrm{ClNO}_{2}, \mathrm{~N}_{2} \mathrm{O}_{5}$ and $\mathrm{Cl}_{2}$ [24,35,37,45-47,74,75], and other studies have described in detail the operation and application of the Aerodyne CIMS. Species reported here that were detected with the iodide CIMS included $\mathrm{CINO}_{2}, \mathrm{Cl}_{2}$ and $\mathrm{N}_{2} \mathrm{O}_{5}$; the ions monitored for these species were $\mathrm{IClNO}_{2}{ }^{-}, \mathrm{ICl}_{2}{ }^{-}$, and $\mathrm{IN}_{2} \mathrm{O}_{5}{ }^{-}$.

A sample flow of 2 LPM was introduced into the instrument through a $\frac{1}{4}$ " outer diameter perfluoroalkoxy (PFA) Teflon®sample line connected to the stainless steel inlet by a $\frac{1}{4}$ " to $10 \mathrm{~mm}$ stainless steel Swagelok union. The sample flow then passed into the ion-molecule reaction (IMR) chamber of the instrument, where it was mixed with a reagent ion flow of $2 \mathrm{LPM}$. Ionization in the IMR took place at a pressure of $200 \mathrm{mbar}$ and temperature of $34^{\circ} \mathrm{C}$ (controlled by an IMR heater). $\mathrm{I}\left(\mathrm{H}_{2} \mathrm{O}\right)_{n}{ }^{-}$reagent ions were generated by passing a flow of ultra-high purity (UHP) $\mathrm{N}_{2}$ over a methyl iodide $\left(\mathrm{CH}_{3} \mathrm{I}\right.$, Sigma Aldrich, 99\%) permeation tube. The $\mathrm{N}_{2}$ flow first passed through purified water (Milli-Q, model Advantage-A10) upstream of the $\mathrm{CH}_{3} \mathrm{I}$ permeation tube in order to provide the humidity necessary for sufficient production of $\mathrm{H}_{2} \mathrm{OI}^{-}$ ions. This also helped to stabilize the relative humidity within the IMR and resulting variations in sensitivity that have been noted for iodide chemical ionization [35,72]. Inside of the inlet, the sample flow then passed through $44.5 \mathrm{~mm}$ of the $10 \mathrm{~mm}$ outer diameter stainless steel tubing before passing through a critical orifice into the IMR. After mixing in the IMR the combined reagent and sample flow passed through two quadrupoles before entering the Time-of-Flight (ToF) mass analyzer. During the entire measurement period, the ToF was operated in $\mathrm{V}$ mode, providing higher sensitivity but lower resolution than $\mathrm{W}$ mode. 
In order to test for measurement artifacts a flow of ultra-high purity (UHP) $\mathrm{N}_{2}$ was periodically introduced into the sampling inlet. This flushing of the sampling line resulted in a rapid drop to background levels in the signals for $\mathrm{Cl}_{2}, \mathrm{~N}_{2} \mathrm{O}_{5}$ and $\mathrm{ClNO}_{2}$, suggesting that adsorption and subsequent desorption of these species from the inlet line walls was not a significant source of measurement artifacts. When the sampling commenced afterwards ion signals returned rapidly to ambient levels, suggestive of a high transmission efficiency. No explicit tests were performed to assess the $\mathrm{N}_{2} \mathrm{O}_{5}$ transmission efficiency in the field introducing uncertainty in the quantification of $\mathrm{N}_{2} \mathrm{O}_{5}$ which could be lost irreversibly to the inlet walls. The $\mathrm{N}_{2} \mathrm{O}_{5}$ concentrations in this study are reported assuming that transmission efficiency was equivalent to that observed during calibrations. We estimate that this introduces a measurement uncertainty of $20 \%$, a conservative estimate compared to previous work [76] which cited a $\pm 7 \%$ accuracy for $\mathrm{N}_{2} \mathrm{O}_{5}$ measured using a different instrument.

Equations (1) and (2) were used to calculate the production of $\mathrm{Cl}^{\bullet}$ and $\mathrm{OH}^{\bullet}$ consistent with box modeling simulation results. The production of $\mathrm{OH}^{\bullet}$ was calculated from the reactions of $\mathrm{O}\left({ }^{1} \mathrm{D}\right)$ with $\mathrm{H}_{2} \mathrm{O}, \mathrm{N}_{2}$, and $\mathrm{O}_{2}$ (Equation (1)), in a manner similar to a previous observational study [45]. The rate of $\mathrm{O}_{3}$ photolysis was used to calculate the rate of $\mathrm{O}\left({ }^{1} \mathrm{D}\right)$ generation, and additional details on the derivation of Equation (1) are described in the supplementary material. The production of $\mathrm{Cl}^{\bullet}$ proceeded directly from the photolysis of $\mathrm{Cl}_{2}$ (Equation (2)).

$$
\begin{gathered}
P_{\mathrm{OH}}=2 J_{\left(\mathrm{O}_{3}\right)}\left[\mathrm{O}_{3}\right] k_{\mathrm{H}_{2} \mathrm{O}}\left[\mathrm{H}_{2} \mathrm{O}\right] /\left(k_{\mathrm{H} 2 \mathrm{O}}\left[\mathrm{H}_{2} \mathrm{O}\right]+k_{\mathrm{N} 2}\left[\mathrm{~N}_{2}\right]+k_{\mathrm{O} 2}\left[\mathrm{O}_{2}\right]\right) \\
P_{\mathrm{Cl}}=2 \times j_{\mathrm{Cl}_{2}}\left[\mathrm{Cl}_{2}\right]
\end{gathered}
$$

Additional simulations were performed to assess the impact of observed $\mathrm{ClNO}_{2}$ concentrations on $\mathrm{Cl}^{\bullet}$ production and atmospheric reactivity, and Equation (3) was used to quantify the production of $\mathrm{Cl}^{\bullet}$ from $\mathrm{ClNO}_{2}$.

$$
P_{\mathrm{Cl}}=j_{\mathrm{ClNO} 2}\left[\mathrm{ClNO}_{2}\right]
$$

\section{Conclusions}

During the month of September 2013, concentrations of $\mathrm{Cl}_{2}$ and $\mathrm{ClNO}_{2}$ regularly reached or exceeded $2 \mathrm{ppt}$ and $60 \mathrm{ppt}$, respectively, at an inland monitoring site in Southeast Texas. The concurrent presence or absence of $\mathrm{N}_{2} \mathrm{O}_{5}$ at the site depended on the source of the sampled air, and longer paths over land corresponded to higher concentrations of $\mathrm{N}_{2} \mathrm{O}_{5}$. Peak $\mathrm{ClNO}_{2}$ concentrations in the early mornings when little to no $\mathrm{N}_{2} \mathrm{O}_{5}$ was present on some days suggest that the production and transport of $\mathrm{ClNO}_{2}$ from a non-local source is occurring. Contemporaneous measurements of PM surface area suggest conditions favorable to heterogeneous conversion of $\mathrm{N}_{2} \mathrm{O}_{5}$ in the advected air masses on these mornings. The presence of $\mathrm{Cl}_{2}$ and $\mathrm{ClNO}_{2}$ at the site 
are significant due to their role as $\mathrm{Cl}^{\bullet}$ sources. Although $\mathrm{Cl}_{2}$ concentrations are lower in magnitude, the fact that $\mathrm{Cl}_{2}$ concentrations consistently peak in the afternoon when photolysis rates are highest suggests a $\mathrm{Cl}_{2}$ source of approximately $30 \mathrm{ppt} \cdot \mathrm{h}^{-1}$ $\mathrm{Cl}_{2}$. Box modeling simulations suggest that such concentrations can contribute to enhanced $\mathrm{O}_{3}$ and $\mathrm{RO}_{2}{ }^{\bullet}$ production during the day. The source of $\mathrm{ClNO}_{2}$ is likely the result of reactions between anthropogenic $\mathrm{NO}_{\mathrm{x}}$ sources and particulate chloride originating along the Gulf Coast, and the reported values are within the range of previous model predictions in the region. Assuming that the surface measurements made in the early morning are representative of the entire nocturnal boundary layer, $\mathrm{ClNO}_{2}$ was also found to contribute to $\mathrm{Cl}^{\bullet}$ production and atmospheric reactivity, particularly in the early morning before significant $\mathrm{OH}^{\bullet}$ production begins. Overall, the results suggest that $\mathrm{Cl}_{2}$ and $\mathrm{ClNO}_{2}$ affect atmospheric reactivity and can impact the formation of ozone and secondary organic aerosol. In a region where attainment of the National Ambient Air Quality Standard for ozone has been an issue in the past decades, quantifying the effects of reactive chlorine chemistry is important for assuring future attainment.

Acknowledgments: This work was funded in part through a grant from the Texas Commission on Environmental Quality (TCEQ), administered by The University of Texas through the Air Quality Research Program (Project 12-012). The contents, findings opinions and conclusions are the work of the authors and do not necessarily represent findings, opinions or conclusions of the TCEQ. The work was also funded in part with funds from the State of Texas as part of the program of the Texas Air Research Center. The contents do not necessarily reflect the views and policies of the sponsor nor does the mention of trade names or commercial products constitute endorsement or recommendation for use. The authors would like to acknowledge the support of the entire DISCOVER-AQ Texas 2013 team. Code for the SAPRC box modeling software can be found at http://www.engr.ucr.edu/ carter/SAPRC. Meteorological data used for model input can be obtained via the TCEQ website (http:/ / www.tceq.state.tx.us).

Author Contributions: Cameron Faxon, Jeffrey Bean, and Lea Hildebrandt Ruiz planned, prepared and conducted the ambient measurements. Cameron Faxon conducted the box model simulations and analyzed ambient data and box model results presented in this work. The manuscript was written by Cameron Faxon with the guidance of Lea Hildebrandt Ruiz.

Conflicts of Interest: The authors declare no conflict of interest.

\section{References}

1. Tanaka, P.L.; Riemer, D.D.; Chang, S.; Yarwood, G.; McDonald-Buller, E.C.; Apel, E.C.; Orlando, J.J.; Silva, P.J.; Jimenez, J.L.; Canagaratna, M.R.; et al. Direct evidence for chlorine-enhanced urban ozone formation in Houston, Texas. Atmos. Environ. 2003, 37, 1393-1400.

2. Wang, L.; Thompson, T.; McDonald-Buller, E.C.; Allen, D.T. Photochemical modeling of emissions trading of highly reactive volatile organic compounds in Houston, Texas. 2. Incorporation of chlorine emissions. Environ. Sci. Technol. 2007, 41, 2103-2107. 
3. Faxon, C.B.; Allen, D.T. Chlorine chemistry in urban atmospheres: A review. Environ. Chem. 2013, 10, 221-233.

4. Wingenter, O.W.; Sive, B.C.; Blake, N.J.; Blake, D.R.; Rowland, F.S. Atomic chlorine concentrations derived from ethane and hydroxyl measurements over the equatorial Pacific Ocean: Implication for dimethyl sulfide and bromine monoxide. J. Geophys. Res. 2005, 110.

5. Graedel, T.E.; Keene, W.C. Tropospheric budget of reactive chlorine. Glob. Biogeochem. Cycles 1995, 9, 47-77.

6. Keene, C.; Aslam, M.; Khalil, K.; Erickson, J.; Archie, I.I.I.; Graedel, E.; Loberr, M.; Aucott, M.L.; Ling, S.; Harper, D.B.; et al. Composite global emissions of reactive chlorine from anthropogenic and natural sources: Reactive chlorine emissions inventory. J. Geophys. Res. 1999, 104, 8429-8440.

7. Sarwar, G.; Bhave, P.V. Modeling the effect of chlorine emissions on ozone levels over the eastern United States. J. Appl. Meteorol. Climatol. 2007, 46, 1009-1019.

8. Liu, C.L.; Smith, J.D.; Che, D.L.; Ahmed, M.; Leone, S.R.; Wilson, K.R. The direct observation of secondary radical chain chemistry in the heterogeneous reaction of chlorine atoms with submicron squalane droplets. Phys. Chem. Chem. Phys. 2011, 13, 8993-9007.

9. Wang, L.; Arey, J.; Atkinson, R. Reactions of chlorine atoms with a series of aromatic hydrocarbons. Environ. Sci. Technol. 2005, 39, 5302-10.

10. Nelson, L.; Rattigan, O.; Neavyn, R.; Sidebottom, H. Absolute and relative rate constants for reactions of hydroxyl radicals and chlorine atoms with series of aliphatic alcohols and ethers at 298K. Int. J. Chem. Kinet. 1990, 22, 1111-1126.

11. Aschmann, S.M.; Atkinson, R. Rate Constants for the Gas-Phase Reactions of alkanes with $\mathrm{Cl}$ atoms at 296. Int. J. Chem. Kinet. 1995, 27, 613-622.

12. Canosa-Mas, C.E.; Hutton-Squire, H.R.; King, M.D.; Stewart, D.J.; Thompson, K.C.; Wayne, R.P. Laboratory Kinetic Studies of the Reactions of $\mathrm{Cl}$ Atoms with Species of Biogenic Origin: $\Delta 3$-Carene, Methacrolein and Methyl Vinyl Ketone. J. Atmos. Chem. 1999, 34, 163-170.

13. Ragains, M.L.; Finlayson-Pitts, B.J. Kinetics and mechanism of the reaction of $\mathrm{Cl}$ atoms with 2-Methyl-1,3-butadiene (Isoprene) at 298 K. J. Phys. Chem. A 1997, 5639, 1509-1517.

14. Chang, S.; McDonald-Buller, E.; Kimura, Y.; Yarwood, G.; Neece, J.; Russell, M.; Tanaka, P.; Allen, D. Sensitivity of urban ozone formation to chlorine emission estimates. Atmos. Environ. 2002, 36, 4991-5003.

15. Tanaka, P.L.; Allen, D.T.; Mullins, C.B. An environmental chamber investigation of chlorine-enhanced ozone formation in Houston, Texas. J. Geophys. Res. 2003, 108.

16. Knipping, E.M.; Dabdub, D. Impact of chlorine emissions from sea-salt aerosol on coastal urban ozone. Environ. Sci. Technol. 2003, 37, 275-284. 
17. Sander, S.P.; Friedl, R.R.; Barker, J.R.; Golden, D.M.; Kurylo, M.J.; Sciences, G.E.; Wine, P.H.; Abbatt, J.P.D.; Burkholder, J.B.; Kolb, C.E.; et al. Chemical Kinetics and Photochemical Data for Use in Atmospheric Studies: Evaluation Number 17. Available online: https://jpldataeval.jpl.nasa.gov/pdf/JPL\%2010-6\%20Final\%2015Jun8011.pdf (accessed on 31 August 2015).

18. Chang, S.; Allen, D.T. Chlorine chemistry in urban atmospheres: Aerosol formation associated with anthropogenic chlorine emissions in southeast Texas. Atmos. Environ. 2006, 40, 512-523.

19. Chang, S.; Tanaka, P.; Mcdonald-buller, E.; Allen, D.T. Emission inventory for atomic chlorine precursors in Southeast Texas. Available online: http://www.tceq.state.tx.us/assets/public/implementation/air/am/contracts/ reports/oth/EmissioninventoryForAtomicChlorinePrecursors.pdf (accessed on 31 August 2015).

20. Atkinson, R.; Baulch, D.L.; Cox, R.A.; Crowley, J.N.; Hampson, R.F.; Hynes, R.G.; Jenkin, M.E.; Rossi, M.J.; Troe, J. Evaluated kinetic and photochemical data for atmospheric chemistry: Volume III-Gas phase reactions of inorganic halogens. Atmos. Chem. Phys. 2007, 7, 981-1191.

21. Pszenny, A.A.P.; Keene, W.C.; Jacob, D.J.; Fan, S.; Maben, J.R.; Zetwo, M.P. Evidence of inorganic chlorine gases other than hydrogen chloride in marine surface air. Geopys. Res. Lett. 1993, 20, 699-702.

22. Spicer, C.; Chapman, E.; Finlayson-Pitts, B.; Plastrige, R.; Hubbe, J.; Fast, J.; Berkowitz, C. Unexpectedly high concentrations of molecular chlorine in coastal air. Nature 1998, 394, 353-356.

23. Finley, B.D.; Saltzman, E.S. Measurement of $\mathrm{Cl}_{2}$ in coastal urban air. Geophys. Res. Lett. 2006, 33.

24. Riedel, T.P.; Bertram, T.H.; Crisp, T.A.; Williams, E.J.; Lerner, B.M.; Vlasenko, A.; Li, S.M.; Gilman, J.; de Gouw, J.; Bon, D.M.; et al. A Nitryl chloride and molecular chlorine in the coastal marine boundary layer. Environ. Sci. Technol. 2012, 46, 10463-10470.

25. Liao, J.; Huey, L.G.; Liu, Z.; Tanner, D.J.; Cantrell, C.A.; Orlando, J.J.; Flocke, F.M.; Shepson, P.B.; Weinheimer, A.J.; Hall, S.R.; et al. High levels of molecular chlorine in the Arctic atmosphere. Nat. Geosci. 2014, 7, 91-94.

26. Chang, S.; Allen, D.T. Atmospheric chlorine chemistry in southeast Texas: impacts on ozone formation and control. Environ. Sci. Technol. 2006, 40, 251-262.

27. Oum, K.W.; Lakin, M.J.; DeHaan, D.O.; Brauers, T.; Finlayson-Pitts, B.J. Formation of molecular chlorine from the photolysis of ozone and aqueous sea-salt particles. Science 1998, 279, 74-76.

28. Herrmann, H.; Majdik, Z.; Ervens, B.; Weise, D. Halogen production from aqueous tropospheric particles. Chemosphere 2003, 52, 485-502.

29. Sadanaga, Y.; Hirokawa, J.; Akimoto, H. Formation of molecular chlorine in dark condition: Heterogeneous reaction of ozone with sea salt in the presence of ferric ion. Geophys. Res. Lett. 2001, 28, 4433-4436. 
30. Knipping, E.M. Experiments and simulations of ion-enhanced interfacial chemistry on aqueous $\mathrm{NaCl}$ aerosols. Science 2000, 288, 301-306.

31. Behnke, W.; Zetzsch, C. Heterogeneous formation of chlorine atoms from various aerosols in the presence of $\mathrm{O}_{3}$ and HCl. J. Aerosol Sci. 1989, 20, 1167-1170.

32. Keene, W.C.; Pszenny, A.A.P.; Jacob, D.J.; Duce, R.A.; Galloway, J.N.; Schultz-Tokos, J.J.; Sievering, H.; Boatman, J.F. The geochemical cycling of reactive chlorine through the marine troposphere. Glob. Biogeochem. Cycles 1990, 4, 407-430.

33. Knipping, E.M. Modeling $\mathrm{Cl}_{2}$ formation from aqueous $\mathrm{NaCl}$ particles: Evidence for interfacial reactions and importance of $\mathrm{Cl}_{2}$ decomposition in alkaline solution. J. Geophys. Res. 2002, 107.

34. George, I.J.; Abbatt, J.P.D. Heterogeneous oxidation of atmospheric aerosol particles by gas-phase radicals. Nat. Chem. 2010, 2, 713-722.

35. Kercher, J.P.; Riedel, T.P.; Thornton, J.A. Chlorine activation by $\mathrm{N}_{2} \mathrm{O}_{5}$ : Simultaneous, in situ detection of $\mathrm{ClNO}_{2}$ and $\mathrm{N}_{2} \mathrm{O}_{5}$ by chemical ionization mass spectrometry. Atmos. Measu. Tech. 2009, 2, 193-204.

36. Thornton, J.A.; Abbatt, J.P.D. $\mathrm{N}_{2} \mathrm{O}_{5}$ reaction on submicron sea salt aerosol: Kinetics, products, and the effect of surface active organics. J. Phys. Chem. A 2005, 109, 10004-10012.

37. Osthoff, H.D.; Roberts, J.M.; Ravishankara, a. R.; Williams, E.J.; Lerner, B.M.; Sommariva, R.; Bates, T.S.; Coffman, D.; Quinn, P.K.; Dibb, J.E.; et al. High levels of nitryl chloride in the polluted subtropical marine boundary layer. Nat. Geosci. 2008, 1, 324-328.

38. Roberts, J.M.; Osthoff, H.D.; Brown, S.S.; Ravishankara, A.R.; Coffman, D.; Quinn, P.; Bates, T. Laboratory studies of products of $\mathrm{N}_{2} \mathrm{O}_{5}$ uptake on $\mathrm{Cl}$-containing substrates. Geophys. Res. Lett. 2009, 36.

39. Sarwar, G.; Simon, H.; Bhave, P.; Yarwood, G. Examining the impact of heterogeneous nitryl chloride production on air quality across the United States. Atmos. Chem. Phys. 2012, 12, 6455-6473.

40. Simon, H.; Kimura, Y.; McGaughey, G.; Allen, D.T.; Brown, S.S.; Coffman, D.; Dibb, J.; Osthoff, H.D.; Quinn, P.; Roberts, J.M. Modeling heterogeneous $\mathrm{ClNO}_{2}$ formation, chloride availability, and chlorine cycling in Southeast Texas. Atmos. Environ. 2010, 44, 5476-5488.

41. Simon, H.; Kimura, Y.; McGaughey, G.; Allen, D.T.; Brown, S.S.; Osthoff, H.D.; Roberts, J.M.; Byun, D.; Lee, D. Modeling the impact of $\mathrm{ClNO}_{2}$ on ozone formation in the Houston area. J. Geophys. Res. 2009, 114.

42. Sarwar, G.; Simon, H.; Xing, J.; Mathur, R. Importance of tropospheric $\mathrm{ClNO}_{2}$ chemistry across the Northern Hemisphere. Geophys. Res. Lett. 2014, 41, 4050-4058.

43. Roberts, J.M.; Osthoff, H.D.; Brown, S.S.; Ravishankara, A.R. $\mathrm{N}_{2} \mathrm{O}_{5}$ oxidizes chloride to $\mathrm{Cl}_{2}$ in acidic atmospheric aerosol. Science 2008, 321, 1059-1059.

44. Thomas, J.L.; Jimenez-Aranda, A.; Finlayson-Pitts, B.J.; Dabdub, D. Gas-phase molecular halogen formation from $\mathrm{NaCl}$ and $\mathrm{NaBr}$ aerosols: when are interface reactions important? J. Phys. Chem. A 2006, 110, 1859-1867. 
45. Phillips, G.J.; Tang, M.J.; Thieser, J.; Brickwedde, B.; Schuster, G.; Bohn, B.; Lelieveld, J.; Crowley, J.N. Significant concentrations of nitryl chloride observed in rural continental Europe associated with the influence of sea salt chloride and anthropogenic emissions. Geophys. Res. Lett. 2012, 39.

46. Thornton, J.A.; Kercher, J.P.; Riedel, T.P.; Wagner, N.L.; Cozic, J.; Holloway, J.S.; Dubé, W.P.; Wolfe, G.M.; Quinn, P.K.; Middlebrook, A.M.; et al. A large atomic chlorine source inferred from mid-continental reactive nitrogen chemistry. Nature 2010, 464, 271-274.

47. Mielke, L.H.; Furgeson, A.; Osthoff, H.D. Observation of $\mathrm{ClNO}_{2}$ in a mid-continental urban environment. Environ. Sci. Technol. 2011, 45, 8889-8896.

48. Behnke, W.; George, C.; Scheer, V.; Zetzsch, C. Production and decay of $\mathrm{ClNO}_{2}$ from the reaction of gaseous $\mathrm{N}_{2} \mathrm{O}_{5}$ with $\mathrm{NaCl}$ solution: Bulk and aerosol experiments. J. Geophys. Res. 1997, 102, 3795-3804.

49. Behnke, W.; Krüger, H.-U.; Scheer, V.; Zetzsch, C. Formation of atomic Cl from sea spray via photolysis of nitryl chloride: Determination of the sticking coefficient of $\mathrm{N}_{2} \mathrm{O}_{5}$ on $\mathrm{NaCl}$ aerosol. J. Aerosol Sci. 1991, 22, S609-S612.

50. Frenzel, A.; Scheer, V.; Sikorski, R.; George, C.; Behnke, W.; Zetzsch, C. Heterogeneous interconversion reactions of $\mathrm{BrNO}_{2}, \mathrm{ClNO}_{2}, \mathrm{Br}_{2}$, and $\mathrm{Cl}_{2}$. J. Phys. Chem. A 1998, 102, 1329-1337.

51. Schweitzer, F.; Mirabel, P.; George, C. Multiphase chemistry of $\mathrm{N}_{2} \mathrm{O}_{5}, \mathrm{ClNO}_{2}$, and $\mathrm{BrNO}_{2}$. J. Phys. Chem. A 1998, 102, 3942-3952.

52. Bertram, T.H.; Thornton, J.A. Toward a general parameterization of $\mathrm{N}_{2} \mathrm{O}_{5}$ reactivity on aqueous particles: The competing effects of particle liquid water, nitrate and chloride. Atmos. Chem. Phys. Discuss. 2009, 9, 15181-15214.

53. Mielke, L.H.; Stutz, J.; Tsai, C.; Hurlock, S.C.; Roberts, J.M.; Veres, P.R.; Froyd, K.D.; Hayes, P.L.; Cubison, M.J.; Jimenez, J.L.; et al. Heterogeneous formation of nitryl chloride and its role as a nocturnal $\mathrm{NO}_{\mathrm{x}}$ reservoir species during CalNex-LA 2010. J. Geophys. Res. Atmos. 2013, 118, 10638-10652.

54. Tham, Y.J.; Yan, C.; Xue, L.; Zha, Q.; Wang, X.; Wang, T. Presence of high nitryl chloride in Asian coastal environment and its impact on atmospheric photochemistry. Chin. Sci. Bull. 2013, 59, 356-359.

55. Riedel, T.P.; Wagner, N.L.; Dubé, W.P.; Middlebrook, A.M.; Young, C.J.; Öztürk, F.; Bahreini, R.; VandenBoer, T.C.; Wolfe, D.E.; Williams, E.J.; et al. Chlorine activation within urban or power plant plumes: Vertically resolved $\mathrm{ClNO}_{2}$ and $\mathrm{Cl}_{2}$ measurements from a tall tower in a polluted continental setting. J. Geophys. Res. Atmos. 2013, 118, 8702-8715.

56. National Aeronautics and Space Administration. Deriving Information on Surface Conditions from Column and Vertically Resolved Observations Relevant to Air Quality (DISCOVER-AQ). Available online: http://discover-aq.larc.nasa.gov/ (accessed on 31 August 2015). 
57. Ng, N.L.; Herndon, S.C.; Trimborn, a.; Canagaratna, M.R.; Croteau, P.L.; Onasch, T.B.; Sueper, D.; Worsnop, D.R.; Zhang, Q.; Sun, Y.L.; et al. An Aerosol Chemical Speciation Monitor (ACSM) for routine monitoring of the composition and mass concentrations of ambient aerosol. Aerosol Sci. Technol. 2011, 45, 780-794.

58. Finley, B.D.; Saltzman, E.S. Observations of $\mathrm{Cl}_{2}, \mathrm{Br}_{2}$, and $\mathrm{I}_{2}$ in coastal marine air. J. Geophys. Res. 2008, 113, 1-14.

59. Jordan, C.E.; Pszenny, A.A.P.; Keene, W.C.; Cooper, O.R.; Deegan, B.; Maben, J.; Routhier, M.; Sander, R.; Young, A.H. Origins of aerosol chlorine during winter over north central Colorado, USA. J. Geophys. Res. Atmos. 2015, 120, 678-694.

60. Young, A.H.; Keene, W.C.; Pszenny, A.A.P.; Sander, R.; Thornton, J.A.; Riedel, T.P.; Maben, J.R. Phase partitioning of soluble trace gases with size-resolved aerosols in near-surface continental air over northern Colorado, USA, during winter. J. Geophys. Res. Atmos. 2013, 118, 9414-9427.

61. Pratt, K.A.; Prather, K.A. Aircraft measurements of vertical profiles of aerosol mixing states. J. Geophys. Res. Atmos. 2010, 115, 1-10.

62. Hildebrandt Ruiz, L.; Yarwood, G.; Koo, B.; Heo, G. Quality assurance project plan project 14-024-Sources of organic particulate matter in Houston: Evidence from DISCOVER-AQ data modeling and experiments. Available online: http://aqrp. ceer.utexas.edu/projectinfoFY14_15\%5C14-024\%5C14-024\%20QAPP.pdf (accessed on 31 August 2015).

63. Draxler, R.R.; Hess, G. Description of the HYSPLIT_4 Modeling System. Available online: https:/ / ready.arl.noaa.gov/HYSPLIT.php; 2010 (accessed on 31 August 2015).

64. Yarwood, G.; Jung, J.; Whitten, G.Z.; Heo, G.; Mellberg, J.; Estes, M. Updates to the Carbon Bond mechanism for version 6 (CB6). In Proceedings of the 9th Annual CMAS Conference, Chapel Hill, NC, USA, 11-13 October 2010; pp. 1-4.

65. Yarwood, G.; Rao, S. Updates to the Carbon Bond Chemical Mechanism: CB05; RT-0400675; Final report to the US EPA; US EPA: Washington, DC, USA, 2005.

66. Tanaka, P.L.; Allen, D.T.; McDonald-Buller, E.C.; Chang, S.; Kimura, Y.; Mullins, C.B.; Yarwood, G.; Neece, J.D. Development of a chlorine mechanism for use in the carbon bond IV chemistry model. J. Geophys. Res. 2003, 108.

67. Carter, W.P.L.; Luo, D.; Malkina, I.L.; Pierce, J.A. Environmental Chamber Studies of Atmospheric Reactivities of Volatile Organic Compounds: Effects of Varying Chamber and Light Source. 1995. Availavle online: https://www.cert.ucr.edu/ carter/pubs/ explrept.pdf (accessed on 31 August 2015).

68. Sokolov, O.; Hurley, M.D.; Wallington, T.J.; Kaiser, E.W.; Platz, J.; Nielsen, O.J.; Berho, F.; Rayez, M.T.; Lesclaux, R. Kinetics and mechanism of the gas-phase reaction of $\mathrm{Cl}$ atoms with benzene. J. Phys. Chem. A 1998, 102, 10671-10681.

69. Wingenter, O.W.; Blake, D.R.; Blake, N.J.; Sive, B.C.; Rowland, F.S.; Atlas, E.; Flocke, F. Tropospheric hydroxyl and atomic chlorine concentrations, and mixing timescales determined from hydrocarbon and halocarbon measurements made over the Southern Ocean. J. Geophys. Res. 1999, 104, 21,819-21,828. 
70. Bertram, T.H.; Kimmel, J.R.; Crisp, T.A.; Ryder, O.S.; Yatavelli, R.L.N.; Thornton, J.A.; Cubison, M.J.; Gonin, M.; Worsnop, D.R. A field-deployable, chemical ionization time-of-flight mass spectrometer. Atmos. Meas. Tech. 2011, 4, 1471-1479.

71. Yatavelli, R.L.N.; Lopez-Hilfiker, F.; Wargo, J.D.; Kimmel, J.R.; Cubison, M.J.; Bertram, T.H.; Jimenez, J.L.; Gonin, M.; Worsnop, D.R.; Thornton, J.A. A chemical ionization high-resolution time-of-flight mass spectrometer coupled to a Micro Orifice Volatilization Impactor (MOVI-HRToF-CIMS) for analysis of gas and particle-phase organic species. Aerosol Sci. Technol. 2012, 46, 1313-1327.

72. Lee, B.H.; Lopez-Hilfiker, F.D.; Mohr, C.; Kurtén, T.; Worsnop, D.R.; Thornton, J.A. An iodide-adduct high-resolution time-of-flight chemical-ionization mass spectrometer: Application to atmospheric inorganic and organic compounds. Environ. Sci. Technol. 2014, 48, 6309-6317.

73. Aljawhary, D.; Lee, A.K.Y.; Abbatt, J.P.D. High-resolution chemical ionization mass spectrometry (ToF-CIMS): Application to study SOA composition and processing. Atmos. Meas. Tech. 2013, 6, 3211-3224.

74. Slusher, D.L.; Huey, L.G.; Tanner, D.J.; Flocke, F.M.; Roberts, J.M. A Thermal Dissociation-Chemical Ionization Mass Spectrometry (TD-CIMS) technique for the simultaneous measurement of peroxyacyl nitrates and dinitrogen pentoxide. J. Geophys. Res. 2004, 109.

75. Zheng, W.; Flocke, F.M.; Tyndall, G.S.; Swanson, A.; Orlando, J.J.; Roberts, J.M.; Huey, L.G.; Tanner, D.J. Characterization of a thermal decomposition chemical ionization mass spectrometer for the measurement of Peroxy Acyl Nitrates (PANs) in the atmosphere. Atmos. Chem. Phys. 2011, 11, 6529-6547.

76. Fuchs, H.; Dube, W.P.; Ciciora, S.J.; Brown, S.S. Determination of inlet transmission and conversion efficiencies for in situ measurements of the nocturnal nitrogen oxides, $\mathrm{NO}_{3}, \mathrm{~N}_{2} \mathrm{O}_{5}$ and $\mathrm{NO}_{2}$, via pulsed cavity ring-down spectroscopy. Anal. Chem. 2008, 80, 6010-6017. 


\title{
CCN Properties of Organic Aerosol Collected Below and within Marine Stratocumulus Clouds near Monterey, California
}

\author{
Akua Asa-Awuku, Armin Sorooshian, Richard C. Flagan, John H. Seinfeld and \\ Athanasios Nenes
}

\begin{abstract}
The composition of aerosol from cloud droplets differs from that below cloud. Its implications for the Cloud Condensation Nuclei (CCN) activity are the focus of this study. Water-soluble organic matter from below cloud, and cloud droplet residuals off the coast of Monterey, California were collected; offline chemical composition, CCN activity and surface tension measurements coupled with Köhler Theory Analysis are used to infer the molar volume and surfactant characteristics of organics in both samples. Based on the surface tension depression of the samples, it is unlikely that the aerosol contains strong surfactants. The activation kinetics for all samples examined are consistent with rapid $\left(\mathrm{NH}_{4}\right)_{2} \mathrm{SO}_{4}$ calibration aerosol. This is consistent with our current understanding of droplet kinetics for ambient CCN. However, the carbonaceous material in cloud drop residuals is far more hygroscopic than in sub-cloud aerosol, suggestive of the impact of cloud chemistry on the hygroscopic properties of organic matter.
\end{abstract}

Reprinted from Atmosphere. Cite as: Asa-Awuku, A.; Sorooshian, A.; Flagan, R.C.; Seinfeld, J.H.; Nenes, A. CCN Properties of Organic Aerosol Collected Below and within Marine Stratocumulus Clouds near Monterey, California. Atmosphere 2015, 6, 1590-1607.

\section{Introduction}

It is well established that organic compounds (especially water-soluble organic compounds, WSOC) are ubiquitous in marine aerosol; they can interact with water and affect aerosol hygroscopicity, droplet surface tension, and Cloud Condensation Nuclei (CCN) activity [1-6]. As a result, marine aerosol organic matter can affect cloud droplet number concentration as much as 15\% [7-10] and may exert a climatically important impact on clouds.

Organic compounds, depending on their source, are classified as "primary" and "secondary". Primary organic marine aerosol (POMA) can include high molecular-weight compounds transferred onto sea-salt aerosol from the surfactant-rich surface ocean during the bubble-bursting process [9]. The presence of POMA is mostly attributed to biological activity, and its concentration varies with 
season $[9,11,12]$. POMA can exhibit low hygroscopic growth factors but maintain high CCN activity [13] or vice versa [14]. Secondary organic marine aerosol (SOMA) can be produced during cloud processing [15-19]; perhaps the most studied chemical pathway for SOMA is glyoxylic acid oxidation [20] via several aqueous phase intermediates [21-23]. Continental biogenic emissions can also contribute to organic mass in marine clouds at high altitudes [24]. Anthropogenic emissions can substantially contribute to marine organics; for example, particulate emissions from ships are composed roughly of up to $10 \%$ carbon $[25,26]$, in the form of sparingly soluble poly-aromatic hydrocarbons, ketones and quinones (PAHs, PAKs, and PAQs, respectively $[13,27])$. "Ship tracks" are a natural laboratory for studying aerosol indirect effects, as clouds with uniform dynamics, are exposed to a strong gradient in emissions concentrations and often exhibit droplet number, effective radius and drizzle rates responses that are consistent with local emission rates [28-32].

In this study, marine aerosol samples influenced by ship emissions are collected in-situ (in and out of cloudy regions) and studied for their cloud-droplet formation properties. Given the complexity of the water-soluble organic fraction, characterization is done by measuring the size-resolved CCN activity of the material, surface tension depression and using Köhler Theory Analysis (KTA) [33-35] to infer the thermodynamic properties (average molar volume, surfactant tension depression) of the organic fraction and its potential impact on droplet growth rate kinetics. These properties are then related to the influence of primary emissions and in-cloud oxidation processes on the CCN activity of the organic compounds.

\section{Experimental Methods}

\subsection{Aerosol Sampling and Chemical Composition}

The samples analyzed in this study were obtained during the Marine Stratus/Stratocumulus Experiments (MASE) that took place near the coast of Monterey, California, from July to August 2005. The airborne platform used the Center for Interdisciplinary Remotely-Piloted Aircraft Studies (CIRPAS) Twin Otter that sampled boundary layer air over 13 flights. A full description of the aerosol and cloud instrument payload aboard the plane during the MASE campaign can be found in Lu et al. [36]. Six of thirteen flights encountered strong, localized perturbations in aerosol concentration, size, and composition consistent with ship emissions. The data presented here were sampled on 13 July in the vicinity of ship tracks. An overview of airborne studies conducted in this region has been summarized by Coggon et al. [18].

Two sample types were collected aboard the aircraft in this study: cloud droplet residuals (CR) from evaporated cloud droplets, and sub-cloud (SC) aerosol sampled below cloud (and occasionally in clear-sky conditions). CR samples were collected with a counter-flow virtual impactor (CVI) [37,38], in which cloud droplets with 
diameter greater than $5 \mu \mathrm{m}$ were inertially separated from interstitial (unactivated) aerosol and evaporated before collection. Analysis of cloud droplet residuals with this approach has been instrumental in understanding the origin of $\mathrm{CCN}$ in ambient clouds [18].

Table 1. Concentrations of Water Soluble Organic Carbon (WSOC) and ions (mg. $\mathrm{L}^{-1}$ sample), and, $\alpha\left(\mathrm{mN} \cdot \mathrm{m}^{-1} \cdot \mathrm{K}^{-1}\right)$ and $\beta\left(\mathrm{L} \cdot \mathrm{mg}^{-1}\right)$ parameters of the Szyszkowski-Langmuir surface tension model ${ }^{*}[45]$ fits for the aerosol samples analyzed.

\begin{tabular}{ccc}
\hline Property & Cloud Residuals (CR) & Sub-Cloud (SC) \\
\hline $\mathrm{WSOC}$ & $220 \pm 14$ & $202 \pm 7$ \\
$\mathrm{Ca}^{2+}$ & 3.10 & 2.75 \\
$\mathrm{Mg}^{2+}$ & 0.07 & 0.58 \\
$\mathrm{Na}^{+}$ & 21.07 & 20.90 \\
$\mathrm{Cl}^{-}$ & 25.30 & 22.61 \\
$\mathrm{NH}_{4}{ }^{+}$ & 21.00 & 36.38 \\
$\mathrm{NO}_{3}{ }^{-}$ & 16.12 & 17.30 \\
$\mathrm{SO}_{4}{ }^{-}$ & 35.30 & 87.85 \\
$\mathrm{Oxalate}^{\alpha}$ & 3.61 & 4.22 \\
$\beta$ & $8.99 \times 10^{-4}$ & $2.91 \times 10^{-3}$ \\
$\beta$ & $3.84 \times 10^{-2}$ & $1.63 \times 10^{-2}$ \\
\hline$*$ Szyszkowski-Langmuir Equation $(1): \sigma=\sigma_{w}-\alpha T \ln (1+\beta c)$.
\end{tabular}

A Brechtel Manufacturing Inc. Particle-Into-Liquid sampler (PILS; [20]) was used to collect the water-soluble fraction of SC and CR by exposing the aerosol to supersaturated steam and growing them into droplets that are subsequently collected by inertial impaction. The liquid stream was then collected in vials over 3.5 to 5 min each. The chemical composition of the ionic species in each sample was measured with a dual Ion Chromatography (IC) system (ICS-2000 with $25 \mu \mathrm{L}$ sample loop, Dionex Inc.); the IC detection limit of aerosol species is less than $0.1 \mu \mathrm{g} \cdot \mathrm{m}^{-3}$ for inorganic ions $\left(\mathrm{Na}^{+}, \mathrm{NH}_{4}{ }^{+}, \mathrm{K}^{+}, \mathrm{Mg}^{2+}, \mathrm{Ca}^{2+}, \mathrm{Cl}^{-}, \mathrm{NO}_{3}{ }^{-}, \mathrm{NO}_{2}{ }^{-}\right.$, and $\mathrm{SO}_{4}{ }^{2-}$ ) and less than $0.01 \mu \mathrm{g} \cdot \mathrm{m}^{-3}$ air for the organic acid ions (dicarboxylic acids $\mathrm{C}_{2}-\mathrm{C}_{9}$, acetic, formic, pyruvic, glyoxylic, maleic, malic, methacrylic, benzoic, and methanesulfonic acids). The WSOC content was also measured off-line with a Total Organic Carbon (TOC) Analyzer (Sievers Model 800 Turbo, Boulder, CO) (Table 1). The contents of the vials were subsequently analyzed for their CCN activity and surfactant characteristics (Sections 2.2-2.4). The aerosol samples analyzed in the study were obtained on 13 July (when the highest organic acid concentrations were reported) from within and below cloud (cloud base and cloud top were measured at 101 and $450 \mathrm{~m}$ altitude, respectively) [36]. During this flight, the aircraft focused on 
sampling air in the vicinity of ship tracks. Backward trajectories calculated using the NOAA HYSPLIT (HYbrid Single-Particle Lagrangian Integrated Trajectory) model (http:/ / www.arl.noaa.gov/ready/hysplit4.html) indicate that the air mass on the 13 July originated from the North Pacific; the vertical profile indicates that the aerosol masses sampled on 13 July originated from the free troposphere before descending into the marine boundary layer (Figure 1).

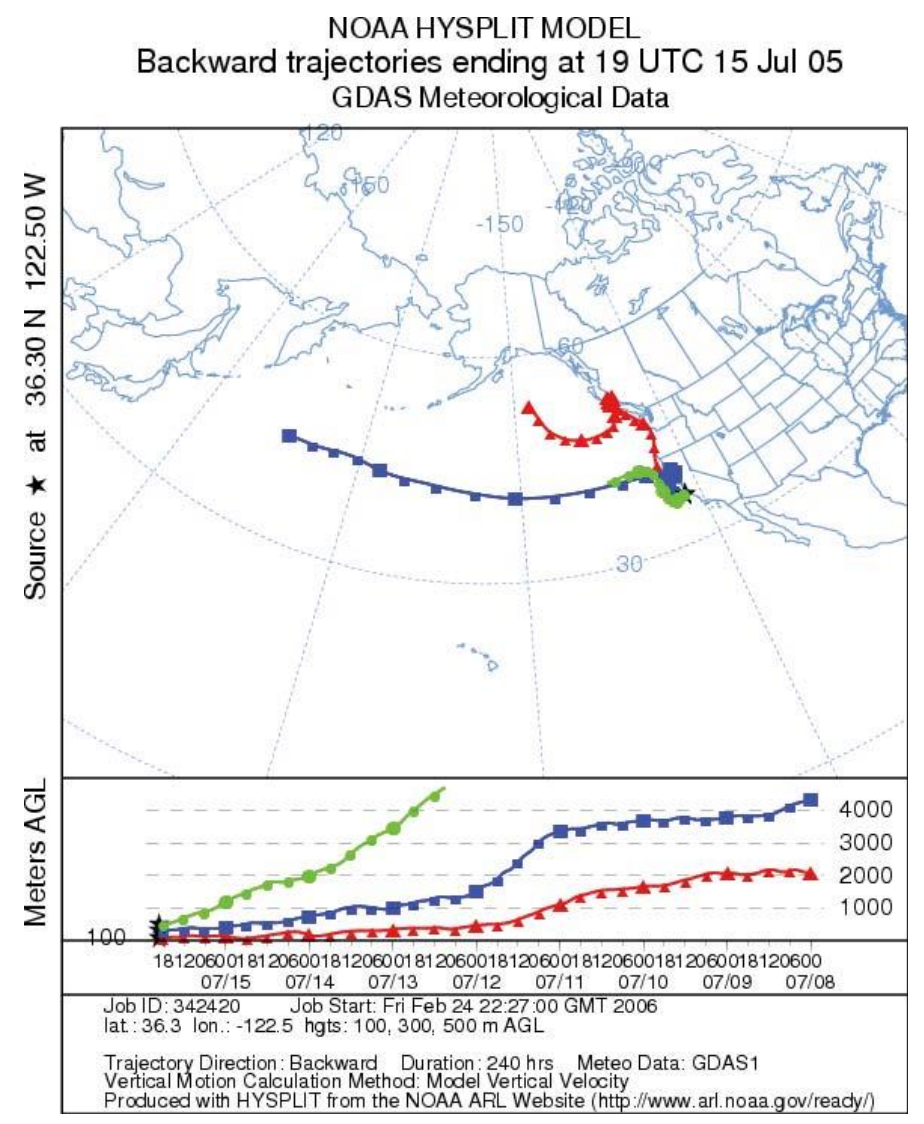

Figure 1. NOAA HYSPLIT (HYbrid Single-Particle Lagrangian Integrated Trajectory) Model Back Trajectories for air masses sampled aboard the Twin Otter on 13 July 2005.

\subsection{CCN Activity of Soluble Material Collected}

The aqueous contents of the PILS vials were atomized with a Collison-type atomizer (Figure 2) operated at 5 psig pressure. The atomized aerosol was then dried through two silica gel diffusional dryers, charged by a Kr-85 bipolar charger, and classified with a Differential Mobility Analyzer (DMA 3081) (Figure 2). The classified 
monodisperse aerosol was then split and passed through a TSI 3025A Condensation Particle Counter (CPC) to measure aerosol number concentration $(\mathrm{CN})$; the other stream was sampled by a Droplet Measurement Technologies Continuous-Flow Streamwise Thermal Gradient CCN Counter (CFSTGC) [39-41]. Given the limited amount of sample, size-resolved $\mathrm{CCN}$ activity and growth kinetics measurements were obtained using scanning mobility CCN Analysis (SMCA) [42]. The SMCA process couples CFSTGC measurements with a scanning mobility particle sizer (SMPS). An inversion procedure was used to compute the ratio of CCN to $\mathrm{CN}$ as a function of aerosol size as the SMPS scans from 10 and $250 \mathrm{~nm}$ dry mobility diameter for a fixed supersaturation, $s$. The data were fit to a sigmoidal function, corrected for diffusion and multiple charges in the DMA [42]; the particle dry diameter size, $d$, for which $50 \%$ of the particles activated into droplets, represents the dry diameter of the particle with critical supersaturation, $s_{\mathcal{C}}$, equal to the instrument supersaturation. The activation experiments were repeated (a minimum of four times) for each $s$ level, which varied from $0.2 \%$ to $1.2 \%$. The compositional data, aerosol surfactant behavior, and the dependence of $d$ with respect to $s_{c}$, were used to infer the molar volume and surfactant characteristics of the organic fraction with Köhler Theory Analysis [33-35,43] (Section 3). The CFSTGC was calibrated using $\left(\mathrm{NH}_{4}\right)_{2} \mathrm{SO}_{4}$ (density $=1.77 \mathrm{~g} \cdot \mathrm{cm}^{-3}$, and molar mass of $132 \mathrm{~g} \cdot \mathrm{mol}^{-1}$ ) generated with the same experimental setup, and operating the DMA with a sheath:aerosol flow-rate ratio of $10: 1 ; d$ for ammonium sulfate was then related to critical supersaturation by applying classical Köhler theory, using an effective van't Hoff factor of $2.5[41,44]$. The constant van't Hoff value is an approximation that can be improved with the use of the Pitzer Method. Multiple calibrations of the instrument were performed over the period of measurements, and each supersaturation was within $10 \%$ of the average.

\subsection{Surface Tension Measurements}

A CAM 200 pendant drop goniometer was used to measure bulk surface tension, $\sigma$, of the original samples and prescribed dilutions of them, following the approach of $[33,35]$. The instrument uses 5-6 $\mathrm{mL}$ of sample to form a drop at the end of a needle. The optical goniometer captures 100 images of the droplet and computes droplet surface tension through application of the Young-Laplace equation; the standard deviations for $\sigma_{\mathrm{s} / \mathrm{a}}$ are $<0.05 \mathrm{mN} \cdot \mathrm{m}^{-1}$ for a given sample at one concentration. The measurements were then fit to the Szyskowski-Langmuir equation [45]:

$$
\sigma=\sigma_{w}-\alpha T \ln (1+\beta c)
$$

where $\alpha, \beta$ are optimally fitted constants, $T$ is temperature, $\sigma_{w}$ is the surface tension of pure water and $c$ is the dissolved carbon concentration $\left(\mathrm{mg} \cdot \mathrm{C} \cdot \mathrm{L}^{-1}\right)$. Each pendant drop was suspended $60 \mathrm{~s}$ before surface tension was measured, in order to allow 
organics in the bulk to equilibrate with the droplet surface layer [46]. Table 1 provides a summary of the $\alpha$ and $\beta$ parameters for all samples considered. Bulk measurements are known to not represent the droplet activation regime. Thus parameters derived from bulk measurements (Table 1) are applied to inferred droplet concentrations at activation [33-35,47].

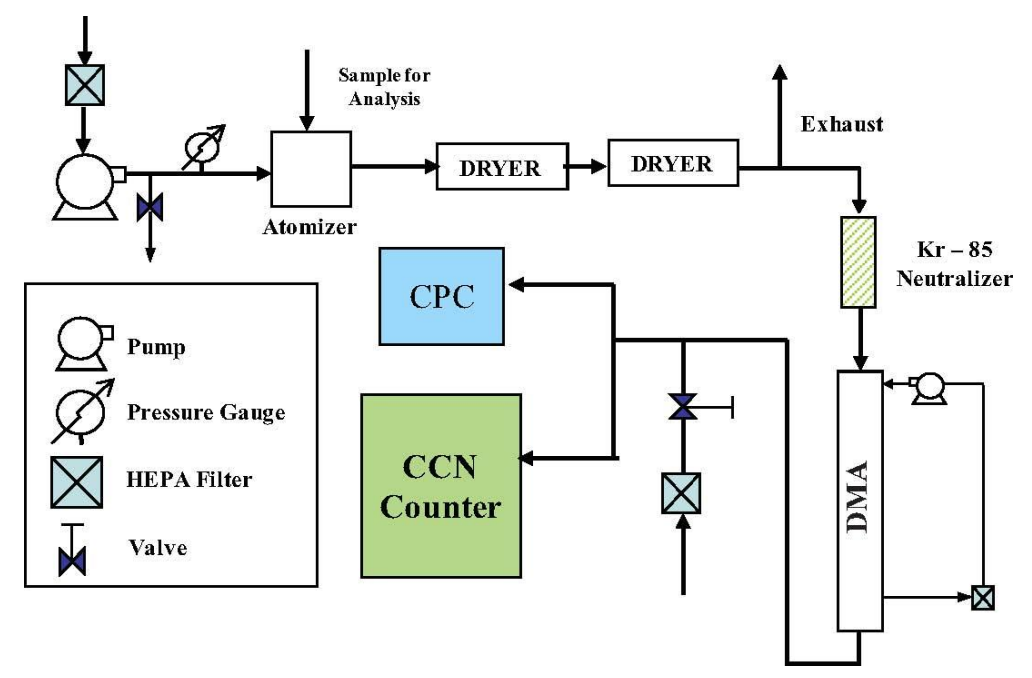

Figure 2. Experimental setup for characterizing size-resolved Cloud Condensation Nuclei activity of samples analyzed in this study.

\subsection{Droplet Size Measurements of Activated CCN}

The optical particle counter used for detection of CCN concentrations also provides the size of the activated droplets; thus the SMCA procedure can determine the size of activated CCN with known dry diameter at the exit of the CFSTGC. We adopt the method of Threshold Droplet Growth Analysis (TDGA) (e.g., but not limited to $[39,48,49])$ to detect the possible presence of droplets growing more slowly than calibration $\left(\mathrm{NH}_{4}\right)_{2} \mathrm{SO}_{4}$ aerosol [50]. If present then, a coupled measurement modeling approach can be used to infer the effective water vapor uptake coefficient $[14,51]$. TDGA compares the $D_{p}$ of CCN with $s_{c}$ equal to the instrument saturation (i.e., $\mathrm{CCN}$ with a dry diameter equal to the cutoff diameter, $d$ ) against the wet diameter, $D_{p,(\mathrm{NH} 4) 2 \mathrm{SO} 4}$ of $\mathrm{CCN}$ with identical $s_{c}$, but composed of $\left(\mathrm{NH}_{4}\right)_{2} \mathrm{SO}_{4}$. If the presence of organics does not delay droplet growth, $D_{p}$ $\sim D_{p,(\mathrm{NH} 4) 2 S O 4}$ (e.g., $\left.[34,52]\right)$. Supersaturation depression is observed at high CCN concentration and can affect droplet size [53]. Thus both $\mathrm{CCN}$ measurement and analysis should account for CCN concentrations. 


\section{Analytical Theory}

\subsection{Köhler Theory}

Köhler Theory Analysis (KTA) [33] can be used to infer the average molar volume (molecular weight, $M_{j}$, over density $\rho_{j}$ ) of the organic fraction, $j$, of CCN. It has been shown to work well for low molecular weight species, such as those presented here. Using measurements of $s_{c \text { versus }} d$ to determine the Fitted CCN Activity parameter (FCA), $\omega=s_{c} d^{-1.5}$, KTA infers the average molar volume of the WSOC, $\frac{\mathrm{M}_{j}}{\rho_{j}}[33,43]$,

$$
\frac{M_{j}}{\rho_{j}}=\frac{\varepsilon_{j} v_{j}}{\frac{256}{27}\left(\frac{M_{w}}{\rho_{w}}\right)^{2}\left(\frac{1}{R T}\right)^{3} \sigma^{3} \omega^{-2}-\sum_{i \neq j} \frac{\rho_{i}}{M_{i}} \varepsilon_{i} v_{i}}
$$

where $M_{w}, \rho_{w}$ are the molecular weight and density of water, respectively, $R$ is the universal gas constant, $T$ is the ambient temperature, $\sigma$ is the droplet surface tension at the point of activation, $\varepsilon$ is the volume fraction, and $v$ is the effective van't Hoff factor. Subscripts $I$ and $j$ refer to the inorganic and organic components, respectively. $\varepsilon_{k}$ is related to the mass fraction, $m_{k}$, of solute $k$ ( $k$ being either of $i$ or $j$ ) as:

$$
\varepsilon_{k}=\frac{m_{k} / \rho_{k}}{\sum_{i \neq j} m_{i} / \rho_{i}+m_{j} / \rho_{j}}
$$

Two measures of molar volume uncertainty are used: (i) the standard deviation of all the $\frac{\mathrm{M}_{j}}{\rho_{j}}$ inferences (Equation (2)), and, (ii) estimates determined from, $\Delta \frac{M_{j}}{\rho_{j}}=$ $\sqrt{\sum_{x}\left(\Phi_{x} \Delta x\right)^{2}}$, where $\Delta x$ is the uncertainty of each of the measured parameters $x$, (i.e., any of $\sigma, \omega, \varepsilon_{i}, \varepsilon_{j}, v_{i}$, and $v_{j}$ ) and $\Phi_{x}$ is the sensitivity of molar volume to $x$, $\Phi_{x}=\frac{\partial}{\partial x}\left(\frac{M_{0}}{\rho_{0}}\right)$, using the formulas of $[33,34,43]$. The maximum of both estimates is the reported uncertainty of $\frac{M_{j}}{\rho_{j}}$. KTA has been shown to constrain molecular weight estimates for laboratory aerosol (having organic mass fraction between 20 and 50\%) with an average error of $20 \%$ [33], 40\% for complex biomass burning aerosol [43], $30 \%$ for secondary organic matter [35], and to within $25 \%$ for primary marine organic matter [34].

\subsection{Inferring Surface Tension}

The low concentration of WSOC in the PILS samples limits the determination (using direct measurements) of their surface tension depression for CCN-relevant concentrations. However, if CCN activity data are available for mixtures of WSOC and a salt (e.g., $\left.\left(\mathrm{NH}_{4}\right)_{2} \mathrm{SO}_{4}\right), \mathrm{KTA}$ can be used to concurrently infer $\frac{M_{j}}{\rho_{j}}$ and $\sigma$ using 
an iterative procedure [34]. If enough salt is present in the sample, the contribution of organic solute to $s_{C}$ is small, and an iterative procedure is not required; the effect of the organic on $\mathrm{CCN}$ activity amounts to its impact on surface tension, and can then be inferred as [35],

$$
\sigma=\sigma_{w}\left(\frac{s_{c}}{s_{c}^{*}}\right)^{\frac{2}{3}}
$$

where $s_{c}$ is the measured critical supersaturation, and $s_{c}{ }^{*}$ is the predicted value (from Köhler theory). Assuming $\sigma=\sigma_{w}$, the surface tension of pure water computed at the average CFSTGC column temperature [35], then

$$
s_{c}^{*}=\frac{2}{3}\left(\frac{4 M_{w} \sigma_{w}}{R T \rho_{w}}\right)^{\frac{3}{2}}\left(\frac{M_{w} d^{3}}{\rho_{w}} \sum_{i} \frac{\varepsilon_{i} v_{i} \rho_{i}}{M_{i}}\right)^{\frac{-1}{2}}
$$

where " $i$ " denotes all inorganic solutes present in the aerosol. Each surface tension inference is then related to the WSOC concentration at the critical diameter (Equation (6) of [34], and fit to the Szyskowski-Langmuir adsorption isotherm (Equation (1)). The partitioning of the surfactant from the bulk to the droplet monolayer should be considered. This method of inferring surface tension depression has been shown to work well for dissolved organic matter isolated from seawater [34]. Thus in the case of marine POMA, where the surfactant may partition mostly to the surface, partitioning effects are less important than bulk properties and why Moore et al. [34] were able to achieve good closure.

\section{Results and Discussion}

\subsection{Surface Tension}

For the low WSOC concentrations measured in the PILS samples, organics have minimal effect on surface tension (Figure 3). Hence, Equation (4) is used to infer $\sigma$ at carbon concentrations relevant for CCN activation (roughly $1000 \mathrm{mg} \cdot \mathrm{C} \cdot \mathrm{L}^{-1}$ ). WSOC from biomass burning aerosol and marine aerosol have been shown to contain strong surfactants that depress surface tension by $25 \%-42 \%[43,47,54,55]$ at similar concentration. The inferred $\sigma$ values for both SC and CR aerosol (Figure 3) exhibit weak surface tension depression $(-\Delta \sigma / \sigma \simeq 5 \%)$ at concentrations $>1000 \mathrm{mg} \cdot \mathrm{C} \cdot \mathrm{L}^{-1}$. The surface tension depression results are similar to results from dissolved organic marine matter [34]. Both CR and SC samples were influenced by ship emissions, SOMA, and POMA and contained soluble organics. The solubility of the organics is unknown, however the potential effects of limited solubility on CCN activity for SC samples may be outweighed by significant depression in surface tension at the droplet surface (Figure 3). Finding the average organic molar mass from KTA 
will help constrain if the SC and CR samples indeed have different organic aerosol compositions that affect aerosol solubility and surfactant properties.

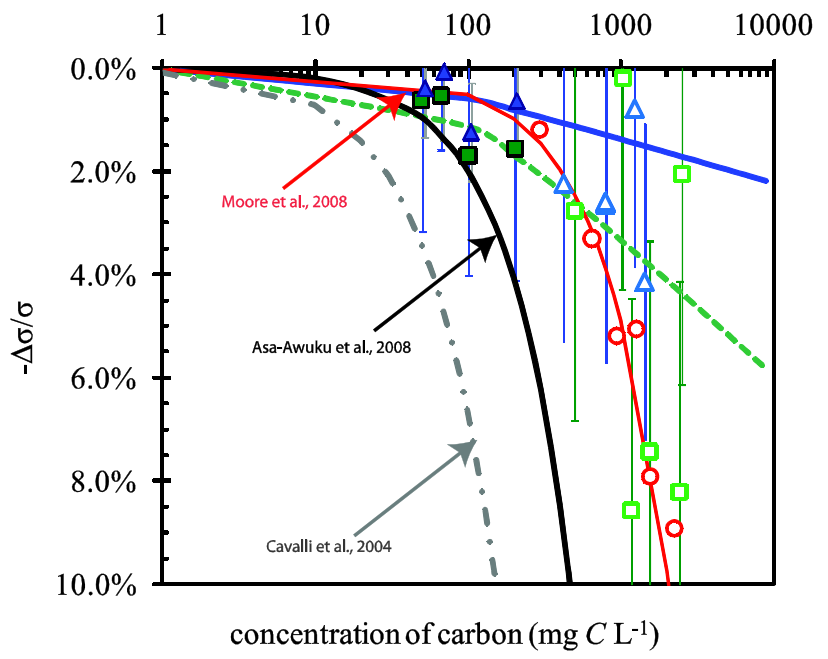

Figure 3. Surface tension depression as a function of dissolved carbon concentration. Measurements (closed symbols) and Inferred values (open symbols) of the CR (blue triangles) and SC aerosol (green squares) are shown as data points. For comparison, curves are added with data from freshly emitted biomass burning aerosol (solid black; [43], marine organic aerosol (grey dash-dot; [54]) and dissolved marine organic matter (solid red; [34]).

\subsection{CCN Activity}

Figures 4 and 5 show the critical supersaturation, $s_{c}$, as a function of dry diameter, $d$, for aerosol atomized from both $\mathrm{CR}$ and $\mathrm{SC}$ samples. The data for $\left(\mathrm{NH}_{4}\right)_{2} \mathrm{SO}_{4}$ aerosol have been added for comparison. Data for $\left(\mathrm{NH}_{4}\right)_{2} \mathrm{SO}_{4}$ are consistent with the expectation that the aerosol is highly CCN active, lies to the left of both CR and SC data sets, and behaves as classical Kohler CCN without surface tension depression. The activation slope of particles composed of soluble and insoluble material should exhibit a $s_{c}$ that scales with $d^{-1.5}$ (e.g., $\left(\mathrm{NH}_{4}\right)_{2} \mathrm{SO}_{4}$ data in Figure 4). At low supersaturations $\left(s_{c} \leq 0.6 \%\right)$, the WSOC concentration at the point of activation was low $\left(<1000 \mathrm{mg} \cdot \mathrm{C} \cdot \mathrm{L}^{-1}\right)$ and surface tension depression was small (Figure 3), hence $s_{c} \sim d^{-1.5}$ for both samples, and KTA is applied for this region of the $\mathrm{CCN}$ spectrum. The $\mathrm{CR}$ sample contained material that was less hygroscopic than sulfate, but more hygroscopic than in the SC sample (i.e., for a given s, the $d$ of the $\mathrm{CR}$ is greater than SC, Figure 4). SC and CR activation curves converge at high $s$, likely because the WSOC concentration is high enough to notably decrease surface depression, more for organics in SC than for CR (Figure 3). The difference in CCN 
activity is consistent with studies to date (e.g., [16,56]), showing that hygroscopic components tend to be incorporated in cloud droplets where their less hygroscopic counterparts prefer to remain in the interstitial air. It is also noted that the CR samples excluded droplets $<5 \mu \mathrm{m}$ diameter thus the excluded smaller droplets may potentially contain less hygroscopic materials.

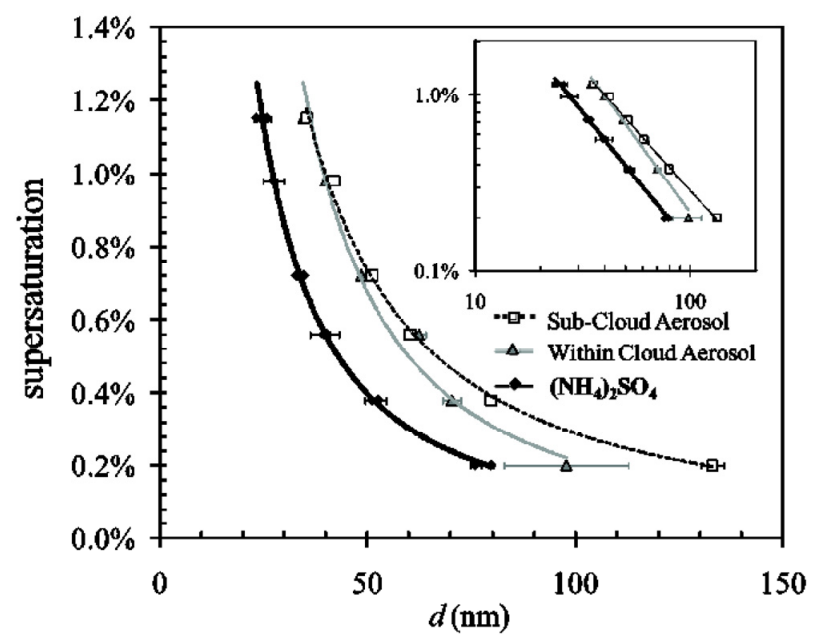

Figure 4. $\mathrm{CCN}$ activity of $\mathrm{SC}$ and $\mathrm{CR}$ samples. $\left(\mathrm{NH}_{4}\right)_{2} \mathrm{SO}_{4}$ (solid black line) is added for comparison. The $\mathrm{CCN}$ activity curve exponents for $\left(\mathrm{NH}_{4}\right)_{2} \mathrm{SO}_{4}, \mathrm{CR}$, and SC are $-1.51,-1.66$, and -1.35 , respectively.
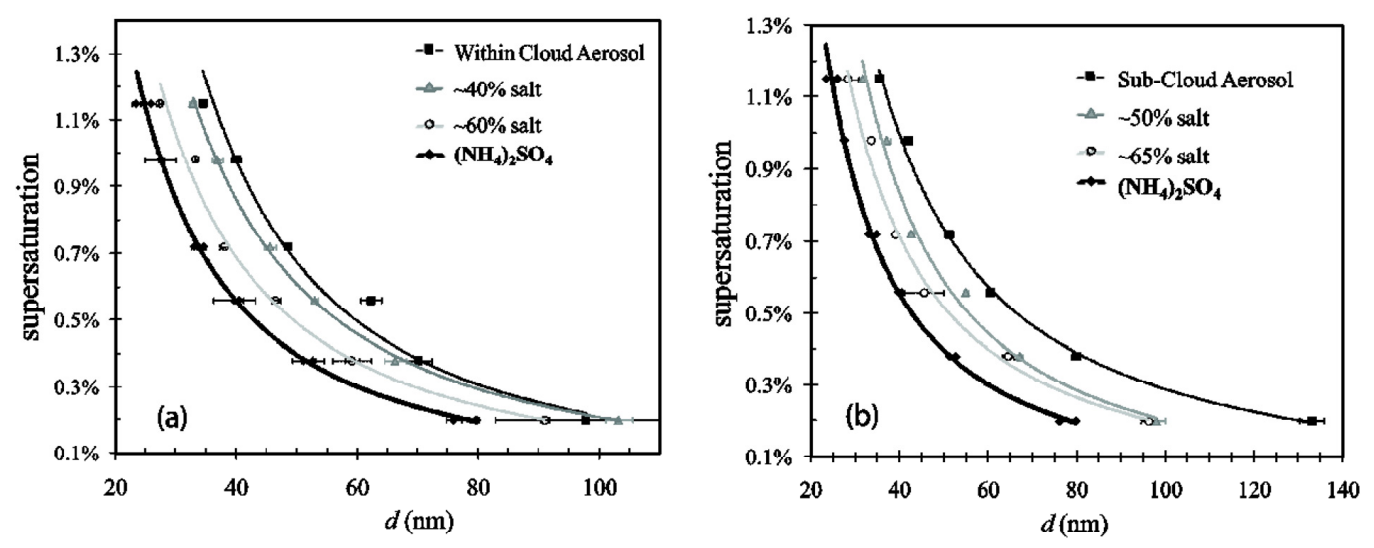

Figure 5. $\mathrm{CCN}$ activity of MASE samples with the addition of $\left(\mathrm{NH}_{4}\right)_{2} \mathrm{SO}_{4}$. Salt contents are expressed in terms of mass fraction. (a) Cloud Residuals and (b) Sub-Cloud samples. 


\subsection{Inferred Molar Volumes and Uncertainties}

Application of KTA requires the input of the organic mass concentration, $m_{\text {organic }}$, which is obtained by dividing the WSOC carbon concentration (Table 1) by the carbon-to-organic mass ratio, $\mathrm{C} / \mathrm{OC}$. In this study, $\mathrm{C} / \mathrm{OC} \approx 0.27$ is applied, the value for oxalic acid $\left(\mathrm{C}_{2} \mathrm{H}_{6} \mathrm{O}_{4}, 90 \mathrm{~g} \cdot \mathrm{moL}^{-1}\right)$, the most abundant organic acid measured in our samples (Table 1 ). $C / O C \approx 0.27$ is very close to 0.29 , the value for dissolved primary organic marine matter and marine coarse mode aerosol [34,54]. Here we expect it to apply to the whole sample. The van't Hoff factor for the organic fraction is assumed to be unity. The presence of organics from ship emissions are assumed to introduce little variability to the organic mass fractions estimated. The ionic composition taken from the chemical analysis of the PILS vials is then converted into a mixture of inorganic salts and organics with the ISORROPIA II aerosol thermodynamic equilibrium model [57]. Lu et al. [36] find the composition of aerosol sampled to be bimodal, composed of ammonium bisulfate and sea-salt; when samples are mixed in the PILS, chloride depletion is both expected and observed in the samples (Table 2). The results of the KTA analysis for each sample are summarized in Tables 3 and 4 . Assuming an organic density of $1.4 \mathrm{~g} \cdot \mathrm{cm}^{-3}[35,58]$, the SC sample has a high inferred molecular weight $\left(\mathrm{M}_{\mathrm{j}}=2413 \pm 536 \mathrm{~g} \cdot \mathrm{mol}^{-1}\right.$; Table 4$)$, possibly long-chained aliphatic compounds within primary organic matter transferred to the aerosol from bubble bursting at the seawater surface $[16,34,54,59,60]$. The marine nature of the SC sample is further supported by its inferred surface tension depression (Figure 3, which is remarkably consistent with dissolved organic matter [34]. The average molecular weight inferred for CR aerosol is substantially less (143 $\pm 25 \mathrm{~g} \cdot \mathrm{mol}^{-1}$; Table 3), consistent with the presence of low molecular weight carboxylic acids (i.e., $\mathrm{C}_{2}-\mathrm{C}_{9}$ mono and dicarboxylic acids) measured in cloud-processed marine aerosol [15]. Water-soluble oxidation products from ship VOC emissions can also contribute to the WSOC, although the information at hand is not sufficient to conclusively show this. As in previous KTA studies (e.g., [33]), inferred average molar volume is subject to an estimated $30 \%$ error; the greatest source of uncertainty stems from the FCA parameter (Tables 3 and 4). The low depression in surface tension (Figure 3) suggests that the molecular weight distribution in the WSOC may not contain compounds characteristic of HULIS $\left(300-750 \mathrm{~g} \cdot \mathrm{mol}^{-1}\right)[43,47]$, but instead can be described by the superposition of two "modes", one from SOMA (low molecular weight compounds), and one from POMA (higher molecular weight compounds). For the SC sample, we assume that the SOMA is primarily composed of oxalic acid $\left(90 \mathrm{~g} \cdot \mathrm{mol}^{-1}\right.$, the predominant compound identified in the PILS samples and constitutes $0.6 \%$ of the organic mass, Table 1), and the remaining mass $(99.4 \%)$ is POMA characteristic of Redfield-type molecules, $\left(\mathrm{CHO}_{2}\right)_{106}\left(\mathrm{NH}_{3}\right)_{16} \mathrm{H}_{3} \mathrm{PO}_{4}, 3444 \mathrm{~g} \cdot \mathrm{mol}^{-1}$ [61,62]. With this assumed two-component model composition, the average $\mathrm{M}_{j}$ in $\mathrm{SC}$ is equal to $2852 \mathrm{~g} \cdot \mathrm{mol}^{-1}$, and consistent (to within uncertainty) with the inferred KTA value 
(Table 4). Similarly, if most of the organic in the CR aerosol is a mixture of ship emissions and oxalate, and that the POMA mode is consistent with phenathrene (178 $\mathrm{g} \cdot \mathrm{mol}^{-1}$ and $99.6 \%$ of the organic mass), and SOMA with oxalic acid $\left(90 \mathrm{~g} \cdot \mathrm{mol}^{-1}\right.$ and $0.4 \%$ of the organic mass, Table 1 ), the average molar mass for the organic distribution yields $177 \mathrm{~g} \cdot \mathrm{mol}^{-1}$, a value within $25 \%$ of our KTA estimate.

Table 2. Mass fraction (\%) of constituents in the aerosol samples considered in this study. Composition of the inorganic fraction was obtained by an aerosol thermodynamic equilibrium model (ISORROPIA-II), using the ionic composition of Table 1.

\begin{tabular}{ccc}
\hline & Cloud Residuals (CR) & Sub-Cloud (SC) \\
\hline Organic & 88.3 & 82 \\
$\mathrm{NH}_{4}$ & 3.4 & 3.6 \\
$\mathrm{NaCl}$ & 0.6 & 0 \\
$\left(\mathrm{NH}_{4}\right)_{2} \mathrm{SO}_{4}$ & 0.0 & 5.1 \\
$\mathrm{NaNO}_{3}$ & 2.3 & 0.0 \\
$\mathrm{NH}_{4} \mathrm{NO}_{3}$ & 0.0 & 2.4 \\
$\mathrm{Na}_{2} \mathrm{SO}_{4}$ & 4.1 & 7.0 \\
$\mathrm{CaSO}_{4}$ & 1.1 & 0.0 \\
\hline
\end{tabular}

Table 3. Köhler Theory Analysis for Cloud-Residual (CR) samples.

\begin{tabular}{|c|c|c|c|c|}
\hline Property $x$ (units) & Average Value of $x$ & Uncertainty $\Delta x$ & $\begin{array}{l}\text { Sensitivity, } \Phi_{x} \\
\left(\mathbf{m}^{3} \cdot \mathbf{m o l}^{-1} \cdot \mathbf{x}^{-1}\right)\end{array}$ & $\begin{array}{c}\frac{\mathbf{M}_{j}}{\rho_{j}} \text { Contribution } \\
(\%)\end{array}$ \\
\hline$\sigma\left(\mathrm{N} \cdot \mathrm{m}^{-1}\right)$ & $6.89 \times 10^{-2}$ & $1.38 \times 10^{-3}$ & $3.83 \times 10^{-3}$ & 5.72 \\
\hline$\omega\left(\mathrm{m}^{1.5}\right)$ & $6.80 \times 10^{-2}$ & $5.53 \times 10^{-15}$ & $2.59 \times 10^{9}$ & 12.42 \\
\hline$v_{\mathrm{NH}_{4} \mathrm{Cl}}$ & 2 & 0.5 & $6.43 \times 10^{-6}$ & 3.49 \\
\hline$v_{\mathrm{NaCl}}$ & 2 & 0.5 & $1.54 \times 10^{-6}$ & 0.83 \\
\hline$v_{\mathrm{NaNO}_{3}}$ & 2 & 0.5 & $4.18 \times 10^{-6}$ & 2.27 \\
\hline$v_{\mathrm{Na}_{2} \mathrm{SO}_{4}}$ & 3 & - & - & - \\
\hline$v_{\text {organic }}$ & 1 & 0.20 & $4.39 \times 10^{-5}$ & 9.52 \\
\hline$\varepsilon_{\mathrm{NaNO}_{3}}$ & 0.05 & - & $\begin{array}{c}- \\
-10\end{array}$ & - \\
\hline$\varepsilon_{\mathrm{Na}_{2} \mathrm{SO}_{4}}$ & 0.07 & - & - & - \\
\hline$\varepsilon_{\mathrm{NH}_{4} \mathrm{Cl}}$ & 0.10 & $1.95 \times 10^{-3}$ & $8.50 \times 10^{-4}$ & 0.80 \\
\hline$\varepsilon_{\text {organic }}$ & 0.88 & $6.36 \times 10^{-3}$ & $4.10 \times 10^{-3}$ & 2.82 \\
\hline$\rho_{\mathrm{NH}_{4} \mathrm{Cl}}$ & 1.53 & - & - & - \\
\hline$\rho_{\mathrm{NaCl}}$ & 2.16 & - & - & - \\
\hline$\rho_{\mathrm{NaNO}_{3}}$ & 2.3 & - & - & - \\
\hline$\rho_{\mathrm{Na}_{2} \mathrm{SO}_{4}}$ & 2.68 & - & - & - \\
\hline$\rho_{\text {organic }}$ & 1.4 & - & - & - \\
\hline$\rho_{\text {aerosol }}$ & 1.59 & - & - & - \\
\hline $\begin{array}{c}M_{J} / \rho_{J} \\
\left(\mathrm{~cm}^{3} \cdot \mathrm{mol}^{-1}\right)\end{array}$ & $1.60 \times 10^{-4}$ & $17.5 \%$ & - & - \\
\hline$M_{I}\left(\mathrm{~g} \cdot \mathrm{mol}^{-1}\right)$ & 143 & 25 & - & - \\
\hline
\end{tabular}


Table 4. Köhler Theory Analysis for Sub-Cloud (SC) samples.

\begin{tabular}{|c|c|c|c|c|}
\hline Property $x$ (units) & Average Value of $x$ & Uncertainty $\Delta x$ & $\begin{array}{l}\text { Sensitivity, } \Phi_{x} \\
\left(\mathrm{~m}^{3} \cdot \mathrm{mol}^{-1} \cdot \mathrm{x}^{-1}\right)\end{array}$ & $\begin{array}{c}\frac{M_{j}}{\rho_{j}} \text { Contribution } \\
(\mathbf{\%})\end{array}$ \\
\hline$\sigma\left(\mathrm{N} \cdot \mathrm{m}^{-1}\right)$ & $6.58 \times 10^{-2}$ & $1.32 \times 10^{-3}$ & $1.97 \times 10^{-3}$ & 8.37 \\
\hline$\omega\left(\mathrm{m}^{1.5}\right)$ & $8.75 \times 10^{-14}$ & $4.43 \times 10^{-15}$ & $9.89 \times 10^{9}$ & 14.13 \\
\hline$v_{\mathrm{NH}_{4} \mathrm{Cl}}$ & 2 & 0.5 & $6.35 \times 10^{-5}$ & 10.24 \\
\hline$v_{\left(\mathrm{NH}_{4}\right)_{2} \mathrm{SO}_{4}}$ & 2.5 & 0.5 & $3.74 \times 10^{-6}$ & 0.60 \\
\hline$v_{\mathrm{NH}_{4} \mathrm{NO}_{3}}$ & 2 & 0.5 & $2.82 \times 10^{-6}$ & 0.45 \\
\hline$v_{\mathrm{Na}_{2} \mathrm{SO}_{4}}$ & 3 & - & - & - \\
\hline $\begin{array}{l}\mathrm{Na}_{2} \mathrm{SU}_{4} \\
v_{\text {organic }}\end{array}$ & 1 & 0.20 & $7.21 \times 10^{-5}$ & 4.65 \\
\hline$\varepsilon_{N_{4} N_{3}}$ & 0.06 & - & - & - \\
\hline$\varepsilon_{\mathrm{Na}_{2} \mathrm{SO}_{4}}$ & 0.10 & - & - & - \\
\hline$\varepsilon_{\mathrm{NH}_{4} \mathrm{Cl}}$ & 0.09 & $1.95 \times 10^{-3}$ & $4.21 \times 10^{-3}$ & 2.64 \\
\hline 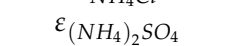 & 0.12 & $2.03 \times 10^{-3}$ & $4.10 \times 10^{-3}$ & 2.69 \\
\hline$\varepsilon_{\text {organic }}$ & 0.82 & $6.36 \times 10^{-3}$ & $4.63 \times 10^{-3}$ & 9.50 \\
\hline$\rho_{\mathrm{NH}_{4} \mathrm{Cl}}^{\circ}$ & 1.53 & - & - & - \\
\hline$\rho_{\left(\mathrm{NH}_{4}\right)_{2} \mathrm{SO}_{4}}$ & 1.77 & - & - & - \\
\hline$\rho_{N_{4} N_{3} O_{3}}$ & 1.73 & - & - & - \\
\hline$\rho_{\mathrm{Na}_{2} \mathrm{SO}_{4}}$ & 2.68 & - & - & - \\
\hline $\begin{array}{c}M_{J} / \rho_{J} \\
\left(\mathrm{~cm}^{3} \cdot \mathrm{mol}^{-1}\right)\end{array}$ & $1.72 \times 10^{-3}$ & $22.2 \%$ & - & - \\
\hline$M_{J}\left(\mathrm{~g} \cdot \mathrm{mol}^{-1}\right)$ & 2413 & 536 & - & - \\
\hline
\end{tabular}

\subsection{The Effect of Organics on Droplet Growth Kinetics}

Figure 6 illustrates the OPC droplet size measurements for all supersaturations and samples considered. The flow rate within the instrument was maintained at $0.5 \mathrm{~L} \cdot \mathrm{min}^{-1}$ and the sheath to aerosol flow rate ratio was 10:1 to so that particles have the same residence time in the CFSTGC. Similar to the WSOC droplet data presented in $[34,35,52,63]$, almost all of the growth droplet data lie within the measurement uncertainty and are in agreement with $\left(\mathrm{NH}_{4}\right)_{2} \mathrm{SO}_{4}$ calibration aerosol. According to TDGA, the water uptake coefficient is similar to that of the water uptake coefficient of $\left(\mathrm{NH}_{4}\right)_{2} \mathrm{SO}_{4}\left(\alpha_{\mathrm{c}} \sim(\mathrm{NH} 4) 2 \mathrm{SO} 4\right)$. Given that the water uptake coefficient, (NH4)2SO4 $\sim 0.2[50,51]$, this suggest that the WSOC would not slow down activation kinetics of $\mathrm{CCN}$ compared to $\left(\mathrm{NH}_{4}\right)_{2} \mathrm{SO}_{4}$. This is consistent with the analysis of CCN data collected from a wide range of environments [51]. The WSOC does not appreciably impact droplet growth kinetics (or the effective water vapor mass transfer coefficient) as aerosol particles produced from the SC and CR grow like $\left(\mathrm{NH}_{4}\right)_{2} \mathrm{SO}_{4}$. 


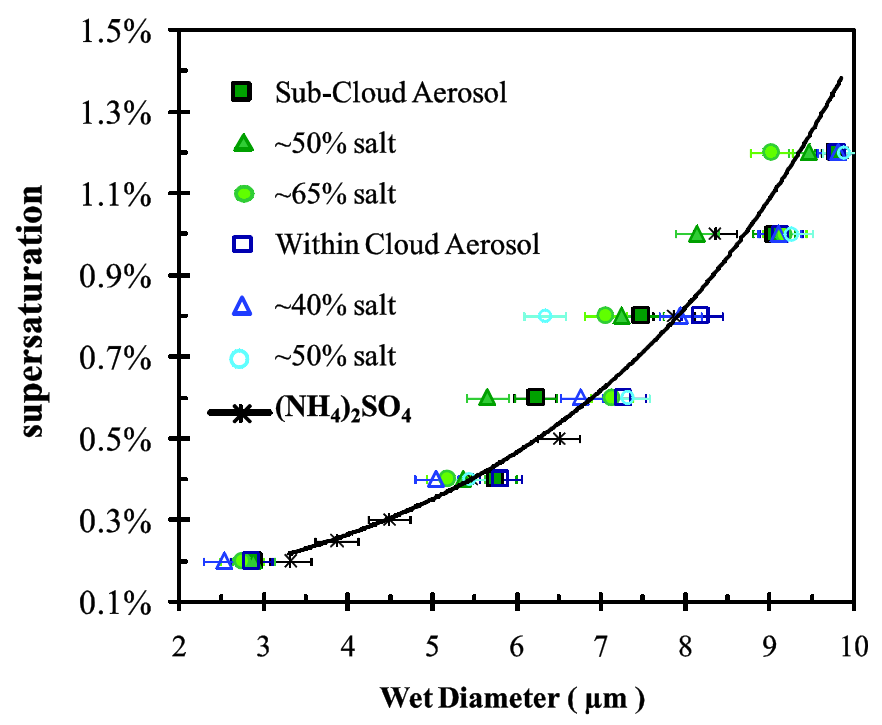

Figure 6. Size of activated CCN generated from the cloud residual samples (CR, solid symbols), sub-cloud samples ( $\mathrm{SC}$, open symbols) and $\left(\mathrm{NH}_{4}\right)_{2} \mathrm{SO}_{4}$ (solid black line). Droplet sizes are presented for CCN with $s_{c}$ equal to the instrument supersaturation.

\section{Summary and Implications}

$\mathrm{CCN}$ activity, chemical composition and droplet growth measurements coupled with Köhler Theory Analysis are used to characterize the cloud droplet formation potential of water-soluble organic matter collected from cloud droplet (CR) residuals and sub-cloud (SC) aerosol during the MASE 2005 campaign over the eastern Pacific Ocean off the coast of California. The organics within CR samples were found to be more hygroscopic than in the SC sample, most likely from the enhanced levels of soluble organic acids (e.g., oxalic) formed during cloud processing. Both the direct and inferred surface tension measurements show neither sample contained strong surfactants; in fact, surface tension depression is consistent with the effect from primary marine organic matter.

Inferred average molecular weights of both CR and SC samples are consistent with a bimodal molecular weight distribution; one composed of oxalic acid (produced through glyoxylic oxidation or the oxidative decay of larger diacids in cloud droplets [15]) with primary organic matter (SC sample), or, organics from ship emissions (CR sample). These results are consistent with recently published work $[18,64]$ that shows SOMA as a significant contributor to CCN activity in marine regions. Finally, all samples display similar droplet growth kinetics to CCN composed of $\left(\mathrm{NH}_{4}\right)_{2} \mathrm{SO}_{4}$, suggesting that water-soluble organics do not significantly 
impact the growth rate of CCN. Hence, CCN activity of the organics affects CCN activity through their contribution of solute from SOMA and possibly a slight surface tension depression from POMA. In both cases, increasing the size of CCN will also have a major impact, which is not shown here.

Acknowledgments: The work in this study was supported by an NSF CAREER Award. In addition, we would like to thank Dr. Rodney Weber and his students at the Georgia Institute of Technology for the use of the Total Organic Carbon (TOC) Turbo Siever Analyzer and Dr. Christos Fountoukis for his help in running the ISORROPIA II code.

Author Contributions: Akua Asa-Awuku is co-corresponding author and conducted laboratory experiments, collected and analyzed data sets, and played a significant role in the writing of the manuscript. Armin Sorooshian collected samples in the field, aided in analysis and writing. Richard C. Flagan and John H. Seinfeld devised flight experiments. Athanasios Nenes is co-corresponding author and conceived and devised experiments, analysis, and significantly contributed to writing.

Conflicts of Interest: The authors declare no conflict of interest.

\section{References}

1. Saxena, P.; Hildemann, L.M.; McMurry, P.H.; Seinfeld, J.H. Organics alter hygroscopic behavior of atmospheric particles. J. Geophys. Res. 1995, 100, 18755-18770.

2. Facchini, M.C.; Fuzzi, S.; Zappoli, S.; Andracchio, A.; Gelencsér, A.; Kiss, G.; Krivácsy, Z.; Mészáros, E.; Hansson, H.-C.; Alsberg, T.; et al. Partitioning of the organic aerosol component between fog droplets and interstitial air. J. Geophys. Res. 1999, 104, 26821-26832.

3. Nenes, A.; Charlson, R.J.; Facchini, M.C.; Kulmala, M.; Laaksonen, A.; Seinfeld, J.H. Can chemical effects on cloud droplet number rival the first indirect effect? Geophys. Res. Lett. 2002, 29, 29-21.

4. Decesari, S.; Facchini, M.C.; Mircea, M.; Cavalli, F.; Fuzzi, S. Solubility properties of surfactants in atmospheric aerosol and cloud/fog water samples. J. Geophys. Res. 2003, 108, 1984-2012.

5. Ervens, B.; Feingold, G.; Clegg, S.L.; Kreidenweis, S.M. A modeling study of aqueous production of dicarboxylic acids: 2. Implications for cloud microphysics. J. Geophys. Res. 2004, 109, D15206.

6. Saxena, P.; Hildemann, L.M. Water-soluble organics in atmospheric particles: A critical review of the literature and application of thermodynamics to identify candidate compounds. J. Atmos. Chem. 1996, 24, 57-109.

7. Mircea, M.; Facchini, M.C.; Decesari, S.; Fuzzi, S.; Charlson, R.J. The influence of the organic aerosol component on CCN supersaturation spectra for different aerosol types. Tellus. B Chem. Phys. Meteorol. 2002, 54, 74-81.

8. Alfonso, L.; Raga, G.B. The influence of organic compounds on the development of precipitation acidity in maritime clouds. Atmos. Chem. Phys. 2004, 4, 1097-1111. 
9. O’Dowd, C.D.; Facchini, M.C.; Cavalli, F.; Ceburnis, D.; Mircea, M.; Decesari, S.; Fuzzi, S.; Yoon, Y.J.; Putaud, J.-P. Biogenically driven organic contribution to marine aerosol. Nature 2004, 431, 676-680.

10. Zhao, C.; Ishizaka, Y.; Peng, D. Numerical Study on Impacts of Multi-Component Aerosols on Marine Cloud Microphysical Properties. J. Meteorol. Soc. Jpn. Ser. II 2005, 83, 977-986.

11. Kanakidou, M.; Seinfeld, J.H.; Pandis, S.N.; Barnes, I.; Dentener, F.J.; Facchini, M.C.; Van Dingenen, R.; Ervens, B.; Nenes, A.; Nielsen, C.J.; et al. Organic aerosol and global climate modelling: A review. Atmos. Chem. Phys. 2005, 5, 1053-1123.

12. Yoon, Y.J.; Ceburnis, D.; Cavalli, F.; Jourdan, O.; Putaud, J.P.; Facchini, M.C.; Decesari, S.; Fuzzi, S.; Sellegri, K.; Jennings, S.G.; et al. Seasonal characteristics of the physicochemical properties of North Atlantic marine atmospheric aerosols. J. Geophys. Res. 2007, 112, D04206.

13. Ovadnevaite, J.; Ceburnis, D.; Martucci, G.; Bialek, J.; Monahan, C.; Rinaldi, M.; Facchini, M.C.; Berresheim, H.; Worsnop, D.R.; O’Dowd, C. Primary marine organic aerosol: A dichotomy of low hygroscopicity and high CCN activity. Geophys. Res. Lett. 2011, 38, L21806.

14. Moore, R.H.; Raatikainen, T.; Langridge, J.M.; Bahreini, R.; Brock, C.A.; Holloway, J.S.; Lack, D.A.; Middlebrook, A.M.; Perring, A.E.; Schwarz, J.P.; et al. CCN spectra, hygroscopicity, and droplet activation kinetics of secondary organic aerosol resulting from the 2010 Deepwater Horizon oil spill. Environ. Sci. Technol. 2012, 46, 3093-3100.

15. Sorooshian, A.; Lu, M.-L.; Brechtel, F.J.; Jonsson, H.; Feingold, G.; Flagan, R.C.; Seinfeld, J.H. On the source of organic acid aerosol layers above clouds. Environ. Sci. Technol. 2007, 41, 4647-4654.

16. Ceburnis, D.; O’Dowd, C.D.; Jennings, G.S.; Facchini, M.C.; Emblico, L.; Decesari, S.; Fuzzi, S.; Sakalys, J. Marine aerosol chemistry gradients: Elucidating primary and secondary processes and fluxes. Geophys. Res. Lett. 2008, 35, L07804.

17. Rinaldi, M.; Decesari, S.; Finessi, E.; Giulianelli, L.; Carbone, C.; Fuzzi, S.; O’Dowd, C.D.; Ceburnis, D.; Facchini, M.C. Primary and secondary organic marine aerosol and oceanic biological activity: Recent results and new perspectives for future studies. Adv. Meteorol. 2010, 2010, 1-10.

18. Coggon, M.M.; Sorooshian, A.; Wang, Z.; Metcalf, A.R.; Frossard, A.A.; Lin, J.J.; Craven, J.S.; Nenes, A.; Jonsson, H.H.; Russell, L.M.; et al. Ship impacts on the marine atmosphere: Insights into the contribution of shipping emissions to the properties of marine aerosol and clouds. Atmos. Chem. Phys. 2012, 12, 8439-8458.

19. Sorooshian, A.; Wang, Z.; Coggon, M.M.; Jonsson, H.H.; Ervens, B. Observations of sharp oxalate reductions in stratocumulus clouds at variable altitudes: Organic acid and metal measurements during the 2011 E-PEACE campaign. Environ. Sci. Technol. 2013, 47, 7747-7756. 
20. Sorooshian, A.; Varutbangkul, V.; Brechtel, F.J.; Ervens, B.; Feingold, G.; Bahreini, R.; Murphy, S.M.; Holloway, J.S.; Atlas, E.L.; Buzorius, G.; et al. Oxalic acid in clear and cloudy atmospheres: Analysis of data from International Consortium for Atmospheric Research on Transport and Transformation 2004. J. Geophys. Res. D: Atmos. 2006, 111, D10S27.

21. Ervens, B.; Feingold, G.; Frost, G.J.; Kreidenweis, S.M. A modeling study of aqueous production of dicarboxylic acids: 1 . Chemical pathways and speciated organic mass production. J. Geophys. Res. 2004, 109, D15205.

22. Lim, H.; Carlton, A.G.; Turpin, B.J. Isoprene forms secondary organic aerosol in Atlanta: Results from time-resolved measurements during the Atlanta supersite experiment. Environ. Sci. Technol. 2005, 39, 4441-4446.

23. Carlton, A.G.; Turpin, B.J.; Lim, H.-J.; Altieri, K.E.; Seitzinger, S. Link between isoprene and secondary organic aerosol (SOA): Pyruvic acid oxidation yields low volatility organic acids in clouds. Geophys. Res. Lett. 2006, 33, L06822.

24. Coggon, M.M.; Sorooshian, A.; Wang, Z.; Craven, J.S.; Metcalf, A.R.; Lin, J.J.; Nenes, A.; Jonsson, H.H.; Flagan, R.C.; Seinfeld, J.H. Observations of continental biogenic impacts on marine aerosol and clouds off the coast of California. J. Geophys. Res. D: Atmos. 2014, 119, 6724-6748.

25. Eyring, V.; Köhler, H.W.; van Aardenne, J.; Lauer, A. Emissions from international shipping: 1. The last 50 years. J. Geophys. Res. 2005, 110, D17305.

26. Zheng, Z.; Tang, X.; Asa-Awuku, A.; Jung, H.S. Characterization of a method for aerosol generation from heavy fuel oil (HFO) as an alternative to emissions from ship diesel engines. J. Aerosol Sci. 2010, 41, 1143-1151.

27. Russell, L.M.; Noone, K.J.; Ferek, R.J.; Pockalny, R.A.; Flagan, R.C.; Seinfeld, J.H. Combustion Organic Aerosol as Cloud Condensation Nuclei in Ship Tracks. J. Atmos. Sci. 2000, 57, 2591-2606.

28. Twomey, S. Pollution and the planetary albedo. Atmos. Environ. 1974, 8, 1251-1256.

29. Coakley, J.A.; Bernstein, R.L.; Durkee, P.A. Effect of ship-stack effluents on cloud reflectivity. Science 1987, 237, 1020-1022.

30. Albrecht, B.A. Aerosols, cloud microphysics, and fractional cloudiness. Science 1989, 245, 1227-1230.

31. Ackerman, A.S.; Toon, O.B.; Taylor, J.P.; Johnson, D.W.; Hobbs, P.V.; Ferek, R.J. Effects of aerosols on cloud albedo: Evaluation of Twomey's parameterization of cloud susceptibility using measurements of ship tracks. J. Atmos. Sci. 2000, 57, 2684-2695.

32. Moore, R.H.; Cerully, K.; Bahreini, R.; Brock, C.A.; Middlebrook, A.M.; Nenes, A. Hygroscopicity and composition of California CCN during summer 2010. J. Geophys. Res. 2012, 117, D00V12.

33. Padró, L.T.; Asa-Awuku, A.; Morrison, R.; Nenes, A. Inferring thermodynamic properties from $\mathrm{CCN}$ activation experiments: Single-component and binary aerosols. Atmos. Chem. Phys. 2007, 7, 5263-5274. 
34. Moore, R.H.; Ingall, E.D.; Sorooshian, A.; Nenes, A. Molar mass, surface tension, and droplet growth kinetics of marine organics from measurements of $\mathrm{CCN}$ activity. Geophys. Res. Lett. 2008, 35, L07801.

35. Asa-Awuku, A.; Nenes, A.; Gao, S.; Flagan, R.C.; Seinfeld, J.H. Water-soluble SOA from Alkene ozonolysis: Composition and droplet activation kinetics inferences from analysis of CCN activity. Atmos. Chem. Phys. 2010, 10, 1585-1597.

36. Lu, M.-L.; Conant, W.C.; Jonsson, H.H.; Varutbangkul, V.; Flagan, R.C.; Seinfeld, J.H. The Marine Stratus/Stratocumulus Experiment (MASE): Aerosol-cloud relationships in marine stratocumulus. J. Geophys. Res. D: Atmos. 2007, 112, D10209.

37. Marple, V.A.; Chien, C.M. Virtual impactors: A theoretical study. Environ. Sci. Technol. 1980, 14, 976-985.

38. Noone, K.J.; Ogren, J.A.; Heintzenberg, J.; Charlson, R.J.; Covert, D.S. Design and calibration of a counterflow virtual impactor for sampling of atmospheric fog and cloud droplets. Aerosol Sci. Technol. 1988, 8, 235-244.

39. Roberts, G.C.; Nenes, A. A continuous-flow streamwise thermal-gradient CCN chamber for atmospheric measurements. Aerosol Sci. Technol. 2005, 39, 206-221.

40. Lance, S.; Nenes, A.; Medina, J.; Smith, J.N. Mapping the operation of the DMT continuous flow CCN counter. Aerosol Sci. Technol. 2006, 40, 242-254.

41. Rose, D.; Gunthe, S.S.; Mikhailov, E.; Frank, G.P.; Dusek, U.; Andreae, M.O.; Pöschl, U. Calibration and measurement uncertainties of a continuous-flow cloud condensation nuclei counter (DMT-CCNC): CCN activation of ammonium sulfate and sodium chloride aerosol particles in theory and experiment. Atmos. Chem. Phys. 2008, 8, 1153-1179.

42. Moore, R.H.; Nenes, A.; Medina, J. Scanning mobility CCN analysis-A method for fast measurements of size-resolved CCN distributions and activation kinetics. Aerosol Sci. Technol. 2010, 44, 861-871.

43. Asa-Awuku, A.; Sullivan, A.P.; Hennigan, C.J.; Weber, R.J.; Nenes, A. Investigation of molar volume and surfactant characteristics of water-soluble organic compounds in biomass burning aerosol. Atmos. Chem. Phys. 2008, 8, 799-812.

44. Brechtel, F.J.; Kreidenweis, S.M. Predicting particle critical supersaturation from hygroscopic growth measurements in the humidified TDMA. Part I: Theory and sensitivity studies. J. Atmos. Sci. 2000, 57, 1854-1871.

45. Langmuir, I. The constitution and fundamental properties of solids and liquids. II. liquids.1. J. Am. Chem. Soc. 1917, 39, 1848-1906.

46. Taraniuk, I.; Graber, E.R.; Kostinski, A.; Rudich, Y. Surfactant properties of atmospheric and model humic-like substances (HULIS). Geophys. Res. Lett. 2007, 34, L16807.

47. Giordano, M.R.; Short, D.Z.; Hosseini, S.; Lichtenberg, W.; Asa-Awuku, A.A. Changes in droplet surface tension affect the observed hygroscopicity of photochemically aged biomass burning aerosol. Environ. Sci. Technol. 2013, 47, 10980-10986. 
48. Lance, S.; Nenes, A.; Mazzoleni, C.; Dubey, M.K.; Gates, H.; Varutbangkul, V.; Rissman, T.A.; Murphy, S.M.; Sorooshian, A.; Flagan, R.C.; et al. Cloud condensation nuclei activity, closure, and droplet growth kinetics of Houston aerosol during the Gulf of Mexico Atmospheric Composition and Climate Study (GoMACCS). J. Geophys. Res. D: Atmos. 2009, 114, D00F15.

49. Asa-Awuku, A.; Moore, R.H.; Nenes, A.; Bahreini, R.; Holloway, J.S.; Brock, C.A.; Middlebrook, A.M.; Ryerson, T.B.; Jimenez, J.L.; DeCarlo, P.F.; et al. Airborne cloud condensation nuclei measurements during the 2006 Texas Air Quality Study. J. Geophys. Res. D: Atmos. 2011, 116, D11201.

50. Raatikainen, T.; Moore, R.H.; Lathem, T.L.; Nenes, A. A coupled observation-modeling approach for studying activation kinetics from measurements of CCN activity. Atmos. Chem. Phys. 2012, 12, 4227-4243.

51. Raatikainen, T.; Nenes, A.; Seinfeld, J.H.; Morales, R.; Moore, R.H.; Lathem, T.L.; Lance, S.; Padró, L.T.; Lin, J.J.; Cerully, K.M.; et al. Worldwide data sets constrain the water vapor uptake coefficient in cloud formation. Proc. Natl. Acad. Sci. USA 2013, 110, 3760-3764.

52. Engelhart, G.J.; Asa-Awuku, A.; Nenes, A.; Pandis, S.N. CCN activity and droplet growth kinetics of fresh and aged monoterpene secondary organic aerosol. Atmos. Chem. Phys. 2008, 8, 3937-3949.

53. Lathem, T.L.; Nenes, A. Water vapor depletion in the DMT continuous-flow CCN chamber: Effects on supersaturation and droplet growth. Aerosol Sci. Technol. 2011, 45, 604-615.

54. Cavalli, F.; Facchini, M.C.; Decesari, S.; Mircea, M.; Emblico, L.; Fuzzi, S.; Ceburnis, D.; Yoon, Y.J.; O'Dowd, C.D.; Putaud, J.-P.; et al. Advances in characterization of size-resolved organic matter in marine aerosol over the North Atlantic. J. Geophys. Res. D: Atmos. 2004, 109, D24215.

55. Kiss, G.; Tombácz, E.; Hansson, H.-C. Surface tension effects of humic-like substances in the aqueous extract of tropospheric fine aerosol. J. Atmos. Chem. 2005, 50, 279-294.

56. Hallberg, A.; Ogren, J.A.; Noone, K.J.; Okada, K.; Heintzenberg, J.; Svenningsson, I.B. The Influence of Aerosol Particle Composition on Cloud Droplet Formation. In The Kleiner Feldberg Cloud Experiment 1990; Springer Netherlands: Houten, the Netherlands, 1994; pp. 153-171.

57. Fountoukis, C.; Nenes, A. ISORROPIA II: A computationally efficient thermodynamic equilibrium model for $\mathrm{K}^{+}-\mathrm{Ca}^{2+}-\mathrm{Mg}^{2+}-\mathrm{NH}^{4+}-\mathrm{Na}^{+}-\mathrm{SO}_{4}{ }^{2-}-\mathrm{NO}_{3}{ }^{-}-\mathrm{Cl}^{-}-\mathrm{H}_{2} \mathrm{O}$ aerosols. Atmos. Chem. Phys. 2007, 7, 4639-4659.

58. Turpin, B.J.; Lim, H.-J. Species contributions to $\mathrm{PM}_{2.5}$ mass concentrations: Revisiting common assumptions for estimating organic MASS. Aerosol Sci. Technol. 2001, 35, 602-610.

59. Oppo, C.; Bellandi, S.; Degli Innocenti, N.; Stortini, A.M.; Loglio, G.; Schiavuta, E.; Cini, R. Surfactant components of marine organic matter as agents for biogeochemical fractionation and pollutant transport via marine aerosols. Mar. Chem. 1999, 63, 235-253.

60. Hansell, D.A.; Carlson, C.A. Biogeochemistry of Marine Dissolved Organic Matter; Academic Press: London, UK, 2002. 
61. Redfield, A.C.; Ketchum, B.H.; Richards, F.A. The influence of organisms on the composition of sea-water. Sea 1963, 2, 26-77.

62. Schulz, H.D.; Zabel, M. Marine Geochemistry; Springer Science \& Business Media: Berlin, Germany, 2013.

63. Asa-Awuku, A.; Engelhart, G.J.; Lee, B.H.; Pandis, S.N.; Nenes, A. Relating CCN activity, volatility, and droplet growth kinetics of $\beta$-caryophyllene secondary organic aerosol. Atmos. Chem. Phys. Disc. 2008, 8, 10105-10151.

64. Wonaschütz, A.; Coggon, M.; Sorooshian, A.; Modini, R.; Frossard, A.A.; Ahlm, L.; Mülmenstädt, J.; Roberts, G.C.; Russell, L.M.; Dey, S.; et al. Hygroscopic properties of smoke-generated organic aerosol particles emitted in the marine atmosphere. Atmos. Chem. Phys. 2013, 13, 9819-9835. 


\title{
The Influence of Sandstorms and Long-Range Transport on Polycyclic Aromatic Hydrocarbons (PAHs) in $\mathbf{P M}_{2.5}$ in the High-Altitude Atmosphere of Southern China
}

\author{
Minmin Yang, Yan Wang, Qiang Liu, Aijun Ding and Yuhua Li
}

Abstract: $\mathrm{PM}_{2.5}$ (Particulate Matter 2.5) samples were collected at Mount Heng and analyzed for polycyclic aromatic hydrocarbons (PAHs). During sampling, a sandstorm from northern China struck Mount Heng and resulted in a mean $\mathrm{PM}_{2.5}$ concentration of $150.61 \mu \mathrm{g} / \mathrm{m}^{3}$, which greatly exceeded the concentration measured under normal conditions (no sandstorm: $58.50 \mu \mathrm{g} / \mathrm{m}^{3}$ ). The average mass of PAHs in $\mathrm{PM}_{2.5}$ was $30.70 \mu \mathrm{g} / \mathrm{g}$, which was much lower than in the non-sandstorm samples $(80.80 \mu \mathrm{g} / \mathrm{g})$. Therefore, the sandstorm increased particle levels but decreased PAH concentrations due to dilution and turbulence. During the sandstorm, the concentrations of 4- and 5-ring PAHs were below their detection limits, and 6-ring PAHs were the most abundant. Under normal conditions, the concentrations of 2-, 3- and 6-ring PAHs were higher, and 4- and 5-ring PAHs were lower relative to the other sampling sites. In general, the PAH contamination was low to medium at Mount Heng. Higher LMW (low molecular weight) concentrations were primarily linked to meteorological conditions, and higher HMW (high molecular weight) concentrations primarily resulted from long-range transport. Analysis of diagnostic ratios indicated that $\mathrm{PM}_{2.5}$ PAHs had been emitted during the combustion of coal, wood or petroleum. The transport characteristics and origins of the PAHs were investigated using backwards Lagrangian particle dispersion modeling. Under normal conditions, the "footprint" retroplumes and potential source contributions of PAHs for the highest and lowest concentrations indicated that local sources had little effect. In contrast, long-range transport played a vital role in the levels of $\mathrm{PM}_{2.5}$ and PAHs in the high-altitude atmosphere.

Reprinted from Atmosphere. Cite as: Yang, M.; Wang, Y.; Liu, Q.; Ding, A.; Li, Y. The Influence of Sandstorms and Long-Range Transport on Polycyclic Aromatic Hydrocarbons (PAHs) in $\mathrm{PM}_{2.5}$ in the High-Altitude Atmosphere of Southern China. Atmosphere 2015, 6, 1633-1651. 


\section{Introduction}

Haze pollution in China has occurred frequently in recent decades, with a vital contribution from $\mathrm{PM}_{2.5}$ (Particulate Matter 2.5). Sandstorms also frequently occur in China, resulting in serious air pollution. $\mathrm{PM}_{2.5}$ and PAHs (polycyclic aromatic hydrocarbons) have become the focus of governments and researchers. Many studies have been conducted on PAHs and $\mathrm{PM}_{2.5}$ in the suburban and urban atmosphere; however, very few studies have evaluated the PAH concentrations associated with $\mathrm{PM}_{2.5}$ at high altitudes in China. Further studies of the PAH concentration distributions and sources in $\mathrm{PM}_{2.5}$ were conducted in this research. Furthermore, the influence of long-range transport on $\mathrm{PAH}$ concentrations in the atmosphere of a heavily polluted area is also discussed.

Regarding $\mathrm{PM}_{2.5}$ pollution, the standard established by the U.S. Environmental Protection Agency (US EPA) has been progressively tightened and is currently set at $35 \mu \mathrm{g} / \mathrm{m}^{3}$ for a $24-\mathrm{h}$ period, with an annual average of $12 \mu \mathrm{g} / \mathrm{m}^{3} . \mathrm{PM}_{2.5}$ originates from both natural and anthropogenic sources, which can directly emit particles or emit precursor gases that subsequently form particles in the atmosphere [1]. $\mathrm{PM}_{2.5}$ can reduce visibility because its particle diameter is near the wavelength of light, in addition to exerting climate and human health effects, particularly in the form of respiratory system disease due to its diverse chemical composition. Furthermore, $\mathrm{PM}_{2.5}$ is a carrier for toxic pollutants, such as PAHs or polychlorinated biphenyls (PCBs). $\mathrm{PM}_{2.5}$ particles have a lifespan of days to weeks and can be transported over distances of thousands of kilometers due to their small size [2]. Therefore, it is important to study the distribution of $\mathrm{PM}_{2.5}$ concentrations and their emission sources.

PAHs, especially those associated with fine particles $\left(\mathrm{PM}_{2.5}\right)$, are mutagenic and carcinogenic [3]. During incomplete combustion or the pyrolysis of organic materials such as wood, fossil fuels and coal, PAHs, a group of semi-volatile compounds, are formed. In terms of environmental effects, PAHs can also inhibit growth of diatoms and even affect the global carbon cycle [4,5]. In terms of human health, PAHs, especially those with high molecular weight, are associated with several pathologies and may cause acute health effects, adverse birth outcomes or higher levels of risk for lung cancer $[3,6,7]$. In China, the limit for the daily average concentration of $\mathrm{BaP}$ in ambient air is $0.01 \mu \mathrm{g} / \mathrm{m}^{3}$. In the 1970s, the US EPA designated 16 PAHs as priority pollutants. In particular, high molecular weight (HMW) PAHs (MW $\geq 202$ ) with 4-6 aromatic rings frequently result from combustion [8]. In addition, PAHs can disperse regionally and travel to remote places through long-range atmospheric transport; the influence of this process is analyzed in this study. PAHs are primarily emitted from anthropogenic sources, including petrogenic and pyrogenic sources [9]. Petrogenic sources are introduced to the atmosphere through oil spills of crude and refined petroleum, and pyrogenic sources are released through the combustion of 
coal, petroleum or biomass [9-11]. The major sources in China are biofuel burning, domestic coal combustion, and industrial emissions [12]. Therefore, it is important to analyze the concentrations, distributions and potential emission sources of PAHs associated with $\mathrm{PM}_{2.5}$, particularly in the atmosphere, to effectively control air pollution caused by $\mathrm{PM}_{2.5}$.

Most studies on PAHs have been conducted in low-lying urban or rural areas around the world [13-15]. However, few studies have described PAH concentrations in $\mathrm{PM}_{2.5}$, especially concerning sandstorms and long-range transport at high mountain sites far from ground-level pollution sources. Areas such as Mount Heng (the sampling site in this study), particularly for those located in the free troposphere of heavily polluted areas, are unique environments for investigating $\mathrm{PM}_{2.5} \mathrm{PAH}$ concentrations. Although high mountain sites are usually considered the most pristine continental areas [16], they may receive significant amounts of PAHs from the deposition of particulates after long-range atmospheric transport $[17,18]$. The particulate $\left(\mathrm{PM}_{2.5}\right)$ samples were collected at the summit of Mount Heng (1269 m asl), located in the transition region between the boundary layer and free troposphere. The concentrations and distributions of chemical compounds at high mountain sites can provide valuable information regarding the long-range transport and accumulation of such pollutants. The objectives of this study were to determine the $\mathrm{PM}_{2.5}$ and particulate PAHs levels and to identify potential emission sources of PAHs and $\mathrm{PM}_{2.5}$ at Mount Heng during the spring of 2009.

\section{Materials and Methods}

\subsection{Sampling}

$\mathrm{PM}_{2.5}$ samples were collected at the top of Mount Heng $\left(27.3^{\circ} \mathrm{N}, 112.7^{\circ} \mathrm{E}\right)$ continuously every day from March to May of 2009, except on rainy or very foggy days. Mount Heng is located in the Hunan province of southern China and is approximately $500 \mathrm{~km}$ from the East China Sea and South China Sea. Sampling was conducted at the Mount Heng Meteorological Station, which is located at an elevation of $1269 \mathrm{~m}$ asl near Zhurong Peak, located within the free troposphere as shown in Figure 1. Measurements were performed in 2009 on the roof of a small house that is part of the meteorological observatory. The local climate is characterized by distinct seasons and influenced by air masses that originate from various directions. Although Mount Heng is less developed than coastal cities in China, it has experienced rapid industrialization and urbanization in the last two decades [19]. Furthermore, its high elevation and geographic location are suitable for studying the long-range transport of pollutants.

A medium-volume PM air sampler (TH150A, Tianhong, Wuhan) with a $\mathrm{PM}_{2.5}$-selective inlet was used for ambient sample collection at a calibrated flow 
rate of $100 \mathrm{~mL} / \mathrm{min} . \mathrm{PM}_{2.5}$ particles were collected on quartz fiber filters $(90 \mathrm{~mm}$ in diameter) that had been pre-baked in a muffle furnace for $24 \mathrm{~h}$ at $600{ }^{\circ} \mathrm{C}$ to minimize the concentration in the blank. Then, the quartz fiber filters were stored in a constant temperature and humidity incubator (WS $150 \mathrm{III}$ ) at $25^{\circ} \mathrm{C}$ and a relative humidity of $50 \%$ for $48 \mathrm{~h}$ before weighing.

A sandstorm in northern China struck Mount Heng on 25 April 2009 (25-1: 8:30-20:15 and 25-2: 21:00-8:05 the next day) and 26 April 2009 (26-1: 8:30-12:30, 26-2:12:50-16:30, 26-3: 16:50-20:40 and 26-4: 20:50-8:40 the next day). During this time, six of the particulate samples were collected. As shown in Figure 1b, when the sandstorm occurred on 25 and 26 April 2009, the UV (ultraviolet) aerosol index increased. However, this index was lower when no sandstorm occurred on 19 and 20 May 2009 (Figure 1a).

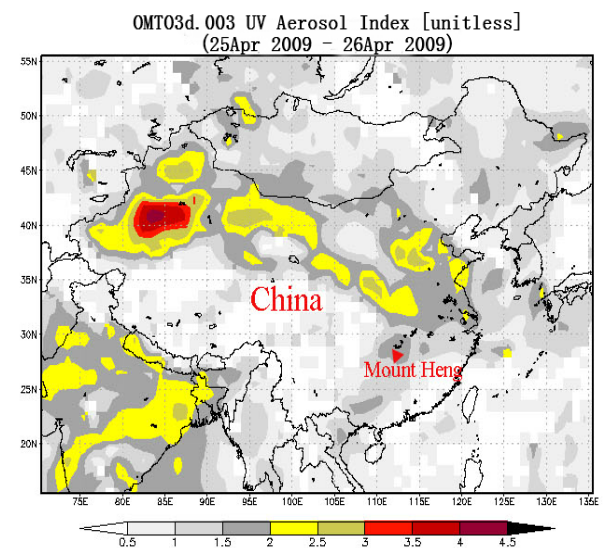

(a)

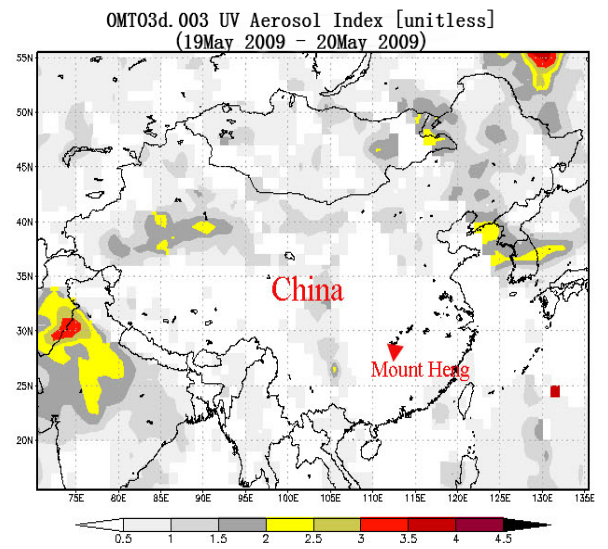

(b)

Figure 1. Sampling locations and UV (ultraviolet) aerosol index under normal conditions (a) and during the sandstorm (b).

\subsection{Analytical Techniques}

After field sampling, all filters were immediately sealed in aluminum foil and stored in a freezer at $-20{ }^{\circ} \mathrm{C}$ before analysis. Next, the compounds collected on the quartz fiber filters were extracted in an accelerated solvent extractor (ASE 3000, Dionex) using a solvent mixture of acetone and $n-h e x a n e(v / v=1: 1)$ at an extraction temperature of $100{ }^{\circ} \mathrm{C}$ for $5 \mathrm{~min}$. Perylene-D12 was added to the samples as a surrogate before extraction to estimate the extraction efficiency and losses during the sample extraction and concentration steps. The extracts were rotary evaporated to a volume of approximately $10 \mathrm{~mL}$ before concentrating in a concentrator (N-EVAP 112) to approximately $1 \mathrm{~mL}$. Finally, each extract was diluted with $1 \mathrm{~mL}$ of $\mathrm{n}$-hexane 
and spiked with phenanthrene-d10 as an internal standard for analysis by gas chromatography/mass spectrometry (GC/MS).

Sixteen priority PAH species listed by the US EPA were quantified using GC/MS (QP2010, SHIMADZU) with a capillary GC column $(30 \mathrm{~m} \times 0.25 \mathrm{~mm} \times 0.25 \mu \mathrm{m}$, DB-5 ms). The ion source, injector and interface temperatures were 200.0, 300.0 and $250.0^{\circ} \mathrm{C}$, respectively. The column oven temperature was initially $45.0^{\circ} \mathrm{C}$ for $1.0 \mathrm{~min}$ before increasing to $130.0^{\circ} \mathrm{C}$ at a rate of $45.0^{\circ} \mathrm{C} / \mathrm{min}$, to $240.0^{\circ} \mathrm{C}$ at a rate of $7.0^{\circ} \mathrm{C} / \mathrm{min}$ and to $320.0^{\circ} \mathrm{C}$ at a rate of $12.0^{\circ} \mathrm{C} / \mathrm{min}$, which was then held for $8.0 \mathrm{~min}$. The analytical method was based on US EPA Method 8270 [20]. Sixteen PAHs were measured and quantified. Although naphthalene is on this list, its levels are affected by many factors, and it was not investigated in this study due to its high volatility in particles and its presence in the ambient air and carrier gas. Therefore, only the sums of the other 15 PAHs were analyzed, as in many previous studies [21,22]. The fifteen priority PAH species are acenaphthene (Ace), acenaphthylene (Acy), fluorene (Flu), phenanthrene (PhA), anthracene (AnT), fluoranthene (FluA), pyrene (Pyr), benz $(a)$ anthracene $(\mathrm{BaA})$, chrysene $(\mathrm{Chr})$, benzo $(b)$ fluoranthene $(\mathrm{BbF})$, benzo $(k)$ fluoranthene $(\mathrm{BkF})$, benzo $(a)$ pyrene $(\mathrm{BaP})$, indeno $(1,2,3-c d)$ pyrene $(\mathrm{InP})$, dibenz $(a, h)$ anthracene $(\mathrm{DbA})$ and benzo $(g, h, i)$ perylene $(\mathrm{BP})$.

\subsection{Quality Assurance and Control}

The analyses were performed in accordance with the technical specifications of the US EPA. Data were acquired in single-ion monitoring mode (SIM) and quantified using the internal standard method. For qualitative analysis, compounds were matched using the retention times and ion mass fragments of standard mixtures of PAHs from the National Institute of Standards and Technology (NIST). Calibration curves were created based on the response factors of certain PAH species in the standard solution versus phenanthrene-d10, the internal standard used to quantify the individual PAH species. A standard mixture of 16 PAHs was diluted to six concentrations and analyzed by GC/MS using the same procedure. The mean extraction recoveries for the $\mathrm{PM}_{2.5}$ surrogates varied from 83.3 to $100.3 \%$. The field and laboratory blanks were treated in parallel with the particulate matter samples during sampling, storage and chemical analysis. The blank levels were subtracted to obtain the reported PAH concentrations in the $\mathrm{PM}_{2.5}$.

\subsection{Modeling Tools and Methodology}

In this study, a Lagrangian dispersion model, the hybrid single-particle Lagrangian integrated trajectory (HYSPLIT) model, was used to simulate transport and dispersion [23]. The positions of the particles were calculated based on the mean wind and turbulence transport components after their release at a receptor for backward runs [24]. Detailed information regarding this model was presented 
by Draxler and Hess [23] and Draxler [25]. This model was used to conduct hourly backward simulations of particle dispersion to calculate the potential source contributions (PSCs) of $\mathrm{PM}_{2.5}$ (i.e., the sources that contribute to the simulated mixing ratios at the receptor) based on the methods used by various authors [24,26-28]. In addition, $\mathrm{PM}_{2.5}$ was the carrier of the PAHs detected in this study and was emitted from the same potential sources. Here, the word "potential" indicates that this calculation was based on transport alone [29] and that the generation of secondary organic aerosols was not considered as a factor during transport. We calculated the residence time at an altitude of $0-100 \mathrm{~m}$ to obtain a "footprint" of the retroplume of the released air particles, which represented the probability distribution or residence time of a simulated air mass based on the method developed by Stohl et al. [29,30]. The gridded contributions of $\mathrm{PM}_{2.5}$ (i.e., the PSCs) to an observed air mass were calculated by multiplying the emission rate by the footprint retroplume with an emissions inventory [24] at a spatial resolution of $0.1^{\circ}$ latitude and $0.1^{\circ}$ longitude. The LPDM (Lagrangian Particle Dispersion Model) was developed on a horizontal grid of $0.1^{\circ} \times 0.1^{\circ}$ (latitude and longitude) and was fully capable of representing the regional transport of air pollutants to the Mount Heng region. An updated $\mathrm{PM}_{2.5}$ emissions inventory (INTEX_B; 2006) was used to calculate the PSCs of $\mathrm{PM}_{2.5}$ [31] at a horizontal resolution of $0.2^{\circ} \times 0.2^{\circ}$ (latitude and longitude) over China. Emissions may not significantly vary from year to year, and the same inventory was applied in this study. Details regarding the application of the LPDM were presented by Ding et al. [24].

\section{Results and Discussion}

\subsection{PM 2.5 and PAH Concentrations and Distributions at Mount Heng}

Figure 2 shows the daily $\mathrm{PM}_{2.5}$ levels measured at Mount Heng during the spring of 2009. When the sandstorm struck Mount Heng on 25 and 26 April 2009, the $\mathrm{PM}_{2.5}$ concentration ranged from 86.65 to $212.66 \mu \mathrm{g} / \mathrm{m}^{3}$ with a mean concentration of $156.61 \mu \mathrm{g} / \mathrm{m}^{3}$. In the absence of a sandstorm, the $\mathrm{PM}_{2.5}$ concentration ranged from $32.69 \mu \mathrm{g} / \mathrm{m}^{3}$ to $92.50 \mu \mathrm{g} / \mathrm{m}^{3}$ with a mean of $58.50 \mu \mathrm{g} / \mathrm{m}^{3}$. The average mass concentrations measured at Mount Heng were greater than those measured at Mount Tai, with a mean concentration of $42.24 \mu \mathrm{g} / \mathrm{m}^{3}$ in the autumn-winter of 2008 [22]. When the sandstorm struck Mount Heng, the $\mathrm{PM}_{2.5}$ concentration rapidly increased, indicating that the sandstorm largely affected the air quality. 


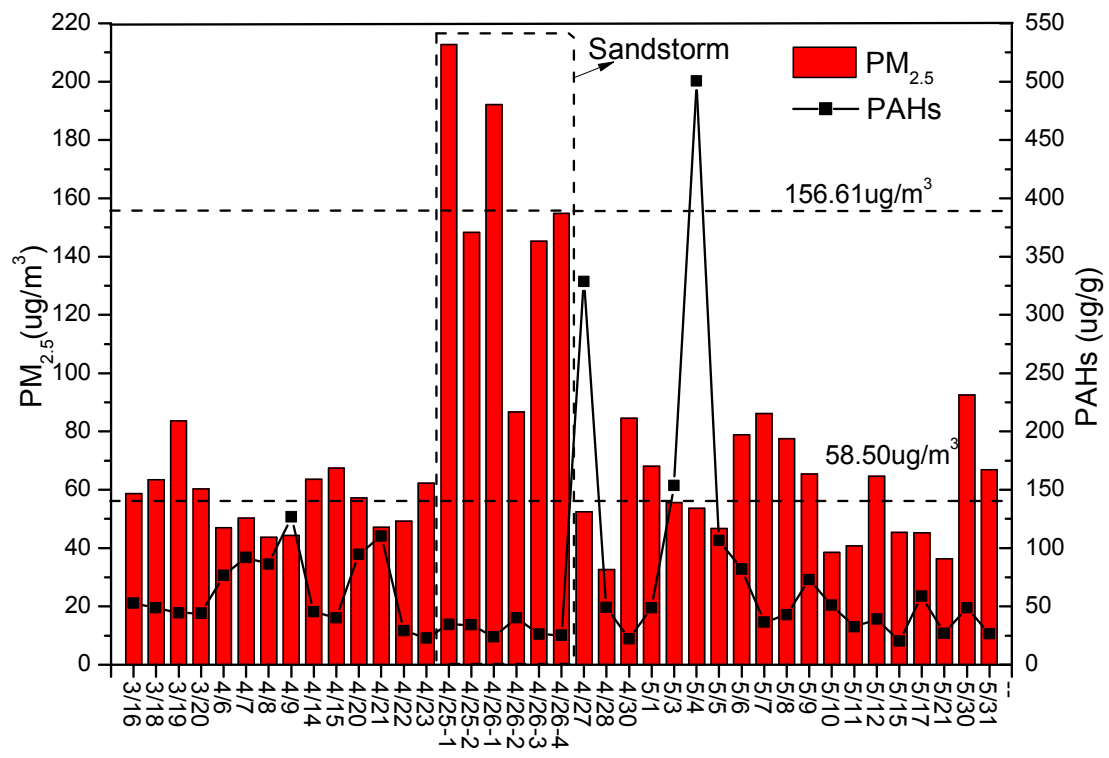

Date

Figure 2. $\mathrm{PM}_{2.5}$ and total daily PAH levels measured at Mount Heng.

Table 1. Concentrations of the individual polycyclic aromatic hydrocarbons (PAHs) during the sandstorm (25 and 26 April 2009) and under normal conditions (no sandstorm) $(\mu \mathrm{g} / \mathrm{g})$.

\begin{tabular}{ccccccccc}
\hline \multirow{2}{*}{ PAH } & \multicolumn{4}{c}{ Sandstorm } & \multicolumn{5}{c}{ No Sandstorm } \\
\cline { 2 - 9 } & Max & Min & Average & Stdev & Max & Min & Average & Stdev \\
\hline Ace & 1.9 & 1.14 & 1.52 & 0.3 & 2.39 & 0.3 & 1.14 & 0.58 \\
Acy & 6.46 & 2.18 & 3.99 & 1.49 & 34.91 & 1.32 & 5.2 & 5.65 \\
Flu & 4.82 & 1.49 & 2.72 & 1.26 & 30.04 & 0.67 & 4.58 & 4.81 \\
PhA & 9.85 & 3.42 & 5.35 & 2.33 & 31.69 & 1.52 & 9.59 & 6.46 \\
AnT & 3.16 & 0.82 & 2.05 & 1 & 8.12 & 0.82 & 2.24 & 1.48 \\
FluA & 5.87 & 1.68 & 3.54 & 1.47 & 71.21 & ND & 9.74 & 14.44 \\
Pyr & 5.06 & 1.22 & 2.65 & 1.35 & 61.91 & ND & 8.17 & 11.85 \\
BaA & ND & ND & ND & ND & 31.08 & ND & 3.48 & 6.95 \\
Chr & ND & ND & ND & ND & 57.07 & ND & 5.42 & 10.34 \\
BbF & 3.6 & ND & 1.19 & 1.84 & 82.76 & ND & 9.14 & 15.66 \\
BkF & 3.14 & ND & 0.79 & 1.32 & 22.67 & ND & 4.64 & 5.21 \\
BaP & 3.89 & ND & 1.21 & 1.88 & 27.6 & ND & 4.76 & 6.21 \\
InP & 2.47 & ND & 0.68 & 1.09 & 37.65 & ND & 4.71 & 7.55 \\
DbA & ND & ND & ND & ND & 12.96 & ND & 1.06 & 2.3 \\
BP & 6.6 & 3.7 & 5.02 & 0.99 & 54.01 & ND & 6.92 & 10.2 \\
SUM & 56.83 & 15.64 & 30.7 & 16.31 & 566.08 & 4.63 & 80.8 & 109.7 \\
\hline
\end{tabular}

ND: not detected. 
The mass concentrations of the individual PAH species under the influence of the sandstorm at Mount Heng are shown in Table 1. When the sandstorm struck Mount Heng, the total PAH mass concentrations in $\mathrm{PM}_{2.5}$ ranged from 15.64 to $56.83 \mu \mathrm{g} / \mathrm{g}$ with a mean concentration of $30.70 \mu \mathrm{g} / \mathrm{g}$. In addition, PhA was the predominant compound $(5.35 \mu \mathrm{g} / \mathrm{g}$ ), followed by BP, Acy and Pyr, which contributed nearly $60 \%$ to the total concentration during the sandstorm. Under normal conditions, the total PAH mass concentration ranged from 4.63 to $566.08 \mu \mathrm{g} / \mathrm{g}$ with a mean of approximately $80.15 \mu \mathrm{g} / \mathrm{g}$; this variation is large and resulted from the varying meteorological conditions and emissions sources during the sampling campaign. The most abundant PAH species observed were FluA, PhA, BbF, and Pyr, which accounted for nearly $50 \%$ of the total.

Figure 3 compares the published data on PAH concentrations in $\mathrm{PM}_{2.5}$ with the values observed at Mount Heng. The volume concentrations of the total PAHs in $\mathrm{PM}_{2.5}$ were comparable under sandstorm $\left(4.70 \mathrm{ng} / \mathrm{m}^{3}\right)$ and normal $\left(4.50 \mathrm{ng} / \mathrm{m}^{3}\right)$ conditions, as shown in the top left corner of Figure 3. The total PAH concentrations at Mount Heng were much greater than those reported in southwestern urban Atlanta [3] and were generally lower than those reported at Mount Tai [22] in northern China and in the city of Beijing [15]. However, these values were comparable to the mean concentrations reported in Hong Kong [32]. In this study, PAHs with 2-6 rings were measured. Figure 3 shows that the concentrations of the 2- and 3-ring PAHs were generally higher at Mount Heng than at Mount Tai [22], except for FluA. The 6-ring PAH concentrations were comparable to the values reported at Mount Tai [22] and were much higher than those in Hong Kong [32] and Atlanta [3] under normal (no sandstorm) conditions. The concentrations of 4- and 5-ring PAHs were lower than those at the other sites, except Atlanta. In this study, 2-6 ring PAHs displayed the same distributions during the sandstorm as the 2-, 3- and 6-ring PAHs, which had greater concentrations. However, the 4- and 5-ring PAHs had lower concentrations than at the other sampling sites, and the overall PAH contamination at Mount Heng was low to medium.

In terms of mass concentrations, PAHs with three and six rings were the predominant compounds, accounting for $51.5 \%$ of the total under normal conditions and $66.8 \%$ during the sandstorm. In contrast, the mass concentrations of PAHs were generally lower during the sandstorm, as shown in Figure 2. The potentially carcinogenic PAHs, particularly $\mathrm{BaA}, \mathrm{BbF}, \mathrm{BkF}, \mathrm{BaP}, \mathrm{InP}$ and $\mathrm{DbA}$ (four and five rings) (according to the International Agency for Research on Cancer (IARC), were present at high concentrations and accounted for $12.0 \%$ of the total at Mount Heng. 


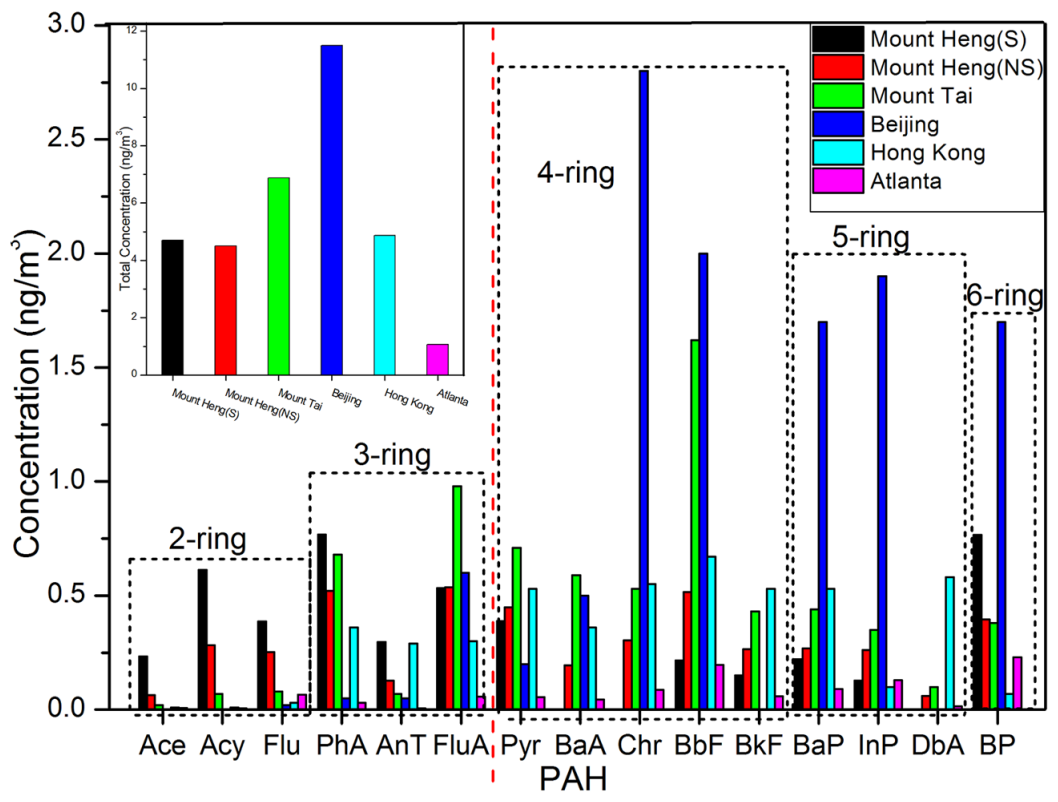

Figure 3. Average concentrations of PAHs in $\mathrm{PM}_{2.5}$ at Mount Heng and other sites (S: sandstorm, NS: no sandstorm).

As shown in Figure 2, the mass concentrations of PAHs were generally lower during the sandstorm. When the sandstorm struck the site on 25 and 26 April 2009, the $\mathrm{PM}_{2.5}$ volume concentrations were much greater, whereas the total PAHs were present at lower mass concentrations. Six samples were collected at various times, and the concentration profiles of the $15 \mathrm{PAH}$ species and the corresponding $\mathrm{PM}_{2.5}$ levels and ring distributions are shown in Figure 3. The average daily concentrations of the individual PAH species were all higher on 26 April than on 25 April 2009, except for $\mathrm{BaA}, \mathrm{Chr}, \mathrm{BbF}, \mathrm{BkF}, \mathrm{BaP}, \mathrm{InP}$ and $\mathrm{DbA}$, which were below the detection limit. The concentration of Acy was greater during the night than during the day, whereas the total PAHs were present at comparable levels. Acy is generally emitted from cement [33], diesel vehicle emissions [34,35] and combustion sources [36]. As shown in Figure 3, the concentrations of the 2-ring PAHs were greater during the night than during the day. On 26 April 2009, four particle samples were collected, and the concentrations of $\mathrm{PhA}$ and Flu were higher in the particulate sample collected between 12:50 and 16:34. PhA primarily results from incomplete combustion and the pyrolysis of fuels [34], and Flu results from diesel and gasoline vehicle emissions, general combustion, industrial oil burning and coal combustion [33-35]. These sources are consistent with the findings presented in the third section. During nighttime, the 2-ring PAH concentrations were higher and equaled those on 
25 April 2009. Therefore, we can conclude that the 2-ring PAH concentrations increased due to their volatility when the temperature fell at night. Furthermore, PAHs with five aromatic rings were not detected on 26 April 2009, but contributed $16.08 \%$ and $12.97 \%$ of the total on 25 April 2009.

During the sandstorm, $\mathrm{BaA}, \mathrm{Chr}$ and $\mathrm{DbA}$ were not detected in the samples, as shown in Table 1. In general, BaA originates from the steel industry [33], and Chr primarily originates from gasoline vehicle emissions [34,35] and industrial oil burning [33,35]. Thus, the steel industry and gasoline-powered vehicles potentially did not contribute to the PAH concentrations under sandstorm conditions, which is supported by the diagnostic ratio analysis of PAHs in the third section.

\subsection{Diagnostic Ratio Analysis of PAHs}

As indicated above, emission sources were estimated based on specific pollutants, and diagnostic ratios were analyzed to clarify their dominant sources. Due to high volatility of 2-3-ring PAHs, only 4-6-ring PAHs were used for source identification in this study. Linear regression analyses were conducted between various aromatic PAH concentrations to estimate their emission sources, as shown in Figure 4. Based on Figure 4a,b, the concentrations of 4-, 5- and 6-ring PAHs were strongly correlated with each other $\left(\mathrm{R}^{2}=0.97,0.97\right.$ and 0.93 , respectively). These good correlations indicate that HMW (4-, 5- and 6-ring) PAHs could be emitted from sources that are similar and distinct from those of the 2-ring PAHs. Portions of the PAHs with three and four aromatic rings resulted from similar emissions sources with a similar transport pathway.
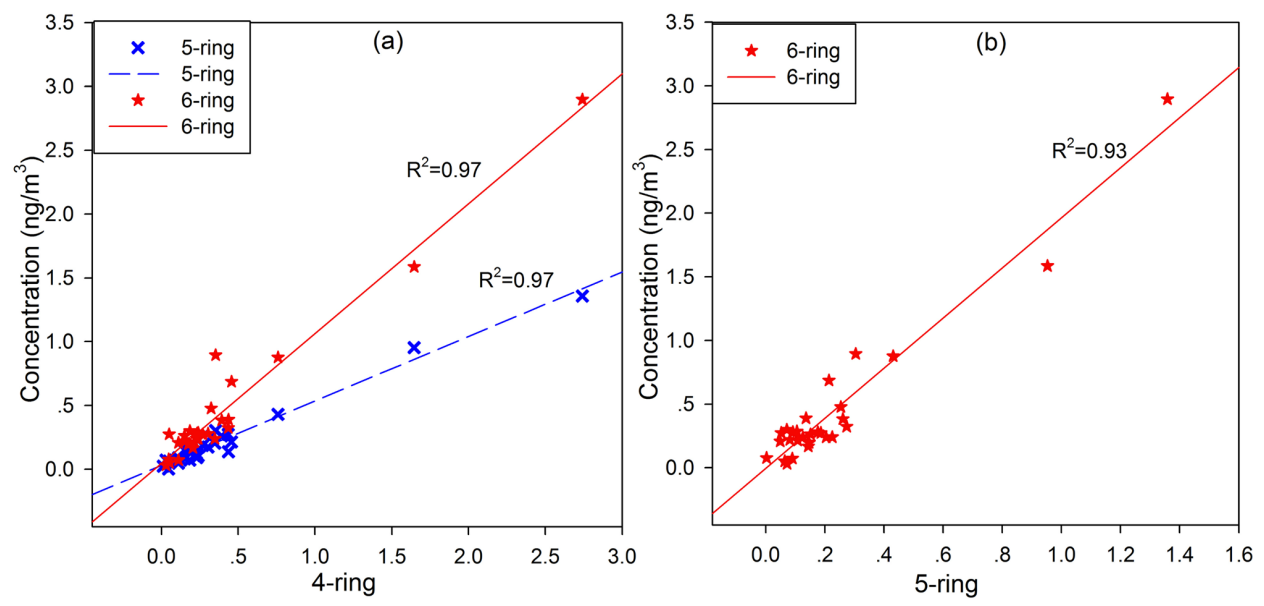

Figure 4. (a) Relationships between the 4-ring PAH concentrations and those of 5- and 6-ring PAHs; (b) relationship between the concentrations of the 5- and 6-ring PAHs. 
The concentrations of certain individual marker PAHs and their ratios have been widely used as indicators to identify PAH sources [8,22,37-41]. Common diagnostic ratios of LMW (low molecular weight, 2-3-ring PAHs)/HMW (4-6-ring $\mathrm{PAHs}), \mathrm{BaA} /(\mathrm{BaA}+\mathrm{Chr})$ and $\mathrm{InP} /(\mathrm{InP}+\mathrm{BP})$ were calculated in this study for source evaluation. LMW/HMW ratios of less than 1.0 indicate pyrogenic sources that include incomplete combustion of fossil fuels or wood [42-44], and LMW/HMW ratios greater than 1.0 (indicating petrogenic sources) include refined oil or petroleum products [44]. In this study, the LMW/HMW ratios ranged from 0.45 to 8.93, which implies that pyrogenic and petrogenic sources contributed to the contamination at the site. PAH isomer pairs with similar molecular weights are further investigated to distinguish between petrogenic and pyrogenic sources. The LMW/HMW ratios were plotted against the $\mathrm{BaA} /(\mathrm{BaA}+\mathrm{Chr})$ and $\mathrm{InP} /(\mathrm{InP}+\mathrm{BP})$ ratios to identify the emission sources affecting Mount Heng, as shown in Figure 5. The $\mathrm{BaA} /(\mathrm{BaA}+\mathrm{Chr})$ ratio has been used to indicate dominant fossil fuel emissions sources [15]. Petroleum combustion dominates $\mathrm{PAH}$ profiles when the $\mathrm{BaA} /(\mathrm{BaA}+\mathrm{Chr})$ ratio is less than 0.2 , a ratio between 0.20 and 0.35 results from a mixture of petroleum and coal combustions, and a ratio higher than 0.35 primarily result from coal combustion $[8,44]$. In this study, according to Figure 5a, most of the ratios are concentrated near 0.35 , indicating that the dominant source was petroleum or coal combustion. The BaA and Chr concentrations were below the detection limit; thus, their ratios were not analyzed during the sandstorm. Furthermore, $\mathrm{InP} /(\mathrm{InP}+\mathrm{BP})$ ratios smaller than 0.2 suggest a petroleum source, and this ratio ranged from 0.2 to 0.5 , indicative of petroleum combustion; if this ratio is greater than 0.5 , combustion of biomass, including grass, wood and coal, is inferred [8,45]. At Mount Heng, the main sources were petroleum combustion (according to Figure $5 b$ ).

The PAH compositional ratios presented above indicate that the dominant PAH sources affecting Mount Heng were coal, wood and petroleum combustion. As indicated in the correlation analysis, PAHs with 4, 5 and 6 rings were emitted from the same sources based on the correlation analysis, and the ratios of $(\mathrm{BaA}+\mathrm{Chr})$ and $\mathrm{InP} /(\mathrm{InP}+\mathrm{BP})$ confirmed that these PAHs primarily resulted from the combustion of petroleum. No large differences were attributed to the sandstorm. 


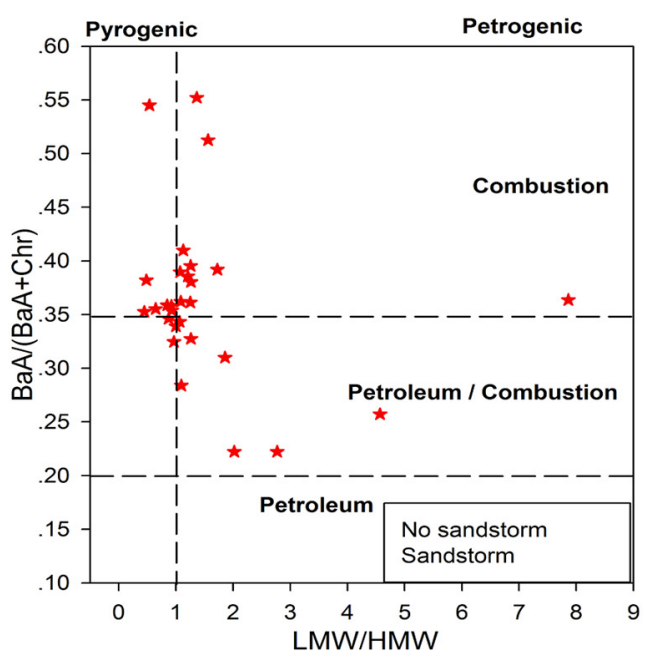

(a)

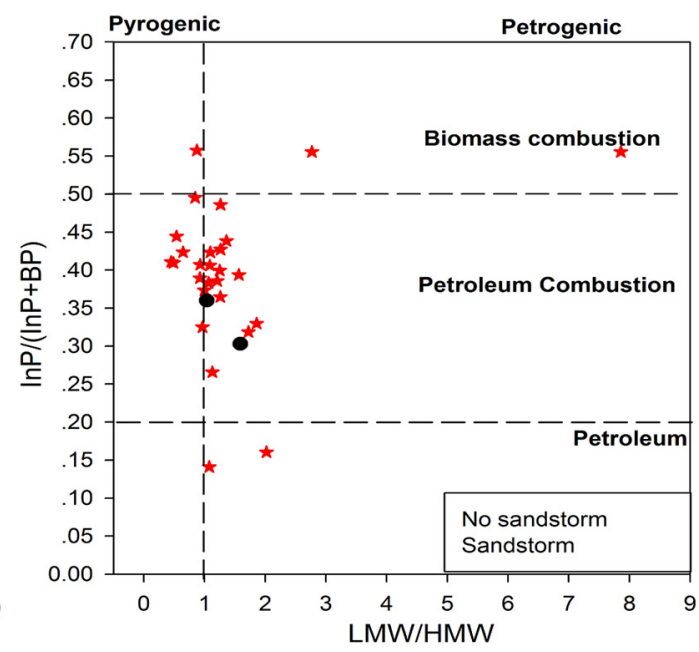

(b)

Figure 5. The ratios of LMW/HMW PAHs versus the ratios of $\mathrm{BaA} /(\mathrm{BaA}+\mathrm{Chr})$ (a) and $\operatorname{InP} /(\operatorname{InP}+\mathrm{BP})(\mathbf{b})$ in $\mathrm{PM}_{2.5}$ for source identification.

\subsection{Implications of Long-Range Transport for Air Pollution}

A ratio analysis was used to estimate the ages of the air masses. Generally, BaA is degraded more easily and rapidly than its isomer Chr during transport due to the higher reactivity of the former species [21,46-48]. The ratios of more-reactive to less-reactive PAHs can be used to estimate whether an air mass is fresh or aged [21]. A higher ratio ( $>0.7)$ indicates that the air mass underwent relatively little photochemical processing and that the major pollutants were from local emissions, whereas a lower ratio suggests that the PAHs were primarily from long-range transport. The ratios of BaA/Chr were calculated in this study. During the sandstorm, the $\mathrm{BaA}$ and $\mathrm{Chr}$ concentrations were below the detection limit, which suggests that the air masses were relatively aged. Under normal (no sandstorm) conditions, most of the ratios were below 0.70 . Therefore, the sampled air masses primarily resulted from remote emissions sources.

Pollutants emitted from distant regions in the free troposphere can travel long distances and strongly influence variations in pollutant concentrations [49]. Under normal conditions, the PAH concentrations varied greatly, and several special samples were analyzed to determine their LMW ( $\sum 2-3$ ring) and HMW ( $\sum 4-6$ ring) PAH concentrations. The backward trajectories of the air masses were calculated using an online version of the HYSPLIT (Hybrid Single Particle Lagrangian Integrated Trajectory) model from the NOAA (National Oceanic and Atmospheric Administration) Resources Laboratory, as shown in Figure A1. The 
total concentrations of the LMW and HMW PAHs in the $\mathrm{PM}_{2.5}$ were calculated. The concentrations of the LMW PAHs were 4-8 times greater than those of the HMW PAHs on 21, 31, 15 and 11 May and 9 April 2009. In particular, the 2-ring PAH concentrations were higher and accounted for $52.6 \%$ of the total PAHs on 9 April 2009. The concentrations of the HMW PAHs were approximately equal to the concentrations of the LMW PAHs on 19 and 20 March 2009, 20 and 22 April 2009 and 5 May 2009. The concentrations of the LMW PAHs were approximately 50\% of the HMW PAHs on 27 April and 3, 4 and 9 May 2009, These variations were potentially caused by differences in sources and air mass transport. On 9 April 2009, the particle samples were collected before a rainstorm, and the air masses were traveling from the sea to the east. The samples collected between 11 May and 15 May 2009 were collected before rainy or foggy weather. Therefore, the higher LMW PAH concentrations on these days were potentially linked to the meteorological conditions. The air masses associated with higher HMW concentrations primarily traveled from eastern coastal cities (e.g., Shanghai, Shandong, and Guangdong Province), including two of China's main industrial regions in southern China, one centered in Shanghai and the other centered near Guangzhou. Thus, the higher HMW concentrations resulted from the transport of pollutants to the site, and the air mass that traveled from the south on 9 May 2009 had been impacted by a non-ferrous metal smelting plant in Hunan Province, which also contributed to the higher concentrations. The LMW concentrations were approximately equal to (or slightly higher than) the HMW concentrations and originated from three sources near coastal areas. On 4 May 2009, the total PAH concentrations were the highest, with the corresponding air masses originating in Shanghai three days earlier. In addition, many nearby factories also contributed to the PAH concentrations. Most of the PAH species collected on 4 May 2009 had undergone little photochemical processing, which explains their higher total concentrations. The LMW PAH concentrations were all higher than the HMW PAH concentrations during the sandstorm.

Based on the observed ratios $(\mathrm{BaA} / \mathrm{Chr}$ ), the $\mathrm{PAH}$ concentrations were primarily affected by emissions located far from the site and by some local sources. Figure $6 a-h$ show the footprint retroplumes (residence times) and PSCs of the $\mathrm{PM}_{2.5}$ samples collected on 25 and 26 April 2009. In the simulation, particle transport was simulated backwards in time over a 72-hour period.

The residence times at an altitude of $100 \mathrm{~m}$ were calculated to obtain the "footprint" retroplumes of released air particles in Figure 6a-f during the sandstorm, which represent the distribution of probability or the residence time of a simulated air mass $[29,30]$. The longer residence time (red and yellow colors in the figure) indicates a higher probability of influence on the sampling site. In addition, $\mathrm{PM}_{2.5}$ was the primary vehicle for PAHs and was assumed to originate from the same sources discussed in this study. The air masses all originated in northern China and carried 
the pollutants to the site during the sandstorm. The nighttime samples collected on 26 April 2009 (Figure 6h) displayed the lowest probability influence from the surroundings. The $\mathrm{PM}_{2.5}$ concentrations in the samples on 26 April 2009 (12:50-16:30) were lower than those in the other samples collected during the sandstorm (as shown in Figure 2), which is consistent with the results of the residence time.
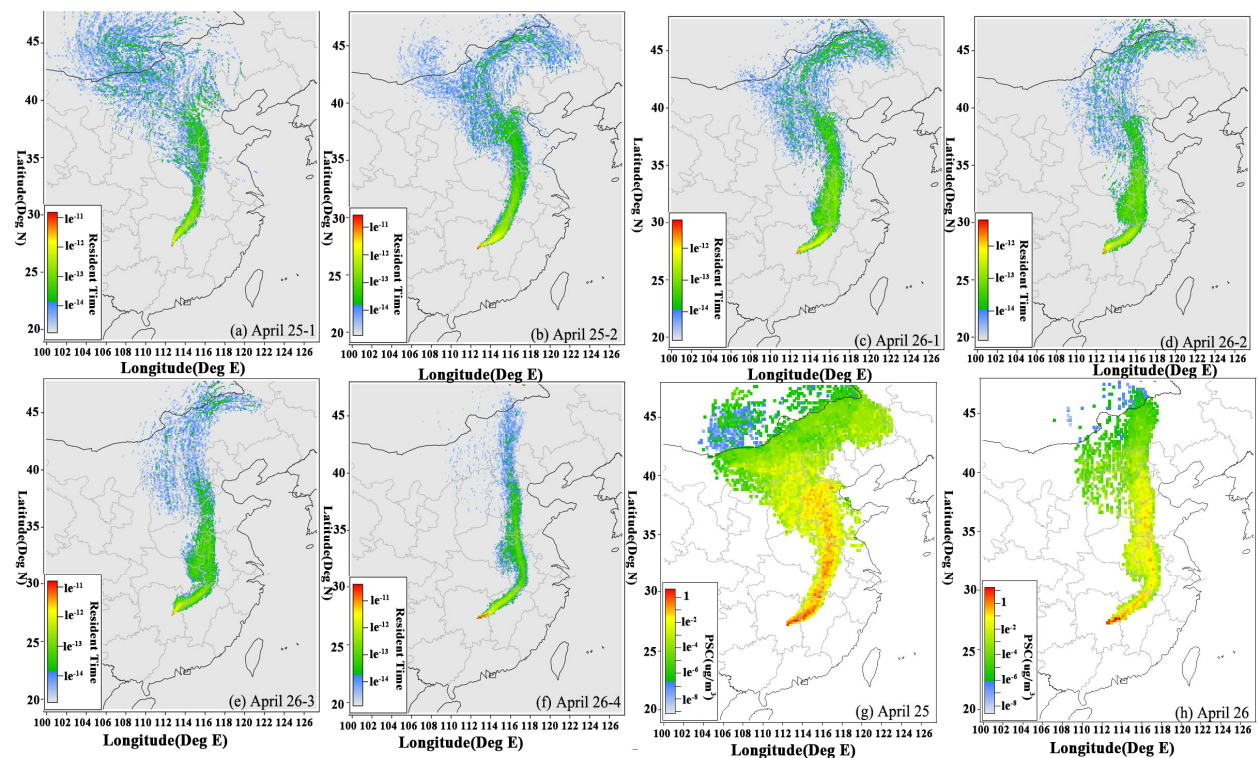

Figure 6. Illustrations of three-day backward retroplumes and potential source contributions (PSCs) during the sandstorm. (a-f) Map of the "footprint" (i.e., $100 \mathrm{~m}$ ) retroplume (residence time) and $(\mathbf{g}-\mathbf{h})$ maps of the PSCs of $\mathrm{PM}_{2.5}$ (PAHs) at Mount Heng.

In addition, the PSCs of the PAHs were also calculated (Figure $6 \mathrm{~g}-\mathrm{h}$ ) in this study. Air pollutants from northern China, including eastern Mongolia, could be transported to Mount Heng within three days, and significant source contributions in several regions were present along the route on 26 April 2009, including central eastern Hunan Province, northwestern Jiangxi Province and the nearby Shandong and Shanxi Provinces in northern China (Figure 6g). In addition, high emission source contributions on 26 April 2009 (Figure 6h) occurred along a similar route with a smaller area and lower concentrations in these regions. The emission sources were primarily in northeastern Hunan Province. Therefore, the $\mathrm{PM}_{2.5}$ concentrations were higher on 25 April than on 26 April 2009 based on the PSC of the $\mathrm{PM}_{2.5}$. The average measured concentration of $\mathrm{PM}_{2.5}$ was $180.49 \mu \mathrm{g} / \mathrm{m}^{3}$ on 25 April 2009, which was greater than the concentration of $144.67 \mu \mathrm{g} / \mathrm{m}^{3}$ on 26 April 2009. This finding is consistent with the PSC results. In addition, the total PAH concentrations were 
higher on 25 April 2009, and most of the individual PAH concentrations were higher on 25 April than on 26 April 2009.
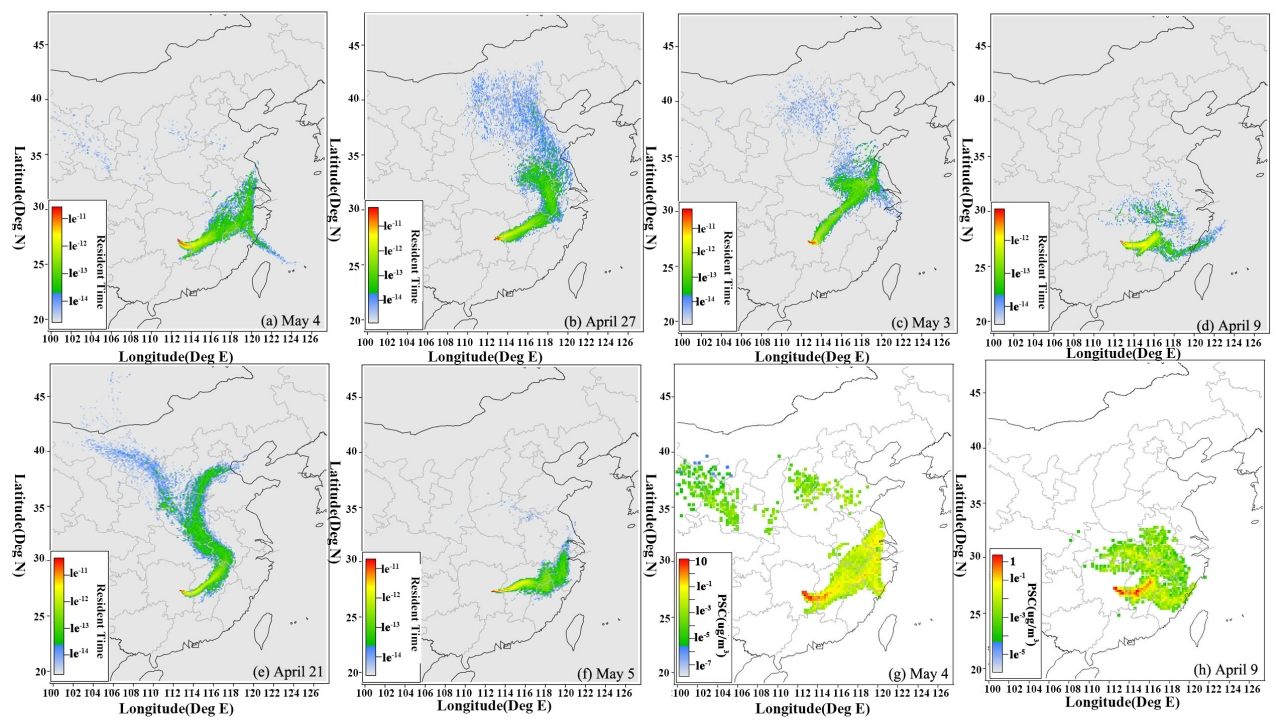

Figure 7. Illustrations of three-day backward retroplumes and the potential source contributions (PSCs) of higher PAH levels. (a-f) Map of the "footprint" (i.e., $100 \mathrm{~m}$ ) retroplume (residence time) and $(\mathbf{g}-\mathbf{h})$ maps of the PSCs of $\mathrm{PM}_{2.5}$ (PAHs) at Mount Heng.

The highest total PAH concentrations were measured on 4 May, 27 April, 3 May, 9 April, and 21 April 2009 and the lowest on 15 May, 30 April, 23 April, 31 May and 21 May 2009 in the absence of sandstorm conditions, as shown in Figure 2. Their "footprint" retroplumes (residence times) of released air particles and the PSCs of the $\mathrm{PM}_{2.5}$ (PAHs) are shown in Figures 7 and 8. The air masses associated with higher concentrations evidently all originated east of the site and passed over urban areas, particularly the air mass of 4 May 2009, which is shown as a large yellow area and extends across Jiangxi Province from west to east. Therefore, the total concentration was the highest on 4 May 2009 (as shown in Figure 2), which is consistent with the simulation results. The influences of emissions from the coastal cities of eastern China were able to reach the site within three days, and the dominant pollutants traveled from eastern Hunan Province and western Jiangxi Province, as indicated by Figure $7 \mathrm{a}-\mathrm{h}$. The large amounts of pollution emitted from the coastal cities of eastern China were carried to the site via atmospheric transport and resulted in higher $\mathrm{PM}_{2.5}$ (PAH) concentrations. In contrast, to demonstrate the influences of the transport of lower concentrations, their "footprint" retroplumes (residence times) of released air particles and the PSCs of $\mathrm{PM}_{2.5}$ (PAHs) are shown in Figure 8a-h. The air masses 
associated with the two lowest concentrations all originated from the ocean to the south, and their corresponding areas depicted in red and yellow are clearly smaller, which indicates that almost no pollutants were carried to this site. The paths of the last three air masses (31 and 21 May, 22 April 2009) originated from near the site, which indicates that the pollutants were primarily from local emissions sources. Their concentrations were lower than those of the other samples. Therefore, the air masses originating from the ocean and carrying few pollutants resulted in lower $\mathrm{PM}_{2.5}$ (PAHs) concentrations, and the local emission sources contributed little to the pollutant concentrations. The pollutants from long-range transport contributed more to the site.
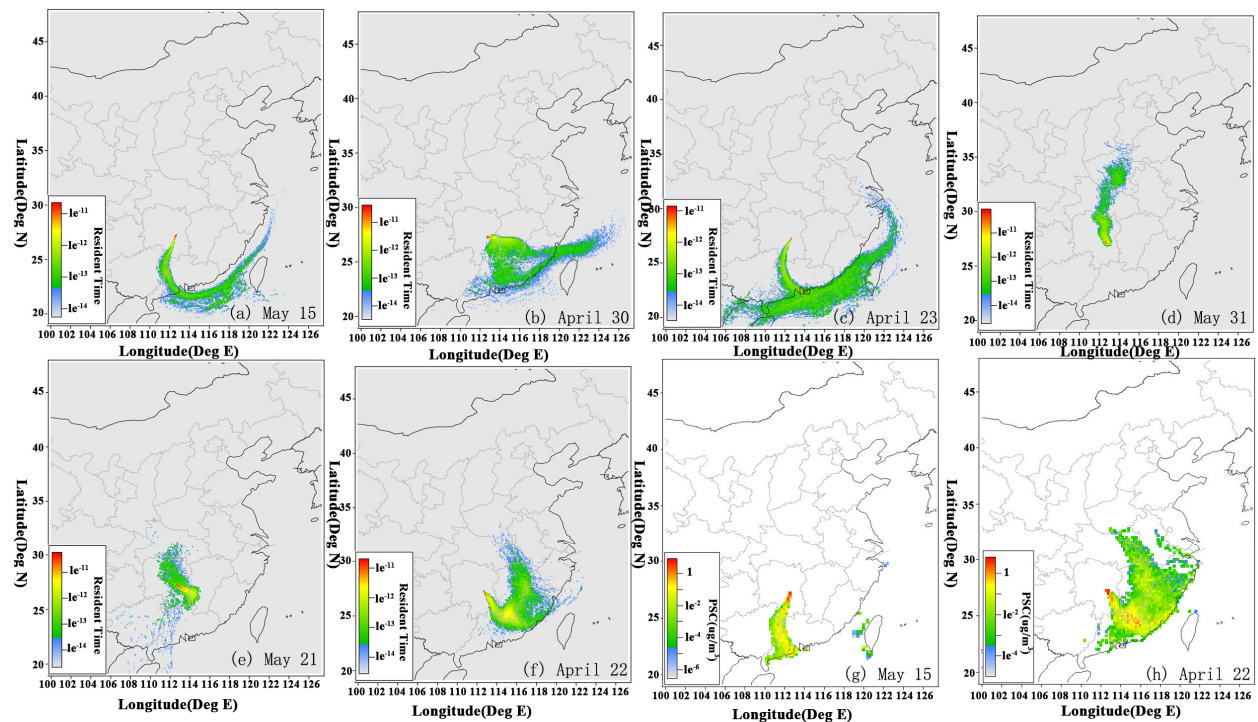

Figure 8. Illustrations of three-day backward retroplumes and potential source contributions (PSCs) of lower PAH levels. (a-f) Map of the "footprint" (i.e., $100 \mathrm{~m}$ ) retroplume (residence time) and $(\mathbf{g}-\mathbf{h})$ maps of the PSCs of $\mathrm{PM}_{2.5}$ (PAHs) at Mount Heng.

Regardless of the presence of the sandstorm, the combination of backward simulation dispersion via the PSCs of the $\mathrm{PM}_{2.5}$ (PAHs) revealed the importance of transport for the pollutant concentrations. During the sandstorm, the air masses all originated from northern China, and the distribution of the PSCs affected the $\mathrm{PM}_{2.5}$ (PAHs) concentrations. Under normal (no sandstorm) conditions, the air masses associated with higher or lower concentrations originated from the same respective directions, and their variations in route resulted in higher or lower concentrations. The higher and lower concentrations demonstrate that the atmospheric transport significantly influenced the pollutant concentrations at Mount Heng. 


\section{Conclusions}

A study of PAHs in $\mathrm{PM}_{2.5}$ was conducted in northern China between March and May in 2009. A sandstorm from northern China struck Mount Heng on 25 and 26 April 2009 during the sampling campaign, and the sandstorm greatly affected the $\mathrm{PM}_{2.5}$ and particulate PAH concentrations. During the sandstorm, the $\mathrm{PM}_{2.5}$ levels rapidly increased to $156.61 \mu \mathrm{g} / \mathrm{m}^{3}$, whereas before or after the sandstorm, the normal value was $58.50 \mu \mathrm{g} / \mathrm{m}^{3}$. The mean mass concentration of the PAHs in $\mathrm{PM}_{2.5}$ was $30.70 \mu \mathrm{g} / \mathrm{g}$, and those of $\mathrm{BaA}, \mathrm{Chr}$ and $\mathrm{DbA}$ were below the detection limits during the sandstorm. Overall, PhA, BP, Acy and Pyr were the predominant compounds, which together accounted for approximately $60 \%$ of the total PAH concentration. The mass PAH concentration at Mount Heng varied greatly around a mean of $80.15 \mu \mathrm{g} / \mathrm{g}$, and the most abundant compounds were FluA, PhA, BbF and Pyr, which accounted for nearly $50 \%$ of the total under normal (no sandstorm) conditions. The individual PAH species were present at low to medium concentrations at Mount Heng. The 4- and 5-ring PAH concentrations were lower at Mount Heng than at the other sites during the sandstorm. In addition, the 3- and 6-ring PAHs were the most abundant, accounting for $66.8 \%$ of the total PAH concentration during the sandstorm and $51.5 \%$ under normal conditions. Under normal conditions, special samples were selected for analysis of the LMW and HMW PAH concentrations. Higher LMW PAH concentrations were linked to meteorological conditions, and the higher HMW concentrations were primarily affected by the transport of pollutants.

Linear regression analyses were performed between the total PAH concentrations and the corresponding $\mathrm{PM}_{2.5}$ levels, and no clear relationship existed between these values. The relationships among the various-ring PAH concentrations suggested that 4-, 5- and 6-ring PAHs were potentially emitted from a similar source. The ratios of $\mathrm{LMW} / \mathrm{HMW}, \mathrm{BaA} /(\mathrm{BaA}+\mathrm{Chr})$ and $\mathrm{InP} /(\mathrm{InP}+\mathrm{BP})$ were used to identify the emissions sources, and indicated that the PAHs had primarily been emitted from the combustion of wood, coal or petroleum. The isomer pair of $\mathrm{BaA} / \mathrm{Chr}$ suggested that most of the air masses collected at Mount Heng resulted from long-range transport. The combination of "footprint" retroplumes of released air particles (residence time) and the PSCs of the $\mathrm{PM}_{2.5}$ (PAHs) with higher and lower concentrations indicated that local emission sources had little effect on the PAH concentrations (i.e., the concentrations in the air masses that were primarily collected from local sources were much lower) and that long-range transport played a vital role in the pollutant concentrations at Mount Heng under normal conditions.

Acknowledgments: This study is based on work supported by funding from the National Natural Science Foundation of China (grants 21177073) and the China National Basic Research Program (2005CB422203). We are grateful to the Mount Heng Meteorological Station for providing access to the experimental site, to Jinan Academy of Environmental sciences (Zaifeng 
Wang and Houyong Zhang) for providing experimental instruments, and to AJE (American Journal Experts) for editing the paper.

Author Contributions: Minmin Yang and Yan Wang wrote the article and design the study, Qiang Liu and Aijun Ding provided the figures of resident time and PSCs. Yuhua Li helped perform the statistical analysis.

Conflicts of Interest: The authors declare no conflict of interest.

\section{Appendix A.}

In this appendix, concentrations of HMW (4-6 ring) and LMW (2-6 ring) PAHs were compared and the backward trajectories of the air masses collected were presented in Figure A1. The HYSPLIT model was used to calculate backward trajectories for each day. The air masses were categorized into three categories based on concentrations. Figure A1a presented the backward trajectories that the concentrations of LMW PAHs were much higher than those of HMW and the backward trajectories that concentrations of HMW PAHs were approximately equal to the concentrations of LMW PAHs were presented in Figure A1b. Backward trajectories of HMW PAH concentrations smaller than the LMW PAH concentrations were shown in Figure A1c. The aim of this section is to indicate that the potential influence on concentrations of HMW and LMW PAHs respectively, including meteorological conditions and long range transport.
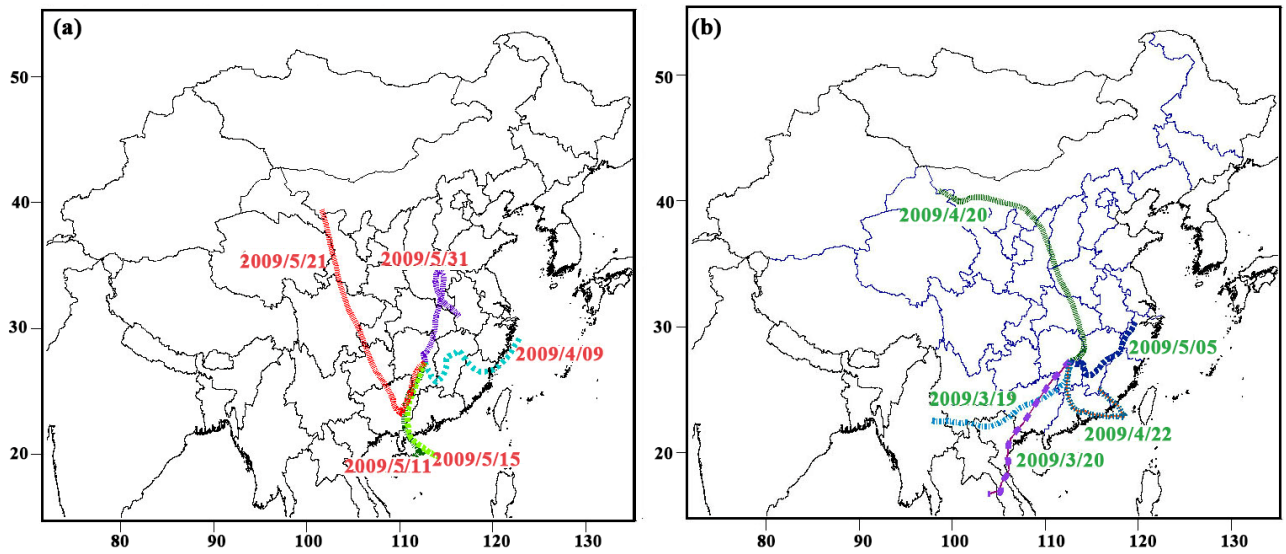

Figure A1. Cont. 


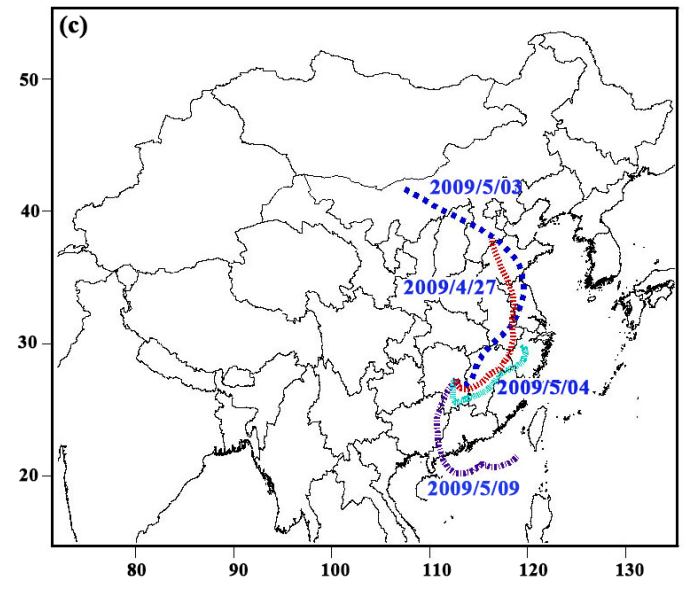

Figure A1. The backward trajectories of the selected air masses based on the LMW and HMW PAH concentrations, (a) LMW »HMW, (b) LMW $\approx \mathrm{HMW}$, (c) LMW « HMW.

\section{References}

1. Wang, Y.; Hopke, P.K.; Xia, X.; Rattigan, O.V.; Chalupa, D.C.; Utell, J.E. Source apportionment of airborne particulate matter using inorganic and organic species as tracers. Atmos. Environ. 2012, 55, 525-532.

2. Pui, D.Y.H.; Chen, S.-C.; Zuo, Z. PM 2.5 in China: Measurements, sources, visibility and health effects and mitigation. Particuology 2013, 13, 1-26.

3. Li, Z.; Porter, E.N.; Sjödin, A.; Needham, L.L.; Lee, S.; Russell, A.G.; Mulholland, J.A. Characterization of $\mathrm{PM}_{2.5}$ bound polycyclic aromatic hydrocarbons in Atlanta-Seasonal variations at urban, suburban, and rural ambient air monitoring sites. Atmos. Environ. 2009, 43, 4187-4193.

4. Neff, J. Polycyclic aromatic hydrocarbons in the aquatic environment and cancer risk to aquatic organisms and man. Am. Environ. Prot. Agency Rep. 1982, 600, 9-82.

5. Bopp, S.K.; Lettieri, T. Gene regulation in the marine diatom thalassiosira pseudonana upon exposure to polycyclic aromatic hydrocarbons (PAHs). Gene 2007, 396, $293-302$.

6. Wilhelm, M.; Ghosh, J.K.; Su, J.; Cockburn, M.; Jerrett, M.; Ritz, B. Traffic-related air toxics and preterm birth: A population-based case-control study in Los Angeles County, California. Environ. Health 2011, 10, 89-89.

7. Straif, K.; Baan, R.; Grosse, Y.; Secretan, B.; El Ghissassi, F.; Cogliano, V. Carcinogenicity of household solid fuel combustion and of high-temperature frying. Lancet Oncol. 2006, 7, 977-978.

8. Yunker, M.B.; Macdonald, R.W.; Vingarzan, R.; Mitchell, R.H.; Goyette, D.; Sylvestre, S.; Mitchelld, D. PAHs in the Fraser River basin: A critical appraisal of PAH ratios as indicators of PAH source and composition. Org. Geochem. 2002, 33, 489-515. 
9. Zakaria, M.P.; Takada, H.; Tsutsumi, S.; Ohno, K.; Yamada, J.; Kouno, E.; Kumata, H. Distribution of polycyclic aromatic hydrocarbons (PAHs) in rivers and estuaries in Malaysia: A widespread input of petrogenic PAHs. Environ. Sci. Technol. 2002, 36, 1907-1918.

10. Zhang, Y.; Tao, S. Global atmospheric emission inventory of polycyclic aromatic hydrocarbons (PAHs) for 2004. Atmos. Environ. 2009, 43, 812-819.

11. Chang, K.-F.; Fang, G.-C.; Chen, J.-C.; Wu, Y.-S. Atmospheric polycyclic aromatic hydrocarbons (PAHs) in Asia: A review from 1999 to 2004. Environ. Pollut. 2006, 142, 388-396.

12. Wang, Y.; Li, P.H.; Li, H.L.; Liu, X.H.; Wang, W.X. PAHs distribution in precipitation at Mount Taishan China. Identification of sources and meteorological influences. Atmos. Res. 2010, 95, 1-7.

13. Liu, F.; Xu, Y.; Liu, J.; Liu, D.; Li, J.; Zhang, G.; Li, X.; Zou, S.; Lai, S. Atmospheric deposition of polycyclic aromatic hydrocarbons (PAHs) to a coastal site of Hong Kong, South China. Atmos. Environ. 2013, 69, 265-272.

14. Tian, F.; Chen, J.; Qiao, X.; Wang, Z.; Yang, P.; Wang, D.; Ge, L. Sources and seasonal variation of atmospheric polycyclic aromatic hydrocarbons in Dalian, China: Factor analysis with non-negative constraints combined with local source fingerprints. Atmos. Environ. 2009, 43, 2747-2753.

15. Wu, Y.; Yang, L.; Zheng, X. Characterization and source apportionment of particulate PAHs in the roadside environment in Beijing. Sci. Total. Environ. 2014, 470, 76-83.

16. Arellano, L.; Fernández, P.; Tatosova, J.; Stuchlik, E.; Grimalt, J.O. Long-range transported atmospheric pollutants in snowpacks accumulated at different altitudes in the Tatra Mountains (Slovakia). Environ. Sci. Technol. 2011, 45, 9268-9275.

17. Carrera, G.; Fernández, P.; Grimalt, J.O.; Ventura, M.; Camarero, L.; Catalan, J.; Nickus, U.; Thies, H.; Psenner, R. Atmospheric deposition of organochlorine compounds to remote high mountain lakes of Europe. Environ. Sci. Technol. 2002, 36, 2581-2588.

18. Fernández, P.; Carrera, G.; Grimalt, J.O.; Ventura, M.; Camarero, L.; Catalan, J.; Nickus, U.; Thies, H.; Psenner, R. Factors governing the atmospheric deposition of polycyclic aromatic hydrocarbons to remote areas. Environ. Sci. Technol. 2003, 37, 3261-3267.

19. Sun, M.; Wang, Y.; Wang, T.; Fan, S.; Wang, W.; Li, P.; Guo, J.; Li, Y. Cloud and the corresponding precipitation chemistry in south China: Water-soluble components and pollution transport. J. Geophys. Res.: Atmos. 2010, 115, D22.

20. Techniquesb, A.P. Method 8270c Semivolatile Organic Compounds by Gas Chromatography/Mass Spectrometry (Gc/Ms). 1996; Available online: http://mx. gemc.gov.cn/upload/20120201035748.pdf (accessed on 22 October 2015).

21. Ding, X.; Wang, X.-M.; Xie, Z.-Q. Atmospheric polycyclic aromatic hydrocarbons observed over the North Pacific Ocean and the Arctic area: Partial distribution and source identification. Atmos. Environ. 2007, 41, 2061-2072.

22. Li, P.H.; Wang, Y.; Li, Y.H.; Wang, Z.F.; Zhang, H.Y. Characterization of polycyclic aromatic hydrocarbons deposition in $\mathrm{PM}_{2.5}$ and cloud/fog water at Mount Taishan (China). Atmos. Environ. 2010, 44, 1996-2003. 
23. Draxler, R.R.; Hess, G.D. An overview of the HYSPLIT_4 modelling system for trajectories dispersion, and deposition. Aus. Meteorol. Maga. 1998, 47, 295-308.

24. Ding, A.; Wang, T.; Fu, C. Transport characteristics and origins of carbon monoxide and ozone in Hong Kong, South China. J. Geophys. Res.: Atmos. 2013, 118, 9475-9488.

25. Draxler, R.R. Demonstration of a global modeling methodology to determine the relative importance of local and long-distance sources. Atmos. Environ. 2007, 41, 776-789.

26. Custódio, D.; Ferreira, C.; Alves, C.; Duarte, M.; Nunes, T.; Cerqueira, M.; Pio, C.; Frosini, D.; Colombi, C.; Gianelle, V. Urban aerosol in Oporto, Portugal: Chemical characterization of $\mathrm{PM}_{10}$ and $\mathrm{PM}_{2.5}$. Available online: http:/ / adsabs.harvard.edu/abs/ 2014EGUGA.16.2454C (accessed on 22 October 2015).

27. Ding, A.; Fu, C.; Yang, X.; Sun, J.; Zheng, L.; Xie, Y.; Herrmann, E.; Nie, W.; Petäjä, T.; Kerminen, V.-M. Ozone and fine particle in the western Yangtze River Delta: An overview of 1 year data at the SORPES station. Atmos. Chem. Phys. 2013, 13, 5813-5830.

28. Nie, W.; Ding, A.; Wang, T.; Kerminen, V.-M.; George, C.; Xue, L.; Wang, W.; Zhang, Q.; Petäjä, T.; Qi, X. Polluted dust promotes new particle formation and growth. Sci. Rep. 2014, 4 .

29. Ding, A.; Wang, T.; Xue, L.; Gao, J.; Stohl, A.; Lei, H.; Jin, D.; Ren, Y.; Wang, X.; Wei, X. Transport of north China air pollution by midlatitude cyclones: Case study of aircraft measurements in summer 2007. J. Geophys. Res.: Atmos. 2009, 114, D8.

30. Stohl, A.; Forster, C.; Eckhardt, S.; Spichtinger, N.; Huntrieser, H.; Heland, J.; Schlager, H.; Wilhelm, S.; Arnold, F.; Cooper, O. A backward modeling study of intercontinental pollution transport using aircraft measurements. J. Geophys. Res.: Atmos. 2003, 108, 4370-4370.

31. Zhang, Q.; Streets, D.G.; Carmichael, G.R.; He, K.; Huo, H.; Kannari, A.; Klimont, Z.; Park, I.; Reddy, S.; Fu, J. Asian emissions in 2006 for the NASA INTEX-B mission. Atmos. Chem. Phys. 2009, 9, 5131-5153.

32. Guo, H.; Lee, S.C.; Ho, K.F.; Wang, X.M.; Zou, S.C. Particle-associated polycyclic aromatic hydrocarbons in urban air of Hong Kong. Atmos. Environ. 2003, 37, 5307-5317.

33. Yang, H.-H.; Lee, W.-J.; Chen, S.-J.; Lai, S.-O. PAH emission from various industrial stacks. J. Hazard. Mater. 1998, 60, 159-174.

34. Ho, K.F.; Lee, S.C.; Chiu, G.M. Characterization of selected volatile organic compounds, polycyclic aromatic hydrocarbons and carbonyl compounds at a roadside monitoring station. Atmos. Environ. 2002, 36, 57-65.

35. Kulkarni, P.; Venkataraman, C. Atmospheric polycyclic aromatic hydrocarbons in Mumbai, India. Atmos. Environ. 2000, 34, 2785-2790.

36. Park, S.S.; Kim, Y.J.; Kang, C.H. Atmospheric polycyclic aromatic hydrocarbons in Seoul, Korea. Atmos. Environ. 2002, 36, 2917-2924.

37. Brown, J.N.; Peake, B.M. Sources of heavy metals and polycyclic aromatic hydrocarbons in urban stormwater runoff. Sci. Total. Environ. 2006, 359, 145-155.

38. Budzinski, H.; Jones, I.; Bellocq, J.; Pierard, C.; Garrigues, P.H. Evaluation of sediment contamination by polycyclic aromatic hydrocarbons in the Gironde estuary. Mar. Chem. 1997, 58, 85-97. 
39. Dunbar, J.C.; Lin, C.-I.; Vergucht, I.; Wong, J.; Durant, J.L. Estimating the contributions of mobile sources of PAH to urban air using real-time PAH monitoring. Sci. Total. Environ. 2001, 279, 1-19.

40. Feng, J.; Hu, M.; Chan, C.K.; Lau, P.S.; Fang, M.; He, L.; Tang, X. A comparative study of the organic matter in $\mathrm{PM}_{2.5}$ from three Chinese megacities in three different climatic zones. Atmos. Environ. 2006, 40, 3983-3994.

41. Zhang, W.; Zhang, S.; Wan, C.; Yue, D.; Ye, Y.; Wang, X. Source diagnostics of polycyclic aromatic hydrocarbons in urban road runoff, dust, rain and canopy throughfall. Environ. Pollut. 2008, 153, 594-601.

42. Mai, B.; Qi, S.; Zeng, E.Y.; Yang, Q.; Zhang, G.; Fu, J.; Sheng, G.; Peng, P.; Wang, Z. Distribution of polycyclic aromatic hydrocarbons in the coastal region of Macao, China: Assessment of input sources and transport pathways using compositional analysis. Environ. Sci. Technol. 2003, 37, 4855-4863.

43. Mai, B.-X.; Fu, J.-M.; Sheng, G.-Y.; Kang, Y.-H.; Lin, Z.; Zhang, G.; Min, Y.-S.; Zeng, E.Y. Chlorinated and polycyclic aromatic hydrocarbons in riverine and estuarine sediments from Pearl River Delta, China. Environ. Pollut. 2002, 117, 457-474.

44. Soclo, H.H.; Garrigues, P.H.; Ewald, M. Origin of polycyclic aromatic hydrocarbons (PAHs) in coastal marine sediments: Case studies in Cotonou (Benin) and Aquitaine (France) areas. Mar. Pollut. Bull. 2000, 40, 387-396.

45. Ravindra, K.; Sokhi, R.; van Grieken, R. Atmospheric polycyclic aromatic hydrocarbons: Source attribution, emission factors and regulation. Atmos. Environ. 2008, 42, 2895-2921.

46. Butler, J.D.; Crossley, P. Reactivity of polycyclic aromatic hydrocarbons adsorbed on soot particles. Atmos. Environ. 1981, 15, 91-94.

47. Kamens, R.M.; Guo, Z.; Fulcher, J.N.; Bell, D.A. The influence of humidity, sunlight, and temperature on the daytime decay of polyaromatic hydrocarbons on atmospheric soot particles. Environ. Sci. Technol. 1988, 22, 103-108.

48. Schauer, J.J.; Rogge, W.F.; Hildemann, L.M.; Mazurek, M.A.; Cass, G.R.; Simoneit, B.R. Source apportionment of airborne particulate matter using organic compounds as tracers. Atmos. Environ. 1996, 30, 3837-3855.

49. Gao, J.; Wang, T.; Ding, A.; Liu, C. Observational study of ozone and carbon monoxide at the summit of mount Tai (1534m asl) in central-eastern China. Atmos. Environ. 2005, 39, 4779-4791. 


\title{
Effect of Nearby Forest Fires on Ground Level Ozone Concentrations in Santiago, Chile
}

\author{
María A. Rubio, Eduardo Lissi, Ernesto Gramsch and René D. Garreaud
}

\begin{abstract}
On 4 and 8 January 2014, at the height of the austral summer, intense wildfires in forests and dry pastures occurred in the Melipilla sector, located about $70 \mathrm{~km}$ to the southwest of Santiago, the Chilean capital, affecting more than 6 million inhabitants. Low level winds transported the forest fire plume towards Santiago causing a striking decrease in visibility and a marked increase in the concentration of both primary ( $\mathrm{PM}_{10}$ and $\mathrm{CO}$ ) and secondary (Ozone) pollutants in the urban atmosphere. In particular, ozone maximum concentrations in the Santiago basin reached hourly averages well above $80 \mathrm{ppb}$, the national air quality standard. This ozone increase took place at the three sampling sites considered in the present study. These large values can be explained in terms of high NOx concentrations and $\mathrm{NO}_{2} / \mathrm{NO}$ ratios in biomass burning emissions.
\end{abstract}

Reprinted from Atmosphere. Cite as: Rubio, M.A.; Lissi, E.; Gramsch, E.; Garreaud, R.D. Effect of Nearby Forest Fires on Ground Level Ozone Concentrations in Santiago, Chile. Atmosphere 2015, 6, 1926-1938.

\section{Introduction}

Air plumes originating in forest fires are rich in primary pollutants such as particles, carbon monoxide (CO), non-methane volatile organic compounds (VOCs), and nitrogen oxides (NOx) [1-3] Photochemical transformations of those air masses can produce secondary pollutants, including ozone [4], that are transported over large distances [1,3,5-9]. These plumes can also affect nearby cities leading to ground level concentrations that largely surpass air quality standards $[8,10,11]$. Nevertheless, there are few evaluations regarding ozone behavior in large cities exposed to polluted masses transported from nearby forest fires $[3,8,11,12]$ Results range from moderate decreases at Arizona and Central Texas, US, and western Mexico, when the cities are located less than $400 \mathrm{~km}$ from fire [12], to large increases as in the city of Edmonton, Canada, when the city is located $300 \mathrm{~km}$ from the fire [11]. On the other hand, measurements in Mexico City (MCMA) did not demonstrate significant differences in ground level ozone concentrations in periods with active fires [13]. Evaluation of the effect of nearby wild fires on the concentration of pollutants in the air at ground level of large cities is particularly complex due to the mixing of local emissions with the fire derived polluted plumes [14]. Interestingly, it has been reported that in western 
U.S, the ozone was significantly correlated with forest fires in the surrounding $5^{\circ} \times 5^{\circ}$ and $10^{\circ} \times 10^{\circ}$ grids, but not with wild fires in the nearest $1^{\circ} \times 1^{\circ}$ region $(110 \times 110 \mathrm{~km}$, approximately), reflecting a subtle balance between ozone production and destruction in NOx rich environments [15].

Santiago, the Chilean capital, is a large city (with just over 6 million inhabitants, accounting for $40 \%$ of the national population with a density of 393 inhabitants per $\mathrm{km}^{2}$ ) located at a subtropical latitude $\left(33^{\circ} \mathrm{S}\right)$, about $100 \mathrm{~km}$ from the Pacific coast and just to the east of the Andes cordillera (Figure 1). It features a semi-arid climate with annual mean precipitation of $310 \mathrm{~mm}$, almost exclusively concentrated in winter months [16]. During the dry summer months (November to March), the city is exposed to relatively high concentrations of secondary oxidants $[17,18]$, particularly in its east side where $35 \%$ of the summer days surpassed the ozone national safety standard of $60 \mathrm{ppbv}$ for an $8 \mathrm{~h}$ mobile running average or 80 ppbv for one hour $[19,20]$.

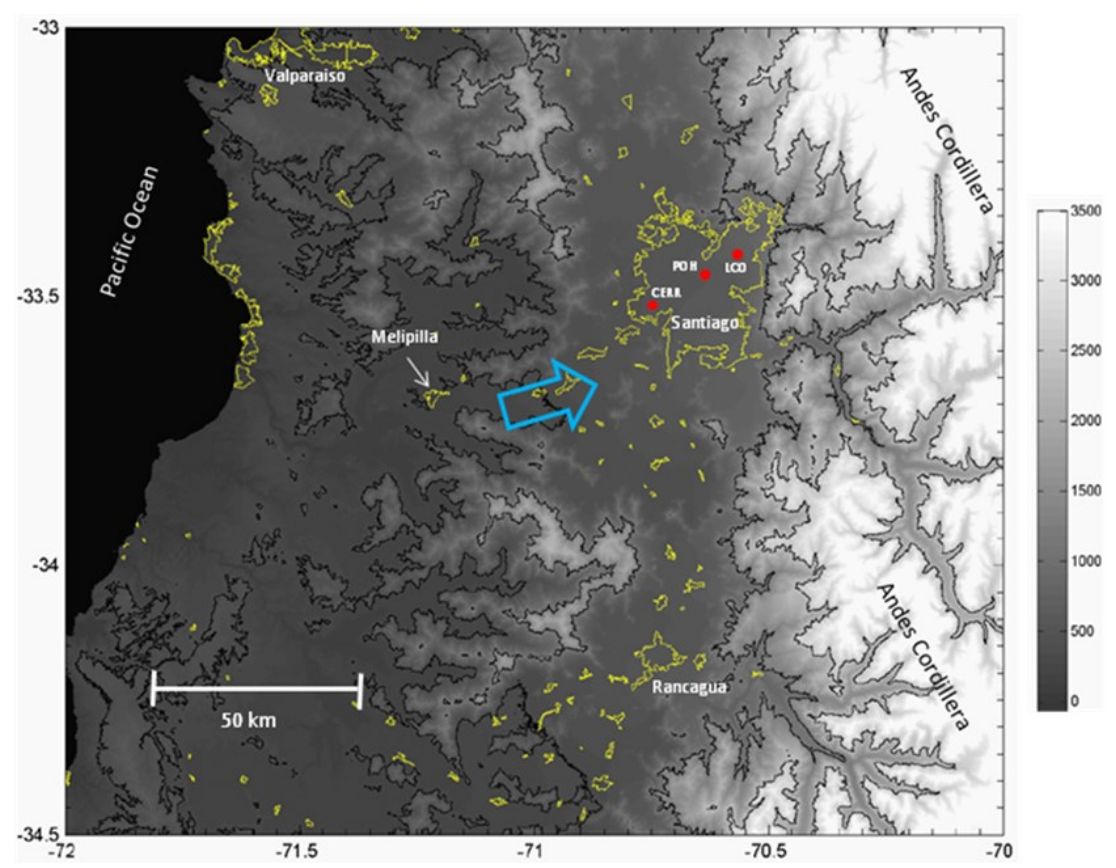

Figure 1. Topographic map of central Chile. Gray shading in m ASL; also indicated the 300, 1200 and $2400 \mathrm{~m}$ above sea level (ASL) topographic contours (black lines). Yellow lines outline main cities, red dots indicate the location of the MACAM (Air Quality Monitoring Program in Santiago Metropolitan Area) stations Cerrillos, Parque O'Higgins and Las Condes. The blue thick arrow indicates the predominant low-level winds from the SW during summer afternoon (adapted from [21], see also Figure 3). 
During 4 and 8 January 2014, two sizable forest fires took place in the Melipilla sector located about $70 \mathrm{~km}$ from downtown Santiago. These fires covered approximately $15 \mathrm{~km}^{2}$ of a Mediterranean landscape characterized by a mixture of pasture, open woodland and shrubland, including some Eucalyptus and Acacia caven trees $[22,23]$. The dominant vegetation and the changes introduced in recent years have been discussed by Schulz et al. [23]. The pre-Columbian vegetation of Central Chile is dominated by pastures and Acacia caven shrubland that have been replaced by vineyard and exotic trees such Pinus radiata and Eucalyptus globulus. These fires produced a noticeable increase in visible particles in the urban atmosphere of Santiago. In this short contribution, we present a critical discussion of ozone and other pollutants' behavior during those days. Given the possible effect of the large amounts of oxidants in the urban air on Santiago's inhabitants [24-26], we analyze the ozone concentrations and discuss the possible reasons for the extremely high values found in different areas of Santiago's basin.

\section{Observations and Methods}

Meteorological data (air temperature, relative humidity, incoming solar radiation and wind direction and speed) in the central part of Santiago (near downtown) was obtained from two automatic weather stations (USACH and DGF-UCh) recording $15 \mathrm{~min}$ averages. Station USACH $\left(33.45^{\circ} \mathrm{S}, 70.68^{\circ} \mathrm{W}, 528 \mathrm{~m}\right.$. above sea level (ASL)) is a Novalynx Corp. Station, model 110-WS-16. Station DGF-UCh $\left(33.46^{\circ} \mathrm{S}, 70.66^{\circ} \mathrm{W}\right.$, $542 \mathrm{~m}$. ASL) includes a standard Campbell Scientific AWS and a laser ceilometer (model VAISALA CL-31) retrieving backscatter reflectivity profiles from the surface up to $6 \mathrm{~km}$ above ground level every $30 \mathrm{~s}$. The laser reflectivity has been related with the aerosol loading in the mixed layer [27]. One-minute average wind speed and wind direction at $10 \mathrm{~m}$ above the ground were also available from station El Paico $\left(33.72^{\circ} \mathrm{S}, 71.02^{\circ} \mathrm{W}, 312 \mathrm{~m}\right.$. ASL), operated by the National Weather Service (DMC). Station El Paico is located in the Maipo valley connecting the Melipilla sector (where the fires took place) with Santiago.

Ozone, $\mathrm{NO}_{2}, \mathrm{NO}, \mathrm{PM}_{10}, \mathrm{PM}_{2.5}$ and $\mathrm{CO}$ concentrations at ground level were obtained from the Air Quality Monitoring Program in Santiago Metropolitan Area (MACAM-2) operated by the Environmental Ministry (SINCA, 2014) [28]. Specifically, hourly average values were gathered from three sampling stations located in Santiago (Figure 1): Cerrillos (CERR) in the west side of the city, $\left(33.49^{\circ} \mathrm{S} ; 70.71^{\circ} \mathrm{W}\right.$; $528 \mathrm{~m}$. ASL); Parque $\mathrm{O}^{\prime}$ Higgins $(\mathrm{POH})$ in downtown $\left(33.46^{\circ} \mathrm{S}, 70.66^{\circ} \mathrm{W}, 562 \mathrm{~m}\right.$. ASL) and Las Condes (LC) at the North East side of the city $\left(33.37^{\circ} \mathrm{S}, 70.52^{\circ} \mathrm{W} ; 811 \mathrm{~m}\right.$. ASL). The map in Figure 1 also shows the location of Melipilla, the small town around which the forest fires took place in January 2014.

In all the stations, ozone was measured using a Thermo UV-Photometric ozone analyzer, model $49 \mathrm{i}$ (measuring range: $0-0.5 \mathrm{ppm}$ ). $\mathrm{NO}-\mathrm{NO}_{2}-\mathrm{NO}$ X were measured 
using a Thermo chemiluminsescent gas analyzer, model 42i (measuring range $0.05-100 \mathrm{ppm}$ with a detection limit of $0.40 \mathrm{ppb}$ ). CO was analyzed using a Thermo CO Analyzer, model 40i (measuring range $0-50 \mathrm{mg} / \mathrm{m}^{3}$ with a detection limit of $4.0 \mathrm{ppm}$ ). $\mathrm{PM}_{10}$ was measured using a $\mathrm{PM}_{10}$ Monitor TEOM 1405 (measuring range of $0-1,000,000 \mu \mathrm{g} / \mathrm{m}^{3}$. $\mathrm{PM}_{2.5}$ was measured using a MET-ONE BAM-1020 monitor (measuring range of $0.1-10 \mu \mathrm{g} / \mathrm{m}^{3}$, with a detection limit $\leqslant 1.0 \mu \mathrm{g} / \mathrm{m}^{3}$.

\section{Results and Discussion}

\subsection{Meteorological Aspects}

Ground level concentrations of primary and secondary pollutants in the proximity of wildfires strongly depend on the direction and intensity of the low-level flow. As evident in the satellite imageries given in Figure 2, the forest fires in the Melipilla sector released a considerable amount of pollutants (ash, particulate matter, gasses) that were transported to Santiago by the southwesterly low-level flow that regularly develops during the afternoon in summer months (Figure 3) [21]. On both 4 and 8 January 2014, the near-surface wind speed at El Paico reached about $10 \mathrm{~m} / \mathrm{s}$ by noon (not shown). Thus, the fire plume that originated during the morning hours took less than $3 \mathrm{~h}$ to reach the city of Santiago, located about $70 \mathrm{~km}$ upwind of the Melipilla sector. The arrival of the polluted plume produced a striking reduction in visibility, readily evident for the general population (especially for the case of 4 January), as illustrated by the time-height diagram of the backscatter reflectivity from the laser ceilometer at DGF-UCh (Figure 4). The reflectivity, indicative of the aerosol loading in the mixed layer [27], exhibits a sharp increase around 2 PM, 4 January, encompassing the first $500 \mathrm{~m}$ above ground level. The marked increase in aerosol loading was accompanied by a simultaneous stalling of the air temperature (that often maximize around $4 \mathrm{PM}$ ) and a reduction of the global solar radiation relative to the previous day (when very similar synoptic conditions prevailed), which integrated over the course of the afternoon represents a deficit of about $15 \%$ of the insolation for a typical clear sky day in Santiago. 


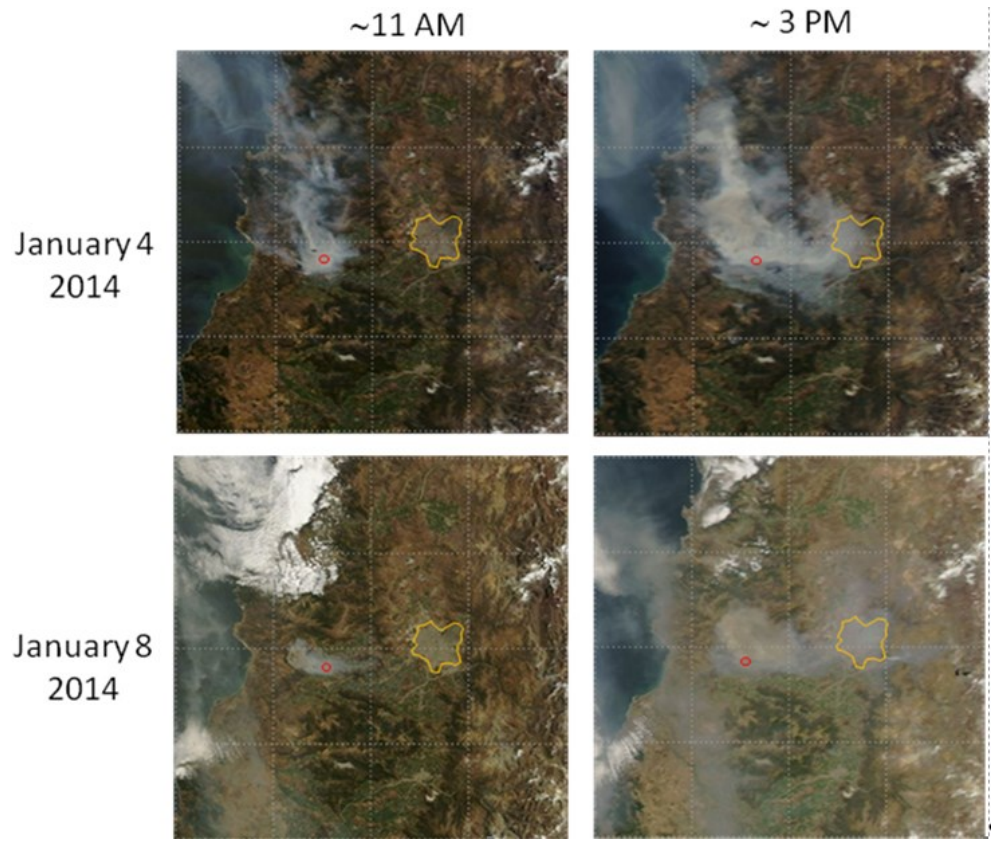

Figure 2. Corrected reflectance (True Color) scenes from MODIS for 4 and 8 January 2014, over central Chile (same region as in Figure 1). The plume of the forest fires in the Melipilla sector (red circle) reaching Santiago (yellow polygon) is evident in all the images. The left column shows images from the AQUA satellite at about 11 a.m., the right column shows images from the TERRA satellite at about 3 p.m.
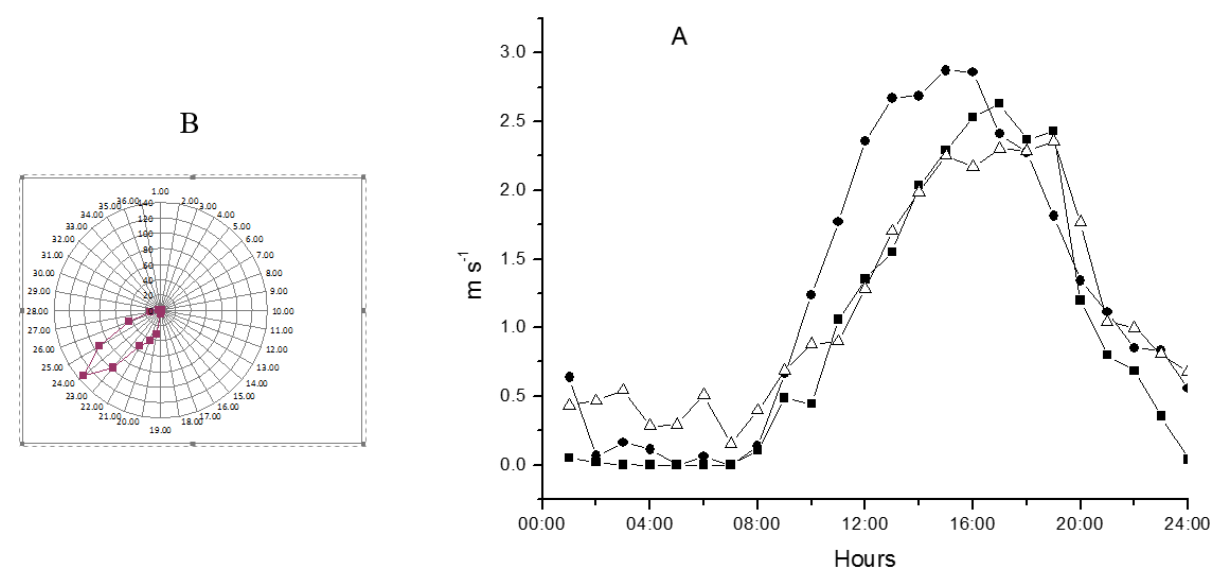

Figure 3. (a) Wind speed and wind in days with active fires (- January 8; $\bullet$ January 4; and days without fires $\Delta ;(\mathbf{b})$ Wind rose for 4 January, 14:00-24:00. 


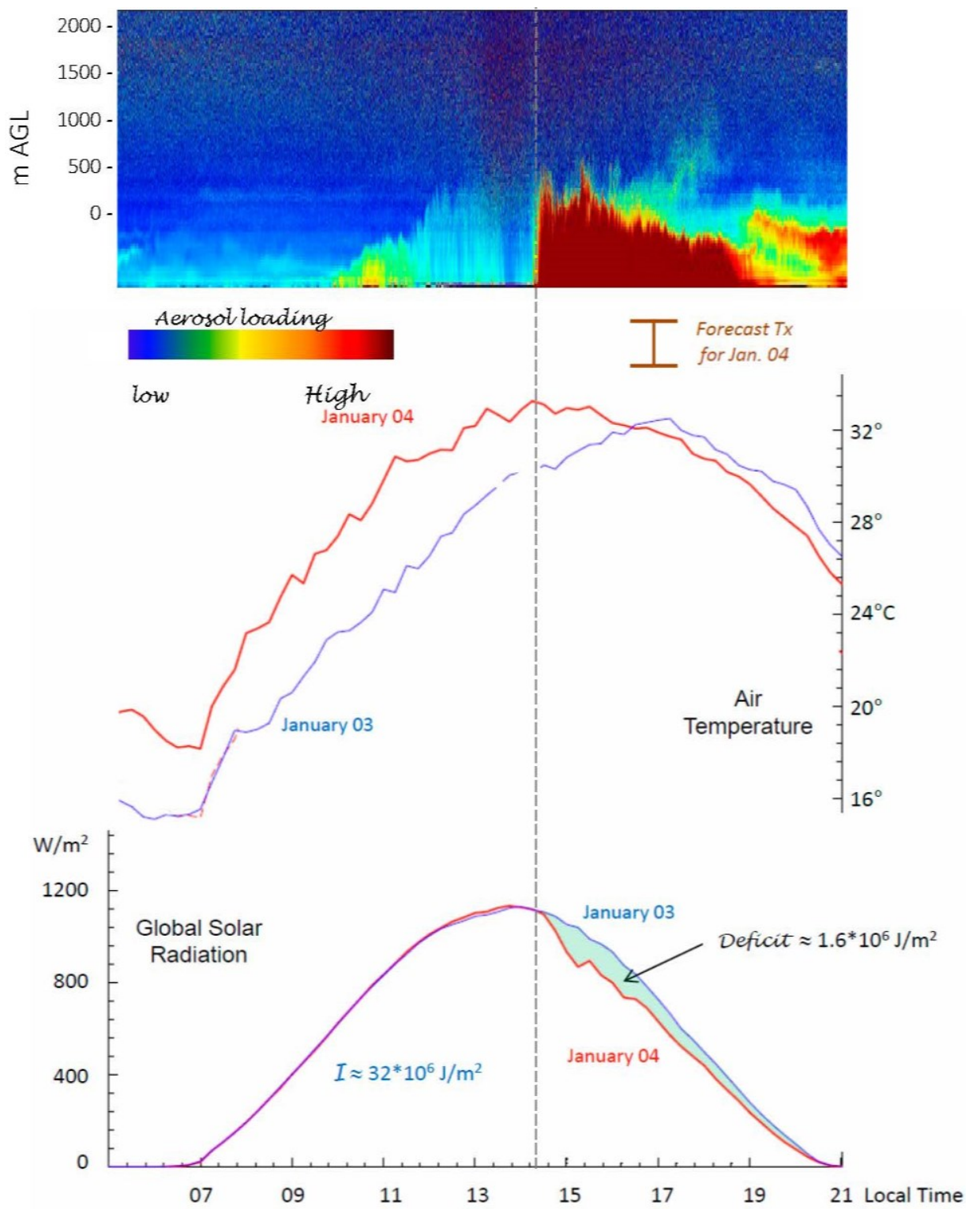

Figure 4. Upper panel: Time-height diagram of the backscatter reflectivity from a laser ceilometer (VAISALA CL-31) located at Santiago downtown during 4 January 2014 (active fire). The reflectivity has been related with the aerosol loading in the mixed layer [27] which is qualitatively indicated here from low to high. Middle panel: Air temperature 4 January (solid red line) and the previous day (3 January, blue line). Lower panel: Global solar radiation reaching the surface at Santiago for 3 January (blue line) and 4 January (red line). 


\subsection{Impacts on Primary Pollutants}

The arrival of the forest fire plumes in Santiago markedly increased the concentration of pollutants measured at ground level relative to non-fire days, as illustrated by the time series of $\mathrm{MP}_{10}$ in CERR and LC (Figure 5). A similar behavior is observed for different pollutants in the three sampling sites summarized in Table 1. This table shows the maximum daily concentrations of $\mathrm{CO}, \mathrm{PM}_{10}$ and $\mathrm{O}_{3}$ during days with active fires in the Melipilla sector and the average daily maximum for non-fire days during summer (days from 2011, 2012 and 2013). Note that the presence of active fires in Melipilla increased nearly three times the maximum CO concentration in Santiago relative to the background reference values. Similar differences were obtained in fires near to Cordoba city (Argentina) in a scenario with characteristics similar to those of Santiago [29].

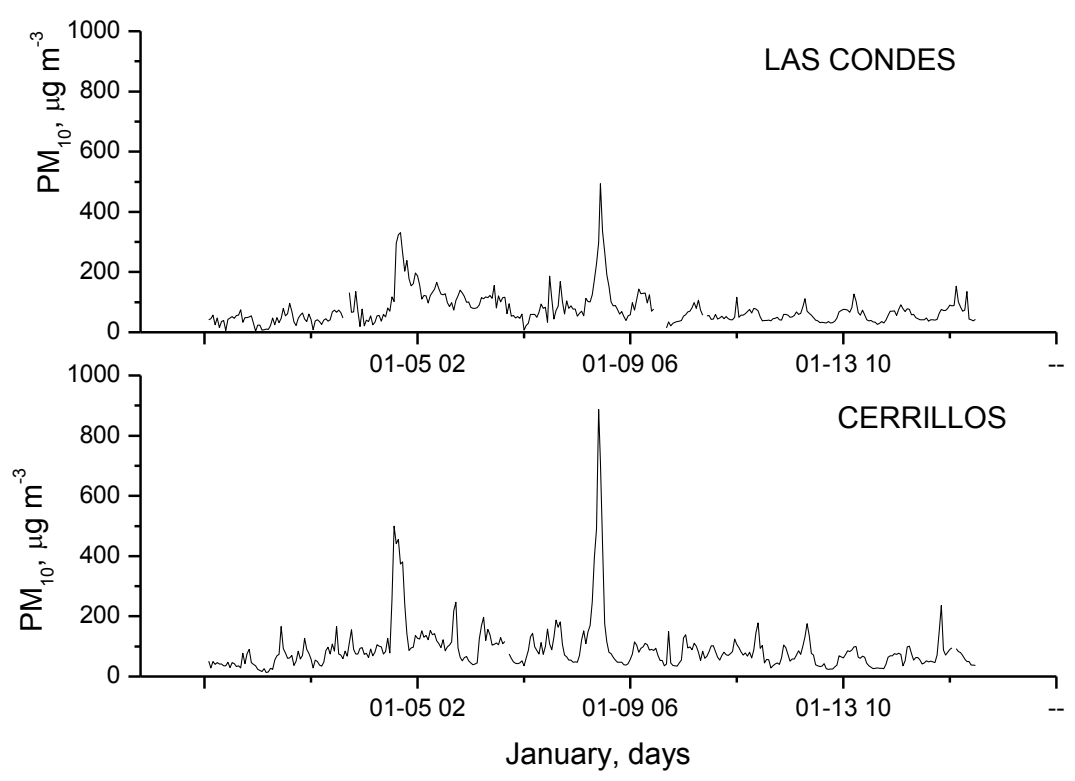

Figure 5. $\mathrm{PM}_{10}$ time profile measured in Cerrillos and las Condes from 1 to 14 January 2014.

The occurrence of nearby wildfires also changed the diurnal cycle of the pollutants. In absence of fires, the highest $\mathrm{CO}$ and $\mathrm{PM}_{10}$ concentrations in $\mathrm{POH}$ and CERR occur during the morning rush hours (7:00-8:00 a.m.) consistent with the elevated emissions from mobile sources in that period, while the maximum concentrations at LC occur around 11:00 a.m., as expected for air masses transported from downtown (Figure 6). In contrast, during days with active wildfires, the maximum $\mathrm{CO}$ and $\mathrm{PM}_{10}$ levels were attained between 15:00 and 16:00, irrespective 
of the sampling location, suggesting the dominant role of the transport processes (Figure 6).

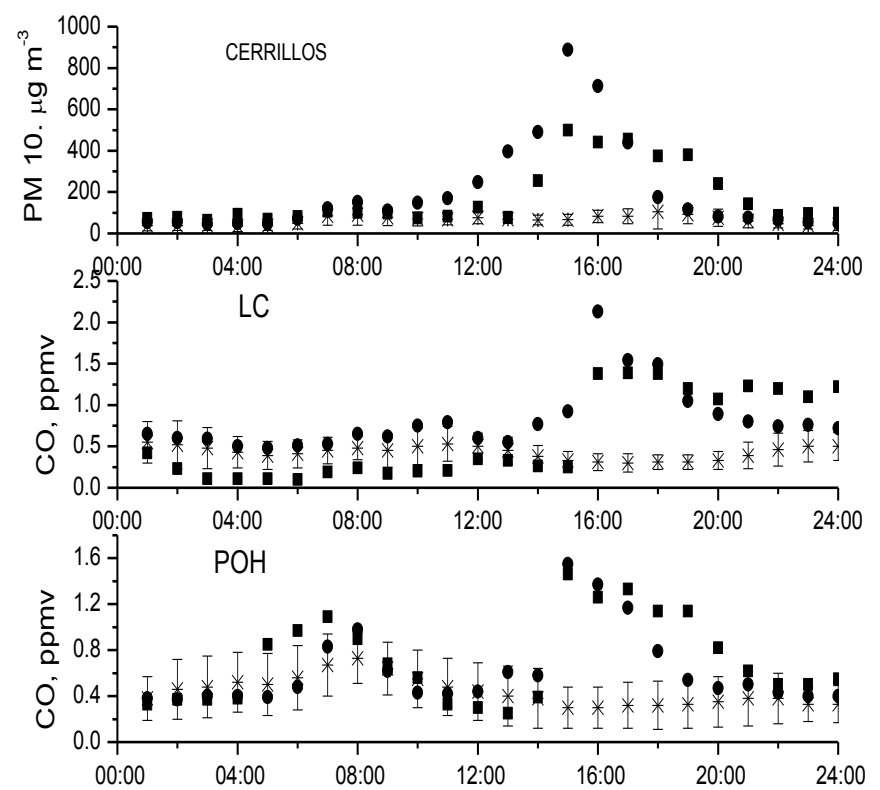

Figure 6. Comparison of data obtained in days with active fires (4 January 2014 and 8 January 2014) $(\bullet, \bullet)$ and the average of background data for days without fires during January $2014(*)$.

Table 1. Historical average maximum values (January days from years 2011, 2012 and 2013) and maximum values in days with active fires (4 January 2014 and 8 January 2014).

\begin{tabular}{|c|c|c|c|c|}
\hline Site & Day & $\mathrm{CO}$ (ppmv) & $\mathrm{PM}_{10}\left(\mu \mathrm{g} \cdot \mathrm{m}^{-3}\right)$ & $\mathrm{O}_{3}$ (ppbv) \\
\hline \multirow{3}{*}{ Cerrillos } & $\begin{array}{l}\text { Historical } \\
\text { Average }\end{array}$ & $0.61 \pm 0.09$ & $116 \pm 19$ & $45.1 \pm 4.7$ \\
\hline & 4 January 2014 & 1.84 & 493 & 127 \\
\hline & 8 January 2014 & 2.26 & 888 & 92 \\
\hline \multirow{3}{*}{$\begin{array}{l}\text { Parque } \\
\text { O'higgins }\end{array}$} & $\begin{array}{c}\text { Historical } \\
\text { Average }\end{array}$ & $0.50 \pm 0.24$ & $83.8 \pm 22.4$ & $43.4 \pm 20.0$ \\
\hline & 4 January 2014 & 1.46 & 433 & 133 \\
\hline & 8 January 2014 & 1.55 & 493 & 91 \\
\hline \multirow{3}{*}{ Las Condes } & $\begin{array}{l}\text { Historical } \\
\text { Average }\end{array}$ & $0.57 \pm 0.18$ & $109 \pm 32$ & $76 \pm 17$ \\
\hline & 4 January 2014 & 1.39 & 331 & 142 \\
\hline & 8 January 2014 & 2.13 & 493 & 105 \\
\hline
\end{tabular}




\subsection{Ozone in Wildfire Days}

Ozone concentrations were also considerably enhanced during the upwind forest fires. Since ozone concentrations depend upon the sampling place and the maximum daily temperature [30-32] the data are plotted as a function of the air temperature in Santiago at each location (Figure 7). This figure shows that nearby fires significantly contributed to the high ozone concentration all over the Santiago basin. The values obtained when fires were active are well outside the $95 \%$ confidence limit of the data obtained using the historical background reference days. The contribution of nearby fires to ozone urban levels can be estimated from the difference between measured values and those expected from the maximum daily temperature [33] included in Table 2. The extra urban ozone associated to Melipilla's wildfires went up to $65 \mathrm{ppb}$, amounting to a $100 \%$ increase. This is a value considerably larger than those reported elsewhere [3]. Furthermore, ozone concentration during wildfire days consistently surpassed the $80 \mathrm{ppb}$ limit in the three locations considered, a level considered harmful for humans, animals and vegetation $[34,35]$ even during photochemically inactive seasons [8].
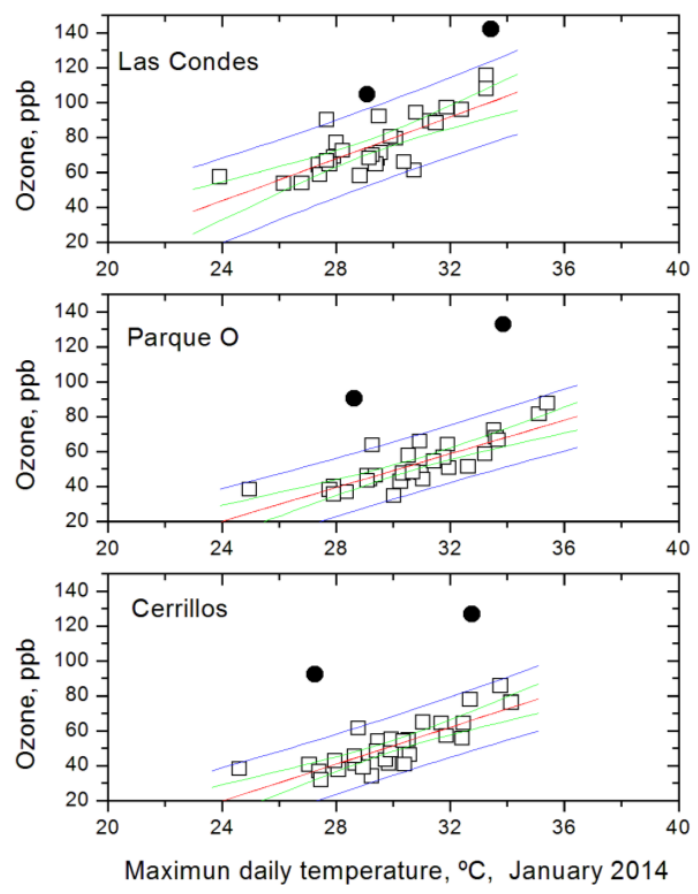

Figure 7. Lineal relationship between Ozone maximum one hour average and daily maximum temperature. ( $\square$ ) with Confidence band and Prediction band (95\%). Measurements in days with nearby fires (•). 
Similar to the evolution of other pollutants, ozone maximum concentrations took place during the late afternoon of the days with wildfire in Melipilla, approximately two hours later than in background reference days (Figure 8). Notably, at these times, there is a noticeable increase in $\mathrm{NO}_{2}$ concentrations in the aging plume (Figure 9) that can push up the ground level ozone concentration as discussed below.

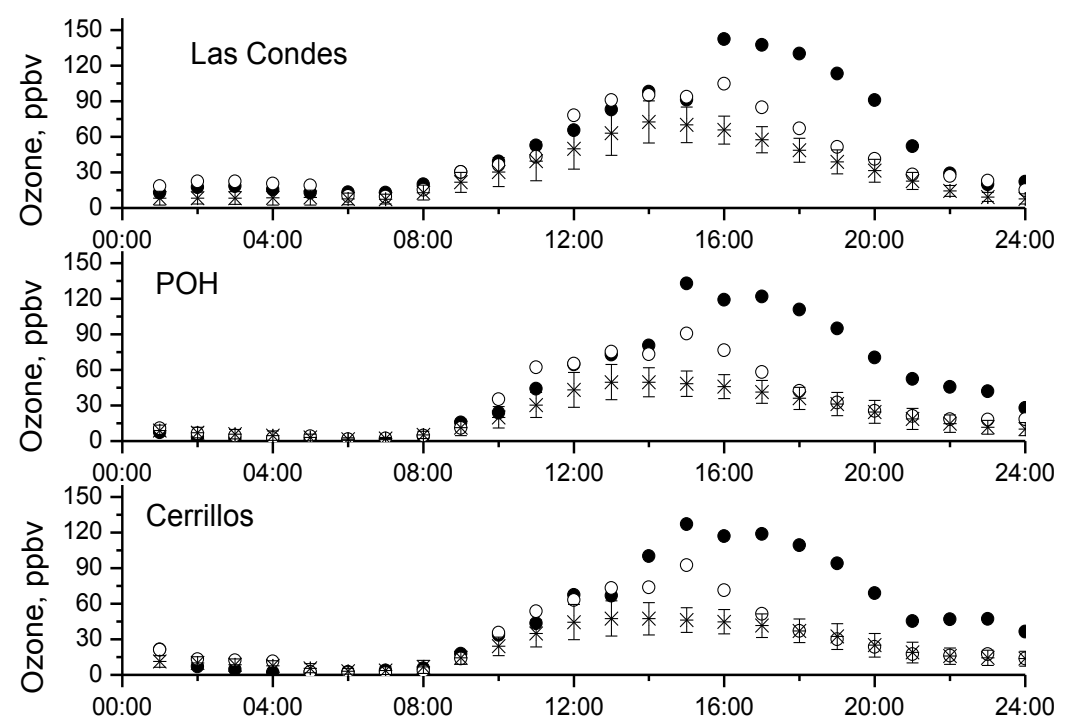

Figure 8. Ozone time profiles in background reference days $(*)$ and days with active fires in Melipilla region $(\bigcirc)(\bullet)$.

Table 2. Contribution of Melipilla's fires to ozone maximum concentration in Santiago's atmosphere (ground level).

\begin{tabular}{ccccc}
\hline Sites & $\begin{array}{c}\text { Fire } \\
\text { Day }\end{array}$ & $\begin{array}{c}\text { Calculated } \\
\text { (ppbv) }\end{array}$ & $\begin{array}{c}\text { Measured } \\
\text { (ppbv) }\end{array}$ & $\begin{array}{c}\text { Difference } \\
\text { (ppbv) }\end{array}$ \\
\hline Las Condes & 4 & 100.19 & 142.15 & 41.96 \\
ParqueO'higgins & 8 & 74.20 & 104.7 & 30.5 \\
& 4 & 67.66 & 132.8 & 65.14 \\
Cerrillos & 8 & 42.40 & 90.55 & 48.15 \\
& 4 & 66.10 & 126.83 & 60.73 \\
& 8 & 36.95 & 92.32 & 55.37 \\
\hline
\end{tabular}




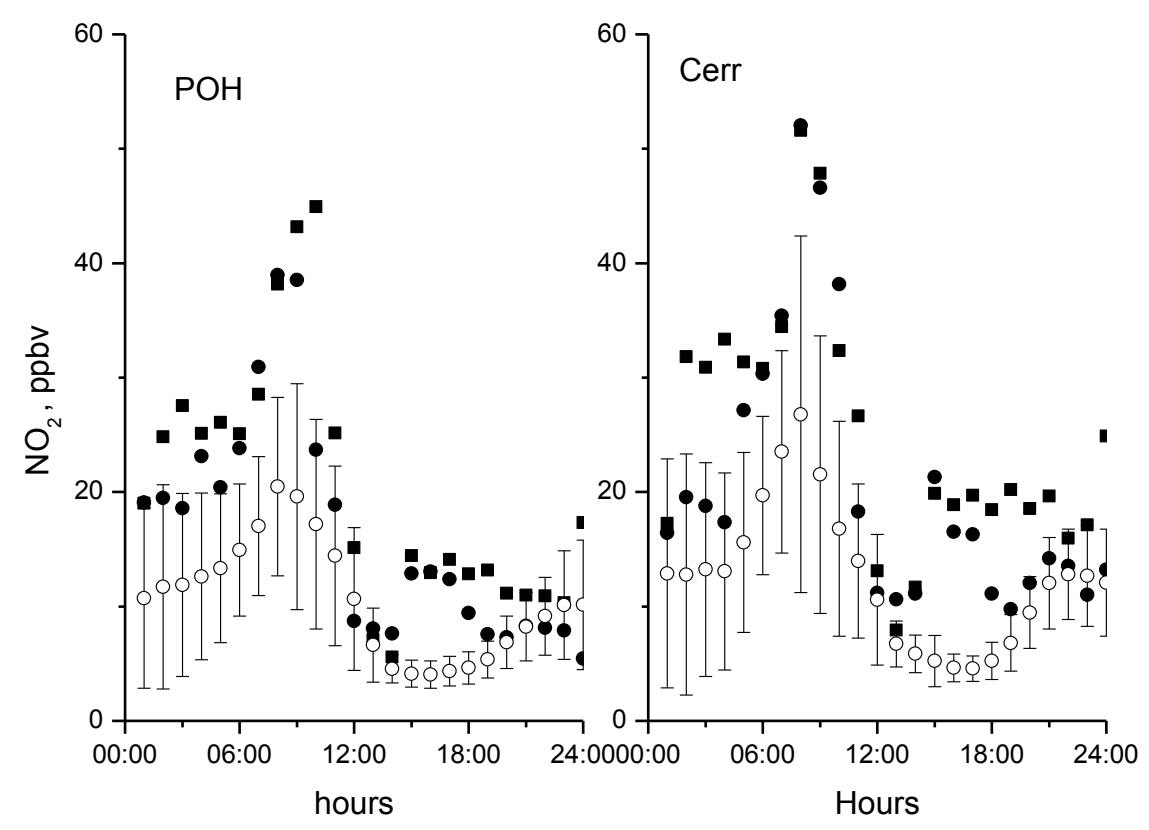

Figure 9. $\mathrm{NO}_{2}$ daily profile, reference background days ( $\square$ ) and days with active wildfires in Melipilla region. (•), (•).

\section{Discussion}

Ozone concentration in the traveling plume increases with ageing but decreases with dilution. The interplay of these two factors can increase or decrease the concentrations of the oxidants with the fire to receptor distance [15], and the ozone concentration in the plumes is depleted as often as it is enhanced [36]. Furthermore, it could be expected that, due to back reflection of solar radiation by the increased amounts of particles (Figure 4), formation of ozone in the plume originating from wildfires could be reduced, particularly at ground level. However, the data obtained in this work and in previous studies [8] show a clear increase in ozone concentrations in plumes from wildfires and at ground level in nearby cities [11].This increase can be related to:

(i) An increase in the rate of the chemical ozone production [3].

(ii) High emissions of ozone precursors, such as VOCs and NOx [4,37-39].

(iii) A reduced rate of ozone capture due to closure of plants stomata [2].

(iv) PAN decomposition as a late source of $\mathrm{NO}_{2}$ [4]. PAN is rapidly formed in smoke plumes, with $c a .40 \%$ if the initial emitted $\mathrm{NO}_{\mathrm{X}}$ being converted to PAN in the first few hours after emission, contributing to downwind ozone formation [36]. 
(v) Significant emissions of formaldehyde [37] and HONO [40-42] relevant sources of hydroxyl radicals in Santiago's atmosphere [18].

Point (i) and point (iii) should not be enough to explain the large differences observed. Since, Melipilla fires involve dry pastures, acacia caven and some eucalyptus globulus with very low ozone capture rates.

$\mathrm{NO}_{2}$ arising from PAN decomposition can be an important promoter of high ozone levels in aged plumes [4,36] but its contribution to ozone levels in cities nearby the wildfire is unlikely, given the high NOx emission rates. In fact, the "direct" emission of $\mathrm{NO}_{2}$ from wildfires has been estimated to have a $\mathrm{NO}_{2} / \mathrm{CO}$ ratio of $0.005 \pm 0.002 \mathrm{~mol} / \mathrm{mol}$ [37]. NOx emissions are determined by the amount of nitrogen present in the fuel [43] and is emitted both at the flaming and smoldering stages $[43,44]$.

Regarding the role of precursors (NOx, VOCS, formaldehyde and HONO), it has to be considered that daytime ozone steady state concentrations near to wildfires can be considered to be approximately given by Leighton's relationship:

$$
\left[\mathrm{O}_{3}\right]_{\mathrm{ss}}=\left(\mathrm{J}_{\mathrm{NO} 2} / \mathrm{k}_{\mathrm{NO}}\right)\left(\left[\mathrm{NO}_{2}\right] /[\mathrm{NO}]\right)
$$

where $\mathrm{J}_{\mathrm{NO} 2}$ is the $\mathrm{NO}_{2}$ photolysis constant and $\mathrm{k}_{\mathrm{NO}}$ is specific rate constant of $\mathrm{NO}$ plus $\mathrm{O}_{3}$ reaction. The role of the precursors in the Los Angeles type photochemical smog is to transform $\mathrm{NO}$ (the main primary NOx emitted by mobile sources) into $\mathrm{NO}_{2}$ allowing an increase in ozone concentrations. Emission $\mathrm{NO}_{2} / \mathrm{NOx}$ ratios in biomass burning are, for a variety of fuels, in the $0.1-0.3$ range [40,45]. These values are larger than those arising from vehicles, the most important NOx source in large cities [46]. During the year 2006 at the monitoring station in Wuppertal (Germany), an annual average $\mathrm{NO}_{2} / \mathrm{NOx}$ emission ratio of $0.12 \pm 0.01$ was reported by Kurtenbach et al., [47]. The same group reported, from a traffic tunnel study in 1997, a much smaller emission ratio $(0.04 \pm 0.01)$. Similarly, in mainly ozone free road tunnels in Hong Kong, an $\mathrm{NO}_{2} / \mathrm{NOx}$ ratio smaller than 0.02 was reported [48]. The relatively high $\mathrm{NO}_{2}$ / NOx emitted in biomass burning implies that high ozone concentrations could be achieved independently of precursor emissions since they are no longer necessary to photochemically generate large $\left[\mathrm{NO}_{2}\right] /[\mathrm{NO}]$ ratios and, hence, high ozone concentrations. In agreement with these considerations, at the morning rush hour of days with active fires, the ratio $\mathrm{NO}_{2} / \mathrm{NO}$ in Cerrillos was 35 and 7.5, values much higher than those measured in background reference days (3.5 and 1.4, respectively). NOx directly measured upon the flames contains approximately $10 \%$ of $\mathrm{NO}_{2}$ [43] suggesting that $\mathrm{NO}$ emission is the dominant process. However, in the fire and in the plume, local NO concentrations are very high and, at the current high temperatures, oxidation of $\mathrm{NO}$ to $\mathrm{NO}_{2}$ can be a fast process that 
increases the $\mathrm{NO}_{2} / \mathrm{NOx}$ ratio during the plume travel from the fire locus to the city (about 4-6 h), Figure 9.

The effect of the fire-derived plume upon urban ozone is complex due to incorporation of local emissions to contaminated air masses. This incorporation is not straightforward and sometimes is difficult to establish [29] and modeling has been minimally successful [14,49-51]. However, this incorporation of contaminants in the travelling plume does not seem to be relevant in the present scenario. The reported data show that ozone values measured in Cerrillos are very similar to those found at $\mathrm{POH}$. If the location of Cerrillos at the entrance of the plume to Santiago's basin is considered, it can be concluded that the urban ozone is mostly explained in terms of the chemistry taking place during the transfer from the fire to the city and is minimally influenced by local emissions. In this regard, it is interesting to note that on normal days the maximum ozone in the city occurs about six hours after the rush hours. This delay time is of the same order of magnitude as that necessary to bring to the city the air masses charged with wildfire emissions. Interestingly, the presence of the plume decreases the difference between LC and the other stations (Table 2) that generally present smaller ozone concentrations.

\section{Conclusions}

Major forest fires in the Melipilla sector in central Chile increased ozone concentrations in the urban atmosphere of Santiago, reaching values higher than $80 \mathrm{ppb}$ (the national one hour average limit). This increase took place at the three sampling sites considered and is explained in terms of a large $\mathrm{NO}_{2} / \mathrm{NO}$ ratio in biomass burning emissions, the age of the plume reaching Santiago, and the high initial concentrations of ozone precursors, such as $\mathrm{NOx}, \mathrm{VOC}$ and $\mathrm{OH} \bullet$ radical sources, such as formaldehyde and $\mathrm{HONO}$, present in the urban atmosphere.

Acknowledgments: This work has been supported by DICYT-USACH 021541RC and CEDENNA-USACH. RG is partially supported by FONDAP Grant 15110009.

Author Contributions: Rubio, Lissi and Gramsch performed the air-chemistry analysis. Garreaud provided the meteorological context.

Conflicts of Interest: The authors declare no conflict of interest.

\section{References}

1. Real, E.; Law, K.S.; Weinzierl, B.; Fiebig, M.; Petzold, A.; Wild, O.; Methven, J.; Arnold, S.; Stohl, A.; Huntrieser, H.; et al. Processes influencing ozone levels in Alaskan forest fire plumes during long-range transport over the North Atlantic. J. Geophys. Res. 2007, 112, $1-19$. 
2. Hodnebrog, O.; Solberg, S.; Stordal, F.; Svendby, T.M.; Simpson, D.; Gauss, M.; Hilboll, A.; Pfister, G.G.; Turquety, S.; Richter, A.; et al. Impact of forest fires, biogenic emissions and high temperatures on the elevated Eastern Mediterranean ozone levels during the hot summer of 2007. Atmos. Chem. Phys. 2012, 12, 8727-8750.

3. Martins, V.; Miranda, A.I.; Carvalho, A.; Schaap, M.; Borrego, C.; Sá, E. Impact of forest fires on particulate matter and ozone levels during the 2003, 2004 and 2005 fire seasons in Portugal. Sci. Total Environ. 2012, 414, 53-62.

4. Jaffe, D.A.; Wigder, N.L. Ozone production from wildfires: A critical review. Atmos. Environ. 2012, 51, 1-10.

5. Val Martin, M.; Honrath, R.E.; Owen, R.C.; Pfister, G.; Fialho, P.; Barata, F. Significant enhancements of nitrogen oxides, black carbon, and ozone in the North Atlantic lower free troposphere resulting from North American boreal wildfire. J. Geophys. Res. 2006, 111, D23S60.

6. Suarez, L.; Castillo, L.; Marín, M.; Carrillo, G.; Rímac, L.; Pomalaya, J.; Penacho, R. Study of the seasonal variation of tropospheric ozone and aerosols related to the biomass burning in Amazonian. Mosaico. Cient. 2006, 3, 1-5.

7. Morris, G.A.; Hersey, S.; Thompson, A.M.; Pawson, S.; Nielsen, J.E.; Colarco, P.R.; Wallace, M.S.; McMillan, W.W.; Stohl, A.; Turquety, S.; et al. Alaskan and Canadian forest fires exacerbate ozone pollution over Houston, Texas, on 19 and 20 July 2004. J. Geophys. Res. 2006, 111.

8. Pfister, G.G.; Wiedinmyer, C.; Emmons, L.K. Impacts of the fall 2007 California wildfires on surface ozone: Integrating local observations with global model simulations. Geophys. Res. Lett. 2008, 35.

9. Mebust, A.K.; Cohen, R.C. Space-based observations of fire NOx emission coefficients: A global biome-scale comparison. Atmos. Chem. Phys. 2014, 14, 2509-2524.

10. Evans, L.F.; King, N.K.; Packham, D.R.; Stephens, E.T. Ozone measurements in smoke from forest fire. Environ. Sci. Technol. 1974, 8, 75-76.

11. Cheng, L.; McDonald, K.M.; Angle, R.P.; Sandhu, H.S. Forest fire enhanced photochemical air pollution. A case study. Atmos. Environ. 1998, 32, 673-681.

12. Chalbot, M.C.; Kavouras, I.C.; Dubois, D.W. Assessment of the contribution of wildfires in ozone concentrations in the Central U: Mexico Border Region. Aerosol Air Qual. Res. 2013, 13, 838-848.

13. Lei, W.; Molina, L.T. Modelling the impact of biomass burning on air quality in and around Mexico City. Atmos. Chem. Phys. 2013, 13, 2299-2319.

14. Crounse, J.D.; DeCarlo, P.F.; Blake, D.R.; Emmons, L.K.; Campos, E.C.; Apel, E.C.; Clarke, A.D.; Weinheimer, A.J.; McCabe, D.C.; Yokelson, R.J.; et al. Biomass burning and urban air pollution over the Central Mexican Plateau. Atmos. Chem. Phys. 2009, 9, 4929-4944.

15. Jaffe, D.A.; Chand, D.; Hafner, W.; Westerling, A.; Spracklen, D. Influence of fires on $\mathrm{O}_{3}$ concentrations in the Western U.S. Environ. Sci. Technol. 2008, 42, 5885-5891.

16. Garreaud, R. The Andes climate and weather. Adv. Geosci. 2009, 7, 1-9. 
17. Rappengluek, B.; Schmitz, R.; Bauerfeind, M.; Cereceda-Balic, F.; von Baer, D.; Jorquera, H.; Silva, Y.; Oyola, P. An urban photochemistry study in Santiago de Chile. Atmos. Environ. 2005, 39, 2913-2931.

18. Elshorbany, Y.F.; Kleffmann, J.; Kurtenbach, R.; Lissi, E.; Rubio, M.A.; Villena, G.; Gramsch, E.; Rickard, A.R.; Pilling, M.J.; Wiesen, P. Summertime photochemical ozone formation in Santiago, Chile. Atmos. Environ. 2009, 43, 6398-6407.

19. Romero, H.; Ihl, M.; Rivera, A.; Zalazar, P.; Azocar, P. Rapid urban growth, land-use changes and air pollution in Santiago, Chile. Atmos. Environ. 1999, 33, 4039-4047.

20. Rubio, M.A.; Lissi, E.; Villena, G.; Caroca, V.; Gramsch, E.; Ruiz, A. Estimation of hydroxyl and hydroperoxyl radicals concentrations in the urban atmosphere of Santiago. J. Chil. Chem. Soc. 2005, 50, 375-379.

21. Rutllant, J.; Garreaud, R. Episodes of strong flow down the western slope of the subtropical Andes. Mon. Weather Rev. 2004, 132, 611-622.

22. Armesto, J.; Arroyo, M.T.K.; Hinojosa, L.F. The Mediterranean enviroment of central Chile. In The Physical Geography of South America; Veblen, T., Young, K., Orme, A., Eds.; Oxford University Press: Oxford, UK, 2007.

23. Schulz, J.J.; Cayuela, L.; Echeverria, C.; Salas, J. Monitoring land cover change of the dryland landscape of Central Chile (1975-2008). Appl. Geogr. 2010, 30, 436-447.

24. Krishna, M.T.; Chauhan, A.J.; Freww, A.J.; Holgate, S.T. Toxicological mechanisms underling oxidant pollutants-induced airway injury. Rev. Environ. Health 1998, 13, 59-61.

25. Yang, W.; Omaye, S.T. Air Pollutants oxidative stress and human health. Mutat. Res. Genet. Toxicol. Environ. Mutagen. 2009, 674, 45-54.

26. Shindell, D.; Kuylenstierna, J.C.I.; Vignati, E.; van Dingenen, R.; Amann, M.; Klimont, Z.; Anenberg, S.C.; Muller, N.; Janssens-Maenhout, G.; Raes, F.; et al. Simultaneous mitigating near-term climate change and improving human health and food security. Science 2012, 335, 183-189.

27. Muñoz, R.C.; Alcafuz, R. Variability of urban aerosols over Santiago, Chile: Comparison of surface PM10 concentrations and remote sensing with ceilometer and lidar. Aerosol Air Qual. Res. 2012, 12, 8-19.

28. SINCA 2014. National Service of Air Quality Information. Available online: http:// SINCA.mma.gob.cl (accessed on 1 November 2015).

29. Olcese, L.E.; Toselli, B.M. Unexpected high levels of ozone measured in Cordoba, Argentina. J. Atmos. Chem. 1998, 31, 269-279.

30. Rubio, M.A.; Oyola, P.; Gramsch, E.; Lissi, E.; Pizarro, J.; Villena, G. Ozone and peroxyacetylnitrate in downtown Santiago, Chile. Atmos. Environ. 2004, 38, 4931-4939.

31. Khoder, M.I. Diurnal seasonal and weekdays-weekend variations of ground level ozone concentrations in an urban area in greater Cairo. Environ. Monit. Assess. 2009, 149, 349-362.

32. Im, U.; Markakis, K.; Poupkou, A.; Melas, D.; Unal, A.; Gerasopoulos, E.; Daskalakis, N.; Kindap, T.; Kanakidou, M. The impact of temperature changes on summer time ozone and its precursors in the Eastern Mediterranean. Atmos. Chem. Phys. 2011, 11, 3847-3864. 
33. Rubio, M.A.; Lissi, E.A. Temperature as thumb rule predictor of ozone levels in Santiago de Chile ground air. J. Chil. Chem. Soc. 2014, 59, 2427-2431.

34. Krupa, S.V.; Kickert, R.V. The greenhouse effect: Impact of ultraviolet B radiation, carbon dioxide and ozone on vegetation. Environ. Pollut. 1989, 61, 263-393.

35. Bell, M.L.; McDermontt, A.; Zeger, S.L.; Samet, J.M.; Dominici, F. Ozone and short term mortality in 95 U.S. urban communities. JAMA 2004, 292, 2372-2378.

36. Alvarado, M.J.; Logan, J.A.; Mao, J.; Apel, E.; Riemer, D.; Blake, D.; Cohen, R.C.; Min, K.E.; Perrin, A.E.; Browne, E.C.; et al. Nitrogen oxides and PAN in plumes from boreal fires during ARCTAS-B and their impact on ozone: An integrated analysis of aircraft and satellite observations. Atmos. Chem. Phys. 2010, 10, 9739-9760.

37. Young, E.; Paton-Walsh, C. Emission ratios of the tropospheric Ozone precursors nitrogen dioxide and formaldehyde from Australia's black Saturday fires. Atmosphere 2011, 2, 617-632.

38. Konovalov, I.B.; Beeckmann, M.; Kuznetsova, I.N.; Yurova, A.; Zvyagintsev, A.M. Atmospheric impacts of the 2010 Russian wildfires: Integrating modeling and measurements of an extreme air pollution episode in the Moscow region. Atmos. Chem. Phys. 2011, 11, 10031-10056.

39. Schreier, S.F.; Richter, A.; Kaiser, J.W.; Burrows, J.P. The empirical relationship between satellite-derived tropospheric $\mathrm{NO}_{2}$ and fire radiative power and possible implications for fire emissions rates of NOx. Atmos. Chem. Phys. 2014, 14, 2447-2466.

40. Burling, I.R.; Yokelson, R.J.; Griffith, D.W.T.; Johnson, T.J.; Veres, P.; Roberts, J.M.; Warneke, C.; Urbanski, S.P.; Reardon, J.; Weise, D.R.; et al. Laboratory measurements of trace gas emissions from biomass burning of fuel types from the south eastern and south western United States. Atmos. Chem. Phys. 2010, 10, 11115-11130.

41. Roberts, J.M.; Veres, P.; Warneke, C.; Neuman, J.A.; Washenfelder, R.A.; Brown, S.S.; Baasandorj, M.; Burkholder, J.B.; Burling, I.R.; Johnson, T.J.; et al. Measurement of $\mathrm{HONO}, \mathrm{HNCO}$, and other inorganic acids by negative ion proton-transfer chemical-ionization mass spectrometry (NI-PT-CIMS): Application to biomass burning emissions. Atmos. Meas. Tech. 2010, 3, 981-990.

42. Nie, W.; Ding, A.J.; Xie, Y.N.; Xu, Z.; Mao, H.; Kerminen, V.; Zheng, L.F.; Qi, X.M.; Yang, X.Q.; Sun, J.N.; et al. Influence of biomass burning plumes on HONO chemistry in eastern China. Atmos. Chem. Phys. Discuss. 2014, 14, 7859-7887.

43. Andreae, M.O.; Merlet, P. Emissions of trace gases and aerosols from biomass burning. Glob. Biogeochem. Cycles. 2001, 15, 955-966.

44. Contreras-Moctezuma, J.; Rodriguez-Trejo, D.A.; Retama-Hernandez, A.; Sanchez-Rodriguez, J.J.M. Smoke gases of wildfires in pinus hartwegii Forest. Agrociencia 2003, 37, 309-316.

45. Yokelson, R.J.; Griffith, D.W.T.; Ward, D.E. Open-path Fourier transforms infrared studies of large scale laboratory biomass fires. J. Geophys. Res. 1996, 101, 21067-21080.

46. Wu, Y.; Zhang, S.J.; Li, L.M.; Ge, Y.S.; Shu, J.W.; Zhou, Y.; Xu, Y.Y.; Hu, J.N.; Liu, H.; $\mathrm{Fu}, \mathrm{L} . \mathrm{X}$; et al. The challenge to NOx emission control for heavy-duty diesel vehicles in China. Atmos. Chem. Phys. 2012, 12, 9365-9379. 
47. Kurtenbach, R.; Kleffmann, J.; Niedojadlo, A.; Wiesen, P. Primary $\mathrm{NO}_{2}$ emissions and their impact on air quality in traffic environments in Germany. Environ. Sci. Eur. 2012, 24, 2-8.

48. Yao, X.; Lau, N.T.; Chan, C.K.; Fang, M. The use of tunnel concentration profile data to determine ratio of NO2/ NOx directly emitted from vehicles. Atmos. Chem. Phys. Discuss. 2005, 5, 12723-12740.

49. Bein, K.J.; Zhao, Y.; Johnston, M.V.; Wexler, A.S. Interactions between boreal wildfire and urban emissions. J. Geophys. Res. 2008, 113, DO 7304.

50. Singh, H.B.; Cai, C.; Kaduwela, A.; Weinheimer, A.; Wisthaler, A. Interaction of fire emissions and urban pollution over California: Ozone formation and air quality simulations. Atmos. Environ. 2012, 56, 45-51.

51. Wigder, N.L.; Jaffe, D.A.; Saketa, F.A. Ozone and particulate matter enhancements from regional wildfires observed at Mount Bachelor during 2004-2011. Atmos. Environ. 2013, 75, 24-31. 


\title{
Frequency and Character of Extreme Aerosol Events in the Southwestern United States: A Case Study Analysis in Arizona
}

\author{
David H. Lopez, Michael R. Rabbani, Ewan Crosbie, Aishwarya Raman, \\ Avelino F. Arellano Jr. and Armin Sorooshian
}

\begin{abstract}
This study uses more than a decade's worth of data across Arizona to characterize the spatiotemporal distribution, frequency, and source of extreme aerosol events, defined as when the concentration of a species on a particular day exceeds that of the average plus two standard deviations for that given month. Depending on which of eight sites studied, between $5 \%$ and $7 \%$ of the total days exhibited an extreme aerosol event due to either extreme levels of $\mathrm{PM}_{10}, \mathrm{PM}_{2.5}$, and/or fine soil. Grand Canyon exhibited the most extreme event days (120, i.e., $7 \%$ of its total days). Fine soil is the pollutant type that most frequently impacted multiple sites at once at an extreme level. $\mathrm{PM}_{10}, \mathrm{PM}_{2.5}$, fine soil, non-Asian dust, and Elemental Carbon extreme events occurred most frequently in August. Nearly all Asian dust extreme events occurred between March and June. Extreme Elemental Carbon events have decreased as a function of time with statistical significance, while other pollutant categories did not show any significant change. Extreme events were most frequent for the various pollutant categories on either Wednesday or Thursday, but there was no statistically significant difference in the number of events on any particular day or on weekends versus weekdays.
\end{abstract}

Reprinted from Atmosphere. Cite as: Lopez, D.H.; Rabbani, M.R.; Crosbie, E.; Raman, A.; Arellano, A.F., Jr.; Sorooshian, A. Frequency and Character of Extreme Aerosol Events in the Southwestern United States: A Case Study Analysis in Arizona. Atmosphere 2016, 7, 1.

\section{Introduction}

Severe aerosol pollution events pose a major threat to society due to significant reductions in visibility and air quality, in addition to adverse impacts on public health and daily operations. Fine particulate matter $\left(\mathrm{PM}_{2.5}\right)$ is linked to various health impacts regardless of whether there is chronic or acute exposure, with effects ranging from lung cancer to cardiovascular disease [1,2]. Extreme pollution events are thought to be especially important with regard to annual acute mortality [3], in addition to leading to temporary shutdown of daily activities such as school and work in parts of the world [4]. Semi-arid and arid regions are particularly vulnerable to such events due to dust emissions, and this is especially dramatic 
during haboob events [5-7]. In recent decades, the Southwestern United States (Southwest) has experienced significant population growth, land use change, and is moving towards a more arid regime with higher temperatures, less precipitation, and lower soil moisture [8]. These changes promote increased dust emissions $[9,10]$ and wildfires [11,12], with a rapidly growing population left vulnerable to the effects of the emissions. These issues coupled to the impact of dust and wildfire emissions on the hydrologic cycle and snowpack behavior at higher altitudes in the Southwest $[9,13]$ warrants an examination of extreme aerosol events.

An ideal location to study extreme aerosol events is Arizona, which represents a state in the Southwest that is impacted by both dust and wildfires, in addition to having one of the fastest growing populations in the United States that is prone to the effects of poor air quality. The absolute population growth between 2000 and 2009, in Tucson and Phoenix, the two largest cities in Arizona, rank as the $33^{\text {rd }}$ and $4^{\text {th }}$ largest in the United States, respectively (U.S. Census Bureau, 2009). Sources of wind-blown dust impacting this and other Southwest states include naturally un-vegetated or anthropogenically disturbed soil surfaces, such as dry lakes ("playas"), dry washes, gravel pits, construction sites, oil and gas development sites, fields (after harvest), and long-range transport of Asian dust [14-20].

Aside from the ubiquity of dust in the Southwest, the greater Western United States is becoming increasingly vulnerable to the effects of wildfires owing to both a warmer climate and fire-control strategies over past decades resulting in conditions that promote larger and more frequent fires $[11,21]$. Depending on the fuel type and burning conditions, biomass burning leads to extensive emissions of various gaseous (e.g., nitrogen oxides $\left(\mathrm{NO}_{\mathrm{x}}\right)$, ozone $\left(\mathrm{O}_{3}\right)$, carbon monoxide $(\mathrm{CO})$, Volatile Organic Compounds (VOCs)) and particulate species (e.g., Elemental Carbon (EC), Organic Carbon (OC), inorganics), but also soil emissions due to lofting of soil in areas of turbulent mixing surrounding flames [22-24].

The goal of this study is to examine long-term data (2001-2014) from the EPA IMPROVE network across Arizona to characterize the frequency, spatial range, and origin of extreme aerosol events. The following questions are addressed: (i) what is the frequency of extreme aerosol events across Arizona and how many are due to EC-enriched air masses, Asian dust, non-Asian dust, or some other source? (ii) how frequently do these events occur at all or subsets of the study sites on the same day? and (iii) how are these events distributed between months of the year, days of the week, and inter-annually? 


\section{Experimental Methods}

\subsection{EPA IMPROVE}

This study utilizes aerosol composition data from the Interagency Monitoring of Protected Visual Environments (IMPROVE) network [25]. IMPROVE aerosol monitoring stations are located primarily in National Parks and Wilderness Areas and collect ambient aerosol on filters over a period of $24 \mathrm{~h}$ every third day. Samples are analyzed for ions, metals, Organic Carbon (OC) and Elemental Carbon (EC). Among the elemental measurements, X-Ray Fluorescence (XRF) is used for Fe and heavier elements while Particle-Induced X-Ray Emission (PIXE) is used for elements $\mathrm{Na}$ to $\mathrm{Mn}$. Fine soil concentrations reported in this study are calculated using the following equation [25]:

$$
\text { Fine Soil }\left(\mu \mathrm{g} \cdot \mathrm{m}^{-3}\right)=2.2[\mathrm{Al}]+2.49[\mathrm{Si}]+1.63[\mathrm{Ca}]+2.42[\mathrm{Fe}]+1.94[\mathrm{Ti}]
$$

with regard to this equation, the components and their contributions were previously confirmed in comparisons of local re-suspended soils and ambient particles in the Western United States [25]. As this study is concerned with extreme concentrations of fine soil, it is expected that this equation can successfully capture all soil-rich air masses regardless of whether minor variations exist in the factors used in Equation (1). Species mass concentrations discussed in this study are from the fine fraction of aerosols $\left(\mathrm{PM}_{2.5}\right)$. Sampling protocols and additional details are provided elsewhere [26].

In this study, we use data from eight sites in Arizona (see map in Figure 1) and over time spans ranging from as early as data were possible starting in January 2001 until August 2014 (Table 1). Three of the eight sites are impacted more significantly by urban emissions owing to their closer proximity to populated cities; the Phoenix site is centrally located in the metropolitan area of the most populated city in Arizona, while Saguaro National Monument and Saguaro West are separated by $\sim 50 \mathrm{~km}$ and are on the east and west sides, respectively, of Tucson, which is the second largest city. Chiricahua National Monument is a high-altitude site that is in a remote vegetated area with the nearest major urban area being Tucson ( 150 km to the west). Nearby aerosol sources include the Willcox Playa and the Apache Power Plant, which are $\sim 45 \mathrm{~km}$ to the west. This site is the closest to the Chihuahuan Desert. Tonto is $\sim 90 \mathrm{~km}$ to the east/northeast of Phoenix and Queen Valley is $\sim 60 \mathrm{~km}$ to the east of Phoenix, and thus these two sites are vulnerable to emissions transported from Phoenix. Organ Pipe is near the border of the United States and Mexico and is vulnerable to dust emissions and anthropogenic emissions from the nearby town of Sonoita, Mexico ( 10,000 inhabitants). Grand Canyon is in a remote site in Northern Arizona and is removed from anthropogenic emissions. 
Table 1. Summary of IMPROVE sites and date ranges over which data are analyzed.

\begin{tabular}{lcccc}
\hline Site Name & Latitude $^{\left({ }^{\circ}\right.}$ ) & Longitude $\left(^{\circ}\right)$ & Altitude $(\mathbf{m})$ & Date Range \\
\hline Chiricahua (Chi) & 32.0994 & -109.389 & 1554 & January 2001-August 2014 \\
Grand Canyon (GC) & 35.9731 & -111.9841 & 2267 & January 2001-August 2014 \\
Organ Pipe (OP) & 31.9506 & -112.8016 & 504 & December 2002-August \\
Phoenix (Ph) & 33.5038 & -112.096 & 342 & April 2001-August 2014 \\
Queen Valley (QV) & 33.2939 & -111.2858 & 661 & April 2001-August 2014 \\
Saguaro NM (SNM) & 32.1746 & -110.737 & 941 & April 2001-August 2014 \\
Saguaro West (SW) & 32.2486 & -111.2178 & 714 & October 2001-August 2014 \\
Tonto (Ton) & 33.6548 & -111.1068 & 775 & January 2001-August 2014 \\
\hline
\end{tabular}

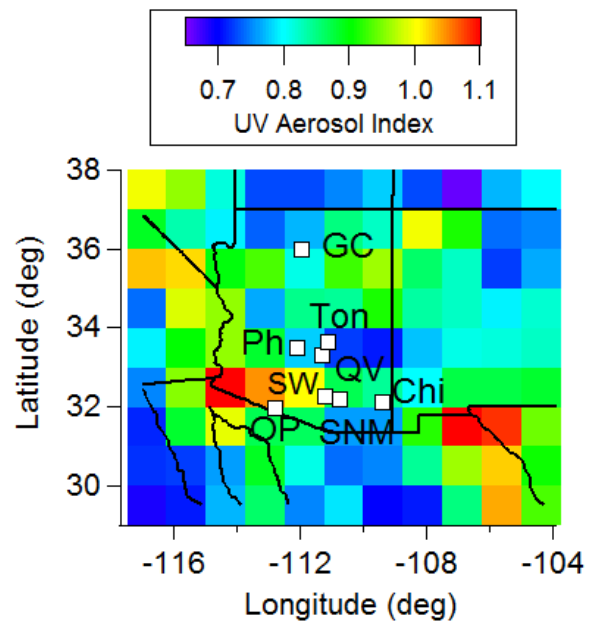

Figure 1. Spatial map of the eight EPA IMPROVE stations examined in Arizona overlaid on a four year average (2005-2008) of OMI ultraviolet aerosol index data, which includes influence from light-absorbing aerosol constituents, such as dust and smoke.

\subsection{NAAPS Aerosol Model}

Simulation data providing information about long-range dust transport are obtained from the Navy Aerosol Analysis and Prediction system (NAAPS; http:/ / www.nrlmry.navy.mil/aerosol_web/). NAAPS relies on global meteorological fields from the Navy Operational Global Atmospheric Prediction System (NOGAPS) $[27,28]$ analyses and provides output at a spatial resolution of $1^{\circ} \times 1^{\circ}$, at six hour intervals, and with 24 vertical levels reaching $100 \mathrm{mb}$ [29]. NAAPS has been used extensively to study intercontinental transport of dust to North America e.g., [18,29-32]. Sources of dust are defined in NAAPS using the USGS Land Cover Characteristics Database, which was created with Advanced Very High Resolution Radiometer (AVHRR) data. TOMS aerosol index data was used to further refine dust 
source regions. Dust emission occurs when the friction velocity exceeds a threshold value (value depending on land type) and when the surface moisture and snow depth are lower than a critical value ( 0.3 and $0.4 \mathrm{~cm}$, respectively). The model operationally assimilates remotely-sensed aerosol optical depth (AOD) data from MODIS [33].

\subsection{Satellite Data}

Ultraviolet aerosol index (UV AI) data are obtained from the Ozone Monitoring Instrument (OMI). Data were obtained at a resolution $1^{\circ} \times 1.25^{\circ}$ using a minimum threshold value of 0.5 [34]. The UV AI parameter serves as a proxy for absorbing aerosol particles [35], which are predominantly comprised of smoke and dust. Figure 1 shows a spatial map of a four-year average of OMI data to provide a backdrop of where light-absorbing aerosol particles (primarily dust in study region) are most abundant relative to where the eight IMPROVE stations are located.

\subsection{Criteria for Events}

In past work, criteria to define an extreme aerosol event have included the use of a cutoff threshold of a parameter value (i.e., average $\pm i \times$ standard deviation, with $i$ starting at 1 and increasing; e.g., [36]) or when parameter values were below and above specific quantile values (e.g., 3). Here we take a similar approach to define an extreme event for specific aerosol parameters (e.g., $\mathrm{PM}_{2.5}, \mathrm{PM}_{10}$, and fine soil) as when the measured concentration on a given day at any of the eight sites exceeds the average concentration plus two times the standard deviation for the month in which the event occurred over the time range of data used for that particular site. This criterion leads to concentrations that exceed the $90^{\text {th }}$ percentile of mass concentrations in each category. The choice of this criterion reflects a balance between removing sensitivity to month-dependent factors and being sufficiently strict to isolate only a few cases that were the most polluted. The conclusions of this study, especially the number of extreme days in the various categories presented, are sensitive to the criteria definition. The numerical threshold criteria values (i.e., average $+2 \times$ standard deviation) for each site and month are shown in Table S1 (Supplementary Material).

Those events with extreme fine soil concentrations are referred to hereafter as extreme dust events. However, it is noted that extreme $\mathrm{PM}_{10}$ events that did not reach extreme fine soil levels could have also been due to dust that was concentrated in coarse aerosol $\left(D_{p} \geqslant 2.5 \mu \mathrm{m}\right)$. Extreme fine soil events are further classified as having influence from Asia or not using output from the NAAPS model to validate long-range transport to the study region. The criteria for Asian dust was to observe a clear aerosol plume being advected from Asia to Arizona with multiple repeated NAAPS output plots as depicted in Figure S1 of the Supplement. It is cautioned that this classification scheme using NAAPS has limitations in that (i) the dust transport 
results are driven by a model rather than fully by observations, and (ii) the relative influence of Asian dust versus local sources is uncertain. Thus, although the term "Asian dust" is used subsequently, this is not meant to indicate that the fine soil measurement is fully due to long-range transport of dust from Asia. A suite of previous studies discussing source attribution of aerosol to long-range transport from Asia to North America have relied on NAAPS. For example, Cottle et al. [31] used NAAPS with HYSPLIT back-trajectories, and sunphotometer and lidar data to show that springtime dust plumes from Asia reached North America. Wu et al. [32], more recently, used NAAPS and remotely-sensed data from CALIPSO to study a trans-Pacific Asian dust event and its impact on the east coast of the United States. McKendry et al. [30] relied on the internal consistency between NAAPS and variety of other tools such as another global chemical model (GEOS-Chem) and surface and satellite observations to trace large dust plumes to their sources in areas, such as North Africa. The consistency between NAAPS and the other aforementioned resources provides confidence in the former for the purposes of source attribution of dust to Asia.

A category termed "High EC" is defined as when both $\mathrm{PM}_{2.5}$ and EC exhibit extreme levels. These events likely stem from anthropogenic sources and biomass burning events owing to the high levels of EC (as compared to other emission sources) and predominantly accumulation mode particles in wildfires [37]. Events that do not qualify as being extreme fine soil or High EC events are considered as "Other".

It is cautioned that the number of extreme events reported between 2001 and 2014 represents an underestimate since data is used only up through August 2014 and only starts in January 2001 for three sites with the most delayed start time being for Organ Pipe in December 2002.

\section{Results and Discussion}

\subsection{Frequency and Categorization of Events}

Of the total number of days when data were available in the time ranges in Table 1 (i.e., 1431-1664 depending on site), between 76 and 120 total days were characterized by some type of extreme event (i.e., $\mathrm{PM}_{10}, \mathrm{PM}_{2.5}$, and/or fine soil) depending on the site (Table 2). This number of days of extreme events corresponds to between $5 \%$ and $7 \%$ of the total days examined. Grand Canyon exhibited the most extreme event days $(120$, i.e., $7 \%$ of its total days), which is coincident with it being one of the most recognized tourism spots in the Southwest.

Relative to the total days with extreme $\mathrm{PM}_{2.5}$ levels, Grand Canyon exhibited the highest percentage in the High EC category ( $47 \%$ versus $13 \%-25 \%$ for other sites). Of the total number of days with extreme fine soil (54-69 days depending on the site), the number of these events being linked to Asian dust ranged from 19\% to $29 \%$ 
(i.e., 10-20 days). The total number of days with extreme events classified as Other (i.e., not High EC or fine soil events) ranged from 17 to 30 days, which represents between $21 \%$ and $33 \%$ of the total extreme days depending on the site. The fact that the highest percentage of Other days were at Phoenix (31\%) and Saguaro West (33\%), the most urban-impacted sites among those studied, suggests that anthropogenic pollution, including anthropogenic dust, contributes to these events. Queen Valley also reached $31 \%$, reflective of possible impact from transported pollution from the major nearby urban center Phoenix. Between 7 and 22 days in the Other category also registered extreme values of Coarse Mass $\left(\mathrm{CM}=\mathrm{PM}_{10}-\mathrm{PM}_{2.5}\right)$, supporting the possibility of influence from locally generated dust.

To gain a sense of the spatial extent of pollution registering as extreme events, Table 3 shows how many sites experienced an extreme event for a specific pollutant category on the same day. Locally produced aerosol would not be expected to impact multiple sites at an extreme level as compared to a transported plume such as from Asia. Grand Canyon is farther removed from the other seven sites that are clustered closer in Southern Arizona, and, thus, Grand Canyon exhibits the highest number of days where an extreme event only impacted that site. Computed as a percentage of all extreme days registered for a particular pollutant type, Grand Canyon was the only site impacted out of $65 \%, 64 \%, 51 \%, 100 \%$, and $84 \%$ of its extreme events for $\mathrm{PM}_{10}, \mathrm{PM}_{2.5}$, fine soil, High EC, and Other, respectively. The categories with the least number of extreme events impacting five or more sites were High EC (0 days for all sites) and Other ( $0-1$ day depending on site). This result is thought to be due to locally generated pollution from either (i) some combination of biomass burning and anthropogenic activity (for High EC) or (ii) dust (for Other) that was not regional in nature. Fine soil events conversely impacted five or more sites on between 13 and 23 days, accounting for between $16 \%$ and $38 \%$ of all fine soil extreme events, depending on the site. Therefore, for the study region, fine soil is the pollutant type that most successfully impacts multiple sites at once at an extreme level.

The region-wide average for the $\mathrm{PM}_{2.5}: \mathrm{PM}_{10}$ ratio was $0.37,0.35$, and 0.23 for non-Asian dust, Asian dust, and Other-CM, respectively. Unexpectedly, the ratios for non-Asian dust events exceeded those for Asian dust events for half the sites (i.e., Chiricahua, Phoenix, Saguaro National Monument, Saguaro West). Also of interest is that non-Asian dust event averages for $\mathrm{PM}_{2.5}: \mathrm{PM}_{10}$ were well above 0.35 at the two sites in Tucson, Arizona (Saguaro National Monument $=0.57 \pm 0.36$; Saguaro West $=0.46 \pm 0.26$ ). A plausible explanation for these unexpected results is interference of background anthropogenic emissions at these urban-impacted sites in Tucson (Saguaro National Monument and Saguaro West), in addition to Phoenix which exhibited an average ratio of 0.35. The same explanation can be applied to the Fe:Ca results, which do not show a clear reduction in value for Asian dust events as compared to the more locally-relevant 
pollution categories for Chiricahua, Saguaro National Monument, and Saguaro West. However, the region-wide average for Fe:Ca was lowest for Asian dust (0.88), followed by non-Asian dust (0.96), and Other-CM (1.03). The values were generally low and close to the threshold value applied by past work to classify dust as purely Asian dust. These results suggest that caution should be exercised with the use of such ratios to distinguish between dust sources owing to mixing between distant and local sources.

Due to the nature of Asian dust pollution being more geographically widespread than other forms of pollution, this category registered the highest frequency of its events impacting $\geqslant 5$ sites. Depending on the site, $30 \%-57 \%$ of extreme Asian dust events (i.e., $3-8$ days) impacted $\geqslant 5$ sites.

It is of interest to compare the Asian dust extreme event data to criteria used previously to distinguish Asian dust events in the study region, including mass concentration ratios of both Fe:Ca and $\mathrm{PM}_{2.5}: \mathrm{PM}_{10}$. Previous work showed that Fe:Ca ratios below 1 are considered to be $100 \%$ Asian dust and values above 2 are $100 \%$ local dust [38]. A threshold ratio value of 0.35 for $\mathrm{PM}_{2.5}: \mathrm{PM}_{10}$ has been applied in other work to remove contamination of non-local dust sources in the study region [39]. This ratio generally increases with dust plume age and, thus, values higher than 0.35 are assumed to be contaminated with sources such as transported Asian dust. Values between 0.15 and 0.26 are associated with soil dust emissions from human activities according to the EPA [39]. Table 4 examines statistics associated with the two aforementioned ratios for non-Asian dust, Asian dust, and also the subset of Other extreme events that also had extreme values of $\mathrm{CM}\left(\mathrm{PM}_{10}-\mathrm{PM}_{2.5}\right)$. The latter are presumed to be due to locally generated dust.

Table 2. Statistics associated with the number of days with extreme events observed in the date range shown in Table 1 for each site. (NAAPS global data were unavailable for the following dates in 2001 that are omitted from categorization into Asian and non-Asian dust: *16 October 2001, 26 August 2001, 9 November 2001; ${ }^{* *} 16$ October 2001, 31 October 2001, 21 November 2001; ${ }^{* * *} 16$ October 2001, 9 November 2001; ${ }^{* * *} 9$ November 2001). Values in the Total category represent days with any type of extreme event (i.e., $\mathrm{PM}_{10}, \mathrm{PM}_{2.5}$, fine soil).

\begin{tabular}{|c|c|c|c|c|c|c|c|c|c|}
\hline Site Name & $\begin{array}{c}\text { Total Days } \\
\text { Data } \\
\text { Available }\end{array}$ & \multicolumn{8}{|c|}{ Extreme Event Types } \\
\hline Grand Canyon & 1664 & 120 & 82 & 64 & 69 & 30 & 49 & 20 & 25 \\
\hline Organ Pipe & 1431 & 76 & 45 & 55 & 54 & 7 & 41 & 13 & 17 \\
\hline Saguaro NM *** & 1628 & 85 & 49 & 56 & 56 & 8 & 40 & 14 & 23 \\
\hline Saguaro West **** & 1563 & 91 & 56 & 55 & 55 & 10 & 44 & 10 & 30 \\
\hline Tonto *** & 1664 & 103 & 67 & 65 & 67 & 12 & 47 & 18 & 25 \\
\hline
\end{tabular}




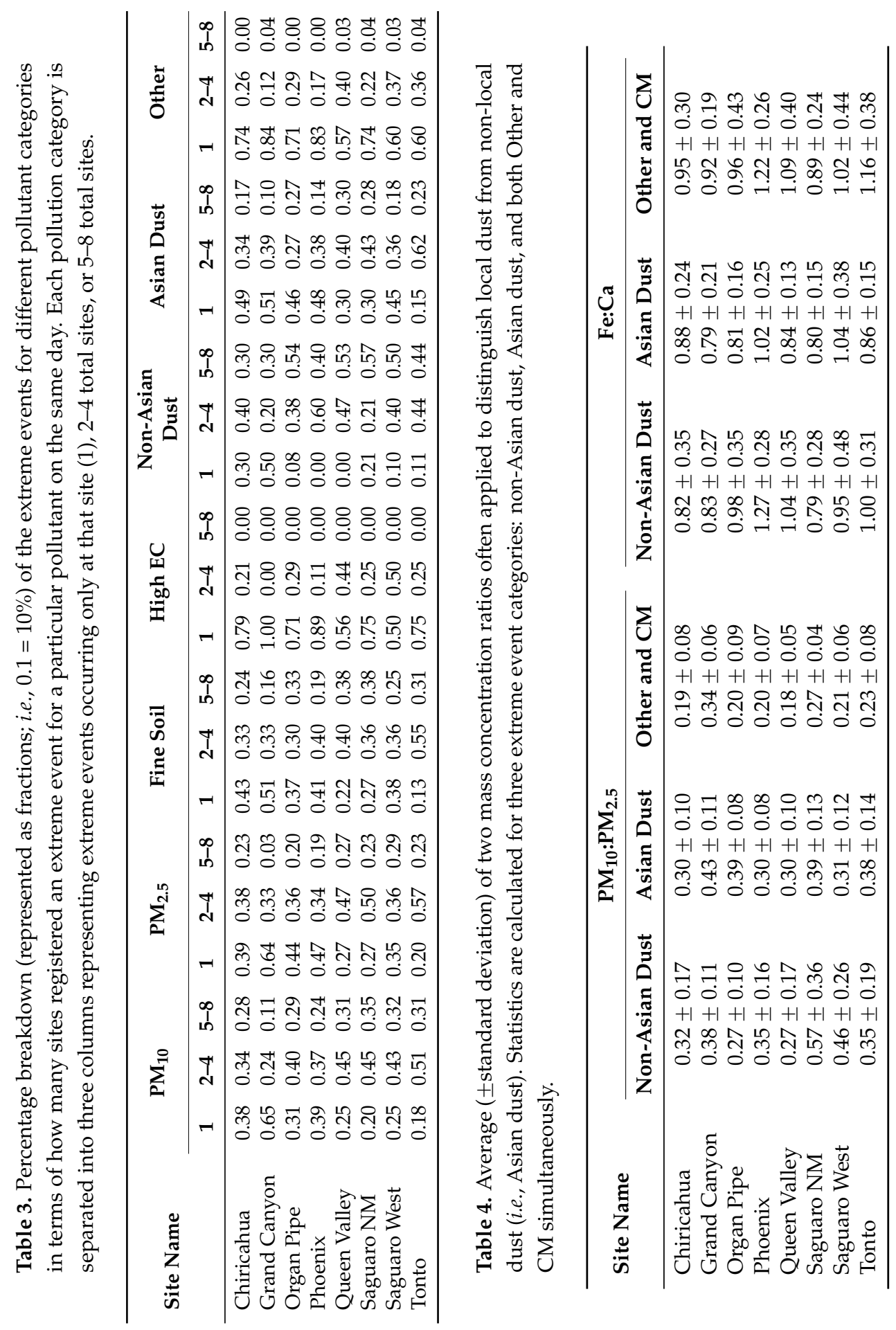




\section{2. "Other" Events}

The Other category was investigated in more detail to gain insight about the source of these extreme events (Table 5). Between $47 \%$ and $84 \%$ of the Other events exhibited extreme $\mathrm{PM}_{10}$ levels, which is suggestive of the presence of locally-generated coarse matter (i.e., dust) since fine soil levels did not reach extreme levels. To gain confidence in this reasoning, the percent frequency of extreme CM days was calculated and is similar to the percent frequency of extreme $\mathrm{PM}_{10}$ days (i.e., within two days) with the exception of Grand Canyon and Phoenix, which had nine and seven fewer extreme $\mathrm{CM}$ days as compared to $\mathrm{PM}_{10}$, respectively. Since the ratio of extreme CM:Other days ranges from 41\% (Organ Pipe) to as high as $74 \%$ (Chiricahua), with the average among all sites being $56 \%$, locally generated CM (i.e., dust) accounted for a significant amount of the Other events.

$\mathrm{PM}_{2.5}$ levels reached extreme levels in $30 \%-76 \%$ of the Other extreme events, with Organ Pipe being the only site with a higher percentage for $\mathrm{PM}_{2.5}$ being extreme versus $\mathrm{PM}_{10}$. The $\mathrm{PM}_{2.5}$ constituents only reached extreme levels in an average of $10 \%$ (OC), 11\% (K), 17\% (nitrate), and 22\% (sulfate) of the Other events. Among these four $\mathrm{PM}_{2.5}$ constituents, sulfate reached extreme levels in $48 \%$ and $35 \%$ of the Other events in Tonto and Organ Pipe, respectively, which were the highest values among all species and sites. This is likely due to anthropogenic emissions near those two sites such as from smelting [40-42]. Between $0 \%$ and $28 \%$ of Other events exhibited extreme levels of nitrate, OC, and potassium, which are all associated with wintertime pollution and fine soil emissions. These relatively low percentages for $\mathrm{PM}_{2.5}$ constituents are consistent with the majority of the Other events being due to CM.

Table 5. Percentage frequency summary (represented as fractions; i.e., $0.1=10 \%$ ) of how many of the Other events at each site exhibited extreme levels of $\mathrm{PM}_{10}, \mathrm{PM}_{2.5}$, coarse mass $\left(\mathrm{CM}=\mathrm{PM}_{10}-\mathrm{PM}_{2.5}\right)$ and individual $\mathrm{PM}_{2.5}$ constituents (potassium, organic carbon, nitrate, sulfate).

\begin{tabular}{cccccccc}
\hline Species & $\begin{array}{c}\text { Chiricahua } \\
(\mathbf{N}=\mathbf{2 3})\end{array}$ & $\begin{array}{c}\text { Grand Canyon } \\
\mathbf{( N = 2 5 )}\end{array}$ & $\begin{array}{c}\text { Organ Pipe } \\
\mathbf{( N = 1 7 )}\end{array}$ & $\begin{array}{c}\text { Phoenix } \\
(\mathbf{N}=\mathbf{3 0})\end{array}$ & $\begin{array}{c}\text { Queen Valley } \\
\mathbf{( N = 3 0 )}\end{array}$ & $\begin{array}{c}\text { Saguaro NM } \\
(\mathbf{N}=\mathbf{2 3})\end{array}$ & $\begin{array}{c}\text { Tonto } \\
(\mathbf{N}=\mathbf{2 5})\end{array}$ \\
\hline $\mathrm{PM}_{10}$ & 0.78 & 0.84 & 0.47 & 0.70 & 0.63 & 0.65 & 0.73 \\
$\mathrm{PM}_{2.5}$ & 0.30 & 0.52 & 0.76 & 0.50 & 0.43 & 0.43 & 0.30 \\
$\mathrm{CM}$ & 0.74 & 0.48 & 0.41 & 0.47 & 0.63 & 0.57 & 0.73 \\
$\mathrm{~K}$ & 0.09 & 0.24 & 0.12 & 0.17 & 0.10 & 0.09 & 0.03 \\
$\mathrm{OC}$ & 0.04 & 0.04 & 0.12 & 0.23 & 0.13 & 0.00 & 0.07 \\
$\mathrm{NO}_{3}{ }^{-}$ & 0.13 & 0.20 & 0.18 & 0.17 & 0.10 & 0.17 & 0.10 \\
$\mathrm{SO}_{4}{ }^{-}$ & 0.17 & 0.08 & 0.35 & 0.07 & 0.20 & 0.26 & 0.13 \\
\hline
\end{tabular}

\subsection{Temporal Nature of Events}

Figure 2 displays the monthly distribution of cumulative (i.e., summed for all years and sites) extreme events broken into the various pollutant categories. The 
month of August experienced the highest number of extreme events in the study region for $\mathrm{PM}_{10}, \mathrm{PM}_{2.5}$, fine soil, non-Asian dust, High EC, and Other (also had an equal peak in June). In contrast to all other pollutant categories, Asian dust events mainly occurred in the spring months of March-June (41 out of 42 days, i.e., $98 \%$ ) with only one event in February. The Other category exhibited a relatively constant amount in each month (12-16 days). Unlike $\mathrm{PM}_{10}, \mathrm{PM}_{2.5}$ exhibited a secondary mode in the winter month of January, driven mostly by High EC and Other events, suggestive of the importance of anthropogenic emissions and biomass burning, and secondary production of aerosol species that are favorably produced in wintertime conditions such as ammonium nitrate.

Figure 3 represents the interannual distribution of extreme events for different pollutant categories. It is cautioned that the time range with full years of data at all eight sites is from 2003 to 2013 (refer to Table 1 for data time ranges for each site). All categories exhibited the most events in either 2002 or 2003 with the exception of Asian dust which reached 10 events in 2007 and followed a distinctly different temporal pattern than all other categories due to its distant source. An interesting feature of Figure 3 is the cyclical pattern of there being a peak every few years for $\mathrm{PM}_{10}, \mathrm{PM}_{2.5}$, fine soil, non-Asian dust, and Other, specifically in the years 2002-2003, 2006-2007, 2009, and 2011-2012. It is unclear with the dataset as to what explains these recurring peaks, and future work is warranted, with a longer term record, to identify what an explanation could be for these features in the data.

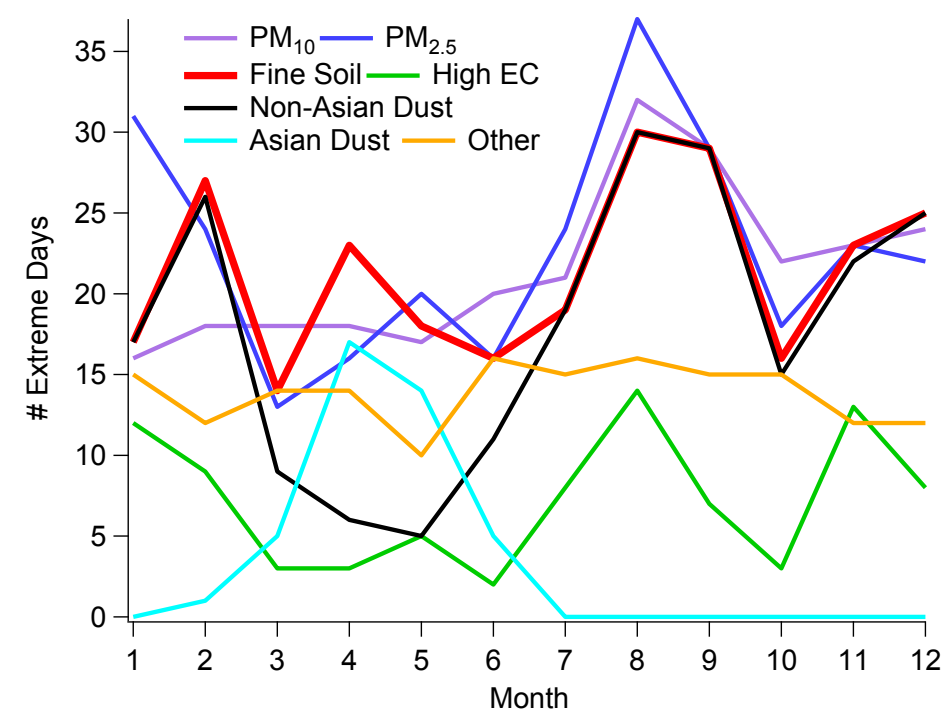

Figure 2. Monthly distribution of extreme events (cumulative for all years and sites) for different pollution categories. 


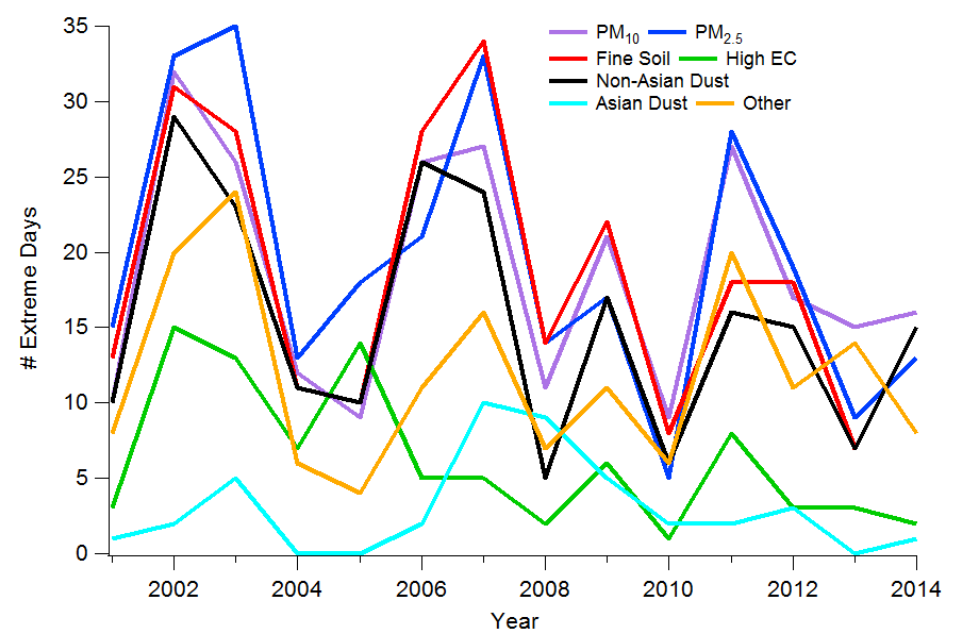

Figure 3. Time series of total extreme events as a function of year for different pollution categories. It is cautioned that the time range with full years of data at all eight sites is from 2003 to 2013 (refer to Table 1 for data time ranges for each site).

A simple linear regression was used to obtain the best-fit line for each pollutant type in Figure 3 using data between 2003 and 2013 when data were available for all sites for full years. Most all slopes were negative except for the Other category, which was only barely positive. The slopes, reported in units of number of events per year, and $\mathrm{p}$ values (in parenthesis) are as follows: $\mathrm{PM}_{10}=-0.19(0.80), \mathrm{PM}_{2.5}=-1.13$ (0.23), fine soil $=-0.95(0.29)$, High EC $=-0.83(0.03)$, non-Asian dust $=-0.85(0.25)$, Asian dust $=-0.11(0.76)$, Other $=0.03$ (0.97). The only statistically significant trend at $95 \%$ confidence was for the High EC category. This is thought to be due to reduced anthropogenic emissions since other work for the study region examining 2005-2009 has shown that the fastest rate of decline in EC levels was in Phoenix [43], which is the most populated area. Another study analyzing IMPROVE data between 1990 and 2004 across the United States, including the Southwest, showed that there has been a $\sim 25 \%$ reduction in EC attributed mostly to emissions controls, with the reduction being most dramatic in the winter as compared to summer [44].

The distribution of extreme events across days of the week is of interest for a few reasons. For example, EC rooted in anthropogenic emissions is thought to lead to higher concentrations around Thursday with minimum values on the weekend [44], and thus examining the frequency of High EC events as a function of the day of the week could help determine if the source of these events is anthropogenic in nature versus biomass burning. When normalized by total number of days on either the weekend (Saturday-Sunday) or weekday (Monday-Friday), High EC events occurred more frequently during weekdays (13.2 versus 10.5$)$. All other categories 
exhibited more events during weekdays too. No air pollutant category exhibited a statistically significant difference in the number of events (normalized by number of either weekend or weekday days) on either the weekend or weekdays (or on any specific day) using a chi-square statistical test at the $95 \%$ confidence level. The day of the week with the most extreme events for the various pollutant categories was either Wednesday or Thursday.

Table 6. Day of week distribution of extreme events combining data from all eight sites over the entire time duration of the study. The number of extreme events on weekends and weekdays are shown with the values in parenthesis being the normalized values relative to the total number of weekend (2) and weekday (5) days. The day of the week with the most and least events are also shown with values in parenthesis being the actual number of occurrences on that particular day.

\begin{tabular}{|c|c|c|c|c|c|c|c|c|}
\hline - & Total & $\mathrm{PM}_{10}$ & $\mathrm{PM}_{2.5}$ & $\begin{array}{l}\text { Fine } \\
\text { Soil }\end{array}$ & $\underset{\text { EC }}{\text { High }}$ & $\begin{array}{l}\text { Non-Asian } \\
\text { Dust }\end{array}$ & $\begin{array}{c}\text { Asian } \\
\text { Dust }\end{array}$ & Other \\
\hline $\begin{array}{l}\text { Weekend } \\
\text { (Saturday-Sunday) }\end{array}$ & $\begin{array}{c}57 \\
(28.5)\end{array}$ & 65 (32.5) & $60(30)$ & $\begin{array}{c}21 \\
(10.5)\end{array}$ & $52(26)$ & $9(4.5)$ & $44(22)$ & $\begin{array}{c}57 \\
(28.5)\end{array}$ \\
\hline $\begin{array}{l}\text { Weekday } \\
\text { (Monday-Friday) }\end{array}$ & $\begin{array}{c}201 \\
(40.2)\end{array}$ & $\begin{array}{c}208 \\
(41.6)\end{array}$ & $197(39.4)$ & $\begin{array}{c}66 \\
(13.2)\end{array}$ & $\begin{array}{c}162 \\
(32.4)\end{array}$ & $33(6.6)$ & $\begin{array}{c}122 \\
(24.4)\end{array}$ & $\begin{array}{c}201 \\
(40.2)\end{array}$ \\
\hline $\begin{array}{l}\text { Day With Most } \\
\text { Events }\end{array}$ & W (53) & Th (55) & $\begin{array}{l}\text { W/Th } \\
(50 / 50)\end{array}$ & Th (19) & Th (42) & W (11) & W (35) & W (53) \\
\hline $\begin{array}{l}\text { Day with Least } \\
\text { Events }\end{array}$ & $\mathrm{Tu}(23)$ & $\mathrm{Tu}(25)$ & $\mathrm{Tu}(21)$ & $\begin{array}{l}\mathrm{M} / \mathrm{F} \\
(11)\end{array}$ & $\mathrm{Tu}(19)$ & $\mathrm{Tu}(1)$ & $\mathrm{Tu}(11)$ & $\mathrm{Tu}(23)$ \\
\hline
\end{tabular}

\section{Conclusions}

The study examined long-term aerosol data for the Arizona region to describe the frequency and character of extreme aerosol events. The results are as follows in order of the questions raised at the end of Section 1:

(i) Between 5\% and 7\% of the total days (i.e., 1431-1664 depending on site) examined at the various sites exhibited an extreme aerosol event due to either extreme levels of $\mathrm{PM}_{10}, \mathrm{PM}_{2.5}$, and/or fine soil. Grand Canyon exhibited the most extreme event days (120, i.e., $7 \%$ of its total days), which is coincident with it being one of the most recognized tourism spots in the Southwest. Relative to the total number of extreme days, Grand Canyon exhibited the highest percentage in the High EC category (47\% versus 13\%-25\% for other sites). "Other" events accounted for between $2 \%$ and $33 \%$ of the total extreme days, with most of these being associated with extreme $\mathrm{PM}_{10}$ levels (i.e., locally-generated dust). Of the total number of days with extreme fine soil (54-69 days depending on the site), the number of these events being linked to Asian dust, based on NAAPS analysis, ranged from 19\% to 29\% (i.e., 10-20 days). The analysis highlighted the complexity of using NAAPS and various mass concentration ratios to distinguish between transported and local dust owing 
to likely mixing effects, especially in urban-impacted areas, such as Tucson and Phoenix.

(ii) Fine soil is the pollutant type that most frequently impacted multiple sites simultaneously on the same day at an extreme level. Five or more sites reached extreme fine soil levels on the same day for $16 \%-38 \%$ of all possible fine soil extreme events depending on the site. Within the fine soil category, Asian dust events impacted five or more sites between $30 \%$ and $57 \%$ of the time when they occurred. The pollutant categories with the least number of extreme events impacting five or more sites on the same day were High EC (0 days for all sites) and Other ( $0-1$ day depending on site) due to locally generated emissions that were not regional in nature. Grand Canyon exhibited the highest number of days where an extreme event only impacted that site since it is farther removed from the other seven sites that are clustered closer in Southern Arizona.

(iii) Most pollutant categories $\left(\mathrm{PM}_{10}, \mathrm{PM}_{2.5}\right.$, fine soil, non-Asian dust, High EC, Other) exhibited the highest number of extreme events in August. The Asian dust category was unique in its monthly pattern with its events occurring in the spring months of March-June (41 out of 42 days, i.e., 98\%) with only one event in February. Unlike the other pollutant categories, High EC was the only one to show a statistically significant change in frequency of occurrence between 2003 and 2013. While extreme events were most frequent for the various pollutant categories on either Wednesday or Thursday, there was no statistically significant difference in the number of events on any particular day or on weekend days versus weekdays.

Supplementary Materials: The following are available online at http:/ /www.mdpi.com/ 2073-4433/7/1/1/s1

Table S1: $\quad$ Summary of criteria concentrations for $\mathrm{PM}_{10}, \mathrm{PM}_{2.5}$, fine soil, and elemental carbon (EC) as a function of month for eight EPA IMPROVE sites in Arizona.

Figure S1: Case examples demonstrating how NAAPS was used as a tool to identify which extreme fine soil events qualified as Asian dust events. The examples below are for extreme fine soil events occurring on 15 May 2003, 12 April 2007, and 5 June 2008, which qualified as extreme events at four, six, and five sites, respectively.

Acknowledgments: This work was funded by Grant 2 P42 ES04940-11 from the National Institute of Environmental Health Sciences (NIEHS) Superfund Research Program, NIH and the Center for Environmentally Sustainable Mining through the TRIF Water Sustainability Program at the University of Arizona. The authors acknowledge Andrew Huerta and the UROC-PREP program in the Graduate College at the University of Arizona. The authors gratefully acknowledge data provided by EPA IMPROVE and the NRL NAAPS model. Some of the analyses and visualizations used in this study were produced with the Giovanni online data system, developed and maintained by the NASA GES DISC. 
Author Contributions: David H. Lopez and Michael R. Rabbani collected and analyzed data. David H. Lopez and Armin Sorooshian wrote the manuscript. Ewan Crosbie, Avelino F. Arellano Jr. and Aishwarya Raman provided useful comments and contributed to the manuscript.

Conflicts of Interest: The authors declare no conflict of interest.

\section{References}

1. Dockery, D.W.; Pope, C.A.; Xu, X.P.; Spengler, J.D.; Ware, J.H.; Fay, M.E.; Ferris, B.G.; Speizer, F.E. An Association between Air-Pollution and Mortality in 6 United-States Cities. New Engl. J. Med. 1993, 329, 1753-1759.

2. Pope, C.A.; Ezzati, M.; Dockery, D.W. Fine-particulate air pollution and life expectancy in the United States. New Engl. J. Med. 2009, 360, 376-386.

3. Porter, W.C.; Heald, C.L.; Cooley, D.; Russell, B. Investigating the observed sensitivities of air-quality extremes to meteorological drivers via quantile regression. Atmos. Chem. Phys. 2015, 15, 10349-10366.

4. Crosbie, E.; Sorooshian, A.; Monfared, N.A.; Shingler, T.; Esmaili, O. A multi-year aerosol characterization for the greater Tehran area using satellite, surface, and modeling data. Atmosphere 2014, 5, 178-197.

5. Sutton, L.J. Haboobs. Q. J. Roy. Meteor. Soc. 1925, 51, 25-30.

6. Miller, S.D.; Kuciauskas, A.P.; Liu, M.; Ji, Q.; Reid, J.S.; Breed, D.W.; Walker, A.L.; Al Mandoos, A. Haboob dust storms of the southern Arabian Peninsula. J. Geophys. Res. 2008, 113.

7. Raman, A.; Arellano, A.F.; Brost, J.J. Revisiting haboobs in the southwestern United States: An observational case study of the 5 July 2011 Phoenix dust storm. Atmos. Environ. 2014, 89, 179-188.

8. Seager, R.; Ting, M.F.; Held, I.; Kushnir, Y.; Lu, J.; Vecchi, G.; Huang, H.P.; Harnik, N.; Leetmaa, A.; Lau, N.C.; et al. Model projections of an imminent transition to a more arid climate in southwestern North America. Science 2007, 316, 1181-1184.

9. Neff, J.C.; Ballantyne, A.P.; Farmer, G.L.; Mahowald, N.M.; Conroy, J.L.; Landry, C.C.; Overpeck, J.T.; Painter, T.H.; Lawrence, C.R.; Reynolds, R.L. Increasing eolian dust deposition in the western United States linked to human activity. Nat. Geosci. 2008, 1, 189-195.

10. Field, J.P.; Belnap, J.; Breshears, D.D.; Neff, J.C.; Okin, G.S.; Whicker, J.J.; Painter, T.H.; Ravi, S.; Reheis, M.C.; Reynolds, R.L. The ecology of dust. Front. Ecol. Environ. 2010, 8, 423-430.

11. Dennison, P.E.; Brewer, S.C.; Arnold, J.D.; Moritz, M.A. Large wildfire trends in the western United States, 1984-2011. Geophys. Res. Lett. 2014, 41, 2928-2933.

12. Stavros, E.N.; Abatzoglou, J.T.; McKenzie, D.; Larkin, N.K. Regional projections of the likelihood of very large wildland fires under a changing climate in the contiguous Western United States. Clim. Chang. 2014, 126, 455-468. 
13. Painter, T.H.; Barrett, A.P.; Landry, C.C.; Neff, J.C.; Cassidy, M.P.; Lawrence, C.R.; McBride, K.E.; Farmer, G.L. Impact of disturbed desert soils on duration of mountain snow cover. Geophys. Res. Lett. 2007, 34.

14. Husar, R.B.; Tratt, D.M.; Schichtel, B.A.; Falke, S.R.; Li, F.; Jaffe, D.; Gasso, S.; Gill, T.; Laulainen, N.S.; Lu, F.; et al. Asian dust events of April 1998. J. Geophys. Res. 2001, 106, 18317-18330.

15. Tratt, D.M.; Frouin, R.J.; Westphal, D.L. April 1998 Asian dust event: A southern California perspective. J. Geophys. Res. 2001, 106, 18371-18379.

16. VanCuren, R.A.; Cahill, T.A. Asian aerosols in North America: Frequency and concentration of fine dust. J. Geophys. Res. 2002, 107.

17. Jaffe, D.; Snow, J.; Cooper, O. The 2001 Asian Dust Events: Transport and impact on surface aerosol concentrations in the U.S. EOS 2003, 84, 501-516.

18. Wells, K.C.; Witek, M.; Flatau, P.; Kreidenweis, S.M.; Westphal, D.L. An analysis of seasonal surface dust aerosol concentrations in the western US (2001-2004): Observations and model predictions. Atmos. Environ. 2007, 41, 6585-6597.

19. Kavouras, I.G.; Etyemezian, V.; DuBois, D.W.; Xu, J.; Pitchford, M. Source reconciliation of atmospheric dust causing visibility impairment in Class I areas of the western United States. J. Geophys. Res. 2009, 114.

20. Creamean, J.M.; Spackman, J.R.; Davis, S.M.; White, A.B. Climatology of long-range transported Asian dust along the West Coast of the United States. J. Geophys. Res. 2014, 119, 12171-12185.

21. Westerling, A.L.; Hidalgo, H.G.; Cayan, D.R.; Swetnam, T.W. Warming and earlier spring increase western US forest wildfire activity. Science 2006, 313, 940-943.

22. Kavouras, I.G.; Nikolich, G.; Etyemezian, V.; DuBois, D.W.; King, J.; Shafer, D. In situ observations of soil minerals and organic matter in the early phases of prescribed fires. J. Geophys. Res. 2012, 117.

23. Popovicheva, O.; Kistler, M.; Kireeva, E.; Persiantseva, N.; Timofeev, M.; Kopeikin, V.; Kasper-Giebl, A. Physicochemical characterization of smoke aerosol during large-scale wildfires: Extreme event of August 2010 in Moscow. Atmos. Environ. 2014, 96, 405-414.

24. Maudlin, L.C.; Wang, Z.; Jonsson, H.H.; Sorooshian, A. Impact of wildfires on size-resolved aerosol composition at a coastal California site. Atmos. Environ. 2015, 119, 59-68.

25. Malm, W.C.; Sisler, J.F.; Huffman, D.; Eldred, R.A.; Cahill, T.A. Spatial and Seasonal Trends in Particle Concentration and Optical Extinction in the United-States. J. Geophys. Res. 1994, 99, 1347-1370.

26. IMPROVE Particulate Monitoring Network Standard Operating Procedures. Available online: http://vista.cira.colostate.edu/improve/Publications/SOPs/UCDavis_SOPs/ IMPROVE_SOPs.htm (accessed on 19 December 2015).

27. Hogan, T.F.; Rosmond, T.E. The description of the navy operational global atmospheric prediction systems spectral forecast model. Mon. Weather Rev. 1991, 119, 1786-1815. 
28. Hogan, T.F.; Brody, L.R. Sensitivity studies of the Navy's global forecast model parameterizations and evaluation of improvements to NOGAPS. Mon. Wea. Rev. 1993, 121, 2373-2395.

29. Witek, M.L.; Flatau, P.J.; Quinn, P.K.; Westphal, D.L. Global sea-salt modeling: Results and validation against multicampaign shipboard measurements. J. Geophys. Res. 2007, 112.

30. McKendry, I.G.; Strawbridge, K.B.; O’Neill, N.T.; Macdonald, A.M.; Liu, P.S.K.; Leaitch, W.R.; Anlauf, K.G.; Jaegle, L.; Fairlie, T.D.; Westphal, D.L. Trans-Pacific transport of Saharan dust to western North America: A case study. J. Geophys. Res. 2007, 112.

31. Cottle, P.; Strawbridge, K.; McKendry, I.; O'Neill, N.; Saha, A. A pervasive and persistent Asian dust event over North America during spring 2010: Lidar and sunphotometer observations. Atmos. Chem. Phys. 2013, 13, 4515-4527.

32. Wu, Y.H.; Han, Z.; Nazmi, C.; Gross, B.; Moshary, F. A trans-Pacific Asian dust episode and its impacts to air quality in the east coast of US. Atmos. Environ. 2015, 106, 358-368.

33. Zhang, J.L.; Reid, J.S.; Westphal, D.L.; Baker, N.L.; Hyer, E.J. A system for operational aerosol optical depth data assimilation over global oceans. J. Geophys. Res. 2008, 113.

34. Hsu, N.C.; Herman, J.R.; Torres, O.; Holben, B.N.; Tanre, D.; Eck, T.F.; Smirnov, A.; Chatenet, B.; Lavenu, F. Comparisons of the TOMS aerosol index with Sun-photometer aerosol optical thickness: Results and applications. J. Geophys. Res. 1999, 104, 6269-6279.

35. Torres, O.; Bhartia, P.K.; Herman, J.R.; Ahmad, Z.; Gleason, J. Derivation of aerosol properties from satellite measurements of backscattered ultraviolet radiation: Theoretical basis (vol 103, pg 17099, 1998). J. Geophys. Res. 1998, 103, 23321-23321.

36. Gkikas, A.; Hatzianastassiou, N.; Mihalopoulos, N. Aerosol events in the broader Mediterranean basin based on 7-year (2000-2007) MODIS C005 data. Ann. Geophys.-Germany 2009, 27, 3509-3522.

37. Reid, J.S.; Koppmann, R.; Eck, T.F.; Eleuterio, D.P. A review of biomass burning emissions part II: Intensive physical properties of biomass burning particles. Atmos. Chem. Phys. 2005, 5, 799-825.

38. VanCuren, R.A.; Cliff, S.S.; Perry, K.D.; Jimenez-Cruz, M. Asian continental aerosol persistence above the marine boundary layer over the eastern North Pacific: Continuous aerosol measurements from Intercontinental Transport and Chemical Transformation 2002 (ITCT 2K2). J. Geophys. Res. 2005, 110.

39. Tong, D.Q.; Dan, M.; Wang, T.; Lee, P. Long-term dust climatology in the western United States reconstructed from routine aerosol ground monitoring. Atmos. Chem. Phys. 2012, 12, 5189-5205.

40. Prabhakar, G.; Sorooshian, A.; Toffol, E.; Arellano, A.F.; Betterton, E.A. Spatiotemporal distribution of airborne particulate metals and metalloids in a populated arid region. Atmos. Environ. 2014, 92, 339-347.

41. Sorooshian, A.; Csavina, J.; Shingler, T.; Dey, S.; Brechtel, F.J.; Saez, A.E.; Betterton, E.A. Hygroscopic and Chemical Properties of Aerosols Collected near a Copper Smelter: Implications for Public and Environmental Health. Environ. Sci. Technol. 2012, 46, 9473-9480. 
42. Sorooshian, A.; Shingler, T.; Harpold, A.; Feagles, C.W.; Meixner, T.; Brooks, P.D. Aerosol and precipitation chemistry in the southwestern United States: Spatiotemporal trends and interrelationships. Atmos. Chem. Phys. 2013, 13, 7361-7379.

43. Sorooshian, A.; Wonaschutz, A.; Jarjour, E.G.; Hashimoto, B.I.; Schichtel, B.A.; Betterton, E.A. An aerosol climatology for a rapidly growing arid region (southern Arizona): Major aerosol species and remotely sensed aerosol properties. J. Geophys. Res. 2011, 116.

44. Murphy, D.M.; Chow, J.C.; Leibensperger, E.M.; Malm, W.C.; Pitchford, M.; Schichtel, B.A.; Watson, J.G.; White, W.H. Decreases in elemental carbon and fine particle mass in the United States. Atmos. Chem. Phys. 2011, 11, 4679-4686. 


\title{
Windblown Dust Deposition Forecasting and Spread of Contamination around Mine Tailings
}

\author{
Michael Stovern, Héctor Guzmán, Kyle P. Rine, Omar Felix, Matthew King, \\ Wendell P. Ela, Eric A. Betterton and Avelino Eduardo Sáez
}

\begin{abstract}
Wind erosion, transport and deposition of windblown dust from anthropogenic sources, such as mine tailings impoundments, can have significant effects on the surrounding environment. The lack of vegetation and the vertical protrusion of the mine tailings above the neighboring terrain make the tailings susceptible to wind erosion. Modeling the erosion, transport and deposition of particulate matter from mine tailings is a challenge for many reasons, including heterogeneity of the soil surface, vegetative canopy coverage, dynamic meteorological conditions and topographic influences. In this work, a previously developed Deposition Forecasting Model (DFM) that is specifically designed to model the transport of particulate matter from mine tailings impoundments is verified using dust collection and topsoil measurements. The DFM is initialized using data from an operational Weather Research and Forecasting (WRF) model. The forecast deposition patterns are compared to dust collected by inverted-disc samplers and determined through gravimetric, chemical composition and lead isotopic analysis. The DFM is capable of predicting dust deposition patterns from the tailings impoundment to the surrounding area. The methodology and approach employed in this work can be generalized to other contaminated sites from which dust transport to the local environment can be assessed as a potential route for human exposure.
\end{abstract}

Reprinted from Atmosphere. Cite as: Stovern, M.; Guzmán, H.; Rine, K.P.; Felix, O.; King, M.; Ela, W.P.; Betterton, E.A.; Sáez, A.E. Windblown Dust Deposition Forecasting and Spread of Contamination around Mine Tailings. Atmosphere 2016, 7,16 .

\section{Introduction}

Wind erosion, transport and deposition of particulate matter from contaminated sites may have significant effects on the surrounding environment, especially in arid and semi-arid regions, which are especially susceptible to erosion because of the dry climate and lack of vegetation. Wind erosion occurs on a variety of spatial scales from very large dust storms that can travel thousands of kilometers in the atmosphere [1] to small local sources whose impact is regionally confined. Some of the human health 
concerns associated with elevated concentrations of particulate matter include fungi and bacteria transport [2] and respiratory stress and cardiovascular disease [3]. Local sources of windblown dust such as dry lake beds, plowed fields and mine tailings can regularly produce windblown particulate matter [4,5]. In semi-arid mining regions, such as the US Southwest, mine tailings impoundments can be a significant anthropogenic local source of windblown particulate matter [4].

Modeling wind erosion, transport and deposition of particulate matter from mine tailings impoundments on scales of a few kilometers is challenging. Typical regulatory models, such as CALPUFF and AERMOD, have difficulty simulating aerosol transport in topographically complex regions on such small scales. Recent advancements in computational capabilities have made Computational Fluid Dynamic (CFD) modeling a viable approach for simulating the erosion and transport of aerosols but minimal research has been done on the modeling of wind erosion and aerosol transport from mine tailings impoundments. Previous studies that have investigated wind erosion of tailings impoundments through the use of CFD modeling [6-9] focused on the erosion process and not the transport and deposition of the windblown particulate matter. In a previous work [10], we utilized a CFD model to simulate wind erosion of a tailings impoundment in a topographically complex region, in order to understand the mechanisms that influence deposition.

The prediction of transport and deposition of windblown dust from mine tailings impoundments is vital in determining the exposure risks in neighboring communities. In a previous work [11] we developed a windblown dust Deposition Forecasting Model (DFM) that was designed to be used in conjunction with operational weather models to forecast deposition of windblown particulate matter for the Iron King Mine (IK) and Humboldt smelter tailings impoundments in Dewey-Humboldt, Arizona. The DFM is a hybrid model that uses both empirical relations derived from direct observations and physical model simulations of aerosol trajectories. The IK and Humboldt smelter tailings impoundments are part of a US Environmental Protection Agency Superfund site that has elevated concentrations of toxic species such as lead and arsenic [12].

In this work, we assess predictions of the DFM by validating the deposition forecasts against a variety of field measurements at the mine site and surrounding areas, including: (i) spatially distributed measurements of dust deposition; (ii) metals composition; and (iii) lead isotope analysis. The spatially distributed measurements of dust deposition were collected using inverted-disc samplers during two month-long field sampling campaigns in April and June 2014. The sampling periods were selected to coincide with spring and early summer, which represent the windy season in southern Arizona. Dust collected during the sampling campaigns was analyzed for total weight, chemical composition and lead isotopes and directly compared to forecasted deposition patterns generated by the DFM. We hypothesize 
that forecast spatial deposition patterns generated by the DFM will agree with arsenic and lead tracers captured by the inverted-disc samplers, which will help in establishing routes of contaminant exposure in local communities. In addition, we investigate how topsoil measurements of arsenic and lead performed in this work based on techniques developed in a previous work [13] correlate with the dust sampler contaminant concentrations and isotopic signatures, which serves to quantify the spread of contamination from the mine tailings site.

\section{Methodology}

\subsection{Site Description}

The Iron King Mine Tailings and Humboldt Smelter Superfund area is located in central Arizona in the vicinity of Dewey-Humboldt (Figure 1). The smelter produced lead, gold, silver, zinc and copper in the period 1906-1969. The area was classified as a Superfund site by the US EPA in 2008 [12] after it was discovered that it is contaminated with lead and arsenic.

The region is classified as semiarid with an annual rainfall of about $480 \mathrm{~mm}$. The vegetation is Pinyon Juniper woodlands with limited desert grasses and other bushes [14]. Most of the land adjacent to the northern, western and southern edges of the tailings and mine operations property is publicly owned state trust and grazing land. Arizona State Highway 69 separates the mine tailings area from the town of Dewey-Humboldt, which has an elevation of $1396 \mathrm{~m}$.

The tailings impoundment consists of two areas: the main tailings impoundment with a total aerial extent of $96,000 \mathrm{~m}^{2}$ and the lower tailings region located directly adjacent to the main tailings impoundment with a total aerial extent of $84,000 \mathrm{~m}^{2}$. The tailings impoundment is devoid of vegetation except where a phytostabilization project is attempting to reestablish native vegetation. The revegetation project has been in progress since May 2010 and is confined to an area of $7200 \mathrm{~m}^{2}$ on top of the tailings [15]. The surface of the impoundment is made up of $34.7 \%$ sand, $44.8 \%$ silt, and $20.4 \%$ clay and has patches of reddish coloration attributed to iron minerals, mostly ferrihydrite [15]. This type of tailings material composition is highly skewed towards the fine particle size range with $65.2 \%$ being silt size $(<50 \mu \mathrm{m})$. The tailings are typically covered by crusted soil with patchy efflorescence that usually forms following rain events. The crust can be broken up and results in very fine powdery material that is easily eroded by the wind. The average arsenic and lead concentrations measured in bulk samples of the mine tailing material are about $0.12 \%$ and $0.10 \%$ by mass, respectively. 


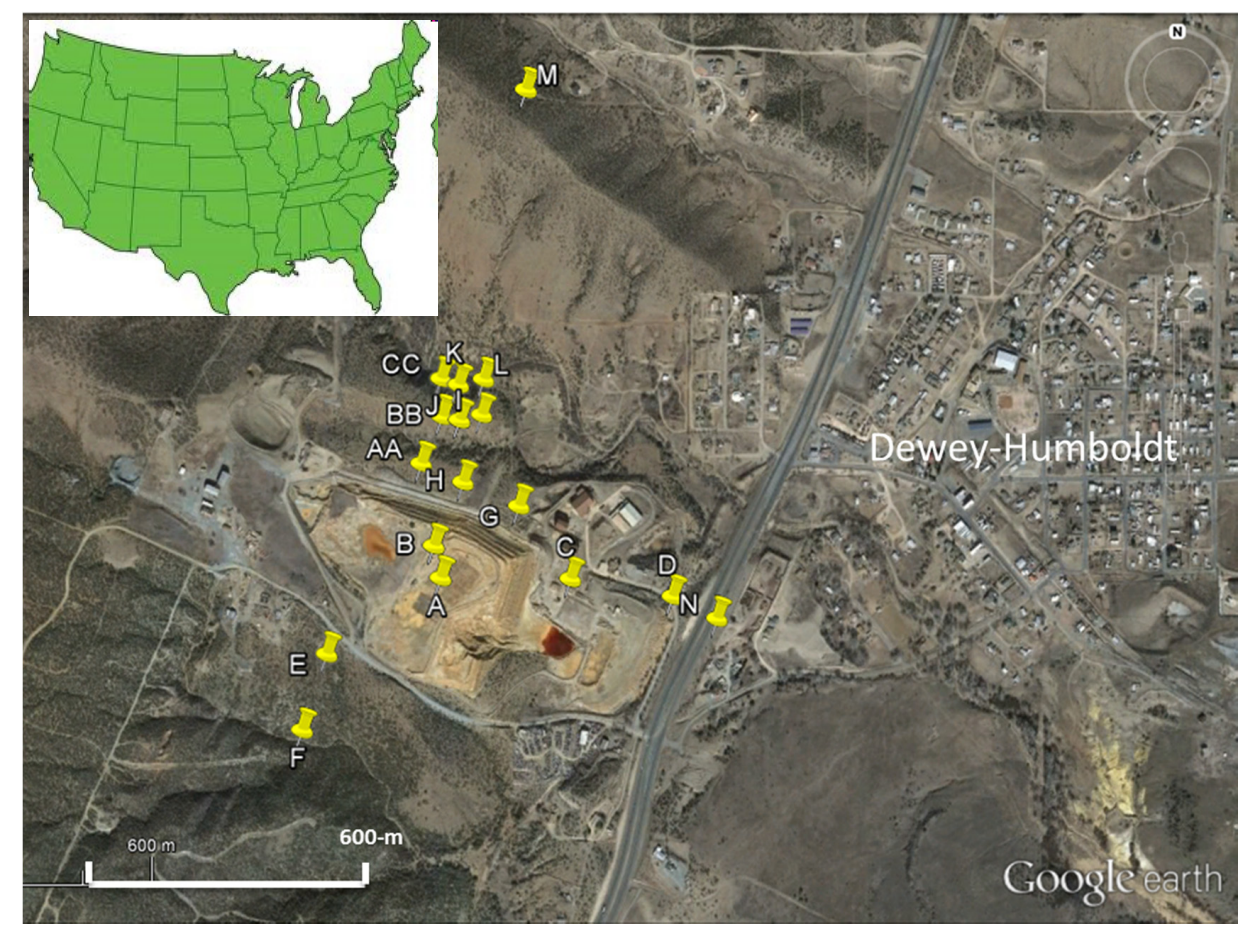

Figure 1. Google Earth visible satellite image of the Iron King Mine tailings impoundment and the town of Dewey-Humboldt (March 2014). The locations of the inverted-disc samplers are denoted by yellow pins (image from Google Earth).

A suite of meteorological and dust monitoring instruments was installed on the tailings impoundment in 2009. The setup consists of two eddy flux towers, equipped with six DUSTTRAK dust monitors for $\mathrm{PM}_{27}$ (particulate matter with an aerodynamic diameter less than $27 \mu \mathrm{m}$,) anemometers, wind vanes, thermometers and hygrometers. In addition, a Micro-Orifice Uniform-Deposit Impactor (MOUDI) was deployed to measure size fractionated distribution of aeolian dust from April 2013 to January 2014. Four month-long MOUDI samples were collected during this period. The MOUDI (MSP Corp) has eleven stages that collect particles with aerodynamic cut point diameters of: 18- $\mu \mathrm{m}, 9.9-\mu \mathrm{m}, 6.2-\mu \mathrm{m}, 3.1-\mu \mathrm{m}, 1.8-\mu \mathrm{m}, 1-\mu \mathrm{m}$, $0.55-\mu \mathrm{m}, 0.32-\mu \mathrm{m}, 0.18-\mu \mathrm{m}, 0.1-\mu \mathrm{m}, 0.054-\mu \mathrm{m}$ and ultrafine particles collected on an "after filter". The MOUDI was located along the northern edge of the tailings impoundment with the inlet located $1 \mathrm{~m}$ above ground level. Observations collected by the DUSTTRAK and the MOUDI were used to generate empirical relations between dust concentration (discriminated by particle size), wind speed at 10-m height and relative humidity. These correlations were employed in the calculation of dust fluxes in the DFM [11]. In addition, the meteorological observations are 
used to determine systematic biases between observed conditions and operational WRF forecasts.

\subsection{Deposition Forecasting Model Description}

The DFM predicts the deposition of $\mathrm{PM}_{27}$ generated by windblown dust from the Iron King tailings impoundment. This size range has been chosen since it corresponds with the measurements made by the DUSTTRAK dust monitors. The hybrid model utilizes both empirically derived relations and particle transport simulations. Details of model development and validation are presented elsewhere [11].

The nearly two years (2012-2013) of meteorological and dust monitoring data collected from the eddy flux towers were used to derive empirical relationships between meteorological conditions and windblown dust generation. These empirical relations include the effects of wind speed and relative humidity on airborne dust, particle size fractionation and the vertical profile of dust concentration measured on the tailings and wind speed effects on wind direction. Here, we will summarize the empirical correlations presented in our previous work [11]. We correlated dust concentrations in the atmosphere over the tailings surface to obtain

$$
C_{d u s t}=A_{1} A_{2} U^{B}
$$

where $C_{\text {dust }}$ is the mass concentration $\left(\mu \mathrm{g} / \mathrm{m}^{3}\right)$ of dust in the atmosphere at a specific height and for a specific particle size range, $U$ is the $10-m$ height wind speed, $A_{1}$ and $B$ are constants that are fitted to measurements for each specific height and particle size range (see [11] for values and details), and $A_{2}$ is a function of atmospheric relative humidity, empirically found to be:

$$
A_{2}=\left\{\begin{array}{c}
1 \text { for } R H \leqslant 25 \% \\
(50-(R H-25)) / 50 \text { for } 25 \%<R H \leqslant 75 \% \\
0 \text { for } R H>75 \%
\end{array}\right.
$$

where $\mathrm{RH}$ is relative humidity (\%). Concentrations, wind speeds and relative humidities in these empirical equations correspond to 1-h averages.

The DFM forecasts a dust emission flux from the tailings as proportional to the integral of the concentration given by Equation (1) over height. The flux is assumed to be uniform over the whole surface area of tailings $\left(95,000 \mathrm{~m}^{2}\right)$.

The DFM forecasts deposition in three particle size ranges. The size ranges are defined by particles that have aerodynamic diameters $\left(D_{p}\right)$ that fall within: $27 \mu \mathrm{m}>$ $\mathrm{D}_{\mathrm{p}}>18 \mu \mathrm{m}$ (coarse), $18 \mu \mathrm{m}>\mathrm{D}_{\mathrm{p}}>3.1 \mu \mathrm{m}$ (medium), and $\mathrm{D}_{\mathrm{p}}<3.1 \mu \mathrm{m}$ (fine). The size ranges were determined by MOUDI and DUSTTRAK observation capabilities: The coarse range represents the difference between DUSTTRAK and total MOUDI mass measured in a given event (that is, for the same amount of air treated). The 
medium and fine ranges were obtained by adding the mass of collected sample in corresponding stages in the MOUDI. The cut point $(3.1 \mu \mathrm{m})$ was selected based on the fact that, on average, total mass collected by the MOUDI above and below that particle size was approximately the same. In addition, the same size ranges were used in deriving the empirical relationships between meteorological conditions and wind erosion flux within the DFM model [11].

The DFM also includes the effect that topography has on aerosol deposition. Stovern et al. [10] simulated windblown transport of fugitive aerosols from the Iron King Mine tailings using computational fluid dynamics. They found that windblown dust preferentially deposits in regions of topographic upslope relative to the mean flow. Due to the complex topography of the site, these effects are included in the model.

The DFM results for each forecast period (see Section 3) are produced for three particle size ranges and are modelled on a spatial grid that is approximately $25 \mathrm{~km}^{2}$ in area with $10.3 \mathrm{~m}$ spatial resolution. These simulations include the effects that a convectively turbulent boundary layer has on particle trajectories. Simulation area includes all of the Iron King tailings and most of the adjacent town of Dewey-Humboldt. The model is initialized using the 48-h forecasts from an operational version of the WRF model produced by the department of Atmospheric Sciences at the University of Arizona.

\subsection{WRF Model Forecasts}

The WRF model is configured with two nested grids. The inner grid covers the entire state of Arizona, portions of Southeastern California, Western New Mexico and Northern Mexico. The outer grid covers from Northern Nevada to the tip of the Baja California peninsula, and from Western Texas to portions of the Eastern Pacific Ocean. The inner and outer domains have horizontal resolutions of 1.8-km and 5.4-km, respectively. Model forecasts are produced daily at $12 \mathrm{Z}$ and $6 \mathrm{Z}$, using both Global Forecast System (GFS) and North American Mesoscale Forecast System (NAM) initializations. Each forecast run is $48 \mathrm{~h}$ long at 1 - $\mathrm{h}$ intervals. The DFM was initialized using the WRF forecast conditions predicted $28-39 \mathrm{~h}$ in advance.

We used the daily $12 Z$ GFS WRF runs during the periods 21 April to 22 May and 11 June to 9 July 2014 to tabulate the horizontal components of the 10-m height winds, 2-m specific humidity, the 2-m height temperature and the surface pressure for each hour between 9 A.M. and 9 P.M. We then calculated the wind speed and wind direction and relative humidity for each hourly interval by averaging the five nearest-neighbor WRF grid points at the tailings location, which was used to initialize the deposition forecasting model.

WRF model forecasts and observed meteorological conditions on the tailings were compared to test for systematic biases over a period of 163 days (June 2012 
to August 2013). This period coincides with the same observing period used to determine the empirical relations used in the creation of the deposition forecasting model [11]. As described later, forecast and observed wind speed and relative humidity were corrected for systematic bias. Results will be summarized in Section 3.1. To calculate directional bias we first calculated the residual for each model and observation pair which fall within the range of -180 to +180 degrees. A positive residual indicates counter-clockwise rotation between the WRF model and observed wind direction and vice versa. A perfectly nonbiased data set would produce an average residual of zero.

\subsection{Dust Deposition Sampling}

Inverted-disc samplers have been used in dust deposition experiments, including aeolian deposition near an eroding source field [16] and dry deposition of polychlorinated organics [17]. The collection efficiencies of inverted-disc samplers have been studied extensively [18-22] and are dependent on wind speed and particle diameter. However, there appears to be general consensus that the collection efficiency falls within the range of $5 \%-40 \%$ for all grain sizes [21]. For this study, collection efficiency is unimportant because we base our comparisons on relative deposition amounts (Section 3.3.2). Hall and Waters [19] showed that an inverted-disc sampler has a significantly higher blowout wind speed than both the flat disc and the British Standard deposit gauge. This reduces resuspension and loss of particulate matter.

The plastic discs (Frisbees) were purchased from discountmugs.com. They have a diameter of $233 \mathrm{~mm}$ and a depth of $25 \mathrm{~mm}$. The discs were glued to the lids of high density polyethylene 500-mL sample bottles (Thermo Scientific) using Loctite plastic bonder. An $8.5 \mathrm{~mm}$ hole was drilled through the disc into the bottle. The samplers were mounted on iron stakes and placed $1 \mathrm{~m}$ above the ground.

A set of 20 inverted-disc samplers was placed around the Iron King tailings impoundment and surrounding area. The disc samplers were placed along three transects to measure deposition in the northward, eastward and southward directions, starting from the main tailings and extending up to $1 \mathrm{~km}$ away. A majority of the samplers were located north of the main tailings, along the dominant wind direction (Figure 1). There is significant topographic variation north of the tailings, which is used for testing the effect that topography has on dust deposition.

\subsection{Soil Sampling}

Soil samples were taken during a single sampling campaign in 2014 at different distances from the mine tailings to assess the extent of contamination. A total of nine soil samples were collected with the first sampling point located on the mine tailings and eight more sampling points in a straight line NE transect, which corresponds 
to the prevailing wind direction. Samples at different depths were taken in order to obtain a vertical profile of the contamination at the following depth intervals: 0-3 mm, 3-6 mm, 6-9 mm and finally $100 \mathrm{~mm}$. Details of the sampling technique are provided elsewhere [13]. Final samples at each site and depth were a composite of three different samples. The composite samples were dried for $10 \mathrm{~h}$ at a temperature of $110{ }^{\circ} \mathrm{C}$ and then sieved through a $0.84 \mathrm{~mm}$ sieve to discard coarser fractions. Distances from the tailings to the sampling points along the NE transect were $13 \mathrm{~m}$, $70 \mathrm{~m}, 130 \mathrm{~m}, 355 \mathrm{~m}, 1100 \mathrm{~m}, 1128 \mathrm{~m}, 1150 \mathrm{~m}$ and $5032 \mathrm{~m}$.

\subsection{Sample Analysis}

In the field, deionized water was used to flush all the dust captured on the inverted-disc samplers into the attached $500 \mathrm{~mL}$ bottle. The bottles were unscrewed and capped for transportation to the laboratory where each sample was partially dried in the $500 \mathrm{~mL}$ bottle in an oven $\left(60^{\circ} \mathrm{C}\right)$ and then transferred into a pre-weighed $50 \mathrm{~mL}$ glass vial. The vial samples were then completely dried and sieved to remove particles $>500 \mu \mathrm{m}$. The samples were then weighed using a Mettler AE100 balance $( \pm 0.1 \mathrm{mg}$ ). The sample masses were normalized to the area of the disc, which yields the mass deposition per unit area for each sample location. The handling of MOUDI samples has been described in detail elsewhere [4].

Both inverted-disc and soil samples were prepared for metal and lead isotopes analysis by extraction with $15 \mathrm{~mL}$ of aqua regia $\left(1.03 \mathrm{M} \mathrm{HNO}_{3} / 2.23 \mathrm{M} \mathrm{HCl}\right.$, trace-metal grade) with sonication at $80{ }^{\circ} \mathrm{C}$ for $60 \mathrm{~min}$. Aliquots of $1.2 \mathrm{~mL}$ of solution were extracted and diluted to $4 \mathrm{~mL}$ with deionized water before the analysis. Due to the relatively low concentrations of lead in some of the samples, the lead isotope analysis samples were concentrated on a hot plate [13]. An ICP-MS (Agilent 7700X with an Octopole Reaction System) was used to analyze for metal concentrations and lead isotopic composition. MiliQ water, $0.669 \mathrm{HCl}$ (Fisher, trace-metal grade) and $0.309 \mathrm{M}$ $\mathrm{HNO}_{3}$ (EMD, Omnitrace) were used to create the certified calibration standards from Accustandard. In addition to each sample, the National Institute of Standards and Technology (NIST) standard reference material (SRM 1643e trace elements in water) was also analyzed. We used the same operating condition for the analysis of both elemental concentrations and the lead isotopic ratios. NIST SRM 981 (Lead isotopic standard) was used for validation and calibration and the analytical precision of lead isotopic ratios was under $0.5 \%$ [13].

\section{Results}

\subsection{WRF Model Verification}

Biases in the 24-h WRF forecast of wind speed, wind direction and relative humidity are determined by direct comparison to in situ observations. The forecast 
of relative humidity showed good agreement with the observations (Figure 2). The results show that the WRF model slightly under predicts the observed relative humidity, which may be caused by precipitation events that the WRF model failed to forecast since potential dust mitigation due to remnant soil moisture from past rain events are not included in the model. To adjust for this slight bias in our calculations, the model-predicted relative humidity is multiplied by a correction factor of $1 / 0.97$, product of a least-squared error fit of the parity plot in Figure 2.

The forecast and observed hourly averaged wind speeds are also correlated but with a high degree of scatter (Figure 3A). The results show that the WRF forecast winds are systematically lower than those observed (slope $<1$ ). The low $\mathrm{R}^{2}$ value is indicative of the difficulties when comparing model forecasts using $1.8-\mathrm{km}$ grid spacing to point observations where boundary layer mechanics and surface roughness play an important role. The frequency distribution of the model forecast and the observed wind speeds, Figure 3B, shows that over multiple hourly periods the distributions are very similar. The model-predicted wind speed is corrected in our calculations by multiplying by $1 / 0.84$, which is the fitted slope of the parity plot (Figure 3A).

The histogram of the hourly wind direction residuals is shown in Figure 4 . The histogram was generated using 10 degree bins from $-180^{\circ}$ to $180^{\circ}$. The histogram is shifted to the right of zero, which means the model-predicted wind direction is systematically biased counter clockwise from the observed. By adding $14.7^{\circ}$ of clockwise rotation to the forecast wind direction (first moment of the histogram) we account for the apparent bias between model forecast and observed conditions.

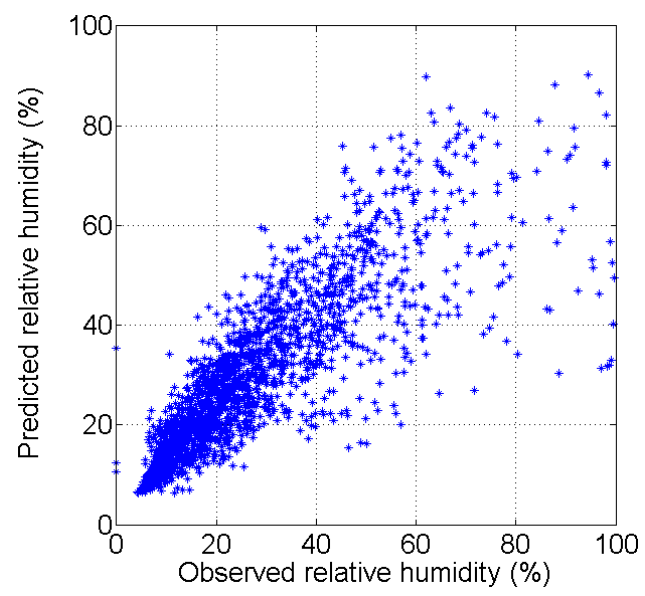

Figure 2. Comparison of hourly averaged observed relative humidity and WRF model forecast predictions for the period of 29 May 2012 to 4 August 2013. A linear fit yields a slope of 0.97 with $R^{2}=0.59$. 

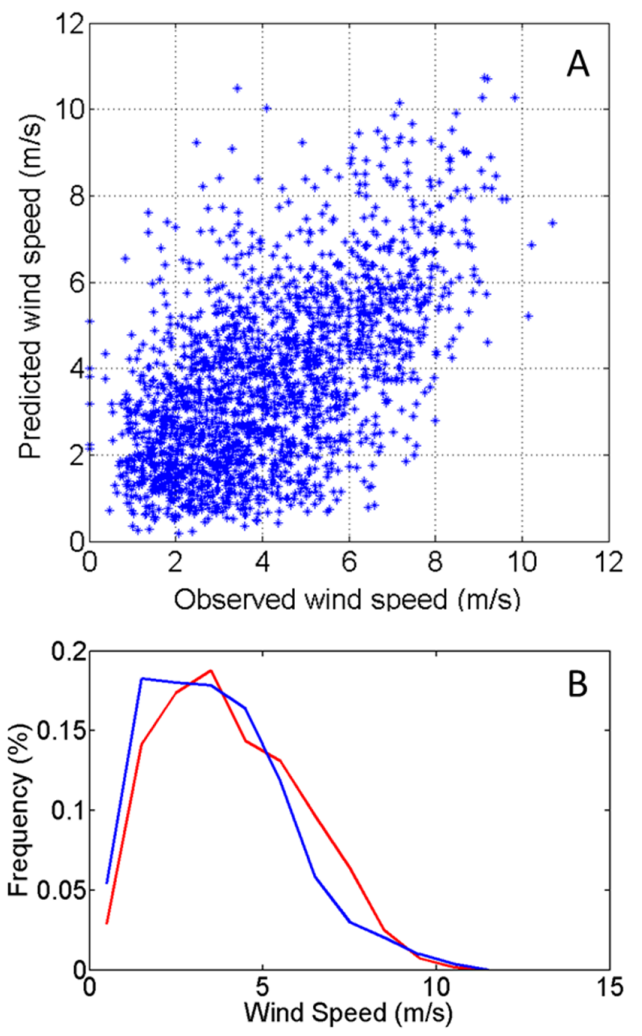

Figure 3. (A) Direct comparison of hourly averaged 10-m height observed wind speed and WRF model predictions for the period of 29 May 2012 to 4 August 2013. A linear fit yields a slope of 0.84 with $\mathrm{R}^{2}=0.23$; (B) Frequency distribution of hourly averaged 10-m height observed wind speed and WRF model predictions for the same period.

\subsection{Deposition Model Predictions}

The DFM simulations were initialized using the corrected WRF model forecasts. Figure 5 shows deposition patterns predicted by the DFM for the fine $\left(\mathrm{PM}_{3.1}\right)$, medium $\left(\mathrm{PM}_{18}-\mathrm{PM}_{3.1}\right)$, coarse $\left(\mathrm{PM}_{27}-\mathrm{PM}_{18}\right)$ and total suspended fine particulate $\left(\mathrm{PM}_{27}\right)$ for the period 21 April to 22 May 2014. A majority of the deposition occurs in the northward direction, which is the prevailing wind direction for the period. However, there is significant predicted deposition in the southeastward and southwestward directions, which was a result of several synoptic scale troughs that shifted the daytime wind direction. One of these troughs was accompanied by precipitation on 27 April 2014. The WRF model predicted the strong wind speeds associated with the trough but failed to accurately forecast the precipitation that was 
observed at the site. The increased soil moisture caused by the precipitation greatly reduces windblown erosion. Hence, the deposition in the southwesterly direction is thought to be significantly overestimated.

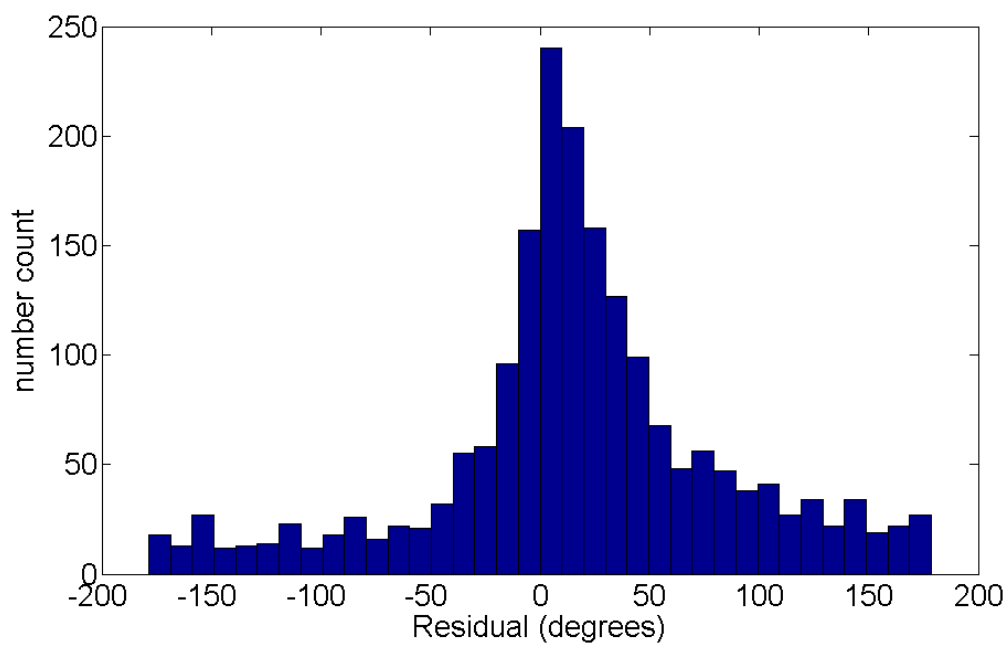

Figure 4. Histogram of residuals of measured wind direction (1-h averages) and WRF model predictions for the period of 29 May 2012 to 4 August 2013. The displaced maximum is at $+14.7^{\circ}$, which indicates a counter clockwise bias in the model results.

The maximum coarse particle deposition was mainly constrained to the immediate vicinity of the tailings due to their large size and fast settling times, which do not allow for long distance transport. The fine particles have a much smaller terminal velocity and are transported much further downwind. Particles of $3-\mu \mathrm{m}$ size have a maximum deposition location that is about three hundred meters from the tailings in the northward direction. Smaller particles may travel longer distances. There are slight variations in the deposition patterns caused by the impact of topography of the surrounding region.

The resulting DFM simulations for the sampling period 11 June to 9 July 2014 can be seen in Figure 6. Compared to the May sampling period, the forecast weather conditions at the site were consistent with predominantly southerly winds that caused almost all the deposition to be in the northward direction. 

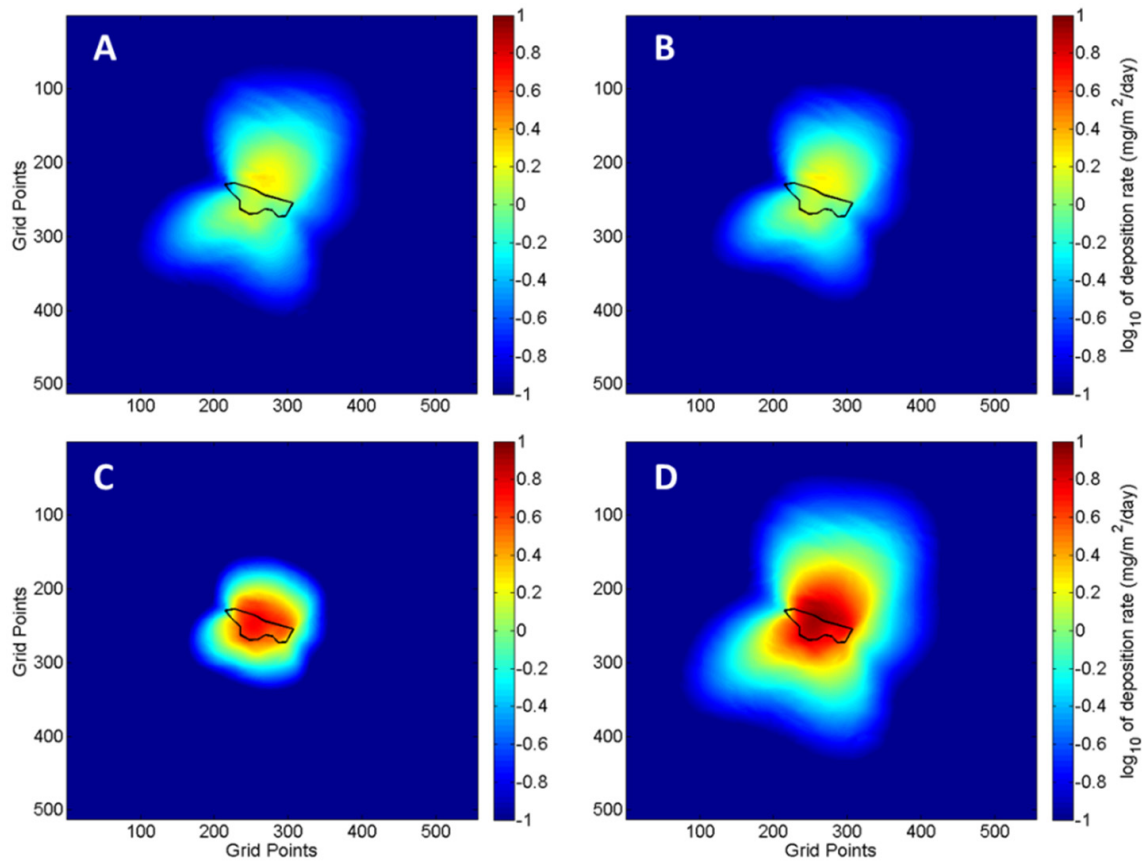

Figure 5. Maps of predicted dust deposition around the IK mine tailings for three size fractions and $\mathrm{PM}_{27}$ predicted by the DFM for the forecast period 21 April to 22 May 2014. Particle size ranges: (A) $\mathrm{PM}_{3.1}$; (B) $\mathrm{PM}_{3.1}-\mathrm{PM}_{18}$; (C) $\mathrm{PM}_{18}-\mathrm{PM}_{27}$; (D) $\mathrm{PM}_{27}$. The tailings impoundment is outlined in black. The grid points are spaced by $10.3 \mathrm{~m}$ and the domain has a total horizontal extent of $34.47639^{\circ}$ to $34.52380^{\circ}$ latitude and $-112.27639^{\circ}$ to $-112.22491^{\circ}$ longitude. Calculated dust deposition corresponds to dust emitted from the mine tailings.

\subsection{Inverted Disc and Soil Sampling Results}

\subsubsection{Mass Analysis}

Inverted-disc sampling occurred during the sampling periods 21 April to 22 May 2014; and 11 June to 9 July 2014. Table 1 shows the deposition fluxes (mass deposited per unit time and sampler surface area) for each disc sampler during both periods and the corresponding model predicted $\mathrm{PM}_{27}$ deposition. It is important to point out that the model developed here predicts deposition of dust generated on the mine tailings and not total deposition, since the region has multiple potential dust sources. It is clear from the data in Table 1 that the model under predicts total deposition, as expected. 

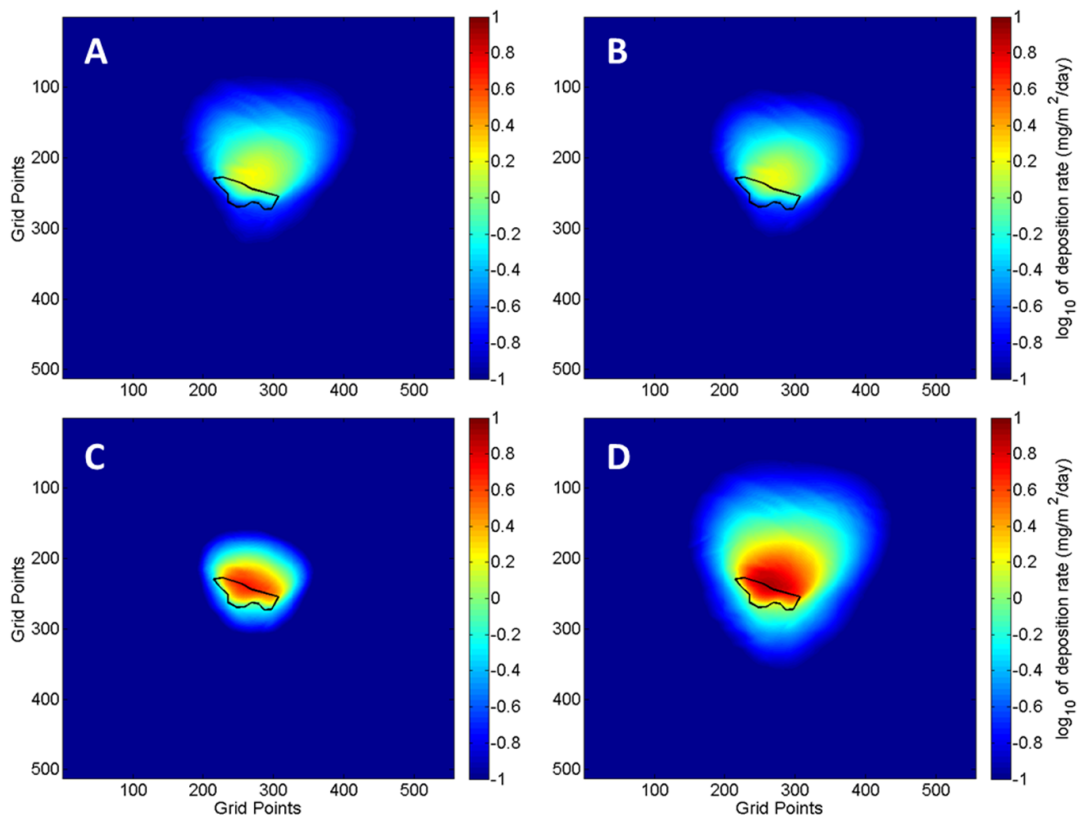

Figure 6. Maps of predicted dust deposition around the IK mine tailings for three size fractions and $\mathrm{PM}_{27}$ predicted by the DFM for the forecast period 11 June to 9 July 2014. Particle size ranges: (A) $\mathrm{PM}_{3.1}$; (B) $\mathrm{PM}_{3.1}-\mathrm{PM}_{18}$; (C) $\mathrm{PM}_{18}-\mathrm{PM}_{27}$; (D) $\mathrm{PM}_{27}$. The tailings impoundment is outline in black. The grid points are spaced by $10.3 \mathrm{~m}$ and the domain has a total horizontal extent of $34.47639^{\circ}$ to $34.52380^{\circ}$ latitude and $-112.27639^{\circ}$ to $-112.22491^{\circ}$ longitude. Calculated dust deposition corresponds to dust emitted from the mine tailings.

The highest deposition fluxes were measured at location $\mathrm{N}$ (Figure 1) for the May sampling period and location B for the June sampling period. Sample N is located adjacent to the highway while sample B is located on the main tailings impoundment. Because roadways are well documented as production source of atmospheric aerosols, it follows that we would expect larger amounts of deposition to be captured with the $\mathrm{N}$ sampler.

The observed deposition flux for the two sampling periods is within an order of magnitude of the forecast deposition fluxes. The peak forecast deposition flux is approximately half of that measured using the samplers. However, the model forecast deposition fluxes drops dramatically for samplers located far from the tailings source (i.e., samplers $\mathrm{F}, \mathrm{M}$ and $\mathrm{N}$ ) while the total mass of dust measured by the samplers remains similar no matter their location. It is important to consider the fact that wind erosion occurs from a variety of sources within the region and each deposition 
sampler is collecting dust from all of them not just aerosols resulting from the tailings, although the exposed, elevated tailings are thought to be an important contributor.

Table 1. Measured dust $\left(\mathrm{PM}_{27}\right)$ mass deposition fluxes in inverted-disc samplers.

\begin{tabular}{|c|c|c|c|c|}
\hline Location & $\begin{array}{c}4 / 21 \text { to } 5 / 22 \text { Observed } \\
\text { Deposition } \\
\left(\mathrm{mg} / \mathrm{m}^{2} / \text { day }\right)\end{array}$ & $\begin{array}{c}4 / 21 \text { to } 5 / 22 \text { Predicted } \\
\text { Deposition } \\
\left(\mathrm{mg} / \mathrm{m}^{2} / \text { day) }\right.\end{array}$ & $\begin{array}{c}\text { 6/11 to } 7 / 09 \text { Observed } \\
\text { Deposition } \\
\left(\mathrm{mg} / \mathrm{m}^{2} / \text { day }\right)\end{array}$ & $\begin{array}{l}\text { 6/11 to } 7 / 09 \text { Predicted } \\
\text { Deposition } \\
\text { (mg/m²/day) }\end{array}$ \\
\hline $\mathrm{A}$ & 15.6 & 7.69 & 17.0 & 4.48 \\
\hline B & 24.2 & 7.96 & 37.1 & 6.11 \\
\hline C & 6.9 & 6.04 & 26.1 & 4.66 \\
\hline D & 26.0 & 1.82 & 29.7 & 1.36 \\
\hline E & 9.4 & 4.48 & 12.1 & 0.53 \\
\hline $\mathrm{F}$ & 14.2 & 2.45 & 10.6 & 0.27 \\
\hline G & 12.3 & 8.11 & 8.5 & 8.21 \\
\hline $\mathrm{H}$ & 18.2 & 8.75 & 8.1 & 8.67 \\
\hline I & 10.1 & 7.39 & 12.6 & 7.02 \\
\hline $\mathrm{J}$ & 16.2 & 7.76 & 9.0 & 7.54 \\
\hline $\mathrm{K}$ & 30.0 & 5.47 & 17.3 & 5.16 \\
\hline $\mathrm{L}$ & 16.3 & 5.45 & 15.5 & 5.15 \\
\hline $\mathrm{M}$ & 16.0 & 1.06 & $\mathrm{~N} / \mathrm{A}$ & $\mathrm{N} / \mathrm{A}$ \\
\hline $\mathrm{N}$ & 32.5 & 1.16 & 6.4 & 0.68 \\
\hline AA & $\mathrm{N} / \mathrm{A}$ & $\mathrm{N} / \mathrm{A}$ & 12.0 & 7.77 \\
\hline BB & $\mathrm{N} / \mathrm{A}$ & $\mathrm{N} / \mathrm{A}$ & 8.7 & 7.43 \\
\hline $\mathrm{CC}$ & $\mathrm{N} / \mathrm{A}$ & $\mathrm{N} / \mathrm{A}$ & 11.0 & 4.81 \\
\hline
\end{tabular}

As mentioned, the deposition forecasting model only simulates the transport of windblown particulate matter from the tailing impoundment and for particles with an aerodynamic diameter $\leqslant 27 \mu \mathrm{m}$. Comparing forecasts of $\mathrm{PM}_{27}$ to bulk deposition samples has inherent errors caused by the potential of the samplers to collect larger particles and skewing the mass fluxes. In order to minimize these issues certain steps were taken to reduce the impact of large particles. The inverted-disc samplers were shown in wind tunnel tests to have the highest collection efficiency, up to $60 \%$, for particles with diameter 10 to $31 \mu \mathrm{m}$. Their collection efficiency significantly drops for particles up to $89-\mu \mathrm{m}$ [21]. The samplers were placed at 1-m height to minimize the capture of large saltating particles. Additionally, mass fractions of dust measured by the MOUDI at 1-m height [11] were 30.1\%, 30.0\%, and 39.9\% for the $>18-\mu \mathrm{m}$, 18 to $3.1-\mu \mathrm{m}$ and $<3.1-\mu \mathrm{m}$ size fractions. The large percentage of mass observed in the smaller size fractions gives us confidence that the inverted-disc samplers are not significantly affected by larger particles and their results are reasonable approximations of $\mathrm{PM}_{27}$ aerosol deposition.

Additional errors in the forecast depositions can arise from the DFM dependency on the WRF wind speed forecast. The WRF model provides us with the highest resolution weather forecasts of the region but it is still susceptible to errors including those experienced during short term high wind events. The compounding factors of multiple dust sources, DFM forecast of $\mathrm{PM}_{27}$ and errors in the WRF forecast makes the under estimated DFM deposition fluxes measured by the inverted-disc samplers 
reasonable, Table 1. Through the use of elemental analysis we can partition the captured dust and determine the relative influence the tailings have on each sampler and the relative spatial distributions.

\subsubsection{Lead and Arsenic Analysis}

The tailings and surrounding soil have significantly elevated arsenic and lead concentrations when compared to the natural surroundings [14]. This is illustrated by the results in Figure 7, where topsoil concentrations of lead and arsenic are shown as a function of distance from the tailings along a NE transect. Significant arsenic and lead contamination extend at least to $1 \mathrm{~km}$ from the tailings, while the $5 \mathrm{~km}$ site exhibits relatively low concentrations, which could correspond to background levels in the region (around $10 \mathrm{ppm}$ for both arsenic and lead).

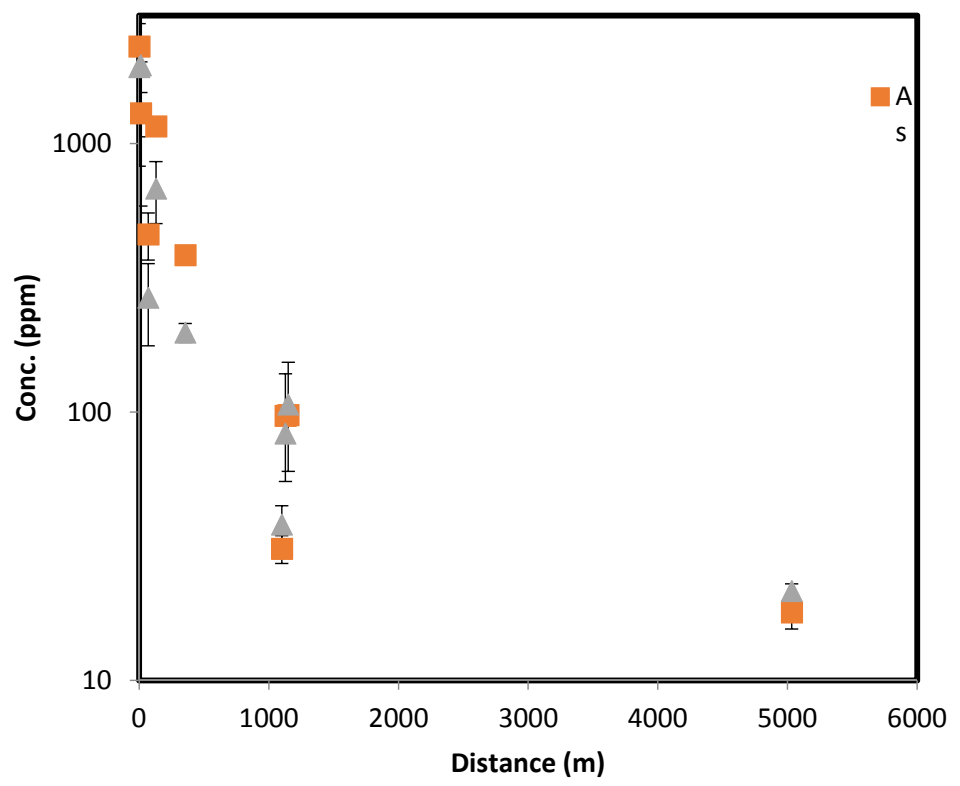

Figure 7. Arsenic and lead concentrations in topsoil (0-3 mm depth) samples at different distances from the mine tailings following a NE transect. Error bars show standard deviation of triplicate samples at the same location.

The arsenic and lead concentrations measured in inverted-disc samplers are presented in Table 2. Figures 8 and 9 show the DFM predictions of average deposition fluxes for the May and June sample periods. A way to assess the results is to compare transects of relative observed concentrations of $\mathrm{As}$ and $\mathrm{Pb}$ with forecast deposition of $\mathrm{PM}_{27}$. Three transects are evaluated in the southwestward, eastward and northward directions. The relative $\mathrm{Pb}$ and $\mathrm{As}$ concentration transects are 
calculated by normalizing each sampler to the average concentration measured by inverted-disc samplers A and B located on the tailings pile. Equation (1) shows the normalization process for a sample concentration $\left(C_{\text {sample }}\right)$ where $C_{A}$ and $C_{B}$ are the concentrations measured at samplers $A$ and $B$, respectively. Transects of the DFM $\mathrm{PM}_{27}$ are normalized by the forecast deposition at the location of sampler $\mathrm{B}$ (34.50087 ${ }^{\circ}$ latitude and $-112.25305^{\circ}$ longitude). All distances of the sample locations are calculated from sample point $B$.

$$
C_{\text {sample norm }}=\frac{C_{\text {sample }}}{\left(C_{A}+C_{b}\right) / 2}
$$

The southward transect is generated using the normalized samples A and B, E (366-m downwind) and $\mathrm{F}$ (538-m downwind). The eastward transect was generated using the normalized samples A and B, C (377-m downwind), D (657-m downwind) and $\mathrm{N}$ (786-m downwind). For the June sample period, the northward transect was generated by first averaging the sample concentrations collected at different downwind ranges, which included the AA samples ( 205-m downwind), I, J, and BB samples ( 300-m downwind) and the K, L and CC samples ( 379-m downwind). The sample averaged concentrations at tailings, 205-m, 300-m and 379-m downwind were then normalized and used to generate the northward transect.

Table 2. Measured total $\mathrm{As}$ and $\mathrm{Pb}$ concentrations measured in the dust collected by the inverted-disc samplers. Locations are shown in Figure 1.

\begin{tabular}{cccc}
\hline Location & $\begin{array}{c}\mathbf{4 / 2 1} \text { to 5/22 Period Pb } \\
\text { (ppm) }\end{array}$ & $\begin{array}{c}\mathbf{6 / 1 1} \text { to } \mathbf{7 / 0 9} \text { Period } \mathbf{P b} \\
\text { (ppm) }\end{array}$ & $\begin{array}{c}\mathbf{6 / 1 1} \text { to } \mathbf{7 / 0 9} \text { Period As } \\
\text { (ppm) }\end{array}$ \\
\hline A & 332 & 903 & 1826 \\
B & 981 & 1743 & 3856 \\
C & 317 & 1037 & 1406 \\
D & 454 & 775 & 1389 \\
E & 130 & 317 & 132 \\
F & 39.3 & 71.2 & 46.4 \\
G & 506 & 1614 & 1311 \\
H & 482 & 838 & 588 \\
I & 217 & 435 & 586 \\
J & 218 & 383 & 504 \\
K & 234 & 342 & 465 \\
L & 292 & 439 & 649 \\
M & 41.9 & N/A & N/A \\
N & 41.2 & 138 & 156 \\
AA & N/A & 384 & 449 \\
BB & N/A & 453 & 595 \\
CC & N/A & 302 & 435 \\
\hline
\end{tabular}




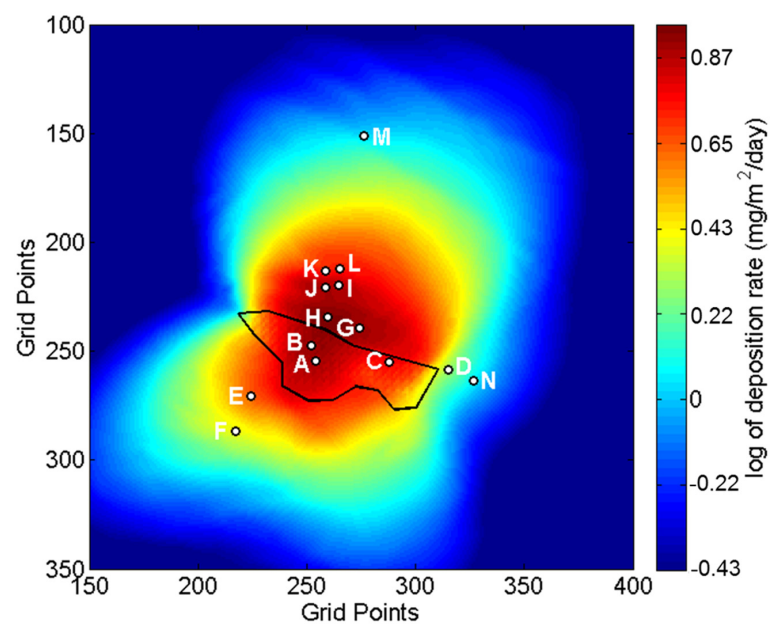

Figure 8. Map of $\mathrm{PM}_{27}$ deposition predicted by the DFM for the forecast period 21 April to 22 May 2014. The color scale represents the natural log of the deposition flux. The tailings impoundment is outlined in black and the locations of the inverted-disc samplers are indicated. The grid points are spaced by $10.3-\mathrm{m}$ and the domain has a total horizontal extent of $34.49142^{\circ}$ to $34.51452^{\circ}$ latitude and $-112.26247^{\circ}$ to $-112.23939^{\circ}$ longitude.

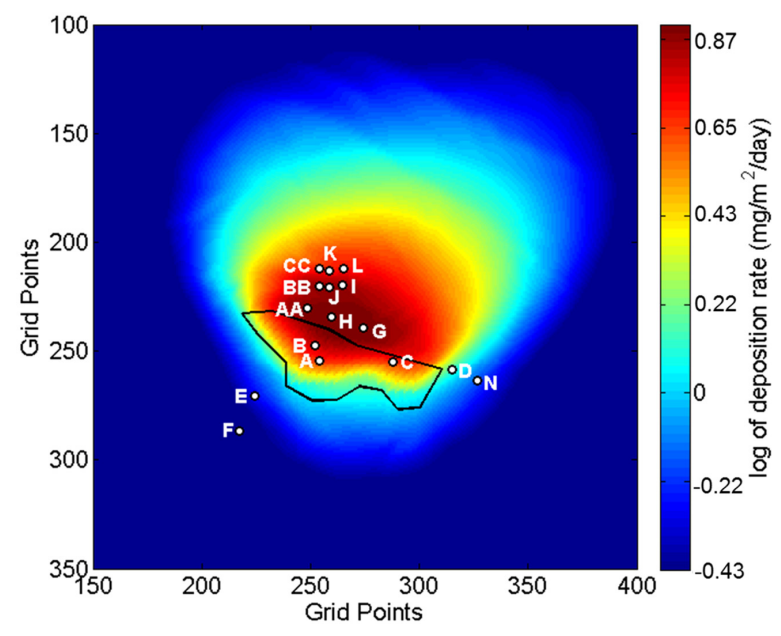

Figure 9. Map of $\mathrm{PM}_{27}$ deposition predicted by the DFM for the forecast period 11 June to 9 July 2014. The color scale represents the natural log of deposition. The tailings impoundment is outlined in black and the location and sample label of the inverted-disc samplers are indicated. The grid points are spaced by $10.3-\mathrm{m}$ and the domain has a total horizontal extent of $34.49142^{\circ}$ to $34.51452^{\circ}$ latitude and $-112.26247^{\circ}$ to $-112.23939^{\circ}$ longitude. 
For the May sampling period we first averaged the sample concentrations of I and J ( 300-m downwind) and K and L ( 379-m downwind). The northward transect was then generated using the normalized samples A and B, H (205-m downwind), 300-m downwind average, 379-m downwind average, and M (1027-m downwind).

Results for the southward, eastward and northward cross sections for the May and June sample periods can be seen in Figure 10. The southward cross sections of the model-predicted fractional reduction in deposition follow similar trends to the observed fractional reduction of $\mathrm{As}$ and $\mathrm{Pb}$ concentrations measured in the inverted-disc samplers. For the May sampling period, the forecast overestimated the relative amount of deposition located at the sample locations $\mathrm{E}$ and $\mathrm{F}$. This was most likely caused by strong northeasterly winds associated with a synoptic scale weather system. This weather system produced precipitation in the region, which the WRF model failed to predict. The erroneous WRF weather forecast yielded an overestimation of windblown dust transport in the southwestward direction. For June, the DFM model accurately forecasts the relative reduction in $\mathrm{As}$ and $\mathrm{Pb}$ at the E and F sample locations.

The eastward transect shows that the model-predicted fractional reduction in deposition was similar to the observed fractional reduction of $\mathrm{As}$ and $\mathrm{Pb}$ concentrations for both the May and June sampling periods for samplers $C$ and $\mathrm{N}$. However, the DFM underestimated the fractional reduction sampler D for both monthly sampling periods (Table 1). Sampler D is located just 10-m from the eastern edge of the lower tailings area and approximately 4-m lower in elevation. This proximity and lower elevation of sampler $\mathrm{D}$ to the lower tailings area increased the likelihood of eroded particles to gravitationally settle into this sampler. Table 1 shows that sampler D had systematically large deposition fluxes measured during both sample periods. Size distributions of the collected dust could not be determined due to the lack of total material captured by the sampler. The increased likelihood of capturing tailing material most likely skewed the fractional $\mathrm{As}$ and $\mathrm{Pb}$ deposition measured by the sampler.

The northward transect shows a similar downwind pattern of reduction in the fractional As and $\mathrm{Pb}$ concentration (Figure 10). The DFM overestimates the fractional reductions of $\mathrm{Pb}$ for the May sampling period and both $\mathrm{As}$ and $\mathrm{Pb}$ for the June sampling period. However, the DFM was significantly better estimating the relative reduction of $\mathrm{As}$ and $\mathrm{Pb}$ for the May sampling period when compared to June. For the May sampling period the DFM accurately predicted the fractional reduction in $\mathrm{Pb}$ concentrations for sampler $\mathrm{M}$ located approximately 1-km north of the tailings. 

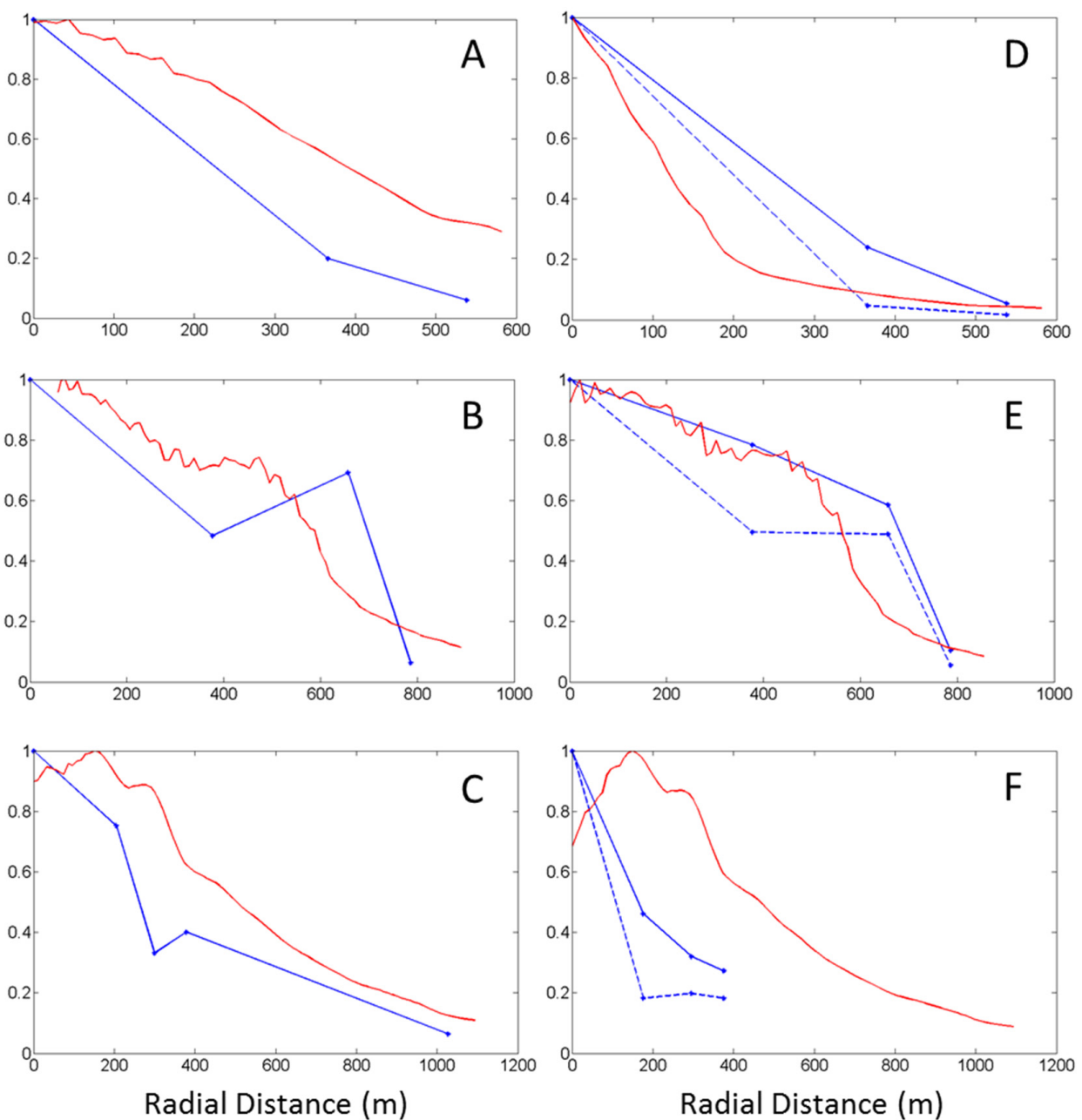

Figure 10. Comparison of relative decreases in arsenic (blue dashed lines) and lead (blue solid lines) mass concentrations measured by the samplers versus the relative decreases of deposition flux forecast by the DFM (red lines) for the southward (A) eastward (B) and northward (C) cross sections for the 21 April to 22 May 2014 sample period and the southward (D) eastward (E) and northward (F) cross sections for the 11 June to 9 July 2014 sample periods.

The highest concentrations of both $\mathrm{As}$ and $\mathrm{Pb}$ were measured by the inverted-disc samplers located on the tailings (A and B). In comparison, the DFM model predicts that the highest amount of tailing dust deposition should occur approximately $150 \mathrm{~m}$ north of sample point B. However the inverted-disc samplers 
show significant reduction in relative $\mathrm{As}$ and $\mathrm{Pb}$ between the tailings ( $\mathrm{A}$ and $\mathrm{B}$ ) and the points located about 205-m north (H and AA).

The DFM includes topographic slopes when calculating deposition rates to the surface. Using computational fluid dynamics modeling, Stovern et al. [10] showed that the slope of the ground significantly impacts deposition in this topographically complex region. The inverted-disc samplers were strategically placed in locations that were sloped for the northward transect. The samples located at 205-m, 300-m and 379-m were in down-sloping, up-sloping and down-sloping regions, respectively. The slope of the ground at $205-\mathrm{m}, 300-\mathrm{m}$ and $379-\mathrm{m}$ is $-5.7^{\circ},+15.1^{\circ}$, and $-22.3^{\circ}$ respectively with a slope length of $50 \mathrm{~m}$. Thus, we would expect that the samples collected at $300 \mathrm{~m}$ should have systematically more deposition than the samplers at 379-m. However, we appear to see the opposite occurring in the May sampling period and relatively equal amounts of deposition in the June sampling period. One reason why the effects of topographic slope are not evident in the deposition patterns may be due to changes in surface roughness between the sampler locations at $300-\mathrm{m}$ and $379-\mathrm{m}$. The samplers located on the up-sloping terrain at $300 \mathrm{~m}$ are surrounded by very sparse vegetation, usually less than a meter in height with large barren patches of soil. On the other hand, the samplers located in the down sloping region at 379-m are surrounded by significantly more vegetation including shrubs, bushes and trees that are typically 2-3 $\mathrm{m}$ in height. The model, however, uses a constant surface roughness of $0.1-\mathrm{m}$. Large surface roughness and obstructing objects capture airborne dust in two ways, it removes momentum from the mean flow slowing transport of airborne particulates allowing them to gravitationally settle, as well as directly capturing airborne dust through direct contact and impaction of the airborne dust. This severe change in surface roughness may explain the counterintuitive results from the samplers. Another possible reason might be the high natural variability in deposition may overwhelm the topographic effect.

\subsubsection{Lead Isotope Analysis}

Figures 11 and 12 show the lead isotopic ratios for the dust collected on the inverted-disc samplers for the May and June sampling periods, respectively. Dust samples A and B located on the tailings themselves have the same isotopic composition as the bulk tailings sample, implying the airborne lead captured in the samplers originated exclusively from the tailings, as expected.

Samples with lower isotopic ratios are the consequence of mixing between the tailings source and regional background. For the May sampling period, the samples that have the lowest isotope ratio are F and N. Sample F is the most southern sample located approximately 300-m from the southern edge of the tailings while $\mathrm{N}$ the eastern most sample is located approximately $150-\mathrm{m}$ from the eastern-most edge of the lower tailings area. The small contribution of tailings lead measured in these 
samplers matches the monthly wind patterns that predominantly transported dust northward, away from the samplers. Samples M and E also have a significantly lower isotopic ratios than the tailing samples. It is interesting to note that sample $\mathrm{E}$ which is located only about $200 \mathrm{~m}$ from the southern edge of the tailings has the same fractional contribution of tailings lead as the sampler located $1 \mathrm{~km}$ north, as expected from the prevailing winds. Also, the source of lead captured by the inverted-disc sampler N, located along AZ highway 69 which separates the Iron King tailings and the town of Dewey-Humboldt, had a smaller tailings contribution than the sampler located $1 \mathrm{~km}$ north of the tailings.

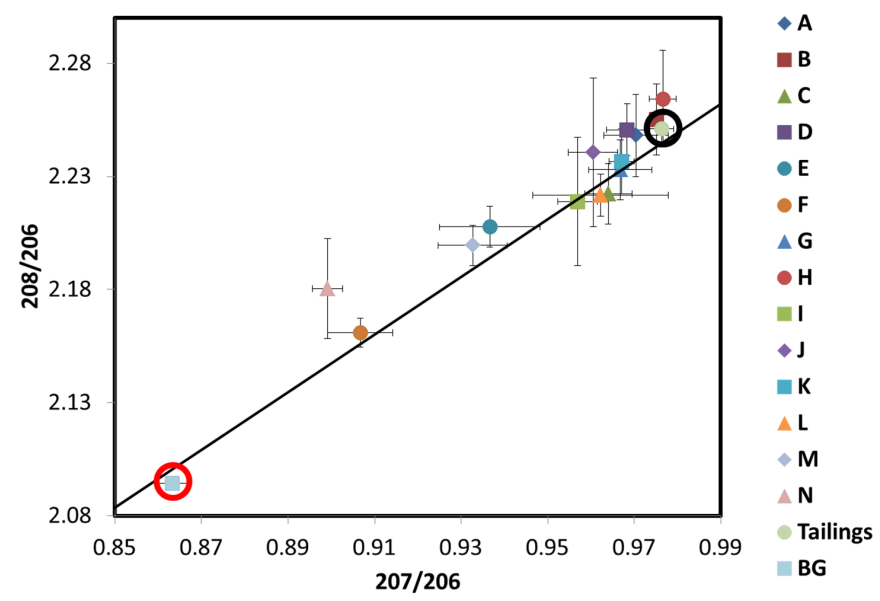

Figure 11. Lead isotopic ratios ${ }^{208} \mathrm{~Pb} /{ }^{206} \mathrm{~Pb}$ and ${ }^{207} \mathrm{~Pb} /{ }^{206} \mathrm{~Pb}$ for each inverted-disc sampler and a bulk sample of tailings material for the 21 April to 22 May 2014 sampling period. The letters represent the inverted-disc sample locations from Figure 1. Tailings (surrounded by a black circle), represent "fingerprint" ratios of the source. The background sample (BG, surrounded by a red circle) corresponds to topsoil ( $0-3 \mathrm{~mm}$ ) collected $5 \mathrm{~km}$ from the source and represents the natural $\mathrm{Pb}$ isotopic "fingerprint" of the region. Error bars represent standard deviations from triplicate samples. Additionally included is the growth curve (solid line) adapted from Chen et al. [23], that represents changes in lead isotopic composition with time due to radiogenic production from isotopes of uranium and thorium, which encompasses all possible Earth samples.

For the June sampling period, the lead isotopic signatures were significantly closer to the tailings bulk sample when compared to the May sampling period. The samples with the lowest isotopic ratios included samples F, N and E. Sample F had the lowest lead contribution from the tailings, this matches the results from the May sampling period. However, more of the lead measured in sample F was sourced from the tailings compared to May. For the eastern most sampler, $\mathrm{N}$ and southern 
sampler E, the isotopic ratios were closer to the tailings signature as well, while still maintaining the lowest isotopic ratios of all the June samplers.

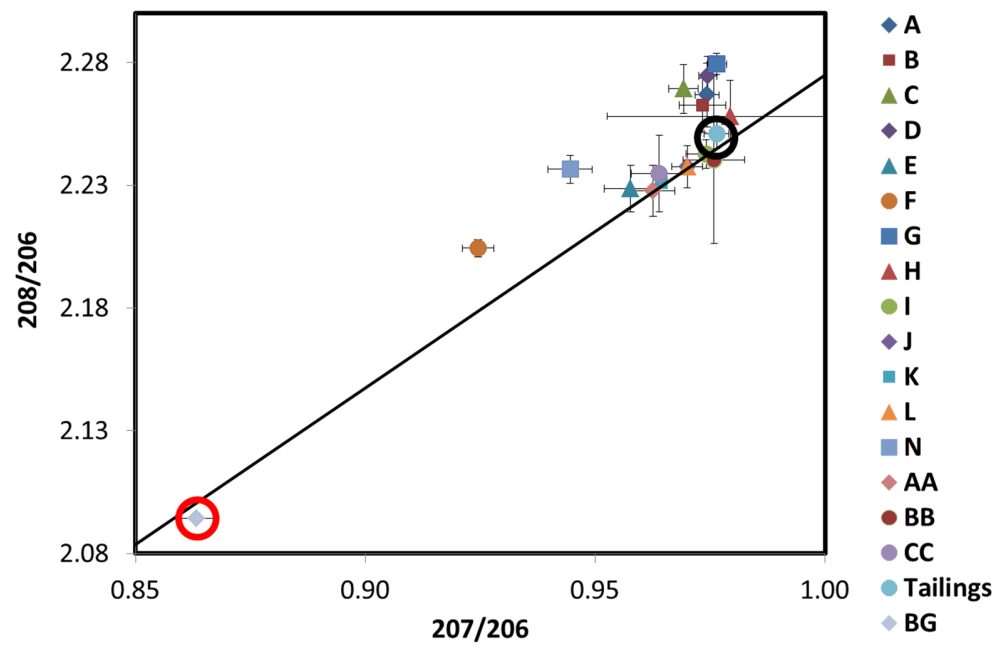

Figure 12. Lead isotopic ratios ${ }^{208} \mathrm{~Pb} /{ }^{206} \mathrm{~Pb}$ and ${ }^{207} \mathrm{~Pb} /{ }^{206} \mathrm{~Pb}$ for each inverted-disc sampler and a sample of tailings topsoil for the 11 June to 9 July 2014 sampling period. The letters represent the inverted-disc sample locations from Figure 1. Tailings (surrounded by a black circle), represent "fingerprint" ratios of the source. The background sample (BG, surrounded by a red circle), corresponds to topsoil (0-3 $\mathrm{mm}$ ) collected $5 \mathrm{~km}$ from the source and represents the natural $\mathrm{Pb}$ isotopic "fingerprint" of the region. Error bars represent standard deviations from triplicate samples. Additionally included is the growth curve (solid line) adapted from Chen et al. [23].

It is interesting to note that the June samplers had significantly higher lead concentrations and isotopic ratios when compared to May. This was caused by a precipitation event that occurred on April 27. This precipitation event significantly increased the tailings moisture content, minimizing wind erosion and reducing windblown lead deposition for the May sample period. In the June sample period the tailings had not received precipitation in over a month which significantly increasing the erosion potential, which resulted in more tailings sourced lead deposition causing the increase in lead concentrations and also shifting isotopic fingerprints closer to the tailings isotopic signature. This shows that local weather patterns including predominant wind directions and precipitation have a significant effect on the deposition of windblown dust from the Iron King tailings impoundment.

The lead isotope ratios can be used to calculate the contribution of deposited dust that originates in the tailings. Assuming only two different lead sources (tailings 
and background), the calculated fractional $\mathrm{Pb}$ tailings contribution was defined here as the ratio between the difference in the $207 / 206$ ratio between the samples and the background, divided by the difference between the tailings and the background. Results are plotted as a function of predicted dust deposition fluxes in Figure 13, for the two different sampling periods. Despite the scatter, a clear increasing relation is obtained between the proportion of dust originating in the tailings and the total deposition flux of tailings materials predicted by the model.

Lead isotopic ratios for the soil samples at different levels collected along the NE transect (Figure 7) are shown in Figure 14. Samples located $130 \mathrm{~m}$ from the tailings were collected in up-sloped terrain and their lead signatures are close to the tailings, indicating that the relatively high concentrations of lead (Figure 7) are a consequence of dust transport from the tailings. At this distance, results from the DFM model also point to a high deposition of tailings dust (Figure 10). It is interesting to point out that even at a depth of $100 \mathrm{~mm}$, lead isotope analysis points to the tailings as main contributor of the metal in the soil. At longer distances from the tailing $(1150 \mathrm{~m})$, topsoil and 100-mm depth soil have significantly different signatures, with deep soil reflecting a higher contribution from the $5-\mathrm{km}$ background. The sampling point located $13 \mathrm{~m}$ from the tailing has the same lead isotope ratios as the tailing topsoil, as expected, but isotopic ratios decrease monotonically with distance towards the background site located $5 \mathrm{~km}$ from the tailings.

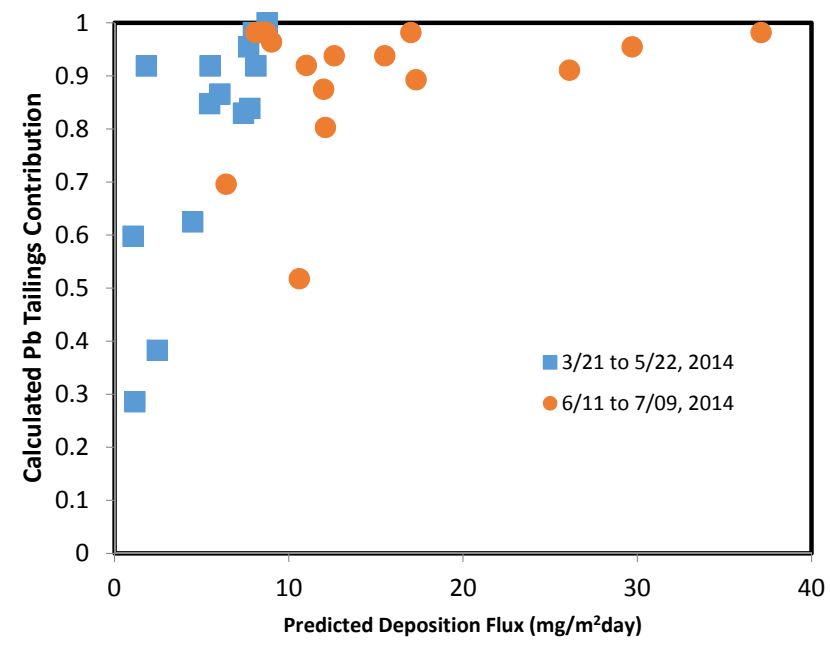

Figure 13. Calculated tailings contribution of deposited lead in inverted-disc samplers as a function of the model-predicted dust deposition flux for the two different sampling periods in 2014. Tailings contribution ( 1 for tailings, 0 for background) are calculated from the 207/206 isotopic ratios reported in Figures 11 and 12. 


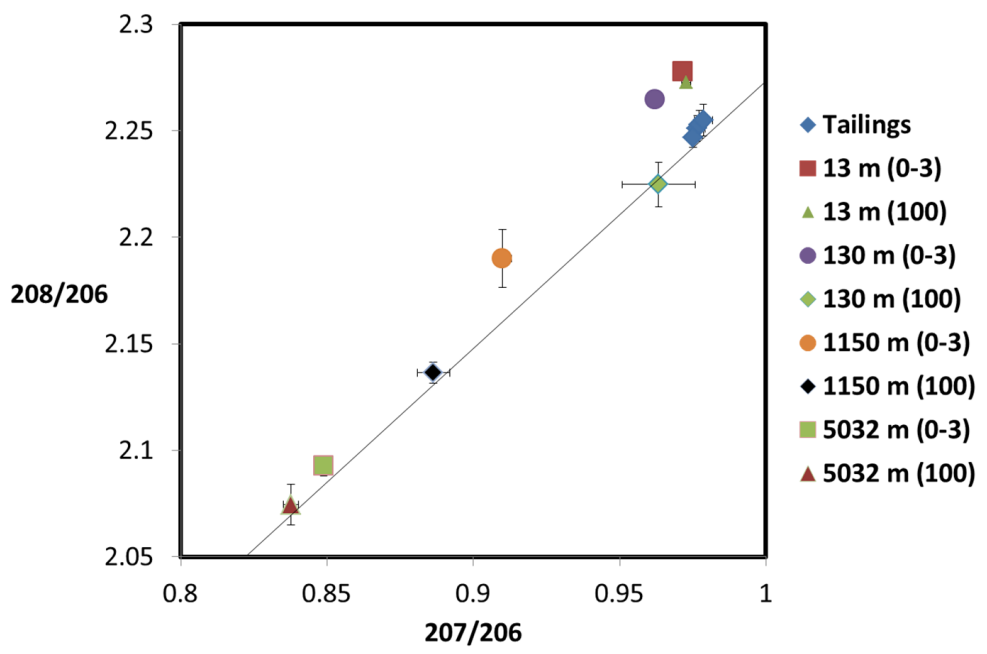

Figure 14. Lead isotopic ratios for soil samples at different distances from the mine tailings (indicated). Numbers in parentheses represent the depth of the sample in $\mathrm{mm}$. Error bars represent standard deviations from triplicate samples. Additionally included is the growth curve (solid line) adapted from Chen et al. [23].

\section{Conclusions}

The DFM is designed to utilize weather forecasts to predict the deposition of fugitive $\mathrm{PM}_{27}$ dust originating from the Iron King tailings impoundment. By comparing the DFM predicted $\mathrm{PM}_{27}$ deposition to arsenic and lead tracers collected by the inverted-disc samplers, it has been shown that the DFM captures trends on spatial variations of the deposition patterns in the surrounding region up to $1 \mathrm{~km}$ distance from the tailings. The effects of topography on deposition still need adjustment due to the complex variations of surface roughness within the region. In addition, the difficulties associated in directly quantifying $\mathrm{PM}_{27}$ deposition using inverted-disc samplers leaves room for additional investigation into the absolute deposition quantities provided by the DFM. However, combining the deposition patterns generated by the DFM and the known concentrations of arsenic and lead in tailing dust we can provide relative estimates of arsenic and lead deposition rates near the tailings impoundment. These estimates of deposition should improve the characterization of potential health impacts caused by windblown transport from the tailings. The methodology employed in this work can be generalized to other contaminated sites from which dust transport to surrounding communities can be assessed in terms of a potential route for human exposure. Dust emission quantification relied on weather forecasting and empirical relations for emitted dust fluxes while transport and deposition were predicted by following particle 
trajectories in the forecast wind field. This approach led to a model that can be used to forecast the rate of transport and deposition of contaminants from the tailings to the local environment.

Acknowledgments: This work was supported by grant number P42 ES04940 from the National Institute of Environmental Health Sciences (NIEHS), National Institutes of Health (NIH). The views of authors do not necessarily represent those of the NIEHS, NIH.

Author Contributions: Michael Stovern developed the model, performed model calculations, designed and collected inverted-disc sample data and contributed to the writing and analysis. Hector Guzman and Omar Felix collected and analyzed soil samples. Kyle Rine and Matthew King contributed to field data collection and experimental design. Wendell Ela, Eric Betterton and Eduardo Sáez contributed to experiment and model design, data analysis and writing.

Conflicts of Interest: The authors declare no conflict of interest.

\section{References}

1. Wilkening, K.E.; Barrie, L.A.; Engle, M. Atmospheric science: Trans-Pacific air pollution. Science 2000, 290, 65-67.

2. Sprigg, W.A.; Nickovica, S.; Galgianic, J.N.; Pejanovic, G.; Petkovic, S.; Vujadinovic, M.; Vukovic, A.; Dacic, M.; DiBiase, S.; Prasad, A.; et al. Regional dust storm modeling for health services: The case of valley fever. Aeolian Res. 2014, 14, 53-73.

3. Brook, R.D.; Rajagopalan, S.; Pope, C.A.; Brook, J.R.; Bhatnagar, A.; Diez-Roux, A.V.; Holguin, F.; Hong, Y.; Luepker, R.V.; Mittleman, M.A.; et al. Particulate matter air pollution and cardiovascular disease an update to the scientific statement from the American Heart Association. Circulation 2010, 121, 2331-2378.

4. Csavina, J.; Field, J.D.; Taylor, M.P.; Gao, S.; Landázuri, A.; Betterton, E.A.; Sáez, A.E. A review on the importance of metals and metalloids in atmospheric dust and aerosol from mining operations. Sci. Total Environ. 2012, 433, 58-73.

5. Csavina, J.; Field, J.D.; Félix, O.; Corral-Avitia, A.Y.; Sáez, A.E.; Betterton, E.A. Effect of wind speed and relative humidity on atmospheric dust concentrations in semi-arid climates. Sci. Total Environ. 2014, 487, 82-90.

6. Badr, T.J.; Harion, L. Numerical modeling of flow over stockpiles: Implications on dust emissions. J. Atmos. Environ. 2005, 39, 5576-5584.

7. Turpin, C.; Harion, J.L. Effect of the topography of an industrial site on dust emissions from open storage yards. Environ. Fluid Mech. 2010, 10, 677-690.

8. Turpin, C.; Harion, J.L. Numerical modelling of flow structures over an industrial site: Effect of the surrounding buildings on dust emissions. Glob. NEST J. 2010, 12, 40-45.

9. Diego, I.; Pelegry, A.; Torno, S.; Torana, J.; Menendez, M. Simultaneous CFD evaluation of wind flow and dust emission in open storage piles. J. Appl. Math. Model. 2009, 33, 3197-3207.

10. Stovern, M.; Felix, O.; Csavina, J.; Rine, K.P.; Russell, M.R.; Jones, R.M.; King, M.; Betterton, E.A.; Sáez, A.E. Simulation of windblown dust transport from a mine tailings impoundment using a computational fluid dynamics model. Aeolian Res. 2014, 14, 75-83. 
11. Stovern, M.K.; Rine, K.P.; Russell, M.R.; Felix, O.; King, M.; Sáez, A.E.; Betterton, E.A. Development of a dust deposition forecasting model for a mine tailings impoundment using in situ observations and idealized particle simulations. Aeolian Res. 2015, 18, 155-167.

12. EPA. Iron King Mine and Humboldt Smelter. Available online: Http:/ / yosemite.epa. gov/r9/sfund/r9sfdocw.nsf/db29676ab46e80818825742600743734/316161edfc4699a 5882574ab0001d1c0!OpenDocument\#descr (accessed on 2 July 2014).

13. Félix, O.I.; Csavina, J.; Field, J.; Rine, K.P.; Sáez, A.E.; Betterton, E.A. Use of lead isotopes to identify sources of metal and metalloid contaminants in atmospheric aerosol from mining operations. Chemosphere 2015, 122, 219-226.

14. EA. Engineering, Science and Technology. Remedial Investigation Report Iron King Mine-Humboldt Smelter Superfund Site Dewey-Humboldt, Yavapai County, Arizona, 2010; US Environmental Protection Agency Region 6: Dallas, TX, USA, 2010.

15. Solís-Dominguez, F.A.; White, S.A.; Hutter, T.B.; Amistadi, M.K.; Root, R.A.; Chorover, J.; Maier, R.M. Response of key soil parameters during compost-assisted phytostabilization in extremely acidic tailings: Effect of plant species. Environ. Sci. Technol. 2012, 46, 1019-1027.

16. Hagen, L.J.; Pelt, S.V.; Zobeck, T.M.; Retta, A. Dust deposition near an eroding source field. Earth Surf. Process. Landf. 2007, 32, 281-289.

17. Koester, C.J.; Hites, R.A. Wet and dry deposition of chlorinated dioxins and furans. Environ. Sci. Technol. 1992, 26, 1375-1382.

18. Hall, D.J.; Upton, S.L. A wind tunnel study of the particle collection efficiency of an inverted Frisbee used as a dust deposition gauge. Atmos. Environ. 1988, 22, 1383-1394.

19. Hall, D.J.; Waters, R.A. An improved readily available dustfall gauge. Atmos. Environ. 1986, 20, 219-222.

20. Vallack, H.W. A field evaluation of Frisbee-type dust deposit gauges. Atmos. Environ. 1995, 29, 1465-1469.

21. Sow, M.; Goossens, D.; Rajot, J.L. Calibration of the MDCO dust collector and of four versions of inverted Frisbee dust deposition sampler. Geomorphology 2006, 82, 360-375.

22. Goossen, D.; Rajot, J.L. Techniques to measure the dry aeolian deposition of dust in arid and semi-arid landscapes: A comparative study in West Niger. Earth Surf. Process. Landf. 2008, 33, 178-195.

23. Chen, J.; Tan, M.; Li, Y.; Zheng, J.; Zhang, Y.; Shan, Z.; Zhang, G.; Li, Y. Characteristics of trace elements and lead isotope ratios in $\operatorname{PM}(2.5)$ from four sites in Shanghai. J. Hazard. Mater. 2008, 156, 1-3. 


\title{
Decreasing Aerosol Loading in the North American Monsoon Region
}

\author{
Aishwarya Raman, Avelino F. Arellano Jr. and Armin Sorooshian
}

Abstract: We examine the spatio-temporal variability of aerosol loading in the recent decade (2005-2014) over the North American Monsoon (NAM) region. Emerging patterns are characterized using aerosol optical depth (AOD) retrievals from the NASA Terra/Moderate Resolution Imaging Spectroradiometer (MODIS) instrument along with a suite of satellite retrievals of atmospheric and land-surface properties. We selected 20 aerosol hotspots and classified them into fire, anthropogenic, dust, and NAM alley clusters based on the dominant driver influencing aerosol variability. We then analyzed multivariate statistics of associated anomalies during pre-, monsoon, and post-monsoon periods. Our results show a decrease in aerosol loading for the entire NAM region, confirming previous reports of a declining AOD trend over the continental United States. This is evident during pre-monsoon and monsoon for fire and anthropogenic clusters, which are associated with a decrease in the lower and upper quartile of fire counts and carbon monoxide, respectively. The overall pattern is obfuscated in the NAM alley, especially during monsoon and post-monsoon seasons. While the NAM alley is mostly affected by monsoon precipitation, the frequent occurrence of dust storms in the area modulates this trend. We find that aerosol loading in the dust cluster is associated with observed vegetation index and has only slightly decreased in the recent decade.

Reprinted from Atmosphere. Cite as: Raman, A.; Arellano, A.F., Jr.; Sorooshian, A. Decreasing Aerosol Loading in the North American Monsoon Region. Atmosphere 2016, 7, 24.

\section{Introduction}

Aerosols play a critical role in global and regional climate, monsoonal circulation, hydrological cycle, air quality, and public health (e.g., [1-4]). Such is the case for aerosols in the semi-arid regions of North America. There is growing concern about aerosols in the North American monsoon (NAM) region as studies have shown (and projected) a warmer and drier southwest United States [5], leading to increased wildfire risks and occurrence of dust storms ([6-8], and references therein). Studies have also reported the ability of aerosols in modifying NAM precipitation through direct or indirect effects [9-11] and epidemiological outbreaks from pollution [12]. While there has been increased attention in recent decades directed to aerosols in the Asian monsoon region $[2,3,13,14]$, limited studies have examined local-to-regional 
characteristics and trends of aerosols in the NAM region, precluding our ability to accurately predict its response to projections of changes in environmental conditions.

NAM is a notable feature in the atmospheric circulation over North America. It is characterized by a shift in the circulation pattern due to warmer land surfaces in the southwestern United States and northwestern Mexico during May and June, resulting in an upper-level anti-cyclone over western Mexico and a pronounced increase in precipitation from convective storms over these regions (including western Texas) during July and August. Although the NAM location is centered at the Sierra Madre Occidental in Mexico, its influence on monsoon precipitation extends widely into the areas of Arizona and New Mexico. In fact, NAM provides $70 \%$ of the annual precipitation in the region [15]. We refer the reader to several studies [16-18] on further details of its spatial extent and the underlying meteorological processes. For this study, we define the NAM region to include: northern Mexico, Arizona, southern California (SoCal), New Mexico, and western Texas. In terms of aerosols, the NAM region exhibits distinct spatio-temporal patterns due to diversity in aerosol sources and sinks during its pre-monsoon (PRM: May-June), monsoon (MON: July-August), and post-monsoon (POM: September-October) phases. During May and June, the warmer and drier conditions result in fires in vegetated areas of NAM. Large dust events typically occur in the desert areas (and abandoned agricultural fields) during MON as a result of mesoscale convective storms. NAM is not only characterized by natural aerosols (dust and fire) but also by anthropogenic aerosols [19,20]. This region in fact has the top five most polluted cities for particulate matter [21]. In terms of aerosol removal, NAM experiences significant rainfall events between July and August that are quite distinct across the year for this semi-arid environment.

Past studies of aerosol trends in the United States (US) have mainly focused on the entire North American continent or the eastern US (e.g., [22,23]). For example, a recent study [24] examined the decadal trend (2000-2009) of aerosol optical depth (AOD) in the US based on NASA Terra Moderate Resolution Imaging Spectroradiometer (MODIS) and from Advanced Very high Resolution Radiometer (AVHRR) aerosol retrievals along with simulations from the Goddard Chemistry Aerosol Radiation and Transport (GOCART) model as part of a global model/data trend analyses. They found a significant decrease in AOD [24], which they attributed to the reductions in anthropogenic (combustion-related) emissions. This finding is consistent with an earlier model study [25] using the Model of Atmospheric Transport and Chemistry and the Dust Entrainment and Deposition models that reported a significant decline of the AOD trend from anthropogenic aerosol sources over the period 1980-2006. This study also revealed that the long-term trends in natural aerosol sources over the US were not significant for the given time period. Thus, more local-to-regional studies are needed as competing sources can obscure the reported global/regional trend [24]. 
Over the southwest US, a climatology of aerosol loading in several areas across the state of Arizona using a suite of aerosol ground-based measurements (e.g., Interagency Monitoring of PROtected Visual Environments or IMPROVE), remotely-sensed aerosol products (e.g., MODIS), as well as model output from GOCART, showed a significant range in spatio-temporal variations of aerosols within the state, depending on the proximity of the measurement site to the dominant drivers (e.g., dust, rural activities, urban pollution, etc.) [19]. Recently, another study in this region noted that AOD has increased by $19 \%$ over Tucson, Arizona, in the last 35 years based on two sets of AOD (400-900 nm) measurements over Tucson (i.e., 1975-1977 versus 2010-2012) [26]. They postulated that the increase might have been contributed by urbanization and the near tripling of population in the city. These studies highlight the importance of examining the aerosol variations across the southwestern US in detail, given mixtures of drivers on aerosol loading across the region and its unique environmental conditions (including complex topography) relative to the southeast US. In light of decadal records of remotely-sensed retrievals of atmospheric composition, precipitation, and land-surface properties, there is also a unique opportunity to conduct a multivariate analysis for a more comprehensive and consistent picture of aerosol trends in the region.

In this study, we focus on assessing the spatio-temporal trends in aerosol loading across the NAM region by examining several aerosol hotpots and their associated trends during pre-monsoon (PRM), monsoon (MON), and post-monsoon (POM) seasons over the recent decade. In particular, we aim to: (1) analyze the trends in MODIS AOD from 2005-2014 over areas characterized by a dominant source or sink (i.e., fire, anthropogenic, dust, and a region affected by monsoon rainfall); (2) elucidate controlling factors of these trends using correlative information from Tropical Rainfall Measuring Mission (TRMM) precipitation products (on aerosol removal), MODIS vegetation index (on dust aerosols), fire products (on fire aerosols), and CO from Measurement of Pollution in The Troposphere (MOPITT) instrument (on anthropogenic aerosols).

\section{Methodology}

\subsection{Satellite Products}

All satellite datasets were downloaded through NASA GIOVANNI web portal developed by Goddard Earth Sciences Data and Information Science Center (GES DISC) [27]. These datasets are monthly averages and were regridded to $1^{\circ} \times 1^{\circ}$ to match the MODIS spatial resolution. A brief description of each data is given below, together with Table 1 which summarizes relevant information about the datasets. 
Table 1. Analysis datasets.

\begin{tabular}{|c|c|c|}
\hline Instrument and Dataset & Resolution & $\begin{array}{l}\text { Relevance to Study } \\
\text { (Main Product Reference) }\end{array}$ \\
\hline $\begin{array}{l}\text { NASA Terra and Aqua L3 MODIS } \\
\text { Aerosol Optical Depth (AOD) } 550 \mathrm{~nm}\end{array}$ & $1^{\circ} \times 1^{\circ}$ & $\begin{array}{l}\text { aerosol loading } \\
\text { (Levy et al., 2007 [29]) }\end{array}$ \\
\hline $\begin{array}{l}\text { NASA Terra L3 MODIS Fire Radiative } \\
\text { Power (FRP) and fire counts }\end{array}$ & $1^{\circ} \times 1^{\circ}$ & $\begin{array}{l}\text { fire sources } \\
\text { (Wooster et al., } 2005 \text { [36]) }\end{array}$ \\
\hline $\begin{array}{l}\text { NASA Terra L3 MODIS Normalized } \\
\text { Vegetation Index (NDVI) }\end{array}$ & $0.05^{\circ} \times 0.05^{\circ}$ & $\begin{array}{l}\text { biogenic and dust sources } \\
\text { (Lunetta et al., 2006 [38]) }\end{array}$ \\
\hline $\begin{array}{l}\text { NASA OMI L2G UV Aerosol } \\
\text { Index (AI) } 354 \mathrm{~nm}\end{array}$ & $0.25^{\circ} \times 0.25^{\circ}$ & $\begin{array}{l}\text { aerosol cluster identification } \\
\text { (Torres et al., 2007 [39]) }\end{array}$ \\
\hline $\begin{array}{l}\text { NASA MOPITT L3 TIR/NIR } \\
\text { Total Column CO }\end{array}$ & $1^{\circ} \times 1^{\circ}$ & $\begin{array}{l}\text { combustion sources } \\
\text { (Deeter } \text { et al., } 2012 \text { [41]) }\end{array}$ \\
\hline $\begin{array}{l}\text { NASA TRMM Best Estimate } \\
\text { Precipitation Rate (BEPR) }\end{array}$ & $0.25^{\circ} \times 0.25^{\circ}$ & $\begin{array}{l}\text { aerosol removal } \\
\text { (Huffman et al., } 2007 \text { [40]) }\end{array}$ \\
\hline $\begin{array}{l}\text { UMBC Anthropogenic Biomes } \\
\text { V2 (2000) (ecotope.org) }\end{array}$ & $0.083^{\circ} \times 0.083^{\circ}$ & $\begin{array}{l}\text { aerosol cluster identification } \\
\text { (Ellis et al., } 2010 \text { [45]) }\end{array}$ \\
\hline $\begin{array}{l}\text { NASA SEDAC Global Rural Urban } \\
\text { Mapping Project version } 1 \text { (GRUMPv1) } \\
\text { Population Density } \\
\text { (sedac.ciesin.columbia.edu) }\end{array}$ & $1 \mathrm{~km} \times 1 \mathrm{~km}$ & $\begin{array}{l}\text { aerosol cluster identification } \\
\text { (Balk et al., } 2009 \text { [43]) }\end{array}$ \\
\hline
\end{tabular}

\subsubsection{MODIS}

The MODIS instrument is an imaging spectroradiometer on board two polar orbiting satellites, NASA EOS/Terra (Febraury 2000-present) and NASA EOS/Aqua (June 2002-present). It provides global coverage every one to two days with an equatorial overpass time around 10:30 am (descending) for Terra and 1:30 pm (ascending) for Aqua. MODIS acquires data in 36 spectral bands from 0.41 to $14 \mu \mathrm{m}$. It has a swath of $2330 \mathrm{~km}$ at cross-track and $10 \mathrm{~km}$ at nadir [28].

Aerosol Optical Depth (AOD). We use the Level 3 (gridded) MODIS $1^{\circ} \times 1^{\circ}$ Collection 5.1 (M3 mean_mean) AOD retrievals at $550 \mathrm{~nm}$ for both Terra and Aqua (MOD08_M3_V051) ([28] and references therein; [29,30]) to investigate trends of aerosol loading across the period 2005-2014. The Level 2 (swath) version of these retrievals are produced using three spectral wavelengths $(470,650,2100 \mathrm{~nm})$ and two algorithms ("dark target" for dark/vegetated surfaces and "deep blue" for bright surfaces such as the desert). The main aerosol product at $550 \mathrm{~nm}$ is derived by matching the reflectance values at different channels to the atmospheric properties using a look-up table. These retrievals have been widely used in the studies pertinent to the spatio-temporal variability of aerosols ([31,32] and references therein). We note, however, that evaluation against AERONET in-situ measurements of Collection 5.1 AOD products as derived using the "dark target" algorithm show overestimation 
of AOD [29,30]. We use the dark target for this study since the Collection 5.1 AOD retrievals using the "deep blue" algorithm were not available after December 2007 due to polarization issues in the sensor [33]. Our initial comparison of AOD between "deep blue" (from Collection 6) and "dark target" over the NAM region shows similarity in spatio-temporal patterns with "deep blue" AOD values (on average) being less by 0.1 . We further note that previous studies report a shift from high to low bias of AOD from the Terra satellite after 2004 due to the degradation of Terra/MODIS's optical response [29]. Further information about these products is discussed in detail in $[29,34]$.

Fire. We use Terra/MODIS monthly mean and cloud-corrected Fire Radiative Power (FRP in units of million watts) along with MODIS fire counts (FC, counts $\mathrm{m}^{-2}$ day $^{-1}$ ) at $1^{\circ} \times 1^{\circ}$ resolution (MODIS Active Fire Product version 005 MOD14CM1) to indicate fires in the region. MODIS identifies a candidate pixel as being affected by fires if the $4 \mu \mathrm{m}$ brightness temperature and the difference between $4 \mu \mathrm{m}$ and $11 \mu \mathrm{m}$ brightness temperature depart substantially from non-fire pixels. The estimated accuracy of FRP from MODIS is $15 \%$ [35,36].

Normalized Difference Vegetation Index (NDVI). Aerosol loading in regions dominated by dust aerosols highly depend on the landcover characteristics (e.g., large-scale vegetation reduces dust lofting) (e.g., [37]). We use the information from NDVI to indicate potential dust mobilization in the region. MODIS/Terra provides cloud-free composites of NDVI obtained using corrected surface reflectances in visible and near infrared channels. Here, we use MODIS/Terra Level 3 monthly mean NDVI at 0.05 degree (MOD13C2) [38]. This product is based on the spatial and temporal averages of 16 day $1 \mathrm{~km}$ NDVI retrievals.

\subsubsection{Ozone Monitoring Instrument (OMI) Ultraviolet Aerosol Index (UV AI)}

OMI, which is on board the NASA EOS-Aura satellite, uses backscattered UV radiation measured at two wavelengths (354 and $388 \mathrm{~nm}$ ) to derive UV AI. OMI has a local equator crossing time at $\sim 1: 45 \mathrm{pm}$. OMI has an advantage of being more sensitive to atmospheric aerosol loading since the reflectance of most terrestrial surfaces are low at UV wavelengths [39]. We use the Level 2G OMI UV AI retrievals at $354 \mathrm{~nm}$ mainly to identify aerosol hotspots in the region and partly to supplement MODIS AOD for aerosol loading trend analysis. The parent resolution of the product is $0.25^{\circ} \times 0.25^{\circ}$. Due to its higher spatial resolution compared to MODIS, OMI AI is more useful in identifying localized high aerosol loading across our domain. Also, OMI UV AI is more sensitive to smoke and dust compared to MODIS AOD.

\subsubsection{Tropical Rainfall Measuring Mission (TRMM) Precipitation Rate}

We use the monthly mean gridded "best estimate precipitation rate" (BEPR) product obtained from TRMM multi-satellite precpitation analyses (3B43 Version 7) 
to understand the potential impact of rainfall on the aerosol loading in the NAM region. TRMM best estimate precipitation rate dataset is a combination of data from rain gauge stations and satellite sensors providing precipitation estimates [40]. The spatial resolution of this product is $0.25^{\circ} \times 0.25^{\circ}$.

\subsubsection{Measurements of Pollution in the Troposphere (MOPITT)} Carbon Monoxide (CO)

The MOPITT instrument is a gas correlation radiometer also onboard the NASA EOS/Terra satellite. We use MOPITT Level 3 gridded $\left(1^{\circ} \times 1^{\circ}\right)$ monthly total column multispectral $(2.3$ and $4.7 \mu \mathrm{m}) \mathrm{CO}$ retrievals [41,42] to indicate fire and/or anthropogenic aerosols in the region. As a product of incomplete combustion, $\mathrm{CO}$ is a useful tracer of fire and anthropogenic pollution. We can therefore distinguish between fire and anthropogenic aerosol signatures using the combination of MOPITT and MODIS fire products. This is further facilitated by enhanced sensitivity of MOPITT CO multispectral retrievals (thermal+near-infrared) to CO in the lowermost troposphere [41].

\subsection{Ancillary Datasets}

We use data on population density from Global Rural-Urban Mapping project version 1 (GRUMP V1 2000), which is provided by Socio Economic Data and Application Center (SEDAC) $[43,44]$, to identify populated areas in the region. The data is available at 30 arc-second resolution. Along with GRUMP, we also use the anthropogenic biomes version 2 for year 2000 (also downloaded from SEDAC) to identify urban and rural areas in the region. Anthropogenic biomes or anthromes provide useful information on the alterations to the global ecosystem and biotic communities by human population $[45,46]$. The anthrome product has 21 anthropogenic biomes with the following six major classes: dense settlements, villages, croplands, rangelands, forested and wildlands (see Figure 1C for the different anthromes in NAM).

\subsection{Data Analysis}

Aerosol hotspots and clusters. We have selected a region of study bounded between the coordinates $\left(120.5^{\circ} \mathrm{W}, 95.5^{\circ} \mathrm{W}\right)$ and $\left(22.5^{\circ} \mathrm{N}, 37.5^{\circ} \mathrm{N}\right)$. We use the monthly decadal mean from OMI AI, along with population density and anthromes, to identify 20 aerosol hotspots in the NAM region. First, we find the climatological maximum in OMI UV AI across all six months (May to October) for each grid cell in the NAM domain. The corresponding month at which the maximum occurs for a grid cell is also identified (see Figure 1a,b). Second, we determine if a grid cell is an aerosol hotspot based on the following criteria: (1) grid value of OMI UV AI is greater than a high aerosol loading threshold value set here as AI of 1.0; (2) population 
density is higher than the neighboring grid cells; (3) grid cell is unique in terms of anthropogenic biome classification, topography, and/or meteorology (see Table 2). For example, although a lot of grid cells in the northwestern Mexico area satisfy the first criteria, we chose only those high aerosol cells which (a) may be affected by monsoon; (b) represent a relatively populated area that can be impacted by high aerosol loading; and (c) have distinct topographic features or anthrome classification. Our goal is to find aerosol hotspots representative of different aerosol environments. OMI UV AI is particularly useful for this purpose as it can locate smaller areas with high aerosol loading that cannot be easily detected using MODIS AOD. We note also that OMI UV AI has higher sensitivity to absorbing aerosols (e.g., black carbon and dust). Once a hotspot is identified, we re-grid OMI AI (using simple averaging) to match the $1^{\circ} \times 1^{\circ}$ resolution of the MODIS AOD that we are using for this study. We define the hotspot region to include the nearest grid cells surrounding the hotspot grid cell that has climatological maximum within \pm 2 standard deviations of the value of the hotspot grid cell. Finally, we group the hotspot regions into four main aerosol clusters, where each cluster exhibits a distinct characteristic of aerosol variability within the NAM region. Our four clusters include: dust, NAM alley, fire, and anthropogenic (see Figure 1 and Table 2 for locations of these hotspot regions).
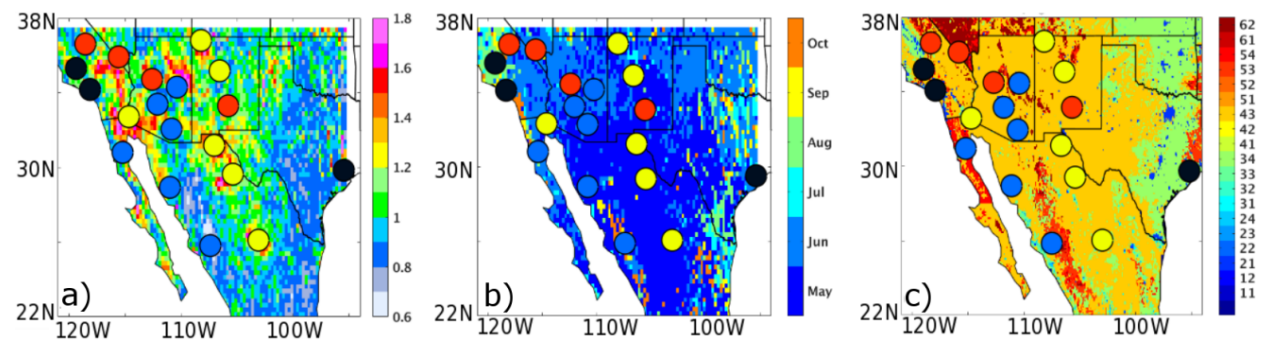

Figure 1. Maps of hotspot regions and aerosol clusters in NAM region. The three panels represent (a) the OMI UV AI climatological maximum across 2005-2014; (b) the month of climatological maximum; and (c) the anthropogenic biomes where different numbers on the colorbar indicate biome categories (11-12 are urban, 21-26 are villages, $31-35$ are croplands, $41-43$ are rangelands, $51-52$ are forests, and 61-63 are considered wildlands). Superimposed on all three plots are the locations of 20 hotspot regions (colored circles) with the color fill indicating the aerosol cluster they belong to (red: fire, yellow: dust, blue: NAM alley, and black: anthro).

Anomalies. We start our analysis by first calculating the decadal monthly mean across 2005-2014 and the corresponding monthly standardized anomalies for each 
year of OMI UV AI, MODIS AOD, MODIS fire counts and FRP, MODIS NDVI, MOPITT CO, and TRMM precipitation rate. These are calculated as follows:

$$
\begin{gathered}
\bar{x}_{m, n}=\frac{1}{N} \sum_{y=1}^{N}\left(x_{m, n, y}\right) \\
\sigma_{m, n}=\sqrt{\frac{\sum_{y=1}^{N}\left(x_{m, n, y}-\bar{x}_{m, n}\right)^{2}}{N-1}} \\
x_{m, n, y}^{\prime}=\frac{\left(x_{m, n, y}-\bar{x}_{m, n}\right)}{\sigma_{m, n}}
\end{gathered}
$$

where $\bar{x}_{m, n}$ is the 10-year average $(y=1$ to $N=10)$ of a certain quantity $\left(x_{m, n, y}\right)$ in each $1^{\circ} \times 1^{\circ}$ grid $(n)$ in our domain for a particular month $(m)$ (May to October) of a given year $(y)$. The standardized anomaly $\left(x_{m, n, y}^{\prime}\right)$ is calculated as the difference between $x_{m, n, y}$ and $\bar{x}_{m, n}$ normalized by the 10-year standard deviation $\left(\sigma_{m, n}\right)$. Positive (negative) anomalies for each grid indicate values higher (lower) than its mean. We standardized the anomalies to facilitate comparison with quantities having different units (similar to [25]).

Table 2. Hotspot sites and associated aerosol cluster information.

\begin{tabular}{lllll}
\hline Site No. & Site Name & Latitude (deg N) & Longitude (deg W) & Aerosol Cluster \\
\hline 1 & Mt. Whitney, CA & 36.557 & 118.50 & Fire \\
2 & Charleston Peak, CA & 36.29 & 115.69 & Fire \\
3 & Tucson, AZ & 32.22 & 110.93 & NAM alley \\
4 & Baja, CA & 30.98 & 115.38 & NAM alley \\
5 & Phoenix, AZ & 33.45 & 112.08 & NAM alley \\
6 & Yuma, AZ & 32.69 & 114.63 & Dust \\
7 & LA County, CA & 34.35 & 118.37 & Anthro \\
8 & Bakersfield, CA & 35.37 & 119.02 & Anthro \\
9 & Prescott, AZ & 34.54 & 112.46 & Fire \\
10 & Petrified Forest, AZ & 34.41 & 110.65 & NAM alley \\
11 & White Mountain, NM & 33.41 & 105.74 & Fire \\
12 & Farmington, NM & 36.73 & 108.22 & Dust \\
13 & Albuquerque, NM & 35.01 & 106.61 & Dust \\
14 & Ejido El Vergel, Mex & 31.20 & 106.59 & Dust \\
15 & Chihuahuan Desert, Mex & 29.52 & 105.48 & Dust \\
16 & Hermosillo, Mex & 29.07 & 110.97 & NAM alley \\
17 & Sierra Madre Occidental, Mex & 25.96 & 107.53 & NAM alley \\
18 & Sierra Madre Oriental, Mex & 26.12 & 103.10 & Dust \\
19 & Houston, TX & 29.74 & 95.36 & Anthro \\
20 & Waco, TX & 31.55 & 97.15 & Dust \\
\hline
\end{tabular}

\section{Results and Discussion}

In this section, we present our results on the spatial variability in aerosol loading and decadal trends of the changes in aerosol loading over different aerosol clusters 
in the NAM region. The temporal trend is examined for the period 2005-2014. We express these changes as standardized anomalies to account for the variability in aerosol loading. We can interpret these anomalies to represent local rather than regional to global changes. This attempts to minimize the influence of the changes in aerosol loading due to background or transported aerosols in the region. Our analysis focuses on intra-seasonal periods corresponding to pre-monsoon, monsoon, and post-monsoon phases since the aerosol patterns vary within the monsoon season. The overall trend is further elucidated by specific trends exhibited by each cluster (fire, anthropogenic, NAM alley, and dust). We note, however, that the only aerosol dataset that shows statistically significant decadal trends in aerosol anomalies is Terra/MODIS AOD. Other aerosol datasets, such as Aqua/MODIS and Aura/OMI which both have local overpass times in the early afternoon, show considerable interannual variations of aerosol anomalies with trends (not shown) that are not statistically significant. The inability of Aqua/MODIS and Aura/OMI to exhibit significant trends can be associated with its large variability possibly due to sampling issues, especially on observing aerosols at times when their abundance is most sensitive to cloud formation, boundary layer mixing, and convection in the region (e.g., [39]). The use of a longer period should provide sufficient samples for decadal trend analysis. For this study, however, we focus on the trends as can be inferred from Terra/MODIS.

\subsection{Spatial Variability}

The decadal mean AODs $\left(\bar{x}_{\text {Aod }}\right)$ for PRM, MON, and POM are shown in Figure 2. Three regions with the highest AOD patterns are apparent in these plots. The first region lies in northern California and Nevada bounded between $36^{\circ} \mathrm{N}$ and $38^{\circ} \mathrm{N}$, and $118^{\circ} \mathrm{W}$ and $115^{\circ} \mathrm{W}$. This region is mostly dominated by fires, especially during PRM and POM. Also, the fire cluster in this region shows the maximum mean AOD and maximum variability during PRM (Table 3). The second maxima in aerosol loading is seen in the southwestern Arizona and northern Mexico regions $\left(30^{\circ} \mathrm{N}, 33^{\circ} \mathrm{N}\right.$, and $\left.108^{\circ} \mathrm{W}, 41^{\circ} \mathrm{N}, 109^{\circ} \mathrm{W}\right)$. This region has been identified as an important source of dust by other studies (e.g., [19]). This region is characterized by haboob-type dust storms during MON and frontal dust storms during POM. The third source region is found between $25^{\circ} \mathrm{N}$ and $31^{\circ} \mathrm{N}$, and $105^{\circ} \mathrm{W}$ and $107^{\circ} \mathrm{W}$. This includes the Chihuahuan desert and Sierra Madre Oriental mountains. In all the three source regions, aerosol loading peaks in PRM and MON and decreases in POM (Table 3). In addition to these source regions, a minor region of activity, centered on LA county $\left(34.35^{\circ} \mathrm{N}\right.$ and $\left.118.37^{\circ} \mathrm{W}\right)$, is also observed. While this region is noted for its anthropogenic pollution, recent investigations have suggested the mixing of anthropogenic and biomass burning pollution in Southern California regions (e.g., [47]). 

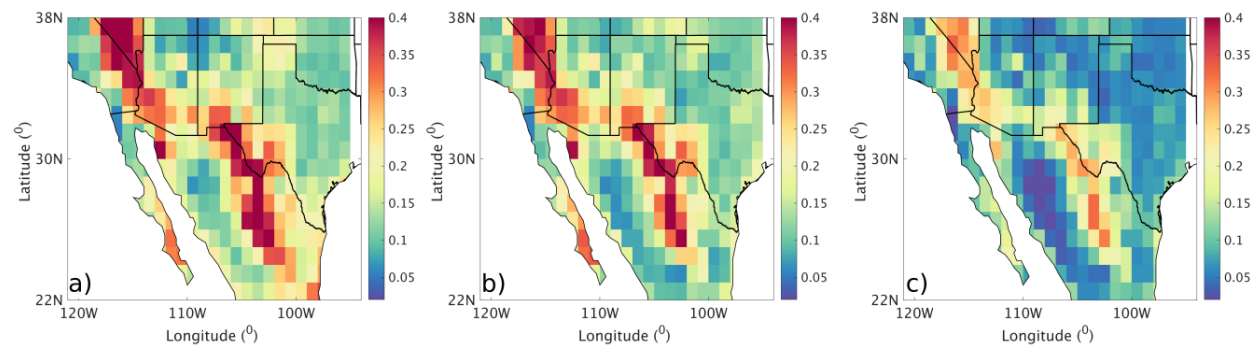

Figure 2. Maps of decadal mean (2005-2014) AOD from Terra-MODIS. The three panels represent (a) mean AOD during PRM; (b) MON; and (c) POM.

Table 3. Decadal mean and standard deviation of AOD for cluster regions.

\begin{tabular}{llll}
\hline Cluster & Season & Mean $\left(\overline{\boldsymbol{x}}_{\text {Aod }}\right)$ & Standard Deviation $\left(\boldsymbol{\sigma}_{A O D}\right)$ \\
\hline \multirow{4}{*}{ Fire } & PRM & $\mathbf{0 . 2 3}$ & $\mathbf{0 . 1 2}$ \\
& MON & $\mathbf{0 . 2 1}$ & $\mathbf{0 . 1 0}$ \\
\multirow{5}{*}{ NAM alley } & POM & 0.15 & 0.09 \\
& PRM & 0.19 & 0.08 \\
\multirow{5}{*}{ Dust } & POM & 0.18 & 0.09 \\
& PRM & 0.11 & 0.07 \\
\multirow{5}{*}{ anthro } & MON & 0.23 & 0.11 \\
& POM & 0.16 & 0.11 \\
& PRM & 0.13 & 0.08 \\
& MON & 0.14 & 0.02 \\
& POM & 0.10 & 0.02 \\
\hline
\end{tabular}

We also estimated the spatial correlation for these source regions by correlating AOD anomalies over a few selected places with AOD over the rest of the domain. The spatial patterns in correlation coefficients vary with season and cluster (Figure 3). The places selected in these source regions are: (1) Charleston peak (fire); (2) Tucson (NAM alley); (3) Hermosillo (dust); (4) Bakersfield (anthropogenic). Plots of correlation coefficients for these places are shown in Figure 3. For example, the peak of the regions of high correlation in AOD anomalies around Charleston is highest during PRM and decreases in MON and POM. In the case of Tucson and Hermosillo, regions of high correlation extend further into Mexico during MON and the higher correlations follow the monsoon track. We notice that, in the case of Bakersfield, regions of higher correlation extend into northern California in all the seasons. This suggests the potential similarity in frequency or magnitude of sources in between these regions. We also show spatial correlation matrices between selected aerosol hotspots (identified in Figure 1) in the supplementary material (Figure S1). 

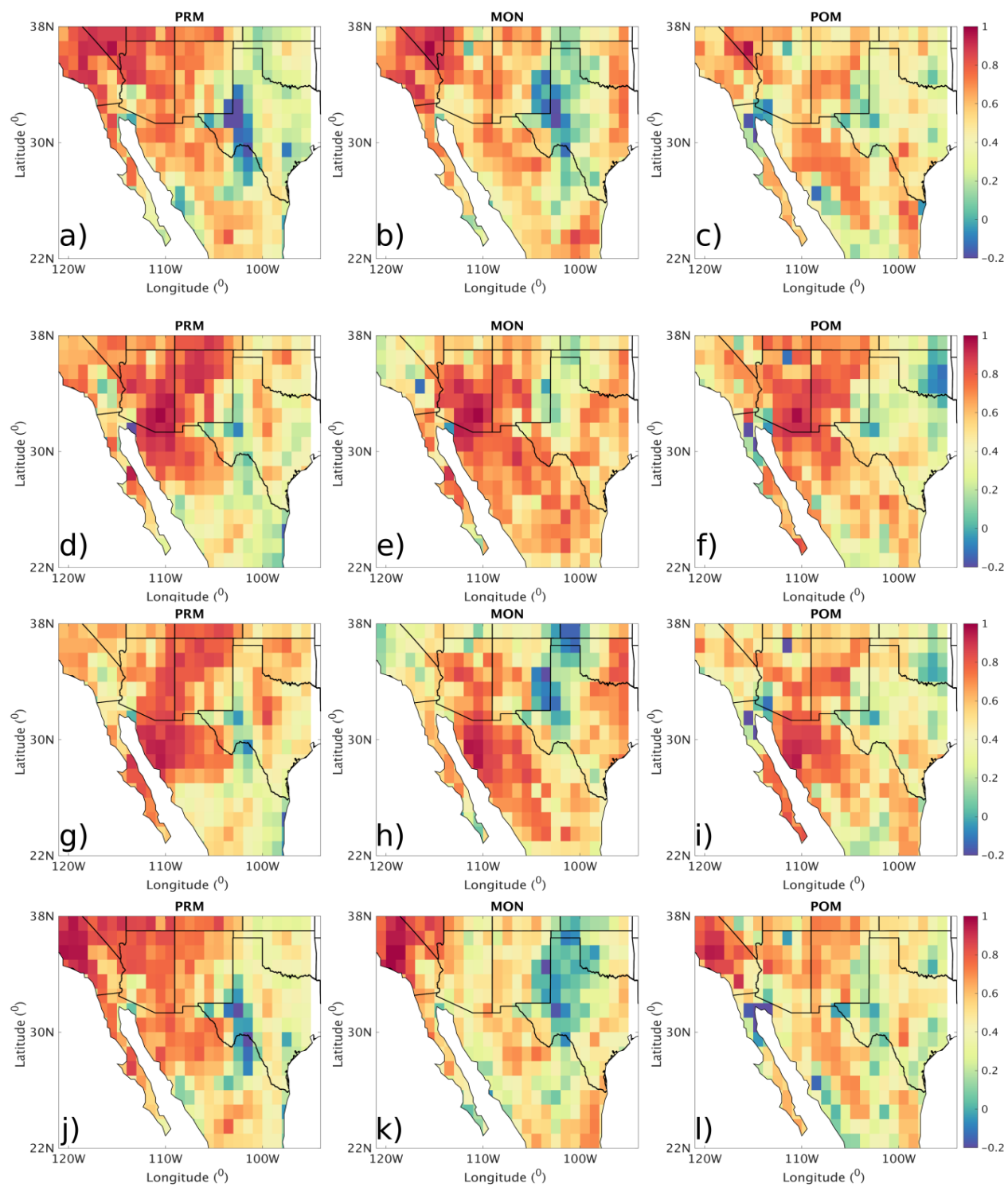

Figure 3. Correlation coefficient of AOD anomalies during PRM, MON, and POM. The panels $(\mathbf{a}-\mathbf{c})$ correspond to spatial correlation of all the grid points with respect to Charleston peak; (d-f) Tucson; (g-i) Hermosillo; (j-1) Bakersfield.

\subsection{Overall Aerosol Trend}

We show in Figure 4 the yearly statistics of anomalies $\left(x_{\text {aod }}^{\prime}\right)$ across the entire land region (region as shown in Figure 1) during PRM, MON, and POM. The statistics 
are summarized here using box plots where the top, central, and bottom edges of the box correspond to the 75th $\left(q_{3}\right)$, 50th (median), and 25th $\left(q_{1}\right)$ percentiles, respectively, while the whiskers correspond to \pm 1.5 of the interquartile range $\left(I Q R=q_{3}-q_{1}\right)$. The interannual variability in these AOD anomalies during all seasons mostly arises from natural aerosol sources such as dust and fires. We explain this further in the following sections for different aerosol clusters. The median of the anomalies during the latter part of the decade (2010-2014) is generally negative (and lower) compared to the earlier part of the decade (2005-2009), which is generally positive (and higher) (Figure 4). This is most evident for the monsoon season. This is also the case for the interquartile range, where a shrinking of the spread in $x_{a o d}^{\prime}$ is observed in the latter part of the decade. Although there appears to be an oscillating pattern across the decade, there is a clear decreasing trend in the median and spread pointing to relatively lower aerosol loading and variability in recent years. Here, we estimate the linear trends of $x_{a o d}^{\prime}$ to be -0.14 for PRM, -0.12 for MON, and -0.15 for POM. In other words, the yearly average decrease in $x_{\text {aod }}^{\prime}$ is greater than $10 \%$ for PRM, MON and POM, with larger decreases during PRM and POM.

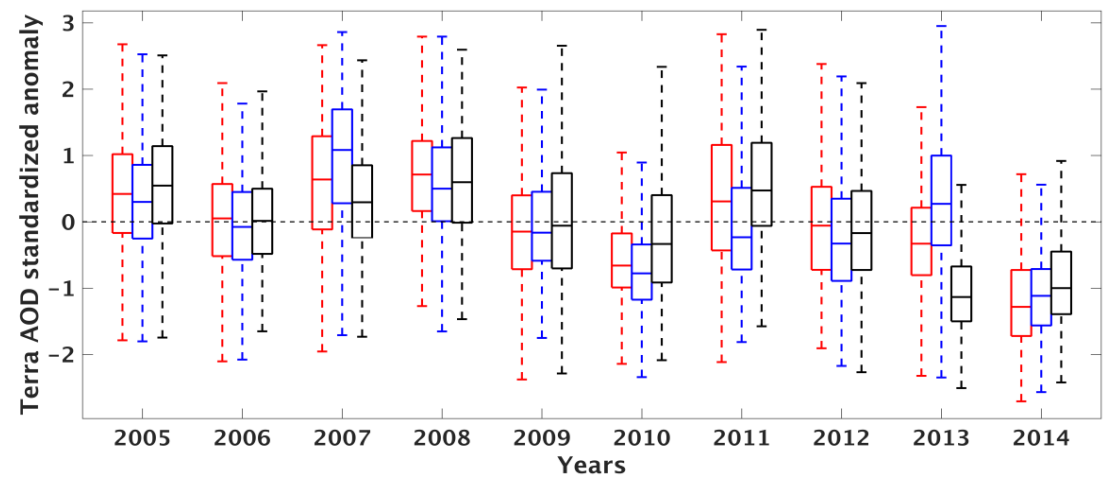

Figure 4. Box plots of yearly Terra/MODIS AOD standardized anomaly over the entire NAM region for the years 2005-2014. PRM (May-June), MON (July-August), and POM (September-October) periods are indicated in red, blue, and black. Whiskers represent 1.5 (IQR) above/below the upper/lower quartiles.

\subsection{Aerosol Trends Across Clusters}

We can further examine this decadal change by looking at the trends in the aerosol hotspot clusters. We expect more enhanced signatures of aerosol changes at these hotspot sites. In Figure 5 we show the decadal variations in $x_{\text {aod }}^{\prime}$ within fire, dust, NAM alley, and anthro clusters. We also summarize our estimates of linear trends in Table 4 . The interannual variability of $x_{a o d}^{\prime}$ is more pronounced in the fire, dust, and NAM alley, which are mostly driven by natural sources/sinks, 
than in the anthro cluster. This is expected as the "natural" aerosol clusters are mainly influenced by periodic atmospheric variations such as droughts and El Nino Southern Oscillation (ENSO) [48-50]. The fire and dust clusters, in particular, exhibit this periodic pattern, which can be linked to larger sources of aerosols (organic and black carbon in fires) under more arid and warmer conditions (PRM and POM). We also find a more pronounced decreasing trend (>20\%) in all clusters during PRM relative to the overall trend $(>10 \%)$. However, during $\mathrm{MON}$, the difference is less pronounced, except for the anthro cluster, which is consistently $>20 \%$ and appears to not be influenced by the monsoon. Except for the NAM alley cluster during the monsoon season, all the linear trends are significant at the $95 \%$ confidence interval (see Table 4).

The maximum decrease in $x_{a o d}^{\prime}$ can be seen in the fire cluster $(27 \%)$ followed by anthro (25\%). We also see a larger difference in $x_{a o d}^{\prime}$ statistics between the early and latter part of the decade in these two clusters. We see a more positive $x_{a o d}^{\prime}$ in 2005-2009 and a more negative $x_{\text {aod }}^{\prime}$ in 2010-2014 for all three periods (PRM, MON, and POM). This decrease is most evident in the anthro cluster, which shows an increase until 2007 and then a continuous drop (both median and spread) after 2007, regardless of the monsoon season. This is consistent with the findings of [24] and [25] (albeit from a different study period) of a decreasing trend in aerosol loading which they attributed to a decreasing trend in anthropogenic aerosols. Further, a recent study on extreme events in the southwestern US also pointed out a decrease in extreme elemental carbon events between 2003 and 2013 [51], which compliments the decreasing trend in AOD anomalies in the NAM region. Our results show that the $x_{a o d}^{\prime}$ trend for the entire NAM region is mainly tied to aerosol hotspots driven mostly by anthropogenic sources that have become weaker in recent years. This is also supported (not shown here) by the decreasing trend in combustion-related aerosol emissions based on recent emission inventories (e.g., [52]) and surface measurements of particulate matter ([53]).

Table 4. Linear trends in Terra/MODIS AOD anomalies for different aerosol clusters.

\begin{tabular}{llll}
\hline Clusters & PRM $(n=10)$ & MON $(n=10)$ & POM $(n=10)$ \\
\hline Fire & $-0.27(0.0002)$ & $-0.19(0.02)$ & $-0.21(0.01)$ \\
NAM alley & $-0.21(0.01)$ & $-0.15(0.11)$ & $-0.13(0.06)$ \\
Dust & $-0.21(0.007)$ & $-0.17(0.03)$ & $-0.17(0.02)$ \\
Anthro & $-0.25(0.0007)$ & $-0.21(0.009)$ & $-0.22(0.0001)$ \\
entire domain & $-0.14(0.02)$ & $-0.12(0.07)$ & $-0.15(0.01)$ \\
\hline
\end{tabular}

Note: values in the brackets refer to the $p$-value for the trends. 

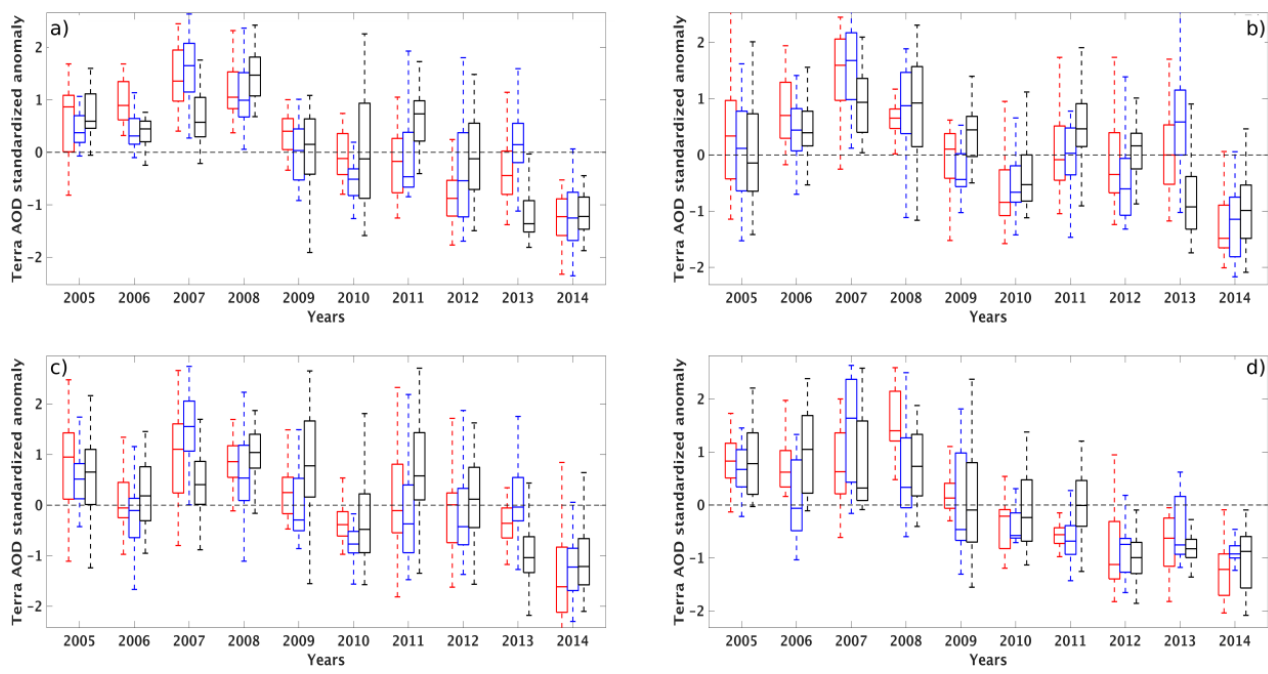

Figure 5. Similar to Figure 4 but for (a) fire; (b) NAM alley; (c) dust; and (d) anthro clusters.

In the NAM alley and dust cluster, we see a very similar trend, especially during PRM. This trend, however, is lower than in the fire and anthro clusters. Differences in the trends between NAM alley and dust clusters can be seen during MON and POM, where the trend in $x_{a o d}^{\prime}$ in the dust cluster is relatively higher than in the NAM alley (see Table 4). The oscillating pattern (as seen in [54]) is also less pronounced in the NAM alley than in the dust cluster. This difference can be attributed to an apparent modulation of $x_{a o d}^{\prime}$ in the NAM alley cluster by more frequent occurrences of convective dust storms or haboobs in this region than in the dust cluster. These storms are typically produced from isolated thunderstorms that merge to form cold pools during monsoon season. These cold pools result in severe downburst winds that lift massive quantities of dust off the surface ([8] and references therein). Based on the storm event database [55] over Arizona, we find that, although the trends in haboob-type dust storms across this study period are not statistically significant, there appears to be an increase in the frequency of dust storms over Arizona in most recent years, especially during the monsoon period. Despite the increase in rainfall (aerosol removal) during this period (which is common to both NAM alley and dust, albeit with a slight shift in timing), we infer that the increase in dust sources in the NAM alley obscures the decrease in the trend as seen in the dust cluster.

\subsection{Multivariate Correlations}

As noted earlier, we use a suite of satellite products (see Table 1) to corroborate the trends that we found in NAM and aerosol clusters. We present here the 
correlations between $x_{a o d}^{\prime}$ and the following anomalies across different aerosol clusters and monsoon periods: Aqua/MODIS AOD $\left(x_{\text {aod_aqua }}^{\prime}\right)$, Aura/OMI AI $\left(x_{a i}^{\prime}\right)$, Terra/MODIS FRP $\left(x_{f r p}^{\prime}\right), \mathrm{FC}\left(x_{f c}^{\prime}\right)$, and NDVI $\left(x_{n d v i}^{\prime}\right)$, Terra/MOPITT CO $\left(x_{c o}^{\prime}\right)$, and TRMM BEPR $\left(x_{\text {rain }}^{\prime}\right)$. These quantities provide unique first-order information on aerosol source/sink types. We note that the trends in $x_{a o d}^{\prime}$ can be influenced by several confounding factors other than those mentioned here, such as trans-Pacific transport of aerosols [56,57], mixing of aerosol emissions from within North America, removal efficiency, injection height, and frequency of emissions. Although we expect that the actual relationship between $x_{a o d}^{\prime}$ and these quantities may be nonlinear, we only examine the linear component of this relationship as a first-order approximation. More robust and quantitiative assessments of aerosol trends including source attribution require modeling of aerosol sources, transport, and sinks, which is beyond the scope of this study. We note, however, that our analysis can be made useful to show observational constraints of these trends.
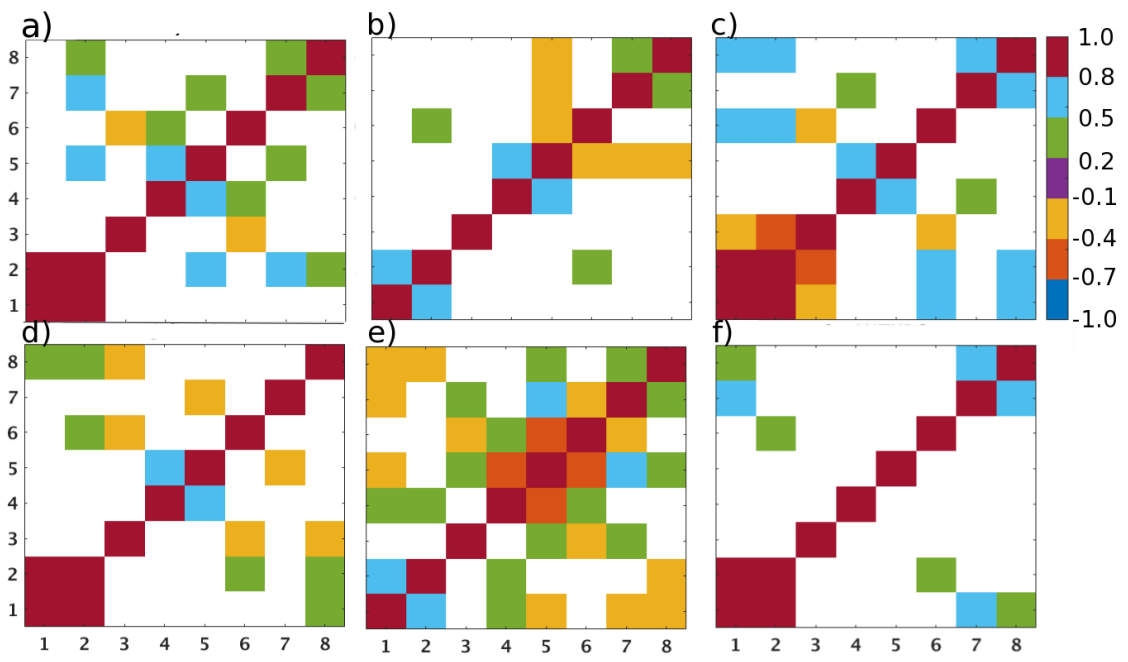

Figure 6. Anomaly correlation matrices between different aerosol-related quantities. The plots show correlation only during months that show significant correlation between the variables. The standardized anomalies considered here are the following: (1) Terra/MODIS AOD; (2) Aqua/MODIS AOD; (3) Aura/OMI UV AI; (4) Terra/MODIS FRP (in MW); (5) Terra/MODIS fire counts; (6) Terra/MOPITT CO (in ppbv); (7) Terra/MODIS NDVI; and (8) TRMM precipitation rate (in mm/day). Positive correlations are shown only if they are greater than 0.3 and significant at $90 \%$ confidence. Negative correlations are shown if they are significant at $90 \%$ confidence. The plots correspond to (a) fire cluster in May; (b) dust cluster in July; (c) anthro cluster in July; (d) NAM alley cluster in July; (e) dust cluster in September; and (f) anthro cluster in October. 
Our results are presented in Figure 6. The correlation coefficient indicates how closely the changes in aerosol loading mirror the changes in these quantities. Here, we focus our analysis on large and significant correlations between anomalies. While high correlation between $x_{a o d}^{\prime}$ and $x_{a o d \_a q u a}^{\prime}$ is expected, we find a lesser correlation in the dust cluster (Figure 6b,e). This may be due to differences in retrievals of aerosols in Terra and Aqua. In the fire cluster, however, $x_{a o d \_a q u a}^{\prime}$ is correlated with $x_{f c}^{\prime}$ and $x_{n d v i}^{\prime}(>0.5)$ in May (see Figure 6a). This is also seen from the decrease in the fire counts during June for the period 2005-2014 (Figure S2). The high correlation implies that most of the anomalies in aerosol loading in this cluster are directly tied to fire aerosols. As the fuel available from biomass increases, the probability of fires also increases, especially when the region tends to get drier in the period. This is usually the case for the summer fires in California [58]. There is also high correlation between $x_{f c}^{\prime}$ and $x_{f r p}^{\prime}$ and to a lesser extent between $x_{a i}^{\prime}$ and $x_{c o}^{\prime}$ which may indicate higher organic (and black) carbon aerosol emissions during mixtures of intense/less intense (flaming/smoldering) fires. This is fairly consistent with the previous studies that suggest that the interannual variability in natural AOD across the US is due to the variability of organic carbon emissions from droughts and biomass burning events $[24,50]$. However, this result needs to be supported by surface measurements and other datasets.

During MON, correlations in the NAM alley cluster show a strong positive relationship between $x_{a o d}^{\prime}, x_{\text {rain }}^{\prime}$, and $x_{\text {aod_aqua }}^{\prime}$ (July: Figure 6b,d) which is not apparent in the dust cluster. This implies that the anomalies in aerosol loading and precipitation rates are moving in the same direction in the NAM alley cluster during the monsoon season. Although, in general, we expect negative correlation between $x_{\text {rain }}^{\prime}$ and $x_{\text {aod }}^{\prime}$, this result suggests that the dust sources, especially from dust storms during the monsoon period, offset the aerosol removal due to rain. This can be contrasted with the dust cluster in September (Figure 6e) where there is a strong negative correlation between $x_{\text {rain }}^{\prime} x_{n d v i}^{\prime}$ (see Figure S3 for time series of NDVI) and $x_{\text {aod }}^{\prime}$, implying the stronger influence of precipitation in the absence of dust storms (and lesser vegetation) (e.g., [59,60]) and despite potentially more intense fires in this region (i.e., negative correlation between $x_{c o}^{\prime}, x_{f c}^{\prime}$ and $x_{f r p^{\prime}}^{\prime}$ positive correlation between $x_{a o d}^{\prime}$ and $x_{f r p}^{\prime}$, and between $x_{f r p}^{\prime}$ and $x_{a i}^{\prime}$ implies more intense flaming fires-less CO, high FRP at lower fire counts, and high black carbon (e.g., [61]).

In the anthro cluster during July, we find strong positive correlations between $x_{a o d}^{\prime}, x_{a o d \_a q u a}^{\prime}$ and $x_{c o}^{\prime}, x_{\text {rain }}^{\prime}$ and a negative correlation between $x_{a o d}^{\prime}$ and $x_{a i}^{\prime}$ (Figure 6c). Although UV AI has been noted for its sensitivity to both dust and black carbon (BC) aerosols, previous studies have also indicated that UV AI is more sensitive to dust aerosols than smoke or BC [19]. In the absence of a positive correlation with fire indicators (FRP and FC), it is evident that anomalies in aerosol loading in the anthro cluster are directly related to anthropogenic combustion. A positive correlation 
between $x_{\text {rain }}^{\prime}$ and $x_{\text {aod }}^{\prime}$ in MON (Figure 6c) and POM (Figure 6f) explains, to some extent, the lesser impact of precipitation to $x_{a o d}^{\prime}$ in the anthro cluster. This indicates that this region is mostly driven by anthropogenic $\left(x_{c o}^{\prime}\right)$ and biogenic $\left(x_{n d v i}^{\prime}\right)$ aerosol sources than precipitation (aerosol sinks) in October.

\subsection{AOD Sensitivity}

Here, we examine the difference in standardized anomalies between the maximum within the early (2005-2009) and latter parts of the decade (2010-2014). This is shown in Figure 7 for selected clusters and years with distinct sensitivities. We focus on contrasting the local maximum between these two segments of the decadal period to support the trend and correlation analysis previously discussed. Together, they provide information on the main factors of change in aerosol loading for different clusters. In Figure 7a we show the sensitivity of $x_{a o d}^{\prime}$ to $x_{f c}^{\prime}$ in the fire cluster, suggesting a similar shift in $x_{a o d}^{\prime}$ and $x_{f c}^{\prime}$ from positive to negative anomalies in the latter part of the decade (2010-2014) for all monsoon periods (also see Figure S2). This is consistent with the strong correlation between $x_{a o d \_a q u a}^{\prime}$ and $x_{f_{c}}^{\prime}$ shown in Figure 7. Although the largest decrease in $x_{f c}^{\prime}$ occurs in PRM, the maximum local sensitivity (steeper slope) in $x_{f c}^{\prime}$ with $x_{a o d}^{\prime}$ is observed during POM (i.e., large change in fire counts is tied with smaller change in AOD). This reveals that AOD is more associated with smoldering fire aerosols (more emissions) during POM than in PRM. This is also indicated in Figure $7 \mathrm{~d}$ where $x_{c o}^{\prime}$ shifts from a negative to positive anomaly during POM and shows high sensitivity with $x_{\text {aod }}^{\prime}$. However, the apparent decrease in fire counts shown in Figure 7a cannot be directly linked to a decrease in intensity or frequency of fires since this scatterplot is only a snapshot of this change.

During MON and POM in the NAM alley cluster, the $x_{a o d}^{\prime}$ decrease is associated with an increase in $x_{\text {rain }}^{\prime}$ (Figure $7 \mathrm{~b}$ and Figure S4). This sensitivity is mostly influenced by data in August (for MON) rather than July where dust storms may modulate this relationship (as we have seen in Figure 6d). Similarly, we find the decrease in $x_{a o d}^{\prime}$ is associated with the decrease in $x_{n d v i}^{\prime}$, with the highest sensitivity during POM in the dust cluster. The small change in AOD despite a large change in NDVI points to confounding factors such as fires, aerosol transport, and atmospheric moisture during POM in this region.

Finally, in the anthro cluster, we see a consistent shift in $x_{a o d}^{\prime}$ and $x_{c o}^{\prime}$ from positive to negative anomalies across the monsoon season (Figure 7e and Figure S5). This is very consistent with the trend and correlation results for this cluster. 

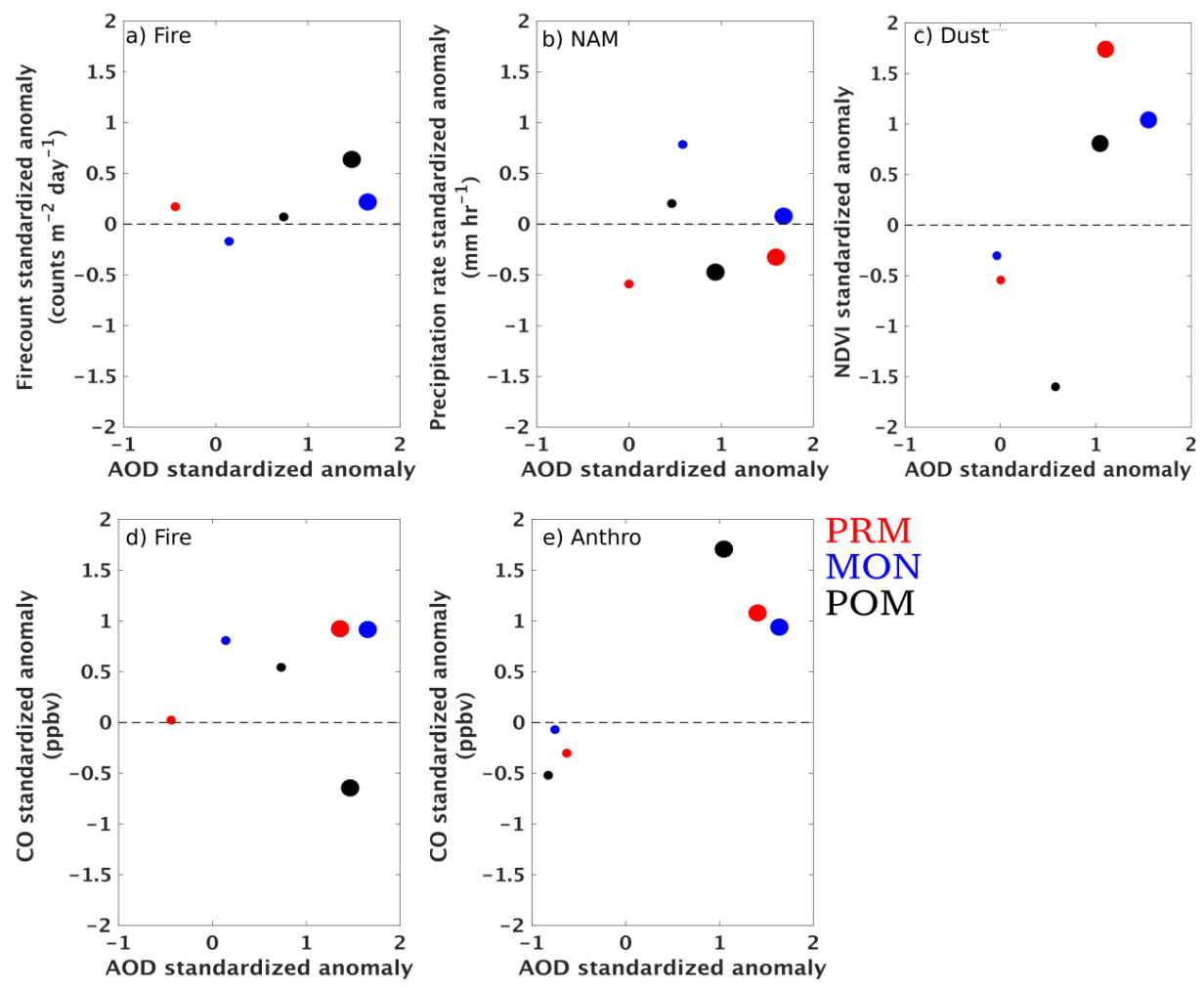

Figure 7. Scatterplots of standardized anomalies for selected clusters and monsoon periods. The filled circles represent local maxima values, with the larger and smaller circles corresponding to local maxima within 2005-2009 and 2010-2014, respectively. The colors represent different periods (red for PRM, blue for MON and black for $\mathrm{POM}$ ). The different plots correspond to AOD anomalies versus (a) fire counts for fire cluster; (b) precipitation for NAM alley cluster; (c) NDVI for dust cluster; (d) CO for fire cluster; and (e) CO for anthro cluster.

\subsection{Comparison between OMI UV AI and MODIS AOD during MON}

As noted already, the UV AI product is useful in aerosol hotspot identification because of its finer spatial resolution. Although UV AI did not show significant trends, previous studies have demonstrated the capability of UV AI in capturing smoke and dust aerosols. We present in Figure 8 the scatterplot between $x_{a i}^{\prime}$ and $x_{a o d}^{\prime}$ during MON to demonstrate the utility of UV AI for periods exhibiting large AOD variability. The different symbols represent different clusters with the smaller sizes indicating the last local maxima value in the time segment 2010-2014, and larger sizes indicating the first local maxima values in the time segment 2005-2010. Although these two quantities cannot be directly compared in terms of magnitude 
(hence the standardized anomalies), we expect these variables to move in the same direction. In contrast, $x_{a i}^{\prime}$ and $x_{a o d}^{\prime}$ show an inverse relationship during MON in all the source regions. One possible reason for the apparent increase in $x_{a i}^{\prime}$ during MON (and POM) could again be the difference in equatorial crossing times of the satellites. Convection builds up in the morning and winds increase in intensity in the afternoon over these regions during the monsoon season. Therefore, windblown dust captured by Aura/OMI UV AI is not seen in Terra/MODIS AOD. Other potential reasons for the discrepancies between Terra/AOD and OMI UV AI are differences in spatial resolution, sampling differences due to overpass times, retrieval characteristics and sensitivities (OMI UV AI retrieval is sensitive to black carbon and dust) (e.g., [62] and references therein). However, the results in Figure 8 show that OMI UV AI can provide additional constraints on aerosols in this region where mixed (confounding) processes are involved, making it challenging to infer aerosol trends. Future studies, based on the emission database and particulate matter concentrations, are required in the NAM region to provide a deeper understanding of the discrepancies in between these products.

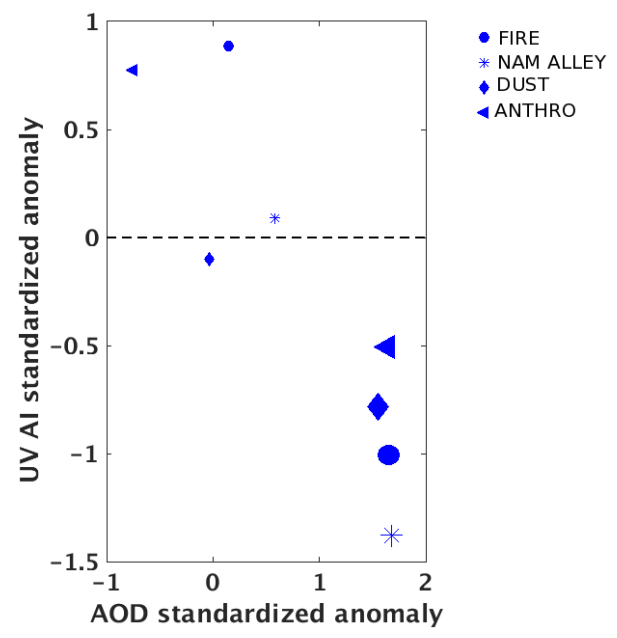

Figure 8. AOD versus UV AI standardized anomalies during the monsoon period (July-August). As in Figure 7, the larger (smaller) size symbols correspond to local maximum within 2005-2009 (2010-2014).

\section{Conclusions}

We investigate the spatial and temporal variability of aerosol loading in the NAM region using retrievals of AOD from Terra-MODIS for the years 2005-2014 during the monsoon season (May-October). We interpret the trends in AOD using other correlative information such as NDVI, CO, rainfall rate and active fire products. 
The decadal average of AOD over the study area shows that the maximum aerosol loading occurs during May and June (PRM) in active source regions. Major aerosol source regions are northern California (fires), southwestern Arizona and northern Mexico (dust), and southern California (anthropogenic). Monsoonal rainfall in Arizona and Mexico acts as a major sink during this period.

We identified four aerosol clusters in the NAM region and selected 21 aerosol hotspots in the entire study area. We conducted a series of statistical analyses for these four cluster regions (fires, dust, NAM alley, and anthropogenic) and for three periods of the monsoon season (pre-, monsoon, and post-monsoon). We correlated the anomalies of AOD to fire counts and FRP (for fires), CO (for combustion), NDVI (for dust) and precipitation rates (for aerosol removal) to explain the trends in aerosol loading. Our results show a significant interannual variability in AOD anomalies in fire, NAM alley and dust clusters which could be linked to climatic events. However, these speculations warrant further analyses of climatic products. The temporal trends in AOD anomaly exhibit a statistically significant decreasing trend in fire (19\%-27\%) and anthropogenic clusters $(17 \%-21 \%)$. The trends in the fire cluster can be attributed to the decreasing trend in the fire counts. The decreasing trends in the NAM alley $(13 \%-21 \%)$ and dust clusters although statistically significant, are influenced by compensating or nullifying processes such as haboobs, increased moisture during the monsoon period, rainfall, etc. While rainfall removes aerosol in this region, processes such as convective dust storm cause massive enhancements of dust loading.

In summary, this study highlights the need for augmenting and integrating observing systems of aerosols (and correlative measurements) in this region with high spatio-temporal resolution datasets. While anthropogenic aerosols show a clear statistically significant decreasing trend, trends in natural aerosol clusters such as dust and the NAM alley are still unclear due to the complex interplay between sources and sinks in this region. A next step would be to corroborate these results with surface observations of $\mathrm{PM}_{10}$ and $\mathrm{PM}_{2.5}$ and the emission database. We would also like to note that we have not fully considered the associated biases of the retrieval products, which is an important limitation of this study. We plan to investigate these limitations in our future work using chemical transport models and ground observations.

Supplementary Materials: The following are available online at http://www.mdpi.com/ 2073-4433/7/2/24/s1.

Figure S1: Correlation matrices for AOD anomalies all the hotspots between the months May-October. The hotspots considered here are: (1) Mt.Whitney, CA; (2) Charleston Peak, CA; (3) Tucson, AZ; (4) Baja, CA; (5) Phoenix, AZ; (6) Phoenix, AZ; (7) Yuma, AZ; (8) LA county, CA; (9) Bakersfield, CA; (10) Prescott, AZ; (11) Petrified forest, AZ; (12) White mountain, NM; (13) Farmington, NM; (14) Albuquerque, NM; (15) Ejido El Vergel, Mex; (16) Chihuahuan Desert, Mex; (17) Hermosillo, Mex; (18) Sierra Madre Occidental, 
Mex; (19) Sierra Madre Oriental, Mex; (20) Houston, TX; (21) Waco, TX. The panels correspond to (A) May; (B) June; (C) July; (D) August; (E) September; (F) October.

Figure S2: Box plots of Terra/MODIS firecount standardized anomaly over the fire cluster for the years 2005-2014 between the months May-October. Whiskers represent 1.5 (IQR) above/below the upper/lower quartiles. The panels correspond to (A) May; (B) June; (C) July; (D) August; (E) September; (F) October.

Figure S3: Box plots of Terra/MODIS NDVI standardized anomaly over the dust cluster for the years 2005-2014 between the months May-October. The panels correspond to (A) May; (B) June; (C) July; (D) August; (E) September; (F) October.

Figure S4: Box plots of TRMM rainfall rate standardized anomaly over the NAM alley for the years 2005-2014 between the months May-October. The panels correspond to (A) May; (B) June; (C) July; (D) August; (E) September; (F) October.

Figure S5: Box plots of MOPITT CO standardized anomaly over the anthropogenic cluster for the years 2005-2014 between the months May-October. The panels correspond to (A) May; (B) June; (C) July; (D) August; (E) September; (F) October.

Acknowledgments: We acknowledge all teams (MODIS, OMI, MOPITT, TRMM, Anthromes, GRUMP) and GIOVANNI for the data. The work performed at the Department of Atmospheric Sciences, University of Arizona and was funded by NASA Grant NNX13AK24G. We also acknowledge support from Grant 2 P42 ES04940-11 from the National Institute of Environmental Health Sciences (NIEHS) Superfund Research Program, NIH. The authors are grateful to the support of National Weather Service, Tucson, for their insightful discussions on North American Monsoon and dust storms. The authors also thank Wenfu Tang and Nick Dawson (Department of Atmospheric Sciences, University of Arizona) for discussions on the topic.

Author Contributions: A.R. downloaded and analyzed the data. A.R. wrote the manuscript and A.F.A. provided guidance and edited the manuscript. A.S. provided comments and suggestions for the manuscript.

Conflicts of Interest: The authors declare no conflicts of interest.

\section{References}

1. Hansen, J.; Sato, M.; Ruedy, R.; Lacis, A.; Oinas, V. Global warming in the twenty-first century: An alternative scenario. Proc. Natl. Acad. Sci. USA 2000, 97, 9875-9880.

2. Lau, K.M.; Tsay, S.C.; Hsu, C. The Joint Aerosol-Monsoon Experiment: A new challenge for monsoon climate research. Bull. Am. Meteorol. Soc. 2008, 89, 369-383.

3. Meehl, G.A.; Arblaster, J.M.; Collins, W.D. Effects of black carbon aerosols on the Indian monsoon. J. Clim. 2008, 21, 2869-2882.

4. Ramanathan, V.; Carmichael, G. Global and regional climate changes due to black carbon. Nat. Geosci. 2008, 1, 221-227.

5. Seager, R.; Ting, M.; Held, I.; Kushnir, Y.; Lu, J.; Vecchi, G.; Huang, H.P.; Harnik, N.; Leetmaa, A.; Lau, N.C.; et al. Model projections of an imminent transition to a more arid climate in southwestern North America. Science 2007, 316, 1181-1184. 
6. Westerling, A.L.; Bryant, B.P. Climate change and wildfire in California. Clim. Chang. 2008, 87, 231-249.

7. Dennison, P.E.; Brewer, S.C.; Arnold, J.D.; Moritz, M.A. Large wildfire trends in the western United States, 1984-2011. Geophys. Res. Lett. 2014, 41, 2928-2933.

8. Raman, A.; Arellano, A.F.; Brost, J.J. Revisiting haboobs in the southwestern United States: An observational case study of the 5 July 2011 Phoenix dust storm. Atmos. Environ. 2014, 89, 179-188.

9. Zhao, Z.; Pritchard, M.S.; Russell, L.M. Effects on precipitation, clouds, and temperature from long-range transport of idealized aerosol plumes in WRF-Chem simulations. J. Geophys. Res. Atmos. 2012, 117.

10. Crosbie, E.; Youn, J.S.; Balch, B.; Wonaschütz, A.; Shingler, T.; Wang, Z.; Conant, W.; Betterton, E.; Sorooshian, A. On the competition among aerosol number, size and composition in predicting CCN variability: A multi-annual field study in an urbanized desert. Atmos. Chem. Phys. 2015, 15, 6943-6958.

11. Sorooshian, A.; Shingler, T.; Harpold, A.; Feagles, C.W.; Meixner, T.; Brooks, P.D. Aerosol and precipitation chemistry in the southwestern United States: Spatio-temporal trends and interrelationships. Atmos. Chem. Phys. 2013, 13, 7361-7379.

12. Sprigg, W.A.; Nickovic, S.; Galgiani, J. N.; Pejanovic, G.; Petkovic, S.; Vujadinovic, M.; Vukovic, A.; Dacic, M.; DiBiase, S.; Prasad, A.; et al. Regional dust storm modeling for health services: the case of valley fever. Aeolian Res. 2014, 14, 53-73.

13. Gautam, R.; Hsu, N.C.; Lau, K.M.; Kafatos, M. Aerosol and rainfall variability over the Indian monsoon region: Distributions, trends and coupling. Ann. Geophys. 2009, 27, 3691-3703.

14. Krishnamurti, T.N.; Chakraborty, A.; Martin, A.; Lau, W.K.; Kim, K.M.; Sud, Y.; Walker, G. Impact of Arabian Sea pollution on the Bay of Bengal winter monsoon rains. J. Geophys. Res. Atmos. 2009, 114, D06213.

15. Douglas, M.W.; Maddox, R.A.; Howard, K.; Reyes, S. The Mexican monsoon. J. Clim. 1993, 6, 1665-1677.

16. Adams, D.K.; Comrie, A.C. The North American Monsoon. Bull. Am. Meteorol. Soc. 1997, 78, 2197-2213.

17. Gochis, D.J.; Shuttleworth, W.J.; Yang, Z.L. Sensitivity of the modeled North American monsoon regional climate to convective parameterization. Mon. Weather Rev. 2002, 130, 1282-1298.

18. Higgins, R.W.; Yao, Y.; Wang, X.L. Influence of the North American monsoon system on the US summer precipitation regime. J. Clim. 1997, 10, 2600-2622.

19. Sorooshian, A.; Wonaschütz, A.; Jarjour, E.G.; Hashimoto, B.I.; Schichtel, B.A.; Betterton, E.A. An aerosol climatology for a rapidly growing arid region (southern Arizona): Major aerosol species and remotely sensed aerosol properties. J. Geophys. Res. Atmos. 2011, 116, D19205.

20. Wise, E.K.; Comrie, A.C. Meteorologically adjusted urban air quality trends in the Southwestern United States. Atmos. Environ. 2005, 39, 2969-2980. 
21. American Lung Association Ranking. Available online: http://www.stateoftheair.org/ 2015/city-rankings/ most-polluted-cities.html (accessed on 13 November 2015).

22. Hand, J.L.; Schichtel, B.A.; Pitchford, M.; Malm, W.C.; Frank, N.H. Seasonal composition of remote and urban fine particulate matter in the United States. J. Geophys. Res. Atmos. 2012, 117.

23. Malm, W.C.; Schichtel, B.A.; Pitchford, M.L.; Ashbaugh, L.L.; Eldred, R.A. Spatial and monthly trends in speciated fine particle concentration in the United States. J. Geophys. Res. Atmos. 2004, 109.

24. Chin, M.; Diehl, T.; Tan, Q.; Prospero, J.M.; Kahn, R.A.; Remer, L.A.; Yu, H.; Sayer, A.M.; Bian, H.; Geogdzhayev, I.V.; et al. Multi-decadal aerosol variations from 1980 to 2009: A perspective from observations and a global model. Atmos. Chem. Phys. 2014, 14, 3657-3690.

25. Streets, D.G.; Yan, F.; Chin, M.; Diehl, T.; Mahowald, N.; Schultz, M.; Wild, M.; Wu, Y.; $\mathrm{Yu}, \mathrm{C}$. Anthropogenic and natural contributions to regional trends in aerosol optical depth, 1980-2006. J. Geophys. Res. Atmos. 2009, 114.

26. Prabhakar, G.; Betterton, E.A.; Conant, W.; Herman, B.M. Effect of Urban Growth on Aerosol Optical Depth-Tucson, Arizona, 35 Years Later. J. Appl. Meterol. Clim. 2014, 53, 1876-1885.

27. GIOVANNI. Available online: http://giovanni.gsfc.nasa.gov/giovanni (accessed on 13 November 2015).

28. Remer, L.A.; Kaufman, Y.J.; Tanré, D.; Mattoo, S.; Chu, D.A.; Martins, J.V. The MODIS aerosol algorithm, products, and validation. J. Atmos. Sci. 2005, 62, 947-973.

29. Levy, R.C.; Remer, L.A.; Kleidman, R.G.; Mattoo, S.; Ichoku, C.; Kahn, R.; Eck, T.F. Global evaluation of the Collection 5 MODIS dark-target aerosol products over land. Atmos. Chem. Phys. 2010, 10, 10399-10420.

30. Levy, R.C.; Remer, L.A.; Mattoo, S.; Vermote, E.F.; Kaufman, Y.J. Second-generation operational algorithm: Retrieval of aerosol properties over land from inversion of Moderate Resolution Imaging Spectroradiometer spectral reflectance. J. Geophys. Res. Atmos. 2007, 112.

31. Papadimas, C.D.; Hatzianastassiou, N.; Mihalopoulos, N.; Querol, X.; Vardavas, I. Spatial and temporal variability in aerosol properties over the Mediterranean basin based on 6-year (2000-2006) MODIS data. J. Geophys. Res. Atmos. 2008, 113.

32. Remer, L.A.; Kleidman, R.G.; Levy, R.C.; Kaufman, Y.J.; Tanre, D.; Mattoo, S. Global aerosol climatology from the MODIS satellite sensors. J. Geophys. Res. Atmos. 2008, 113.

33. MODIS-Terra Collection 5.1 Atmospheric Data. Available online: http://disc.sci.gsfc. nasa.gov/gesNews/ new_modis_version_5.1_data (accessed on 13 November 2015).

34. Hubanks, P.; King, M. NASA MODIS Moderate Resolution Imaging Spectroradiometer, Atmosphere Home Page [Fact Sheet]. 2007. Available online: http://modis-atmos.gsfc. nasa.gov/MOD04_L2/index.html (accessed on 13 November 2015).

35. Giglio, L.; Csiszar, I.; Justice, C.O. Global distribution and seasonality of active fires as observed with the Terra and Aqua Moderate Resolution Imaging Spectroradiometer (MODIS) sensors. J. Geophys. Res. Biogeosci. 2006, 111, G02016. 
36. Wooster, M.J.; Roberts, G.; Perry, G.L. W.; Kaufman, Y.J. Retrieval of biomass combustion rates and totals from fire radiative power observations: FRP derivation and calibration relationships between biomass consumption and fire radiative energy release. J. Geophys. Res. Atmos. 2005, 110, D24311.

37. Peng, S.; Piao, S.; Ciais, P.; Fang, J.; Wang, X. Change in winter snow depth and its impacts on vegetation in China. Glob. Chang. Biol. 2010, 16, 3004-3013.

38. Lunetta, R.S.; Knight, J.F.; Ediriwickrema, J.; Lyon, J.G.; Worthy, D. Land-cover change detection using multi-temporal MODIS NDVI data. Remote. Sen. Environ. 2006, 105, 142-154.

39. Torres, O.; Tanskanen, A.; Veihelmann, B.; Ahn, C.; Braak, R.; Bhartia, P.K.; Veefkind, P.; Levelt, P. Aerosols and surface UV products from Ozone Monitoring Instrument observations: An overview. J. Geophys. Res. Atmos. 2007, 112, D2108.

40. Huffman, G.J.; Adler, R.F.; Bolvin, D.T.; Gu, G.; Nelkin, E.J.; Bowman, K.P.; Hong, Y.; Stocker, E.F.; Wolff, D.B. The TRMM multisatellite precipitation analysis (TMPA): Quasi-global, multiyear, combined-sensor precipitation estimates at fine scales. J. Hydrometeorol. 2007, 8, 38-55.

41. Deeter, M.N.; Martínez-Alonso, S.; Edwards, D.P.; Emmons, L.K.; Gille, J.C.; Worden, H.M.; Pittman, J.E.; Daube, B.C.; Wofsy, S.C. Validation of MOPITT Version 5 thermal-infrared, near-infrared, and multispectral carbon monoxide profile retrievals for 2000-2011. J. Geophys. Res. Atmos. 2013, 118, 6710-6725.

42. Worden, H.M.; Deeter, M.N.; Edwards, D.P.; Gille, J.; Drummond, J.; Emmons, L.K.; Francis, G.; Martínez-Alonso, S. 13 years of MOPITT operations: Lessons from MOPITT retrieval algorithm development. Ann Geophys. 2014, 56.

43. Balk, D.; Montgomery, M.R.; McGranahan, G.; Kim, D.; Mara, V.; Todd, M.; Buettner, T.; Dorélien, A. Mapping urban settlements and the risks of climate change in Africa, Asia and South America. In Population Dynamics and Climate Change; Guzmán, J.M., Martine, G., McGranahan, G., Schensul, D., Tacoli, C., Eds.; International Institute for Environment and Development: London, UK, 2009.

44. SEDAC Data Portal: Population Density. Available online: http://sedac.ciesin.columbia. edu/data/set/ grump-v1-population-density (accessed on 13 November 2015).

45. Ellis, E.C.; Klein Goldewijk, K.; Siebert, S.; Lightman, D.; Ramankutty, N. Anthropogenic transformation of the biomes, 1700 to 2000. Global Ecol. Biogeogr. 2010, 19, 589-606.

46. SEDAC Data Portal: Anthropogenic Biomes. Available online: http://sedac.ciesin. columbia.edu/data/set/anthromes-anthropogenic-biomes-world-v2-1900 (accessed on 13 November 2015).

47. Singh, H.B.; Anderson, B.E.; Brune, W.H.; Cai, C.; Cohen, R.C.; Crawfold, J.H. Pollution influences on atmospheric composition and chemistry at high northern latitudes: Boreal and California forest fire emissions. Atmos. Environ. 2010, 44, 4553-4564.

48. Van der Werf, G.R.; Randerson, J.T.; Giglio, L.; Collatz, G.J.; Kasibhatla, P.S.; Arellano, A.F. Interannual variability in global biomass burning emissions from 1997 to 2004. Atmos. Chem. Phys. 2006, 6, 3423-3441. 
49. Marlier, M.E.; DeFries, R.S.; Voulgarakis, A.; Kinney, P.L.; Randerson, J.T.; Shindell, D.T.; Chen, Y.; Faluvegi, G. El Nino and health risks from landscape fire emissions in southeast Asia. Nat. Clim. Chang. 2013, 3, 131-136.

50. Wang, Y.; Xie, Y.; Cai, L.; Dong, W.; Zhang, Q.; Zhang, L. Impact of the 2011 Southern US Drought on Ground-Level Fine Aerosol Concentration in Summertime. J. Atmos. Sci. 2015, 72, 1075-1093.

51. Lopez, D.H.; Rabbani, M.R.; Crosbie, E.; Raman, A.; Arellano, A.F.; Sorooshian, A. Frequency and Character of Extreme Aerosol Events in the Southwestern United States: A Case Study Analysis in Arizona. Atmosphere 2016, 7, 1.

52. Emissions of Atmospheric Compounds and Compilation of Ancilliary Data. Available online: http:/ / eccad.sedoo.fr (accessed on 13 November 2015).

53. Air trends. Available online: http://www3.epa.gov/airtrends/pm.html (accessed on 13 November 2015).

54. Tong, D.Q.; Dan, M.; Wang, T. Long-term dust climatology in the western United States reconstructed from routine aerosol ground monitoring. Atmos. Chem. Phys. 2012, 12, 5189-5205.

55. Storm Events Database. Available online: https://www.ncdc.noaa.gov/stormevents (accessed on 13 November 2015).

56. Yu, H.; Remer, L.A.; Chin, M.; Bian, H.; Tan, Q.; Yuan, T.; Zhang, Y. Aerosols from overseas rival domestic emissions over North America. Science 2012, 337, 566-569.

57. Lin, M.; Fiore, A.M.; Horowitz, L.W.; Cooper, O.R.; Naik, V.; Holloway, J.; Johnson, B.J.; Middlebrook, A.M.; Oltmans, S.J.; Pollack, I.B.; et al. Transport of Asian ozone pollution into surface air over the western United States in spring. J. Geophys. Res. Atmos. 2012, 117, D00V07.

58. Jin, Y.; Randerson, J.T.; Faivre, N. Contrasting controls on wildland fires in Southern California during periods with and without Santa Ana winds. J. Geophys. Res Biogeosci. 2014, 119, 432-450.

59. Huang, M.; Tong, D.; Lee, P.; Pan, L.; Tang, Y.; Stajner, I.; Pierce, R.B.; MacQueen, J.; Wang, J. Toward enhanced capability for detecting and predicting dust events in the western United States: The Arizona case study. Atmos. Chem. Phys., 2015, 15, 12595-12610.

60. Moreno-Rodríguez, V.; Del Rio-Salas, R.; Adams, D.K.; Ochoa-Landin, L.; Zepeda, J.; Gómez-Alvarez, A.; Palafox-Reyes, J.; Meza-Figueroa, D. Historical trends and sources of TSP in a Sonoran desert city: Can the North America Monsoon enhance dust emissions? Atmos. Environ. 2015, 110, 111-121.

61. Kondo, Y.; Matsui, H.; Moteki, N.; Sahu, L.; Takegawa, N.; Kajino, M.; Zhao, Y.; Cubison, M.J.; Jimenez, J.L.; Vay, S.; et al. Emissions of black carbon, organic, and inorganic aerosols from biomass burning in North America and Asia in 2008. J. Geophys. Res. Atmos. 2011, 116.

62. OMI Version 4.2x Data Quality Document. Available online: http://disc.sci.gsfc. nasa.gov/Aura/additional/ documentation/Aura-MLS_DataQuality_v4-2x-rev1-0.pdf (accessed on 15 January 2016). 


\title{
Characterization of Fine Particulate Matter Emitted from the Resuspension of Road and Pavement Dust in the Metropolitan Area of São Paulo, Brazil
}

\author{
Ivan Gregorio Hetem and Maria de Fatima Andrade
}

\begin{abstract}
Many studies have been performed in order to characterize the sources of airborne particles in the Metropolitan Area of São Paulo (MASP), in Brazil. Those studies have been based on receptor modeling and most of the uncertainties in their results are related to the emission profile of the resuspended road dust particles. In this study, we analyzed the composition of resuspended road dust particles in different environments: local streets, paved roads inside traffic tunnels, and high traffic streets. We analyzed the samples to quantify the concentrations of trace elements and black carbon. On the basis of that analysis, we developed emission profiles of the resuspended road dust that are representative of the different types of urban pavement in the MASP. This study is important given the international efforts in improving emissions factors with local characteristics, mainly in South America and other regions for which there is a lack of related information. This work presents emission profiles derived from resuspended road dust samples that are representative of the different types of urban pavement in the Metropolitan Area of São Paulo.
\end{abstract}

Reprinted from Atmosphere. Cite as: Hetem, I.G.; de Fatima Andrade, M. Characterization of Fine Particulate Matter Emitted from the Resuspension of Road and Pavement Dust in the Metropolitan Area of São Paulo, Brazil. Atmosphere 2016, 7,31 .

\section{Introduction}

Airborne particles that originate specifically from vehicle emissions are known to cause a variety of deleterious health effects, such as cardiorespiratory diseases and intrauterine mortality $[1,2]$. The particles emitted by vehicles can originate from exhaust and non-exhaust emissions. Non-exhaust particles comprise those generated from brake and tire wear; road surface abrasion; and corrosion. The resuspended road dust identified in the source apportionment of the atmospheric aerosol is composed of particles originating from the abrasion of different pavements, including bare soil and asphalt. In urban areas, road surfaces are contaminated by the deposition of pollutants from anthropogenic sources, mainly vehicle emissions. The resuspension 
of urban road dust affects not only the concentration of particulate matter (PM) smaller than 10 microns $\left(\mathrm{PM}_{10}\right)$ but also that of fine particles-those smaller than 2.5 microns $\left(\mathrm{PM}_{2.5}\right)$. It has been estimated that urban road dust resuspension is responsible for $8 \%$ of the total $\mathrm{PM}_{2.5}$ concentration in the Metropolitan Area of São Paulo (MASP), in southeastern Brazil, [3]. Pant and Harrison [4] presented a review of road traffic emissions of particulate matter. The authors concluded that road traffic can make a significant contribution to airborne concentrations of particulate matter, and that the particles arise not only from engine exhaust but also from the abrasion of tires, of the road surface, and of brake components. The particles arising from dust emissions can be classified as urban dust, which is comprised of soluble inorganics, carbonaceous compounds, and inert species (including metals). Some compounds derive from the composition of the soil itself, whereas others are derived from the deposition on the soil of particles and gases emitted by anthropogenic sources. Abbasi [5] performed experiments simulating different tires and driving patterns, as well as testing the brake system, to determine the mass and composition of the particles produced. Although most of those particles are in the coarse fraction of particulate matter, the abrasion process can generate the fine fraction $\left(\mathrm{PM}_{2.5}\right)$. Studies performed in road tunnels in the MASP showed a large contribution of metals that are not known to be emitted during the exhaust process: $\mathrm{Ba}, \mathrm{Cd}$, and $\mathrm{Sb}$, from brake wear [6]; and $\mathrm{Zn}, \mathrm{Sr}, \mathrm{Co}$, and $\mathrm{W}$ from tire wear [7,8]. Studies have shown that a significant proportion of the coarse fraction of particulate matter is road dust produced by the vehicles traveling over paved or unpaved roads [9].

In the MASP, there has long been a need to quantify the contributions that vehicle emissions make to the total concentration of particulate matter, differentiating between that coming from exhaust and that generated by the mechanical process of road dust resuspension. It has been a challenge to determine the contribution of each of these sources to the $\mathrm{PM}_{2.5}$ concentration using trace-elements because of the difficulty in finding element characteristics of one specific source. Determining the contribution that vehicle emissions make to the concentration of particles in the atmosphere of the MASP has been the theme of many studies. Many authors have evaluated the role that urban sources play in determining the concentration of pollutants, mainly particulate matter. In studies applying multivariate analysis, specifically factor analysis, Ynoue and Andrade [10] and Sanchez-Ccoyllo et al. [11], considering the elements $\mathrm{Fe}, \mathrm{Al}, \mathrm{Si}$, and $\mathrm{Ti}$ as tracers of road dust, found that the proportion of resuspended road dust in the $\mathrm{PM}_{2.5}$ varied from $15 \%$ to $25 \%$. These trace elements are also emitted by other processes, such as the mechanical action of tires on road surfaces, evaporation, and fuel combustion. To distinguish between particles derived from exhaust and those derived from mechanical abrasion, we analyzed samples of urban road dust from inside and just outside road tunnels. These analyses were performed in the context of a more comprehensive project that had the objective of evaluating the emission factors 
of gaseous and particulate emissions by vehicles. In that project, the emission factors of pollutants were determined using measurements taken inside and outside road tunnels. Tunnel measurements were taken in order to characterize the true nature of the vehicle fleet in the MASP. In one tunnel study, Pérez-Martínez et al. [12] showed that the emission factors of regulated pollutants were higher than those presented in official reports (CETESB, 2014). The authors compared their data with those of analyses performed under the same conditions in 2004 [11,13]. The CO and nitrogen oxide $\left(\mathrm{NO}_{x}\right)$ emission factors reported by Pérez-Martínez et al. [12] for light-duty vehicles in 2011 were both significantly (2.2 times) lower than those reported by Martins et al. [13] for light-duty vehicles in 2004, whereas they were five times and 2.5 times lower for $\mathrm{CO}$ and $\mathrm{NO}_{x}$, respectively, from heavy-duty vehicles. Analyzing the number and mass size distribution of particles, Pérez-Martínez et al. [12] calculated the $\mathrm{PM}_{2.5}$ emission factor to be $20 \mathrm{mg} / \mathrm{km}$ for light-duty vehicles and $277 \mathrm{mg} / \mathrm{km}$ for heavy-duty vehicles, showing the great contribution of diesel to the emission of fine particles. The authors also showed that black carbon accounted for $40 \%$ of the $\mathrm{PM}_{2.5}$ diesel emissions, compared with 50\% in 2004 [13]. The decreases in the emission factors were due to the implementation of a program for controlling vehicle emissions in Brazil. Although the vehicle fleet in the MASP has increased from 4 million in 2004 to almost 8 million in 2011, total emissions have decreased [11-14]. Brito et al. [15] showed that the burning of diesel by heavy-duty vehicles produced particles that were more numerous and for which the mean geometric diameter was smaller than that reported for particles produced by light-duty vehicles: the average particle count found for light-duty vehicles was $73,000 \mathrm{~cm}^{-3}$ with an average diameter of $48 \mathrm{~nm}$, compared with $366,000 \mathrm{~cm}^{-3}$, with an average diameter of $39 \mathrm{~nm}$, for heavy-duty vehicles.

Quantification of the contributions that road dust and vehicle emissions make to the concentration of particulate matter is a major step in developing a road dust profile for the urban area of the MASP. The objective is to distinguish, within the $\mathrm{PM}_{2.5}$ fraction, between the metals and black carbon that originate from the exhaust process and those that originate from the resuspension of dust from paved roads. The same approach was applied by [16], who presented an analysis of the enrichment factors of atmospheric particulate pollutants at the roadside, calculating the contribution that each different source made to the total particle concentration.

In this study, we attempted to provide emission profiles for road dust contaminated by vehicle emissions in the MASP. We present the composition of particles collected on a local street near an air quality monitoring station, as well as inside and outside two road tunnels. These data are a rich resource for the characterization of urban dust profiles that are highly influenced by vehicle emissions. The road dust resuspension source profile will be used in receptor modeling studies in the MASP and can also be a reference for other urban areas that are particularly affected by vehicle emissions. 


\section{Material and Methods}

\subsection{Sample Collection}

Road dust samples were collected in bags at five different sites: inside and just outside the Jânio Quadros road tunnel (JQ_i and JQ_o, respectively; $23^{\circ} 35^{\prime}$ S, $46^{\circ} 41^{\prime} \mathrm{W}$ ), where only light-duty and small diesel utility vehicles are allowed to travel; inside and just outside the Rodoanel road tunnel (RA_i and RA_o, respectively; $23^{\circ} 27^{\prime} \mathrm{S}, 46^{\circ} 47^{\prime} \mathrm{W}$ ), which is part of the beltway running outside the main area of the city, with a significant contribution by heavy-duty vehicles, mainly trucks; and on a local street, traveled by a mix of light- and heavy-duty vehicles, with a significant contribution by buses, near the Institute of Astronomy, Geophysics, and Atmospheric Sciences (IAG; $23^{\circ} 33^{\prime} \mathrm{S}, 46^{\circ} 44^{\prime} \mathrm{W}$ ), which is on the main campus of the University of São Paulo, within the MASP.

Light-duty vehicles consist of those running on gasohol, ethanol, or diesel, with a gross weight of less than $3900 \mathrm{~kg}$. In Brazil, most of the light-duty vehicles run on gasohol and ethanol. Passenger cars are not allowed to use diesel as fuel. Heavy-duty vehicles in Brazil consist of diesel vehicles with a gross weight of more than $3900 \mathrm{~kg}$. The collection of samples inside and outside tunnels was part of a project to determine the emission factors of vehicles [12]. The JQ_i/JQ_o and RA_i/RA_o samples were collected in May and June 2011, respectively. Table 1 provides details of the sampling sites. Samples were collected by broom sweeping paved roads at five different spots, $10 \mathrm{~m}$ apart, collectively representing one site. The samples were collected in plastic bags, totaling $200 \mathrm{~g}$ of material per site.

Table 1. Characteristics of the roadways sampled.

\begin{tabular}{lccc}
\hline Characteristic & JQ & RA & IAG \\
\hline Location & $23^{\circ} 35^{\prime} \mathrm{S} ;$ & $23^{\circ} 27^{\prime} \mathrm{S} ;$ & $23^{\circ} 33^{\prime} \mathrm{S} ;$ \\
LDVs per hour, mean & $46^{\circ} 41^{\prime} \mathrm{W}$ & $46^{\circ} 47^{\prime} \mathrm{W}$ & $46^{\circ} 44^{\prime} \mathrm{W}$ \\
HDVs per hour, mean & 2356 & 1511 & 140 \\
Hierarchy & 18 & 634 & 32 \\
& Arterial & Freeway & Local \\
\hline
\end{tabular}

JQ, Jânio Quadros (road tunnel, inside and outside measurements); RA, Rodoanel (road tunnel, inside and outside measurements); IAG, Institute of Astronomy, Geophysics, and Atmospheric Sciences (local street); LDVs, light-duty vehicles; HDVs, heavy-duty vehicles.

\subsection{Sample Preparation}

The samples were stored in bags at $4{ }^{\circ} \mathrm{C}$ until use, and the material in each bag was manually sieved. The structure of the resuspension chamber and dichotomous sampler are illustrated in Figure 1, together with the protocol for the procedure. After being sieved, each sample was transferred to a resuspension chamber belonging to the 
São Paulo State Environmental Protection Agency. The chamber consists of a virtual impactor (dichotomous sampler; Sierra-Andersen, Smyrna, GA, USA), coupled to a stainless steel vat (Figure 1). The vat is cylindrical, with a diameter of $40 \mathrm{~cm}$ and a height of $100 \mathrm{~cm}$. The dichotomous sampler uses a $\mathrm{PM}_{10}$ inlet operating at $16.7 \mathrm{~L} / \mathrm{min}$ to provide the D50 particle size cutoff at 10 microns in diameter. The virtual impactor is located after the inlet, and there are two separate flow controllers that maintain the fine particle air flux at $15.0 \mathrm{~L} / \mathrm{min}$ and the coarse particle air flux at $1.6 \mathrm{~L} / \mathrm{min}$.

We inserted $100 \mathrm{mg}$ of the dust sample in the vat and then scattered it with filtered laboratory compressed air. Using laboratory procedures, we determined that, after 3 min of scattering, the volume within the vat was homogeneously filled with the dust particles. The dust was collected on 37-mm Teflon-membrane filters. Each filter was exposed to four resuspension cycles, and samples from each site were collected on four filters, corresponding to one sample for each resuspension cycle. Each filter sampled a total of $0.4 \mathrm{~m}^{3}$ of air. The elemental analysis was performed in the fine mode fraction $\left(\mathrm{PM}_{2.5}\right)$, which is the focus of this study.

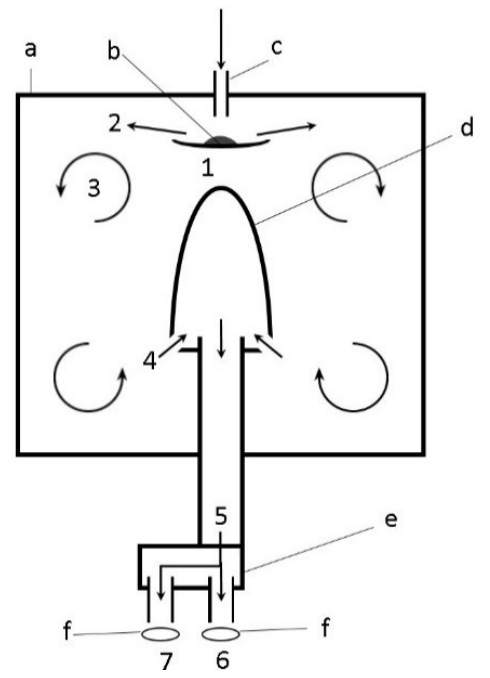

1) Structure

a) Steel vat

b) Sample

c) Compressed air injector

d) PM 10 inlet

e) Virtual impactor

f) Teflon filters

II) Procedure

1) Setting of Sample

2) Scattering

3) Homogenization

4) D50 cutoff

5) Flow separation

6) Coarse particle collection

7) Fine particle collection

Figure 1. Structure of the resuspension chamber, including the dichotomous sampler, and the procedure for collecting the material on the filters.

\section{Analysis}

The speciated emission inventory for road dust was constructed based on the material deposited on the filters. Although the particles may be shattered, the material deposited on the membrane filters retains the characteristics of the road dust that were generated by the abrasion of tires and wind on the road at the sampling site. 
For the evaluation of the mass deposited on the filters, we submitted the samples to gravimetric analysis using a balance with a resolution of $1 \mu \mathrm{g}$ (Mettler-Toledo MX-5). The filters were stored for $48 \mathrm{~h}$ in a temperature- and humidity-controlled environment $\left(24^{\circ} \mathrm{C} ; 40 \%\right.$ relative humidity). To quantify the accumulated mass, each filter was weighed before and after sampling. Measurements were corrected by subtracting the fluctuation observed on a set of blank filters. Ten blank filters were used for each set of samples. Concentrations of black carbon were evaluated through reflectance analysis of each filter with a smoke stain reflectometer (model 43; Diffusion Systems Ltd., London, UK). In brief, the filters were exposed to known amounts of visible light and a sensor registered the reflected fraction, thus inferring the superficial concentration of light-absorbing material, which can be classified as equivalent black carbon (BCe), as described by Bond et al. [17]. The material deposited on the filters was also submitted to analysis in an energy-dispersive X-ray fluorescence spectrometer (Epsilon 5; PANalytical, Almelo, The Netherlands). The spectrometer has three-dimensional, polarizing geometry with a supercritical water anode X-ray tube, up to 15 secondary targets as the polarizing scatterers. Details of the methodology for analyzing the trace-elements using this setup were described previously [15]. The typical detection limits for X-ray fluorescence analysis can be found elsewhere [18].

\section{Data Processing Analysis}

The profiles, composed of the mean concentrations for each element and for each sampling site, were defined as representing distinct sources. We then compared those sources with each other by calculating the coefficient of divergence (COD). The COD, as presented by [19], was defined as a measure of the data point spread between the road dust resuspension data sets and was applied to help determine whether any two profiles could be considered similar. The COD was calculated through the use of the following expression:

$$
\operatorname{COD}_{j k}=\sqrt{\frac{1}{p}} \sum_{i=1}^{p}\left(\frac{x_{i j}-x_{i k}}{x_{i j}+x_{i k}}\right)^{2}
$$

where $j$ and $k$ represent two source profiles; $p$ is the number of investigated components; and $x_{i j}$ and $x_{i k}$ represent the average mass concentrations of a compound $i$ for the profiles $j$ and $k$, respectively. The sources $j$ and $k$ can be considered similar if the $C O D_{j k}$ is close to zero and significantly different if the $C O D_{j k}$ is close to 1 . In this analysis, we considered the following compounds: $\mathrm{BCe}, \mathrm{Na}, \mathrm{Mg}, \mathrm{Al}, \mathrm{Si}, \mathrm{P}, \mathrm{S}, \mathrm{Cl}, \mathrm{K}$, $\mathrm{Ca}, \mathrm{Ti}, \mathrm{V}, \mathrm{Cr}, \mathrm{Mn}, \mathrm{Fe}, \mathrm{Ni}, \mathrm{Cu}, \mathrm{Zn}, \mathrm{Se}, \mathrm{Br}, \mathrm{Rb}, \mathrm{Sr}, \mathrm{Zr}, \mathrm{Cd}, \mathrm{Sb}$ and $\mathrm{Pb}$. Following the example set by [20], we excluded elements that accounted for less than $0.001 \%$ of the total mass, which resulted in the exclusion of As. According to those authors, 
a COD above the threshold of 0.3 indicates that the profiles were different from each other, whereas COD values that were mostly lower than or near 0.3 indicate that the profiles are mostly similar to each other and can be substituted for each other.

\section{Results and Discussion}

Table 2 shows the composition of the road dust samples collected from each of the sampling sites according to their order of importance in explaining the mass concentration. The trace elements $\mathrm{Al}_{2} \mathrm{O}_{3}, \mathrm{SiO}_{2}, \mathrm{CaCO}_{3}, \mathrm{~K}_{2} \mathrm{O}, \mathrm{Fe}_{2} \mathrm{O}_{3}$ and $\mathrm{ZnO}$ were considered to be oxides and collectively accounted for an average of $60 \%$ of the total mass, the main contributors being black carbon and the oxides $\mathrm{Al}, \mathrm{Si}, \mathrm{Fe}$ and $\mathrm{Ca}$. The tunnel profiles present some remarkable differences: at the JQ site, Fe was a major contributor, accounting for $10 \%$ of the mass concentration; and at the RA site, the contribution of BCe was ten times higher inside the tunnel than outside of it.

Some elements, such as $\mathrm{Na}$ and $\mathrm{P}$, were not considered to be in the oxidized state due to the uncertainties in the composition. The unexplained portion of the mass was due to components not measured in the samples, mainly water and organic compounds. In atmospheric samples, Ynoue and Andrade [10] demonstrated that organic material accounted for approximately $50 \%$ of the mass and black carbon (measured by optical reflectance) accounted for 30\%, and they also showed that sulfate is present in the form of $\left(\mathrm{NH}_{4}\right)_{2} \mathrm{SO}_{4}$. Those authors showed that, within the explained fraction, crustal elements were the most abundant species, and that oxides of aluminum and silicon explained more than $10 \%$ and $20 \%$ of the mass, respectively. The compounds $\mathrm{BCe}, \mathrm{Na},\left(\mathrm{NH}_{4}\right)_{2} \mathrm{SO}_{4}, \mathrm{Cl}, \mathrm{CuO}, \mathrm{ZnO}, \mathrm{As}, \mathrm{Se}, \mathrm{Br}, \mathrm{Sb}$ and $\mathrm{Pb}$ were more abundant in samples from inside the tunnels, whereas $\mathrm{MgO}, \mathrm{Al}_{2} \mathrm{O}_{3}, \mathrm{SiO}_{2}, \mathrm{TiO}$ and $\mathrm{Rb}$ were more abundant in samples from outside of the tunnels, as well as in those from the IAG site.

Compounds such as BCe and sulfur are characteristic of vehicle emissions in São Paulo, mainly from heavy-duty vehicles. This is similar to the situation in other parts of the world where policies to control diesel exhaust (mandatory use of diesel particle filters, for instance) have not been implemented. Strict regulations on diesel emissions, involving the mandatory use of retrofit systems, have been put in place only in recent years. One important program to control pollutant emissions from the light-duty fleet was the implementation of policies mandating the use of three-way catalysts, although the catalysts may also produce ammonia under reducing conditions. Typically, $\mathrm{Cu}$ and $\mathrm{Zn}$ are used as tracers of gasoline and ethanol emissions, respectively. Silva et al., [21], analyzing $\mathrm{PM}_{10}$ collected from ethanol and gasohol exhaust emissions in a dynamometer study performed with the fuels consumed in São Paulo, found different groups of metal elements in gasohol exhaust: $\mathrm{Mn}, \mathrm{Pt}, \mathrm{Ni}, \mathrm{Cu}, \mathrm{Pb}, \mathrm{Cr}$ and $\mathrm{Zn}$. Copper is added as an antioxidant, and $\mathrm{Zn}$ is 
associated with the use of additives and lubricants, as found also by Morawska and Zhang [22].

Within the road tunnels, vehicle emissions are the dominant sources. Some elements, such as $\mathrm{Al}, \mathrm{Si}$ and $\mathrm{Ti}$, are mostly associated with road dust composition. Outside-tunnel samples were rich in those elements, because the outdoor pavement is more likely to have a dust component, due to soil transport mechanisms, than is the pavement within the tunnels. It is of note that diesel fuel contains Si and emissions of Si are therefore also associated with the burning of diesel $[23,24]$.

Table 2. Composition of the road dust as a percentage of the total mass of the resuspended material collected on the Teflon filters in the resuspension chamber.

\begin{tabular}{|c|c|c|c|c|c|}
\hline Compound & IAG & RA_i & RA_o & $\overline{J Q \_i}$ & JQ_o \\
\hline $\mathrm{BCe}$ & $1.96 \pm 0.39$ & $10.75 \pm 4.3$ & $1.52 \pm 0.17$ & $3.99 \pm 1.99$ & $1.04 \pm 0.19$ \\
\hline $\mathrm{Al}_{2} \mathrm{O}_{3}$ & $32.22 \pm 6.44$ & $19.27 \pm 7.71$ & $32.57 \pm 3.58$ & $13.99 \pm 7$ & $32.76 \pm 5.9$ \\
\hline $\mathrm{SiO}_{2}$ & $33.41 \pm 6.68$ & $12.53 \pm 5.01$ & $30.68 \pm 3.37$ & $19.52 \pm 9.76$ & $29.36 \pm 5.29$ \\
\hline $\mathrm{Fe}_{2} \mathrm{O}_{3}$ & $4.54 \pm 0.91$ & $3.76 \pm 1.5$ & $6.18 \pm 0.68$ & $9.74 \pm 4.87$ & $4.75 \pm 0.85$ \\
\hline $\mathrm{K}_{2} \mathrm{O}$ & $2.15 \pm 0.43$ & $1.41 \pm 0.56$ & $1.78 \pm 0.2$ & $1.55 \pm 0.78$ & $1.32 \pm 0.24$ \\
\hline $\mathrm{CaCO}_{3}$ & $2.85 \pm 0.57$ & $6.27 \pm 2.51$ & $3.98 \pm 0.44$ & $8.78 \pm 4.39$ & $7.77 \pm 1.4$ \\
\hline $\mathrm{MgO}$ & $2.34 \pm 0.47$ & $1.11 \pm 0.44$ & $2.23 \pm 0.24$ & $1.97 \pm 0.98$ & $2.29 \pm 0.41$ \\
\hline$\left(\mathrm{NH}_{4}\right)_{2} \mathrm{SO}_{4}$ & $1.68 \pm 0.34$ & $10.99 \pm 4.4$ & $1.51 \pm 0.17$ & $6.77 \pm 3.39$ & $1.14 \pm 0.21$ \\
\hline $\mathrm{Na}$ & $0.34 \pm 0.07$ & $0.73 \pm 0.29$ & $0.27 \pm 0.03$ & $0.7 \pm 0.35$ & $0.2 \pm 0.04$ \\
\hline $\mathrm{TiO}$ & $0.61 \pm 0.12$ & $0.23 \pm 0.09$ & $0.57 \pm 0.06$ & $0.43 \pm 0.22$ & $0.65 \pm 0.12$ \\
\hline $\mathrm{ZnO}$ & $0.11 \pm 0.02$ & $0.44 \pm 0.18$ & $0.2 \pm 0.02$ & $0.73 \pm 0.36$ & $0.13 \pm 0.02$ \\
\hline $\mathrm{MnO}_{2}$ & $0.07 \pm 0.01$ & $0.11 \pm 0.05$ & $0.12 \pm 0.01$ & $0.16 \pm 0.08$ & $0.06 \pm 0.01$ \\
\hline $\mathrm{P}$ & $0.06 \pm 0.01$ & $0.26 \pm 0.11$ & $0.44 \pm 0.05$ & $0.14 \pm 0.07$ & $0.04 \pm 0.01$ \\
\hline $\mathrm{Cl}$ & $0.06 \pm 0.01$ & $0.74 \pm 0.3$ & $0.19 \pm 0.02$ & $0.53 \pm 0.27$ & $0.02 \pm 0.004$ \\
\hline $\mathrm{CuO}$ & $0.04 \pm 0.01$ & $0.23 \pm 0.09$ & $0.06 \pm 0.01$ & $0.28 \pm 0.14$ & $0.05 \pm 0.01$ \\
\hline $\mathrm{V}_{2} \mathrm{O}_{5}$ & $0.01 \pm 0.002$ & -- & $0.02 \pm 0.002$ & $0.02 \pm 0.011$ & $0.01 \pm 0.002$ \\
\hline $\mathrm{Cr}$ & -- & -- & $0.05 \pm 0.005$ & $0.04 \pm 0.018$ & $0.03 \pm 0.005$ \\
\hline $\mathrm{NiO}$ & $0.01 \pm 0.002$ & $0.04 \pm 0.016$ & $0.03 \pm 0.004$ & $0.01 \pm 0.006$ & $0.02 \pm 0.003$ \\
\hline $\mathrm{Rb}$ & $0.01 \pm 0.002$ & - & $0.01 \pm 0.001$ & $0.004 \pm 0.002$ & $0.01 \pm 0.001$ \\
\hline $\mathrm{Sr}$ & $0.02 \pm 0.004$ & $0.02 \pm 0.007$ & $0.03 \pm 0.004$ & $0.06 \pm 0.032$ & $0.02 \pm 0.004$ \\
\hline $\mathrm{Cd}$ & $0.01 \pm 0.001$ & -- & $0.01 \pm 0.001$ & $0.02 \pm 0.011$ & $0.01 \pm 0.001$ \\
\hline $\mathrm{Sb}$ & $0.01 \pm 0.003$ & $0.08 \pm 0.033$ & $0.01 \pm 0.001$ & $0.1 \pm 0.048$ & $0.02 \pm 0.004$ \\
\hline $\mathrm{Pb}$ & $0.03 \pm 0.005$ & $0.07 \pm 0.028$ & $0.01 \pm 0.001$ & $0.05 \pm 0.026$ & $0.01 \pm 0.002$ \\
\hline As & $0.001 \pm 0.0002$ & $0.01 \pm 0.0041$ & $0.002 \pm 0.0003$ & $0.009 \pm 0.0047$ & $0.0004 \pm 0.0001$ \\
\hline Se & $0.002 \pm 0.0005$ & $0.03 \pm 0.01$ & -- & $0.01 \pm 0.01$ & $0.003 \pm 0.001$ \\
\hline $\mathrm{Br}$ & $0.001 \pm 0.0003$ & $0.005 \pm 0.0021$ & $0.001 \pm 0.0001$ & $0.005 \pm 0.0027$ & $0.002 \pm 0.0003$ \\
\hline
\end{tabular}

Values are expressed as average \pm standard deviation. JQ_i and JQ_o, respectively, inside and outside the Jânio Quadros Tunnel (traveled primarily by light-duty and small, diesel-powered utility vehicles); RA_i and RA_o, respectively, inside and outside the Rodoanel Tunnel (traveled by a significant number of heavy-duty vehicles); and IAG, Institute of Astronomy, Geophysics, and Atmospheric Sciences (local street traveled by a mix of light- and heavy-duty vehicles).

The COD results, shown in Table 3, present another form of grouping to these profiles. To define which groups could be considered similar and could be substitutes for each other, being considered representative of the resuspended road dust, we used the classification system devised by Kong et al. [20]. According to the COD values, 
the pairings between the IAG, JQ_o, and RA_o sites show that they are similar, even though they were collected from roadways that were quite different (hierarchy-wise) and in distinct areas of the MASP. This result is relevant, given that different sampling sites show the same profile for pavement dust emission. Previous studies applying principal component analysis to fine particle concentrations in various capital cities in Brazil, including São Paulo [3], have shown that it is very difficult to distinguish among road dust resuspension, vehicle exhaust, and the different urban sources. This was the motivation to improve the knowledge of resuspended road dust in order to subtract this source from the database before performing the receptor model analysis.

Table 3. Coefficient of divergence values for the different profiles analyzed, considering a similarity threshold of 0.3 , as proposed by Kong et al. [20].

\begin{tabular}{ccccc}
\hline Site & RA_i & RA_o & JQ_i & JQ_o \\
\hline IAG & 0.557 & $\mathbf{0 . 3 4 0}$ & 0.533 & $\mathbf{0 . 3 0 5}$ \\
RA_i & X & 0.450 & $\mathbf{0 . 2 7 9}$ & 0.555 \\
RA_o & $X$ & $X$ & 0.414 & $\mathbf{0 . 3 2 1}$ \\
JQ_i & $X$ & $X$ & $X$ & 0.501 \\
\hline
\end{tabular}

Profile pairings with a COD below (or near) 0.3 are similar, whereas pairings with a COD above 0.3 are different. RA_i and RA_o, respectively, inside and outside the Rodoanel Tunnel (traveled by a significant number of heavy-duty vehicles); JQ_i and JQ_o, respectively, inside and outside the Jânio Quadros Tunnel (traveled primarily by light-duty and small, diesel-powered utility vehicles); and IAG, Institute of Astronomy, Geophysics, and Atmospheric Sciences (local street traveled by a mix of light- and heavy-duty vehicles).

The pairing of the profiles from inside the tunnels (RA_i and JQ_i) also resulted in COD values $<0.3$, and those profiles are therefore also similar, despite the differences shown in Table 2, and could represent the dust profile inside traffic tunnels in São Paulo [19,20,25,26]. The pairings of the respective in- and outside-tunnel profiles for both tunnels resulted in COD values $>0.3$. The concentrations of fine particles were higher in the in-tunnel samples than in the outside-tunnel samples, which also differed in composition, despite being collected from roadways traveled by the same types of vehicles. The profiles derived from in-tunnel samples are different from those derived from outside-tunnel samples, including those collected at the IAG site. The compounds presenting the highest contribution to the mass concentration were black carbon, $\mathrm{Ca}$ and $\mathrm{Pb}$. Pairings of the mismatched profiles RA_o-JQ_i and RA_i-JQ_o resulted in COD values $>0.3$, as did pairings of the IAG profile with both in-tunnel profiles, emphasizing how distinct they are. Considering the results obtained with the COD analysis, it was possible to establish a representative profile for road dust resuspension by using the average values for the JQ_o, RA_o and IAG samples, as presented in Table 4. 
In the samples collected from the three outside-tunnel sites, we observed high contributions of the crustal elements $\mathrm{Al}_{2} \mathrm{O}_{3}, \mathrm{SiO}_{2}, \mathrm{Fe}_{2} \mathrm{O}_{3}, \mathrm{~K}_{2} \mathrm{O}, \mathrm{CaCO}_{3}$ and $\mathrm{MgO}$ (Table 4). These compounds are also important tracers for tires, break wear and pavement abrasion which are the main sources of road dust $[8,9]$.

In future multivariate analyses, that profile will likely be used as a standard road dust resuspension profile for the MASP. The contribution of BCe to that profile was one order of magnitude less than was its contribution to the in-tunnel profile. The profile encountered here was compared with data found for other urban sites in the United States and Spain. In its emissions profile database (SPECIATE 4.0), the United States Environmental Protection Agency (EPA) presented a composite of five paved road dust profiles from the cities of San Antonio and Laredo, Texas (BVPVRD01, BVPVRD02, BVPVRD03, BVPVRD04 and BVPVRD05). Amato et al. [27] analyzed road dust at different sites in Spain and compiled a profile for an urban site in the city of Seville. Table 4 compares the urban profile for the MASP (derived from the present study), the EPA composite urban profile, and the urban profile for Seville. 
Table 4. Profile of resuspended road dust from the three outside-tunnel sites evaluated in Brazil, together with comparable profiles for cities in Texas and for the city of Seville, Spain.

\begin{tabular}{|c|c|c|c|}
\hline \multirow{3}{*}{ Compound/Element } & \multicolumn{3}{|c|}{ Urban Resuspended Road Dust Profiles } \\
\hline & Brazil & United States & Spain \\
\hline & $\%$ of Total $\mathrm{PM}_{2.5}$ & $\%$ of Total $\mathrm{PM}_{2.5}$ & $\%$ of Total $\mathrm{PM}_{2.5}$ \\
\hline $\mathrm{BCe}$ & $1.50 \pm 0.16$ & $2.54 \pm 0.28$ & $5.3 \pm 2.1$ \\
\hline $\mathrm{Si}$ & $10.37 \pm 1.72$ & $19.13 \pm 2.10$ & -- \\
\hline $\mathrm{Al}$ & $8.61 \pm 1.42$ & $6.81 \pm 0.75$ & $2.1 \pm 0.6$ \\
\hline $\mathrm{Fe}$ & $5.15 \pm 0.82$ & $2.63 \pm 0.29$ & $2.5 \pm 0.5$ \\
\hline $\mathrm{Ca}$ & $2.81 \pm 0.47$ & $30.11 \pm 3.30$ & $11.2 \pm 3.1$ \\
\hline $\mathrm{Mg}$ & $1.38 \pm 0.23$ & $0.54 \pm 0.06$ & $1.9 \pm 1.3$ \\
\hline $\mathrm{K}$ & $1.32 \pm 0.22$ & $1.42 \pm 0.16$ & $0.7 \pm 0.2$ \\
\hline $\mathrm{Ti}$ & $0.61 \pm 0.10$ & $0.31 \pm 0.03$ & $1.6 \pm 0.3$ \\
\hline S & $0.35 \pm 0.06$ & $3.04 \pm 0.33$ & $0.4 \pm 0.1$ \\
\hline $\mathrm{Na}$ & $0.27 \pm 0.04$ & $0.02 \pm 0.002$ & $0.3 \pm 0.1$ \\
\hline $\mathrm{P}$ & $0.18 \pm 0.02$ & $0.28 \pm 0.03$ & $0.1 \pm 0.0$ \\
\hline $\mathrm{Zn}$ & $0.14 \pm 0.02$ & $0.22 \pm 0.02$ & $1.3 \pm 0.3$ \\
\hline $\mathrm{Mn}$ & $0.07 \pm 0.01$ & $0.05 \pm 0.01$ & $0.37 \pm 0.13$ \\
\hline $\mathrm{Cu}$ & $0.04 \pm 0.01$ & $0.03 \pm 0.003$ & $0.77 \pm 0.27$ \\
\hline $\mathrm{Cl}$ & $0.04 \pm 0.005$ & $0.15 \pm 0.02$ & $0.9 \pm 1.5$ \\
\hline $\mathrm{Cr}$ & $0.03 \pm 0.002$ & $0.02 \pm 0.002$ & $0.145 \pm 0.061$ \\
\hline $\mathrm{Sr}$ & $0.0254 \pm 0.0040$ & $0.1537 \pm 0.0169$ & - \\
\hline $\mathrm{Pb}$ & $0.0158 \pm 0.0027$ & -- & -- \\
\hline $\mathrm{Sb}$ & $0.0118 \pm 0.0020$ & -- & -- \\
\hline $\mathrm{Ni}$ & $0.0113 \pm 0.0016$ & $0.0090 \pm 0.0010$ & -- \\
\hline $\mathrm{V}$ & $0.01 \pm 0.001$ & $0.02 \pm 0.002$ & $0.0057 \pm 0.001$ \\
\hline
\end{tabular}

Values are expressed as average \pm standard deviation. Data for the United States (five sites within the cities of San Antonio and Laredo, Texas) were obtained from the SPECIATE 4.0 emissions profile database of the United States Environmental Protection Agency. Data for Spain (an urban site in the city of Seville) were obtained from Amato et al. [27].

Comparing the profiles obtained for the fine fraction of resuspended road dust in urban areas, we can see that the MASP profile presents more aluminum and iron and less calcium than do the profiles obtained in the United States and Spain. The contributions of $\mathrm{S}, \mathrm{Na}, \mathrm{P}$ and $\mathrm{Mg}$ in the MASP were similar to those reported for Spain, whereas the contributions of $\mathrm{K}, \mathrm{Mn}, \mathrm{Zn}$ and $\mathrm{Cu}$ were similar to those reported for the United States. Although comparable for some species, the MASP sites had their own characteristics, demonstrating the need to expand the current road dust emission profiles. That would lead to better identification of this source and the evaluation of its contribution to the concentration of fine particulate matter. 


\section{Conclusions}

Here, we have presented the inorganic composition of the fine $\left(\mathrm{PM}_{2.5}\right)$ fraction of resuspended road dust. The road dust samples were collected from (inside and outside) two tunnels and from one suburban street. The profiles defined here are representative of the inorganic fraction of resuspended urban road dust in the MASP. Our study was part of an initiative to provide more information regarding emission profiles in cities in South America. These profiles are important to the estimation of the sources responsible for particulate air pollution. We demonstrated that the profiles inside the tunnels were different from those outside the tunnels, as well as from that defined for the local street. We also found that the outside-tunnel profiles were similar and can be substitutes for each other. Therefore, the average profile for resuspended urban dust might be useful in quantifying the contribution of other vehicle emissions, including exhaust emissions. The in-tunnel profiles will improve the identification of this source of fine particulate matter. The profile found here provides important information for the identification of compounds in the fine fraction of particulate matter originating from the resuspension of road dust and from vehicle exhaust. This profile can be used in receptor model studies to estimate the impact that resuspended dust has on the fine particle mass concentration. Our results can be valuable for studies analyzing the toxicity of the PM content of road dust in urban areas that are especially affected by vehicle emissions.

Acknowledgments: The authors thank the Research Program on Global Climate Change of the Fundação de Amparo à Pesquisa do Estado de São Paulo (FAPESP, São Paulo Research Foundation) for the financial support provided (Grant No. 2008/58104-8, NUANCE project). This study was also supported by the Brazilian agencies Conselho Nacional de Desenvolvimento Científico e Tecnológico (CNPq, National Council for Scientific and Technological Development) and Coordenação de Aperfeiçoamento de Pessoal de Nível Superior (CAPES, Office for the Advancement of Higher Education). We are also grateful to the São Paulo State Companhia de Tecnologia de Saneamento Ambiental (CETESB, Environmental Protection Agency) for providing logistical support.

Author Contributions: Ivan Hetem conceived the experimental setup and Maria de Fatima Andrade with Ivan Hetem analysed the data.

Conflicts of Interest: The authors declare no conflict of interest.

\section{References}

1. Saiki, M.; Santos, J.O.; Alves, E.R.; Genezini, F.A.; Marcelli, M.P.; Saldiva, P.H.N. Correlation study of air pollution and cardio-respiratory diseases through NAA of an atmospheric pollutant biomonitor. J. Radioanal. Nucl. Chem. 2014, 299, 773-779.

2. Loomis, D.; Pereira, L.A.; Conceição, G.M.; Arcas, R.M.; Kishi, H.S.; Singer, J.M.; Böhm, G.M.; Saldiva, P.H.N. Association Between Air Pollution and Intrauterine Mortality in São Paulo, Brazil. Environ. Health Perspect. 1998, 106, 325-329. 
3. Andrade, M.F.; Miranda, R.M.; Fornaro, A.; Kerr, A.; Oyama, B.; André, P.A.; Saldiva, P.H. Vehicle emissions and $\mathrm{PM}_{2.5}$ mass concentrations in six Brazilian cities. Air Qual. Atmos. Health 2012, 5, 79-88.

4. Pant, P.; Harrison, R. Estimation of the contribution of road traffic emissions to particulate matter concentration form field measurements: A review. Atmos. Environ. 2013, 77, 78-97.

5. Abbasi, S. Technical note: Experiences of studying airborne wear particles from road and rail transport. Aerosol Air Qual. Res. 2013, 13, 1161-1169.

6. Grieshop, A.P.; Lipsky, E.M.; Pekney, N.J.; Takahama, S.; Robinson, A.L. Fine particle emission factors from vehicles in a highway tunnel: Effects of fleet composition and season. Atmos. Environ. 2006, 40, 287-298.

7. Apeagyei, E.; Bank, M.S.; Spengler, J.D. Distribution of heavy metals in road dust along an urban-rural gradient in Massachusetts. Atmos. Environ. 2011, 45, 2310-2323.

8. Wåhlin, P.; Berkowicz, R.; Palmgren, F. Characterization of traffic-generated particulate matter in Copenhagen. Atmos. Environ. 2006, 40, 2151-2159.

9. Kupiainen, K.J.; Tervahattu, H.; Räisänen, M.; Mäkelä, T.; Aurela, M.; Hillamo, R. Size and composition of airborne particles from pavement wear, tires, and traction sanding. Environ. Sci. Technol. 2005, 39, 699-706.

10. Ynoue, R.Y.; Andrade, M.F. Size resolved mass balance of aerosol particles over São Paulo Metropolitan Area, Brazil. J. Aerosol Sci. Technol. 2004, 38, 52-62.

11. Sánchez-Ccoyllo, O.R.; Ynoue, R.Y.; Martins, L.D.; Astolfo, R.; Miranda, R.M.; Freitas, E.D.; Borges, A.S.; Fornaro, A.; Freitas, H.; Moreira, A.; et al. Vehicular particulate matter emissions in road tunnels in Sao Paulo, Brazil. Environ. Monit. Assess. 2009, 149, 241-249.

12. Pérez-Martínez, P.J.; Miranda, R.M.; Nogueira, T.; Guardani, M.L.; Fornaro, A.; Ynoue, R.; Andrade, M.F. Emission factors of air pollutants from vehicles measured inside road tunnels in São Paulo: Case study comparison. Int. J. Environ. Sci. Tech. 2014, 11, 2155-2168.

13. Martins, L.D.; Andrade, M.F.; Freitas, E.D.; Pretto, A.; Gatti, L.V.; Albuquerque, E.L.; Tomaz, E.; Guardani, M.L.; Martins, M.; Junior, O.M.A. Emission factors for gas-powered vehicles traveling through road tunnels in Sao Paulo, Brazil. Environ. Sci. Technol. 2006, 40, 6722-6729.

14. São Paulo State Companhia de Tecnologia de Saneamento Ambiental. Qualidade Do Ar No Estado De São Paulo 2013; Série Relatórios; CETESB Report: Sao Paulo, Brazil, 2014.

15. Brito, J.; Rizzo, L.V.; Herckes, P.; Vasconcellos, P.C.; Caumo, S.E.S.; Fornaro, A.; Ynoue, R.Y.; Artaxo, P.; Andrade, M.F. Physical-chemical characterisation of the particulate matter inside two road tunnels in the São Paulo Metropolitan Area. Atmos. Chem. Phys. 2013, 13, 12199-12213.

16. Amato, F.; Viana, M.; Richard, A.; Furger, M.; Prevot, A.S.H.; Nava, S.; Lucarelli, F.; Bukoviwecki, N.; Alastuey, A.; Reche, C.; et al. Size and time-resolved roadside enrichment of atmospheric particulate pollutants. Atmos. Chem. Phys. 2011, 11, 2917-2931. 
17. Bond, T.C.; Doherty, S.J.; Fahey, D.W.; Forster, P.M.; Berntsen, T.; DeAngelo, B.J.; Flanner, M.G.; Ghan, S.; Krächer, B.; Koch, D.; et al. Bounding the role of black carbon in the climate system: A scientific assessment. J. Geophys. Res. Atmos. 2013, 118, 5380-5552.

18. Spolnik, Z.; Belikov, K.; van Meel, K.; Adriaenssens, E.; de Roeck, F.; van Grieken, R. Optimization of measurement conditions of an energy dispersive $\mathrm{X}$-ray fluorescence spectrometer with high-energy polarized beam excitation for analysis of aerosol filters. Appl. Spectrosc. 2005, 59, 1465-1469.

19. Wongphatarakul, V.; Friedlander, S.K.; Pinto, J.P. A comparative study of $\mathrm{PM}_{2.5}$ ambient aerosol chemical databases. Environ. Sci. Technol. 1998, 32, 3926-3934.

20. Kong, S. Similarities and Differences in $\mathrm{PM}_{2.5}, \mathrm{PM}_{10}$ and TSP Chemical Profiles of Fugitive Dust Sources in a Coastal Oilfield City in China. Aerosol Air Qual. Res. 2014, 14.

21. Silva, M.F.; Assunção, J.V.; Andrade, M.F.; Pesquero, C.R. Characterization of Metal and Trace Element Contents of Particulate Matter $\left(\mathrm{PM}_{10}\right)$ Emitted by Vehicles Running on Brazilian Fuels-Hydrated Ethanol and Gasoline with 22\% of Anhydrous Ethanol. J. Toxicol. Environ. Health A 2010, 73, 901-909.

22. Morawska, L.; Zhang, J.J. Combustion sources of particles. 1. Health relevance and source signatures. Chemosphere 2002, 49, 1045-1058.

23. Taylor, D. Fuel Oils, Lubricant Oils and Their Treatment. In Introduction to Marine Engineering; Butterworth-Heinemann: Oxford, UK, 1996; pp. 150-162. Available online: http:/ /books.google.com/ (accessed on 20 October 2015).

24. Giordano, S.; Adamo, P.; Spagnuolo, V.; Vaglieco, B.M. Instrumental and bio-monitoring of heavy metal and nanoparticle emissions from diesel engine exhaust in controlled environment. J. Environ. Sci. 2010, 22, 1357-1363.

25. Feng, Y.C.; Xue, Y.H.; Chen, X.H.; Wu, J.H.; Zhu, T.; Bai, Z.P. Source apportionment of Ambient Total Suspended Particulates and Coarse Particulate Matter in Urban Areas of Jiaozuo, China. J. Air Waste Manag. Assoc. 2007, 57, 561-575.

26. Kong, S.F.; Shi, J.W.; Lu, B.; Qiu, W.G.; Zhang, B.S.; Peng, Y.; Zhang, B.W.; Bai, Z.P. Characteristic of PAHs with $\mathrm{PM}_{10}$ Fraction for Ashes from Coke Production, Iron Smelt, Heating Station and Power Plant Stacks in Liaoning Province, China. Atmos. Environ. 2011, 45, 3777-3785.

27. Amato, F.; Alastuey, A.; de la Rosa, J.; Gonzalez Castanedo, Y.; Sánchez de la Campa, A.M.; Pandolfi, M.; Lozano, A.; Contreras González, J.; Querol, X. Trends of road dust emissions contributions on ambient air particulate levels at rural, urban and industrial sites in southern Spain. Atmos. Chem. Phys. 2014, 14, 3533-3544. 
MDPI AG

St. Alban-Anlage 66

4052 Basel, Switzerland

Tel. +41616837734

Fax +41 613028918

http://www.mdpi.com

Atmosphere Editorial Office

E-mail: atmosphere@mdpi.com

http://www.mdpi.com/journal/atmosphere

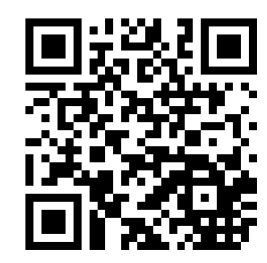



MDPI AG

St. Alban-Anlage 66

4052 Basel

Switzerland

Tel: +41 616837734

Fax: +41 613028918 